\title{
International Study of Aluminum Impacts on Crystallization in U.S. High Level Waste Glass
}

K. M. Fox, D. K. Peeler, T. B. Edwards, D. R. Best, I. A. Reamer, R. J. Workman and J.C. Marra

Pacific Northwest National Laboratory

B. J. Riley, J. D. Vienna, J. V. Crum, J. Matyas, A. B. Edmondson, J. B. Lang, N. M. Ibarra and A. Fluegel

V.G. Khlopin Radium Institute

A. Aloy, A.V. Trofimenko and R. Soshnikov

September 2008

Environmental \& Chemical Process Technology Savannah River National Laboratory Aiken, SC 29808

This document was prepared in conjunction with work accomplished under Contract No. DE-AC09-08SR22470 with the U.S. Department of Energy. 
SRNS-STI-2008-00057

Revision 0

\section{DISCLAIMER}

This work was prepared under an agreement with and funded by the U.S. Government. Neither the U.S. Government or its employees, nor any of its contractors, subcontractors or their employees, makes any express or implied: 1 . warranty or assumes any legal liability for the accuracy, completeness, or for the use or results of such use of any information, product, or process disclosed; or 2. representation that such use or results of such use would not infringe privately owned rights; or 3. endorsement or recommendation of any specifically identified commercial product, process, or service. Any views and opinions of authors expressed in this work do not necessarily state or reflect those of the United States Government, or its contractors, or subcontractors.

This document was prepared in conjunction with work accomplished under Contract No. DE-AC09-08SR22470 with the U.S. Department of Energy. 
Keywords: waste glass formulation, crystallization, aluminum retention

Retention: permanent

\section{International Study of Aluminum Impacts on Crystallization in U.S. High Level Waste Glass}

K. M. Fox, D. K. Peeler, T. B. Edwards, D. R. Best, I. A. Reamer, R. J. Workman and J.C. Marra

Pacific Northwest National Laboratory

B. J. Riley, J. D. Vienna, J. V. Crum, J. Matyas, A. B. Edmondson, J. B. Lang, N. M. Ibarra and A. Fluegel

V.G. Khlopin Radium Institute

A. Aloy, A.V. Trofimenko and R. Soshnikov

September 2008

Environmental \& Chemical Process Technology Savannah River National Laboratory Aiken, SC 29808

This document was prepared in conjunction with work accomplished under Contract No. DE-AC09-08SR22470 with the U.S. Department of Energy. 


\section{EXECUTIVE SUMMARY}

The objective of this task was to develop glass formulations for (Department of Energy) DOE waste streams with high aluminum concentrations to avoid nepheline formation while maintaining or meeting waste loading and/or waste throughput expectations as well as satisfying critical process and product performance related constraints. Liquidus temperatures and crystallization behavior were carefully characterized to support model development for higher waste loading glasses. The experimental work, characterization, and data interpretation necessary to meet these objectives were performed among three partnering laboratories: the V.G. Khlopin Radium Institute (KRI), Pacific Northwest National Laboratory (PNNL) and Savannah River National Laboratory (SRNL).

Projected glass compositional regions that bound anticipated Defense Waste Processing Facility (DWPF) and Hanford high level waste (HLW) glass regions of interest were developed and used to generate glass compositions of interest for meeting the objectives of this study. A thorough statistical analysis was employed to allow for a wide range of waste glass compositions to be examined while minimizing the number of glasses that had to be fabricated and characterized in the laboratory. The glass compositions were divided into two sets, with 45 in the test matrix investigated by the U.S. laboratories and 30 in the test matrix investigated by KRI. Fabrication and characterization of the US and KRI-series glasses were generally handled separately. This report focuses mainly on the US-series glasses.

Glasses were fabricated and characterized by SRNL and PNNL. Crystalline phases were identified by X-ray diffraction (XRD) in the quenched and canister centerline cooled (CCC) glasses and were generally iron oxides and spinels, which are not expected to impact durability of the glass. Nepheline was detected in five of the glasses after the CCC heat treatment.

Chemical composition measurements for each of the glasses were conducted following an analytical plan. A review of the individual oxides for each glass revealed that there were no errors in batching significant enough to impact the outcome of the study. A comparison of the measured compositions of the replicates indicated an acceptable degree of repeatability as the percent differences for most of the oxides were less than $5 \%$ and percent differences for all of the oxides were less than $10 \mathrm{wt} \%$.

Chemical durability was measured using the Product Consistency Test (PCT). All but two of the study glasses had normalized leachate for boron (NL [B]) values that were well below that of the Environmental Assessment (EA) reference glass. The two highest NL [B] values were for the CCC versions of glasses US-18 and US-27 (10.498 g/L and $15.962 \mathrm{~g} / \mathrm{L}$, respectively). Nepheline crystallization was identified by qualitative XRD in five of the US-series glasses. Each of these five glasses (US-18, US-26, US-27, US-37 and US-43) showed a significant increase in NL [B] values after the CCC heat treatment. This reduction in durability can be attributed to the formation of nepheline during the slow cooling cycle and the removal of glass formers from the residual glass network.

The liquidus temperature $\left(\mathrm{T}_{\mathrm{L}}\right)$ of each glass in the study was determined by both optical microscopy and XRD methods. The correlation coefficient of the measured XRD $T_{L}$ data versus the measured optical $T_{L}$ data was very good $\left(R^{2}=0.9469\right)$. Aside from a few outliers, the two datasets aligned very well across the entire temperature range $\left(829^{\circ} \mathrm{C}\right.$ to $1312^{\circ} \mathrm{C}$ for optical data and $813^{\circ} \mathrm{C}$ to $1310^{\circ} \mathrm{C}$ for XRD crystal fraction data). The data also correlated well with the predictions of a PNNL $\mathrm{T}_{\mathrm{L}}$ model. The correlation between the measured and calculated data had 
a higher degree of merit for the XRD crystal fraction data than for the optical data (higher $\mathrm{R}^{2}$ value of 0.9089 versus 0.8970 for the optical data).

The SEM-EDS analysis of select samples revealed the presence of undissolved $\mathrm{RuO}_{2}$ in all glasses due to the low solubility of $\mathrm{RuO}_{2}$ in borosilicate glass. These particles tended to form agglomerates with varying sizes and shapes that were located close to the bottom of crucibles.

The results of this study provide further insight into the ability of borosilicate waste glass to incorporate increased $(>16 \mathrm{wt} \%)$ concentrations of aluminum. The glass composition and properties data will be incorporated into a database of glass composition-property relationships (ComPro) to support further optimization of waste glass compositions at DOE sites. 


\section{TABLE OF CONTENTS}

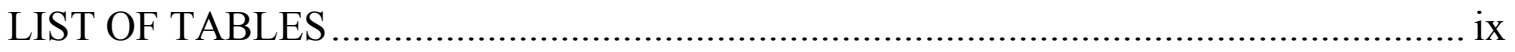

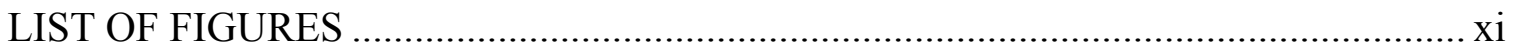

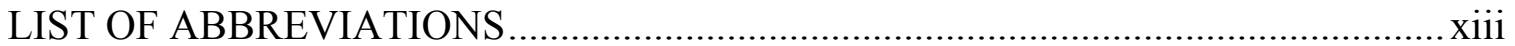

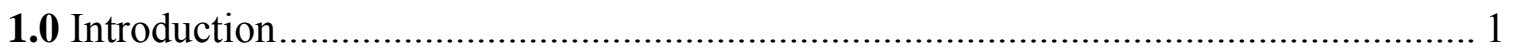

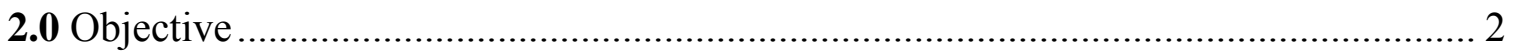

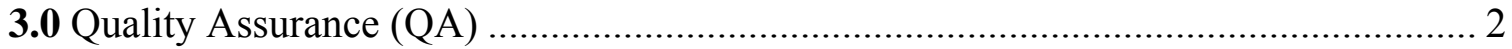

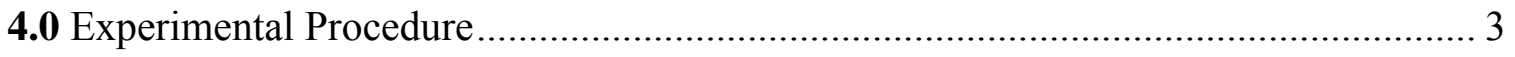

4.1 Selection of Study Glass Compositions............................................................. 3

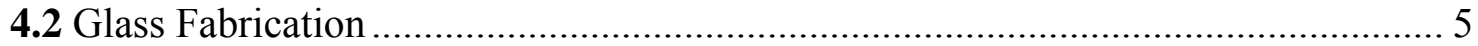

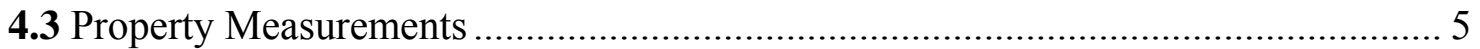

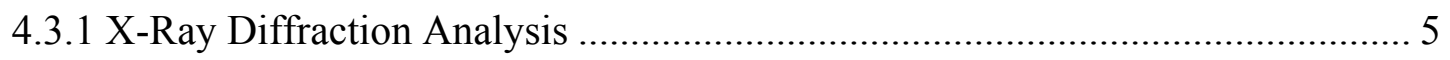

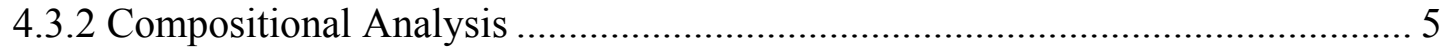

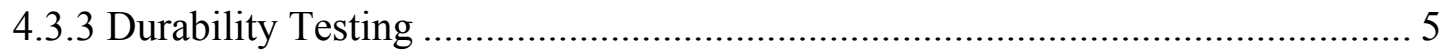

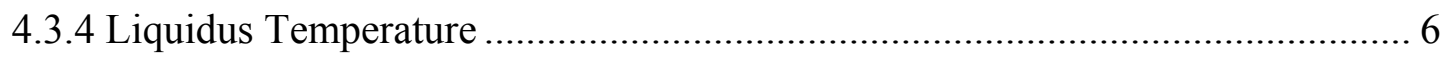

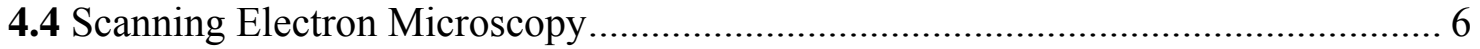

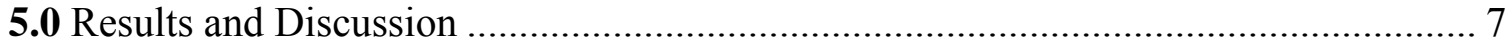

5.1 Visual Observations and XRD Results............................................................. 7

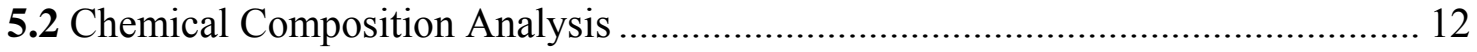

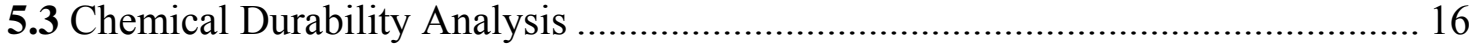

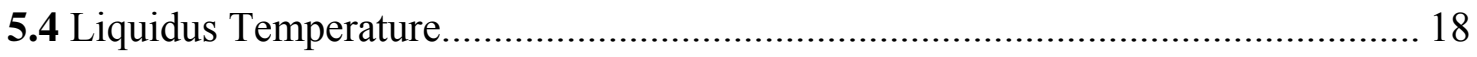

5.4.1 Select Visual Observations of the Quenched Glasses ...................................... 18

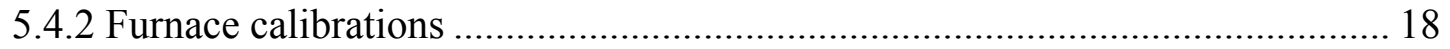

5.4.3 Initial Optical Microscopy Observations ..................................................... 19

5.4.4 Uniform Temperature Heat Treatments and Observations ............................. 34

5.5 Crystallinity vs. Temperature Using XRD (Crystal Fraction Method) .................. 35

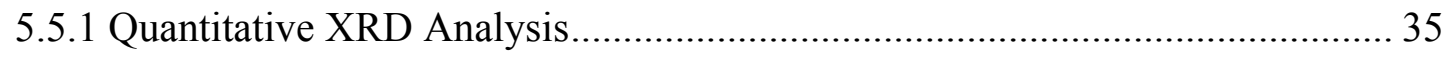

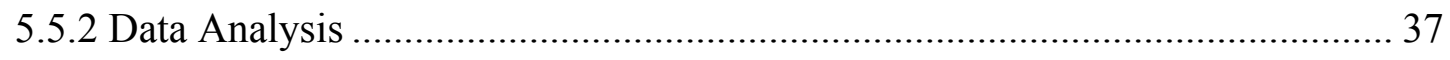

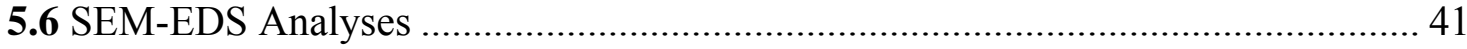

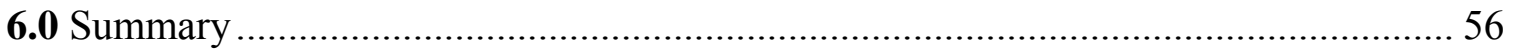

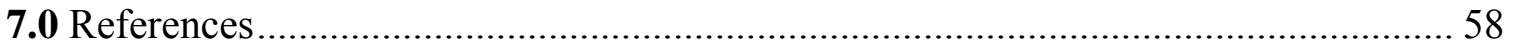


SRNS-STI-2008-00057

Revision 0

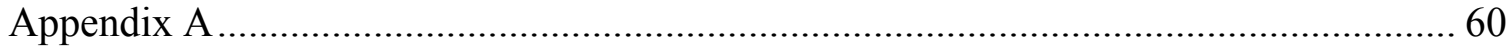

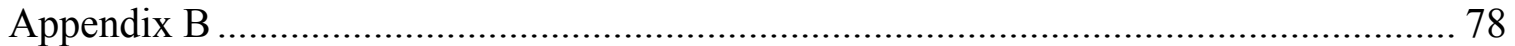

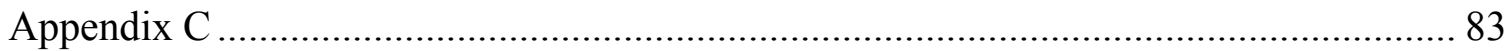




\section{LIST OF TABLES}

Table 3-1. Target Compositions of the US-Series Glasses. ....................................................... 4

Table 4-1. Visual Observations and XRD Results for the US-Series Glasses. ............................ 8

Table 4-2. Bias-Corrected Chemical Composition Data for the US-Series Glasses and Percent

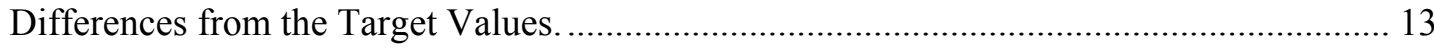

Table 4-3. PCT Results for Each of the US-Series Glasses by Heat Treatment, Normalized Using the Bias-Corrected Composition Data................................................................................... 16

Table 4-4. Furnace calibrations at PNNL. The $\mathrm{T}_{\mathrm{L}}$ of SRM-773 is $990^{\circ} \mathrm{C}$ (standard certificate). 19

Table 4-5. $\mathrm{T}_{\mathrm{L}}$ of ARG-1 and Zr-9 from a Round Robin Study with Monarch Laboratories and INL.

Table 4-6. Initial LROM Observations of Surface and Bulk Crystallization in the US-Series Glasses.

Table 4-7. Predicted and Measured Liquidus Temperatures.

Table 4-8. Identified Crystalline Phases and Their Concentrations (vol\%) in the CCC Glasses. 36

Table 4-9. Regression Coefficients for Optical and Crystal Fraction Methods. 38

Table 4-10. Summary of measured $\mathrm{T}_{\mathrm{L}}$ data for optical and crystal fraction methods for all glasses containing spinel as the primary phase as well as the calculated $\mathrm{T}_{\mathrm{L}}$ values for both sets using Equation 1 with data presented in Table 3-1 and the coefficients presented in Table 4-9.... 39

Table 4-11. Phases identified in borosilicate glasses US-01, US-03 to US-09, and US-14. 41

Table 4-12. Composition of Crystals, Particles, and the Surrounding Glass in US-01 Measured with EDS After Heat Treating at $1008^{\circ} \mathrm{C}$ for $24 \mathrm{~h}$.

Table 4-13. Composition of Particles and the Surrounding Glass in US-03 Measured with EDS After Heat Treating at $1107^{\circ} \mathrm{C}$ for $24 \mathrm{~h}$.

Table 4-14. Composition of Particles and the Surrounding Glass in US-04 Measured with EDS After Heat Treating at $1107^{\circ} \mathrm{C}$ for $24 \mathrm{~h}$.

Table 4-15. Composition of Particles and the Surrounding Glass in US-05 Measured by EDS After Heat Treating at $1107^{\circ} \mathrm{C}$ for $24 \mathrm{~h}$.

Table 4-16. Composition of Particles and the Surrounding Glass in US-06 Measured by EDS After Heat Treating at $1107^{\circ} \mathrm{C}$ for $24 \mathrm{~h}$.

Table 4-17. Composition of Particles and the Surrounding Glass in US-07 Measured by EDS After Heat Treating at $1058^{\circ} \mathrm{C}$ for $24 \mathrm{~h}$.

Table 4-18. Composition of Particles and the Surrounding Glass in US-08 Measured by EDS After Heat Treating at $1058^{\circ} \mathrm{C}$ for $24 \mathrm{~h}$. 
Table 4-19. Composition of Particles and the Surrounding Glass in US-09 Measured by EDS After Heat Treating at $1107^{\circ} \mathrm{C}$ for $24 \mathrm{~h}$.

Table 4-20. Composition of Particles and Spinel Crystals in Glass US-14 Measured by EDS After Heat Treating at $954{ }^{\circ} \mathrm{C}$ for $24 \mathrm{~h}$. 


\section{LIST OF FIGURES}

Figure 4-1. US-40 as-received after quenching on a steel plate. Crystallization is visible in the bulk of the glass, while a homogeneous region formed in contact with the steel plate at the bottom of the sample.

Figure 4-2. Brown crystal striations in the bulk of glass US-03 after heat treating at $854{ }^{\circ} \mathrm{C}$ (sample length approximately $12 \mathrm{~mm}$ ).

Figure 4-3. Surface of glass US-08 covered with grey crystals after heat treating at $904{ }^{\circ} \mathrm{C}$ (sample length approximately $12 \mathrm{~mm}$ ).

Figure 4-4. Glittering crystals in the bulk of glass US-11 after heat treating at $904{ }^{\circ} \mathrm{C} \ldots . .21$

Figure 4-5. White crystal agglomerates on the surface of glass US-13 while still in the platinum crucible after heat treating at $1056{ }^{\circ} \mathrm{C}$ (exaggerated colors, black glass)....... 21

Figure 4-6. Tiny, bright crystals on the surface and brown crystal striations in the bulk of glass US-19 while still in the platinum crucible after heat treating at $904{ }^{\circ} \mathrm{C}$ (sample length approximately $12 \mathrm{~mm}$ ).

Figure 4-7. Many black crystal agglomerates in the bulk of glass US-26 while still in the platinum crucible after heat treating at $1157^{\circ} \mathrm{C}$.

Figure 4-8. Surface of glass US-41 covered by hexagonal crystals while still in the platinum crucible after heat treating at $1007^{\circ} \mathrm{C}$.

Figure 4-9. Hematite in glass US-09 after heat treating at $1157^{\circ} \mathrm{C}$. Colors in the image are not original: the crystals appear very dark red.

Figure 4-10. Large, black agglomerate of undissolved noble metals in glass US-09 after heat treating at $1157^{\circ} \mathrm{C}$. Colors in the image are not original: the glass appears light yellow and the crystal agglomerate is black.

Figure 4-11. Comparison of $\mathrm{T}_{\mathrm{L}}$ data obtained using XRD and optical methods.

Figure 4-12. Calculated versus measured data obtained for both optical (left) and crystal fraction

(right) methods.

Figure 4-13. Comparison of calculated $\mathrm{T}_{\mathrm{L}}$ data for both crystal fraction and optical methods.... 41

Figure 4-14. Spinel, Nepheline and Undissolved $\mathrm{RuO}_{2}$ Agglomerates in Glass US-01 After Heat

Treating at $1008^{\circ} \mathrm{C}$ for $24 \mathrm{~h}$.

Figure 4-15. Undissolved $\mathrm{RuO}_{2}$ Agglomerates in Glass US-03 After Heat Treating at $1107{ }^{\circ} \mathrm{C}$ for

$24 \mathrm{~h}$.

Figure 4-16. Undissolved $\mathrm{RuO}_{2}$ Agglomerates in Glass US-04 After Heat Treating at $1107{ }^{\circ} \mathrm{C}$ for $24 \mathrm{~h}$.

Figure 4-17. Undissolved $\mathrm{RuO}_{2}$ Agglomerates in Glass US-05 After Heat Treating at $1107^{\circ} \mathrm{C}$ for

$24 \mathrm{~h}$. 
Figure 4-18. Undissolved $\mathrm{RuO}_{2}$ Agglomerates in Glass US-06 After Heat Treating at $1107{ }^{\circ} \mathrm{C}$ for $24 \mathrm{~h}$. 50

Figure 4-19. Undissolved $\mathrm{RuO}_{2}$ Agglomerates in Glass US-07 After Heat Treating at $1058{ }^{\circ} \mathrm{C}$ for $24 \mathrm{~h}$.

Figure 4-20. Undissolved $\mathrm{RuO}_{2}$ Agglomerates in Glass US-08 After Heat Treating at $1058{ }^{\circ} \mathrm{C}$ for $24 \mathrm{~h}$. 52

Figure 4-21. Undissolved $\mathrm{RuO}_{2}$ Agglomerates in Glass US-09 After Heat Treating at $1107^{\circ} \mathrm{C}$ for $24 \mathrm{~h}$. 53

Figure 4-22. Undissolved $\mathrm{RuO}_{2}$ Agglomerates and Spinel Crystals in Glass US-14 After Heat Treating at $954{ }^{\circ} \mathrm{C}$ for $24 \mathrm{~h}$. 


\section{LIST OF ABBREVIATIONS}

\begin{tabular}{|c|c|}
\hline ARM & Approved Reference Material \\
\hline $\mathrm{CCC}$ & Canister Centerline Cooled \\
\hline DOE & Department of Energy \\
\hline DWPF & Defense Waste Processing Facility \\
\hline EA & Environmental Assessment \\
\hline EDS & Energy Dispersive Spectroscopy \\
\hline $\mathrm{FC}$ & Furnace Controller \\
\hline HLW & High Level Waste \\
\hline HROM & High Resolution Optical Microscopy \\
\hline ICP-AES & Inductively Coupled Plasma - Atomic Emission Spectroscopy \\
\hline INL & Idaho National Laboratory \\
\hline KRI & V.G. Khlopin Radium Institute \\
\hline LM & Lithium Metaborate \\
\hline LROM & Low Resolution Optical Microscopy \\
\hline LWO & Liquid Waste Organization \\
\hline NL & Normalized Leachate \\
\hline NQARD & Nuclear Quality Assurance Requirements and Description \\
\hline PCT & Product Consistency Test \\
\hline $\mathrm{PF}$ & Peroxide Fusion \\
\hline PNNL & Pacific Northwest National Laboratory \\
\hline PSAL & Process Science Analytical Laboratory \\
\hline SEM & Scanning Electron Microscopy \\
\hline QA & Quality Assurance \\
\hline SRNL & Savannah River National Laboratory \\
\hline SRS & Savannah River Site \\
\hline $\mathrm{T}_{\mathrm{a}}$ & Amorphous Temperature \\
\hline $\mathrm{TC}$ & Thermocouple \\
\hline $\mathrm{T}_{\mathrm{c}}$ & Crystallization Temperature \\
\hline ThS & Thin Section \\
\hline $\mathrm{T}_{\mathrm{L}}$ & Liquidus Temperature \\
\hline UT & Uniform Temperature \\
\hline XRD & X-ray Diffraction \\
\hline
\end{tabular}




\subsection{Introduction}

The United States Department of Energy (DOE) is currently processing (or planning to process) high-level waste (HLW) through Joule-heated melters at the Savannah River Site (SRS) and Hanford. The process combines the HLW sludge with prefritted or mined mineral glass forming additives that are subsequently melted. The molten glass is poured into stainless steel canisters to create the final waste form. In preparation for the qualification and receipt of each sludge batch, development and definition of various tank blending and/or washing strategies have been or will be initiated. The various strategies are contemplated in an effort to meet critical site objectives or constraints that include tank volume space, transfer options, and settling issues. Although these objectives or constraints are critical, the ability to meet both process and product performance criteria associated with the final waste form must also be maintained. The product performance issues relate to the durability of the glass waste form. Process related issues (e.g., liquidus temperature, viscosity, electrical conductivity, and melting rate) ultimately dictate the efficiency and effectiveness of the melter operation.

Tank retrieval and blending strategies at both the SRS and Hanford have identified high $\mathrm{Al}_{2} \mathrm{O}_{3}$ concentration waste streams that are scheduled to be processed through their respective HLW vitrification facilities. For example, the Liquid Waste Organization (LWO) at SRS provided compositional projections for the next two sludge batches (Sludge Batch 4 and Sludge Batch 5) to be processed in the Defense Waste Processing Facility (DWPF). These streams have $\mathrm{Al}_{2} \mathrm{O}_{3}$ concentrations of approximately $25-40 \mathrm{wt} \%$ (on a calcined oxide basis). In addition, physical limitations in the Tank Farms and/or settling issues associated with the sludge coupled with the need to maintain feed for DWPF have prevented advanced washing which has resulted in relatively high $\mathrm{Na}_{2} \mathrm{O}$ (approximately 22-26 wt\%) and $\mathrm{SO}_{4}{ }^{2-}$ (approximately 0.8-1.6 wt\%) concentrations. Current Hanford projections suggest that $\mathrm{Al}_{2} \mathrm{O}_{3}$ concentrations in sludge could be much greater than those currently projected for DWPF, with $\mathrm{Al}_{2} \mathrm{O}_{3}$ concentrations as high as $80 \mathrm{wt} \%$.

While it is well known that the addition of small amounts of $\mathrm{Al}_{2} \mathrm{O}_{3}$ to borosilicate glass generally enhances the durability of the waste form (through creation of network-forming tetrahedral $\mathrm{Na}^{+}-$ $\left[\mathrm{AlO}_{4 / 2}\right]^{-}$pairs), nepheline $\left(\mathrm{NaAlSiO}_{4}\right)$ formation, which depends in part on the $\mathrm{Al}_{2} \mathrm{O}_{3}$ concentration, can result in a severe deterioration of the chemical durability of the glass through residual glass compositional changes. The primary driver for this reduction in durability is the fact that nepheline removes three moles of glass forming oxides $\left(\mathrm{Al}_{2} \mathrm{O}_{3}\right.$ and $\left.2 \mathrm{SiO}_{2}\right)$ from the continuous glass phase per each mole of $\mathrm{Na}_{2} \mathrm{O}$ consumed. Nepheline formation produces an $\mathrm{Al}_{2} \mathrm{O}_{3}$ and $\mathrm{SiO}_{2}$ deficient continuous glass matrix (relative to the same composition which is void of crystals), which reduces the durability of the final product. The magnitude of the reduction ultimately depends on the extent of crystallization and on the initial glass composition.

The formation of nepheline and/or other aluminum/silicon-containing crystals is a potential for both DWPF and Hanford due to the projected compositional views recently evaluated coupled with the frit development strategy (e.g., higher alkali frits have lead to enhanced melt rates at DWPF for those sludge batches). Although durability is obviously a critical constraint that the HLW glass must meet, other process related issues must also be considered. Additionally, the inclusion of higher concentrations of $\mathrm{Al}_{2} \mathrm{O}_{3}$ will generally increase the liquidus temperature of the melt and decrease the processing rate. 


\subsection{Objective}

The objective of this task was to develop glass formulations for DOE waste streams with high aluminum concentrations to avoid nepheline formation while maintaining or meeting waste loading and/or waste throughput expectations as well as satisfying critical process and product performance related constraints. Secondary objectives of this task were to assess the $\mathrm{SO}_{4}{ }^{2-}$ solubility limit for the DWPF compositions and spinel settling for the Hanford compositions. Liquidus temperatures and crystallization behavior were carefully characterized to support model development for higher waste loading glasses.

The experimental work, characterization, and data interpretation necessary to meet these objectives were performed among three partnering laboratories: the V.G. Khlopin Radium Institute (KRI), Pacific Northwest National Laboratory (PNNL) and Savannah River National Laboratory (SRNL).

\subsection{Quality Assurance (QA)}

This project was performed in accordance with the applicable criteria of 10 CFR 830.120, Subpart A, Quality Assurance Criteria and DOE/RW-0333P requirements. The PNNL QA Program implemented for this project was the Nuclear Quality Assurance Requirements and Description (NQARD) document. The NQARD is an NQA-1 based program (which meets DOE/RW-0333P requirements) with a graded approach for applying QA controls. The NQARD manual includes a program description section, followed by a set of QA implementing procedures. ${ }^{1}$ The SRNL work scope was performed in accordance with Savannah River Site Manual 1Q, Quality Assurance, with supplemental QA requirements as defined by Manual L1, Section 8.21, Supplemental QA Requirements for DOE/RW-0333P. Additional detail of the QA measures applied to this study can be found in the Test Plan. ${ }^{2}$ 
SRNS-STI-2008-00057

Revision 0

\subsection{Experimental Procedure}

\subsection{Selection of Study Glass Compositions}

A total of 67 individual glass compositions ( 75 glasses including replicates) were selected for this study. A thorough description of the process used to select these glasses is available in a separate document, ${ }^{3}$ although a brief summary will be provided here. Projected glass compositional regions that bound anticipated DWPF and Hanford glass regions of interest were developed and used to generate glass compositions of interest for meeting the objectives of this study (i.e., the incorporation of larger concentrations of aluminum, as well as improved sulfur retention and minimization of detrimental crystallization). A thorough statistical analysis was employed to allow for a wide range of waste glass compositions to be examined while minimizing the number of glasses that had to be fabricated and characterized in the laboratory. The glass compositions were divided into two sets, with 45 in the test matrix investigated by the U.S. laboratories (US-xx series glasses) and 30 in the test matrix investigated by KRI (KRI-xx series glasses). Fabrication and characterization of the US and KRI-series glasses were generally handled separately. SRNL and PNNL supported work on the US-series glasses, while KRI performed all work on the KRIseries glasses. This report will therefore focus mainly on the US-series glasses. A complete description of the results from the KRI-series glasses can be found in the final report from Aloy et al., ${ }^{4}$ which is included in the appendices.

The targeted compositions for the 45 US-series glasses in this study are given in Table 4-1. 
Table 4-1. Target Compositions of the US-Series Glasses.

\begin{tabular}{|c|c|c|c|c|c|c|c|c|c|c|c|c|c|c|c|c|c|c|c|}
\hline $\begin{array}{c}\text { Glass } \\
\text { Identifier }\end{array}$ & $\mathrm{Al}_{2} \mathrm{O}_{3}$ & $\mathbf{B}_{2} \mathbf{O}_{3}$ & $\mathrm{CaO}$ & $\mathrm{Cr}_{2} \mathrm{O}_{3}$ & $\mathrm{Fe}_{2} \mathrm{O}_{3}$ & $\mathrm{~K}_{2} \mathrm{O}$ & $\mathrm{Li}_{2} \mathrm{O}$ & MgO & MnO & $\mathrm{Na}_{2} \mathrm{O}$ & $\mathrm{NiO}$ & PbO & $\mathrm{RuO}_{2}$ & $\mathrm{SO}_{3}$ & $\mathrm{SiO}_{2}$ & SrO & $\mathrm{TiO}_{2}$ & ZnO & $\mathrm{ZrO}_{2}$ \\
\hline US-01 & 0.1493 & 0.0789 & 0.0000 & 0.0030 & $\begin{array}{ll}0.0859 \\
\end{array}$ & 0.0100 & 0.0562 & 0.0050 & 0.0250 & 0.1237 & 0.0006 & 0.0010 & 0.0002 & 0.0000 & 0.4553 & 0.0010 & 0.0000 & 0.0000 & 0.0050 \\
\hline US-02 & 0.1600 & 0.1686 & 0.0000 & 0.0000 & 0.0659 & 0.0000 & 0.0529 & 0.0000 & 0.0063 & 0.1233 & 0.0000 & 0.0000 & 0.0002 & 0.0000 & 0.4168 & 0.0000 & 0.0000 & 0.0010 & 0.0050 \\
\hline US-03 & 0.1600 & 0.1731 & 0.0100 & 0.0000 & 0.0653 & 0.0100 & 0.0407 & 0.0050 & 0.0000 & 0.0788 & 0.0029 & 0.0010 & 0.0002 & 0.0000 & 0.4421 & 0.0010 & 0.0100 & 0.0000 & $\overline{0.0000}$ \\
\hline $\begin{array}{l}\text { US-04 } \\
\end{array}$ & 0.1600 & 0.1129 & 0.0100 & 0.0000 & 0.0827 & 0.0100 & 0.0374 & 0.0050 & 0.0219 & 0.1033 & 0.0000 & 0.0010 & 0.0002 & 0.0050 & 0.4338 & 0.0010 & 0.0100 & 0.0010 & 0.0050 \\
\hline US- 05 & 0.1599 & 0.1670 & 0.0070 & 0.0000 & 0.0816 & 0.0100 & 0.0200 & 0.0050 & 0.0241 & 0.0958 & 0.0000 & 0.0010 & 0.0002 & 0.0050 & 0.4064 & 0.0010 & 0.0100 & 0.0010 & 0.0050 \\
\hline US-06 & 0.1600 & 0.1406 & 0.0100 & 0.0000 & 0.0815 & 0.0100 & 0.0247 & 0.0050 & 0.0300 & 0.1078 & 0.0000 & 0.0000 & 0.0002 & 0.0050 & 0.4142 & 0.0010 & 0.0100 & 0.0000 & 0.0000 \\
\hline US-07 & 0.1600 & 0.1349 & 0.0100 & 0.0000 & 0.0730 & 0.0100 & 0.0266 & 0.0050 & 0.0300 & 0.1067 & 0.0020 & 0.0010 & 0.0002 & 0.0050 & 0.4188 & 0.0010 & 0.0100 & 0.0010 & 0.0050 \\
\hline US-08 & 0.1519 & 0.0956 & 0.0000 & 0.0000 & 0.0975 & 0.0100 & 0.0486 & 0.0000 & 0.0244 & 0.0981 & 0.0000 & 0.0010 & 0.0002 & 0.0050 & 0.4577 & 0.0000 & 0.0100 & 0.0000 & 0.0000 \\
\hline US-09 & 0.1337 & 0.1505 & 0.0100 & 0.0030 & 0.1088 & 0.0000 & 0.0200 & 0.0050 & 0.0000 & 0.1411 & 0.0000 & 0.0010 & 0.0002 & 0.0050 & 0.4117 & 0.0000 & 0.0100 & 0.0000 & 0.0000 \\
\hline US-10 & 0.1412 & 0.1099 & 0.0100 & 0.0000 & 0.1069 & 0.0100 & 0.0200 & 0.0050 & 0.0274 & 0.1267 & 0.0000 & 0.0010 & 0.0002 & 0.0050 & $\begin{array}{l}0.4198 \\
\end{array}$ & 0.0010 & 0.0100 & 0.0010 & 0.0050 \\
\hline US-11 & 0.1370 & 0.1998 & 0.0100 & 0.0000 & 0.0776 & 0.0100 & 0.0200 & 0.0050 & 0.0300 & 0.0669 & 0.0000 & 0.0010 & 0.0002 & 0.0050 & 0.4366 & 0.0000 & 0.0000 & 0.0010 & 0.0000 \\
\hline US-12 & 0.1324 & 0.1608 & 0.0100 & 0.0014 & 0.0874 & 0.0100 & 0.0600 & 0.0000 & 0.0000 & 0.1116 & 0.0184 & 0.0000 & 0.0002 & 0.0050 & 0.3913 & 0.0010 & 0.0050 & 0.0010 & 0.0045 \\
\hline US-13 & 0.1600 & 0.1457 & 0.0100 & 0.0000 & 0.0730 & 0.0100 & 0.0215 & 0.0050 & 0.0280 & 0.1153 & 0.0028 & 0.0010 & 0.0002 & 0.0000 & 0.4165 & 0.0000 & 0.0100 & 0.0010 & 0.0000 \\
\hline US-14 & 0.1302 & 0.1790 & 0.0040 & 0.0000 & 0.0941 & 0.0100 & 0.0574 & 0.0050 & 0.0260 & 0.1122 & 0.0000 & 0.0007 & 0.0002 & 0.0000 & 0.3652 & 0.0000 & 0.0100 & 0.0010 & 0.0050 \\
\hline US-15 & 0.1000 & 0.1780 & 0.0050 & 0.0030 & 0.0500 & 0.0100 & 0.0200 & 0.0000 & 0.0000 & 0.1500 & 0.0000 & 0.0010 & 0.0002 & 0.0050 & 0.4659 & 0.0010 & 0.0100 & 0.0010 & 0.0000 \\
\hline US-16 & 0.1332 & 0.1881 & 0.0100 & 0.0008 & 0.0903 & 0.0100 & 0.0256 & 0.0000 & 0.0248 & 0.0630 & 0.0000 & 0.0000 & 0.0002 & 0.0000 & 0.4391 & 0.0000 & 0.0100 & 0.0000 & 0.0050 \\
\hline US-17 & 0.1166 & 0.1178 & 0.0000 & 0.0011 & 0.0622 & 0.0000 & 0.0536 & 0.0050 & 0.0300 & 0.0858 & 0.0108 & 0.0000 & 0.0002 & 0.0000 & 0.4999 & 0.0010 & 0.0100 & 0.0010 & 0.0050 \\
\hline US-18 & 0.1600 & 0.0913 & 0.0100 & 0.0000 & 0.0975 & 0.0000 & 0.0495 & 0.0000 & 0.0300 & 0.1341 & 0.0000 & 0.0010 & 0.0002 & 0.0000 & 0.4145 & 0.0010 & 0.0100 & 0.0010 & 0.0000 \\
\hline US-19 & 0.1447 & 0.1440 & 0.0070 & 0.0007 & 0.0823 & 0.0078 & 0.0364 & 0.0033 & 0.0199 & 0.1080 & 0.0021 & 0.0007 & 0.0002 & 0.0028 & 0.4281 & 0.0006 & 0.0080 & 0.0007 & 0.0027 \\
\hline US-20 & 0.1447 & 0.1440 & 0.0070 & 0.0007 & 0.0823 & 0.0078 & 0.0364 & 0.0033 & 0.0199 & 0.1080 & 0.0021 & 0.0007 & 0.0002 & 0.0028 & 0.4281 & 0.0006 & 0.0080 & 0.0007 & 0.0027 \\
\hline US-21 & 0.1422 & 0.1375 & 0.0038 & 0.0008 & 0.0845 & 0.0075 & 0.0300 & 0.0037 & 0.0178 & 0.1075 & 0.0050 & 0.0008 & 0.0002 & 0.0012 & 0.4495 & 0.0008 & 0.0054 & 0.0003 & 0.0013 \\
\hline US-22 & 0.1334 & 0.1169 & 0.0075 & 0.0008 & 0.0947 & 0.0075 & 0.0500 & 0.0037 & 0.0225 & 0.1178 & 0.0092 & 0.0003 & 0.0002 & 0.0012 & 0.4240 & 0.0008 & 0.0075 & 0.0008 & 0.0013 \\
\hline US-23 & 0.1450 & 0.1332 & 0.0075 & 0.0008 & 0.0762 & 0.0025 & 0.0350 & 0.0012 & 0.0225 & 0.1168 & 0.0050 & 0.0008 & 0.0002 & 0.0037 & 0.4446 & 0.0003 & 0.0025 & 0.0008 & 0.0013 \\
\hline US-24 & 0.1450 & 0.1225 & 0.0075 & 0.0008 & 0.0779 & 0.0075 & 0.0300 & 0.0037 & 0.0225 & 0.1157 & 0.0050 & & 0.0002 & 0.0037 & 0.4443 & 0.0008 & 0.0075 & 0.0008 & 0.0038 \\
\hline US-25 & 0.1158 & 0.1625 & 0.0075 & 0.0022 & 0.0854 & 0.0029 & 0.0500 & 0.0037 & 0.0216 & 0.1088 & 0.0145 & 0.0003 & 0.0002 & 0.0037 & 0.4079 & 0.0008 & 0.0075 & 0.0008 & 0.0038 \\
\hline US-26 & 0.1883 & 0.1069 & 0.0100 & 0.0055 & 0.0500 & 0.0000 & 0.0600 & 0.0050 & 0.0000 & 0.1102 & 0.0000 & 0.0000 & 0.0002 & 0.0000 & 0.3805 & 0.0300 & 0.0100 & 0.0200 & 0.0235 \\
\hline US-27 & 0.1040 & 0.0648 & 0.0000 & 0.0100 & 0.1518 & 0.0100 & 0.0600 & 0.0050 & 0.0000 & 0.1687 & 0.0000 & 0.0000 & 0.0002 & 0.0050 & 0.4004 & 0.0000 & 0.0000 & 0.0200 & 0.0000 \\
\hline US-28 & 0.1747 & 0.1796 & 0.0000 & 0.0000 & & 0.0100 & 0.0200 & 0.0000 & 0.0000 & 0.1336 & 0.0000 & 0.0000 & 0.0002 & 0.0050 & 0.3597 & 0.0300 & 0.0100 & 0.0200 & 0.0000 \\
\hline US-29 & 0.1000 & 0.0873 & 0.0100 & 0.0067 & 0.0500 & 0.0100 & 0.0600 & 0.0050 & 0.0000 & 0.1868 & 0.0000 & 0.0100 & 0.0002 & 0.0000 & 0.4526 & 0.0118 & 0.0096 & 0.0000 & 0.0000 \\
\hline US-30 & 0.1265 & 0.1819 & 0.0100 & 0.0082 & 0.0533 & 0.0100 & 0.0600 & 0.0000 & 0.0350 & 0.0500 & 0.0000 & 0.0000 & 0.0002 & 0.0050 & 0.3752 & 0.0300 & 0.0100 & 0.0048 & 0.0400 \\
\hline US-31 & 0.1267 & 0.1452 & 0.0000 & 0.0000 & 0.1545 & 0.0000 & 0.0491 & 0.0050 & 0.0074 & 0.0710 & 0.0000 & 0.0100 & 0.0002 & 0.0050 & 0.3860 & 0.0300 & 0.0100 & 0.0000 & 0.0000 \\
\hline US-32 & 0.1000 & 0.1901 & 0.0100 & 0.0000 & 0.0500 & 0.0000 & 0.0600 & 0.0000 & 0.0400 & 0.0750 & 0.0000 & 0.0100 & 0.0002 & 0.0050 & 0.4397 & 0.0000 & 0.0000 & 0.0200 & 0.0000 \\
\hline US-33 & 0.1134 & 0.0500 & 0.0000 & 0.0100 & 0.0500 & 0.0000 & 0.0200 & 0.0050 & 0.0400 & 0.1976 & 0.0097 & 0.0100 & 0.0002 & 0.0050 & 0.4591 & 0.0300 & 0.0000 & 0.0000 & 0.0000 \\
\hline US-34 & 0.1000 & 0.1369 & 0.0000 & 0.0000 & 0.0639 & 0.0100 & 0.0242 & 0.0050 & 0.0400 & 0.2000 & 0.0000 & 0.0000 & 0.0002 & 0.0000 & 0.3499 & 0.0300 & 0.0000 & 0.0000 & 0.0400 \\
\hline US-35 & 0.1002 & 0.1996 & 0.0100 & 0.0052 & 0.1135 & 0.0000 & 0.0200 & 0.0050 & 0.0400 & 0.0794 & 0.0000 & 0.0000 & 0.0002 & 0.0000 & 0.3800 & 0.0270 & 0.0000 & 0.0200 & 0.0000 \\
\hline US-36 & 0.1286 & 0.1551 & 0.0021 & 0.0051 & 0.0976 & 0.0052 & 0.0242 & 0.0005 & 0.0121 & 0.1252 & 0.0056 & 0.0100 & 0.0002 & 0.0000 & 0.3884 & 0.0001 & 0.0001 & 0.0001 & 0.0400 \\
\hline $\begin{array}{l}\text { US-37 } \\
\end{array}$ & 0.1000 & 0.0577 & 0.0100 & 0.0000 & 0.1700 & 0.0000 & 0.0200 & 0.0000 & 0.0000 & 0.1889 & 0.0000 & 0.0100 & 0.0002 & 0.0000 & 0.3733 & 0.0000 & 0.0100 & 0.0200 & 0.0400 \\
\hline US-38 & 0.1000 & 0.0731 & 0.0079 & 0.0000 & 0.0500 & 0.0100 & 0.0200 & 0.0000 & 0.0400 & 0.1972 & 0.0291 & 0.0100 & 0.0002 & 0.0050 & 0.3975 & 0.0300 & 0.0100 & 0.0200 & 0.0000 \\
\hline $\begin{array}{l}\text { US-39 } \\
\end{array}$ & 0.1155 & 0.1635 & 0.0000 & 0.0000 & 0.0750 & 0.0100 & 0.0600 & 0.0050 & 0.0000 & 0.0500 & 0.0183 & 0.0100 & 0.0002 & 0.0000 & 0.4246 & 0.0080 & 0.0000 & 0.0200 & 0.0400 \\
\hline US-40 & 0.1552 & 0.1700 & 0.0000 & 0.0043 & 0.1142 & 0.0100 & 0.025 & 0.0000 & 0.0276 & 0.10 & 0.00 & 0.0100 & 0.0002 & 0.0000 & 0.3730 & 0.0000 & 0.0092 & 0.0000 & 0.0000 \\
\hline US-41 & 0.1000 & 0.1555 & 0.0100 & 0.0000 & 0.1553 & 0.0100 & 0.0372 & 0.0000 & 0.0000 & 0.0652 & 0.0000 & 0.0100 & 0.0002 & 0.0000 & 0.4265 & 0.0300 & 0.0000 & 0.0000 & 0.0000 \\
\hline US-42 & 0.2000 & 0.0619 & 0.0100 & 0.0000 & 0.0500 & 0.0100 & 0.0600 & 0.0000 & 0.0400 & 0.1882 & 0.0000 & 0.0100 & 0.0002 & 0.0000 & 0.3549 & 0.0000 & 0.0000 & 0.0149 & 0.0000 \\
\hline US-43 & 0.1717 & 0.0864 & 0.0100 & 0.0000 & 0.0886 & 0.0100 & 0.0490 & 0.0050 & 0.0081 & 0.1029 & 0.0020 & 0.0100 & 0.0002 & 0.0050 & 0.3970 & 0.0047 & 0.0048 & 0.0045 & 0.0400 \\
\hline US-44 & 0.1280 & 0.1259 & 0.0056 & 0.0031 & 0.0886 & 0.0064 & 0.0405 & 0.0025 & 0.0183 & 0.1273 & 0.0036 & 0.0067 & 0.0002 & 0.0022 & 0.3955 & 0.0162 & 0.0047 & 0.0102 & 0.0146 \\
\hline US-45 & 0.1280 & 0.1259 & 0.0056 & 0.0031 & 0.0886 & 0.0064 & 0.0405 & 0.0025 & 0.0183 & 0.1273 & 0.0036 & 0.0067 & 0.0002 & 0.0022 & 0.3955 & 0.0162 & 0.0047 & 0.0102 & 0.0146 \\
\hline
\end{tabular}




\subsection{Glass Fabrication}

Each study glass was prepared from the proper proportions of reagent-grade metal oxides, carbonates, $\mathrm{H}_{3} \mathrm{BO}_{3}$, and salts in $250 \mathrm{~g}$ batches. The raw materials were thoroughly mixed and placed into a $95 \%$ platinum / 5\% gold, $250 \mathrm{~mL}$ crucible. The crucibles were placed into a hightemperature furnace at a target melt temperature of $1150{ }^{\circ} \mathrm{C}$. The crucibles were removed from the furnace after an isothermal hold at $1150{ }^{\circ} \mathrm{C}$ for 1 hour. The molten glass was quenched by pouring onto a clean, stainless steel plate. Note that some of the glasses did not appear to have melted completely after holding at $1150{ }^{\circ} \mathrm{C}$ for 1 hour. Those glasses were re-melted at $1200{ }^{\circ} \mathrm{C}$ for 1 hour and then quenched.

Approximately $25 \mathrm{~g}$ of each glass was heat-treated to simulate cooling along the centerline of a DWPF-type canister to gauge the effects of thermal history on the product performance. ${ }^{5}$ This cooling schedule is referred to as canister centerline cooling (CCC). Visual observations were recorded for each of the glasses both after quenching and at the completion of the CCC heat treatment.

\subsection{Property Measurements}

\subsubsection{X-Ray Diffraction Analysis}

Representative samples of each of the quenched and CCC glasses were submitted to SRNL Analytical Development for X-ray diffraction (XRD) analysis. Samples were run under conditions providing a detection limit of approximately $0.5 \mathrm{vol} \%$. That is, if crystals (or undissolved batch material) were present at $0.5 \mathrm{vol} \%$ or greater, the diffractometer would not only be capable of detecting the crystals but would also allow a qualitative determination of the type of crystal(s) present. Otherwise, a characteristically high background signal (amorphous hump) devoid of crystalline spectral peaks indicates that the glass product is free of crystallization, suggesting either a completely amorphous product or that the degree of crystallization is below the detection limit.

\subsubsection{Compositional Analysis}

To confirm that the as-fabricated glasses met the target compositions, a representative sample from each quenched glass was submitted to the SRNL Process Science Analytical Laboratory (PSAL) for chemical analysis under the auspices of an analytical plan. ${ }^{6}$ The plan identified the cations to be analyzed and the two dissolution techniques, sodium peroxide fusion (PF) and lithium-metaborate (LM), to be used. The samples prepared by LM were used to measure calcium $(\mathrm{Ca})$, chromium $(\mathrm{Cr})$, potassium $(\mathrm{K})$, magnesium $(\mathrm{Mg})$, manganese $(\mathrm{Mn})$, sodium $(\mathrm{Na})$, lead $(\mathrm{Pb})$, sulfur $(\mathrm{S})$, strontium $(\mathrm{Sr})$, titanium $(\mathrm{Ti})$, zinc $(\mathrm{Zn})$, and zirconium $(\mathrm{Zr})$ concentrations. Samples prepared by PF were used to measure aluminum (Al), boron (B), iron (Fe), lithium ( $\mathrm{Li})$, nickel $(\mathrm{Ni})$, and silicon $(\mathrm{Si})$ concentrations. Each glass was prepared in duplicate for each of the two cation dissolution techniques. All of the prepared samples were analyzed twice for each element of interest by Inductively Coupled Plasma - Atomic Emission Spectroscopy (ICP-AES), with the instrumentation being re-calibrated between the duplicate analyses. The analytical plan was developed in such a way as to provide the opportunity to evaluate potential sources of bias and error. Glass standards were also intermittently measured to assess the performance of the ICP-AES instrument over the course of these analyses.

\subsubsection{Durability Testing}

A 7-day Product Consistency Test (PCT) was performed in triplicate on each quenched and CCC glass to assess chemical durability using Method A of the PCT procedure. ${ }^{7}$ Also included in the 
experimental test matrix was the Environmental Assessment (EA) glass, the Approved Reference Material (ARM) glass, and blanks from the sample cleaning batch. Samples were ground, washed, and prepared according to the standard procedure. The resulting solutions were sampled (filtered and acidified) and analyzed under the auspices of three analytical plans. ${ }^{8-10}$ Samples of a multi-element standard solution were also included in the analytical plans as a check on the accuracy of the ICP-AES instrument. Normalized release rates were calculated based on the target and measured compositions using the average of the logs of the leachate concentrations.

\subsubsection{Liquidus Temperature}

Liquidus temperature $\left(T_{L}\right)$ measurements were performed on the 45 study glasses using the uniform temperature (UT) method as described in PNNL procedure GDL-LQT. ${ }^{11}$ Using this procedure, the quenched glass was crushed and sieved to a size fraction from $4.0 \mathrm{~mm}$ to 0.425 $\mathrm{mm}$ ( -5 to +40 mesh, respectively). The captured chunks were then cleaned three times with ethanol using an ultrasonic bath. The UT analyses for these samples were completed by:

- Placing the glass in a covered, square platinum boat with a side length of about $13 \mathrm{~mm}$

- Heat treating for $24 \pm 2$ hours at temperature (shorter times are used at higher temperatures)

- Quenching the boat on steel plate

- Recording initial observations by optical microscopy with the glass still in the platinum boat

- Grinding the glass with $5 \mathrm{wt} \% \mathrm{CaF}_{2}$ for quantitative $\mathrm{XRD}$ analysis

XRD measurements for all glasses were performed with a Scintag PAD V diffractometer using $\mathrm{Cu} \mathrm{K} \mathrm{K}_{\alpha}$ radiation $(\lambda=1.5406 \AA, 45 \mathrm{kV}$, and $40 \mathrm{~mA})$ and equipped with a Peltier-cooled $\mathrm{Si}(\mathrm{Li})$ solid state detector. The experiments were done using $\theta-2 \theta$ geometry in a step-scan approach from $10^{\circ}$ to $60^{\circ} 2 \theta$ with a step size of $0.04^{\circ} 2 \theta$ and a dwell time of 6 seconds per step. Each sample was loaded into a 12-position automatic sample changer. Jade $6^{\circledR}$ and RIQAS ${ }^{\circledR}$ software $^{\mathrm{a}}$ were used to process and identify phase assemblages. PNNL's standard procedure for XRD analysis ${ }^{12}$ was used to guide these measurements.

Optional portions of the method include thin-sectioning and analysis using high-power optical microscopy or SEM analysis.

\subsection{Scanning Electron Microscopy}

A select group of the US-series glasses, US-01, US-03 to US-09, and US-14 (see compositions in Table 4-1) were analyzed by Scanning Electron Microscopy (SEM) and Energy Dispersive Spectroscopy (EDS) to identify crystalline phases and insoluble metal species. The glasses were subjected to heat-treatments at various temperatures for $24 \pm 2 \mathrm{hrs}$ in $1.2 \times 1.2 \times 1.2 \mathrm{~cm} \mathrm{Pt}-10 \%$ $\mathrm{Rh}$ crucibles. Samples were removed from the crucibles and cut in half. Cross-sections were ground, polished and coated by sputtering with gold-palladium for SEM and EDS analysis.

\footnotetext{
${ }^{a}$ Materials Data, Inc., Livermore, CA.
} 


\subsection{Results and Discussion}

\subsection{Visual Observations and XRD Results}

Visual observations for each of the study glasses were recorded both after quenching and at the completion of the CCC heat treatment. These observations are listed in Table 5-1. Crystallization in the glasses is described as a haze, streaks or swirls. Glasses that are described as clean or homogeneous indicate that no crystallization was observable by the unaided eye. XRD results for the quenched and CCC version of each glass are also provided in Table 5-1. The XRD results generally agree with the visual observations, although there are some cases where crystallization occurred only on the surface of the glass and may not have existed in a significant enough volume fraction to be detected by XRD. The phases identified by XRD are generally iron oxides and spinels, which are not expected to impact durability of the glass. ${ }^{13}$ Nepheline was detected in five of the glasses after the CCC heat treatment (highlighted in the table): US-18, US-26, US-27, US-37 and US-43. The impact of the nepheline crystallization on the durability of the glasses will be explored in the following sections. 
Table 5-1. Visual Observations and XRD Results for the US-Series Glasses.

\begin{tabular}{|c|c|c|c|c|}
\hline Glass & $\begin{array}{l}\mathbf{T}_{\text {Melt }} \\
\left({ }^{\circ} \mathrm{C}\right)\end{array}$ & $\begin{array}{c}\text { Heat } \\
\text { Treatment }\end{array}$ & Visual Observations & XRD Results \\
\hline \multirow{2}{*}{ US-01 } & \multirow{2}{*}{1150} & quenched & $\begin{array}{l}\text { Crucible - clean with bubbles; Patty - black, } \\
\text { shiny, homogeneous }\end{array}$ & amorphous \\
\hline & & $\mathrm{CCC}$ & $\begin{array}{l}\text { Surface - very light shiny black haze; Bulk - } \\
\text { clean }\end{array}$ & $\mathrm{Fe}_{3} \mathrm{O}_{4}, \mathrm{NiFe}_{2} \mathrm{O}_{4}, \mathrm{SiO}_{2}$ \\
\hline \multirow[t]{2}{*}{ US-02 } & \multirow[t]{2}{*}{1150} & quenched & $\begin{array}{l}\text { Crucible - clean with bubbles; Patty - black, } \\
\text { shiny, homogeneous }\end{array}$ & amorphous \\
\hline & & $\mathrm{CCC}$ & Surface - black, shiny, clean; Bulk - clean & $\mathrm{Fe}_{3} \mathrm{O}_{4}, \mathrm{NiFe}_{2} \mathrm{O}_{4}, \mathrm{SiO}_{2}$ \\
\hline \multirow{2}{*}{ US-03 } & \multirow{2}{*}{1150} & quenched & $\begin{array}{l}\text { Crucible - clean with bubbles; Patty - black, } \\
\text { shiny, homogeneous }\end{array}$ & amorphous \\
\hline & & $\mathrm{CCC}$ & $\begin{array}{l}\text { Surface - multiple crystals; Bulk - multiple } \\
\text { crystals }\end{array}$ & $\mathrm{Fe}_{2} \mathrm{O}_{3}$ \\
\hline \multirow{2}{*}{ US-04 } & \multirow{2}{*}{1150} & quenched & $\begin{array}{l}\text { Crucible - clean with bubbles; Patty - black, } \\
\text { shiny, homogeneous }\end{array}$ & amorphous \\
\hline & & $\mathrm{CCC}$ & $\begin{array}{l}\text { Surface - chocolate brown; Bulk - black and } \\
\text { shiny }\end{array}$ & amorphous \\
\hline \multirow[t]{2}{*}{ US-05 } & \multirow[t]{2}{*}{1150} & quenched & $\begin{array}{l}\text { Crucible - several spots of undissolved } \\
\text { material; Patty - black and shiny with some } \\
\text { silvery spots/pits on the surface, thin white film } \\
\text { on bottom }\end{array}$ & amorphous \\
\hline & & $\mathrm{CCC}$ & $\begin{array}{l}\text { Surface - chocolate brown with clusters of } \\
\text { crystals; Bulk - chocolate brown }\end{array}$ & $\mathrm{Fe}_{3} \mathrm{O}_{4}, \mathrm{Fe}_{2} \mathrm{O}_{3}$ \\
\hline \multirow{2}{*}{ US-06 } & \multirow{2}{*}{1150} & quenched & $\begin{array}{l}\text { Crucible - clean with bubbles; Patty - black, } \\
\text { shiny, homogeneous }\end{array}$ & amorphous \\
\hline & & $\mathrm{CCC}$ & $\begin{array}{l}\text { Surface - chocolate brown with clusters of } \\
\text { crystals; Bulk - black and shiny }\end{array}$ & $\mathrm{Fe}_{2} \mathrm{O}_{3}$ (Hematite) \\
\hline \multirow{2}{*}{ US-07 } & \multirow{2}{*}{1150} & quenched & $\begin{array}{l}\text { Crucible - few spots of undissolved material; } \\
\text { Patty - black, shiny, homogeneous }\end{array}$ & amorphous \\
\hline & & $\mathrm{CCC}$ & $\begin{array}{l}\text { Surface - chocolate brown with clusters of } \\
\text { shiny crystals; Bulk - black and shiny }\end{array}$ & $\mathrm{Fe}_{3} \mathrm{O}_{4}, \mathrm{NiFe}_{2} \mathrm{O}_{4}$ \\
\hline \multirow{2}{*}{ US-08 } & \multirow{2}{*}{1150} & quenched & $\begin{array}{l}\text { Crucible - few spots of undissolved material; } \\
\text { Patty - black, shiny, homogeneous }\end{array}$ & amorphous \\
\hline & & $\mathrm{CCC}$ & $\begin{array}{l}\text { Surface - brown with crystals; Bulk - black, } \\
\text { shiny, clean }\end{array}$ & amorphous \\
\hline \multirow[b]{2}{*}{ US-09 } & \multirow[b]{2}{*}{1150} & quenched & $\begin{array}{l}\text { Crucible - few spots of undissolved material; } \\
\text { Patty - black, shiny, homogeneous }\end{array}$ & amorphous \\
\hline & & $\mathrm{CCC}$ & $\begin{array}{l}\text { Surface - clear shellac with crystals under } \\
\text { surface, also circular clusters of crystals; } \\
\text { Bulk - solid medium brown }\end{array}$ & $\mathrm{Fe}_{2} \mathrm{O}_{3}$ \\
\hline \multirow{2}{*}{ US-10 } & \multirow{2}{*}{1150} & quenched & $\begin{array}{l}\text { Crucible - clean with bubbles; Patty - black, } \\
\text { shiny, homogeneous }\end{array}$ & amorphous \\
\hline & & $\mathrm{CCC}$ & $\begin{array}{l}\text { Surface - dull matte with crystals; Bulk - } \\
\text { devitrified }\end{array}$ & $\mathrm{Fe}_{2} \mathrm{O}_{3}$ \\
\hline \multirow[t]{2}{*}{ US-11 } & \multirow[t]{2}{*}{1150} & quenched & $\begin{array}{l}\text { Crucible - few spots of undissolved material; } \\
\text { Patty - some surface pits and white film on } \\
\text { bottom }\end{array}$ & amorphous \\
\hline & & $\mathrm{CCC}$ & Surface and Bulk - totally devitrified & $\mathrm{Fe}_{2} \mathrm{O}_{3}$ \\
\hline
\end{tabular}


Table 5-1. Visual Observations and XRD Results for the US-Series Glasses. (continued)

\begin{tabular}{|c|c|c|c|c|}
\hline Glass & $\begin{array}{l}\mathbf{T}_{\text {Melt }} \\
\left({ }^{\circ} \mathrm{C}\right)\end{array}$ & $\begin{array}{c}\text { Heat } \\
\text { Treatment }\end{array}$ & Visual Observations & XRD Results \\
\hline \multirow{2}{*}{ US-12 } & \multirow{2}{*}{1150} & quenched & $\begin{array}{l}\text { Crucible - clean with bubbles; Patty - black, } \\
\text { shiny, homogeneous }\end{array}$ & amorphous \\
\hline & & $\mathrm{CCC}$ & Surface - black matte; Bulk - crystals & $\mathrm{Fe}_{3} \mathrm{O}_{4}, \mathrm{NiFe}_{2} \mathrm{O}_{4}$ \\
\hline \multirow{2}{*}{ US-13 } & \multirow{2}{*}{1150} & quenched & $\begin{array}{l}\text { Crucible - few spots of undissolved material; } \\
\text { Patty - clean }\end{array}$ & amorphous \\
\hline & & $\mathrm{CCC}$ & $\begin{array}{l}\text { Surface - large metallic circles with crystals; } \\
\text { Bulk - crystals }\end{array}$ & $\mathrm{Fe}_{3} \mathrm{O}_{4}, \mathrm{NiFe}_{2} \mathrm{O}_{4}$ \\
\hline \multirow[b]{2}{*}{ US-14 } & \multirow[b]{2}{*}{1150} & quenched & Crucible - clean; Patty - Clean, homogeneous & amorphous \\
\hline & & $\mathrm{CCC}$ & $\begin{array}{l}\text { Surface - shiny haze with crystals along the } \\
\text { melt line; Bulk - clean }\end{array}$ & $\mathrm{Fe}_{3} \mathrm{O}_{4}, \mathrm{NiFe}_{2} \mathrm{O}_{4}$ \\
\hline \multirow[t]{2}{*}{ US-15 } & \multirow[t]{2}{*}{1200} & quenched & $\begin{array}{l}\text { Crucible - green glass with some brown streaks; } \\
\text { Patty - translucent green glass with some brown } \\
\text { streaks }\end{array}$ & amorphous \\
\hline & & $\mathrm{CCC}$ & Surface - dark, shiny; Bulk - green, clean & $\mathrm{Fe}_{3} \mathrm{O}_{4}$ \\
\hline \multirow{2}{*}{ US-16 } & \multirow{2}{*}{1200} & quenched & $\begin{array}{l}\text { Crucible - two small spots of undissolved } \\
\text { material; Patty - black, shiny, homogeneous }\end{array}$ & $\mathrm{NiFe}_{2} \mathrm{O}_{4}, \mathrm{Fe}_{2} \mathrm{O}_{3}$ \\
\hline & & $\mathrm{CCC}$ & $\begin{array}{l}\text { Surface - green, hazy sheen with small silver } \\
\text { crystals; Bulk - crystals throughout }\end{array}$ & $\mathrm{Fe}_{2} \mathrm{O}_{3}$ \\
\hline \multirow[b]{2}{*}{ US-17 } & \multirow[b]{2}{*}{1150} & quenched & $\begin{array}{l}\text { Crucible - two spots of undissolved material; } \\
\text { Patty - black, shiny, clean }\end{array}$ & amorphous \\
\hline & & $\mathrm{CCC}$ & $\begin{array}{l}\text { Surface - shiny with crystals; Bulk - bottom } \\
\text { layer chocolate brown and crystallized, top layer } \\
\text { black, shiny, clean }\end{array}$ & $\mathrm{Fe}_{3} \mathrm{O}_{4}, \mathrm{NiFe}_{2} \mathrm{O}_{4}$ \\
\hline \multirow[t]{2}{*}{ US-18 } & \multirow[t]{2}{*}{1150} & quenched & $\begin{array}{l}\text { Crucible - four small spots of undissolved } \\
\text { material; Patty - black, shiny, homogeneous }\end{array}$ & amorphous \\
\hline & & $\mathrm{CCC}$ & Surface and Bulk - totally crystallized & $\mathrm{NaAlSiO}_{4}$ \\
\hline \multirow{2}{*}{ US-19 } & \multirow{2}{*}{1150} & quenched & $\begin{array}{l}\text { Crucible - three spots of undissolved material; } \\
\text { Patty - black, shiny, homogeneous }\end{array}$ & amorphous \\
\hline & & $\mathrm{CCC}$ & $\begin{array}{l}\text { Surface - shiny, silver spots of crystals; Bulk - } \\
\text { clean }\end{array}$ & $\mathrm{Fe}_{3} \mathrm{O}_{4}$ \\
\hline \multirow[b]{2}{*}{ US-20 } & \multirow[b]{2}{*}{1150} & quenched & $\begin{array}{l}\text { Crucible - three spots of undissolved material; } \\
\text { Patty - black, shiny, homogeneous }\end{array}$ & amorphous \\
\hline & & $\mathrm{CCC}$ & $\begin{array}{l}\text { Surface - shiny, silver spots of crystals; Bulk - } \\
\text { thin layer of crystals along surfaces in contact } \\
\text { with crucible }\end{array}$ & $\mathrm{Fe}_{3} \mathrm{O}_{4}$ \\
\hline \multirow[t]{2}{*}{ US-21 } & \multirow[t]{2}{*}{1150} & quenched & $\begin{array}{l}\text { Crucible - four small spots of undissolved } \\
\text { material and bubbles; Patty - black, shiny, } \\
\text { homogeneous }\end{array}$ & amorphous \\
\hline & & $\mathrm{CCC}$ & $\begin{array}{l}\text { Surface - brown with a lot of metallic crystals; } \\
\text { Bulk - crystals }\end{array}$ & $\mathrm{Fe}_{3} \mathrm{O}_{4}, \mathrm{NiFe}_{2} \mathrm{O}_{4}$ \\
\hline \multirow{2}{*}{ US-22 } & \multirow{2}{*}{1150} & quenched & $\begin{array}{l}\text { Crucible - two spots of undissolved material; } \\
\text { Patty - black, shiny, homogeneous }\end{array}$ & amorphous \\
\hline & & $\mathrm{CCC}$ & $\begin{array}{l}\text { Surface - hazy sheen with a few metallic } \\
\text { crystals; Bulk - crystals }\end{array}$ & $\mathrm{Fe}_{3} \mathrm{O}_{4}, \mathrm{NiFe}_{2} \mathrm{O}_{4}, \mathrm{SiC}$ \\
\hline
\end{tabular}


Table 5-1. Visual Observations and XRD Results for the US-Series Glasses. (continued)

\begin{tabular}{|c|c|c|c|c|}
\hline Glass & $\begin{array}{l}\mathbf{T}_{\text {Melt }} \\
\left({ }^{\circ} \mathbf{C}\right)\end{array}$ & $\begin{array}{c}\text { Heat } \\
\text { Treatment }\end{array}$ & Visual Observations & XRD Results \\
\hline \multirow[t]{2}{*}{ US-23 } & \multirow[t]{2}{*}{1150} & quenched & $\begin{array}{l}\text { Crucible - four spots of undissolved material } \\
\text { with bubbles; Patty - black, shiny, } \\
\text { homogeneous }\end{array}$ & amorphous \\
\hline & & $\mathrm{CCC}$ & $\begin{array}{l}\text { Surface - brown with metallic crystals; Bulk - } \\
\text { clean }\end{array}$ & $\mathrm{Fe}_{3} \mathrm{O}_{4}, \mathrm{NiFe}_{2} \mathrm{O}_{4}$ \\
\hline \multirow{2}{*}{ US-24 } & \multirow{2}{*}{1150} & quenched & $\begin{array}{l}\text { Crucible - three spots of undissolved material; } \\
\text { Patty - black, shiny, clean, homogeneous }\end{array}$ & amorphous \\
\hline & & $\mathrm{CCC}$ & $\begin{array}{l}\text { Surface - chocolate brown with crystals; Bulk - } \\
\text { clean }\end{array}$ & $\mathrm{Fe}_{3} \mathrm{O}_{4}, \mathrm{NiFe}_{2} \mathrm{O}_{4}$ \\
\hline \multirow{2}{*}{ US-25 } & \multirow{2}{*}{1150} & quenched & $\begin{array}{l}\text { Crucible - two spots of undissolved material; } \\
\text { Patty - clean, black, shiny, homogeneous }\end{array}$ & amorphous \\
\hline & & $\mathrm{CCC}$ & $\begin{array}{l}\text { Surface - hazy sheen; Bulk - crystals } \\
\text { throughout }\end{array}$ & $\mathrm{Fe}_{3} \mathrm{O}_{4}, \mathrm{NiFe}_{2} \mathrm{O}_{4}$ \\
\hline \multirow{2}{*}{ US-26 } & \multirow{2}{*}{1200} & quenched & $\begin{array}{l}\text { Crucible - green glass with some undissolved } \\
\text { material; Patty - dark with some undissolved } \\
\text { solids and some metallic streaks }\end{array}$ & $\mathrm{Fe}_{3} \mathrm{O}_{4}$ \\
\hline & & $\mathrm{CCC}$ & $\begin{array}{l}\text { Surface - brown with pitted crystals; Bulk - } \\
\text { crystals }\end{array}$ & $\begin{array}{c}\mathrm{Fe}_{3} \mathrm{O}_{4}, \mathrm{NiFe}_{2} \mathrm{O}_{4} \\
\mathrm{NaAlSiO}_{4} \\
\end{array}$ \\
\hline \multirow{2}{*}{ US-27 } & \multirow{2}{*}{1150} & quenched & $\begin{array}{l}\text { Crucible - clean with bubbles; Patty - very light } \\
\text { amount of possible salt layer on surface }\end{array}$ & $\mathrm{Fe}_{3} \mathrm{O}_{4}$ \\
\hline & & $\mathrm{CCC}$ & $\begin{array}{l}\text { Surface - gold and yellow crystals; Bulk - } \\
\text { crystals throughout }\end{array}$ & $\begin{array}{c}\mathrm{Fe}_{3} \mathrm{O}_{4}, \mathrm{NiFe}_{2} \mathrm{O}_{4} \\
\mathrm{NaAlSiO}_{4}\end{array}$ \\
\hline \multirow{2}{*}{ US-28 } & \multirow{2}{*}{1150} & quenched & $\begin{array}{l}\text { Crucible - one spot of undissolved material; } \\
\text { Patty - black, shiny, clean, homogeneous }\end{array}$ & amorphous \\
\hline & & $\mathrm{CCC}$ & $\begin{array}{l}\text { Surface - broken bubbles in small section of } \\
\text { melt line, otherwise clean; Bulk - clean }\end{array}$ & amorphous \\
\hline \multirow{2}{*}{ US-29 } & \multirow{2}{*}{1150} & quenched & $\begin{array}{l}\text { Crucible - green glass with some darker green } \\
\text { on bottom; Patty - clean, homogeneous }\end{array}$ & amorphous \\
\hline & & $\mathrm{CCC}$ & $\begin{array}{l}\text { Surface - dark and shiny with five small spots } \\
\text { of crystals; Bulk - clean }\end{array}$ & amorphous \\
\hline \multirow[t]{2}{*}{ US-30 } & \multirow[t]{2}{*}{1200} & quenched & $\begin{array}{l}\text { Crucible - two spots of undissolved material; } \\
\text { Patty - shiny silver streaks on surface, bulk } \\
\text { clean }\end{array}$ & $\mathrm{Fe}_{3} \mathrm{O}_{4}$ \\
\hline & & $\mathrm{CCC}$ & $\begin{array}{l}\text { Surface - hazy sheen with clusters of crystals } \\
\text { along melt line; Bulk - crystals }\end{array}$ & $\mathrm{Fe}_{3} \mathrm{O}_{4}, \mathrm{MnFeO}_{4}$ \\
\hline \multirow{2}{*}{ US-31 } & \multirow{2}{*}{1150} & quenched & $\begin{array}{l}\text { Crucible - clean; Patty - black, shiny, a few } \\
\text { light brown surface streaks }\end{array}$ & amorphous \\
\hline & & $\mathrm{CCC}$ & $\begin{array}{l}\text { Surface - covered with metallic crystals; Bulk - } \\
\text { completely devitrified }\end{array}$ & $\mathrm{Fe}_{2} \mathrm{O}_{3}$ \\
\hline \multirow{2}{*}{ US-32 } & \multirow{2}{*}{1150} & quenched & $\begin{array}{l}\text { Crucible - clean with bubbles; Patty - black, } \\
\text { shiny, homogeneous }\end{array}$ & amorphous \\
\hline & & $\mathrm{CCC}$ & $\begin{array}{l}\text { Surface - black, shiny, homogeneous; Bulk - } \\
\text { clean }\end{array}$ & amorphous \\
\hline \multirow{2}{*}{ US-33 } & \multirow{2}{*}{1150} & quenched & $\begin{array}{l}\text { Crucible - clean; Patty - some silvery, hazy } \\
\text { swirls on surface, bulk black and shiny }\end{array}$ & $\mathrm{Fe}_{3} \mathrm{O}_{4}$ \\
\hline & & $\mathrm{CCC}$ & $\begin{array}{l}\text { Surface - covered with silvery/goldish swirls of } \\
\text { crystals; Bulk - crystals }\end{array}$ & $\mathrm{Fe}_{3} \mathrm{O}_{4}, \mathrm{NiFe}_{2} \mathrm{O}_{4}$ \\
\hline \multirow[b]{2}{*}{ US-34 } & \multirow[b]{2}{*}{1150} & quenched & Crucible - clean; Patty - clean & amorphous \\
\hline & & $\mathrm{CCC}$ & $\begin{array}{l}\text { Surface - covered with shiny crystals; Bulk - } \\
\text { crystals }\end{array}$ & amorphous \\
\hline
\end{tabular}


Table 5-1. Visual Observations and XRD Results for the US-Series Glasses. (continued)

\begin{tabular}{|c|c|c|c|c|}
\hline Glass & $\begin{array}{l}\mathbf{T}_{\text {Melt }} \\
\left({ }^{\circ} \mathbf{C}\right)\end{array}$ & $\begin{array}{c}\text { Heat } \\
\text { Treatment }\end{array}$ & Visual Observations & XRD Results \\
\hline \multirow{2}{*}{ US-35 } & \multirow{2}{*}{1200} & quenched & $\begin{array}{l}\text { Crucible - clean with bubbles; Patty - black, } \\
\text { shiny, homogeneous }\end{array}$ & $\mathrm{Fe}_{3} \mathrm{O}_{4}$ \\
\hline & & $\mathrm{CCC}$ & $\begin{array}{l}\text { Surface - covered with shiny crystalline haze; } \\
\text { Bulk - crystals }\end{array}$ & $(\mathrm{Zn}, \mathrm{Mn}, \mathrm{Fe})(\mathrm{Fe}, \mathrm{Mn})_{2} \mathrm{O}_{4}$ \\
\hline \multirow{2}{*}{ US-36 } & \multirow{2}{*}{1150} & quenched & $\begin{array}{l}\text { Crucible - a few silver streaks; Patty - black, } \\
\text { shiny with some silver swirls on surface }\end{array}$ & $\mathrm{NiFe}_{2} \mathrm{O}_{4}$ \\
\hline & & $\mathrm{CCC}$ & $\begin{array}{l}\text { Surface - covered with shiny crystals, some dull } \\
\text { crystals along melt line; Bulk - crystals }\end{array}$ & $\mathrm{Fe}_{3} \mathrm{O}_{4}, \mathrm{NiFe}_{2} \mathrm{O}_{4}$ \\
\hline \multirow{2}{*}{ US-37 } & \multirow{2}{*}{1150} & quenched & $\begin{array}{l}\text { Crucible - clean with bubbles; Patty - black, } \\
\text { shiny, homogeneous }\end{array}$ & amorphous \\
\hline & & $\mathrm{CCC}$ & $\begin{array}{l}\text { Surface - dull, reddish/brown crystals; Bulk - } \\
\text { devitrified }\end{array}$ & $\mathrm{NaAlSiO}_{4}, \mathrm{Fe}_{3} \mathrm{O}_{4}$ \\
\hline \multirow[t]{2}{*}{ US-38 } & \multirow[t]{2}{*}{1150} & quenched & $\begin{array}{l}\text { Crucible - clean with bubbles; Patty - milky } \\
\text { haze on surface, bulk black, shiny and clean }\end{array}$ & amorphous \\
\hline & & $\mathrm{CCC}$ & Surface - matte finish; Bulk - crystals & $\mathrm{NiO}, \mathrm{Fe}_{3} \mathrm{O}_{4}$ \\
\hline \multirow{2}{*}{ US-39 } & \multirow{2}{*}{1150} & quenched & $\begin{array}{l}\text { Crucible - clean; Patty - black, shiny, } \\
\text { homogeneous }\end{array}$ & amorphous \\
\hline & & $\mathrm{CCC}$ & $\begin{array}{l}\text { Surface - chocolate brown crystals; Bulk - } \\
\text { chocolate brown color with crystals }\end{array}$ & $\mathrm{Fe}_{3} \mathrm{O}_{4}, \mathrm{NiFe}_{2} \mathrm{O}_{4}$ \\
\hline \multirow[t]{2}{*}{ US-40 } & \multirow[t]{2}{*}{1150} & quenched & $\begin{array}{l}\text { Crucible - clean; Patty - dark brown surface, } \\
\text { bulk was a lighter brown with a thin layer of } \\
\text { black shiny glass on the bottom }\end{array}$ & $\mathrm{Fe}_{2} \mathrm{O}_{3}, \mathrm{Fe}_{3} \mathrm{O}_{4}$ \\
\hline & & $\mathrm{CCC}$ & $\begin{array}{l}\text { Surface - shiny crystals under surface; Bulk - } \\
\text { crystals }\end{array}$ & $\mathrm{Fe}_{2} \mathrm{O}_{3}$ \\
\hline \multirow{2}{*}{ US-41 } & \multirow{2}{*}{1150} & quenched & $\begin{array}{l}\text { Crucible - clean; Patty - black, shiny, } \\
\text { homogeneous }\end{array}$ & amorphous \\
\hline & & $\mathrm{CCC}$ & $\begin{array}{l}\text { Surface - Purple, sparkly crystals, pitted; Bulk - } \\
\text { shiny crystals throughout }\end{array}$ & $\mathrm{Fe}_{2} \mathrm{O}_{3}$ \\
\hline \multirow[t]{2}{*}{ US-42 } & \multirow[t]{2}{*}{1150} & quenched & $\begin{array}{l}\text { Crucible - haze on surface, no undissolved; } \\
\text { Patty - dull, brownish haze on surface, bulk } \\
\text { clean }\end{array}$ & amorphous \\
\hline & & $\mathrm{CCC}$ & $\begin{array}{l}\text { Surface - covered with shiny/reddish crystals; } \\
\text { Bulk - completely devitrified }\end{array}$ & nepheline \\
\hline \multirow{2}{*}{ US-43 } & \multirow{2}{*}{1150} & quenched & $\begin{array}{l}\text { Crucible - light beige streaks on surface; Patty - } \\
\text { shiny brown with a few beige streaks }\end{array}$ & amorphous \\
\hline & & $\mathrm{CCC}$ & $\begin{array}{l}\text { Surface - dull, matte, covered with crystals; } \\
\text { Bulk - devitrified }\end{array}$ & $\begin{array}{c}\mathrm{Na}_{6.65} \mathrm{Al}_{6.24} \mathrm{Si}_{9.76} \mathrm{O}_{32} \\
\mathrm{Fe}_{2} \mathrm{O}_{3} \\
\end{array}$ \\
\hline \multirow{2}{*}{ US-44 } & \multirow{2}{*}{1150} & quenched & $\begin{array}{l}\text { Crucible - two spots of undissolved material; } \\
\text { Patty - dark, shiny, homogeneous }\end{array}$ & amorphous \\
\hline & & $\mathrm{CCC}$ & $\begin{array}{l}\text { Surface - black, shiny, a few tiny spots of } \\
\text { crystals; Bulk - crystals }\end{array}$ & $\mathrm{Fe}_{3} \mathrm{O}_{4}$ \\
\hline \multirow{2}{*}{ US-45 } & \multirow{2}{*}{1150} & quenched & $\begin{array}{l}\text { Crucible - three spots of undissolved material; } \\
\text { Patty - dark, shiny, homogeneous }\end{array}$ & amorphous \\
\hline & & $\mathrm{CCC}$ & $\begin{array}{l}\text { Surface - Black, shiny, a few tiny spots of } \\
\text { crystals; Bulk - crystals }\end{array}$ & $\mathrm{Fe}_{3} \mathrm{O}_{4}$ \\
\hline
\end{tabular}




\subsection{Chemical Composition Analysis}

Chemical composition measurements for these glasses were conducted by the SRNL PSAL following an analytical plan. ${ }^{6}$ The elemental concentrations were converted to oxide concentrations by multiplying the values for each element by the gravimetric factor for the corresponding oxide. During this process, each elemental concentration that was determined to be below the detection limit of the analytical procedures used by the PSAL was reduced to half of that detection limit as the oxide concentration was determined.

As detailed in the summary by Edwards, ${ }^{14}$ the oxide measurements of the study glasses were bias corrected for the effect of the ICP-AES calibration on each of the analytical blocks. The four measurements for each oxide for each glass (over both preparation methods) were averaged to determine a representative chemical composition for each glass. These determinations were conducted both for the measured and for the bias-corrected data. A sum of oxides was also computed for each glass based upon both the measured and bias-corrected values.

Table 5-2 provides a summary of the average, bias-corrected concentration of each oxide for each glass. All of the sums of oxides for the study glasses fall within the interval of 95 to $105 \mathrm{wt} \%$, indicating good confidence in the analyses. Table 5-2 also provides the percent difference (when the targeted concentration for the oxide is at least $0.5 \mathrm{wt} \%$ ) between the measured bias-corrected concentrations and the targeted concentrations. A review of the individual oxides for each glass reveals that there were no errors in batching significant enough to impact the outcome of the study.

The chemical compositions of glasses US-44 and US-45 can be compared as a measure of repeatability of the batching process since these glasses were duplicates; that is, they had the same target compositions. A comparison indicates an acceptable degree of repeatability as the percent differences for most of the oxides are less than $5 \%$ and percent differences for all of the oxides are less than $10 \mathrm{wt} \%$. 
Table 5-2. Bias-Corrected Chemical Composition Data for the US-Series Glasses and Percent Differences from the Target Values.

\begin{tabular}{|c|c|c|c|c|c|c|c|c|c|c|c|c|c|c|c|c|c|c|c|}
\hline $\begin{array}{c}\text { Glass } \\
\text { ID }\end{array}$ & $\mathrm{Al}_{2} \mathrm{O}_{3}$ & $\mathrm{~B}_{2} \mathrm{O}_{3}$ & $\mathrm{CaO}$ & $\mathrm{Cr}_{2} \mathrm{O}_{3}$ & $\mathrm{Fe}_{2} \mathrm{O}_{3}$ & $\mathrm{~K}_{2} \mathrm{O}$ & $\mathrm{Li}_{2} \mathrm{O}$ & MgO & MnO & $\mathrm{Na}_{2} \mathrm{O}$ & $\mathrm{NiO}$ & $\mathrm{PbO}$ & $\mathrm{SiO}_{2}$ & $\mathrm{SO}_{3}$ & Sro & $\mathrm{TiO}_{2}$ & Zno & $\mathrm{ZrO}_{2}$ & Sum \\
\hline \multirow{2}{*}{ US-01 } & 15.389 & 8.190 & 0.131 & 0.265 & 8.811 & 0.884 & 5.712 & 0.559 & 2.674 & 13.799 & 0.062 & 0.060 & 44.045 & 0.062 & 0.090 & 0.014 & 0.006 & 0.463 & 101.216 \\
\hline & $3.10 \%$ & $3.80 \%$ & & & $2.60 \%$ & $-11.6 \%$ & $1.70 \%$ & & $7.10 \%$ & $11.50 \%$ & & & $-3.30 \%$ & & & & & & $1.20 \%$ \\
\hline \multirow{2}{*}{ US-02 } & 16.355 & 16.043 & 0.069 & 0.024 & 6.561 & 0.053 & 5.137 & 0.009 & 0.617 & 12.660 & 0.021 & 0.005 & 40.456 & 0.062 & 0.036 & 0.068 & 0.123 & 0.458 & 98.759 \\
\hline & $2.20 \%$ & $-4.80 \%$ & & & $-0.40 \%$ & & $-2.90 \%$ & & $-2.70 \%$ & $2.60 \%$ & & & $-2.90 \%$ & & & & & & $-1.20 \%$ \\
\hline \multirow{2}{*}{ US-03 } & 16.359 & 17.213 & 1.010 & 0.016 & 6.649 & 0.839 & 4.071 & 0.543 & 0.068 & 8.272 & 0.307 & 0.093 & 44.120 & 0.062 & 0.088 & 1.025 & 0.006 & 0.007 & 100.747 \\
\hline & $2.30 \%$ & $-0.60 \%$ & $1.10 \%$ & & $1.90 \%$ & $-16.1 \%$ & $0.00 \%$ & & & $4.90 \%$ & & & $-0.20 \%$ & & & $2.70 \%$ & & & $0.80 \%$ \\
\hline \multirow{2}{*}{ US-04 } & 16.676 & 11.158 & 0.987 & 0.017 & 7.978 & 0.837 & 3.788 & 0.535 & 2.298 & 11.108 & 0.007 & 0.090 & 42.575 & 0.396 & 0.089 & 1.007 & 0.095 & 0.430 & 100.071 \\
\hline & $4.30 \%$ & $-1.10 \%$ & $-1.20 \%$ & & $-3.50 \%$ & $-16.2 \%$ & $1.20 \%$ & & $5.00 \%$ & $7.50 \%$ & & & $-1.90 \%$ & & & $0.90 \%$ & & & $0.10 \%$ \\
\hline \multirow{2}{*}{ US-05 } & 16.456 & 16.370 & 0.696 & 0.017 & 7.948 & 0.885 & 2.020 & 0.539 & 2.545 & 10.162 & 0.007 & 0.091 & 39.287 & 0.284 & 0.113 & 1.061 & 0.097 & 0.495 & 99.072 \\
\hline & $2.90 \%$ & $-2.00 \%$ & $-1.00 \%$ & & $-2.60 \%$ & $-11.4 \%$ & $1.00 \%$ & & $5.70 \%$ & $6.10 \%$ & & & $-3.30 \%$ & & & $6.40 \%$ & & & $-0.90 \%$ \\
\hline \multirow{2}{*}{ US-06 } & 16.523 & 13.986 & 0.984 & 0.018 & 7.925 & 0.880 & 2.513 & 0.537 & 3.223 & 10.564 & 0.007 & 0.005 & 42.120 & 0.351 & 0.107 & 0.982 & 0.018 & 0.024 & 100.765 \\
\hline & $3.30 \%$ & $-0.60 \%$ & $-1.50 \%$ & & $-2.80 \%$ & $-11.9 \%$ & $1.60 \%$ & & $7.50 \%$ & $-2.00 \%$ & & & $1.70 \%$ & & & $-1.70 \%$ & & & $0.80 \%$ \\
\hline \multirow{2}{*}{ US-07 } & 16.305 & 13.114 & 0.981 & 0.013 & 6.767 & 0.850 & 2.598 & 0.540 & 3.086 & 11.082 & 0.208 & 0.090 & 43.442 & 0.360 & 0.087 & 1.011 & 0.097 & 0.423 & 101.054 \\
\hline & $1.90 \%$ & $-2.80 \%$ & $-1.80 \%$ & & $-7.30 \%$ & $-14.9 \%$ & $-2.30 \%$ & & $2.90 \%$ & $3.90 \%$ & & & $3.70 \%$ & & & $1.20 \%$ & & & $1.10 \%$ \\
\hline \multirow{2}{*}{ US-08 } & 15.825 & 9.577 & 0.132 & 0.040 & 9.083 & 0.832 & 4.926 & 0.051 & 2.363 & 10.143 & 0.007 & 0.082 & 44.050 & 0.380 & 0.180 & 0.938 & 0.133 & 0.154 & 98.894 \\
\hline & $4.20 \%$ & $0.20 \%$ & & & $-6.80 \%$ & $-16.8 \%$ & $1.30 \%$ & & $-3.10 \%$ & $3.40 \%$ & & & $-3.80 \%$ & & & $-6.10 \%$ & & & $-1.10 \%$ \\
\hline \multirow{2}{*}{ US-09 } & 14.037 & 15.097 & 0.956 & 0.223 & 10.602 & 0.053 & 2.087 & 0.541 & 0.069 & 12.911 & 0.007 & 0.089 & 41.899 & 0.390 & 0.016 & 1.013 & 0.006 & 0.007 & 100.003 \\
\hline & $5.00 \%$ & $0.30 \%$ & $-4.40 \%$ & & $-2.60 \%$ & & $4.30 \%$ & & & $-8.50 \%$ & & & $1.80 \%$ & & & $1.30 \%$ & & & $0.00 \%$ \\
\hline \multirow{2}{*}{ US-10 } & 14.693 & 11.104 & 0.998 & 0.017 & 10.257 & 0.879 & 2.085 & 0.540 & 2.893 & 12.992 & 0.007 & 0.088 & 40.747 & 0.398 & 0.090 & 1.022 & 0.099 & 0.437 & 99.347 \\
\hline & $4.00 \%$ & $1.00 \%$ & $0.00 \%$ & & $-4.00 \%$ & $-12.0 \%$ & $4.20 \%$ & & $5.70 \%$ & $2.50 \%$ & & & $-2.90 \%$ & & & $2.40 \%$ & & & $-0.60 \%$ \\
\hline \multirow{2}{*}{ US-11 } & 14.713 & 20.008 & 1.036 & 0.015 & 7.014 & 0.867 & 2.057 & 0.555 & 3.284 & 6.916 & 0.007 & 0.093 & 45.619 & 0.249 & 0.006 & 0.009 & 0.102 & 0.012 & 102.561 \\
\hline & $7.40 \%$ & $0.20 \%$ & $3.60 \%$ & & $-9.60 \%$ & $-13.3 \%$ & $2.90 \%$ & & $9.50 \%$ & $3.40 \%$ & & & $4.50 \%$ & & & & & & $2.60 \%$ \\
\hline \multirow{2}{*}{ US-12 } & 13.938 & 15.884 & 1.053 & 0.117 & 8.935 & 0.905 & 6.066 & 0.009 & 0.068 & 12.482 & 1.944 & 0.005 & 40.564 & 0.456 & 0.094 & 0.547 & 0.111 & 0.439 & 103.617 \\
\hline & $5.30 \%$ & $-1.20 \%$ & $5.30 \%$ & & $2.20 \%$ & $-9.50 \%$ & $1.10 \%$ & & & $11.90 \%$ & $5.80 \%$ & & $3.70 \%$ & & & & & & $3.60 \%$ \\
\hline \multirow{2}{*}{ US-13 } & 16.547 & 14.553 & 0.995 & 0.020 & 7.050 & 0.847 & 2.220 & 0.542 & 2.987 & 12.006 & 0.294 & 0.088 & 43.101 & 0.062 & 0.006 & 1.021 & 0.102 & 0.036 & 102.477 \\
\hline & $3.40 \%$ & $-0.10 \%$ & $-0.40 \%$ & & $-3.40 \%$ & $-15.2 \%$ & $3.00 \%$ & & $6.70 \%$ & $4.10 \%$ & & & $3.50 \%$ & & & $2.20 \%$ & & & $2.50 \%$ \\
\hline \multirow{2}{*}{ US-14 } & 13.587 & 17.685 & 0.465 & 0.009 & 10.051 & 0.865 & 5.810 & 0.538 & 2.718 & 11.160 & 0.007 & 0.063 & 37.117 & 0.062 & 0.006 & 1.030 & 0.107 & 0.459 & 101.739 \\
\hline & $4.40 \%$ & $-1.20 \%$ & & & $6.80 \%$ & $-13.5 \%$ & $1.20 \%$ & & $4.60 \%$ & $-0.60 \%$ & & & $1.60 \%$ & & & $3.10 \%$ & & & $1.80 \%$ \\
\hline \multirow{2}{*}{ US-15 } & 10.537 & 17.897 & 0.609 & 0.227 & 5.132 & 0.845 & 2.030 & 0.009 & 0.068 & 15.674 & 0.007 & 0.089 & 46.591 & 0.408 & 0.090 & 0.996 & 0.098 & 0.024 & 101.330 \\
\hline & $5.40 \%$ & $0.60 \%$ & & & $2.70 \%$ & $-15.5 \%$ & $1.50 \%$ & & & $4.50 \%$ & & & $0.00 \%$ & & & $-0.40 \%$ & & & $1.40 \%$ \\
\hline \multirow{2}{*}{ US-16 } & 13.889 & 18.816 & 0.997 & 0.080 & 8.856 & 0.894 & 2.598 & 0.009 & 2.634 & 6.690 & 0.015 & 0.005 & 43.370 & 0.062 & 0.006 & 1.029 & 0.006 & 0.486 & 100.442 \\
\hline & $4.30 \%$ & $0.00 \%$ & $-0.30 \%$ & & $-1.90 \%$ & $-10.5 \%$ & $1.70 \%$ & & $6.10 \%$ & $6.20 \%$ & & & $-1.20 \%$ & & & $2.90 \%$ & & & $0.50 \%$ \\
\hline \multirow{2}{*}{ US-17 } & 11.942 & 11.287 & 0.073 & 0.087 & 7.303 & 0.053 & 5.227 & 0.533 & 3.071 & 8.867 & 1.097 & 0.005 & 47.124 & 0.062 & 0.085 & 1.016 & 0.104 & 0.409 & 98.345 \\
\hline & $2.50 \%$ & $-4.10 \%$ & & & $17.40 \%$ & & $-2.50 \%$ & & $2.40 \%$ & $3.30 \%$ & $1.20 \%$ & & $-5.70 \%$ & & & $1.60 \%$ & & & $-1.60 \%$ \\
\hline \multirow{2}{*}{ US-18 } & 16.864 & 9.267 & 0.925 & 0.011 & 9.544 & 0.053 & 4.998 & 0.009 & 3.240 & 12.607 & 0.007 & 0.090 & 43.066 & 0.062 & 0.096 & 1.030 & 0.112 & 0.006 & 101.989 \\
\hline & $5.40 \%$ & $1.50 \%$ & $-7.40 \%$ & & $-2.10 \%$ & & $0.90 \%$ & & $8.00 \%$ & $-6.00 \%$ & & & $3.90 \%$ & & & $3.00 \%$ & & & $2.00 \%$ \\
\hline US-19 & 14.877 & 13.961 & 0.730 & 0.055 & 7.923 & 0.689 & 3.647 & 0.364 & 2.008 & 11.245 & 0.209 & 0.066 & 40.951 & 0.263 & 0.060 & 0.836 & 0.067 & 0.227 & 98.179 \\
\hline US-19 & $2.80 \%$ & $-3.10 \%$ & $4.30 \%$ & & $-3.70 \%$ & $-11.3 \%$ & $0.30 \%$ & & $1.00 \%$ & $4.10 \%$ & & & $-4.30 \%$ & & & $3.90 \%$ & & & $-1.80 \%$ \\
\hline US-20 & 14.848 & 14.014 & 0.816 & 0.065 & 8.327 & 0.672 & 3.625 & 0.362 & 2.059 & 11.143 & 0.214 & 0.065 & 41.109 & 0.254 & 0.058 & 0.835 & 0.072 & 0.278 & 98.814 \\
\hline US-20 & $2.60 \%$ & $-2.70 \%$ & $16.70 \%$ & & $1.20 \%$ & $-13.5 \%$ & $-0.30 \%$ & & $3.60 \%$ & $3.20 \%$ & & & $-4.00 \%$ & & & $3.70 \%$ & & & $-1.20 \%$ \\
\hline & 15.158 & 13.697 & 0.405 & 0.058 & 7.914 & 0.639 & 3.095 & 0.404 & 1.848 & 11.347 & 0.489 & 0.075 & 46.106 & 0.127 & 0.075 & 0.558 & 0.037 & 0.103 & 102.134 \\
\hline US-21 & $6.60 \%$ & $-0.40 \%$ & & & $-6.40 \%$ & $-14.7 \%$ & $3.10 \%$ & & $3.90 \%$ & $5.50 \%$ & $-2.30 \%$ & & $2.60 \%$ & & & $2.80 \%$ & & & $2.20 \%$ \\
\hline
\end{tabular}


Table 5-2. Bias-Corrected Chemical Composition Data for the US-Series Glasses and Percent Differences from the Target Values. (continued)

\begin{tabular}{|c|c|c|c|c|c|c|c|c|c|c|c|c|c|c|c|c|c|c|c|}
\hline $\begin{array}{c}\text { Glass } \\
\text { ID }\end{array}$ & $\mathrm{Al}_{2} \mathrm{O}_{3}$ & $\mathrm{~B}_{2} \mathrm{O}_{3}$ & $\mathrm{CaO}$ & $\mathrm{Cr}_{2} \mathrm{O}_{3}$ & $\mathrm{Fe}_{2} \mathrm{O}_{3}$ & $\mathrm{~K}_{2} \mathrm{O}$ & $\mathrm{Li}_{2} \mathrm{O}$ & MgO & MnO & $\mathrm{Na}_{2} \mathrm{O}$ & $\mathrm{NiO}$ & $\mathrm{PbO}$ & $\mathrm{SiO}_{2}$ & $\mathrm{SO}_{3}$ & SrO & $\mathrm{TiO}_{2}$ & $\mathrm{ZnO}$ & $\mathrm{ZrO}_{2}$ & Sum \\
\hline \multirow{2}{*}{ US-22 } & 13.878 & 11.602 & 0.768 & 0.078 & 9.440 & 0.650 & 5.025 & 0.416 & 2.316 & 13.016 & 0.947 & 0.030 & 42.670 & 0.140 & 0.071 & 0.797 & 0.087 & 0.123 & 102.053 \\
\hline & $4.00 \%$ & $-0.70 \%$ & $2.60 \%$ & & $-0.30 \%$ & $-13.2 \%$ & $0.50 \%$ & & $3.00 \%$ & $10.50 \%$ & $3.10 \%$ & & $0.60 \%$ & & & $6.40 \%$ & & & $2.10 \%$ \\
\hline \multirow{2}{*}{ US-23 } & 14.860 & 13.111 & 0.746 & 0.065 & 6.941 & 0.223 & 3.488 & 0.137 & 2.350 & 12.454 & 0.505 & 0.078 & 44.318 & 0.331 & 0.030 & 0.273 & 0.091 & 0.137 & 100.139 \\
\hline & $2.50 \%$ & $-1.60 \%$ & $-0.50 \%$ & & $-8.90 \%$ & & $-0.30 \%$ & & $4.50 \%$ & $6.60 \%$ & & & $-0.30 \%$ & & & & & & $0.20 \%$ \\
\hline \multirow{2}{*}{ US-24 } & 15.252 & 12.258 & 0.768 & 0.057 & 7.602 & 0.669 & 3.145 & 0.399 & 2.454 & 12.201 & 0.502 & 0.077 & 43.185 & 0.325 & 0.072 & 0.759 & 0.082 & 0.384 & 100.189 \\
\hline & $5.20 \%$ & $0.10 \%$ & $2.60 \%$ & & $-2.40 \%$ & $-10.6 \%$ & $4.80 \%$ & & $9.10 \%$ & $5.40 \%$ & $0.10 \%$ & & $-2.80 \%$ & & & $1.30 \%$ & & & $0.20 \%$ \\
\hline \multirow{2}{*}{ US-25 } & 12.279 & 16.128 & 0.781 & 0.145 & 8.434 & 0.270 & 5.036 & 0.406 & 2.219 & 11.622 & 1.412 & 0.031 & 40.043 & 0.337 & 0.082 & 0.793 & 0.105 & 0.361 & 100.483 \\
\hline & $6.00 \%$ & $-0.70 \%$ & $4.20 \%$ & & $-1.20 \%$ & & $0.70 \%$ & & $2.50 \%$ & $6.80 \%$ & $-2.60 \%$ & & $-1.80 \%$ & & & $5.70 \%$ & & & $0.50 \%$ \\
\hline \multirow{2}{*}{ US-26 } & 19.201 & 10.429 & 1.003 & 0.402 & 5.114 & 0.053 & 5.923 & 0.522 & 0.068 & 11.285 & 0.007 & 0.005 & 38.642 & 0.062 & 2.528 & 1.002 & 1.696 & 2.040 & 99.982 \\
\hline & $2.00 \%$ & $-2.40 \%$ & $0.30 \%$ & $-26.7 \%$ & $2.30 \%$ & & $-1.30 \%$ & & & $2.40 \%$ & & & $1.50 \%$ & & $-15.7 \%$ & $0.20 \%$ & $-15.2 \%$ & $-13.2 \%$ & $0.00 \%$ \\
\hline \multirow{2}{*}{ US-27 } & 10.812 & 6.676 & 0.030 & 0.820 & 15.146 & 0.905 & 5.879 & 0.530 & 0.068 & 17.868 & 0.007 & 0.019 & 39.803 & 0.483 & 0.006 & 0.011 & 1.733 & 0.420 & 101.217 \\
\hline & $3.90 \%$ & $3.00 \%$ & & $-18.0 \%$ & $-0.20 \%$ & $-9.40 \%$ & $-2.00 \%$ & & & $5.90 \%$ & & & $-0.60 \%$ & & & & $-13.4 \%$ & & $1.20 \%$ \\
\hline \multirow{2}{*}{ US-28 } & 17.717 & 17.637 & 0.038 & 0.007 & 5.618 & 0.841 & 2.051 & 0.009 & 0.067 & 13.709 & 0.007 & 0.005 & 36.896 & 0.381 & 2.481 & 1.019 & 1.758 & 0.013 & 100.254 \\
\hline & $1.40 \%$ & $-1.80 \%$ & & & $-1.80 \%$ & -15.80 & $2.60 \%$ & & & $2.70 \%$ & & & $2.60 \%$ & & $-17.3 \%$ & $1.90 \%$ & $-12.1 \%$ & & $0.30 \%$ \\
\hline \multirow{2}{*}{ US-29 } & 10.441 & 8.543 & 1.012 & 0.542 & 4.875 & 0.874 & 5.934 & 0.537 & 0.068 & 18.814 & 0.007 & 1.301 & 44.137 & 0.062 & 1.076 & 0.979 & 0.008 & 0.007 & 99.214 \\
\hline & $4.40 \%$ & $-2.10 \%$ & $1.20 \%$ & -19.30 & $-2.50 \%$ & $-12.6 \%$ & $-1.10 \%$ & & & $0.70 \%$ & & $30.10 \%$ & $-2.50 \%$ & & $-8.50 \%$ & $1.50 \%$ & & & $-0.80 \%$ \\
\hline \multirow{2}{*}{ US-30 } & 13.165 & 18.374 & 1.053 & 0.569 & 5.403 & 0.879 & 6.053 & 0.009 & 3.752 & 5.453 & 0.007 & 0.005 & 39.059 & 0.458 & 2.690 & 1.050 & 0.454 & 3.576 & 102.009 \\
\hline & $4.10 \%$ & $1.00 \%$ & $5.40 \%$ & $-30.5 \%$ & $1.40 \%$ & $-12.0 \%$ & $0.90 \%$ & & $7.30 \%$ & $9.10 \%$ & & & $4.10 \%$ & & $-10.3 \%$ & $5.00 \%$ & & $-10.6 \%$ & $2.00 \%$ \\
\hline \multirow{2}{*}{ US-31 } & 13.472 & 14.224 & 0.062 & 0.013 & 14.859 & 0.053 & 4.992 & 0.518 & 0.777 & 8.022 & 0.025 & 0.877 & 40.030 & 0.368 & 2.537 & 1.027 & 0.006 & 0.011 & 101.874 \\
\hline & $6.40 \%$ & $-2.00 \%$ & & & $-3.80 \%$ & & $1.70 \%$ & & $4.80 \%$ & $13.00 \%$ & & $-12.2 \%$ & $3.70 \%$ & & $-15.4 \%$ & $2.70 \%$ & & & $1.90 \%$ \\
\hline \multirow{2}{*}{ US-32 } & 10.653 & 18.938 & 1.014 & 0.016 & 5.045 & 0.053 & 6.268 & 0.009 & 4.594 & 7.829 & 0.007 & 0.894 & 44.534 & 0.411 & 0.006 & 0.009 & 1.773 & 0.044 & 102.097 \\
\hline & $6.50 \%$ & $-0.40 \%$ & $1.40 \%$ & & $0.90 \%$ & & $4.50 \%$ & & $14.90 \%$ & $4.40 \%$ & & $-10.5 \%$ & $1.30 \%$ & & & & $-11.3 \%$ & & $2.10 \%$ \\
\hline \multirow{2}{*}{ US-33 } & 12.205 & 5.355 & 0.038 & 0.708 & 5.033 & 0.053 & 2.089 & 0.577 & 4.460 & 19.458 & 0.992 & 0.900 & 47.795 & 0.506 & 2.608 & 0.009 & 0.120 & 0.006 & 102.912 \\
\hline & $7.60 \%$ & $7.10 \%$ & & $-29.2 \%$ & $0.70 \%$ & & $4.50 \%$ & & $11.50 \%$ & $-1.50 \%$ & $2.80 \%$ & $-10.0 \%$ & $4.10 \%$ & & $-13.1 \%$ & & & & $2.90 \%$ \\
\hline \multirow{2}{*}{ US-34 } & 10.492 & 13.621 & 0.034 & 0.009 & 6.398 & 0.929 & 2.442 & 0.537 & 4.294 & 19.339 & 0.007 & 0.005 & 36.505 & 0.062 & 2.685 & 0.009 & 0.006 & 3.505 & 100.879 \\
\hline & $4.90 \%$ & $-0.50 \%$ & & & $0.20 \%$ & $-7.10 \%$ & $0.90 \%$ & & $7.40 \%$ & $-3.30 \%$ & & & $4.30 \%$ & & $-10.5 \%$ & & & $-12.3 \%$ & $0.90 \%$ \\
\hline \multirow{2}{*}{ US-35 } & 10.482 & 19.694 & 1.022 & 0.428 & 11.344 & 0.053 & 2.061 & 0.537 & 4.304 & 8.447 & 0.012 & 0.005 & 38.785 & 0.062 & 2.339 & 0.009 & 1.756 & 0.009 & 101.350 \\
\hline & $4.70 \%$ & $-1.30 \%$ & $2.30 \%$ & $-17.3 \%$ & $-0.10 \%$ & & $3.10 \%$ & & $7.60 \%$ & $6.40 \%$ & & & $2.10 \%$ & & $-13.5 \%$ & & $-12.2 \%$ & & $1.40 \%$ \\
\hline \multirow{2}{*}{ US-36 } & 13.117 & 15.337 & 0.385 & 0.383 & 9.734 & 0.494 & 2.439 & 0.049 & 1.174 & 13.355 & 0.553 & 0.918 & 38.895 & 0.062 & 0.006 & 0.025 & 0.014 & 3.472 & 100.409 \\
\hline & $2.00 \%$ & $-1.10 \%$ & & $-25.1 \%$ & $-0.30 \%$ & $-4.60 \%$ & $0.70 \%$ & & $-2.60 \%$ & $6.70 \%$ & $-1.00 \%$ & $-8.20 \%$ & $0.10 \%$ & & & & & $-13.2 \%$ & $0.40 \%$ \\
\hline \multirow{2}{*}{ US-37 } & 10.711 & 6.196 & 1.013 & 0.009 & 17.569 & 0.053 & 2.049 & 0.009 & 0.068 & 19.151 & 0.013 & 0.881 & 38.701 & 0.062 & 0.041 & 1.020 & 1.746 & 3.431 & 102.724 \\
\hline & $7.10 \%$ & $7.40 \%$ & $1.30 \%$ & & $3.40 \%$ & & $2.40 \%$ & & & $1.40 \%$ & & $-11.8 \%$ & $3.70 \%$ & & & $2.00 \%$ & $-12.7 \%$ & $-14.2 \%$ & $2.70 \%$ \\
\hline \multirow{2}{*}{ US-38 } & 10.468 & 7.361 & 0.798 & 0.013 & 5.477 & 0.886 & 2.083 & 0.009 & 4.204 & 18.343 & 3.165 & 0.883 & 40.195 & 0.483 & 2.649 & 1.011 & 1.764 & 0.019 & 99.810 \\
\hline & $4.70 \%$ & $0.60 \%$ & $1.10 \%$ & & $9.60 \%$ & $-11.4 \%$ & $4.20 \%$ & & $5.10 \%$ & $-7.00 \%$ & $8.90 \%$ & $-11.7 \%$ & $1.10 \%$ & & $-11.7 \%$ & $1.10 \%$ & $-11.8 \%$ & & $-0.20 \%$ \\
\hline \multirow{2}{*}{ US-39 } & 12.079 & 15.852 & 0.023 & 0.011 & 7.325 & 0.927 & 5.993 & 0.538 & 0.068 & 5.006 & 1.915 & 0.911 & 40.840 & 0.062 & 0.729 & 0.009 & 1.785 & 3.526 & 97.596 \\
\hline & $4.60 \%$ & $-3.00 \%$ & & & $-2.40 \%$ & $-7.30 \%$ & $-0.10 \%$ & & & $0.10 \%$ & $4.70 \%$ & $-8.90 \%$ & $-3.80 \%$ & & $-8.60 \%$ & & $-10.7 \%$ & $-11.8 \%$ & $-2.40 \%$ \\
\hline US-40 & 16.088 & 16.968 & 0.051 & 0.308 & 11.157 & 0.887 & 2.554 & 0.009 & 2.945 & 10.661 & 0.007 & 0.921 & 38.486 & 0.062 & 0.006 & 0.945 & 0.012 & 0.007 & 102.072 \\
\hline US-40 & $3.70 \%$ & $-0.20 \%$ & & & $-2.30 \%$ & $-11.3 \%$ & $1.70 \%$ & & $6.70 \%$ & $5.20 \%$ & & $-7.80 \%$ & $3.20 \%$ & & & $2.40 \%$ & & & $2.10 \%$ \\
\hline US-41 & 10.238 & 15.224 & 1.019 & 0.015 & 14.818 & 0.883 & 3.707 & 0.009 & 0.069 & 6.884 & 0.013 & 0.894 & 41.681 & 0.062 & 2.593 & 0.009 & 0.006 & 0.013 & 98.137 \\
\hline $03-41$ & $2.40 \%$ & $-2.10 \%$ & $1.90 \%$ & & $-4.60 \%$ & -11.60 & $-0.50 \%$ & & & $5.60 \%$ & & $-10.6 \%$ & $-2.30 \%$ & & $-13.6 \%$ & & & & $-1.80 \%$ \\
\hline & 20.833 & 6.381 & 0.975 & 0.007 & 5.122 & 0.861 & 6.044 & 0.009 & 4.415 & 18.720 & 0.007 & 0.887 & 36.583 & 0.059 & 0.006 & 0.009 & 1.369 & 0.012 & 102.298 \\
\hline US-4L & $4.20 \%$ & $3.10 \%$ & $-2.50 \%$ & & $2.50 \%$ & $-13.9 \%$ & $0.70 \%$ & & $10.40 \%$ & $-0.50 \%$ & & $-11.3 \%$ & $3.10 \%$ & & & & $-8.00 \%$ & & $2.30 \%$ \\
\hline
\end{tabular}


Table 5-2. Bias-Corrected Chemical Composition Data for the US-Series Glasses and Percent Differences from the Target Values. (continued)

\begin{tabular}{|c|c|c|c|c|c|c|c|c|c|c|c|c|c|c|c|c|c|c|c|}
\hline $\begin{array}{c}\text { Glass } \\
\text { ID }\end{array}$ & $\mathrm{Al}_{2} \mathrm{O}_{3}$ & $\mathrm{~B}_{2} \mathrm{O}_{3}$ & $\mathrm{CaO}$ & $\mathrm{Cr}_{2} \mathrm{O}_{3}$ & $\mathrm{Fe}_{2} \mathrm{O}_{3}$ & $\mathrm{~K}_{2} \mathrm{O}$ & $\mathrm{Li}_{2} \mathrm{O}$ & MgO & $\mathrm{MnO}$ & $\mathrm{Na}_{2} \mathrm{O}$ & $\mathrm{NiO}$ & $\mathrm{PbO}$ & $\mathrm{SiO}_{2}$ & $\mathrm{SO}_{3}$ & SrO & $\mathrm{TiO}_{2}$ & Zno & $\mathrm{ZrO}_{2}$ & Sum \\
\hline \multirow{2}{*}{ US-43 } & 17.964 & 8.692 & 0.987 & 0.007 & 9.099 & 0.866 & 4.968 & 0.529 & 0.752 & 11.014 & 0.203 & 0.888 & 38.865 & 0.409 & 0.416 & 0.494 & 0.441 & 3.367 & 99.959 \\
\hline & $4.60 \%$ & $0.50 \%$ & $-1.30 \%$ & & $2.70 \%$ & $-13.3 \%$ & $1.30 \%$ & & $-7.40 \%$ & $7.00 \%$ & & $-11.2 \%$ & $-2.10 \%$ & & & & & $-15.8 \%$ & $0.00 \%$ \\
\hline \multirow{2}{*}{ US-44 } & 13.826 & 12.378 & 0.606 & 0.255 & 8.775 & 0.567 & 4.462 & 0.275 & 1.866 & 13.354 & 0.374 & 0.607 & 38.240 & 0.228 & 1.407 & 0.488 & 0.965 & 1.308 & 99.980 \\
\hline & $8.00 \%$ & $-1.70 \%$ & $9.20 \%$ & & $-1.00 \%$ & $-11.3 \%$ & $10.20 \%$ & & $1.70 \%$ & $4.90 \%$ & & $-8.90 \%$ & $-3.30 \%$ & & $-13.1 \%$ & & $-5.70 \%$ & $-10.7 \%$ & $0.00 \%$ \\
\hline \multirow{2}{*}{ US-45 } & 13.488 & 12.779 & 0.584 & 0.250 & 8.924 & 0.589 & 4.122 & 0.266 & 1.898 & 12.958 & 0.366 & 0.591 & 40.330 & 0.222 & 1.434 & 0.488 & 0.961 & 1.313 & 101.562 \\
\hline & $5.40 \%$ & $1.50 \%$ & $5.10 \%$ & & $0.70 \%$ & $-7.90 \%$ & $1.80 \%$ & & $3.50 \%$ & $1.80 \%$ & & $-11.3 \%$ & $2.00 \%$ & & $-11.5 \%$ & & $-6.20 \%$ & $-10.3 \%$ & $1.60 \%$ \\
\hline
\end{tabular}




\subsection{Chemical Durability Analysis}

The results of the PCT for each of the study glasses in terms of the normalized leachate (NL) releases for each of the four elements of interest are given in Table 5-3 as a function of heat treatment. All but two of the study glasses have normalized leachate for boron (NL [B]) values that are well below that of the EA reference glass $(16.695 \mathrm{~g} / \mathrm{L}) .{ }^{15}$ The two highest NL [B] values are for the CCC versions of glasses US-18 and US-27 $(10.498 \mathrm{~g} / \mathrm{L}$ and $15.962 \mathrm{~g} / \mathrm{L}$, respectively). Note the similarity of PCT results for US-44 and US-45. These two glasses had the same targeted composition, and their PCT results differ by less than $0.1 \mathrm{~g} / \mathrm{L}$ for any of the four elements over all of the compositional views and heat treatments, indicating good reproducibility in fabrication and characterization of the study glasses.

Nepheline crystallization was identified by qualitative XRD in five of the US-series glasses (see Section 5.1). Each of these five glasses (US-18, US-26, US-27, US-37 and US-43) shows a significant increase in NL [B] after the CCC heat treatment. This reduction in durability can be attributed to the formation of nepheline during the slow cooling cycle and the removal of glass formers from the residual glass network.

Five other glasses in the study showed elevated NL [B] values after both quenching and the CCC heat treatment (glasses US-14, US-15, US-32, US-34 and US-35). While there are likely to be compositional drivers responsible for this reduced durability, no clear trends in composition are apparent for this subset of the study glasses.

Table 5-3. PCT Results for Each of the US-Series Glasses by Heat Treatment, Normalized Using the Bias-Corrected Composition Data.

\begin{tabular}{|c|c|c|c|c|c|}
\hline Glass ID & $\begin{array}{c}\text { Heat } \\
\text { Treatment }\end{array}$ & $\begin{array}{c}\text { NL [B] } \\
(\mathrm{g} / \mathrm{L})\end{array}$ & $\begin{array}{c}\text { NL [Li] } \\
(\mathrm{g} / \mathrm{L})\end{array}$ & $\begin{array}{c}\text { NL [Na] } \\
(\mathrm{g} / \mathrm{L})\end{array}$ & $\begin{array}{c}\text { NL [Si] } \\
(\mathrm{g} / \mathrm{L})\end{array}$ \\
\hline US-01 & $\mathrm{CCC}$ & 0.446 & 0.533 & 0.454 & 0.407 \\
\hline US-01 & quenched & 0.454 & 0.545 & 0.478 & 0.418 \\
\hline US-02 & $\mathrm{CCC}$ & 1.468 & 1.264 & 0.742 & 0.354 \\
\hline US-02 & quenched & 1.821 & 1.562 & 0.871 & 0.354 \\
\hline US-03 & $\mathrm{CCC}$ & 0.326 & 0.475 & 0.157 & 0.256 \\
\hline US-03 & quenched & 0.358 & 0.427 & 0.232 & 0.271 \\
\hline US-04 & $\mathrm{CCC}$ & 0.335 & 0.451 & 0.264 & 0.248 \\
\hline US-04 & quenched & 0.353 & 0.494 & 0.266 & 0.257 \\
\hline US-05 & $\mathrm{CCC}$ & 0.500 & 0.672 & 0.338 & 0.283 \\
\hline US-05 & quenched & 0.347 & 0.688 & 0.186 & 0.249 \\
\hline US-06 & $\mathrm{CCC}$ & 0.308 & 0.441 & 0.279 & 0.220 \\
\hline US-06 & quenched & 0.338 & 0.501 & 0.296 & 0.225 \\
\hline US-07 & $\mathrm{CCC}$ & 0.331 & 0.462 & 0.259 & 0.217 \\
\hline US-07 & quenched & 0.364 & 0.527 & 0.283 & 0.226 \\
\hline US-08 & $\mathrm{CCC}$ & 0.407 & 0.527 & 0.314 & 0.370 \\
\hline US-08 & quenched & 0.433 & 0.600 & 0.314 & 0.395 \\
\hline US-09 & $\mathrm{CCC}$ & 0.441 & 0.510 & 0.286 & 0.240 \\
\hline US-09 & quenched & 0.431 & 0.534 & 0.439 & 0.275 \\
\hline US-10 & $\mathrm{CCC}$ & 0.345 & 0.460 & 0.369 & 0.245 \\
\hline US-10 & quenched & 0.352 & 0.476 & 0.381 & 0.251 \\
\hline US-11 & $\mathrm{CCC}$ & 0.796 & 0.888 & 0.494 & 0.220 \\
\hline US-11 & quenched & 0.328 & 0.561 & 0.246 & 0.186 \\
\hline US-12 & $\mathrm{CCC}$ & 1.765 & 1.478 & 0.979 & 0.370 \\
\hline US-12 & quenched & 1.286 & 1.106 & 0.800 & 0.342 \\
\hline US-13 & $\mathrm{CCC}$ & 0.299 & 0.421 & 0.280 & 0.216 \\
\hline US-13 & quenched & 0.352 & 0.485 & 0.317 & 0.233 \\
\hline
\end{tabular}


Table 5-3. PCT Results for Each of the US-Series Glasses by Heat Treatment, Normalized Using the Bias-Corrected Composition Data. (continued)

\begin{tabular}{|c|c|c|c|c|c|}
\hline Glass ID & $\begin{array}{c}\text { Heat } \\
\text { Treatment }\end{array}$ & $\begin{array}{c}\text { NL [B] } \\
(g / L)\end{array}$ & $\begin{array}{c}\text { NL [Li] } \\
(\mathrm{g} / \mathrm{L})\end{array}$ & $\begin{array}{c}\text { NL [Na] } \\
\text { (g/L) }\end{array}$ & $\begin{array}{c}\text { NL [Si] } \\
(g / L)\end{array}$ \\
\hline US-14 & CCC & 2.650 & 2.200 & 1.577 & 0.333 \\
\hline US-14 & quenched & 3.162 & 2.618 & 1.846 & 0.334 \\
\hline US-15 & $\mathrm{CCC}$ & 3.627 & 3.051 & 2.007 & 0.232 \\
\hline US-15 & quenched & 4.623 & 3.867 & 2.512 & 0.217 \\
\hline US-16 & $\mathrm{CCC}$ & 0.864 & 0.925 & 0.426 & 0.269 \\
\hline US-16 & quenched & 0.640 & 0.788 & 0.292 & 0.254 \\
\hline US-17 & $\mathrm{CCC}$ & 0.503 & 0.579 & 0.302 & 0.399 \\
\hline US-17 & quenched & 0.514 & 0.641 & 0.279 & 0.415 \\
\hline US-18 & CCC & 10.498 & 8.136 & 3.540 & 0.830 \\
\hline US-18 & quenched & 0.516 & 0.590 & 0.620 & 0.389 \\
\hline US-19 & $\mathrm{CCC}$ & 0.427 & 0.533 & 0.328 & 0.312 \\
\hline US-19 & quenched & 0.458 & 0.579 & 0.330 & 0.335 \\
\hline US-20 & $\mathrm{CCC}$ & 0.429 & 0.552 & 0.334 & 0.312 \\
\hline US-20 & quenched & 0.458 & 0.595 & 0.344 & 0.325 \\
\hline US-21 & $\mathrm{CCC}$ & 0.398 & 0.543 & 0.292 & 0.293 \\
\hline US-21 & quenched & 0.419 & 0.587 & 0.301 & 0.350 \\
\hline US-22 & $\mathrm{CCC}$ & 0.536 & 0.545 & 0.517 & 0.414 \\
\hline US-22 & quenched & 0.570 & 0.569 & 0.466 & 0.357 \\
\hline US-23 & $\mathrm{CCC}$ & 0.445 & 0.566 & 0.335 & 0.323 \\
\hline US-23 & quenched & 0.457 & 0.610 & 0.338 & 0.353 \\
\hline US-24 & $\mathrm{CCC}$ & 0.407 & 0.522 & 0.335 & 0.305 \\
\hline US-24 & quenched & 0.395 & 0.532 & 0.320 & 0.299 \\
\hline US-25 & $\mathrm{CCC}$ & 1.423 & 1.228 & 0.865 & 0.432 \\
\hline US-25 & quenched & 1.155 & 1.026 & 0.731 & 0.348 \\
\hline US-26 & $\mathrm{CCC}$ & 3.691 & 2.579 & 1.184 & 0.325 \\
\hline US-26 & quenched & 0.430 & 0.527 & 0.409 & 0.261 \\
\hline US-27 & $\mathrm{CCC}$ & 15.962 & 7.281 & 7.039 & 2.316 \\
\hline US-27 & quenched & 1.213 & 1.050 & 1.475 & 0.835 \\
\hline US-28 & CCC & 1.402 & 1.226 & 0.884 & 0.272 \\
\hline US-28 & quenched & 1.738 & 1.467 & 1.023 & 0.260 \\
\hline US-29 & $\mathrm{CCC}$ & 1.568 & 1.389 & 1.736 & 0.760 \\
\hline US-29 & quenched & 1.868 & 1.486 & 2.074 & 0.791 \\
\hline US-30 & $\mathrm{CCC}$ & 0.634 & 0.616 & 0.405 & 0.244 \\
\hline US-30 & quenched & 0.569 & 0.567 & 0.358 & 0.219 \\
\hline US-31 & $\mathrm{CCC}$ & 0.314 & 0.486 & 0.178 & 0.241 \\
\hline US-31 & quenched & 0.508 & 0.625 & 0.262 & 0.275 \\
\hline US-32 & CCC & 2.828 & 2.258 & 1.444 & 0.334 \\
\hline US-32 & quenched & 3.579 & 2.849 & 1.787 & 0.337 \\
\hline US-33 & $\mathrm{CCC}$ & 0.321 & 0.544 & 0.864 & 0.356 \\
\hline US-33 & quenched & 0.338 & 0.498 & 0.911 & 0.342 \\
\hline US-34 & $\mathrm{CCC}$ & 3.865 & 3.169 & 2.811 & 0.430 \\
\hline US-34 & quenched & 4.176 & 3.389 & 3.067 & 0.450 \\
\hline US-35 & $\mathrm{CCC}$ & 4.012 & 3.440 & 2.367 & 0.215 \\
\hline US-35 & quenched & 0.812 & 0.883 & 0.596 & 0.203 \\
\hline US-36 & $\mathrm{CCC}$ & 0.812 & 0.783 & 0.463 & 0.238 \\
\hline US-36 & quenched & 0.658 & 0.688 & 0.395 & 0.226 \\
\hline US-37 & $\mathrm{CCC}$ & 1.982 & 1.621 & 1.395 & 0.503 \\
\hline US-37 & quenched & 0.676 & 0.645 & 0.919 & 0.384 \\
\hline US-38 & CCC & 0.794 & 0.781 & 1.207 & 0.487 \\
\hline US-38 & quenched & 0.785 & 0.688 & 1.164 & 0.429 \\
\hline US-39 & $\mathrm{CCC}$ & 0.451 & 0.518 & 0.182 & 0.287 \\
\hline US-39 & quenched & 0.511 & 0.552 & 0.220 & 0.285 \\
\hline
\end{tabular}


Table 5-3. PCT Results for Each of the US-Series Glasses by Heat Treatment, Normalized Using the Bias-Corrected Composition Data. (continued)

\begin{tabular}{||c|c|c|c|c|c||}
\hline \hline Glass ID & $\begin{array}{c}\text { Heat } \\
\text { Treatment }\end{array}$ & $\begin{array}{c}\text { NL [B] } \\
(\mathbf{g} / \mathbf{L})\end{array}$ & $\begin{array}{c}\mathbf{N L}[\mathbf{L i}] \\
\mathbf{( g / L )}\end{array}$ & $\begin{array}{c}\mathbf{N L}[\mathbf{N a}] \\
\mathbf{( g / L )}\end{array}$ & $\begin{array}{c}\mathbf{N L}[\mathbf{S i}] \\
\mathbf{( g / L})\end{array}$ \\
\hline US-40 & CCC & 1.543 & 1.366 & 0.794 & 0.283 \\
\hline US-40 & quenched & 1.096 & 1.057 & 0.608 & 0.290 \\
\hline US-41 & CCC & 0.287 & 0.493 & 0.170 & 0.211 \\
\hline US-41 & quenched & 0.333 & 0.493 & 0.198 & 0.217 \\
\hline US-42 & CCC & 0.897 & 1.987 & 3.664 & 0.190 \\
\hline US-42 & quenched & 1.259 & 1.088 & 1.664 & 0.690 \\
\hline US-43 & CCC & 1.745 & 1.755 & 0.491 & 0.352 \\
\hline US-43 & quenched & 0.403 & 0.587 & 0.336 & 0.287 \\
\hline US-44 & CCC & 0.520 & 0.516 & 0.471 & 0.301 \\
\hline US-44 & quenched & 0.532 & 0.514 & 0.477 & 0.292 \\
\hline US-45 & CCC & 0.446 & 0.538 & 0.452 & 0.278 \\
\hline US-45 & quenched & 0.467 & 0.559 & 0.485 & 0.280 \\
\hline
\end{tabular}

\subsection{Liquidus Temperature}

\subsubsection{Select Visual Observations of the Quenched Glasses}

Glass composition US-40 appears to be located outside the glass formation range as it is homogeneous at the bottom of the pour patty and inhomogeneous in all other locations, recognizable through different colors visible by eye (Figure 5-1). US-40 may have crystallized or phase separated quickly during pouring. In addition, glass composition US-43 appears to be incompletely melted because it does not have a homogeneous color. A liquidus temperature therefore cannot be determined for these glasses.

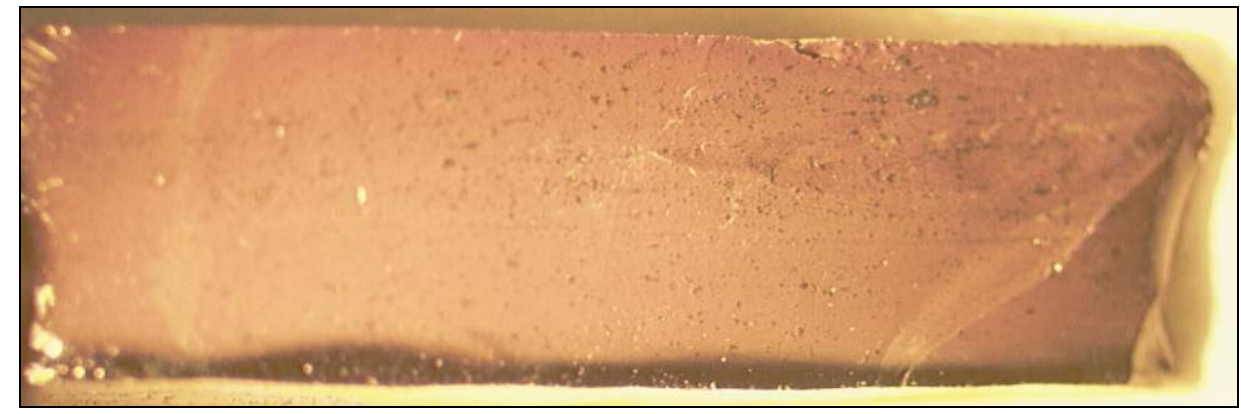

Figure 5-1. US-40 as-received after quenching on a steel plate. Crystallization is visible in the bulk of the glass, while a homogeneous region formed in contact with the steel plate at the bottom of the sample.

\subsubsection{Furnace calibrations}

The calibration of the furnaces used for isothermal $T_{L}$ measurements at PNNL was verified prior to the start of experiments. Temperature calibration data are given in Table 5-4. Homogeneity of the temperature gradient within each furnace was found to be sufficient to provide reliable measurements.

In addition, $\mathrm{T}_{\mathrm{L}}$ standard glasses were measured to verify the correct furnace temperatures and correct execution of the experimental procedure. The $\mathrm{T}_{\mathrm{L}}$ of the ARG-1 and $\mathrm{Zr}-9$ standard glasses 
have been measured in a Round Robin study with Monarch Laboratories and Idaho National Laboratory (INL). Data from the Round Robin study are listed in Table 5-5. The $\mathrm{T}_{\mathrm{L}}$ determination of Zr-9 is complicated by the fact that small crystals appear like air bubbles. Therefore, Zr-9 may be heat-treated several days to allow the crystals to grow. The measured $\mathrm{T}_{\mathrm{L}}$ for each standard glass for each of the furnaces used are given at the bottom of Table 5-4, and are in reasonable agreement with the Round Robin values shown in Table 5-5.

Table 5-4. Furnace calibrations at PNNL. The $T_{L}$ of $S R M-773$ is $990^{\circ} \mathrm{C}$ (standard certificate).

\begin{tabular}{||l|c|c|c|c||}
\hline \multirow{2}{*}{} & \multicolumn{4}{|c||}{ Furnace } \\
\cline { 2 - 5 } & 25 & 12 & 5 & 10 \\
\hline Calibration date using SRM 773 & Sept. 22, 2006 & Oct. 5, 2006 & Nov. 4, 2006 & Nov. 18, 2006 \\
\hline Thermocouple (TC) reading in ${ }^{\circ} \mathrm{C}$ & 993 & 991 & 1009 & 1008 \\
\hline Furnace controller (FC) reading in ${ }^{\circ} \mathrm{C}$ & 984 & 989 & 1006 & 1010 \\
\hline $\mathrm{T}_{\mathrm{L}}(\mathrm{SRM}-773)-\mathrm{TC}$ & -3 & -1 & -19 & -18 \\
\hline $\mathrm{TC}-\mathrm{FC}$ in ${ }^{\circ} \mathrm{C}$ at $\mathrm{FC}=800^{\circ} \mathrm{C}$ & 7 & 5 & 4 & -9 \\
\hline $\mathrm{TC}-\mathrm{FC}$ in ${ }^{\circ} \mathrm{C}$ at $\mathrm{FC}=900^{\circ} \mathrm{C}$ & 6 & 2 & 4 & - \\
\hline $\mathrm{TC}-\mathrm{FC}$ in ${ }^{\circ} \mathrm{C}$ at $\mathrm{FC}=1000^{\circ} \mathrm{C}$ & 9 & 4 & 3 & - \\
\hline $\mathrm{TC}-\mathrm{FC}$ in ${ }^{\circ} \mathrm{C}$ at $\mathrm{FC}=1100^{\circ} \mathrm{C}$ & 10 & 2 & 4 & - \\
\hline $\mathrm{TC}-\mathrm{FC}$ in ${ }^{\circ} \mathrm{C}$ at $\mathrm{FC}=1200^{\circ} \mathrm{C}$ & 8 & -1 & 4 & - \\
\hline $\mathrm{TC}-\mathrm{FC}$ in ${ }^{\circ} \mathrm{C}$ at $\mathrm{FC}=1300^{\circ} \mathrm{C}$ & 6 & -1 & 4 & - \\
\hline $\mathrm{TC}-\mathrm{FC}$ in ${ }^{\circ} \mathrm{C}$ at $\mathrm{FC}=1400^{\circ} \mathrm{C}$ & 5 & 2 & 4 & - \\
\hline $\begin{array}{l}\text { Temperature homogeneity at } \sim 1000^{\circ} \mathrm{C} \\
\text { using Ferro temperature control rings }\end{array}$ & below detection & $\sim 4-8{ }^{\circ} \mathrm{C}$ & $\sim 4-8^{\circ} \mathrm{C}$ & $\sim 4-8^{\circ} \mathrm{C}$ \\
\hline $\mathrm{T}_{\mathrm{L}}$ in ${ }^{\circ} \mathrm{C}, \mathrm{Zr}-9$ & limit $\left(4{ }^{\circ} \mathrm{C}\right)$ & & & 973 \\
\hline $\mathrm{T}_{\mathrm{L}}$ in ${ }^{\circ} \mathrm{C}, \mathrm{ARG}-1$ & 962 & 963 & 965 & 1042 \\
\hline \hline
\end{tabular}

Table 5-5. $T_{L}$ of ARG-1 and Zr-9 from a Round Robin Study with Monarch Laboratories and INL.

\begin{tabular}{||l|c|c||}
\hline \multirow{3}{*}{ PNNL, measured previously } & ARG-1, $\mathrm{T}_{\mathrm{L}}$ in ${ }^{\circ} \mathrm{C}$ & $\mathrm{Zr}-9, \mathrm{~T}_{\mathrm{L}}$ in ${ }^{\circ} \mathrm{C}$ \\
\cline { 2 - 3 } & 1028.3 & 960 \\
\cline { 2 - 3 } & 1030 & 967 \\
\cline { 2 - 3 } & 1031 & 969 \\
\hline \multirow{2}{*}{ INL } & 1038 & 973 \\
\cline { 2 - 3 } & 1046 & 956 \\
& 1046 & 966 \\
\hline
\end{tabular}

\subsubsection{Initial Optical Microscopy Observations}

The glasses were analyzed by both low and high resolution optical microscopy following isothermal heat treatments. Select micrographs are described below, followed by a summary of observations for all of the US-series glasses.

Select Low Resolution Optical Microscopy Observations

Select Low Resolution Optical Microscopy (LROM) observations are provided below. The observations are detailed in the captions accompanying each micrograph. 


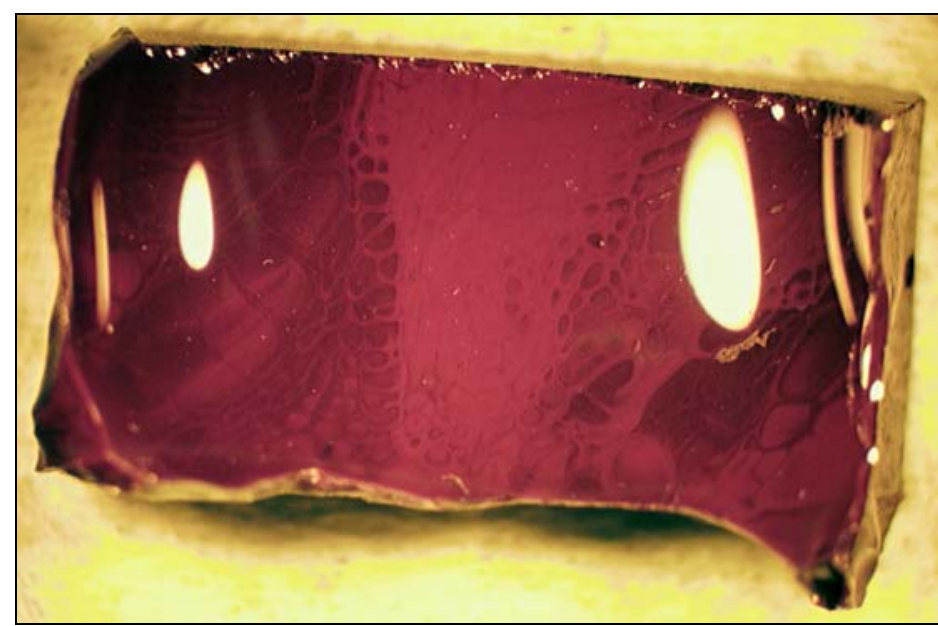

Figure 5-2. Brown crystal striations in the bulk of glass US-03 after heat treating at $854{ }^{\circ} \mathrm{C}$ (sample length approximately $12 \mathrm{~mm}$ ).

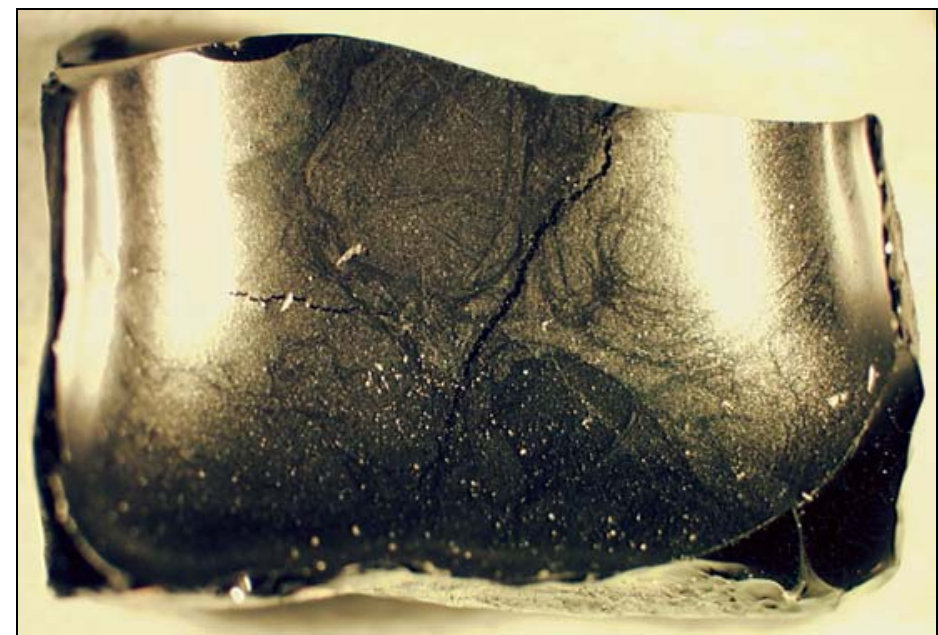

Figure 5-3. Surface of glass US-08 covered with grey crystals after heat treating at $904{ }^{\circ} \mathrm{C}$ (sample length approximately $12 \mathrm{~mm}$ ). 
Figure 5-4. Glittering crystals in the bulk of glass US-11 after heat treating at $904{ }^{\circ} \mathrm{C}$.

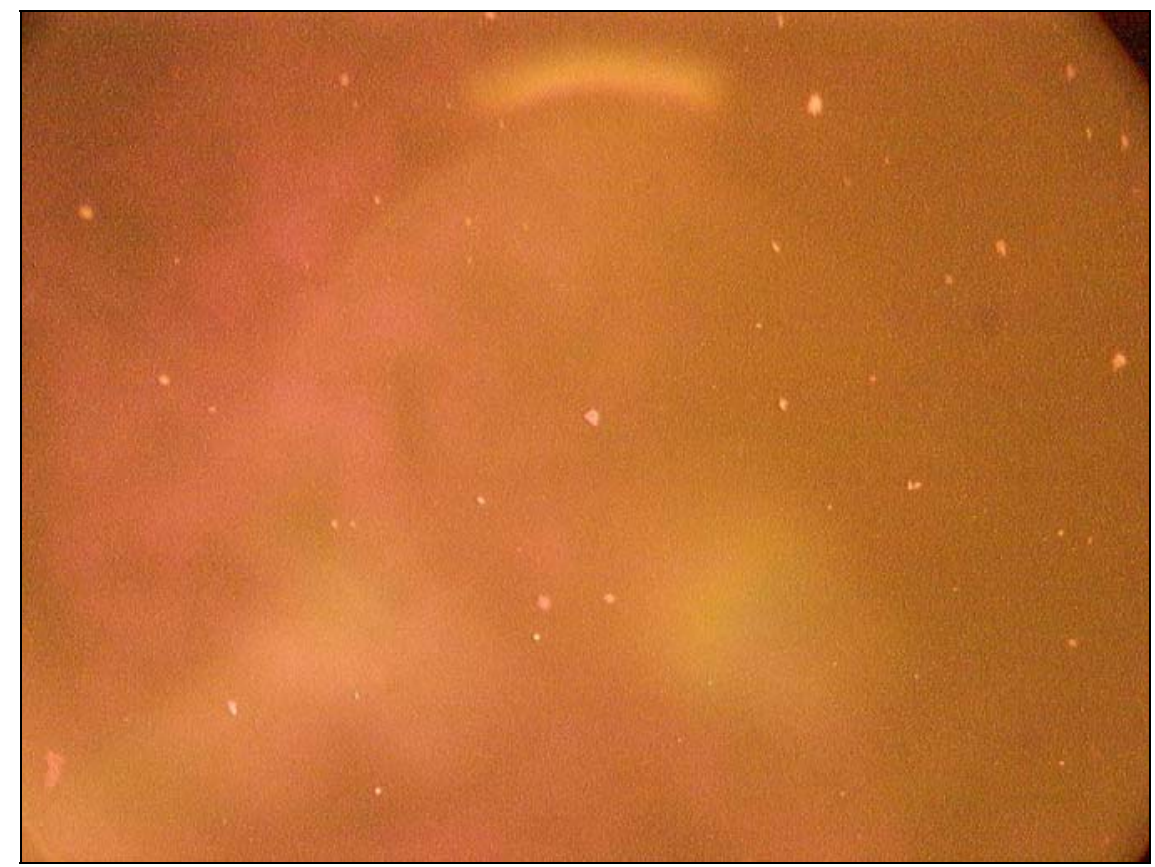

Figure 5-5. White crystal agglomerates on the surface of glass US-13 while still in the platinum crucible after heat treating at $1056{ }^{\circ} \mathrm{C}$ (exaggerated colors, black glass). 


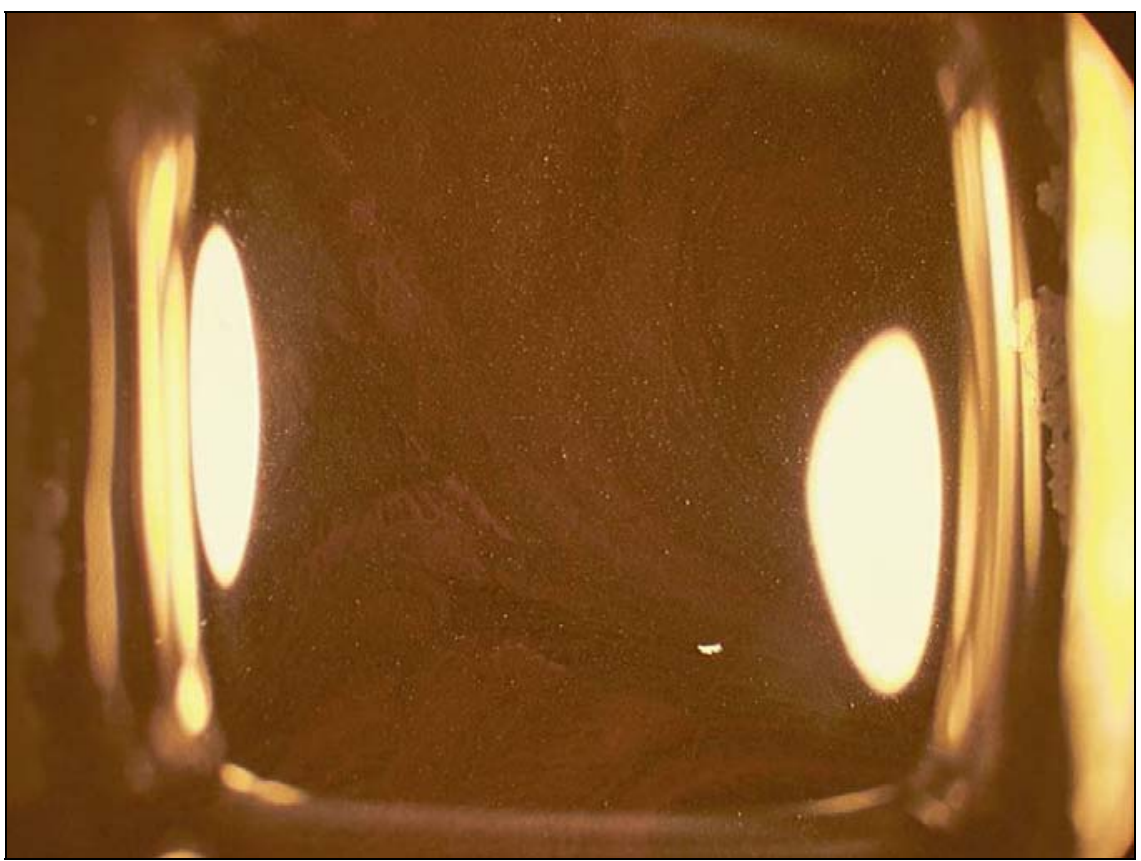

Figure 5-6. Tiny, bright crystals on the surface and brown crystal striations in the bulk of glass US-19 while still in the platinum crucible after heat treating at $904{ }^{\circ} \mathrm{C}$ (sample length approximately $12 \mathrm{~mm}$ ).

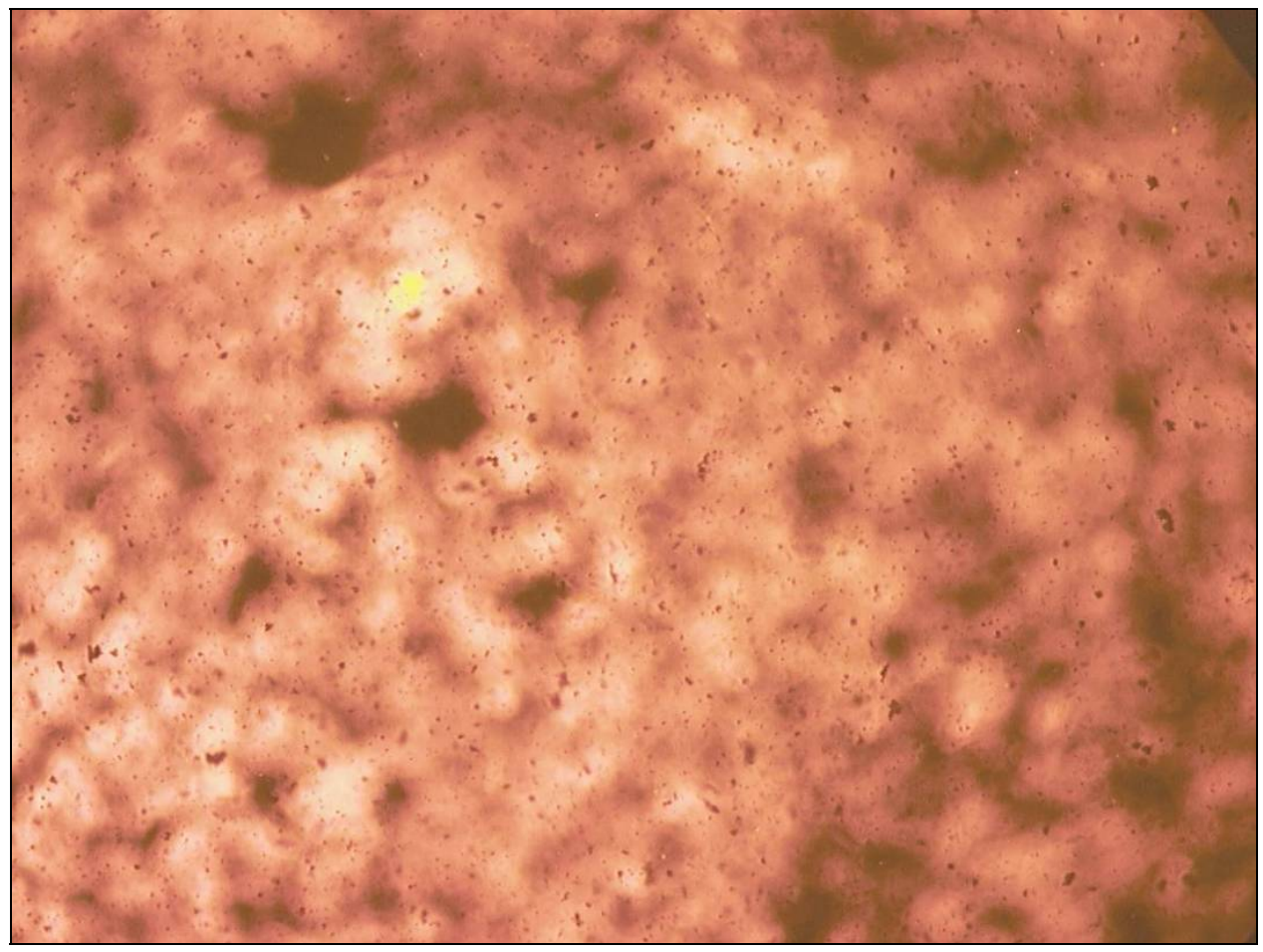

Figure 5-7. Many black crystal agglomerates in the bulk of glass US-26 while still in the platinum crucible after heat treating at $1157^{\circ} \mathrm{C}$. 


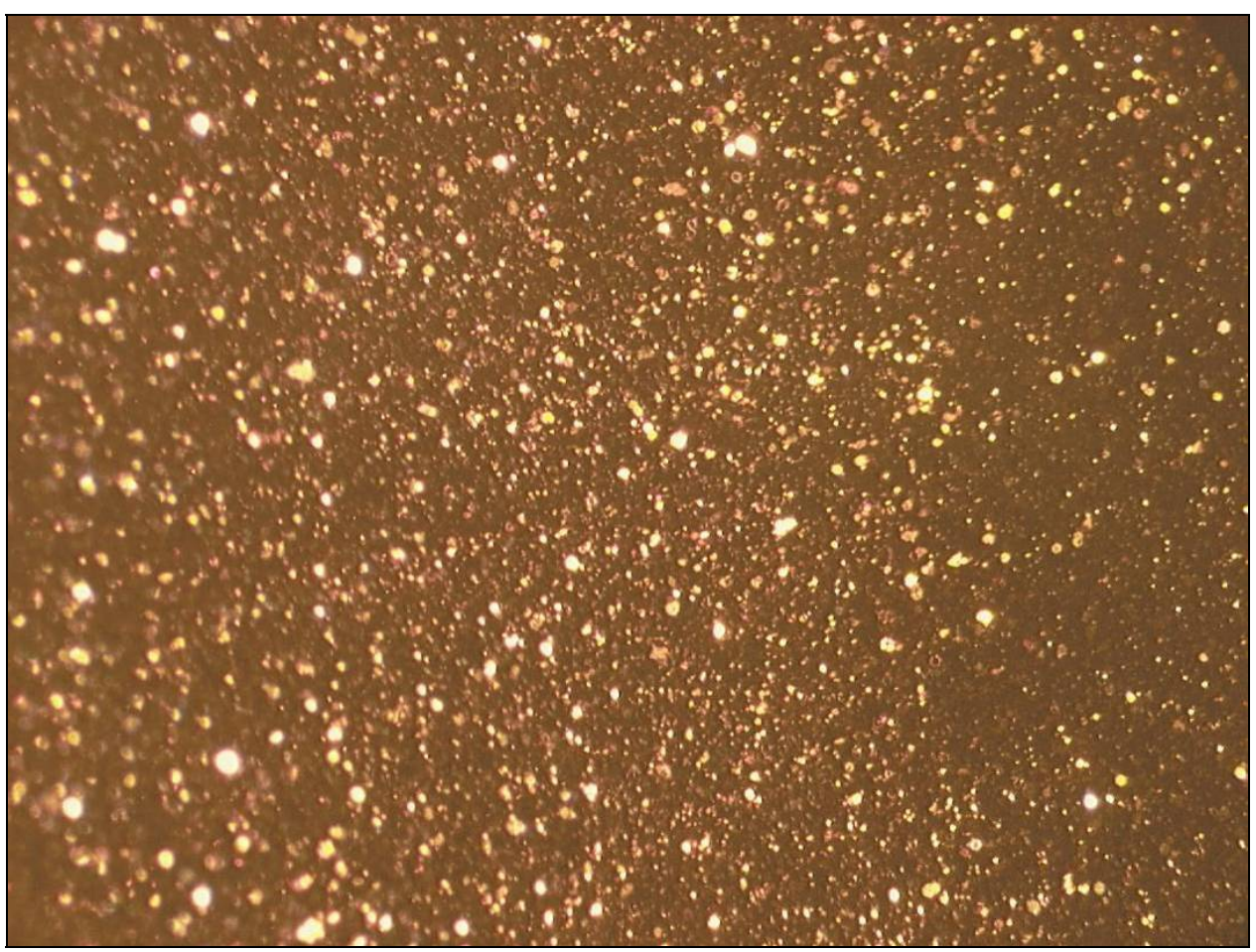

Figure 5-8. Surface of glass US-41 covered by hexagonal crystals while still in the platinum crucible after heat treating at $1007^{\circ} \mathrm{C}$.

Select High Resolution Optical Microscopy Observations

Initial High Resolution Optical Microscopy (HROM) observations are provided below. The observations are detailed in the captions accompanying each micrograph. 


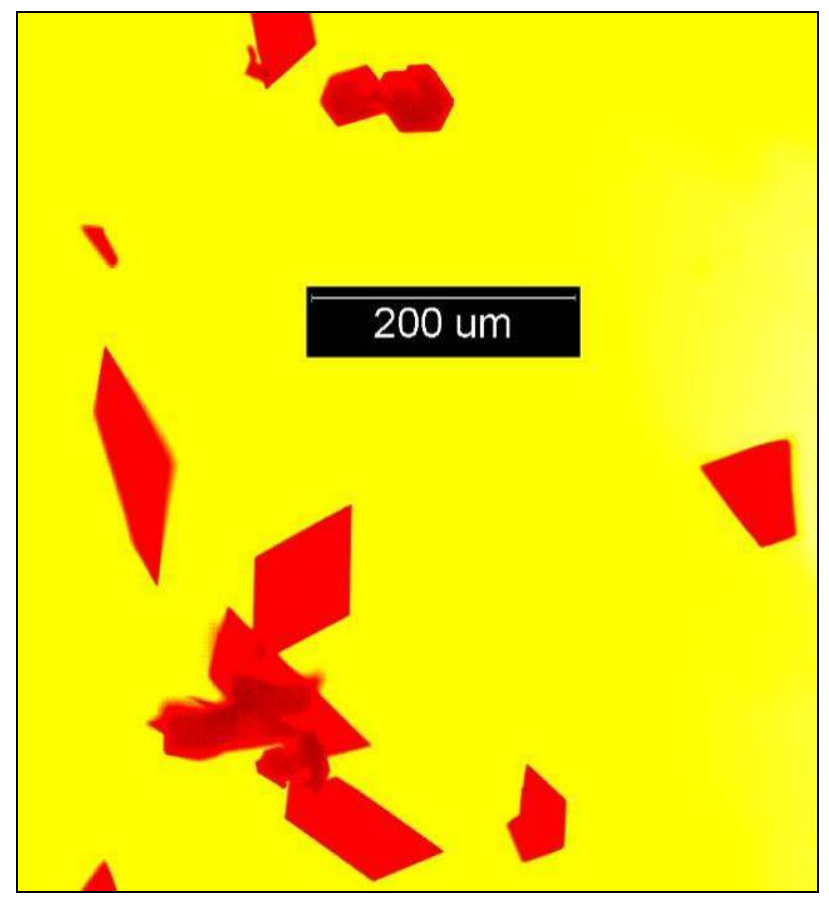

Figure 5-9. Hematite in glass US-09 after heat treating at $1157^{\circ} \mathrm{C}$. Colors in the image are not original: the crystals appear very dark red.

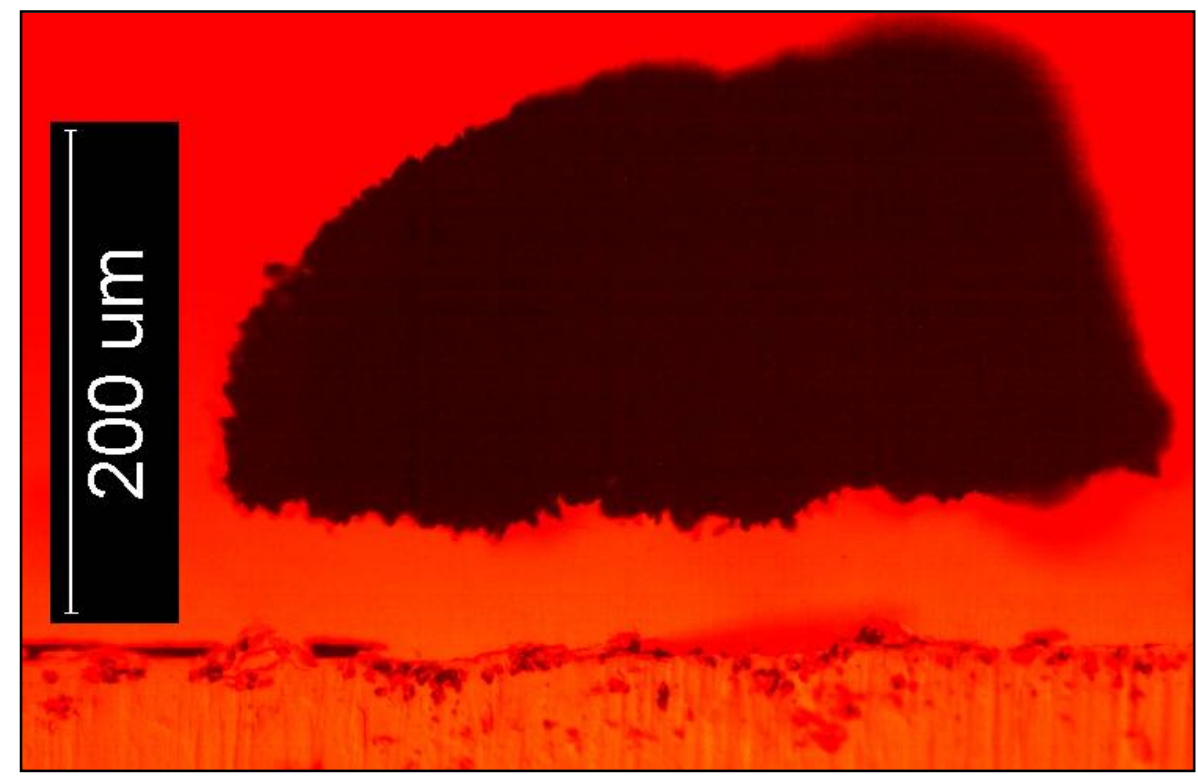

Figure 5-10. Large, black agglomerate of undissolved noble metals in glass US-09 after heat treating at $1157^{\circ} \mathrm{C}$. Colors in the image are not original: the glass appears light yellow and the crystal agglomerate is black.

Summary of Optical Microscopy Observations

The complete optical microscopy observations for each of the US-series glasses are provided in Table 5-6 below as a function of heat treatment temperature. 
Table 5-6. Initial LROM Observations of Surface and Bulk Crystallization in the US-Series Glasses.

\begin{tabular}{|c|c|c|c|}
\hline Glass ID & $853^{\circ} \mathrm{C}$ & $904^{\circ} \mathrm{C}$ & $954^{\circ} \mathrm{C}$ \\
\hline US-01 & grey crystal film on surface & grey crystal film on surface & fine crystal striations on surface \\
\hline US-02 & few black crystals in bulk & few black crystals in bulk & $\begin{array}{l}\text { fine crystal layer on surface, black spots in } \\
\text { bulk }\end{array}$ \\
\hline US-03 & brown crystal striations in bulk & brown crystal striations in bulk & $\begin{array}{l}\text { brown crystal striations, tiny crystals on } \\
\text { surface }\end{array}$ \\
\hline US-04 & grey crystal film on surface & brown crystal striations in bulk & $\begin{array}{l}\text { brown crystal striations, tiny crystals on } \\
\text { surface }\end{array}$ \\
\hline US-06 & grey crystal film on surface & grey crystal film on surface, brown crystals in bulk & $\begin{array}{l}\text { brown crystal striations in bulk, some } \\
\text { glittering crystals on surface }\end{array}$ \\
\hline US-07 & grey crystal film on surface & grey crystals on surface and in bulk & grey crystals on surface and in bulk \\
\hline US-08 & grey crystal film on surface & grey crystal film on surface & partly grey crystal film on surface \\
\hline US-09 & glittering crystals in bulk & glittering crystals in bulk & glittering crystals in bulk \\
\hline US-10 & grey crystal film on surface & grey crystal film on surface & brown crystal striations in bulk \\
\hline US-11 & glittering crystals in bulk & glittering crystals in bulk & glittering crystals in bulk \\
\hline US-13 & partly grey crystal cover on surface & $\begin{array}{l}\text { many tiny bright crystals on surface, in one place } \\
\text { still continuous grey crystal cover }\end{array}$ & many tiny bright crystals on surface \\
\hline US-14 & triangular crystals on surface & homogeneous & 1 \\
\hline US-15 & $\begin{array}{l}\text { crystals on surface and in bulk, } \\
\text { striations }\end{array}$ & black crystal agglomerates in bulk & black crystal agglomerates in bulk \\
\hline
\end{tabular}


Table 5-6. Initial LROM Observations of Surface and Bulk Crystallization in the US-Series Glasses. (continued)

\begin{tabular}{|c|c|c|c|}
\hline GLASS ID & $1007^{\circ} \mathrm{C}$ & $1058{ }^{\circ} \mathrm{C}$ & $1108^{\circ} \mathrm{C}$ \\
\hline US-01 & $\begin{array}{l}\text { white crystal agglomerates on surface } \\
\text { and in bulk }\end{array}$ & very few white crystal agglomerates on surface & $\begin{array}{l}\text { one tiny white crystal agglomerate on surface, } \\
2-3 \text { tiny bright spots }\end{array}$ \\
\hline US-02 & black crystal agglomerates in bulk & few black crystal agglomerates in bulk & homogeneous \\
\hline US-03 & many black crystals in bulk & black crystal agglomerates in bulk & $\begin{array}{l}\text { very few white crystal agglomerates on } \\
\text { surface, black agglomerates in bulk }\end{array}$ \\
\hline US-04 & many white crystals in bulk & few black crystal agglomerates in bulk & few black crystal agglomerates in bulk \\
\hline US-05 & many glittering crystals in bulk & $\begin{array}{l}\text { white crystal agglomerates on surface and in } \\
\text { bulk }\end{array}$ & $\begin{array}{l}\text { few white crystal agglomerates on surface and } \\
\text { in bulk, } 1 \text { bright tiny spot }\end{array}$ \\
\hline US-06 & $\begin{array}{l}\text { white crystal agglomerates on surface } \\
\text { and in bulk }\end{array}$ & $\begin{array}{l}\text { few white crystal agglomerates on surface and in } \\
\text { bulk }\end{array}$ & $\begin{array}{l}\text { few white crystal agglomerates on surface, } \\
\text { black agglomerates in bulk }\end{array}$ \\
\hline US-07 & few glittering crystals in bulk & few white crystal agglomerates on surface & $\begin{array}{l}\text { very few white crystal agglomerates on } \\
\text { surface and in bulk }\end{array}$ \\
\hline US-08 & $\begin{array}{l}\text { few white crystal agglomerates on } \\
\text { surface and in bulk }\end{array}$ & $\begin{array}{l}\text { few white crystal agglomerates on surface and in } \\
\text { bulk }\end{array}$ & $\begin{array}{l}\text { very few white crystal agglomerates on } \\
\text { surface and in bulk }\end{array}$ \\
\hline US-09 & few glittering crystals in bulk & $\begin{array}{l}\text { few black crystal agglomerates in bulk, few } \\
\text { white crystal agglomerates on surface }\end{array}$ & very few black crystal agglomerates in bulk \\
\hline US-10 & $\begin{array}{l}\text { few white crystal agglomerates on } \\
\text { surface and in bulk }\end{array}$ & $\begin{array}{l}\text { few white crystal agglomerates on surface and in } \\
\text { bulk }\end{array}$ & $\begin{array}{l}\text { very few white crystal agglomerates on } \\
\text { surface }\end{array}$ \\
\hline US-11 & $\begin{array}{l}\text { few white and glittering crystals in } \\
\text { bulk }\end{array}$ & $\begin{array}{l}\text { few white crystal agglomerates on surface and in } \\
\text { bulk }\end{array}$ & $\begin{array}{l}\text { very few white crystal agglomerates on } \\
\text { surface and in bulk }\end{array}$ \\
\hline US-12 & $\begin{array}{l}\text { tiny white crystals on surface and in } \\
\text { bulk }\end{array}$ & tiny white crystals on surface and in bulk & extremely few and tiny white spots (crystals?) \\
\hline US-13 & many tiny bright crystals on surface & white crystal agglomerates on surface & $\begin{array}{l}\text { few white crystal agglomerates on surface, } \\
\text { few black crystal agglomerates in bulk }\end{array}$ \\
\hline US-14 & 1 & 1 & 1 \\
\hline US-15 & black crystal agglomerates in bulk & black crystal agglomerates in bulk & few black crystal agglomerates in bulk \\
\hline
\end{tabular}


Table 5-6. Initial LROM Observations of Surface and Bulk Crystallization in the US-Series Glasses. (continued)

\begin{tabular}{||l|l|l||}
\hline \hline Glass ID & \multicolumn{1}{|c|}{$\mathbf{1 1 5 7}^{\circ} \mathbf{C}$} & \multicolumn{1}{|c|}{$\mathbf{1 2 0 6}^{\circ} \mathbf{C}$} \\
\hline US-01 & glass very dark, no crystals on surface, maybe homogeneous & $/$ \\
\hline US-02 & $/$ & $/$ \\
\hline US-03 & very few black crystal agglomerates in bulk & homogeneous \\
\hline US-04 & very few white crystal agglomerates on surface & homogeneous \\
\hline US-05 & homogeneous & $/$ \\
\hline US-06 & very few black crystal agglomerates in bulk & homogeneous \\
\hline US-07 & homogeneous & $/$ \\
\hline US-08 & homogeneous & $/$ \\
\hline US-09 & homogeneous & $/$ \\
\hline US-10 & homogeneous & $/$ \\
\hline US-11 & homogeneous & $/$ \\
\hline US-12 & homogeneous & $/$ \\
\hline US-13 & homogeneous & $/$ \\
\hline US-14 & $/$ & $/$ \\
\hline US-15 & homogeneous & $/$ \\
\hline
\end{tabular}


Table 5-6. Initial LROM Observations of Surface and Bulk Crystallization in the US-Series Glasses. (continued)

\begin{tabular}{|c|c|c|c|}
\hline Glass ID & $853^{\circ} \mathrm{C}$ & $904^{\circ} \mathrm{C}$ & $954^{\circ} \mathrm{C}$ \\
\hline US-16 & glass appears grey, many crystals & cell-like crystal striations on surface & grey, cell-like crystal striations \\
\hline US-17 & silver crystal cover on surface & $\begin{array}{l}\text { partly silver crystal cover on surface, brown } \\
\text { crystal striations in bulk }\end{array}$ & grey-brown crystal striations \\
\hline US-18 & grey crystal cover on surface & silver crystal cover on surface & partly grey crystal cover on surface \\
\hline US-19 & $\begin{array}{l}\text { partly silver crystal cover on surface, brown } \\
\text { crystal striations in bulk }\end{array}$ & $\begin{array}{l}\text { tiny bright crystals on surface, brown } \\
\text { crystal striations in bulk }\end{array}$ & $\begin{array}{l}\text { tiny bright crystals on surface, brown crystal } \\
\text { striations in bulk }\end{array}$ \\
\hline US-20 & $\begin{array}{l}\text { partly grey crystal cover on surface, crystal } \\
\text { striations in bulk }\end{array}$ & $\begin{array}{l}\text { tiny bright crystals on surface, brown } \\
\text { crystal striations in bulk }\end{array}$ & $\begin{array}{l}\text { tiny bright crystals on surface, brown crystal } \\
\text { striations in bulk }\end{array}$ \\
\hline US-21 & $\begin{array}{l}\text { partly grey crystal cover on surface, brown } \\
\text { crystal striations in bulk }\end{array}$ & $\begin{array}{l}\text { tiny bright crystals on surface, brown } \\
\text { crystal striations in bulk }\end{array}$ & many tiny bright crystals on surface \\
\hline US-22 & $\begin{array}{l}\text { partly grey crystal cover on surface, grey } \\
\text { crystal striations in bulk }\end{array}$ & $\begin{array}{l}\text { partly grey crystal cover on surface, grey } \\
\text { crystal striations in bulk }\end{array}$ & grey crystal striations in bulk \\
\hline US-23 & silver crystal cover on surface & partly grey crystal cover on surface & $\begin{array}{l}\text { tiny bright crystals on surface, brown crystal } \\
\text { striations in bulk }\end{array}$ \\
\hline US-24 & $\begin{array}{l}\text { partly grey crystal cover on surface, brown } \\
\text { crystal striations in bulk }\end{array}$ & $\begin{array}{l}\text { tiny bright crystals on surface, brown } \\
\text { crystal striations in bulk }\end{array}$ & $\begin{array}{l}\text { tiny bright crystals on surface, brown crystal } \\
\text { striations in bulk }\end{array}$ \\
\hline US-25 & grey-brown crystal striations in bulk & grey-brown crystal striations in bulk & grey-brown crystal striations in bulk \\
\hline US-26 & brown and white crystals on surface & brown and white crystals on surface & $\begin{array}{l}\text { red-brown crystal striations in bulk, white } \\
\text { crystal agglomerates on surface }\end{array}$ \\
\hline US-27 & brown and white crystals on surface & brown and white crystals on surface & partly grey crystal cover on surface \\
\hline US-28 & $\begin{array}{l}\text { many black crystal agglomerates in bulk, } \\
\text { some white crystal agglomerates on surface }\end{array}$ & many black crystal agglomerates in bulk & black crystal agglomerates in bulk \\
\hline US-29 & $\begin{array}{l}\text { many small black crystal agglomerates in } \\
\text { bulk }\end{array}$ & $\begin{array}{l}\text { black crystal agglomerates in bulk, small } \\
\text { needle-like crystals on surface }\end{array}$ & few black crystal agglomerates in bulk \\
\hline US-30 & many white crystal agglomerates on surface & white crystal agglomerates on surface & white crystal agglomerates on surface \\
\hline
\end{tabular}


Table 5-6. Initial LROM Observations of Surface and Bulk Crystallization in the US-Series Glasses. (continued)

\begin{tabular}{|c|c|c|c|}
\hline Glass ID & $1007^{\circ} \mathrm{C}$ & $1058{ }^{\circ} \mathrm{C}$ & $1108{ }^{\circ} \mathrm{C}$ \\
\hline US-16 & tiny bright crystals on surface, grey striations & $\begin{array}{l}\text { tiny bright crystals on surface, glass } \\
\text { appears grey, many crystals }\end{array}$ & big and very bright spots on surface \\
\hline US-17 & $\begin{array}{l}\text { brown crystal striations in bulk, one big and } \\
\text { very bright crystal agglomerate on surface }\end{array}$ & $\begin{array}{l}\text { few big and very bright spots on } \\
\text { surface }\end{array}$ & homogeneous \\
\hline US-18 & very few black crystal agglomerates in bulk & homogeneous & / \\
\hline US-19 & bright crystals on surface and in bulk & white crystal agglomerates on surface & $\begin{array}{l}\text { white crystal agglomerates on surface, black crystal } \\
\text { agglomerates in bulk }\end{array}$ \\
\hline US-20 & white crystals on surface and in bulk & very bright spots on surface & $\begin{array}{l}\text { few white crystal agglomerates on surface, few } \\
\text { black crystal agglomerates in bulk }\end{array}$ \\
\hline US-21 & many tiny bright crystals on surface & $\begin{array}{l}\text { white crystal agglomerates on surface } \\
\text { and in bulk }\end{array}$ & $\begin{array}{l}\text { white crystal agglomerates on surface, black crystal } \\
\text { agglomerates in bulk }\end{array}$ \\
\hline US-22 & many tiny bright crystals on surface & $\begin{array}{l}\text { many bright agglomerates on surface } \\
\text { and in bulk }\end{array}$ & $\begin{array}{l}\text { glass appear homogeneous on surface and in bulk, } \\
\text { except one white spot on surface }\end{array}$ \\
\hline US-23 & tiny bright crystals on surface and in bulk & $\begin{array}{l}\text { white crystal agglomerates on surface } \\
\text { and in bulk }\end{array}$ & $\begin{array}{l}\text { white crystal agglomerates on surface, black crystal } \\
\text { agglomerates in bulk }\end{array}$ \\
\hline US-24 & $\begin{array}{l}\text { tiny bright crystals on surface, brown crystal } \\
\text { striations in bulk }\end{array}$ & $\begin{array}{l}\text { white crystal agglomerates on surface } \\
\text { and in bulk }\end{array}$ & $\begin{array}{l}\text { few white crystal agglomerates on surface, few } \\
\text { black crystal agglomerates in bulk }\end{array}$ \\
\hline US-25 & many tiny bright crystals on surface & many bright tiny crystals on surface & $\begin{array}{l}\text { very few white crystal agglomerates on surface, too } \\
\text { dark to see in bulk }\end{array}$ \\
\hline US-26 & many white and brown crystals & many white and brown crystals & many black crystal agglomerates in bulk \\
\hline US-27 & $\begin{array}{l}\text { white crystal agglomerates on surface, black } \\
\text { crystal agglomerates in bulk }\end{array}$ & black crystal agglomerates in bulk & black crystal agglomerates in bulk \\
\hline US-28 & black crystal agglomerates in bulk & black crystal agglomerates in bulk & homogeneous \\
\hline US-29 & one small black crystal agglomerate in bulk & homogeneous & 1 \\
\hline US-30 & white crystal agglomerates on surface & many tiny white spots on surface & many black crystal agglomerates in bulk \\
\hline
\end{tabular}


Table 5-6. Initial LROM Observations of Surface and Bulk Crystallization in the US-Series Glasses. (continued)

\begin{tabular}{|c|c|c|c|}
\hline Glass ID & $1157^{\circ} \mathrm{C}$ & $1206^{\circ} \mathrm{C}$ & $1254^{\circ} \mathrm{C}$ \\
\hline US-16 & many big black crystal agglomerates & many big black crystal agglomerates & 1 \\
\hline US-17 & 1 & l & 1 \\
\hline US-18 & 1 & 1 & 1 \\
\hline US-19 & homogeneous & 1 & 1 \\
\hline US-20 & homogeneous & 1 & 1 \\
\hline US-21 & homogeneous & 1 & 1 \\
\hline US-22 & homogeneous & 1 & 1 \\
\hline US-23 & homogeneous & 1 & 1 \\
\hline US-24 & homogeneous & 1 & 1 \\
\hline US-25 & homogeneous & 1 & 1 \\
\hline US-26 & many black crystal agglomerates in bulk & some black crystal agglomerates in bulk & homogeneous \\
\hline US-27 & homogeneous & I & 1 \\
\hline US-28 & 1 & 1 & 1 \\
\hline US-29 & l & I & l \\
\hline US-30 & many big black crystal agglomerates in bulk & many black crystal agglomerates in bulk & big black crystal agglomerates \\
\hline
\end{tabular}


Table 5-6. Initial LROM Observations of Surface and Bulk Crystallization in the US-Series Glasses. (continued)

\begin{tabular}{|c|c|c|c|}
\hline Glass ID & $853^{\circ} \mathrm{C}$ & $904^{\circ} \mathrm{C}$ & $954^{\circ} \mathrm{C}$ \\
\hline US-31 & silver crystal cover on surface & silver crystal cover on surface & white crystal agglomerates cover surface \\
\hline US-32 & $\begin{array}{l}\text { many white crystal agglomerates on surface } \\
\text { and in bulk }\end{array}$ & tiny white crystals on surface and in bulk & $\begin{array}{l}\text { tiny white crystals on surface, black crystal } \\
\text { agglomerates in bulk }\end{array}$ \\
\hline US-33 & silver crystal cover on surface & partly silver crystal cover on surface & white crystal agglomerates on surface \\
\hline US-34 & homogeneous & homogeneous & 1 \\
\hline US-35 & $\begin{array}{l}\text { brown crystal striations, tiny white spots on } \\
\text { surface }\end{array}$ & tiny white and brown crystals on surface & tiny white and brown crystals on surface \\
\hline US-36 & $\begin{array}{l}\text { brown crystal striations, white crystal } \\
\text { agglomerates on surface }\end{array}$ & $\begin{array}{l}\text { brown crystal striations, white crystal } \\
\text { agglomerates on surface }\end{array}$ & $\begin{array}{l}\text { brown crystal striations, white crystal } \\
\text { agglomerates on surface }\end{array}$ \\
\hline US-37 & brown crystal cover on surface & $\begin{array}{l}\text { partly brown crystal cover on surface, } \\
\text { brown crystal striations in bulk, white } \\
\text { crystal agglomerates on surface }\end{array}$ & $\begin{array}{l}\text { many white crystal agglomerates on surface } \\
\text { and in bulk }\end{array}$ \\
\hline US-38 & grey crystal cover on surface & silver crystal cover on surface & silver crystal cover on surface \\
\hline US-39 & $\begin{array}{l}\text { small regular red crystal striations, partly } \\
\text { grey crystal cover on surface }\end{array}$ & $\begin{array}{l}\text { regular circular red crystal striations with } \\
\text { white crystal agglomerates in center }\end{array}$ & $\begin{array}{l}\text { regular circular red crystal striations with white } \\
\text { crystal agglomerates in center }\end{array}$ \\
\hline US-40 & on hold, fast crystallization & & \\
\hline US-41 & surface covered by hexagonal crystals & surface covered by hexagonal crystals & surface covered by hexagonal crystals \\
\hline US-42 & dull grey crystal cover on surface & grey crystal cover on surface & many white crystal agglomerates on surface \\
\hline US-43 & 1 & 1 & / \\
\hline US-44 & partly grey crystal cover on surface & many tiny white spots on surface & many tiny white spots on surface \\
\hline US-45 & partly grey crystal cover on surface & many tiny white spots on surface & many tiny white spots on surface \\
\hline
\end{tabular}


Table 5-6. Initial LROM Observations of Surface and Bulk Crystallization in the US-Series Glasses. (continued)

\begin{tabular}{|c|c|c|c|}
\hline Glass ID & $1007^{\circ} \mathrm{C}$ & $1058{ }^{\circ} \mathrm{C}$ & $1108{ }^{\circ} \mathrm{C}$ \\
\hline US-31 & many single crystals on surface & many single crystals on surface & homogeneous \\
\hline US-32 & two small black crystal agglomerates in bulk & one small black crystal agglomerate in bulk & homogeneous \\
\hline US-33 & many tiny white spots on surface & many tiny white spots on surface & many tiny white spots on surface \\
\hline US-34 & / & 1 & $/$ \\
\hline US-35 & tiny white and brown crystals on surface & tiny white and brown crystals on surface & many tiny white spots on surface \\
\hline US-36 & many tiny white spots on surface & many single crystals on surface & many black crystal agglomerates in bulk \\
\hline US-37 & tiny white spots on surface and in bulk & homogeneous & 1 \\
\hline US-38 & many small white spots on surface & many small white spots on surface & many small white spots on surface \\
\hline US-39 & single crystals cover surface & single crystals cover surface & homogeneous \\
\hline US-40 & on hold, fast crystallization & & \\
\hline US-41 & surface covered by hexagonal crystals & white crystal agglomerates on surface and in bulk & few white crystal agglomerates on surface \\
\hline US-42 & many white crystal agglomerates on surface & many white crystal agglomerates on surface & many white crystal agglomerates on surface \\
\hline US-43 & l & 1 & 1 \\
\hline US-44 & many tiny white spots on surface & few white crystal agglomerates on surface & homogeneous \\
\hline US-45 & many tiny white spots on surface & white crystal agglomerates on surface & homogeneous \\
\hline
\end{tabular}


Table 5-6. Initial LROM Observations of Surface and Bulk Crystallization in the US-Series Glasses. (continued)

\begin{tabular}{|c|c|c|c|}
\hline Glass ID & $1157^{\circ} \mathrm{C}$ & $1206^{\circ} \mathrm{C}$ & $1254^{\circ} \mathrm{C}$ \\
\hline US-31 & 1 & 1 & \\
\hline US-32 & 1 & / & \\
\hline US-33 & many tiny white spots on surface & homogeneous & \\
\hline US-34 & 1 & / & \\
\hline US-35 & $\begin{array}{l}\text { many white crystal agglomerates on surface, } \\
\text { many black crystal agglomerates in bulk }\end{array}$ & $\begin{array}{l}\text { many big black crystal } \\
\text { agglomerates in bulk }\end{array}$ & \\
\hline US-36 & many big black crystal agglomerates in bulk & $\begin{array}{l}\text { many big black crystal } \\
\text { agglomerates in bulk }\end{array}$ & \\
\hline US-37 & 1 & 1 & \\
\hline US-38 & many small white spots on surface & $\begin{array}{l}\text { many small white spots } \\
\text { on surface }\end{array}$ & $\begin{array}{l}\text { many small white } \\
\text { spots on surface }\end{array}$ \\
\hline US-39 & 1 & / & \\
\hline US-40 & on hold, fast crystallization & & \\
\hline US-41 & homogeneous & l & 1 \\
\hline US-42 & many white crystal agglomerates on surface & $\begin{array}{l}\text { many white crystal } \\
\text { agglomerates on surface }\end{array}$ & $\begin{array}{l}\text { grey crystal cover } \\
\text { on surface }\end{array}$ \\
\hline US-43 & / & $\begin{array}{l}\text { many big white crystal } \\
\text { agglomerates in bulk }\end{array}$ & \\
\hline US-44 & 1 & I & 1 \\
\hline US-45 & 1 & 1 & 1 \\
\hline
\end{tabular}




\subsubsection{Uniform Temperature Heat Treatments and Observations}

Uniform temperature heat treatments were made on 44 of the US-series glasses (glass US-40 was excluded) in order to determine $T_{L}$ optically. These data are presented in Table 5-7 where the amorphous temperature $\left(T_{a}\right)$, the crystallization temperature $\left(T_{c}\right)$, and $T_{L}$ are presented along with the predicted $T_{L}$ (using models prior to the commencement of this study). The measured crystal fraction $\mathrm{T}_{\mathrm{L}}$ and the crystalline phases measured using quantitative XRD for each glass (as discussed in the next section) are included in this table as well. Detailed data for optical heat treatment specimens are available in Appendix A.

Table 5-7. Predicted and Measured Liquidus Temperatures.

\begin{tabular}{|c|c|c|c|c|c|}
\hline $\begin{array}{l}\text { Glass } \\
\text { ID }\end{array}$ & $\begin{array}{c}\text { Predicted } T_{L} \\
\left({ }^{\circ} \mathrm{C}\right)\end{array}$ & $\mathbf{T}_{\mathrm{c}}-\mathbf{T}_{\mathrm{a}}\left({ }^{\circ} \mathrm{C}\right)$ & $\begin{array}{l}\text { Optical } T_{L} \\
\quad\left({ }^{\circ} \mathrm{C}\right)\end{array}$ & $\begin{array}{c}T_{L} \text { by XRD } \\
\text { Crystal Fraction } \\
\left({ }^{\circ} \mathrm{C}\right)\end{array}$ & $\begin{array}{l}\text { XRD Primary \& } \\
\text { Secondary Phases }\end{array}$ \\
\hline US-01 & 1048 & $1049-1057$ & 1053 & 1019 & Spinel \\
\hline US-02 & 882 & $753-764$ & 759 & $\mathrm{BDL}^{*}$ & Baddelyite (optical) \\
\hline US-03 & 1009 & $995-1000$ & 997 & 1018 & Spinel \\
\hline US-04 & 1050 & $946-954$ & 950 & 956 & Spinel \\
\hline US-05 & 1050 & $1019-1027$ & 1023 & 1025 & Hematite \\
\hline US-06 & 1037 & 1001-1008 & 1005 & 968 & Spinel \\
\hline US-07 & 1050 & $1022-1029$ & 1026 & 1018 & Spinel \\
\hline US-08 & 1042 & $994-1000$ & 997 & 981 & Spinel \\
\hline US-09 & 1027 & $1157-1169$ & 1163 & 1091 & Hematite \\
\hline US-10 & 1050 & $995-1001$ & 998 & 998 & Spinel \\
\hline US-11 & 1017 & $1000-1006$ & 1003 & 976 & Spinel, hematite \\
\hline US-12 & 1050 & $1082-1086$ & 1084 & 1062 & Spinel \\
\hline US-13 & 1030 & $1022-1029$ & 1026 & 1034 & Spinel \\
\hline US-14 & 882 & $869-879$ & 874 & 880 & Spinel \\
\hline US-15 & 786 & $890-901$ & 895 & 913 & Spinel \\
\hline US-16 & 1050 & $1120-1126$ & 1124 & 1136 & Hematite, spinel \\
\hline US-17 & 1050 & $1040-1049$ & 1045 & 1031 & Spinel \\
\hline US-18 & 1007 & $982-994$ & 988 & 1026 & Spinel \\
\hline US-19 & 1007 & $1029-1040$ & 1035 & 1052 & Spinel \\
\hline US-20 & 1007 & $1001-1007$ & 1004 & 1006 & Spinel \\
\hline US-21 & 1050 & $1070-1074$ & 1072 & 1071 & Spinel \\
\hline US-22 & 1043 & 1086-1095 & 1091 & 1050 & Spinel \\
\hline US-23 & 1021 & $1030-1035$ & 1032 & 1032 & Spinel \\
\hline US-24 & 1050 & $1049-1056$ & 1052 & 1024 & Spinel \\
\hline US-25 & 1050 & $1080-1086$ & 1083 & 1074 & Spinel \\
\hline US-26 & 1150 & $1205-1206$ & 1206 & 1167 & Spinel \\
\hline US-27 & 1113 & $1079-1086$ & 1082 & 1077 & Spinel \\
\hline US-28 & 928 & $826-831$ & 829 & 813 & $\begin{array}{l}\text { Spinel, nepheline, } \\
\text { nosean }\end{array}$ \\
\hline US-29 & 804 & $879-890$ & 885 & $\mathrm{BDL}^{*}$ & Spinel \\
\hline US-30 & 1150 & $1235-1247$ & 1241 & 1251 & Spinel, zircon \\
\hline US-31 & 1121 & $1107-1118$ & 1113 & 1125 & Hematite \\
\hline US-32 & 814 & $753-764$ & 759 & $\mathrm{BDL}^{*}$ & Spinel \\
\hline
\end{tabular}


Table 5-7. Predicted and Measured Liquidus Temperatures. (continued)

\begin{tabular}{||c|c|c|c|c|l||}
\hline $\begin{array}{c}\text { Glass } \\
\text { ID }\end{array}$ & $\begin{array}{c}\text { Predicted } \mathbf{T}_{\mathbf{L}} \\
\left({ }^{\circ} \mathbf{C}\right)\end{array}$ & $\mathbf{T}_{\mathbf{c}}-\mathbf{T}_{\mathbf{a}}\left({ }^{\circ} \mathbf{C}\right)$ & $\begin{array}{c}\text { Optical } \mathbf{T}_{\mathbf{L}} \\
\left({ }^{\circ} \mathbf{C}\right)\end{array}$ & $\begin{array}{c}\mathbf{T}_{\mathbf{L}} \text { by XRD } \\
\text { Crystal Fraction } \\
\left({ }^{\circ} \mathbf{C}\right)\end{array}$ & $\begin{array}{c}\text { XRD Primary \& } \\
\text { Secondary Phases }\end{array}$ \\
\hline US-33 & 1121 & $1001-1007$ & 1004 & 1017 & Spinel, nosean \\
\hline US-34 & 764 & $826-840$ & 832 & BDL $^{*}$ & Spinel \\
\hline US-35 & 1118 & $1309-1318$ & 1312 & 1310 & Spinel \\
\hline US-36 & 1150 & $1235-1247$ & 1238 & 1253 & Spinel \\
\hline US-37 & 1044 & $1001-1007$ & 1004 & 1036 & Spinel \\
\hline US-38 & 1004 & $1056-1067$ & 1062 & 1038 & Spinel, NiO \\
\hline US-39 & 1150 & $1107-1110$ & 1109 & 1105 & Spinel, zircon \\
\hline US-40 & 1144 & $\begin{array}{c}\text { no glass } \\
\text { formation }\end{array}$ & $\begin{array}{c}\text { no glass } \\
\text { formation }\end{array}$ & N/A & N/A \\
\hline US-41 & 1066 & $1058-1067$ & 1063 & 1046 & Hematite \\
\hline US-42 & 895 & $1017-1028$ & 1023 & 1037 & Spinel, nepheline \\
\hline US-43 & 1147 & $1217-1225$ & 1221 & 1245 & $\begin{array}{l}\text { Zircon, Li } \text { SiO }_{3}, \\
\text { spinel }\end{array}$ \\
\hline US-44 & 1038 & $1095-1101$ & 1098 & 1112 & Spinel \\
\hline US-45 & 1038 & $1070-1074$ & 1072 & 1072 & Spinel \\
\hline
\end{tabular}

*Four glasses (US-02, US-29, US-32, and US-34) don't have a value listed for the $\mathrm{T}_{\mathrm{L}}$ calculated from XRD crystal fraction data and therefore are listed as "BDL" for below detection limit where the quantity of crystals in the heat treatments for a particular glass was too low to get a data fit.

\subsection{Crystallinity vs. Temperature Using XRD (Crystal Fraction Method)}

\subsubsection{Quantitative XRD Analysis}

Several heat treatment specimens were analyzed by XRD for each glass in order to obtain a meaningful extrapolation of crystal fraction versus temperature. A summary of the volume fractions of crystalline phases identified in each glass after the $\mathrm{CCC}$ heat treatment is provided in Table 5-8. Detailed data for crystal fraction as a function of temperature for each glass is available in Appendix B. 
Table 5-8. Identified Crystalline Phases and Their Concentrations (vol\%) in the CCC Glasses.

\begin{tabular}{|c|c|c|c|c|c|}
\hline Glass ID & Crystalline Phases & Glass ID & Crystalline Phases & Glass ID & Crystalline Phases \\
\hline US-01 & WC (inhomogeneity from milling) & US-16 & Hematite $\left(\mathrm{Fe}_{2} \mathrm{O}_{3}\right)-2.23$ & US-31 & Hematite $\left(\mathrm{Fe}_{2} \mathrm{O}_{3}\right)-3.19$ \\
\hline US-02 & WC (inhomogeneity from milling) & US-17 & Spinel -0.34 & US-32 & none \\
\hline US-03 & $\begin{array}{l}\text { Hematite }\left(\mathrm{Fe}_{2} \mathrm{O}_{3}\right)-0.15, \\
\text { WC (inhomogeneity from milling) }\end{array}$ & US-18 & Nepheline $\left(\mathrm{Na}_{7.11} \mathrm{Al}_{7.2} \mathrm{Si}_{8.8} \mathrm{O}_{32}\right)-4.7$ & US-33 & Spinel -0.39 \\
\hline US-04 & none & US-19 & none & US-34 & none \\
\hline US-05 & $\begin{array}{l}\text { Spinel - 2.44, Hematite }\left(\mathrm{Fe}_{2} \mathrm{O}_{3}\right)- \\
0.44, \\
\text { Perovskite }\left(\mathrm{CaFe}_{3} \mathrm{Ti}_{4} \mathrm{O}_{12}\right)-0.48 \\
\end{array}$ & US-20 & none & US-35 & Spinel -3.13 \\
\hline US-06 & $\begin{array}{l}\text { Spinel }-0.24, \\
\text { WC (inhomogeneity from milling) }\end{array}$ & US-21 & Spinel -0.15 & US-36 & Spinel -0.93 \\
\hline US-07 & none & US-22 & Spinel -0.34 & US-37 & $\begin{array}{l}\text { Nepheline }\left(\mathrm{Na}_{6.65} \mathrm{Al}_{6.24} \mathrm{Si}_{9.76} \mathrm{O}_{32}\right)- \\
\text { 12.5, Spinel - } 0.78\end{array}$ \\
\hline US-08 & none & US-23 & none & US-38 & $\begin{array}{l}\text { Bunsenite }(\mathrm{NiO})-0.22 \mathrm{vol} \% \text {, Spinel }- \\
0.2\end{array}$ \\
\hline US-09 & none & US-24 & none & US-39 & Spinel $-1.17 \mathrm{vol} \%$ \\
\hline US-10 & Hematite $\left(\mathrm{Fe}_{2} \mathrm{O}_{3}\right)-0.19$ & US-25 & Spinel -0.88 & US-40 & $\begin{array}{l}\text { Spinel }-0.64 \mathrm{vol} \% \text {, Hematite }\left(\mathrm{Fe}_{2} \mathrm{O}_{3}\right) \\
-2.18\end{array}$ \\
\hline US-11 & $\begin{array}{l}\text { Hematite }\left(\mathrm{Fe}_{2} \mathrm{O}_{3}\right)-1.06+\text { another } \\
\text { crystalline phase }(61-2808 \mathrm{LiCl} \text { ?) }\end{array}$ & US-26 & $\begin{array}{l}\text { Spinel - 0.93, } \\
\text { Nepheline }\left(\mathrm{Na}_{6.8} \mathrm{Al}_{6.3} \mathrm{Si}_{9.7} \mathrm{O}_{32}\right)-0.9\end{array}$ & US-41 & Hematite $\left(\mathrm{Fe}_{2} \mathrm{O}_{3}\right)-2.08$ \\
\hline US-12 & Spinel -0.93 & US-27 & $\begin{array}{l}\text { Nepheline }\left(\mathrm{Na}_{2.8} \mathrm{~K}_{0.66} \mathrm{Ca}_{0.32} \mathrm{Al}_{3.82} \mathrm{Si}_{4.18} \mathrm{O}_{15.3}\right)- \\
11.8, \mathrm{Li}_{2} \mathrm{SiO}_{3}-4.05 \\
\text { Nosean }\left(\mathrm{Na}_{8} \mathrm{Al}_{6} \mathrm{Si}_{6} \mathrm{O}_{24} \mathrm{SO}_{4}\right)-1.05 \text {, Spinel - } 0.88\end{array}$ & US-42 & $\begin{array}{l}\text { Nepheline }\left(\mathrm{KNa}_{3}\left(\mathrm{AlSiO}_{4}\right)_{4}\right), \\
\mathrm{Li} 0.88 \mathrm{Mn} 2 \mathrm{O} 4, \text { Tridymite }\left(\mathrm{SiO}_{2}\right) \text {, } \\
\mathrm{Na}_{0.68} \mathrm{Fe}_{0.68} \mathrm{Si}_{0.32} \mathrm{O}_{2}, \mathrm{Al}_{0.5} \mathrm{Si}_{0.75} \mathrm{O}_{0.25}, \\
\text { Hematite }\left(\mathrm{Fe}_{2} \mathrm{O}_{3}\right), \mathrm{Pb}_{2} \mathrm{O}_{3} \\
\end{array}$ \\
\hline US-13 & none & US-28 & none & US-43 & $\begin{array}{l}\text { Nepheline }\left(\mathrm{Na}_{6} \mathrm{~K}_{1.2} \mathrm{Al}_{7.2} \mathrm{Si}_{8.8} \mathrm{O}_{32}\right)- \\
\text { 17.8, Hematite }\left(\mathrm{Fe}_{2} \mathrm{O}_{3}\right)-1.06 \\
\text { Spinel - } 0.49\end{array}$ \\
\hline US-14 & none & US-29 & none & US-44 & Spinel -0.39 \\
\hline US-15 & none & US-30 & Spinel -1.12 & US-45 & Spinel -0.24 \\
\hline
\end{tabular}

${ }^{a} \mathrm{XRD}$ pattern is too busy to determine amounts of crystalline phases with RIQAS

Spinel peak locations and intensities matched XRD patterns of following crystalline phases:

Magnetite $\left(\mathrm{Fe}_{3} \mathrm{O}_{4}\right)$ - Glasses US-06, 12, 17, 21, 22, 25-27, 30, 33, 36-40, 44, and 45

$\mathrm{MnFe}_{2} \mathrm{O}_{4}$ - Glass US-05

$\mathrm{MgFe}_{2} \mathrm{O}_{4}$ - Glass US-43

$\mathrm{Zn}_{0.6} \mathrm{Mn}_{0.4} \mathrm{Fe}_{2} \mathrm{O}_{4}$ - Glass US-35 


\subsubsection{Data Analysis}

The section provides a comparison of the data obtained using optical and crystal fraction (XRD) methods. The correlation coefficient of the measured XRD data versus the measured optical $\mathrm{T}_{\mathrm{L}}$ data was $\mathrm{R}^{2}=0.9469$. Aside from a few outliers, the two datasets aligned very well across the entire temperature range $\left(829{ }^{\circ} \mathrm{C}\right.$ to $1312{ }^{\circ} \mathrm{C}$ for optical data and $813{ }^{\circ} \mathrm{C}$ to $1310{ }^{\circ} \mathrm{C}$ for crystal fraction data). See Figure 5-11 for a comparison of the two measured datasets.

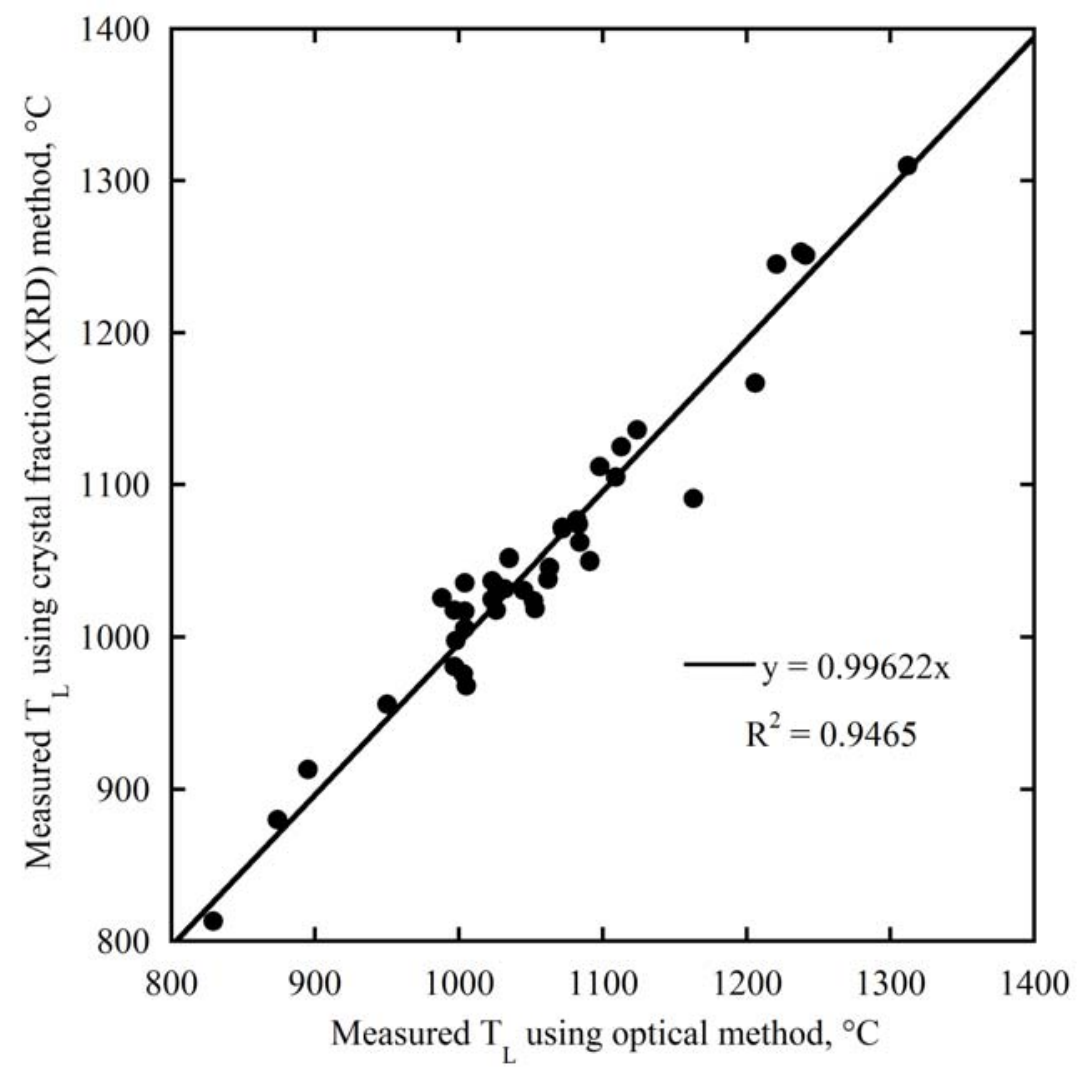

Figure 5-11. Comparison of $T_{L}$ data obtained using XRD and optical methods.

Regression models were fit from the mass fraction data presented in Table 4-1 to both the measured optical and crystal fraction $\mathrm{T}_{\mathrm{L}}$ data presented in Table 5-7 where spinel was listed as the primary phase and regression coefficients were produced. These coefficients are presented in Table 5-9. 
SRNS-STI-2008-00057

Revision 0

Table 5-9. Regression Coefficients for Optical and Crystal Fraction Methods.

\begin{tabular}{|c|c|c||}
\hline Component & Optical & XRD \\
\hline $\mathrm{Al}_{2} \mathrm{O}_{3}$ & 2459 & 2245 \\
\hline $\mathrm{B}_{2} \mathrm{O}_{3}$ & 232.3 & 505.5 \\
\hline $\mathrm{CaO}$ & 9007 & 8226 \\
\hline $\mathrm{Cr}_{2} \mathrm{O}_{3}$ & 34305 & 34764 \\
\hline $\mathrm{Fe}_{2} \mathrm{O}_{3}$ & 2236 & 1605 \\
\hline $\mathrm{K}_{2} \mathrm{O}$ & 5727 & 2622 \\
\hline $\mathrm{Li}_{2} \mathrm{O}$ & -1784 & -853 \\
\hline $\mathrm{MnO}$ & 2467 & 2347 \\
\hline $\mathrm{Na}_{2} \mathrm{O}$ & -806.2 & -705.4 \\
\hline $\mathrm{NiO}^{2}$ & 11034 & 8590 \\
\hline $\mathrm{PbO}_{\mathrm{SiO}}$ & -7884 & 1589 \\
\hline $\mathrm{SrO}$ & 1188 & 1111 \\
\hline $\mathrm{TiO}$ & 688.6 & -1806 \\
\hline $\mathrm{ZnO}$ & -2639 & 1754 \\
\hline $\mathrm{ZrO}$ & 2573 & 3139 \\
\hline $\mathrm{Others}{ }^{*}$ & 2712 & 2465 \\
\hline \hline
\end{tabular}

* Others coefficient includes $\mathrm{MgO}, \mathrm{RuO}_{2}$, and $\mathrm{SO}_{3}$.

The component coefficients, $r_{i}$, presented in Table 5-9 were then used in Equation 1:

$$
T_{L}(\text { calc })=\sum_{i=1}^{n} m_{i} r_{i}
$$

along with the mass fraction of the $i$-th component in the glass, $m_{i}$ (data from Table 4-1), and the resulting sum-product was the calculated $\mathrm{T}_{\mathrm{L}}$ value for the given method (calculated for both optical and crystal fraction $T_{L}$ data). These data were computed and the calculated values for both methods of $\mathrm{T}_{\mathrm{L}}$ measurements are presented in Table 5-10. Outliers are listed in color in the table according to the difference in temperature between the measured and calculated values (red is $>70{ }^{\circ} \mathrm{C}$ variation, orange is $55-70{ }^{\circ} \mathrm{C}$ variation, and yellow is $40-55{ }^{\circ} \mathrm{C}$ variation). 
Table 5-10. Summary of measured $T_{L}$ data for optical and crystal fraction methods for all glasses containing spinel as the primary phase as well as the calculated $T_{L}$ values for both sets using Equation 1 with data presented in Table 4-1 and the coefficients presented in Table 5-9.

\begin{tabular}{||c|c|c|c|c|c|c||}
\hline \multirow{2}{*}{ Glass ID } & \multicolumn{3}{|c|}{ Optical Data } & \multicolumn{2}{c|}{ Crystal Fraction Data } \\
\cline { 2 - 7 } & Meas. & Calc. & $\Delta \mathrm{T}$ & Meas. & Calc. & $\Delta \mathrm{T}$ \\
\hline US-01 & 1053 & 1068 & 15 & 1019 & 1025.5 & 7 \\
\hline US-03 & 997 & 1030 & 33 & 1018 & 1039.0 & 21 \\
\hline US-04 & 950 & 970 & 20 & 956 & 979.8 & 24 \\
\hline US-06 & 1005 & 981 & 24 & 968 & 979.5 & 12 \\
\hline US-07 & 1026 & 995 & 31 & 1018 & 1001.8 & 16 \\
\hline US-08 & 997 & 973 & 24 & 981 & 968.9 & 12 \\
\hline US-10 & 998 & 987 & 11 & 998 & 970.7 & 27 \\
\hline US-11 & 1003 & 1018 & 15 & 976 & 998.2 & 22 \\
\hline US-12 & 1084 & 1126 & 42 & 1062 & 1083.8 & 22 \\
\hline US-13 & 1026 & 1085 & 59 & 1034 & 1069.5 & 35 \\
\hline US-14 & 874 & 870 & 4 & 880 & 901.4 & 21 \\
\hline US-15 & 895 & 868 & 27 & 913 & 904.1 & 9 \\
\hline US-17 & 1045 & 1018 & 27 & 1031 & 1038.0 & 7 \\
\hline US-18 & 988 & 1058 & 70 & 1026 & 1056.2 & 30 \\
\hline US-19 & 1035 & 1003 & 32 & 1052 & 1002.3 & 50 \\
\hline US-20 & 1004 & 1003 & 1 & 1006 & 1002.3 & 4 \\
\hline US-21 & 1072 & 1063 & 9 & 1071 & 1031.8 & 39 \\
\hline US-22 & 1091 & 1076 & 15 & 1050 & 1047.0 & 3 \\
\hline US-23 & 1032 & 1044 & 12 & 1032 & 1025.4 & 7 \\
\hline US-24 & 1052 & 1037 & 15 & 1024 & 1023.9 & 0 \\
\hline US-25 & 1083 & 1045 & 38 & 1074 & 1052.1 & 22 \\
\hline US-26 & 1206 & 1159 & 47 & 1167 & 1143.7 & 23 \\
\hline US-27 & 1082 & 1111 & 29 & 1077 & 1081.9 & 5 \\
\hline US-28 & 829 & 884 & 55 & 813 & 838.3 & 25 \\
\hline US-30 & 1241 & 1298 & 57 & 1251 & 1266.2 & 15 \\
\hline US-33 & 1004 & 1059 & 55 & 1017 & 1060.5 & 44 \\
\hline US-36 & 1238 & 1222 & 16 & 1253 & 1237.5 & 16 \\
\hline US-37 & 1004 & 1036 & 32 & 1036 & 1065.5 & 30 \\
\hline US-38 & 1062 & 1064 & 2 & 1038 & 1026.2 & 12 \\
\hline US-39 & 1109 & 1107 & 2 & 1105 & 1129.5 & 25 \\
\hline US-42 & 1023 & 983 & 40 & 1037 & 1033.4 & 4 \\
\hline US-44 & 1098 & 1037 & 61 & 1112 & 1041.4 & 71 \\
\hline US-45 & 1072 & 1037 & 35 & 1072 & 1041.4 & 31 \\
\hline & & & & & & \\
\hline
\end{tabular}

Using the data presented in Table 5-10, Figure 5-12 shows plots of the calculated versus measured data for both methods. The correlation between the measured and calculated data has a higher degree of merit for the crystal fraction data than for the optical data (higher $\mathrm{R}^{2}$ value of 0.9089 versus 0.8970 for the optical data). The crystal fraction data only have 3 glasses with a calculated value differing from the corresponding measured value by $\geq 40{ }^{\circ} \mathrm{C}$, whereas the optical data have 9 glasses, thus helping to explain the better fit to the crystal fraction data. 

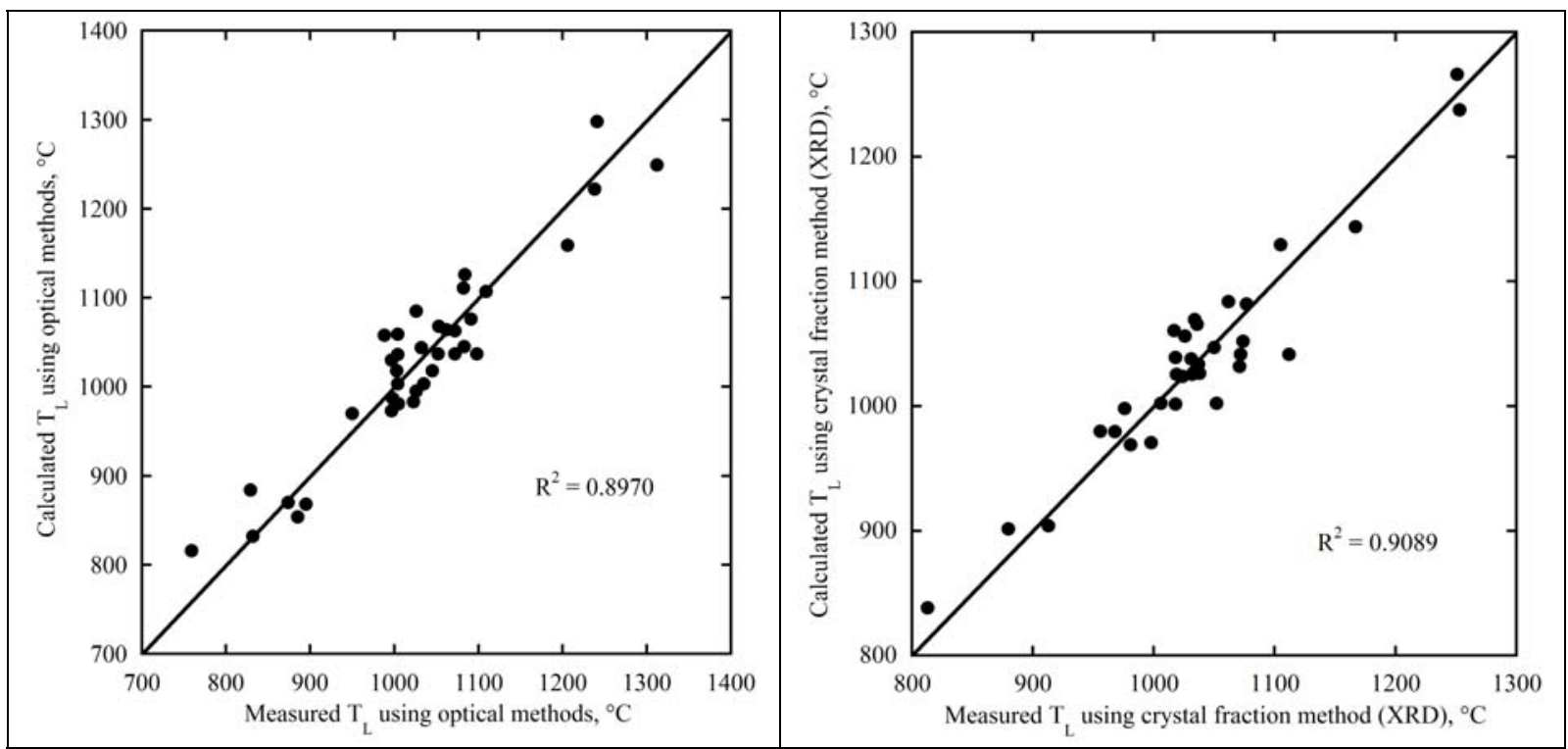

Figure 5-12. Calculated versus measured data obtained for both optical (left) and crystal fraction (right) methods.

The data calculated for the different methods are presented in Figure 5-13. The two methods predict $T_{L}$ values that are in good agreement $\left(R^{2}=0.9135\right)$. 


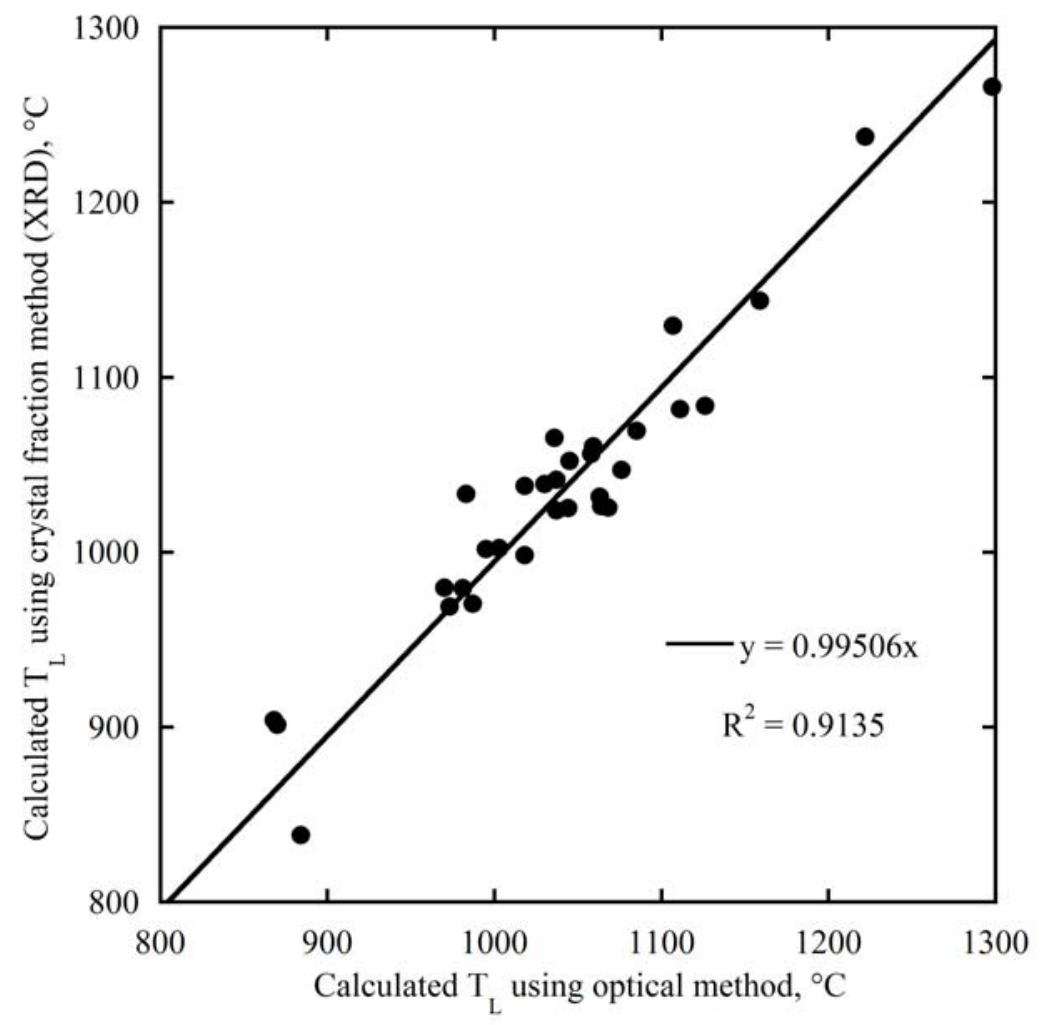

Figure 5-13. Comparison of calculated $T_{L}$ data for both crystal fraction and optical methods.

\subsection{SEM-EDS Analyses}

The SEM-EDS analysis of coated samples revealed the presence of undissolved $\mathrm{RuO}_{2}$ in all glasses due to the low solubility of $\mathrm{RuO}_{2}$ in borosilicate glass. According to Schreiber et al., ${ }^{16}$ the solubility of $\mathrm{RuO}_{2}$ in borosilicate glasses is lower than $0.001 \mathrm{wt} \%$ at melting temperatures from $1050{ }^{\circ} \mathrm{C}$ to $1150{ }^{\circ} \mathrm{C}$. Most of the $\mathrm{RuO}_{2}$ is present as approximately $1 \mu \mathrm{m}$ diameter particles. These particles tend to form agglomerates with varying sizes and shapes that are located close to the bottom of crucible. Table 5-11 summarizes the phases identified and the number of $\mathrm{RuO}_{2}$ agglomerates observed on the polished glass surfaces.

Table 5-11. Phases identified in borosilicate glasses US-01, US-03 to US-09, and US-14.

\begin{tabular}{|c|c|c|c|c|c|c|c|c|c|}
\hline & US-01 & US-03 & US-04 & US-05 & US-06 & US-07 & US-08 & US-09 & US-14 \\
\hline $\begin{array}{c}\text { Identified } \\
\text { phases }\end{array}$ & $\begin{array}{c}\text { Spinel } \\
\mathrm{Nepheline} \\
\mathrm{RuO}_{2}(5)^{\mathrm{b}}\end{array}$ & $\mathrm{RuO}_{2}$ & $\begin{array}{c}\mathrm{RuO}_{2} \\
(5)^{\mathrm{b}}\end{array}$ & $\mathrm{RuO}_{2}$ & $\begin{array}{l}\mathrm{RuO}_{2} \\
(1)^{\mathrm{b}}\end{array}$ & $\frac{\mathrm{RuO}_{2}}{(5)^{\mathrm{b}}}$ & $\begin{array}{c}\mathrm{RuO}_{2} \\
(3)^{\mathrm{b}}\end{array}$ & $\begin{array}{l}\mathrm{RuO}_{2} \\
(2)^{\mathrm{b}}\end{array}$ & $\begin{array}{c}\text { Spinel } \\
\mathrm{RuO}_{2}(6)^{\mathrm{b}}\end{array}$ \\
\hline
\end{tabular}

${ }^{\mathrm{b}}$ number of undissolved $\mathrm{RuO}_{2}$ agglomerates

A series of SEM micrographs detailing the morphology of the $\mathrm{RuO}_{2}$ agglomerates in each of the nine glasses selected for analysis is provided in Figure 5-14 through Figure 5-22. Locations are identified on some of the micrographs where semi-quantitative EDS spectra were recorded. The EDS data are provided after each corresponding figure, constituting Table 5-12 through Table $5-20$. 
There was only one $\mathrm{RuO}_{2}$ agglomerate detected on the polished surface of glass US-06, compared to two on US-09, three on US-08, five on US-01, US-04, and US-07, and six agglomerates on the polished surfaces of glasses US-03, US-05, and US-14. Spinel crystallized in glasses US-01 and US-14. Nepheline crystals were present solely in glass US-01. Figure 5-14 shows spinel and nepheline crystals together with undissolved $\mathrm{RuO}_{2}$ particles identified in glass US-01. The spinel crystals located on the shell of a bubble that formed next to the crucible wall contained a small concentration of $\mathrm{Rh}$ from the crucible. A much higher concentration of $\mathrm{Rh}$ was detected in the spinel crystals deposited along the crucible bottom in glass US-14 (see Table 5-20).

The EDS data shown in the tables below should be considered qualitative only because the detection of light elements such as B and O with EDS is limited. The scintillation counter detector cannot accurately separate and measure the characteristic X-rays of elements with atomic numbers lower than 11. Also, the detected low concentration of $\mathrm{Na}, \mathrm{Al}, \mathrm{Si}, \mathrm{Ti}, \mathrm{Mn}$, and $\mathrm{Fe}$ in the $1 \mu \mathrm{m} \mathrm{RuO}_{2}$ particles is attributed to the surrounding glass due to image drift during spectra acquisition and a large spot size. 


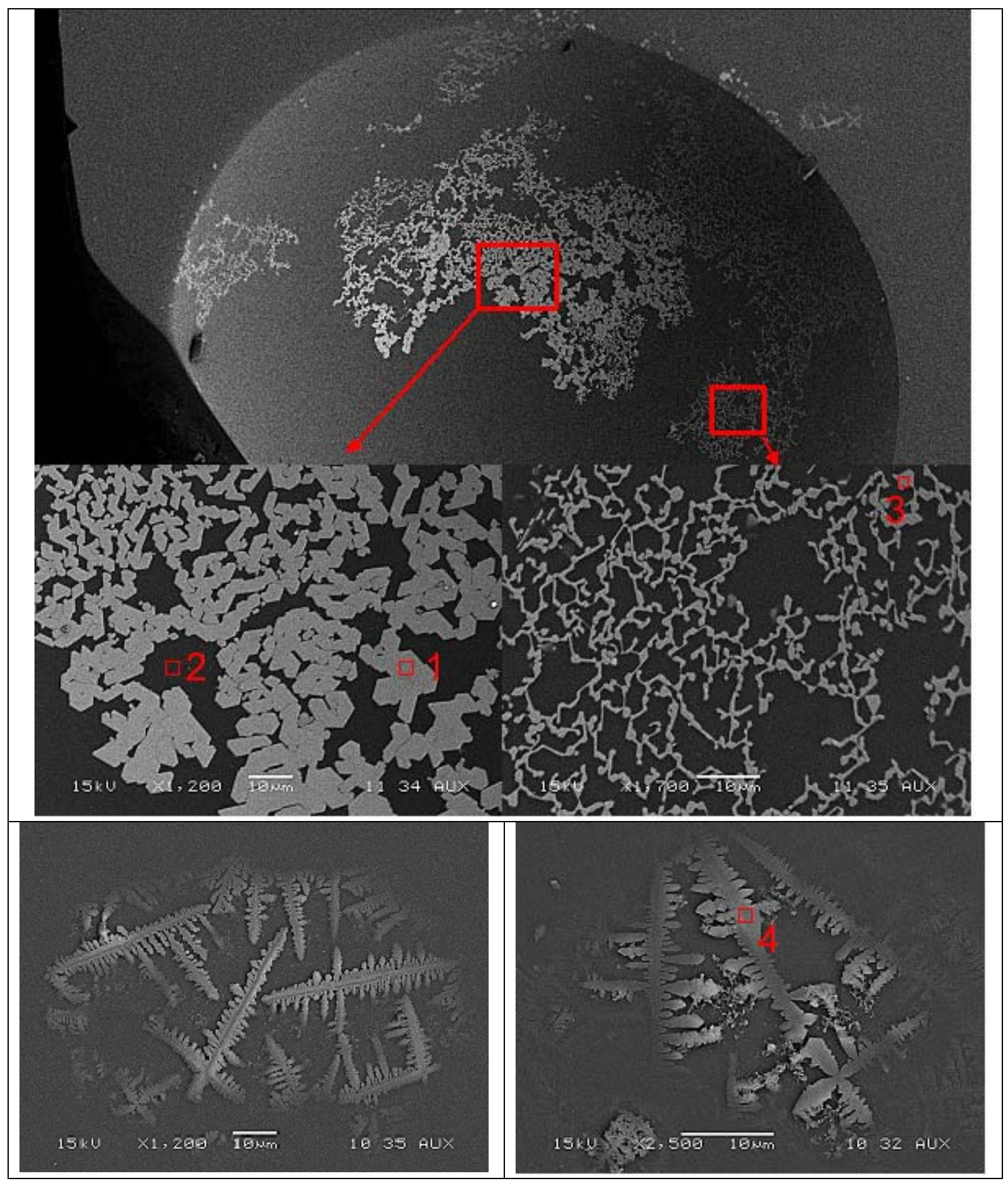

Figure 5-14. Spinel, Nepheline and Undissolved $\mathrm{RuO}_{2}$ Agglomerates in Glass US-01 After Heat Treating at $1008{ }^{\circ} \mathrm{C}$ for $24 \mathrm{~h}$. 


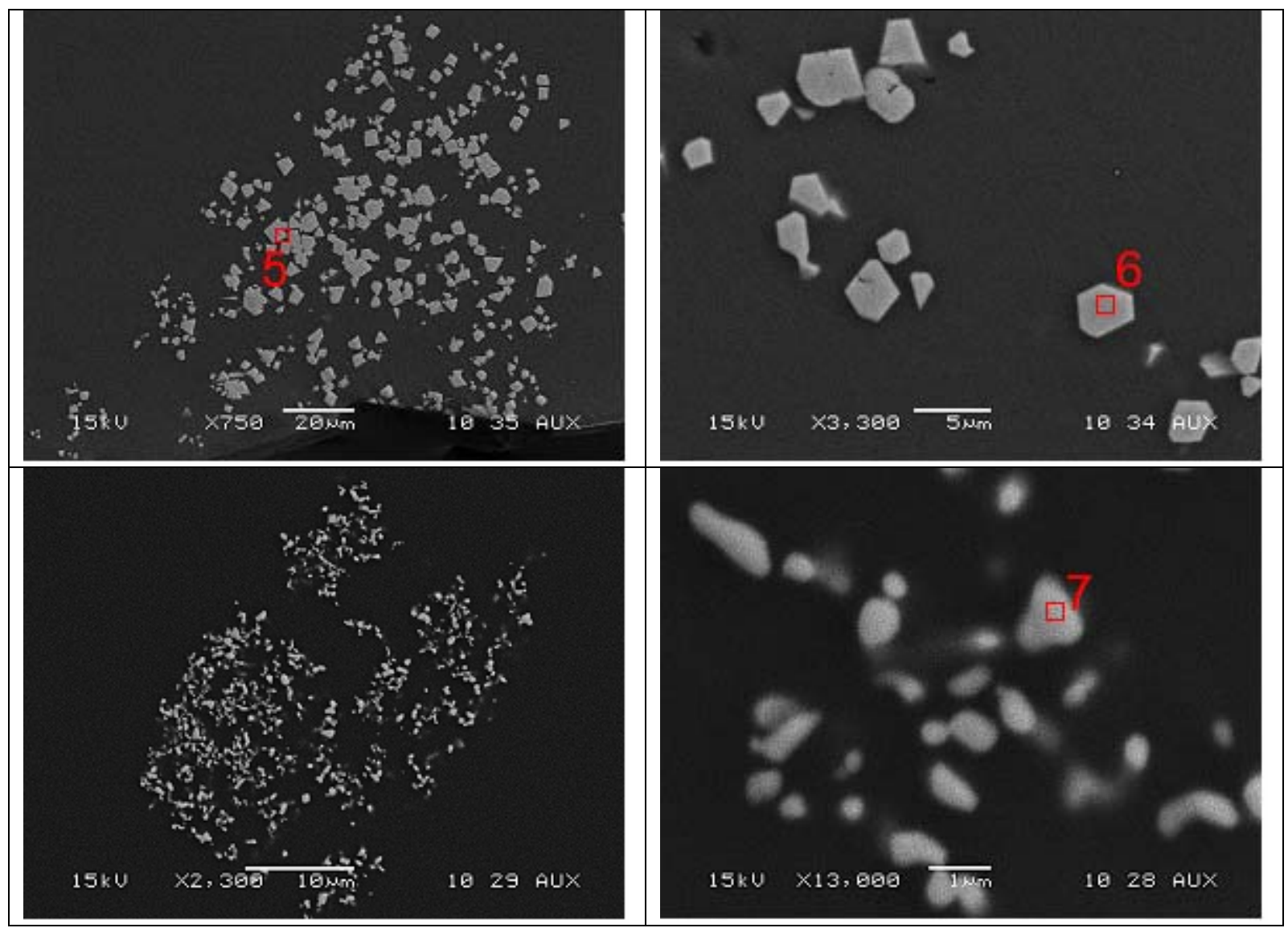

Figure 5-14. Spinel, Nepheline and Undissolved RuO2 Agglomerates in Glass US-01 After Heat Treating at $1008{ }^{\circ} \mathrm{C}$ for $24 \mathrm{~h}$. (continued)

Table 5-12. Composition of Crystals, Particles, and the Surrounding Glass in US-01 Measured with EDS After Heat Treating at $1008{ }^{\circ} \mathrm{C}$ for $24 \mathrm{~h}$.

\begin{tabular}{|c|c|c|c|c|c|c|c|}
\hline \multirow{2}{*}{ Element } & X1200_1 & X1200_2 & X1700_3 & X2500_4 & X750_5 & X3300_6 & X13000_7 \\
\hline & \multicolumn{7}{|c|}{ Atomic $\%$} \\
\hline $\mathrm{O}$ & 21.03 & 26.04 & 28.03 & 7.35 & 18.70 & 24.17 & 29.82 \\
\hline $\mathrm{Na}$ & 1.39 & 10.51 & 4.90 & 8.65 & & & 6.60 \\
\hline $\mathrm{Mg}$ & 1.29 & 0.51 & 1.93 & 0.51 & 2.75 & 2.79 & \\
\hline $\mathrm{Al}$ & 6.14 & 12.99 & 6.06 & 10.81 & 1.10 & 2.08 & 6.02 \\
\hline $\mathrm{Si}$ & 15.21 & 39.63 & 10.74 & 32.82 & 1.00 & 1.25 & 14.59 \\
\hline $\mathrm{Ru}$ & & & & & & & 38.40 \\
\hline $\mathrm{Rh}$ & 3.64 & & 1.26 & & & & \\
\hline $\mathrm{Cl}$ & & & & 15.71 & & & \\
\hline $\mathrm{K}$ & 0.50 & 1.04 & & 17.53 & & & \\
\hline $\mathrm{Cr}$ & 9.74 & & 10.08 & & 47.08 & 33.22 & \\
\hline $\mathrm{Mn}$ & 13.01 & 2.36 & 11.19 & 1.68 & 14.54 & 14.26 & 1.54 \\
\hline $\mathrm{Fe}$ & 26.18 & 6.92 & 24.21 & 4.95 & 13.63 & 20.63 & 3.03 \\
\hline $\mathrm{Ni}$ & 1.88 & & 1.60 & & 1.20 & 1.60 & \\
\hline
\end{tabular}




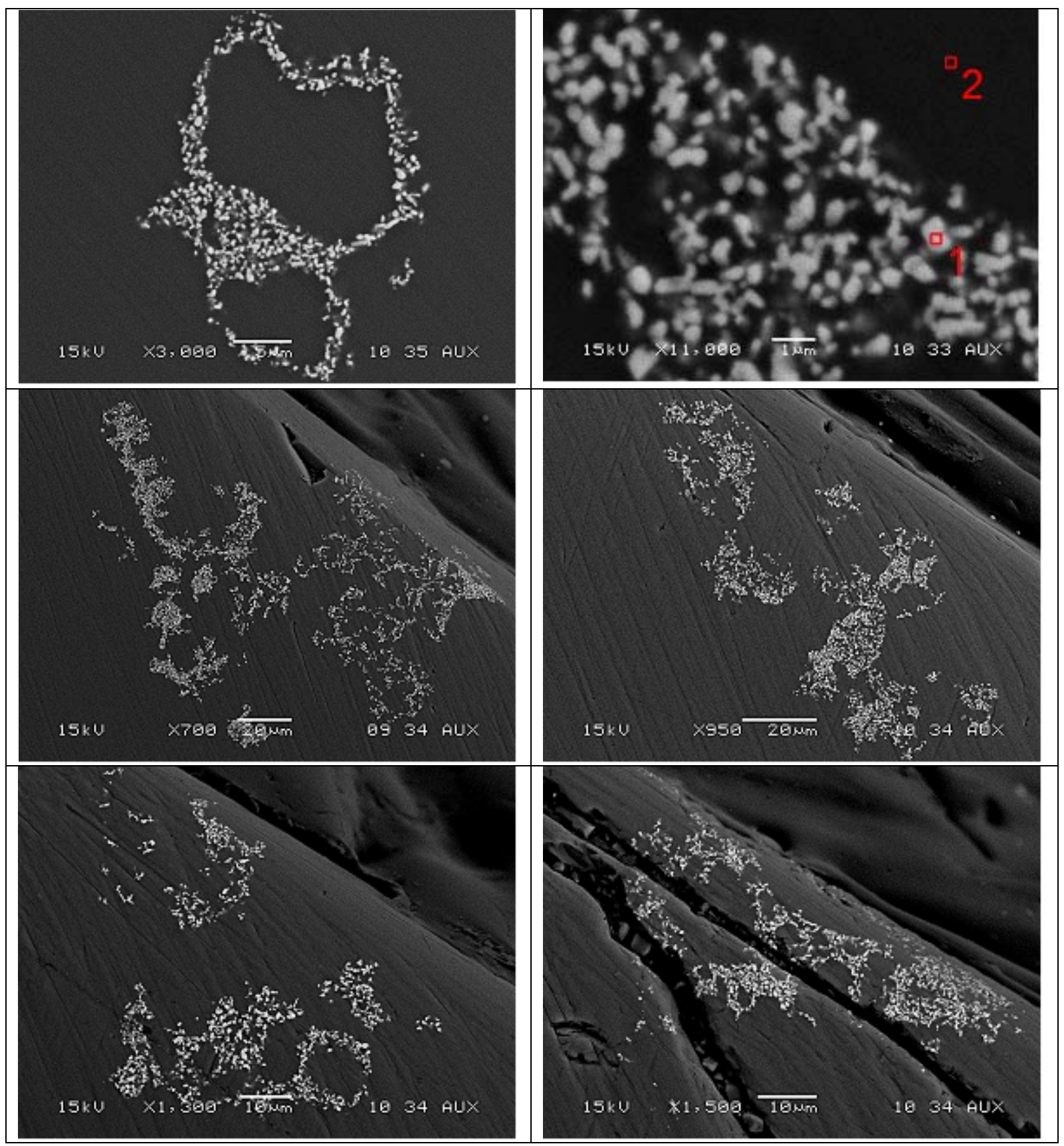

Figure 5-15. Undissolved $\mathrm{RuO}_{2}$ Agglomerates in Glass US-03 After Heat Treating at $1107^{\circ} \mathrm{C}$ for $24 \mathrm{~h}$. 
Table 5-13. Composition of Particles and the Surrounding Glass in US-03 Measured with EDS After Heat Treating at $1107^{\circ} \mathrm{C}$ for $24 \mathrm{~h}$.

\begin{tabular}{|c|c|c||}
\hline \multirow{2}{*}{ Element } & X11000_1 & \multicolumn{1}{|c|}{ X11000_2 } \\
\cline { 2 - 3 } & \multicolumn{2}{|c|}{ Atomic\% } \\
\hline $\mathrm{O}$ & 34.20 & 2.14 \\
\hline $\mathrm{Na}$ & 2.68 & 6.02 \\
\hline $\mathrm{Mg}$ & & 0.55 \\
\hline $\mathrm{Al}$ & 4.63 & 14.25 \\
\hline $\mathrm{Si}$ & 9.33 & 40.34 \\
\hline $\mathrm{K}$ & & 0.78 \\
\hline $\mathrm{Ca}$ & & 0.83 \\
\hline $\mathrm{Ru}$ & 47.43 & \\
\hline $\mathrm{Ti}$ & & 0.56 \\
\hline $\mathrm{Fe}$ & 1.71 & 4.00 \\
\hline $\mathrm{Ni}$ & & 0.53 \\
\hline
\end{tabular}




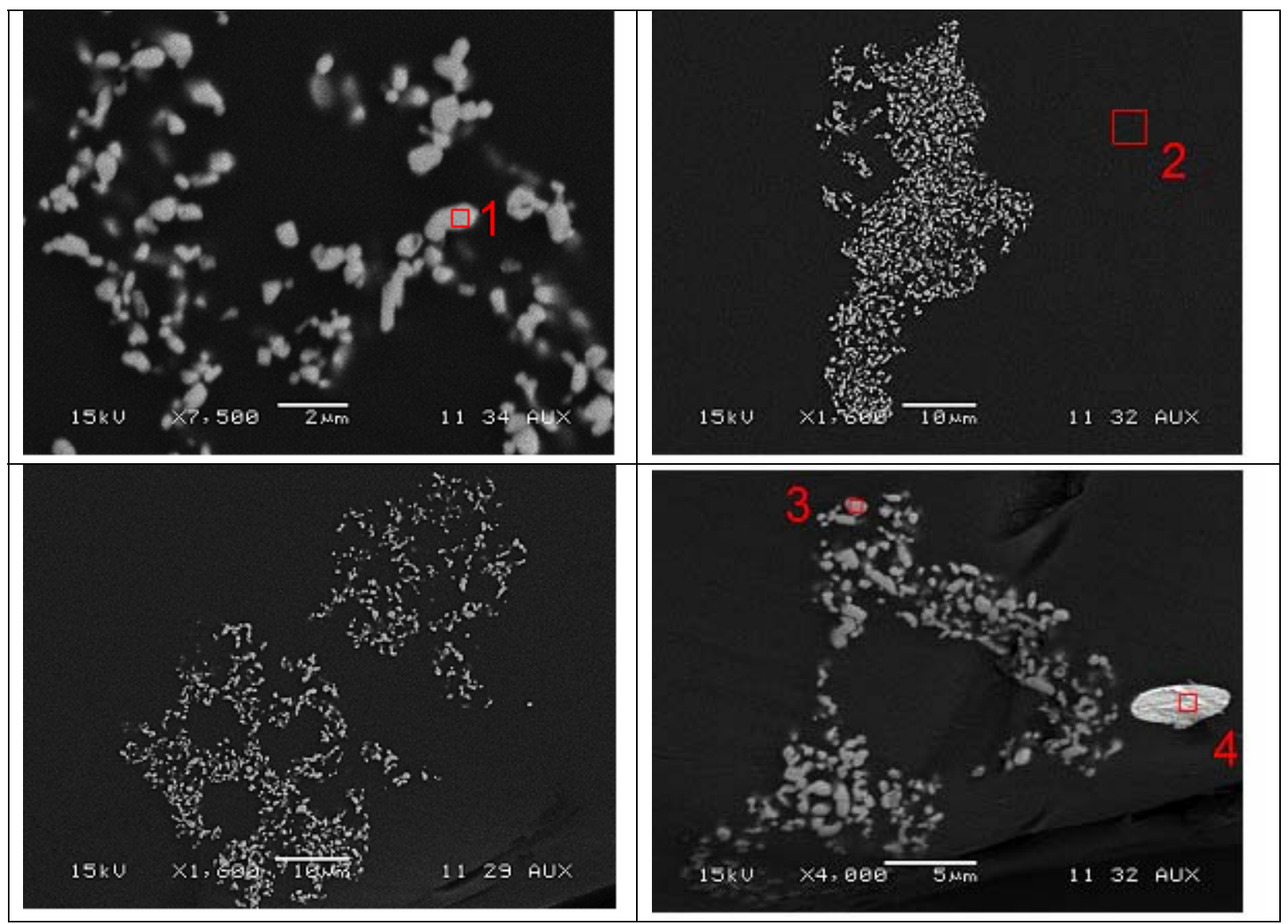

Figure 5-16. Undissolved $\mathrm{RuO}_{2}$ Agglomerates in Glass US-04 After Heat Treating at $1107^{\circ} \mathrm{C}$ for $24 \mathrm{~h}$.

Table 5-14. Composition of Particles and the Surrounding Glass in US-04 Measured with EDS After Heat Treating at $1107^{\circ} \mathrm{C}$ for $24 \mathrm{~h}$.

\begin{tabular}{||c|r|r|r|r||}
\hline \multirow{2}{*}{ Element } & \multicolumn{1}{|c|}{ X7500_1 } & \multicolumn{1}{|c|}{ 1600_2 } & \multicolumn{1}{|c|}{ X4000_3 } & X4000_4 \\
\cline { 2 - 5 } & \multicolumn{5}{|c||}{ Atomic\% } \\
\hline $\mathrm{O}$ & 33.73 & 32.07 & 33.42 & \\
\hline $\mathrm{Na}$ & 4.77 & 9.64 & 4.55 & \\
\hline $\mathrm{Mg}$ & & 0.59 & & \\
\hline $\mathrm{Al}$ & 5.29 & 13.65 & 5.43 & \\
\hline $\mathrm{Si}$ & 10.91 & 36.47 & 11.85 & \\
\hline $\mathrm{Ru}$ & 43.42 & & 41.68 & \\
\hline $\mathrm{Pt}$ & & & & 87.06 \\
\hline $\mathrm{Rh}$ & & & & 12.94 \\
\hline $\mathrm{K}$ & & 0.87 & & \\
\hline $\mathrm{Ca}$ & & 0.76 & & \\
\hline $\mathrm{Ti}$ & & 0.51 & & \\
\hline $\mathrm{Mn}$ & & 1.30 & & \\
\hline $\mathrm{Fe}$ & 1.87 & 4.13 & 3.07 & \\
\hline \hline
\end{tabular}




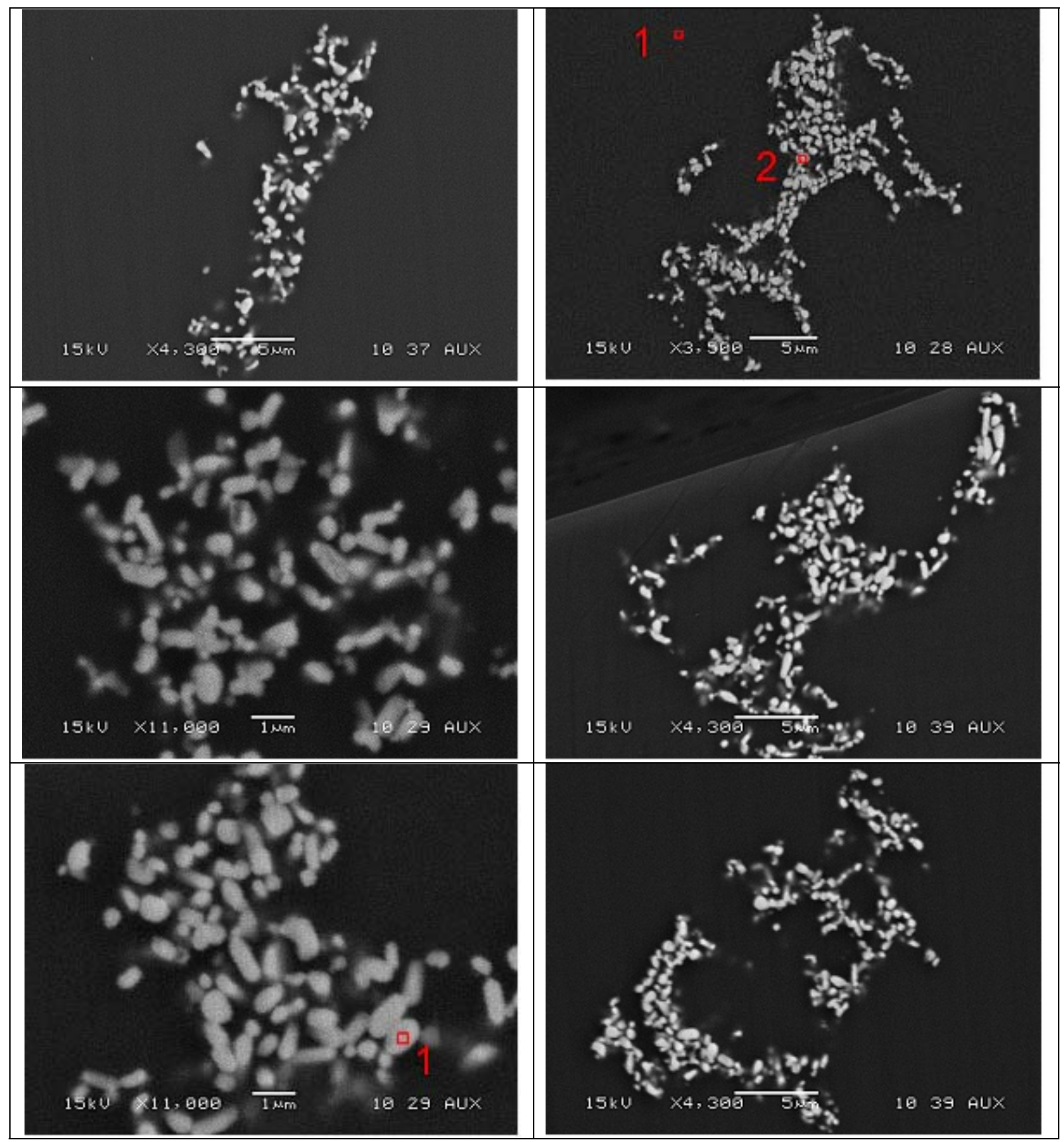

Figure 5-17. Undissolved $\mathrm{RuO}_{2}$ Agglomerates in Glass US-05 After Heat Treating at $1107^{\circ} \mathrm{C}$ for $24 \mathrm{~h}$. 
Table 5-15. Composition of Particles and the Surrounding Glass in US-05 Measured by EDS After Heat Treating at $1107^{\circ} \mathrm{C}$ for $24 \mathrm{~h}$.

\begin{tabular}{|c|c|c|c|}
\hline \multirow{2}{*}{ Element } & X3500_1 & X3500_2 & X11000_1 \\
\hline & \multicolumn{3}{|c|}{ Atomic $\%$} \\
\hline $\mathrm{O}$ & 33.97 & 40.88 & 34.05 \\
\hline $\mathrm{Na}$ & 5.96 & 5.04 & 3.69 \\
\hline $\mathrm{Mg}$ & 0.75 & & \\
\hline $\mathrm{Al}$ & 14.43 & 5.58 & 5.82 \\
\hline $\mathrm{Si}$ & 37.08 & 11.42 & 12.27 \\
\hline $\mathrm{K}$ & 0.86 & & \\
\hline $\mathrm{Ca}$ & 0.53 & & \\
\hline $\mathrm{Ru}$ & & 32.40 & 39.49 \\
\hline $\mathrm{Ti}$ & 0.51 & 0.66 & 0.35 \\
\hline $\mathrm{Mn}$ & 1.54 & 1.31 & 1.29 \\
\hline $\mathrm{Fe}$ & 4.38 & 2.71 & 3.05 \\
\hline
\end{tabular}




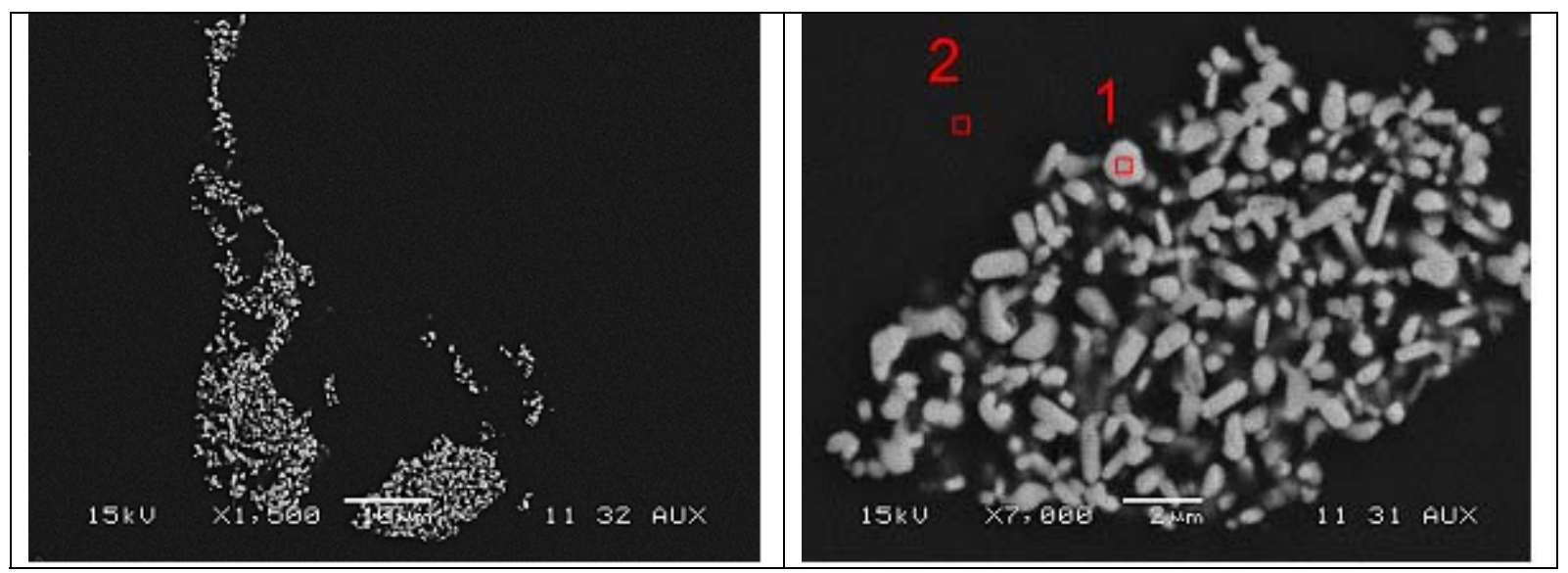

Figure 5-18. Undissolved $\mathrm{RuO}_{2}$ Agglomerates in Glass US-06 After Heat Treating at $1107^{\circ} \mathrm{C}$ for $24 \mathrm{~h}$.

Table 5-16. Composition of Particles and the Surrounding Glass in US-06 Measured by EDS After Heat Treating at $1107^{\circ} \mathrm{C}$ for $24 \mathrm{~h}$.

\begin{tabular}{||c|r|r||}
\hline \multirow{2}{*}{ Element } & \multicolumn{1}{|c|}{ X7000_1 } & \multicolumn{1}{|c|}{ X7000_2 } \\
\cline { 2 - 3 } & \multicolumn{2}{|c|}{ Atomic\% } \\
\hline $\mathrm{O}$ & 43.15 & 33.42 \\
\hline $\mathrm{Na}$ & 5.40 & 8.06 \\
\hline $\mathrm{Mg}$ & & 0.59 \\
\hline $\mathrm{Al}$ & 6.37 & 13.53 \\
\hline $\mathrm{Si}$ & 13.03 & 35.21 \\
\hline $\mathrm{K}$ & & 1.06 \\
\hline $\mathrm{Ca}$ & & 0.92 \\
\hline $\mathrm{Ti}$ & & 0.62 \\
\hline $\mathrm{Ru}$ & 28.88 & \\
\hline $\mathrm{Mn}$ & 1.03 & 2.02 \\
\hline $\mathrm{Fe}$ & 2.15 & 4.56 \\
\hline
\end{tabular}




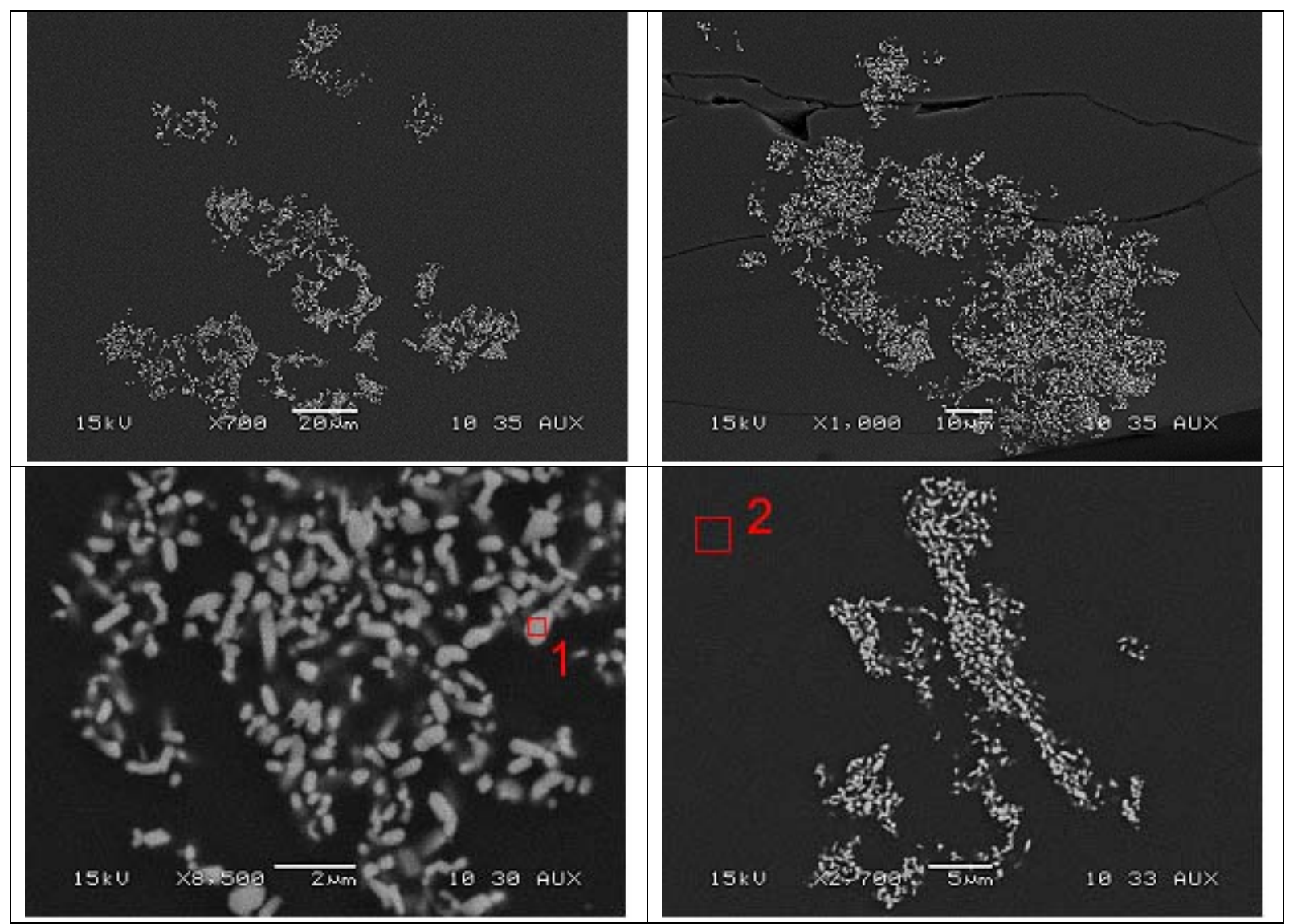

Figure 5-19. Undissolved $\mathrm{RuO}_{2}$ Agglomerates in Glass US-07 After Heat Treating at $1058{ }^{\circ} \mathrm{C}$ for $24 \mathrm{~h}$.

Table 5-17. Composition of Particles and the Surrounding Glass in US-07 Measured by EDS After Heat Treating at $1058{ }^{\circ} \mathrm{C}$ for $24 \mathrm{~h}$.

\begin{tabular}{||c|r|r||}
\hline \multirow{2}{*}{ Element } & \multicolumn{1}{|c|}{ X8500_1 } & \multicolumn{1}{|c|}{ x2700_2 } \\
\cline { 2 - 3 } & \multicolumn{2}{|c|}{ Atomic\% } \\
\hline $\mathrm{O}$ & 38.97 & 30.36 \\
\hline $\mathrm{Na}$ & 7.15 & 8.67 \\
\hline $\mathrm{Mg}$ & & 0.86 \\
\hline $\mathrm{Al}$ & 7.84 & 14.04 \\
\hline $\mathrm{Si}$ & 16.88 & 37.34 \\
\hline $\mathrm{Ru}$ & 26.20 & \\
\hline $\mathrm{K}$ & & 1.00 \\
\hline $\mathrm{Ca}$ & & 0.94 \\
\hline $\mathrm{Ti}$ & & 0.66 \\
\hline $\mathrm{Mn}$ & 0.79 & 1.93 \\
\hline $\mathrm{Fe}$ & 2.16 & 4.21 \\
\hline
\end{tabular}




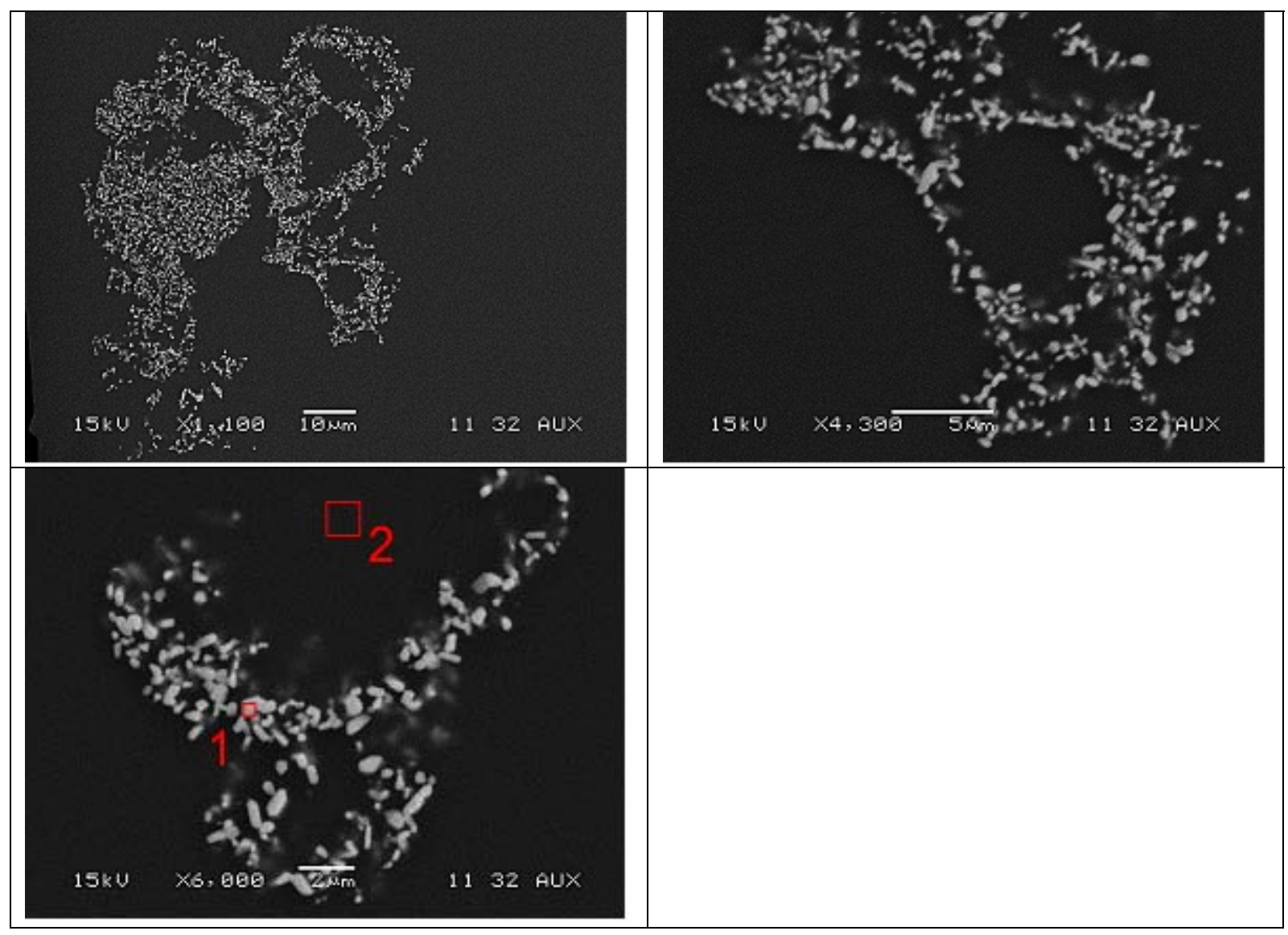

Figure 5-20. Undissolved $\mathrm{RuO}_{2}$ Agglomerates in Glass US-08 After Heat Treating at $1058{ }^{\circ} \mathrm{C}$ for $24 \mathrm{~h}$.

Table 5-18. Composition of Particles and the Surrounding Glass in US-08 Measured by EDS After Heat Treating at $1058{ }^{\circ} \mathrm{C}$ for $24 \mathrm{~h}$.

\begin{tabular}{||c|r|r||}
\hline \multirow{2}{*}{ Element } & \multicolumn{1}{|c|}{ X600__1 } & \multicolumn{1}{|c|}{ X6000_2 } \\
\cline { 2 - 3 } & \multicolumn{2}{|c|}{ Atomic\% } \\
\hline $\mathrm{O}$ & 36.10 & 31.33 \\
\hline $\mathrm{Na}$ & 4.88 & 7.72 \\
\hline $\mathrm{Al}$ & 5.22 & 13.26 \\
\hline $\mathrm{Si}$ & 13.31 & 39.81 \\
\hline $\mathrm{Ru}$ & 36.04 & \\
\hline $\mathrm{K}$ & & 0.82 \\
\hline $\mathrm{Ti}$ & 0.34 & 0.43 \\
\hline $\mathrm{Mn}$ & 1.20 & 1.47 \\
\hline $\mathrm{Fe}$ & 2.91 & 5.15 \\
\hline
\end{tabular}




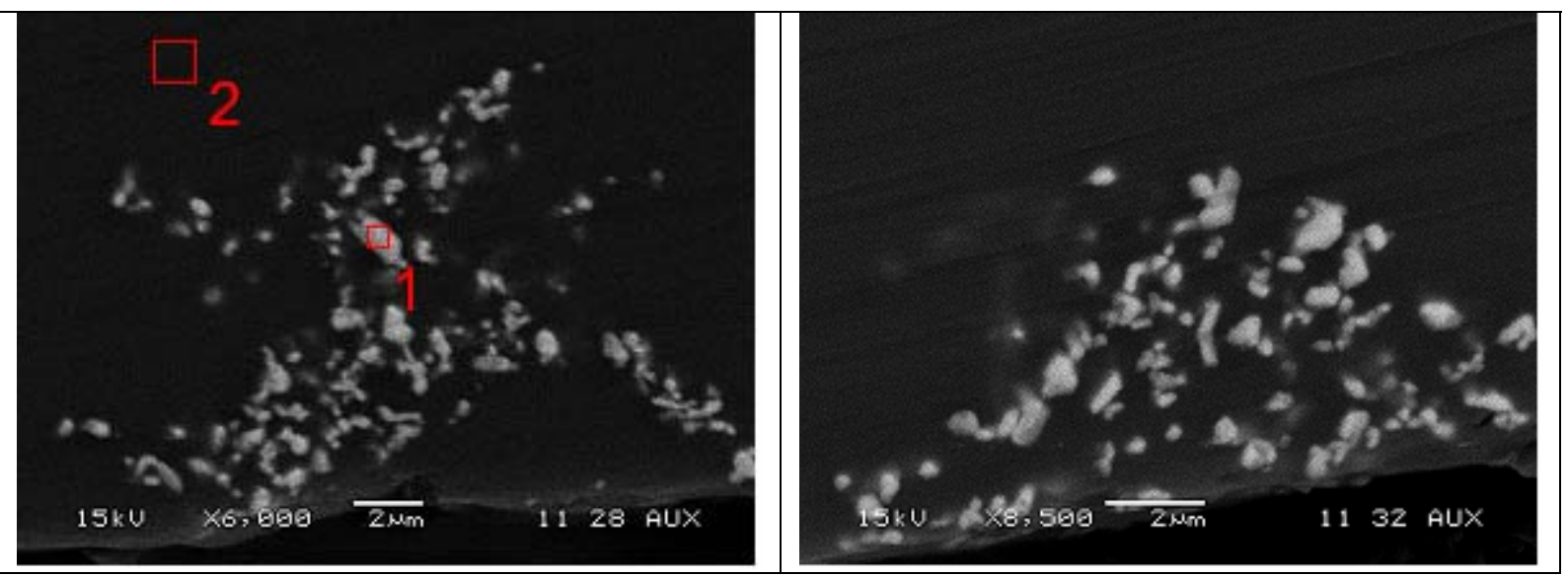

Figure 5-21. Undissolved $\mathrm{RuO}_{2}$ Agglomerates in Glass US-09 After Heat Treating at $1107^{\circ} \mathrm{C}$ for $24 \mathrm{~h}$.

Table 5-19. Composition of Particles and the Surrounding Glass in US-09 Measured by EDS After Heat Treating at $1107^{\circ} \mathrm{C}$ for $24 \mathrm{~h}$.

\begin{tabular}{||l|r|r||}
\hline \multirow{2}{*}{ Element } & \multicolumn{1}{|c||}{ X6000_1 } & \multicolumn{1}{|c|}{ X6000_2 } \\
\cline { 2 - 3 } & \multicolumn{2}{|c|}{ Atomic\% } \\
\hline $\mathrm{O}$ & 26.57 & 20.23 \\
\hline $\mathrm{Na}$ & 5.19 & 5.28 \\
\hline $\mathrm{Mg}$ & & 0.82 \\
\hline $\mathrm{Al}$ & 5.74 & 14.23 \\
\hline $\mathrm{Si}$ & 15.13 & 46.58 \\
\hline $\mathrm{Ru}$ & 42.10 & \\
\hline $\mathrm{Ca}$ & & 1.22 \\
\hline $\mathrm{Ti}$ & & 0.94 \\
\hline $\mathrm{Cr}$ & 1.46 & 0.43 \\
\hline $\mathrm{Fe}$ & 3.81 & 10.26 \\
\hline \hline
\end{tabular}




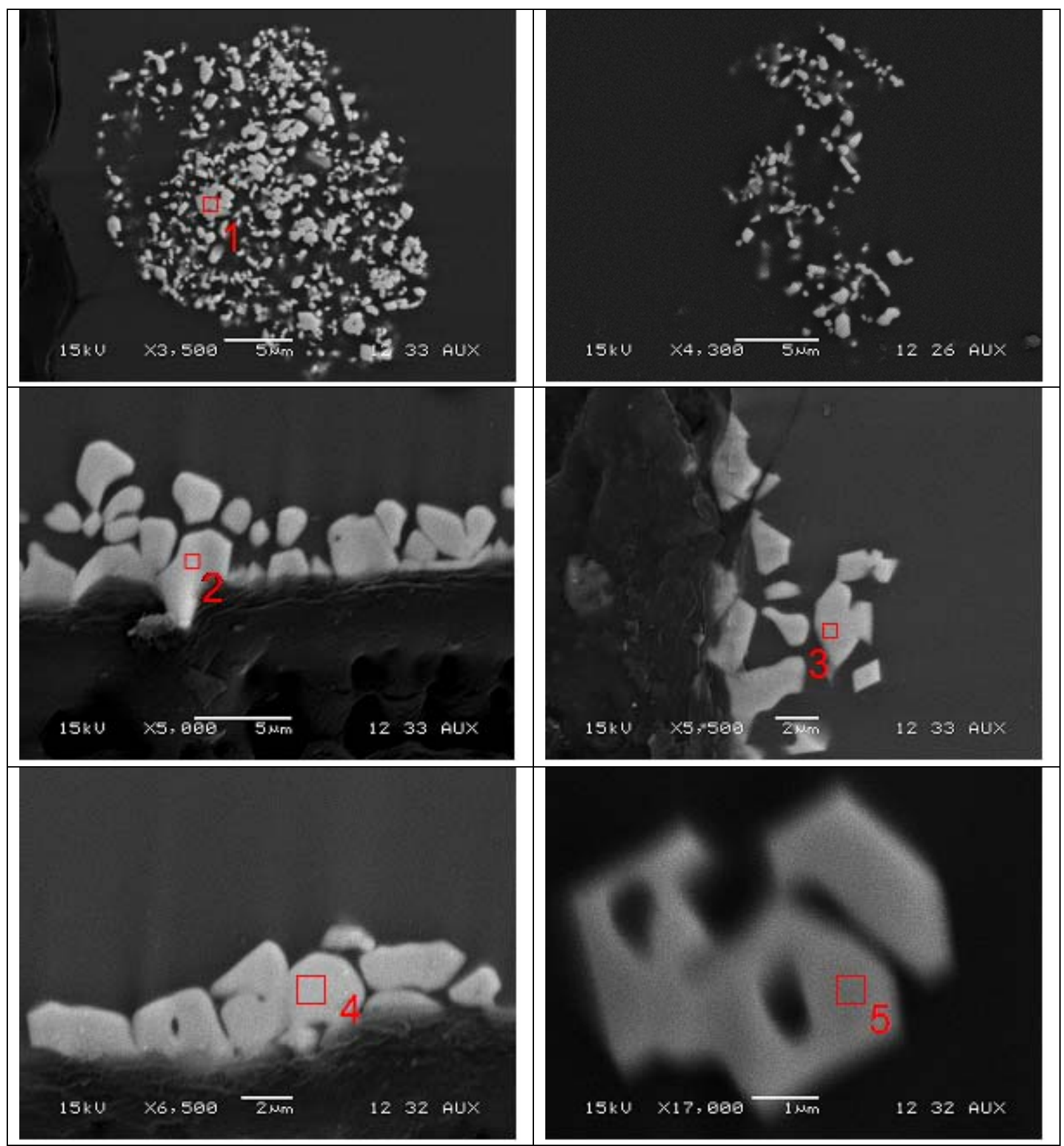

Figure 5-22. Undissolved $\mathrm{RuO}_{2}$ Agglomerates and Spinel Crystals in Glass US-14 After Heat Treating at $954^{\circ} \mathrm{C}$ for $24 \mathrm{~h}$. 
Table 5-20. Composition of Particles and Spinel Crystals in Glass US-14 Measured by EDS After Heat Treating at $954^{\circ} \mathrm{C}$ for $24 \mathrm{~h}$.

\begin{tabular}{||c|r|r|r|r|r||}
\hline \multirow{2}{*}{ Element } & \multicolumn{1}{|c|}{ X3500_1 } & \multicolumn{5}{|c||}{ x5500_4 } & \multicolumn{1}{|c||}{ X5000_2 } & \multicolumn{1}{|c||}{ x5500_3 } & X17000_5 \\
\cline { 2 - 6 } & \multicolumn{5}{|c||}{ Atomic\% } \\
\hline $\mathrm{O}$ & 37.77 & 15.93 & 15.33 & 15.32 & 27.56 \\
\hline $\mathrm{Na}$ & 2.79 & 1.73 & 1.33 & 1.33 & 2.60 \\
\hline $\mathrm{Mg}$ & & 1.20 & 1.38 & 1.40 & \\
\hline $\mathrm{Al}$ & 2.93 & 2.37 & 1.75 & 1.77 & 2.41 \\
\hline $\mathrm{Si}$ & 7.50 & 3.00 & 1.47 & 1.50 & 3.34 \\
\hline $\mathrm{Ru}$ & 46.88 & & & & \\
\hline $\mathrm{Rh}$ & & 15.67 & 17.64 & 17.66 & 15.46 \\
\hline $\mathrm{Ti}$ & & 1.13 & 1.20 & 1.19 & 1.20 \\
\hline $\mathrm{Mn}$ & & 17.81 & 18.81 & 18.78 & 14.95 \\
\hline $\mathrm{Fe}$ & 2.12 & 41.15 & 41.09 & 41.04 & 32.48 \\
\hline \hline
\end{tabular}




\subsection{Summary}

The objective of this task was to develop glass formulations for DOE waste streams with high aluminum concentrations to avoid nepheline formation while maintaining or meeting waste loading and/or waste throughput expectations as well as satisfying critical process and product performance related constraints. Liquidus temperatures and crystallization behavior were carefully characterized to support model development for higher waste loading glasses. The experimental work, characterization, and data interpretation necessary to meet these objectives were performed among three partnering laboratories: KRI, PNNL and SRNL.

Projected glass compositional regions that bound anticipated DWPF and Hanford HLW glass regions of interest were developed and used to generate glass compositions of interest for meeting the objectives of this study. A thorough statistical analysis was employed to allow for a wide range of waste glass compositions to be examined while minimizing the number of glasses that had to be fabricated and characterized in the laboratory. The glass compositions were divided into two sets, with 45 in the test matrix investigated by the U.S. laboratories and 30 in the test matrix investigated by KRI. Fabrication and characterization of the US and KRI-series glasses were generally handled separately. This report has focused mainly on the US-series glasses.

Glasses were fabricated and characterized by SRNL and PNNL. Crystalline phases were identified by XRD in the quenched and CCC glasses and were generally iron oxides and spinels, which are not expected to impact durability of the glass. Nepheline was detected in five of the glasses after the CCC heat treatment.

Chemical composition measurements each of the glasses were conducted following an analytical plan. A review of the individual oxides for each glass revealed that there were no errors in batching significant enough to impact the outcome of the study. A comparison of the measured compositions of the replicates indicated an acceptable degree of repeatability as the percent differences for most of the oxides were less than $5 \%$ and percent differences for all of the oxides were less than $10 \mathrm{wt} \%$.

Chemical durability was measured using the PCT. All but two of the study glasses had NL [B] values that were well below that of the EA reference glass. The two highest NL [B] values were for the CCC versions of glasses US-18 and US-27 (10.498 g/L and $15.962 \mathrm{~g} / \mathrm{L}$, respectively). Nepheline crystallization was identified by qualitative XRD in five of the US-series glasses. Each of these five glasses (US-18, US-26, US-27, US-37 and US-43) shows a significant increase in NL [B] after the CCC heat treatment. This reduction in durability can be attributed to the formation of nepheline during the slow cooling cycle and the removal of glass formers from the residual glass network.

The liquidus temperature of each glass in the study was determined by both optical microscopy and XRD methods. The correlation coefficient of the measured XRD $\mathrm{T}_{\mathrm{L}}$ data versus the measured optical $\mathrm{T}_{\mathrm{L}}$ data was very good $\left(\mathrm{R}^{2}=0.9469\right)$. Aside from a few outliers, the two datasets aligned very well across the entire temperature range $\left(829^{\circ} \mathrm{C}\right.$ to $1312^{\circ} \mathrm{C}$ for optical data and $813^{\circ} \mathrm{C}$ to $1310^{\circ} \mathrm{C}$ for XRD crystal fraction data). The data also correlated well with the predictions of a $\mathrm{T}_{\mathrm{L}}$ model. The correlation between the measured and calculated data had a higher degree of merit for the XRD crystal fraction data than for the optical data (higher $\mathrm{R}^{2}$ value of 0.9089 versus 0.8970 for the optical data). 
The SEM-EDS analysis of select samples revealed the presence of undissolved $\mathrm{RuO}_{2}$ in all glasses due to the low solubility of $\mathrm{RuO}_{2}$ in borosilicate glass. These particles tended to form agglomerates with varying sizes and shapes that were located close to the bottom of crucibles.

The results of this study provide further insight into the ability of borosilicate waste glass to incorporate increased concentrations of aluminum. The glass composition and properties data will be incorporated into a database of glass composition-property relationships $\left(\mathrm{ComPro}^{\mathrm{a}}\right)$ to support further optimization of waste glass compositions at DOE sites.

\footnotetext{
a Taylor, A. S., T. B. Edwards, J. C. George, T. K. Snyder and D. K. Peeler, "The SRNL Composition - Properties $\left(\right.$ ComPro $\left.^{\mathrm{TM}}\right)$ Database," U.S. Department of Energy Report WSRC-RP-2004-00704, Revision 0, Westinghouse Savannah River Company, Aiken, SC (2004).
} 


\subsection{References}

1. Barnes, B. O., "Pacific Northwest National Laboratory Quality Assurance Plan, Project 47607 - Task 6, LAW Glass Studies," U.S. Department of Energy Report PNNL-QAP-47607, Revision 0, Pacific Northwest National Laboratory, Richland, WA (2006).

2. Peeler, D. K. and T. B. Edwards, "Test Plan: Improved Alumina Solubility in US High Level Waste Glasses," U.S. Department of Energy Report SRNL-PSE-2006-00275, Washington Savannah River Company, Aiken, SC 2006).

3. Aloy, A., J. D. Vienna, K. M. Fox, T. B. Edwards and D. K. Peeler, "Glass Selection Strategy: Development of US and KRI Test Matrices," U.S. Department of Energy Report WSRC-STI2006-00205, Revision 0, Washington Savannnah River Company, Aiken, SC (2006).

4. Aloy, A., "Final Report: Improved Retention of Aluminum, Chromium, and Sulfate in U.S. Waste Glasses," U.S. Department of Energy Report SRNS-OS-2008-00001, V.G. Khlopin Radium Institute, St. Petersburg, Russia (2008).

5. Marra, S. L. and C. M. Jantzen, "Characterization of Projected DWPF Glass Heat Treated to Simulate Canister Centerline Cooling," U.S. Department of Energy Report WSRC-TR-92-142, Revision 1, Westinghouse Savannah River Company, Aiken, SC (1993).

6. Edwards, T. B., "An Analytical Plan for Measuring the Chemical Compositions of Glasses from the Task \#6 Study," U.S. Department of Energy Report SRNL-SCS-2006-00036, Washington Savannah River Company, Aiken, SC (2006).

7. Herman, C. C., T. B. Edwards, D. R. Best, D. M. Marsh and R. J. Workman, "Reduction of Constraints: Phase 2 Experimental Assessment for Sludge-Only Processing," U.S. Department of Energy Report WSRC-TR-2002-00482, Revision 0, Westinghouse Savannah River Company, Aiken, SC (2002).

8. Edwards, T. B., "An Analytical Plan for Measuring the PCT Solutions for the Third Set of Glasses from the Task \#6 Study (U)," U.S. Department of Energy Report SRNL-SCS-2006-00041, Washington Savannah River Company, Aiken, SC (2006).

9. Edwards, T. B., "An Analytical Plan for Measuring the PCT Solutions for the Second Set of Glasses from the Task \#6 Study," U.S. Department of Energy Report SRNL-SCS-2006-00037, Washington Savannah River Company, Aiken, SC (2006).

10. Edwards, T. B., "An Analytical Plan for Measuring the PCT Solutions for the First Set of Glasses from the Task \#6 Study," U.S. Department of Energy Report SRNL-SCS-2006-00035, Washington Savannah River Company, Aiken, SC (2006).

11. "Standard Test Methods for Determining the Liquidus Temperature (TL) of Waste Glasses and Simulated Waste Glases," U.S. Department of Energy Report PNNL Technical Procedure GDL-LQT, Revison 3, Pacific Northwest National Laboratory, Richland, WA

12. "Quantitative and Semi-Quantitative Analysis using X-Ray Diffraction," U.S. Department of Energy Report PNNL Technical Procedure GDL-XRD, Revision 0, Pacific Northwest National Laboratory, Richland, WA 
13. Bickford, D. F. and C. M. Jantzen, "Devitrification of SRL Defense Waste Glass," Sci. Basis for Nuclear Waste Management VII, edited by G. L. McVay. Elsevier, New York, pp. 557-565 (1984).

14. Edwards, T. B., "A Statistical Review of the Chemical Composition Measurements and PCT Results for the Glass Fabricated as Part of the US Test Matrix," U.S. Department of Energy Report SRNL-SCS-2007-00029, Washington Savannah River Company, Aiken, SC (2007).

15. Jantzen, C. M., N. E. Bibler, D. C. Beam, C. L. Crawford and M. A. Pickett, "Characterization of the Defense Waste Processing Facility (DWPF) Environmental Assessment (EA) Glass Standard Reference Material," U.S. Department of Energy Report WSRC-TR-92-346, Revision 1, Westinghouse Savannah River Company, Aiken, SC (1993).

16. Schreiber, H. D., F. A. Settle Jr., P. L. Jamison, J. P. Eckenrode and G. W. Headley, "Ruthenium in Glass-Forming Borosilicate Melts," Journal of the Less-Common Metals, 115 145-154 (1986). 
SRNS-STI-2008-00057

Revision 0

Appendix A

Heat Treatment Summaries for Optical Determination of Liquidus Temperature 
The data below include some low resolution optical microscopy (LROM) observations and more detailed information from the high resolution optical microscopy (HROM) analyses using thin sections (ThS). Here, the heat treatments are listed with the type of observation (i.e., HROM or LROM), the temperature, and the observations that were made on that particular sample.

\section{US-01:}

- HROM ThS, $1156^{\circ} \mathrm{C}$ : homogeneous $\gg>>$ lower temperature

- HROM ThS, $1107^{\circ} \mathrm{C}$ : black agglomerates of noble metals at bottom $\gg>>$ lower temperature

- HROM ThS, $1057^{\circ} \mathrm{C}$ : black agglomerates of noble metals $>>>$ lower temperature

- HROM ThS, $905^{\circ} \mathrm{C}$ : cubic crystals (spinels) $\gg>\mathrm{T}_{\mathrm{L}}$ between $905^{\circ} \mathrm{C}$ and $1057^{\circ} \mathrm{C}$

- HROM ThS, $954^{\circ} \mathrm{C}$ : cubic crystals (spinels) $\gg>\mathrm{T}_{\mathrm{L}}$ between $954^{\circ} \mathrm{C}$ and $1057^{\circ} \mathrm{C}$

- HROM ThS, $1008^{\circ} \mathrm{C}$ : black agglomerates of noble metals, few spinels $\gg>\mathrm{T}_{\mathrm{L}}$ between $1008^{\circ} \mathrm{C}$ and $1057^{\circ} \mathrm{C}$

- HROM ThS, $1040^{\circ} \mathrm{C}$ : black agglomerates of noble metals, several spinels at bottom of sample >> $\mathrm{T}_{\mathrm{L}}$ between $1040^{\circ} \mathrm{C}$ and $1057^{\circ} \mathrm{C}$

- HROM ThS, $1049^{\circ} \mathrm{C}$ : black agglomerates of noble metals at bottom of crucible, center of sample contains several spinels and black agglomerates of noble metals $\gg>T_{L}$ between $1049^{\circ} \mathrm{C}$ and $1057^{\circ} \mathrm{C}$

- $\mathrm{T}_{\mathrm{L}}=1053^{\circ} \mathrm{C}$

US-02:

- HROM ThS, $1285^{\circ} \mathrm{C}$ : homogeneous

- LROM, $1108^{\circ} \mathrm{C}$ : large black agglomerates of noble metals at bottom $>>>$ lower temperature

- HROM ThS, $1008^{\circ} \mathrm{C}$ : black agglomerates of noble metals $>>>$ lower temperature

- HROM ThS, $905^{\circ} \mathrm{C}$ : needles of noble metals $\gg>>$ lower temperature

- HROM ThS, $954^{\circ} \mathrm{C}$ : needles of noble metals $\gg>>$ lower temperature

- HROM ThS, $982^{\circ} \mathrm{C}$ : needles of noble metals and black agglomerates of noble metals $>>$ lower temperature

- HROM ThS, $995^{\circ} \mathrm{C}$ : needles of noble metals and black agglomerates of noble metals $>>$ lower temperature

- HROM ThS, $1001^{\circ} \mathrm{C}$ : black agglomerates of noble metals throughout specimen $>>>$ lower temperature

- HROM ThS, $861^{\circ} \mathrm{C}$ : needles of noble metals throughout sample $>>>$ lower temperature

- HROM ThS, $812^{\circ} \mathrm{C}$ : black agglomerates of noble metals throughout, very small undissolved particles everywhere $\gg>\mathrm{T}_{\mathrm{L}}$ less than $812^{\circ} \mathrm{C}$

- HROM ThS, $764^{\circ} \mathrm{C}$ : black agglomerates of noble metals, lots of needles, undissolved material $\gg>\mathrm{T}_{\mathrm{L}}$ less than $764^{\circ} \mathrm{C}$

- HROM ThS, $704^{\circ} \mathrm{C}$ : hematite on surface of sample, hematite throughout bulk, some spinel, black agglomerates of noble metals $\gg>\mathrm{T}_{\mathrm{L}}$ between $704^{\circ} \mathrm{C}$ and $764^{\circ} \mathrm{C}$

- HROM ThS, $731^{\circ} \mathrm{C}$ : large prism-like clear crystals, needles throughout bulk $\gg>\mathrm{T}_{\mathrm{L}}$ between $731^{\circ} \mathrm{C}$ and $764^{\circ} \mathrm{C}$

- HROM ThS, $753^{\circ} \mathrm{C}$ : lots of clear cubic crystals, some needles $>>\mathrm{T}_{\mathrm{L}}$ between $753^{\circ} \mathrm{C}$ and $764^{\circ} \mathrm{C} ; \mathrm{T}_{\mathrm{L}}=759^{\circ} \mathrm{C}$ 


\section{US-03:}

- HROM ThS, $1206^{\circ} \mathrm{C}$ : black agglomerates of noble metals in bulk $\gg>$ lower temperature

- HROM ThS, $1056^{\circ} \mathrm{C}$ : black agglomerates of noble metals in bulk $>>>$ lower temperature

- HROM ThS, $1009^{\circ} \mathrm{C}$ : black agglomerates of noble metals in bulk $>>>$ lower temperature

- HROM ThS, $954^{\circ} \mathrm{C}$ : cubic crystals (spinels) $\gg>\mathrm{T}_{\mathrm{L}}$ between $954^{\circ} \mathrm{C}$ and $1009^{\circ} \mathrm{C}$

- HROM ThS, $982^{\circ} \mathrm{C}$ : large agglomerates of cubic crystals (spinels) $(\sim 50)$, black agglomerates of noble metals at bottom of sample $>>>\mathrm{T}_{\mathrm{L}}$ between $982^{\circ} \mathrm{C}$ and $1009^{\circ} \mathrm{C}$

- HROM ThS, $995^{\circ} \mathrm{C}$ : black agglomerates of noble metals throughout sample $\gg>\mathrm{T}_{\mathrm{L}}$ between $995^{\circ} \mathrm{C}$ and $1009^{\circ} \mathrm{C}$

- HROM ThS, $1000^{\circ} \mathrm{C}$ : several black agglomerates of noble metals in bulk, no crystals visible $\gg>\mathrm{T}_{\mathrm{L}}$ between $995^{\circ} \mathrm{C}$ and $1000^{\circ} \mathrm{C}$

- $\mathrm{T}_{\mathrm{L}}=997^{\circ} \mathrm{C}$

\section{US-04:}

- HROM ThS, $1205^{\circ} \mathrm{C}$ : homogeneous $\gg>>$ lower temperature

- HROM ThS, $1156^{\circ} \mathrm{C}$ : homogeneous $\gg>>$ lower temperature

- HROM ThS, $1056^{\circ} \mathrm{C}$ : black agglomerates of noble metals $\gg>>$ lower temperature

- HROM ThS, $954^{\circ} \mathrm{C}$ : very tiny needles $\gg>>\mathrm{T}_{\mathrm{L}}$ between $954^{\circ} \mathrm{C}$ and $1056^{\circ} \mathrm{C}$

- HROM ThS, $1009^{\circ} \mathrm{C}$ : black agglomerates of noble metals $\gg>\mathrm{T}_{\mathrm{L}}$ between $954^{\circ} \mathrm{C}$ and $1009^{\circ} \mathrm{C}$

- HROM ThS, $980^{\circ} \mathrm{C}$ : black agglomerates of noble metals, needles on side of sample (also, there were lots of really small anomalies in the sample that were too small to distinguish at $1000 \mathrm{x}$ using HROM) $\gg>\mathrm{T}_{\mathrm{L}}$ between $980^{\circ} \mathrm{C}$ and $1009^{\circ} \mathrm{C}$

- HROM ThS, $994^{\circ} \mathrm{C}$ : black agglomerates of noble metals $\gg>\mathrm{T}_{\mathrm{L}}$ between $980^{\circ} \mathrm{C}$ and $994^{\circ} \mathrm{C}$

- HROM ThS, $1000^{\circ} \mathrm{C}$ : black agglomerates of noble metals, undissolved particulates throughout sample $\gg>\mathrm{T}_{\mathrm{L}}$ between $980^{\circ} \mathrm{C}$ and $994^{\circ} \mathrm{C}$

- HROM ThS, $861^{\circ} \mathrm{C}$ : surface crystallization

- HROM ThS, $904^{\circ} \mathrm{C}$ : spinel everywhere, some hematite $>>>T_{L}$ between $904^{\circ} \mathrm{C}$ and $954^{\circ} \mathrm{C}$

- HROM ThS, $931^{\circ} \mathrm{C}$ : surface crystallization, spinel throughout, undissolved material throughout $\gg>\mathrm{T}_{\mathrm{L}}$ between $931^{\circ} \mathrm{C}$ and $954^{\circ} \mathrm{C}$

- HROM ThS, $946^{\circ} \mathrm{C}$ : very large quantity of small undissolved particles, possibly a few cubic crystals (spinels) $\gg>\mathrm{T}_{\mathrm{L}}$ between $946^{\circ} \mathrm{C}$ and $954^{\circ} \mathrm{C}$

- $\mathrm{T}_{\mathrm{L}}=950^{\circ} \mathrm{C}$ 
US-05:

- HROM ThS, $1157^{\circ} \mathrm{C}$ : large black agglomerates of noble metals $\gg>$ lower temperature

- HROM ThS, $1056^{\circ} \mathrm{C}$ : large black agglomerates of noble metals, $\mathrm{T}_{\mathrm{L}}$ possibly above $1056^{\circ} \mathrm{C}$ but verification advisable $\gg>$ temperature between $1056^{\circ} \mathrm{C}$ and $1156^{\circ} \mathrm{C}$

- HROM ThS, $1109^{\circ} \mathrm{C}$ : large black agglomerates of noble metals, $\mathrm{T}_{\mathrm{L}}$ possibly above $1109^{\circ} \mathrm{C}$ but verification advisable $>>>$ temperature possibly between $1109^{\circ} \mathrm{C}$ and $1156^{\circ} \mathrm{C}$

- HROM ThS, $1130^{\circ} \mathrm{C}$ : large black agglomerates of noble metals $\gg \gg \mathrm{T}_{\mathrm{L}}$ between questionable

- HROM ThS, $994^{\circ} \mathrm{C}$ :very crystallized with hematite and spinel, large black agglomerates of noble metals throughout bulk $\gg>\mathrm{T}_{\mathrm{L}}$ between $994^{\circ} \mathrm{C}$ and $1056^{\circ} \mathrm{C}$

- HROM ThS, $1027^{\circ} \mathrm{C}$ : lots of crystals at base (round spinels) $\gg>\mathrm{T}_{\mathrm{L}}$ between $1027^{\circ} \mathrm{C}$ and $1056^{\circ} \mathrm{C}$

- HROM ThS, $1043^{\circ} \mathrm{C}$ : several black agglomerates of noble metals, several clusters with round shaped objects $\gg>\mathrm{T}_{\mathrm{L}}$ between $994^{\circ} \mathrm{C}$ and $1027^{\circ} \mathrm{C}$

- HROM ThS, $1013^{\circ} \mathrm{C}$ : hematite and cubic crystals (spinels) everywhere; few black agglomerates of noble metals $\gg>\mathrm{T}_{\mathrm{L}}$ between $1013^{\circ} \mathrm{C}$ and $1027^{\circ} \mathrm{C}$

- HROM ThS, $1019^{\circ} \mathrm{C}$ : large black agglomerates of noble metals, few cubic crystals (spinels) $\gg>\mathrm{T}_{\mathrm{L}}$ between $1019^{\circ} \mathrm{C}$ and $1027^{\circ} \mathrm{C}$

- $\mathrm{T}_{\mathrm{L}}=1023^{\circ} \mathrm{C}$

US-06:

- HROM ThS, $1206^{\circ} \mathrm{C}$ : black agglomerates of noble metals in bulk $\gg>$ lower temperature

- HROM ThS, $1056^{\circ} \mathrm{C}$ : black agglomerates of noble metals at bottom and in bulk $>>>$ lower temperature

- HROM ThS, $954^{\circ} \mathrm{C}$ : cubic crystals (spinels) $\gg>>$ higher temperature

- HROM ThS, $1008^{\circ} \mathrm{C}$ : large black agglomerates of noble metals $\gg>\mathrm{T}_{\mathrm{L}}$ between $954^{\circ} \mathrm{C}$ and $1008^{\circ} \mathrm{C}$

- HROM ThS, $982^{\circ} \mathrm{C}$ : cubic crystals (spinels) at middle of the bottom ( 100 spinel agglomerate) $\gg>\mathrm{T}_{\mathrm{L}}$ between $982^{\circ} \mathrm{C}$ and $1008^{\circ} \mathrm{C}$

- HROM ThS, $995^{\circ} \mathrm{C}$ : black agglomerates of noble metals, needles at bottom (large but few) $>>>$ $\mathrm{T}_{\mathrm{L}}$ between $995^{\circ} \mathrm{C}$ and $1008^{\circ} \mathrm{C}$

- HROM ThS, $1001^{\circ} \mathrm{C}$ : black agglomerates of noble metals throughout bulk, submicron particles in areas (maybe spinels) $\gg>\mathrm{T}_{\mathrm{L}}$ between $1001^{\circ} \mathrm{C}$ and $1008^{\circ} \mathrm{C}$

- $\mathrm{T}_{\mathrm{L}}=1005^{\circ} \mathrm{C}$

\section{US-07:}

- HROM ThS, $1157^{\circ} \mathrm{C}$ : black agglomerates of noble metals $>>>$ lower temperature

- HROM ThS, $1107^{\circ} \mathrm{C}$ : black agglomerates of noble metals $\gg>>$ lower temperature

- HROM ThS, $1006^{\circ} \mathrm{C}$ : cubic crystals (spinels) $\gg>\mathrm{T}_{\mathrm{L}}$ between $1006^{\circ} \mathrm{C}$ and $1107^{\circ} \mathrm{C}$

- HROM ThS, $1058^{\circ} \mathrm{C}$ : no cubic crystals, black agglomerates of noble metals at bottom of sample and $\sim 10$ throughout the bulk of the sample $>>>\mathrm{T}_{\mathrm{L}}$ between $1006^{\circ} \mathrm{C}$ and $1058^{\circ} \mathrm{C}$

- HROM ThS, $1015^{\circ} \mathrm{C}$ : lots of cubic crystals (spinels), black agglomerates of noble metals $\gg>$ $\mathrm{T}_{\mathrm{L}}$ between $1015^{\circ} \mathrm{C}$ and $1058^{\circ} \mathrm{C}$

- HROM ThS, $1029^{\circ} \mathrm{C}$ : black agglomerates of noble metals, no cubic crystals $>>>\mathrm{T}_{\mathrm{L}}$ between $1015^{\circ} \mathrm{C}$ and $1029^{\circ} \mathrm{C}$

- HROM ThS, $1022^{\circ} \mathrm{C}$ : large and small cubic crystals (spinel), $\mathrm{RuO}_{2}$ agglomerates $\gg>\mathrm{T}_{\mathrm{L}}$ between $1022^{\circ} \mathrm{C}$ and $1029^{\circ} \mathrm{C}$

- $\mathrm{T}_{\mathrm{L}}=1026^{\circ} \mathrm{C}$ 


\section{US-08:}

- HROM ThS, $1157^{\circ} \mathrm{C}$ : large black agglomerates of noble metals $\gg>$ lower temperature

- HROM ThS, $1107^{\circ} \mathrm{C}$ : black agglomerates of noble metals $>>>$ lower temperature

- HROM ThS, $1006^{\circ} \mathrm{C}$ : black agglomerates of noble metals $\gg>>$ lower temperature

- HROM ThS, $903^{\circ} \mathrm{C}$ : cubic crystals (spinels) $\gg>\mathrm{T}_{\mathrm{L}}$ between $903^{\circ} \mathrm{C}$ and $1006^{\circ} \mathrm{C}$

- HROM ThS, $954^{\circ} \mathrm{C}$ : streaks of cubic crystals (spinels) throughout the sample, needles at bottom of sample $\gg>\mathrm{T}_{\mathrm{L}}$ between $954^{\circ} \mathrm{C}$ and $1006^{\circ} \mathrm{C}$

- HROM ThS, $980^{\circ} \mathrm{C}$ : black agglomerates of noble metals, chunks of cubic crystals (spinel) at bottom; small chunks of debris in bulk of sample - too small to distinguish at $1000 \mathrm{x} \gg>>\mathrm{T}_{\mathrm{L}}$ between $980^{\circ} \mathrm{C}$ and $1006^{\circ} \mathrm{C}$

- HROM ThS, $994^{\circ} \mathrm{C}$ : black agglomerates of noble metals, microscopic undissolved material (too small to distinguish at $1000 \mathrm{x}$ ) $\gg>\mathrm{T}_{\mathrm{L}}$ between $994^{\circ} \mathrm{C}$ and $1006^{\circ} \mathrm{C}$

- HROM ThS, $1000^{\circ} \mathrm{C}$ : several black agglomerates of noble metals, small undissolved particulates throughout sample $\gg>\mathrm{T}_{\mathrm{L}}$ between $994^{\circ} \mathrm{C}$ and $1000^{\circ} \mathrm{C}$

- $\mathrm{T}_{\mathrm{L}}=997^{\circ} \mathrm{C}$

US-09:

- HROM ThS, $1157^{\circ} \mathrm{C}$ : large black agglomerates of noble metals, dark red prismatic crystals $>>>$ higher temperature

- HROM ThS, $1202^{\circ} \mathrm{C}$ : homogeneous $\gg>>\mathrm{T}_{\mathrm{L}}$ between $1157^{\circ} \mathrm{C}$ and $1202^{\circ} \mathrm{C}$

- HROM ThS, $1232^{\circ} \mathrm{C}$ : homogeneous $\gg>\mathrm{T}_{\mathrm{L}}$ between $1157^{\circ} \mathrm{C}$ and $1202^{\circ} \mathrm{C}$

- HROM ThS, $1180^{\circ} \mathrm{C}$ : sample clear aside from one black agglomerate of noble metals $\gg>T_{L}$ between $1157^{\circ} \mathrm{C}$ and $1180^{\circ} \mathrm{C}$

- HROM ThS, $1169^{\circ} \mathrm{C}$ : no crystals, few black agglomerates of noble metals $\gg>\mathrm{T}_{\mathrm{L}}$ between $1157^{\circ} \mathrm{C}$ and $1169^{\circ} \mathrm{C}$

- $\mathrm{T}_{\mathrm{L}}=1163^{\circ} \mathrm{C}$

US-10:

- HROM ThS, $1157^{\circ} \mathrm{C}$ : homogeneous $\gg>>$ lower temperature

- HROM ThS, $1107^{\circ} \mathrm{C}$ : few black agglomerates of noble metals $\gg>>$ lower temperature

- HROM ThS, $1006^{\circ} \mathrm{C}$ : black agglomerates of noble metals $>>>$ lower temperature

- HROM ThS, $954^{\circ} \mathrm{C}$ : cubic crystals (spinels) $\gg>\mathrm{T}_{\mathrm{L}}$ between $954^{\circ} \mathrm{C}$ and $1006^{\circ} \mathrm{C}$

- HROM ThS, $982^{\circ} \mathrm{C}$ : streaks of tiny cubic crystals (spinels) throughout the bulk, black agglomerates of noble metals $\gg>\mathrm{T}_{\mathrm{L}}$ between $982^{\circ} \mathrm{C}$ and $1006^{\circ} \mathrm{C}$

- HROM ThS, $995^{\circ} \mathrm{C}$ : black agglomerates of noble metals, needles at bottom, few large cubic clusters (spinels?) at base $\gg>\mathrm{T}_{\mathrm{L}}$ between $995^{\circ} \mathrm{C}$ and $1006^{\circ} \mathrm{C}$

- HROM ThS, $1001^{\circ} \mathrm{C}$ : no cubic crystals, black agglomerates of noble metals throughout sample $\gg>\mathrm{T}_{\mathrm{L}}$ between $995^{\circ} \mathrm{C}$ and $1001^{\circ} \mathrm{C}$

- $\mathrm{T}_{\mathrm{L}}=998^{\circ} \mathrm{C}$ 
US-11:

- HROM ThS, $1157^{\circ} \mathrm{C}$ : few black agglomerates of noble metals $>>>$ lower temperature

- HROM ThS, $1107^{\circ} \mathrm{C}$ : few black agglomerates of noble metals $\gg>$ lower temperature

- HROM ThS, $1006^{\circ} \mathrm{C}$ : black agglomerates of noble metals, some very small undissolved particles throughout bulk of sample $>>>$ lower temperature

- HROM ThS, $903^{\circ} \mathrm{C}$ : many red hexagons (hematite), some cubic crystals $\gg>T_{L}$ between $903^{\circ} \mathrm{C}$ and $1006^{\circ} \mathrm{C}$

- HROM ThS, $954^{\circ} \mathrm{C}$ : lots of crystals including hematite and a few cubic crystals (spinel) $\gg>\mathrm{T}_{\mathrm{L}}$ between $954^{\circ} \mathrm{C}$ and $1006^{\circ} \mathrm{C}$

- HROM ThS, $980^{\circ} \mathrm{C}$ : lots of hematite and cubic crystals (spinels), black agglomerates of noble metals $>>>\mathrm{T}_{\mathrm{L}}$ between $980^{\circ} \mathrm{C}$ and $1006^{\circ} \mathrm{C}$

- HROM ThS, $994^{\circ} \mathrm{C}$ : lots of spinel and hematite (small to moderate in size), some hematite strings, black agglomerates of noble metals $\gg>T_{L}$ between $994^{\circ} \mathrm{C}$ and $1006^{\circ} \mathrm{C}$

- HROM ThS, $1000^{\circ} \mathrm{C}$ : large spinel cluster at bottom of sample, black agglomerates of noble metals throughout bulk $\gg>>\mathrm{T}_{\mathrm{L}}$ between $1000^{\circ} \mathrm{C}$ and $1006^{\circ} \mathrm{C}$

- $\mathrm{T}_{\mathrm{L}}=1003^{\circ} \mathrm{C}$

US-12:

- HROM ThS, $1157^{\circ} \mathrm{C}$ : black agglomerates of noble metals on bottom $>>>$ lower temperature

- HROM ThS, $1107^{\circ} \mathrm{C}$ : homogeneous in small sample section $\gg>$ lower temperature

- HROM ThS, $1006^{\circ} \mathrm{C}$ : cubic crystals (spinels) $\gg>\mathrm{T}_{\mathrm{L}}$ between $1006^{\circ} \mathrm{C}$ and $1107^{\circ} \mathrm{C}$

- HROM ThS, $1030^{\circ} \mathrm{C}$, cubic crystals (spinels) $\gg>\mathrm{T}_{\mathrm{L}}$ between $1030^{\circ} \mathrm{C}$ and $1107^{\circ} \mathrm{C}$

- HROM ThS, $1058^{\circ} \mathrm{C}$ : cubic crystals (spinels) $\gg>\mathrm{T}_{\mathrm{L}}$ between $1058^{\circ} \mathrm{C}$ and $1107^{\circ} \mathrm{C}$

- HROM ThS, $1074^{\circ} \mathrm{C}$ : small cubic crystals (spinels) $\gg>\mathrm{T}_{\mathrm{L}}$ between $1074^{\circ} \mathrm{C}$ and $1107^{\circ} \mathrm{C}$

- HROM ThS, $1086^{\circ} \mathrm{C}$ : sample clear of spinel $\gg>\mathrm{T}_{\mathrm{L}}$ between $1074^{\circ} \mathrm{C}$ and $1086^{\circ} \mathrm{C}$

- HROM ThS, $1082^{\circ} \mathrm{C}$ : some features in agglomerates appear to be cubic at base, large black agglomerates of noble metals $\gg>\mathrm{T}_{\mathrm{L}}$ between $1082^{\circ} \mathrm{C}$ and $1086^{\circ} \mathrm{C}$

- $\mathrm{T}_{\mathrm{L}}=1084^{\circ} \mathrm{C}$

US-13:

- HROM ThS, $1157^{\circ} \mathrm{C}$ : homogeneous $\gg>>$ lower temperature

- LROM, $1108^{\circ} \mathrm{C}$ : few white agglomerates on surface, few black agglomerates of noble metals in bulk $\gg>$ heat treatment between $1108^{\circ} \mathrm{C}$ and $1157^{\circ} \mathrm{C}$

- HROM ThS, $1131^{\circ} \mathrm{C}$ : black agglomerates of noble metals in bulk $\gg>>$ lower temperature

- HROM ThS, $1106^{\circ} \mathrm{C}$ : black agglomerates of noble metals in bulk $>>>$ lower temperature

- HROM ThS, $1005^{\circ} \mathrm{C}$ : cubic crystals (spinels) $\gg>\mathrm{T}_{\mathrm{L}}$ between $1005^{\circ} \mathrm{C}$ and $1106^{\circ} \mathrm{C}$

- HROM ThS, $1056^{\circ} \mathrm{C}$ : black agglomerates of noble materials in bulk $\gg>\mathrm{T}_{\mathrm{L}}$ between $1005^{\circ} \mathrm{C}$ and $1056^{\circ} \mathrm{C}$

- HROM ThS, $1040^{\circ} \mathrm{C}$ : black agglomerates of noble metals $\gg>\mathrm{T}_{\mathrm{L}}$ between $1005^{\circ} \mathrm{C}$ and $1040^{\circ} \mathrm{C}$

- HROM ThS, $1015^{\circ} \mathrm{C}$ : several cubic crystals (spinels) throughout bulk, black agglomerates of noble metals $\gg>\mathrm{T}_{\mathrm{L}}$ between $1015^{\circ} \mathrm{C}$ and $1040^{\circ} \mathrm{C}$

- HROM ThS, $1029^{\circ} \mathrm{C}$ : black agglomerates of noble metals, no spinel $\gg>\mathrm{T}_{\mathrm{L}}$ between $1015^{\circ} \mathrm{C}$ and $1029^{\circ} \mathrm{C}$

- HROM ThS, $1022^{\circ} \mathrm{C}$ : several small cubes (spinel), black agglomerates of noble metals $\gg \gg \mathrm{T}_{\mathrm{L}}$ between $1022^{\circ} \mathrm{C}$ and $1029^{\circ} \mathrm{C}$

- $\mathrm{T}_{\mathrm{L}}=1026^{\circ} \mathrm{C}$ 
US-14:

- HROM ThS, $901{ }^{\circ} \mathrm{C}$ : big black agglomerates of noble metals at bottom and in the bulk, needlelike crystals that often agglomerate to stars $>>>$ higher temperature

- HROM ThS, $1005^{\circ} \mathrm{C}$ : few black agglomerates of noble metals $\gg>>$ lower temperature

- HROM ThS, $952^{\circ} \mathrm{C}$ : many tiny needles $\gg>\mathrm{T}_{\mathrm{L}}$ between $952^{\circ} \mathrm{C}$ and $1005^{\circ} \mathrm{C}$

- HROM ThS, $980^{\circ} \mathrm{C}$ : small needles everywhere in bulk $\gg>\mathrm{T}_{\mathrm{L}}$ between $980^{\circ} \mathrm{C}$ and $1005^{\circ} \mathrm{C}$

- HROM ThS, $994^{\circ} \mathrm{C}$ : needles everywhere $\gg>\mathrm{T}_{\mathrm{L}}$ between $994^{\circ} \mathrm{C}$ and $1005^{\circ} \mathrm{C}$

- HROM ThS, $1000^{\circ} \mathrm{C}$ : several black agglomerates of noble metals, lots of small undissolved needles $\gg>\mathrm{T}_{\mathrm{L}}$ between $994^{\circ} \mathrm{C}$ and $1005^{\circ} \mathrm{C}$

- HROM ThS, $861^{\circ} \mathrm{C}$ : lots of small cubic crystals (spinels) at base, lots of needles, black agglomerates of noble metals $\gg>T_{L}$ between $861{ }^{\circ} \mathrm{C}$ and $901{ }^{\circ} \mathrm{C}$

- HROM ThS, $879^{\circ} \mathrm{C}$ : needles everywhere, black agglomerates of noble metals (discoloration in sample - possibly redox) $\gg>\mathrm{T}_{\mathrm{L}}$ between $861^{\circ} \mathrm{C}$ and $879^{\circ} \mathrm{C}$

- HROM ThS, $869^{\circ} \mathrm{C}$ : black agglomerates of noble metals in bulk at base, needles throughout bulk lots of undissolved particles throughout, small cubic crystals (spinels) in bulk and throughout base $\gg>>\mathrm{T}_{\mathrm{L}}$ between $869^{\circ} \mathrm{C}$ and $879^{\circ} \mathrm{C}$

- $\mathrm{T}_{\mathrm{L}}=874^{\circ} \mathrm{C}$

US-15:

- LROM, $1121^{\circ} \mathrm{C}, 1136^{\circ} \mathrm{C}, 1156^{\circ} \mathrm{C}, 1176^{\circ} \mathrm{C}, 1236^{\circ} \mathrm{C}$ : big black amorphous agglomerates at bottom, glass becomes darker with increasing temperature $\gg>$ SEM

- HROM ThS, $1386^{\circ} \mathrm{C}$ : homogeneous

- SEM-EDS, $1236^{\circ} \mathrm{C}$ : big black amorphous agglomerates at bottom are $\mathrm{RuO}_{2} \gg>$ lower temperature to detect other crystals besides $\mathrm{RuO}_{2}$

- HROM ThS, $1005^{\circ} \mathrm{C}$ : black agglomerates of noble metals $>>>$ lower temperature

- HROM ThS, $901^{\circ} \mathrm{C}$ : needles $\gg>>$ higher temperature

- HROM ThS, $954^{\circ} \mathrm{C}$ : few needles $\gg>>\mathrm{T}_{\mathrm{L}}$ between $954^{\circ} \mathrm{C}$ and $1005^{\circ} \mathrm{C}$

- HROM ThS, $982^{\circ} \mathrm{C}$ : black agglomerates of noble metals $\gg>\mathrm{T}_{\mathrm{L}}$ between $954^{\circ} \mathrm{C}$ and $982^{\circ} \mathrm{C}$

- HROM ThS, $861^{\circ} \mathrm{C}$ : lots of hematite, black agglomerates of noble metals, needles everywhere $\gg \mathrm{T}_{\mathrm{L}}$ between $861^{\circ} \mathrm{C}$ and $901^{\circ} \mathrm{C}$

- HROM ThS, $879^{\circ} \mathrm{C}$ : hematite everywhere, black agglomerates of noble metals, needles throughout, undissolved particles throughout $\gg>>\mathrm{T}_{\mathrm{L}}$ between $879^{\circ} \mathrm{C}$ and $901^{\circ} \mathrm{C}$

- HROM ThS, $890^{\circ} \mathrm{C}$ : several hematite crystals, black agglomerates of noble metals, lots of undissolved material throughout $\gg>\mathrm{T}_{\mathrm{L}}$ between $890^{\circ} \mathrm{C}$ and $901^{\circ} \mathrm{C}$

- $\mathrm{T}_{\mathrm{L}}=895^{\circ} \mathrm{C}$ 


\section{US-16:}

- LROM, $1402^{\circ} \mathrm{C}$ : brown crystal surface layer, fractured surface clear black

- HROM ThS, $1199^{\circ} \mathrm{C}$ : some undissolved materials (appears that they are all noble metals) $>>>$ $\mathrm{T}_{\mathrm{L}}<1199^{\circ} \mathrm{C}$

- HROM ThS, $1247^{\circ} \mathrm{C}$ : sample appears to be clear of crystals but does contain noble metals $>>>$ $\mathrm{T}_{\mathrm{L}}<1199^{\circ} \mathrm{C}$

- HROM ThS, $1000^{\circ} \mathrm{C}$ : sample full of undissolved materials (spinels and noble metals) $\gg>\mathrm{T}_{\mathrm{L}}$ between $1000^{\circ} \mathrm{C}$ and $1199^{\circ} \mathrm{C}$

- HROM ThS, $1098^{\circ} \mathrm{C}$ : lots of undissolved materials (small and large spinels, some noble metals) $\gg>\mathrm{T}_{\mathrm{L}}$ between $1098^{\circ} \mathrm{C}$ and $1199^{\circ} \mathrm{C}$

- HROM ThS, $1126^{\circ} \mathrm{C}$ : no spinel, some undissolved materials present $\gg>\mathrm{T}_{\mathrm{L}}$ between $1099^{\circ} \mathrm{C}$ and $1126^{\circ} \mathrm{C}$

- HROM ThS, $980^{\circ} \mathrm{C}$ : sample full of undissolved materials (noble metals and spinels), some hematite at base of sample $\gg>>\mathrm{T}_{\mathrm{L}}$ between $1099^{\circ} \mathrm{C}$ and $1126^{\circ} \mathrm{C}$ [XRD sample]

- HROM ThS, $1113^{\circ} \mathrm{C}$ : spinel at base, noble metals along base $\gg>\mathrm{T}_{\mathrm{L}}$ between $1113^{\circ} \mathrm{C}$ and $1126^{\circ} \mathrm{C}$

- HROM ThS, $1120^{\circ} \mathrm{C}$ : spinel at base, noble metals along base $\gg>\mathrm{T}_{\mathrm{L}}$ between $1120^{\circ} \mathrm{C}$ and $1126^{\circ} \mathrm{C}$

- $\mathrm{T}_{\mathrm{L}}=1124^{\circ} \mathrm{C}$

\section{US-17:}

- HROM ThS, $1056^{\circ} \mathrm{C}$ : black agglomerates of noble metals $>>>$ lower temperature

- HROM ThS, $1005^{\circ} \mathrm{C}$ : cubic crystals (spinels) $\gg>\mathrm{T}_{\mathrm{L}}$ between $1005^{\circ} \mathrm{C}$ and $1056^{\circ} \mathrm{C}$

- HROM ThS, $1030^{\circ} \mathrm{C}$ : cubic crystals (spinels) $\gg>\mathrm{T}_{\mathrm{L}}$ between $1030^{\circ} \mathrm{C}$ and $1056^{\circ} \mathrm{C}$

- HROM ThS, $1040^{\circ} \mathrm{C}$ : black agglomerates of noble metals, lots of little cubic crystals (spinels) at walls and bottom corner $\gg>\mathrm{T}_{\mathrm{L}}$ between $1040^{\circ} \mathrm{C}$ and $1057^{\circ} \mathrm{C}$

- HROM ThS, $1049^{\circ} \mathrm{C}$ : black agglomerates of noble metals throughout bulk $\gg>\mathrm{T}_{\mathrm{L}}$ between $1040^{\circ} \mathrm{C}$ and $1049^{\circ} \mathrm{C}$

- $\mathrm{T}_{\mathrm{L}}=1045^{\circ} \mathrm{C}$

\section{US-18:}

- HROM ThS, $1005^{\circ} \mathrm{C}$ : few tiny needles $\gg>>$ higher temperature

- HROM ThS, $1056^{\circ} \mathrm{C}$ : black agglomerates of noble metals $\gg>\mathrm{T}_{\mathrm{L}}$ between $1005^{\circ} \mathrm{C}$ and $1056^{\circ} \mathrm{C}$

- HROM ThS, $1030^{\circ} \mathrm{C}$ : black agglomerates of noble metals $\gg>\mathrm{T}_{\mathrm{L}}$ between $1005^{\circ} \mathrm{C}$ and $1030^{\circ} \mathrm{C}$

- HROM ThS, $1015^{\circ} \mathrm{C}$ : needles everywhere $\gg>\mathrm{T}_{\mathrm{L}}$ between $1005^{\circ} \mathrm{C}$ and $1015^{\circ} \mathrm{C}$

- HROM ThS, $861^{\circ} \mathrm{C}$ : surface crystallization

- HROM ThS, $954^{\circ} \mathrm{C}$ : needles everywhere, several small cubic crystals (spinel) at base $>\gg \mathrm{T}_{\mathrm{L}}$ between $954^{\circ} \mathrm{C}$ and $1005^{\circ} \mathrm{C}$

- HROM ThS, $982^{\circ} \mathrm{C}$ : a few large spinel agglomerates at base, needles throughout, $\mathrm{RuO}_{2}$ agglomerates $\gg>>T_{L}$ between $982^{\circ} \mathrm{C}$ and $1005^{\circ} \mathrm{C}$

- HROM ThS, $994^{\circ} \mathrm{C}$ : lots of needles, few $\mathrm{RuO}_{2}$ agglomerates $\gg>\mathrm{T}_{\mathrm{L}}$ between $982^{\circ} \mathrm{C}$ and $994^{\circ} \mathrm{C}$

- $\mathrm{T}_{\mathrm{L}}=988^{\circ} \mathrm{C}$ 


\section{US-19:}

- HROM ThS, $1056^{\circ} \mathrm{C}$ : black agglomerates of noble metals in bulk $>>>$ lower temperature

- HROM ThS, $954^{\circ} \mathrm{C}$ : cubic crystals (spinels) $\gg>\mathrm{T}_{\mathrm{L}}$ between $954^{\circ} \mathrm{C}$ and $1056^{\circ} \mathrm{C}$

- HROM ThS, $1007^{\circ} \mathrm{C}$ : tiny black spots around edges (maybe crystals) $\gg>\mathrm{T}_{\mathrm{L}}$ between $1007^{\circ} \mathrm{C}$ and $1056^{\circ} \mathrm{C}$

- HROM ThS, $1040^{\circ} \mathrm{C}$ : black agglomerates of noble metals in bulk $\gg>\mathrm{T}_{\mathrm{L}}$ between $1007^{\circ} \mathrm{C}$ and $1040^{\circ} \mathrm{C}$

- HROM ThS, $1015^{\circ} \mathrm{C}$ : black agglomerates of noble metals $\gg>\mathrm{T}_{\mathrm{L}}$ between $1007^{\circ} \mathrm{C}$ and $1015^{\circ} \mathrm{C}$

- HROM ThS, $1029^{\circ} \mathrm{C}$ : small spinel cluster at bottom, black agglomerates of noble metals $>>>$ $\mathrm{T}_{\mathrm{L}}$ between $1029^{\circ} \mathrm{C}$ and $1040^{\circ} \mathrm{C}$

- $\mathrm{T}_{\mathrm{L}}=1035^{\circ} \mathrm{C}$

\section{US-20:}

- HROM ThS, $1056^{\circ} \mathrm{C}$ : black agglomerates of noble metals at bottom and in bulk $>>>$ lower temperature

- HROM ThS, $954^{\circ} \mathrm{C}$ : cubic crystals $\gg>>$ higher temperature

- HROM ThS, $1007^{\circ} \mathrm{C}$ : black agglomerates of noble metals $\gg>\mathrm{T}_{\mathrm{L}}$ between $954^{\circ} \mathrm{C}$ and $1007^{\circ} \mathrm{C}$

- HROM ThS, $981^{\circ} \mathrm{C}$ : many cubic crystals in bulk of sample $\gg>\mathrm{T}_{\mathrm{L}}$ between $982^{\circ} \mathrm{C}$ and $1007^{\circ} \mathrm{C}$

- HROM ThS, $995^{\circ} \mathrm{C}$ : black agglomerates of noble metals, lots of spinel in bulk, few needles $\gg>\mathrm{T}_{\mathrm{L}}$ between $995^{\circ} \mathrm{C}$ and $1007^{\circ} \mathrm{C}$

- HROM ThS, $1001^{\circ} \mathrm{C}$ : black agglomerates of noble metals, submicron particles in areas (maybe spinels) $\gg>\mathrm{T}_{\mathrm{L}}$ between $1001^{\circ} \mathrm{C}$ and $1007^{\circ} \mathrm{C}$

- $\mathrm{T}_{\mathrm{L}}=1004^{\circ} \mathrm{C}$

\section{US-21:}

- HROM ThS, $1056^{\circ} \mathrm{C}$ : small cubic crystals $\gg>>$ higher temperature

- HROM ThS, $1106^{\circ} \mathrm{C}$ : black agglomerates of noble metals $>>\mathrm{T}_{\mathrm{L}}$ between $1056^{\circ} \mathrm{C}$ and $1106^{\circ} \mathrm{C}$

- HROM ThS, $1080^{\circ} \mathrm{C}$ : several large black agglomerates of noble metals in bulk, no spinels $\gg>$ $\mathrm{T}_{\mathrm{L}}$ between $1056^{\circ} \mathrm{C}$ and $1080^{\circ} \mathrm{C}$

- HROM ThS, $1067^{\circ} \mathrm{C}$ : black agglomerates of noble metals, very few small spinel $>>>T_{L}$ between $1067^{\circ} \mathrm{C}$ and $1080^{\circ} \mathrm{C}$

- HROM ThS, $1074^{\circ} \mathrm{C}$ : several black agglomerates of noble metals, no spinels $\gg>\mathrm{T}_{\mathrm{L}}$ between $1067^{\circ} \mathrm{C}$ and $1074^{\circ} \mathrm{C}$

- HROM ThS, $1070^{\circ} \mathrm{C}$ : a few small black agglomerates of noble metals at bottom - some appear to spinel and the rest are $\mathrm{RuO}_{2} \gg>\mathrm{T}_{\mathrm{L}}$ between $1070^{\circ} \mathrm{C}$ and $1074^{\circ} \mathrm{C}$

- $\mathrm{T}_{\mathrm{L}}=1072^{\circ} \mathrm{C}$

US-22:

- HROM ThS, $1056^{\circ} \mathrm{C}$ : small cubic crystals $\gg>$ higher temperature

- HROM ThS, $1106^{\circ} \mathrm{C}$ : black agglomerates of noble metals $\gg>\mathrm{T}_{\mathrm{L}}$ between $1056^{\circ} \mathrm{C}$ and $1106^{\circ} \mathrm{C}$

- HROM ThS, $1080^{\circ} \mathrm{C}$ : spinel present (some large, some small) $\gg>\mathrm{T}_{\mathrm{L}}$ between $1080^{\circ} \mathrm{C}$ and $1106^{\circ} \mathrm{C}$

- HROM ThS, $1095^{\circ} \mathrm{C}$ : black agglomerates of noble metals at base, no crystals present $>>\mathrm{T}_{\mathrm{L}}$ between $1080^{\circ} \mathrm{C}$ and $1095^{\circ} \mathrm{C}$

- HROM ThS, $1086^{\circ} \mathrm{C}$ : few clusters of spinel at bottom corner, black agglomerates of noble metals $>>>\mathrm{T}_{\mathrm{L}}$ between $1086^{\circ} \mathrm{C}$ and $1095^{\circ} \mathrm{C}$

- HROM ThS, $1066^{\circ} \mathrm{C}$ : several spinels at base, small and large (other undissolved materials) $>>>$ $\mathrm{T}_{\mathrm{L}}$ between $1086^{\circ} \mathrm{C}$ and $1095^{\circ} \mathrm{C}$ [XRD sample]

- $\mathrm{T}_{\mathrm{L}}=1091^{\circ} \mathrm{C}$ 
US-23:

- HROM ThS, $1056^{\circ} \mathrm{C}$ : black agglomerates of noble metals in bulk $\gg>>$ lower temperature

- HROM ThS, $1007^{\circ} \mathrm{C}$ : cubic crystals (spinels) $\gg>\mathrm{T}_{\mathrm{L}}$ between $1007^{\circ} \mathrm{C}$ and $1056^{\circ} \mathrm{C}$

- HROM ThS, $1030^{\circ} \mathrm{C}$ : very few cubic crystals (spinels) $\gg>\mathrm{T}_{\mathrm{L}}$ between $1030^{\circ} \mathrm{C}$ and $1056^{\circ} \mathrm{C}$

- HROM ThS, $1040^{\circ} \mathrm{C}$ : black agglomerates of noble metals $\gg>>\mathrm{T}_{\mathrm{L}}$ between $1030^{\circ} \mathrm{C}$ and $1040^{\circ} \mathrm{C}$

- HROM ThS, $1035^{\circ} \mathrm{C}$ : black agglomerates of noble metals, no spinels $\gg>\mathrm{T}_{\mathrm{L}}$ between $1030^{\circ} \mathrm{C}$ and $1035^{\circ} \mathrm{C}$

- $\mathrm{T}_{\mathrm{L}}=1032^{\circ} \mathrm{C}$

\section{US-24:}

- HROM ThS, $1056^{\circ} \mathrm{C}$ : black agglomerates of noble metals in bulk $\gg>>$ lower temperature

- HROM ThS, $954^{\circ} \mathrm{C}$ : cubic crystals (spinels) $>>>$ higher temperature

- HROM ThS, $1007^{\circ} \mathrm{C}$ : cubic crystals (spinels) $\gg>\mathrm{T}_{\mathrm{L}}$ between $1007^{\circ} \mathrm{C}$ and $1056^{\circ} \mathrm{C}$

- HROM ThS, $1030^{\circ} \mathrm{C}$ : cubic crystals (spinels) $\gg>\mathrm{T}_{\mathrm{L}}$ between $1030^{\circ} \mathrm{C}$ and $1056^{\circ} \mathrm{C}$

- HROM ThS, $1040^{\circ} \mathrm{C}$ : black agglomerates of noble metals in bulk, lots of little cubic crystals (spinel) throughout sample $\gg>\mathrm{T}_{\mathrm{L}}$ between $1040^{\circ} \mathrm{C}$ and $1056^{\circ} \mathrm{C}$

- HROM ThS, $1049^{\circ} \mathrm{C}$ : clusters of very small cubic crystals (spinels) throughout the sample, black agglomerates of noble metals $\gg>\mathrm{T}_{\mathrm{L}}$ between $1049^{\circ} \mathrm{C}$ and $1056^{\circ} \mathrm{C}$

- $\mathrm{T}_{\mathrm{L}}=1052^{\circ} \mathrm{C}$

US-25:

- HROM ThS, $1007^{\circ} \mathrm{C}$ and $1056^{\circ} \mathrm{C}$ : cubic crystals (spinel) $\gg>$ higher temperature

- HROM ThS, $1106^{\circ} \mathrm{C}$ : black agglomerates of noble metals $\gg>\mathrm{T}_{\mathrm{L}}$ between $1056^{\circ} \mathrm{C}$ and $1106^{\circ} \mathrm{C}$

- HROM ThS, $1080^{\circ} \mathrm{C}$ : lots of cubic crystals (spinel), lots of smaller undefined crystals $>>\mathrm{T}_{\mathrm{L}}$ between $1080^{\circ} \mathrm{C}$ and $1106^{\circ} \mathrm{C}$

- HROM ThS, $1095^{\circ} \mathrm{C}$ : black agglomerates of noble metals $\gg>>\mathrm{T}_{\mathrm{L}}$ between $1080^{\circ} \mathrm{C}$ and $1095^{\circ} \mathrm{C}$

- HROM ThS, $1086^{\circ} \mathrm{C}$ : black agglomerates of noble metals $\gg>\mathrm{T}_{\mathrm{L}}$ between $1080^{\circ} \mathrm{C}$ and $1086^{\circ} \mathrm{C}$

- $\mathrm{T}_{\mathrm{L}}=1083^{\circ} \mathrm{C}$

US-26:

- HROM ThS, $1107^{\circ} \mathrm{C}$ : many tiny cubic crystals (spinel) mostly at crucible bottom $>>>$ higher temperature

- HROM ThS, $1205^{\circ} \mathrm{C}$ : cubic crystals (spinels) $\gg>>$ higher temperature

- HROM ThS, $1254^{\circ} \mathrm{C}$ : black agglomerates of noble metals $\gg>\mathrm{T}_{\mathrm{L}}$ between $1205^{\circ} \mathrm{C}$ and $1254^{\circ} \mathrm{C}$

- HROM ThS, $1232^{\circ} \mathrm{C}$ : black agglomerates of noble metals $\gg>\mathrm{T}_{\mathrm{L}}$ between $1205^{\circ} \mathrm{C}$ and $1232^{\circ} \mathrm{C}$

- HROM ThS, $1217^{\circ} \mathrm{C}$ : sample clear $\gg>\mathrm{T}_{\mathrm{L}}$ between $1205^{\circ} \mathrm{C}$ and $1217^{\circ} \mathrm{C}$

- HROM ThS, $1206^{\circ} \mathrm{C}$ : black agglomerates of noble metals at base of sample $>>>\mathrm{T}_{\mathrm{L}}$ between $1205^{\circ} \mathrm{C}$ and $1206^{\circ} \mathrm{C}$

- $\mathrm{T}_{\mathrm{L}}=1206^{\circ} \mathrm{C}$ 
US-27:

- HROM ThS, $1056^{\circ} \mathrm{C}$ : few cubic crystals (spinels) and black agglomerates of noble metals in bulk $>>>$ higher temperature

- HROM ThS, $1095^{\circ} \mathrm{C}$ : sample clear $\gg>\mathrm{T}_{\mathrm{L}}$ between $1056^{\circ} \mathrm{C}$ and $1095^{\circ} \mathrm{C}$

- HROM ThS, $1086^{\circ} \mathrm{C}$ : sample clear $\gg>\mathrm{T}_{\mathrm{L}}$ between $1056^{\circ} \mathrm{C}$ and $1086^{\circ} \mathrm{C}$

- HROM ThS, $1070^{\circ} \mathrm{C}$ : a few small cubic crystals (spinels) at base, black agglomerates of noble metals at base of sample $\gg>\mathrm{T}_{\mathrm{L}}$ between $1070^{\circ} \mathrm{C}$ and $1086^{\circ} \mathrm{C}$

- HROM ThS, $1079^{\circ} \mathrm{C}$ : sample volatilized out of crucible a lot; layer on bottom of agglomerates, appear to have cubic shape (possibly spinel), some black agglomerates of noble metals in bulk $\gg>\mathrm{T}_{\mathrm{L}}$ between $1079^{\circ} \mathrm{C}$ and $1086^{\circ} \mathrm{C}$

- $\mathrm{T}_{\mathrm{L}}=1082^{\circ} \mathrm{C}$

\section{US-28:}

- HROM ThS, $1006^{\circ} \mathrm{C}$ : black agglomerates of noble metals $\gg>>$ lower temperature

- HROM ThS, $903^{\circ} \mathrm{C}$ : few needles $\gg>\mathrm{T}_{\mathrm{L}}$ between $903^{\circ} \mathrm{C}$ and $1006^{\circ} \mathrm{C}$

- HROM ThS, $952^{\circ} \mathrm{C}$ : black agglomerates of noble metals, several small sets of needles $>\gg \mathrm{T}_{\mathrm{L}}$ between $952^{\circ} \mathrm{C}$ and $1006^{\circ} \mathrm{C}$

- HROM ThS, $980^{\circ} \mathrm{C}$ : small needles through out bulk, black agglomerates of noble metals $>>>$ $\mathrm{T}_{\mathrm{L}}$ between $980^{\circ} \mathrm{C}$ and $1006^{\circ} \mathrm{C}$

- HROM ThS, $994^{\circ} \mathrm{C}$ : small needles throughout sample, black agglomerates of noble metals throughout bulk $\gg>\mathrm{T}_{\mathrm{L}}$ between $994^{\circ} \mathrm{C}$ and $1006^{\circ} \mathrm{C}$

- HROM ThS, $1000^{\circ} \mathrm{C}$ : black agglomerates of noble metals throughout, small needles throughout, undissolved particles throughout $\gg>\mathrm{T}_{\mathrm{L}}$ between $994^{\circ} \mathrm{C}$ and $1006^{\circ} \mathrm{C}$

- HROM ThS, $861^{\circ} \mathrm{C}$ : black agglomerates of noble metals throughout, small needles throughout $\gg>\mathrm{T}_{\mathrm{L}}<861^{\circ} \mathrm{C}$

- HROM ThS, $812^{\circ} \mathrm{C}$ : black agglomerates of noble metals, small spinel everywhere, some hematite, also clear cubic crystals everywhere $>>>T_{L}$ between $812^{\circ} \mathrm{C}$ and $861{ }^{\circ} \mathrm{C}$

- HROM ThS, $840^{\circ} \mathrm{C}$ : black agglomerates of noble metals, lots of needles $\gg>\mathrm{T}_{\mathrm{L}}$ between $812^{\circ} \mathrm{C}$ and $840^{\circ} \mathrm{C}$

- HROM ThS, $826^{\circ} \mathrm{C}$ : a few hematite crystals, a few small spinels in bulk $\gg>\mathrm{T}_{\mathrm{L}}$ between $826^{\circ} \mathrm{C}$ and $840^{\circ} \mathrm{C}$

- HROM ThS, $831^{\circ} \mathrm{C}$ : black agglomerates of noble metals, needles throughout, small undissolved particles in bulk $\gg>\mathrm{T}_{\mathrm{L}}$ between $826^{\circ} \mathrm{C}$ and $831^{\circ} \mathrm{C}$

- $\mathrm{T}_{\mathrm{L}}=829^{\circ} \mathrm{C}$

\section{US-29:}

- HROM ThS, $952^{\circ} \mathrm{C}$ : very few needles $\gg>>$ higher temperature

- HROM ThS, $1006^{\circ} \mathrm{C}$ : possible homogeneous $\gg>>T_{L}$ between $952^{\circ} \mathrm{C}$ and $1006^{\circ} \mathrm{C}$

- HROM ThS, $981^{\circ} \mathrm{C}$ : few black agglomerates of noble metals $>>\mathrm{T}_{\mathrm{L}}$ between $952^{\circ} \mathrm{C}$ and $981^{\circ} \mathrm{C}$

- HROM ThS, $812^{\circ} \mathrm{C}$ : black agglomerates of noble metals, needles everywhere, undissolved material everywhere $>>>\mathrm{T}_{\mathrm{L}}<812^{\circ} \mathrm{C}$

- HROM ThS, $879^{\circ} \mathrm{C}$ : spinel everywhere, black agglomerates of noble metals $\gg>\mathrm{T}_{\mathrm{L}}$ between $879^{\circ} \mathrm{C}$ and $903^{\circ} \mathrm{C}$

- HROM ThS, $890^{\circ} \mathrm{C}$ : a few black agglomerates of noble metals, needles everywhere $>>\mathrm{T}_{\mathrm{L}}$ between $879^{\circ} \mathrm{C}$ and $890^{\circ} \mathrm{C}$

- $\mathrm{T}_{\mathrm{L}}=885^{\circ} \mathrm{C}$ 
US-30:

- LROM, $1402^{\circ} \mathrm{C}$ : brown crystal striations in bulk

- HROM ThS, $1426^{\circ} \mathrm{C}$ : sample clear $\gg>\mathrm{T}_{\mathrm{L}}<1426^{\circ} \mathrm{C}$

- HROM ThS, $1405^{\circ} \mathrm{C}$ : lots of small crystals, looks like they all formed during cooling $\gg>\mathrm{T}_{\mathrm{L}}<$ $1426^{\circ} \mathrm{C}$

- HROM ThS, $1350^{\circ} \mathrm{C}$ : sample clear $\gg>\mathrm{T}_{\mathrm{L}} 1350<{ }^{\circ} \mathrm{C}$

- HROM ThS, $1304^{\circ} \mathrm{C}$ : a few small undissolved agglomerates at base of sample, possible some spinel near one of the agglomerates (5 cubic structures)

- HROM ThS, $1247^{\circ} \mathrm{C}$ : several clusters of noble metals throughout sample, possibly some spinel

- HROM ThS, $1218^{\circ} \mathrm{C}$ : quite a few spinels throughout bulk, some other undissolved materials (noble metals) $>>>\mathrm{T}_{\mathrm{L}}$ between $1218^{\circ} \mathrm{C}$ and $1247^{\circ} \mathrm{C}$

- HROM ThS, $1074^{\circ} \mathrm{C}$ : lots of undissolved materials (noble metals and spinels) $\gg>T_{L}$ between $1218^{\circ} \mathrm{C}$ and $1247^{\circ} \mathrm{C}$ [XRD sample]

- HROM ThS, $1017^{\circ} \mathrm{C}$ : lots of undissolved materials (noble metals and spinels) $\gg>T_{L}$ between $1218^{\circ} \mathrm{C}$ and $1247^{\circ} \mathrm{C}$ [XRD sample]

- HROM ThS, $1232^{\circ} \mathrm{C}$ : few spinels and other undissolved noble metals $>>>\mathrm{T}_{\mathrm{L}}$ between $1232^{\circ} \mathrm{C}$ and $1247^{\circ} \mathrm{C}$

- HROM ThS, $1235^{\circ} \mathrm{C}$ : few spinels and other undissolved noble metals $\gg>\mathrm{T}_{\mathrm{L}}$ between $1235^{\circ} \mathrm{C}$ and $1247^{\circ} \mathrm{C}$

- $\mathrm{T}_{\mathrm{L}}=1241^{\circ} \mathrm{C}$

\section{US-31:}

- HROM ThS, $1056^{\circ} \mathrm{C}$ : cubic crystals (spinels) and red rhombic crystals (hematite) $\gg>$ higher temperature

- HROM ThS, $1107^{\circ} \mathrm{C}$ : black agglomerates of noble metals at bottom, some agglomerates are cubic (spinel) $\gg>>$ higher temperature

- HROM ThS, $1153^{\circ} \mathrm{C}$ : black agglomerates of noble metals $>>>$ lower temperature

- HROM ThS, $1130^{\circ} \mathrm{C}$ : sample looks clear $\gg>\mathrm{T}_{\mathrm{L}}$ between $1107^{\circ} \mathrm{C}$ and $1130^{\circ} \mathrm{C}$

- HROM ThS, $1095^{\circ} \mathrm{C}$ : black agglomerates of noble metals, several clusters of round and cubic spinel along bottom perimeter of sample $\gg>\mathrm{T}_{\mathrm{L}}$ between $1107^{\circ} \mathrm{C}$ and $1130^{\circ} \mathrm{C}$

- HROM ThS, $1118^{\circ} \mathrm{C}$ : sample clear $\gg>\mathrm{T}_{\mathrm{L}}$ between $1107^{\circ} \mathrm{C}$ and $1118^{\circ} \mathrm{C}$

- $\mathrm{T}_{\mathrm{L}}=1113^{\circ} \mathrm{C}$ 
US-32:

- HROM ThS, $903^{\circ} \mathrm{C}$ : some very tiny needles, very difficult to see, verification advisable $>>>$ lower temperature

- HROM ThS, $952^{\circ} \mathrm{C}$ : some very tiny needles, very difficult to see, verification advisable $>>>$ lower temperature

- HROM ThS, $1006^{\circ} \mathrm{C}$ : possibly homogeneous $\gg>>$ lower temperature

- HROM ThS, $981^{\circ} \mathrm{C}$ : black agglomerates of noble metals and needles throughout the sample $>>$ lower temperature

- HROM ThS, $995^{\circ} \mathrm{C}$ : black agglomerates of noble metals, few small needles in bulk $\gg>$ lower temperature

- HROM ThS, $1001^{\circ} \mathrm{C}$ : black agglomerates of noble metals throughout, needles in bulk $>>>$ lower temperature

- HROM ThS, $861^{\circ} \mathrm{C}$ : needles everywhere $\gg>>$ lower temperature

- HROM ThS, $812^{\circ} \mathrm{C}$ : black agglomerates of noble metals, undissolved material everywhere, needles throughout $>>>$ lower temperature

- HROM ThS, $764^{\circ} \mathrm{C}$ : surface crystallization, black agglomerates of noble metals, undissolved material $\gg>\mathrm{T}_{\mathrm{L}}<764^{\circ} \mathrm{C}$

- HROM ThS, $704^{\circ} \mathrm{C}$ : surface crystallization; some spinel in bulk, lots of clear rectangular prism-like crystals with different shapes (some flat plates, some rods), black agglomerates of noble metals $\gg>\mathrm{T}_{\mathrm{L}}$ between $704^{\circ} \mathrm{C}$ and $764^{\circ} \mathrm{C}$

- HROM ThS, $731^{\circ} \mathrm{C}$ : large hematite crystals, few cubic crystals (spinel), few triangle-like crystals, lots of small undissolved particles $>>>\mathrm{T}_{\mathrm{L}}$ between $731^{\circ} \mathrm{C}$ and $764^{\circ} \mathrm{C}$

- HROM ThS, $753^{\circ} \mathrm{C}$ : large spinel at top, streaks of needles throughout bulk, undissolved materials (noble metals) $\gg>\mathrm{T}_{\mathrm{L}}$ between $753^{\circ} \mathrm{C}$ and $764^{\circ} \mathrm{C}$

- $\mathrm{T}_{\mathrm{L}}=759^{\circ} \mathrm{C}$

US-33:

- HROM ThS, $1157^{\circ} \mathrm{C}$ and $1206^{\circ} \mathrm{C}$ : small, black agglomerates of noble metals $\gg>$ repeat treatment at $1206^{\circ} \mathrm{C}$ for obtaining bigger sample

- HROM ThS, $1203^{\circ} \mathrm{C}$ : few black agglomerates of noble metals $\gg>>$ lower temperature

- HROM ThS, $1007^{\circ} \mathrm{C}$ : homogeneous in small sample, black agglomerates of noble metals $\gg>$ lower temperature

- HROM ThS, $854^{\circ} \mathrm{C}$ : cubic crystals (spinel) $\gg>\mathrm{T}_{\mathrm{L}}$ between $854^{\circ} \mathrm{C}$ and $1007^{\circ} \mathrm{C}$

- HROM ThS, $902^{\circ} \mathrm{C}$ : lots of small cubic crystals (spinel), several prism/faceted crystals $\gg \gg \mathrm{T}_{\mathrm{L}}$ between $902^{\circ} \mathrm{C}$ and $1007^{\circ} \mathrm{C}$

- HROM ThS, $954^{\circ} \mathrm{C}$ : spinel everywhere $\gg>\mathrm{T}_{\mathrm{L}}$ between $954^{\circ} \mathrm{C}$ and $1007^{\circ} \mathrm{C}$

- HROM ThS, $980^{\circ} \mathrm{C}$ : spinel everywhere $\gg>>\mathrm{T}_{\mathrm{L}}$ between $980^{\circ} \mathrm{C}$ and $1007^{\circ} \mathrm{C}$

- HROM ThS, $995^{\circ} \mathrm{C}$ : extremely large quantity of noble metal agglomerates $\gg>>\mathrm{T}_{\mathrm{L}}$ between $995^{\circ} \mathrm{C}$ and $1007^{\circ} \mathrm{C}$

- HROM ThS, $1001^{\circ} \mathrm{C}$ : lots of spinels and black agglomerates of noble metals $\gg>\mathrm{T}_{\mathrm{L}}$ between $1001^{\circ} \mathrm{C}$ and $1007^{\circ} \mathrm{C}$

- $\mathrm{T}_{\mathrm{L}}=1004^{\circ} \mathrm{C}$ 
US-34:

- HROM ThS, $854^{\circ} \mathrm{C}$ : many needle-like crystals in bulk $\gg>>$ lower temperature

- HROM ThS, $903^{\circ} \mathrm{C}$ : some needle-like crystals in bulk $\gg>>$ lower temperature

- HROM ThS, $954^{\circ} \mathrm{C}$ : small needles in bulk $\gg>>$ lower temperature

- HROM ThS, $1095^{\circ} \mathrm{C}$ : sample appears to be amorphous $>>>$ lower temperature

- HROM ThS, $994^{\circ} \mathrm{C}$ : one black agglomerate of noble metals - sample appears to be amorphous $\gg>$ lower temperature

- HROM ThS, $1015^{\circ} \mathrm{C}$ : sample clear $\gg>>$ lower temperature

- HROM ThS, $861^{\circ} \mathrm{C}$ : needles everywhere $>>>$ lower temperature

- HROM ThS, $854^{\circ} \mathrm{C}$ : needles everywhere $>>>$ lower temperature

- HROM ThS, $812^{\circ} \mathrm{C}$ : lots of large spinel, some hematite $>>\mathrm{T}_{\mathrm{L}}$ between $812^{\circ} \mathrm{C}$ and $854^{\circ} \mathrm{C}$

- HROM ThS, $840^{\circ} \mathrm{C}$ : lots of undissolved material everywhere, needles throughout $\gg>\mathrm{T}_{\mathrm{L}}$ between $812^{\circ} \mathrm{C}$ and $840^{\circ} \mathrm{C}$

- HROM ThS, $828^{\circ} \mathrm{C}$ : sample too opaque to see through, even when polished really thin $>>>\mathrm{T}_{\mathrm{L}}$ is between $828^{\circ} \mathrm{C}$ and $840^{\circ} \mathrm{C}$

- $\mathrm{T}_{\mathrm{L}}=832^{\circ} \mathrm{C}$

\section{US-35:}

- LROM, $1200^{\circ} \mathrm{C}$ (as received sample from SRNL): many black crystal agglomerates in bulk

- LROM, $1397^{\circ} \mathrm{C}$ : brown crystal surface layer, many white spots on fractured surface

- HROM ThS, $1350^{\circ} \mathrm{C}$ : small crystals formed upon cooling, a few clusters of semi-transparent orange cubic crystals at base with light halo surrounding (possibly undissolved noble metals)

- HROM ThS, $1247^{\circ} \mathrm{C}$ : several spinel throughout bulk, other undissolved materials $\gg>\mathrm{T}_{\mathrm{L}}>$ $1247^{\circ} \mathrm{C}$

- HROM ThS, $1218^{\circ} \mathrm{C}$ : several spinel throughout bulk, other undissolved materials $>>\mathrm{T}_{\mathrm{L}}>$ $1247^{\circ} \mathrm{C}$

- HROM ThS, $1074^{\circ} \mathrm{C}$ : lots of undissolved materials $>>>\mathrm{T}_{\mathrm{L}}>1247^{\circ} \mathrm{C}$ [XRD sample]

- HROM ThS, $1017^{\circ} \mathrm{C}$ : lots of undissolved materials $\gg>\mathrm{T}_{\mathrm{L}}>1247^{\circ} \mathrm{C}$ [XRD sample]

- HROM ThS, $1318^{\circ} \mathrm{C}$ : some undissolved materials at base (most likely noble metals and not spinel $\gg>\mathrm{T}_{\mathrm{L}}$ is between $1247^{\circ} \mathrm{C}$ and $1318^{\circ} \mathrm{C}$

- HROM ThS, $1278^{\circ} \mathrm{C}$ : some undissolved materials at base (noble metals), probably not spinel $\gg>\mathrm{T}_{\mathrm{L}}$ is between $1278^{\circ} \mathrm{C}$ and $1318^{\circ} \mathrm{C}$

- HROM ThS, $1301{ }^{\circ} \mathrm{C}$ : few spinels and some other undissolved materials (noble metals) $\gg>\mathrm{T}_{\mathrm{L}}$ is between $1301^{\circ} \mathrm{C}$ and $1318^{\circ} \mathrm{C}$

- HROM ThS, $1309^{\circ} \mathrm{C}$ : undissolved materials, appears to be a few cubic structures (spinels?) $\gg>\mathrm{T}_{\mathrm{L}}$ is between $1309^{\circ} \mathrm{C}$ and $1318^{\circ} \mathrm{C}$

- $\mathrm{T}_{\mathrm{L}}=1312^{\circ} \mathrm{C}$ 
US-36:

- LROM, $1150^{\circ} \mathrm{C}$ (as received sample from SRNL): many black crystal agglomerates in bulk

- LROM, $1401^{\circ} \mathrm{C}$ : brown crystal striations on surface, many white spots on fractured surface

- HROM ThS, $1426^{\circ} \mathrm{C}$ : redox in sample, small crystals from cooling but no large crystals $\gg>\mathrm{T}_{\mathrm{L}}$ $<1426^{\circ} \mathrm{C}$

- HROM ThS, $1405^{\circ} \mathrm{C}$ : lots of small crystals, looks like they all formed during cooling $\gg>\mathrm{T}_{\mathrm{L}}<$ $1405^{\circ} \mathrm{C}$

- HROM ThS, $1350^{\circ} \mathrm{C}$ : small crystals formed upon cooling, doesn't appear to contain any large crystals $\gg>\mathrm{T}_{\mathrm{L}}<1350^{\circ} \mathrm{C}$

- HROM ThS, $1304^{\circ} \mathrm{C}$ : samples appears to be clear $\gg>\mathrm{T}_{\mathrm{L}}<1304^{\circ} \mathrm{C}$

- HROM ThS, $1328^{\circ} \mathrm{C}$ : one small black structure with a light halo around it (might be spinel or a different crystal) $\gg>\mathrm{T}_{\mathrm{L}}<1304^{\circ} \mathrm{C}$

- HROM ThS, $1199^{\circ} \mathrm{C}$ : a few spinel throughout bulk, some undissolved noble metals $>>>\mathrm{T}_{\mathrm{L}}$ is between $1199^{\circ} \mathrm{C}$ and $1304^{\circ} \mathrm{C}$

- HROM ThS, $1247^{\circ} \mathrm{C}$ : crystallized upon cooling, glass matrix has a light brown color $>>\mathrm{T}_{\mathrm{L}}$ is between $1199^{\circ} \mathrm{C}$ and $1247^{\circ} \mathrm{C}$

- HROM ThS, $1000^{\circ} \mathrm{C}$ : large quantities of spinel and other undissolved materials $\gg>\mathrm{T}_{\mathrm{L}}$ is between $1199^{\circ} \mathrm{C}$ and $1247^{\circ} \mathrm{C}$ [XRD sample]

- HROM ThS, $1098^{\circ} \mathrm{C}$ : several spinel throughout bulk, other undissolved materials (noble metals) $\gg>\mathrm{T}_{\mathrm{L}}$ is between $1199^{\circ} \mathrm{C}$ and $1247^{\circ} \mathrm{C}$ [XRD sample]

- HROM ThS, $1164^{\circ} \mathrm{C}$ : several crystals throughout bulk, some noble metals at base $\gg>\mathrm{T}_{\mathrm{L}}$ is between $1199^{\circ} \mathrm{C}$ and $1247^{\circ} \mathrm{C}$ [XRD sample]

- HROM ThS, $1225^{\circ} \mathrm{C}$ : spinels in sample $\gg>\mathrm{T}_{\mathrm{L}}$ is between $1225^{\circ} \mathrm{C}$ and $1247^{\circ} \mathrm{C}$

- HROM ThS, $1235^{\circ} \mathrm{C}$ : lots of little spinels (formed from cooling), possibly one actual spinel (formed during HT) triangle with halo in bulk $\gg>\mathrm{T}_{\mathrm{L}}$ is between $1235^{\circ} \mathrm{C}$ and $1247^{\circ} \mathrm{C}$

- $\mathrm{T}_{\mathrm{L}}=1238^{\circ} \mathrm{C}$

US-37:

- HROM ThS, $1007^{\circ} \mathrm{C}$ : black agglomerates of noble metals $\gg>>$ lower temperature

- HROM ThS, $952^{\circ} \mathrm{C}$ : cubic crystals (spinels) $\gg>\mathrm{T}_{\mathrm{L}}$ between $952^{\circ} \mathrm{C}$ and $1007^{\circ} \mathrm{C}$

- HROM ThS, $981^{\circ} \mathrm{C}$ : black agglomerates of noble metals at bottom, tiny needles throughout the sample $\gg>>\mathrm{T}_{\mathrm{L}}$ between $981^{\circ} \mathrm{C}$ and $1007^{\circ} \mathrm{C}$

- HROM ThS, $995^{\circ} \mathrm{C}$ : needles throughout sample, black agglomerates of noble metals $>>>\mathrm{T}_{\mathrm{L}}$ between $995^{\circ} \mathrm{C}$ and $1007^{\circ} \mathrm{C}$

- HROM ThS, $1001^{\circ} \mathrm{C}$ : lots of cubic crystals (spinels) and some black agglomerates of noble metals $\gg>>\mathrm{T}_{\mathrm{L}}$ between $1001{ }^{\circ} \mathrm{C}$ and $1007^{\circ} \mathrm{C}$

- HROM ThS, $1021^{\circ} \mathrm{C}$ : undissolved materials (all noble metals?) $\gg>\mathrm{T}_{\mathrm{L}}$ between $1001^{\circ} \mathrm{C}$ and $1007^{\circ} \mathrm{C}$

- $\mathrm{T}_{\mathrm{L}}=1004^{\circ} \mathrm{C}$ 
US-38:

- HROM ThS, $1056^{\circ} \mathrm{C}$ : very few cubic crystals and black agglomerates of noble metals in bulk $>>>$ higher temperature

- HROM ThS, $1095^{\circ} \mathrm{C}$ : a few black agglomerates of noble metals $\gg>\mathrm{T}_{\mathrm{L}}$ between $1056^{\circ} \mathrm{C}$ and $1095^{\circ} \mathrm{C}$

- HROM ThS, $1086^{\circ} \mathrm{C}$ : sample clear $>>>\mathrm{T}_{\mathrm{L}}$ between $1056^{\circ} \mathrm{C}$ and $1086^{\circ} \mathrm{C}$

- HROM ThS, $1070^{\circ} \mathrm{C}$ : one small, black agglomerate of noble metals $\gg>\mathrm{T}_{\mathrm{L}}$ between $1056^{\circ} \mathrm{C}$ and $1070^{\circ} \mathrm{C}$

- HROM ThS, $1067^{\circ} \mathrm{C}$ : sample is clear of crystals $\gg>\mathrm{T}_{\mathrm{L}}$ between $1056^{\circ} \mathrm{C}$ and $1067^{\circ} \mathrm{C}$

- HROM ThS, $1036^{\circ} \mathrm{C}$ : a few 4-pronged stars; like sharp-cornered spinels at top and bottom borders $\gg>\mathrm{T}_{\mathrm{L}}$ between $1056^{\circ} \mathrm{C}$ and $1067^{\circ} \mathrm{C}$

- $\mathrm{T}_{\mathrm{L}}=1062^{\circ} \mathrm{C}$

\section{US-39:}

- HROM ThS, $1056^{\circ} \mathrm{C}$ : many tiny cubic crystals, especially at crucible bottom $\gg>$ higher temperature

- HROM ThS, $1107^{\circ} \mathrm{C}$ : few cubic crystals $\gg>>$ higher temperature

- HROM ThS, $1130^{\circ} \mathrm{C}$ : very few black agglomerates of noble metals in bulk $>>>\mathrm{T}_{\mathrm{L}}$ between $1107^{\circ} \mathrm{C}$ and $1130^{\circ} \mathrm{C}$

- HROM ThS, $1118^{\circ} \mathrm{C}$ : black agglomerates of noble metals, no spinel $\gg>\mathrm{T}_{\mathrm{L}}$ between $1107^{\circ} \mathrm{C}$ and $1118^{\circ} \mathrm{C}$

- HROM ThS, $1110^{\circ} \mathrm{C}$ : black agglomerates of noble metals at base of sample, no spinel $>>>T_{L}$ between $1107^{\circ} \mathrm{C}$ and $1110^{\circ} \mathrm{C}$

- $\mathrm{T}_{\mathrm{L}}=1109^{\circ} \mathrm{C}$

\section{US-40:}

- Didn't form glass upon initial melting so this glass was rejected as a candidate for testing

\section{US-41:}

- HROM ThS, $1108^{\circ} \mathrm{C}$ : black agglomerates of noble metals, few cubic crystals (spinel) $>>>$ higher temperature

- HROM ThS, $1157^{\circ} \mathrm{C}$ : small, black agglomerates of noble metals, appears to be no spinel $>>>$ repeat treatment at $1157^{\circ} \mathrm{C}$ for obtaining bigger sample

- HROM ThS, $1159^{\circ} \mathrm{C}$ : black agglomerates of noble metals at base, some needle-like crystals at bottom with brown coloring surrounding them $\gg>$ SEM

- SEM-EDS, $1159^{\circ} \mathrm{C}$ : black agglomerates of noble metals $>>>$ lower temperature

- HROM ThS, $1058^{\circ} \mathrm{C}$ : red hexagons (hematite) $\gg>>$ higher temperature

- HROM ThS, $1108^{\circ} \mathrm{C}$ : black agglomerates of noble metals $\gg>\mathrm{T}_{\mathrm{L}}$ between $1058^{\circ} \mathrm{C}$ and $1108^{\circ} \mathrm{C}$

- HROM ThS, $1080^{\circ} \mathrm{C}$ : black agglomerates of noble metals $\gg>\mathrm{T}_{\mathrm{L}}$ between $1058^{\circ} \mathrm{C}$ and $1080^{\circ} \mathrm{C}$

- HROM ThS, $1067^{\circ} \mathrm{C}$ : black agglomerates of noble metals $\gg>\mathrm{T}_{\mathrm{L}}$ between $1058^{\circ} \mathrm{C}$ and $1067^{\circ} \mathrm{C}$

- $\mathrm{T}_{\mathrm{L}}=1063^{\circ} \mathrm{C}$ 


\section{US-42:}

- LROM, $1302^{\circ} \mathrm{C}$ and $1401^{\circ} \mathrm{C}$ : grey crystal cover on surface, clear brown fractured surface, bulk may be homogeneous

- HROM ThS, $1405^{\circ} \mathrm{C}$ : bulk is clear/glassy (no redox) strange structures around top and base (maybe crystals) $\gg>>\mathrm{T}_{\mathrm{L}}<1405^{\circ} \mathrm{C}$

- HROM ThS, $1350^{\circ} \mathrm{C}$ : sample looks clear aside from strange transparent diamond/cubic structures at base of sample $\gg>\mathrm{T}_{\mathrm{L}}<1350^{\circ} \mathrm{C}$

- HROM ThS, $1304^{\circ} \mathrm{C}$ : sample clear $\gg>>\mathrm{T}_{\mathrm{L}}<1304^{\circ} \mathrm{C}$

- HROM ThS, $1199^{\circ} \mathrm{C}$ : sample appears to be clear $>>>\mathrm{T}_{\mathrm{L}}<1199^{\circ} \mathrm{C}$

- HROM ThS, $1247^{\circ} \mathrm{C}$ : no spinel, possibly some undissolved noble metals $\gg>\mathrm{T}_{\mathrm{L}}<1199^{\circ} \mathrm{C}$

- HROM ThS, $1000^{\circ} \mathrm{C}$ : spinel throughout bulk and other undissolved materials $\gg>\mathrm{T}_{\mathrm{L}}$ is between $1000^{\circ} \mathrm{C}$ and $1199^{\circ} \mathrm{C}$

- HROM ThS, $1098^{\circ} \mathrm{C}$ : sample clear $\gg>\mathrm{T}_{\mathrm{L}}$ is between $1000^{\circ} \mathrm{C}$ and $1098^{\circ} \mathrm{C}$

- HROM ThS, $1035^{\circ} \mathrm{C}$ : sample clear of crystals $\gg>\mathrm{T}_{\mathrm{L}}$ is between $1000^{\circ} \mathrm{C}$ and $1035^{\circ} \mathrm{C}$

- HROM ThS, $1052^{\circ} \mathrm{C}$ : sample clear $\gg>>\mathrm{T}_{\mathrm{L}}$ is between $1000^{\circ} \mathrm{C}$ and $1035^{\circ} \mathrm{C}$

- HROM ThS, $925^{\circ} \mathrm{C}$ : sample full of undissolved materials (spinel, noble metals, possibly nepheline) $\gg>\mathrm{T}_{\mathrm{L}}$ is between $1000^{\circ} \mathrm{C}$ and $1035^{\circ} \mathrm{C}$

- HROM ThS, $1017^{\circ} \mathrm{C}$ : lots of undissolved materials, some spinels $\gg>\mathrm{T}_{\mathrm{L}}$ is between $1017^{\circ} \mathrm{C}$ and $1035^{\circ} \mathrm{C}$

- HROM ThS, $1028^{\circ} \mathrm{C}$ : no spinel, some undissolved materials present $\gg>\mathrm{T}_{\mathrm{L}}$ is between $1017^{\circ} \mathrm{C}$ and $1028^{\circ} \mathrm{C}$

- $\mathrm{T}_{\mathrm{L}}=1023^{\circ} \mathrm{C}$

\section{US-43:}

- HROM ThS, $1402^{\circ} \mathrm{C}$ : homogeneous $\gg>>$ lower temperature

- HROM ThS, $1282^{\circ} \mathrm{C}$ : homogeneous $\gg>>$ lower temperature

- HROM ThS, $1197^{\circ} \mathrm{C}$ : crystals at crucible bottom, very few of them appear to be cubic $\gg>T_{L}$ between $1197^{\circ} \mathrm{C}$ and $1282^{\circ} \mathrm{C}$

- HROM ThS, $1232^{\circ} \mathrm{C}$ : sample is clear of crystals $\gg>T_{L}$ between $1197^{\circ} \mathrm{C}$ and $1232^{\circ} \mathrm{C}$

- HROM ThS, $1217^{\circ} \mathrm{C}$ : small round crystals at base, black agglomerates of noble metals $\gg>\mathrm{T}_{\mathrm{L}}$ between $1217^{\circ} \mathrm{C}$ and $1232^{\circ} \mathrm{C}$

- HROM ThS, $1225^{\circ} \mathrm{C}$ : some of the sample bubbled out of the crucible, sample looks free of crystals $\gg>\mathrm{T}_{\mathrm{L}}$ between $1217^{\circ} \mathrm{C}$ and $1225^{\circ} \mathrm{C}$

- HROM ThS, $1085^{\circ} \mathrm{C}$ : lots of undissolved transparent material in bulk, undissolved opaque materials at base (noble metals) $\gg>>\mathrm{T}_{\mathrm{L}}$ between $1217^{\circ} \mathrm{C}$ and $1225^{\circ} \mathrm{C}$ [XRD sample]

- $\mathrm{T}_{\mathrm{L}}=1221^{\circ} \mathrm{C}$

\section{US-44:}

- HROM ThS, $1058^{\circ} \mathrm{C}$ : cubic crystals $\gg>$ higher temperature

- HROM ThS, $1108^{\circ} \mathrm{C}$ : black agglomerates of noble metals $>>>\mathrm{T}_{\mathrm{L}}$ between $1058^{\circ} \mathrm{C}$ and $1108^{\circ} \mathrm{C}$

- HROM ThS, $1080^{\circ} \mathrm{C}$ : cubic crystals $\gg>\mathrm{T}_{\mathrm{L}}$ between $1080^{\circ} \mathrm{C}$ and $1108^{\circ} \mathrm{C}$

- HROM ThS, $1095^{\circ} \mathrm{C}$ : small spinel clumps at bottom, black agglomerates of noble metals $>>>$ $\mathrm{T}_{\mathrm{L}}$ between $1095^{\circ} \mathrm{C}$ and $1108^{\circ} \mathrm{C}$

- HROM ThS, $1101^{\circ} \mathrm{C}$ : small, black agglomerates of noble metals $>>>\mathrm{T}_{\mathrm{L}}$ between $1095^{\circ} \mathrm{C}$ and $1101^{\circ} \mathrm{C}$

- $\mathrm{T}_{\mathrm{L}}=1098^{\circ} \mathrm{C}$ 
US-45:

- HROM ThS, $1058^{\circ} \mathrm{C}$ : few cubic crystals in bulk $\gg>>$ higher temperature

- HROM ThS, $1108^{\circ} \mathrm{C}$ : black agglomerates of noble metals $\gg>\mathrm{T}_{\mathrm{L}}$ between $1058^{\circ} \mathrm{C}$ and $1108^{\circ} \mathrm{C}$

- HROM ThS, $1080^{\circ} \mathrm{C}$ : black agglomerates of noble metals $\gg>\mathrm{T}_{\mathrm{L}}$ between $1058^{\circ} \mathrm{C}$ and $1080^{\circ} \mathrm{C}$

- HROM ThS, $1067^{\circ} \mathrm{C}$ : large and small spinels throughout sample, black agglomerates of noble metals $\gg>\mathrm{T}_{\mathrm{L}}$ between $1067^{\circ} \mathrm{C}$ and $1080^{\circ} \mathrm{C}$

- HROM ThS, $1074^{\circ} \mathrm{C}$ : sample clear $\gg>>\mathrm{T}_{\mathrm{L}}$ between $1067^{\circ} \mathrm{C}$ and $1074^{\circ} \mathrm{C}$

- HROM ThS, $1070^{\circ} \mathrm{C}$ : a few small agglomerates at bottom - some are spinel and the rest are black agglomerates of noble metals $\gg>T_{L}$ between $1070^{\circ} \mathrm{C}$ and $1074{ }^{\circ} \mathrm{C}$

- $\mathrm{T}_{\mathrm{L}}=1072^{\circ} \mathrm{C}$ 
SRNS-STI-2008-00057

Revision 0

\section{Appendix B}

\section{Crystal Fraction Summary Table}


Table B-1. Summary of crystalline phases for different heat treated specimens for all glasses along with the data fit information - slope, intercept (or calculated $T_{L}$ ), and correlation coefficient $\left(\mathbf{R}^{2}\right)$ - and the primary phase (listed as “Prim. Ph.”) identified.

Crystalline phases, listed in the table as wt $\%$ abundance, are abbreviated as follows: Trev. = trevorite, Neph. = nepheline, Nos. = nosean, Zirc. = zircon, Hem. = hematite. The term "(OP)" listed after some of the primary phases means that the measured $T_{L}$ for the crystal fraction method for that particular glass is listed as being below the detection limit in Table 5-7 due to a lack of sufficient crystal fraction data.

\begin{tabular}{|c|c|c|c|c|c|c|c|c|c|c|c|c|}
\hline Glass & $\begin{array}{c}\text { Temp. } \\
\left({ }^{\circ} \mathrm{C}\right)\end{array}$ & Trev. & Neph. & $\mathrm{NiO}$ & Nos. & Zirc. & Hem. & $\mathrm{Li}_{2} \mathrm{SiO}_{3}$ & Slope & Int. & $\mathbf{R}^{2}$ & Prim. Ph. \\
\hline US-01 & 905 & 1 & & & & & & & -110.74 & 1019 & 0.997 & Spinel \\
\hline \begin{tabular}{|l|} 
US-01 \\
\end{tabular} & 854 & 1.5 & & & & & & & & & & \\
\hline US-01 & 954 & 0.6 & & & & & & & & & & \\
\hline US-02 & 854 & & & & & & & & N/A & N/A & N/A & $\begin{array}{l}\text { Baddelyite } \\
\text { (OP) }\end{array}$ \\
\hline US-02 & 905 & & & & & & & & & & & \\
\hline US-02 & 954 & & & & & & & & & & & \\
\hline US-02 & 1006 & & & & & & & & & & & \\
\hline US-03 & 854 & 1.1 & & & & & & & -148.28 & 1018 & 0.996 & Spinel \\
\hline \begin{tabular}{|l} 
US-03 \\
\end{tabular} & 954 & 0.4 & & & & & & & & & & \\
\hline US-03 & 904 & 0.8 & & & & & & & & & & \\
\hline US- 03 & 1006 & 0.1 & & & & & & & & & & \\
\hline US-04 & 954 & 0.1 & & & & & & & -62.67 & 956 & 0.984 & Spinel \\
\hline US-04 & 861 & 1.5 & & & & & & & & & & \\
\hline US-04 & 904 & 0.9 & & & & & & & & & & \\
\hline US-04 & 931 & 0.3 & & & & & & & & & & \\
\hline US-05 & 854 & & & & & & 4.6 & & -37.99 & 1025 & 0.984 & Hematite \\
\hline US- 05 & 904 & & & & & & 2.9 & & & & & \\
\hline US-05 & 954 & & & & & & 2.1 & & & & & \\
\hline US-05 & 1006 & 0.2 & & & & & 0.5 & & & & & \\
\hline US-06 & 854 & 3 & & & & & & & -39.39 & 968 & 0.945 & Spinel \\
\hline US-06 & 904 & 1.3 & & & & & & & & & & \\
\hline US-06 & 954 & 0.6 & & & & & & & & & & \\
\hline US-07 & 954 & 1.7 & & & & & & & -37.76 & 1018 & 1.000 & Spinel \\
\hline US-07 & 1006 & 0.3 & & & & & & & & & & \\
\hline US-07 & 1015 & 0.1 & & & & & & & & & & \\
\hline US-08 & 854 & 2.4 & & & & & & & -52.60 & 981 & 1.000 & Spinel \\
\hline US-08 & 903 & 1.5 & & & & & & & & & & \\
\hline US-08 & 954 & 0.5 & & & & & & & & & & \\
\hline US-09 & 854 & & & & & & 0.5 & & -510.00 & 1091 & 0.917 & Hematite \\
\hline US-09 & 903 & & & & & & 0.3 & & & & & \\
\hline US-09 & 1058 & & & & & & 0.1 & & & & & \\
\hline US-10 & 854 & 3.5 & & & & & & & -42.09 & 998 & 0.966 & Spinel \\
\hline US-10 & 903 & 2 & & & & & & & & & & \\
\hline US-10 & 954 & 1.2 & & & & & & & & & & \\
\hline US-11 & 854 & 1.6 & & & & & 2.8 & & -79.29 & 976 & 0.764 & Spinel \\
\hline
\end{tabular}


SRNS-STI-2008-00057

Revision 0

Appendix B

\begin{tabular}{|c|c|c|c|c|c|c|c|c|c|c|c|c|}
\hline Glass & $\begin{array}{c}\text { Temp. } \\
\left({ }^{\circ} \mathrm{C}\right)\end{array}$ & Trev. & Neph. & $\mathrm{NiO}$ & Nos. & Zirc. & Hem. & $\mathrm{Li}_{2} \mathrm{SiO}_{3}$ & Slope & Int. & $\mathbf{R}^{2}$ & Prim. Ph. \\
\hline US-11 & 980 & 0.3 & & & & & & & & & & \\
\hline US-11 & 903 & 0.5 & & & & & 1.7 & & & & & \\
\hline US-12 & 854 & 5.2 & & & & & & & -39.99 & 1062 & 1.000 & Spinel \\
\hline US-12 & 903 & 4 & & & & & & & & & & \\
\hline US-12 & 954 & 2.7 & & & & & & & & & & \\
\hline US-13 & 854 & 2.6 & & & & & & & -69.26 & 1034 & 0.999 & Spinel \\
\hline US-13 & 901 & 1.9 & & & & & & & & & & \\
\hline US-13 & 954 & 1.2 & & & & & & & & & & \\
\hline US-13 & 1005 & 0.4 & & & & & & & & & & \\
\hline US-14 & 861 & 0.5 & & & & & & & -40.00 & 880 & 0.750 & Spinel \\
\hline US-14 & 861 & 0.4 & & & & & & & & & & \\
\hline US-14 & 869 & 0.3 & & & & & & & & & & \\
\hline US-15 & 854 & 0.5 & & & & & & & -117.50 & 913 & 1.000 & Spinel \\
\hline US-15 & 901 & 0.1 & & & & & & & & & & \\
\hline US-16 & 954 & & & & & & 3.7 & & -47.67 & 1136 & 0.964 & Hematite \\
\hline US-16 & 1005 & & & & & & 3.1 & & & & & \\
\hline US-16 & 1000 & & & & & & 2.6 & & & & & \\
\hline US-16 & 1000 & & & & & & 2.8 & & & & & \\
\hline US-16 & 1056 & & & & & & 1.8 & & & & & \\
\hline US-16 & 1106 & & & & & & 0.6 & & & & & \\
\hline US-17 & 903 & 2.2 & & & & & & & -58.39 & 1031 & 0.994 & Spinel \\
\hline US-17 & 974 & 0.9 & & & & & & & & & & \\
\hline US-17 & 999 & 0.6 & & & & & & & & & & \\
\hline US-18 & 901 & 2.1 & & & & & & & -60.62 & 1026 & 0.992 & Spinel \\
\hline US-18 & 954 & 1.1 & & & & & & & & & & \\
\hline US-18 & 1005 & 0.4 & & & & & & & & & & \\
\hline US-19 & 852 & 1.6 & & & & & & & -118.03 & 1052 & 0.950 & Spinel \\
\hline US-19 & 954 & 1 & & & & & & & & & & \\
\hline US-19 & 1007 & 0.3 & & & & & & & & & & \\
\hline US-20 & 852 & 1.5 & & & & & & & -97.09 & 1006 & 0.954 & Spinel \\
\hline US-20 & 902 & 1.2 & & & & & & & & & & \\
\hline US-20 & 954 & 0.5 & & & & & & & & & & \\
\hline US-21 & 852 & 2.2 & & & & & & & -96.13 & 1071 & 0.987 & Spinel \\
\hline US-21 & 902 & 1.8 & & & & & & & & & & \\
\hline US-21 & 954 & 1.3 & & & & & & & & & & \\
\hline US-21 & 1007 & 0.6 & & & & & & & & & & \\
\hline US-22 & 852 & 3.6 & & & & & & & -53.81 & 1050 & 0.977 & Spinel \\
\hline US-22 & 895 & 2.7 & & & & & & & & & & \\
\hline US-22 & 902 & 3 & & & & & & & & & & \\
\hline US-22 & 1007 & 0.8 & & & & & & & & & & \\
\hline US-23 & 852 & 1.4 & & & & & & & -129.25 & 1032 & 1.000 & Spinel \\
\hline US-23 & 902 & 1 & & & & & & & & & & \\
\hline US-23 & 954 & 0.6 & & & & & & & & & & \\
\hline US-23 & 1007 & 0.2 & & & & & & & & & & \\
\hline
\end{tabular}


SRNS-STI-2008-00057

Revision 0

Appendix B

\begin{tabular}{|c|c|c|c|c|c|c|c|c|c|c|c|c|}
\hline Glass & $\begin{array}{l}\text { Temp. } \\
\left({ }^{\circ} \mathrm{C}\right)\end{array}$ & Trev. & Neph. & $\mathrm{NiO}$ & Nos. & Zirc. & Hem. & $\mathrm{Li}_{2} \mathrm{SiO}_{3}$ & Slope & Int. & $\mathbf{R}^{2}$ & Prim. Ph. \\
\hline US-24 & 852 & 2.8 & & & & & & & -58.55 & 1024 & 0.987 & Spinel \\
\hline US-24 & 902 & 2.2 & & & & & & & & & & \\
\hline US-24 & 954 & 1.3 & & & & & & & & & & \\
\hline US-24 & 1007 & 0.2 & & & & & & & & & & \\
\hline US-25 & 954 & 1.9 & & & & & & & -61.79 & 1074 & 0.986 & Spinel \\
\hline US-25 & 994 & 1.4 & & & & & & & & & & \\
\hline US-25 & 1007 & 1 & & & & & & & & & & \\
\hline US- 25 & 1056 & 0.3 & & & & & & & & & & \\
\hline US-26 & 855 & 1.3 & & & & & & & -236.29 & 1167 & 0.967 & Spinel \\
\hline US-26 & 903 & 1.2 & & & & & & & & & & \\
\hline US-26 & 952 & 0.8 & & & & & & & & & & \\
\hline US-26 & 1107 & 0.3 & & & & & & & & & & \\
\hline US-27 & 903 & 1.2 & & & & & & & -153.00 & 1077 & 0.959 & Spinel \\
\hline US-27 & 952 & 0.7 & & & & & & & & & & \\
\hline US-27 & 1056 & 0.2 & & & & & & & & & & \\
\hline US-28 & 752 & 1 & & & 0.6 & & 0.2 & & -61.11 & 813 & 1.000 & Spinel \\
\hline US-28 & 807 & 0.1 & & & 0.9 & & & & & & & \\
\hline US-29 & 903 & 1 & & & & & 0 & & N/A & N/A & N/A & Spinel(OP) \\
\hline US-29 & 700 & 1 & & & 0.7 & & 0.2 & & & & & \\
\hline US-30 & 855 & 1.4 & & & & 0.1 & & & -277.91 & 1251 & 0.996 & Spinel \\
\hline US-30 & 952 & 1.1 & & & & 0.2 & & & & & & \\
\hline US-30 & 1006 & 0.9 & & & & & & & & & & \\
\hline US-30 & 1056 & 0.7 & & & & & & & & & & \\
\hline US-30 & 1107 & 0.5 & & & & & & & & & & \\
\hline US-31 & 903 & & & & & & 5.6 & & -39.59 & 1125 & 1.000 & Hematite \\
\hline US-31 & 952 & & & & & & 4.4 & & & & & \\
\hline US-31 & 1006 & & & & & & 3 & & & & & \\
\hline US-32 & 704 & 0.1 & & & & & & & N/A & N/A & N/A & Spinel(OP) \\
\hline US-32 & 731 & 0.1 & & & & & & & & & & \\
\hline US-33 & 854 & 1 & & & 1.9 & & & & -163.33 & 1017 & 1.000 & Spinel \\
\hline US-33 & 902 & 0.7 & & & 1.5 & & & & & & & \\
\hline US-33 & 952 & 0.4 & & & & & & & & & & \\
\hline US-34 & 828 & 11.2 & & & & & & & N/A & N/A & $\mathrm{N} / \mathrm{A}$ & Spinel(OP) \\
\hline US-34 & 854 & & & & & & & & & & & \\
\hline US-34 & 902 & & & & & & & & & & & \\
\hline US-35 & 952 & 3.6 & & & & & & & -114.02 & 1310 & 0.880 & Spinel \\
\hline US-35 & 1007 & 2 & & & & & & & & & & \\
\hline US-35 & 1205 & 0.8 & & & & & & & & & & \\
\hline US-35 & 1247 & 0.7 & & & & & & & & & & \\
\hline US-35 & 1328 & 0.4 & & & & & & & & & & \\
\hline US-35 & 1017 & 2.7 & & & & & & & & & & \\
\hline US-35 & 1074 & 1.4 & & & & & & & & & & \\
\hline US-35 & 1218 & 0.6 & & & & & & & & & & \\
\hline US-35 & 1278 & 0.4 & & & & & & & & & & \\
\hline
\end{tabular}


SRNS-STI-2008-00057

Revision 0

Appendix B

\begin{tabular}{|c|c|c|c|c|c|c|c|c|c|c|c|c|}
\hline Glass & $\begin{array}{c}\text { Temp. } \\
\left({ }^{\circ} \mathrm{C}\right)\end{array}$ & Trev. & Neph. & $\mathrm{NiO}$ & Nos. & Zirc. & Hem. & $\mathrm{Li}_{2} \mathrm{SiO}_{3}$ & Slope & Int. & $\mathbf{R}^{2}$ & Prim. Ph. \\
\hline US-35 & 1301 & 0.3 & & & & & & & & & & \\
\hline US-36 & 902 & 1.9 & & & & & & & -196.84 & 1253 & 0.804 & Spinel \\
\hline US-36 & 952 & 1.2 & & & & & & & & & & \\
\hline US-36 & 1007 & 0.8 & & & & & & & & & & \\
\hline US-36 & 1199 & 0.4 & & & & & & & & & & \\
\hline US-36 & 1205 & 0.5 & & & & & & & & & & \\
\hline US-36 & 1000 & 1.5 & & & & & & & & & & \\
\hline US-36 & 1098 & 0.7 & & & & & & & & & & \\
\hline US-36 & 1164 & 0.6 & & & & & & & & & & \\
\hline US-37 & 854 & 2.3 & 3.9 & & & & & & -80.59 & 1036 & 0.996 & Spinel \\
\hline US-37 & 902 & 1.6 & & & & & & & & & & \\
\hline US-37 & 952 & 1 & & & & & & & & & & \\
\hline US-37 & 1007 & 0.4 & & & & & & & & & & \\
\hline US-38 & 854 & 2.6 & & 0.4 & 0.7 & & & & -71.32 & 1038 & 0.968 & Spinel \\
\hline US-38 & 902 & 1.7 & & 0.5 & & & & & & & & \\
\hline US-38 & 952 & 1.4 & & 0.7 & & & & & & & & \\
\hline US-38 & 1007 & 0.4 & & 1.1 & & & & & & & & \\
\hline US-39 & 854 & 5.4 & & & & & & & -46.68 & 1105 & 0.999 & Spinel \\
\hline US-39 & 902 & 4.3 & & & & 0.2 & & & & & & \\
\hline US-39 & 952 & 3.3 & & & & 0.3 & & & & & & \\
\hline US-39 & 1007 & 2.1 & & & & 0.2 & & & & & & \\
\hline US-41 & 855 & & & & & & 3.7 & & -49.04 & 1046 & 0.982 & Hematite \\
\hline US-41 & 905 & & & & & & 3.1 & & & & & \\
\hline US-41 & 956 & & & & & & 1.9 & & & & & \\
\hline US-41 & 1008 & & & & & & 0.7 & & & & & \\
\hline US-42 & 925 & 3.1 & 35.3 & & & & & & -36.56 & 1037 & 0.982 & Spinel \\
\hline US-42 & 1035 & 0.3 & & & & & & & & & & \\
\hline US-42 & 1017 & 0.4 & & & & & & & & & & \\
\hline US-42 & 1000 & 0.9 & & & & & & & & & & \\
\hline US-43 & 895 & 2.6 & & & & 1.5 & & 4.1 & -217.69 & 1245 & 0.910 & Zircon \\
\hline US-43 & 994 & 0.8 & & & & 1.3 & & 2.1 & & & & \\
\hline US-43 & 1085 & & & & & 0.7 & & 0.7 & & & & \\
\hline US-44 & 905 & 1 & & & & & & & -203.42 & 1112 & 0.988 & Spinel \\
\hline US-44 & 956 & 0.8 & & & & & & & & & & \\
\hline US-44 & 1008 & 0.5 & & & & & & & & & & \\
\hline US-45 & 905 & 0.8 & & & & & & & -203.42 & 1072 & & Spinel \\
\hline US-45 & 956 & 0.6 & & & & & & & & & & \\
\hline US-45 & 1008 & 0.3 & & & & & & & & & & \\
\hline
\end{tabular}


SRNS-STI-2008-00057

Revision 0

\section{Appendix C}

Report on Fabrication and Testing of KRI Test Matrix Glasses

(SRNS-OS-2008-00001) 


\section{Subcontract AC 55551T}

\section{Task 6: \\ Improved Retention of Aluminum, Chromium, and Sulfate in U.S. Waste Glasses}

Deliverable 6 on Sub-Task T6-12a

Report on Fabrication and Testing of KRI Test Matrix Glasses

(crucible scale)

S. Petersburg

2007 
Introduction

\section{TABLE OF CONTENTS}

1. Fabrication of Glasses under Laboratory Conditions (Crucible Scale)......................... 5

2. Research and Data Processing Methods...................................................................... 7

2.1 X-Ray Diffraction Analysis (XRDA) ……........................................................ 7

2.2 Chemical and Phase Composition Glass Homogeneity Studies ......................... 7

3. Chemical and Phase Composition Glass Homogeneity Studies ....................................... 9

4. CCC Testing of KRI Matrix Glasses ..................................................................... 11

4.1 CCC Testing and Determination of Phase Compositions for KRI Matrix Glasses

5. Liquidus Temperature $\left(T_{L}\right)$ Measurements for KRI Matrix 16

5.1 Muffle Furnace Calibrations and $T_{L}$ Measurements for SRM-773

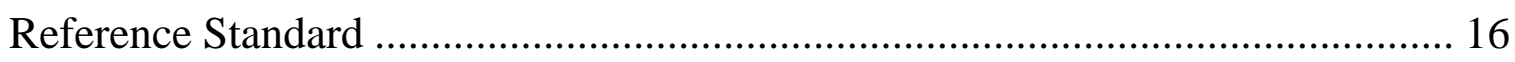

$5.2 \mathrm{~T}_{\mathrm{L}}$ Measurements for KRI Matrix Glasses...................................................... 18

6. PCT Evaluations of Quenched and CCC Treated KRI Matrix Glasses ..................... 21

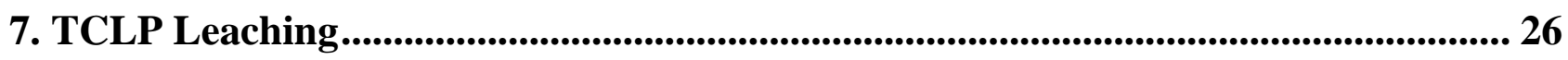

7.1 Selection of Appropriate Extraction Fluid ................................................ 26

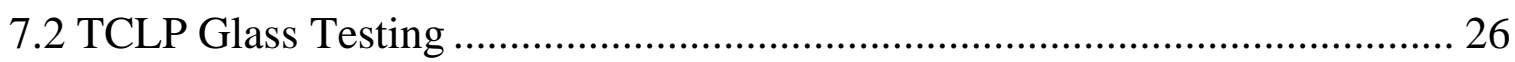

7.3. TCLP Testing Quality Assurance ................................................................. 27

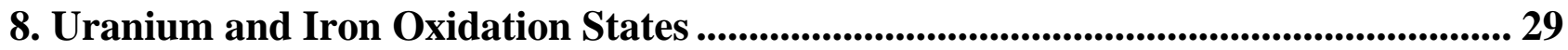

8.1. Uranium Oxidation State in KRI-28 and KRI-30 ....................................... 29

8.2 Iron Oxidation State in Glasses KRI-01, KRI-06, and KRI-28 ....................... 30

9. Viscosity Measurements for Selected KRI Matrix Glasses ........................................... 31

9.1. Viscosity Measurement Procedure...................................................................... 31

9.2. Viscosity Parameters for Studied Glasses ........................................................ 32

10. Selection of Optimal KRI Matrix Glasses.............................................................. 34

11. Conclusions........................................................................................................................................ 36

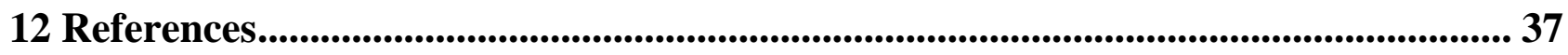

Attachment 1. EPMA Data on Chemical Homogeneity of Glasses.................................... 38 
Attachment 2. SEM Photographs of Quenched Glasses ........................................................ 46

Attachment 3. XRD Data for Quenched Glasses................................................................ 52

Attachment 4 Surface of KRI Glasses after CCC Treatment ........................................... 56

Attachment 5. SEM Photographs of Glass Samples after CCC Treatment .................... 60

Attachment 6. EPMA Data on Chemical Homogeneity of Glasses After CCC

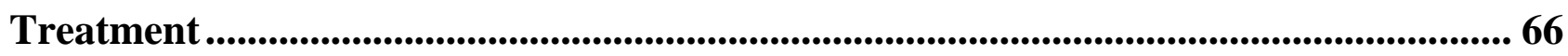

Attachment 7. XRD Data for Glasses after CCC Treatment ............................................ 74

\section{LIST OF ABBREVIATIONS}

HLW High-level waste

U.S. DOE United States Department of Energy

KRI Khlopin Radium Institute

DWPF Defense Waste Processing Facility at Savannah River Site

WTP Waste Treatment Plant at Hanford

SNF spent nuclear fuel

PNNL Pacific North-West National Laboratory

SRNL Savannah River National Laboratory

EPMA electron probe microanalysis

PCT product consistency test (glass leach tests for B, Li, Na, and SI leaching)

XRDA X-ray diffraction analysis

SEM scanning electron microscopy

TCLP Toxicity Characteristics Leaching Procedure

CCC Canister Centerline Cooling

TC thermocouple 


\section{Introduction}

The U.S. Department of Energy (DOE) is currently processing (or planning to process) highlevel waste (HLW) through Joule-heated melters at the Savannah River Site (SRS) and Hanford. The process combines the HLW sludge with a prefritted or mined mineral glass forming additives which are subsequently melted and pouring the molten glass in stainless steel canisters to create the final waste form.

Development of strategies at both the SRS and Hanford have identified high $\mathrm{Al}_{2} \mathrm{O}_{3}$ waste streams that are scheduled to be processed through their respective HLW vitrification facilities. These streams have $\mathrm{Al}_{2} \mathrm{O}_{3}$ concentrations between approximately 25 - 40 wt. \%, $\mathrm{Na}_{2} \mathrm{O}$ - between $\sim 22$ - $26 \mathrm{wt} \%$, and $\mathrm{SO}_{4}$ - between $\sim 0.8$ - 1.6 wt. Current Hanford projections suggest the $\mathrm{Al}_{2} \mathrm{O}_{3}$ concentrations in sludge could be much greater than those currently projected for DWPF with $\mathrm{Al}_{2} \mathrm{O}_{3}$ concentrations as high as $80 \mathrm{wt} \%$

While it is well known that the addition of small amounts of $\mathrm{Al}_{2} \mathrm{O}_{3}$ to borosilicate glasses generally enhances the durability of the waste form (through creation of network-forming tetrahedral $\mathrm{Na}^{+}-\left[\mathrm{AlO}_{4 / 2}\right]^{-}$pairs), nepheline $\left(\mathrm{NaAlSiO}_{4}\right)$ formation, which depends in part on the $\mathrm{Al}_{2} \mathrm{O}_{3}$ content, can result in a severe deterioration of the chemical durability of the slowly cooled glass near the center of the canister through residual glass compositional changes. The primary driver for the reduction in durability is the fact that nepheline removes three moles of glass forming oxides $\left(\mathrm{Al}_{2} \mathrm{O}_{3}\right.$ and $\left.2 \mathrm{SiO}_{2}\right)$ per each mole of $\mathrm{Na}_{2} \mathrm{O}$ from the continuous glass phase. Therefore, nepheline formation produces an $\mathrm{Al}_{2} \mathrm{O}_{3}$ and $\mathrm{SiO}_{2}$ deficient continuous glass matrix (relative to the same composition which is void of crystals) which reduces the durability of the final product. The magnitude of the reduction ultimately depends on the extent (e.g., volume \%) of crystallization and on the initial glass composition.

The formation of nepheline and/or other aluminum/silicon-containing crystals is a potential for both DWPF and Hanford due to the projected compositional views recently evaluated coupled with the frit development strategy (e.g., higher alkali frits have lead to enhanced melt rates at DWPF for those sludge batches). Although durability is obvious a critical constraint that the HLW glass must meet, other process related issues are equally important. Additionally, the addition of higher concentrations of $\mathrm{Al}_{2} \mathrm{O}_{3}$ will generally increase the liquidus temperature of the melt and decrease the processing rate.

The objective of this task is to develop glass formulations for specific DOE waste streams to avoid nepheline formation while maintaining or meeting waste loading and/or waste throughput expectations as well as satisfying critical process and product performance related constraints. Secondary objectives of this are to assess the $\mathrm{SO}_{4}$ solubility limit for the DWPF composition and spinel settling for the PNNL composition. 


\section{Fabrication of Glasses under Laboratory Conditions (Crucible Scale)}

Thirty glass compositions were selected for studies at KRI, and all these glass compositions were synthesized with appropriate ratios of metal oxides, carbonates, $\mathrm{H}_{3} \mathrm{BO}_{3}$, and salts to assure sufficient quantities for subsequent testing (usually 300 grams).

Table 1 lists chemicals that were utilized for feed preparation. The original materials were thoroughly mixed and placed into a platinum crucible. The crucible was then placed into a muffle furnace to be heated to $1,150^{\circ} \mathrm{C}$. After being isothermically held at $1,150^{\circ} \mathrm{C}$ for two hours, the glass was poured onto a clean stainless steel plate for quenching.

Fig. 1 shows a photograph of KRI-07 glass being poured from the Pt crucible. Fig. 2 shows a cooled glass on the stainless steel plate. Table 2 provides compositions of the synthesized glasses.

Table 1. Oxides and Chemicals Utilized for Feed Preparation

\begin{tabular}{|c|c|c|}
\hline Oxides & Chemicals & Grade \\
\hline $\mathrm{Al}_{2} \mathrm{O}_{3}$ & $\mathrm{Al}(\mathrm{OH})_{3}$ & Pure for analysis \\
\hline $\mathrm{B}_{2} \mathrm{O}_{3}$ & $\mathrm{H}_{3} \mathrm{BO}_{3}$ & Chemically pure \\
\hline $\mathrm{CaO}$ & $\mathrm{CaO}$ & Pure for analysis \\
\hline $\mathrm{Cr}_{2} \mathrm{O}_{3}$ & $\mathrm{Cr}_{2} \mathrm{O}_{3}$ & Pure \\
\hline $\mathrm{Fe}_{2} \mathrm{O}_{3}$ & $\mathrm{Fe}_{2} \mathrm{O}_{3}$ & Pure for analysis \\
\hline $\mathrm{K}_{2} \mathrm{O}$ & $\mathrm{K}_{2} \mathrm{CO}_{3}$ & Chemically pure \\
\hline $\mathrm{Li}_{2} \mathrm{O}$ & $\mathrm{Li}_{2} \mathrm{CO}_{3}$ & Chemically pure \\
\hline $\mathrm{MgO}$ & $\mathrm{MgO}$ & Chemically pure \\
\hline $\mathrm{MnO}$ & $\mathrm{MnO}_{2}$ & Pure \\
\hline $\mathrm{Na} 2$ & $\mathrm{Na}_{2} \mathrm{CO}$ & Chemically pure \\
\hline $\mathrm{NiO}$ & $\mathrm{NiO}_{3}$ & Pure \\
\hline $\mathrm{PbO}$ & $\mathrm{PbO} \mathrm{O}$ & Pure for analysis \\
\hline $\mathrm{SO}$ & $\mathrm{Na}_{2} \mathrm{SO}_{4}$ & Chemically pure \\
\hline $\mathrm{SiO}$ & $\mathrm{SiO}_{2}$ & Pure \\
\hline $\mathrm{SrO}$ & $\mathrm{SrCO}_{3}$ & Pure for analysis \\
\hline $\mathrm{TiO}$ & $\mathrm{TiO}_{2}$ & Pure \\
\hline $\mathrm{ZnO}$ & $\mathrm{ZnO}_{2}$ & Pure \\
\hline $\mathrm{ZrO}$ & $\mathrm{ZrO}_{2}$ & Pure \\
\hline
\end{tabular}




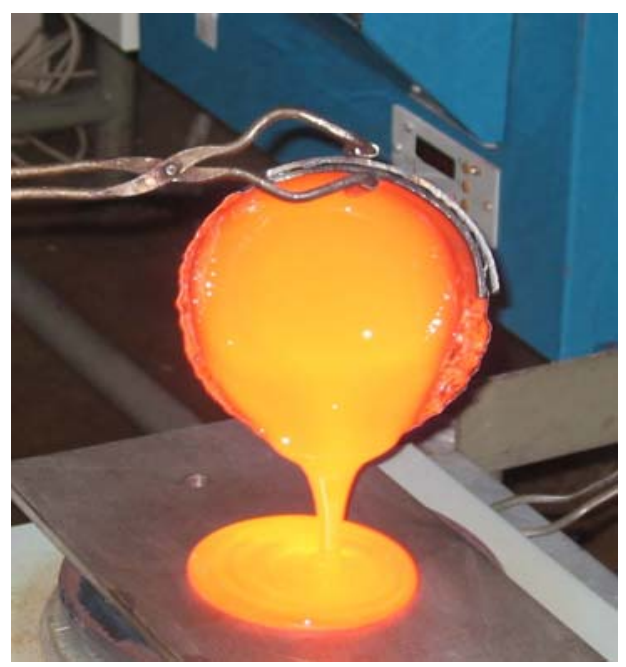

Fig. 1. Glass pouring

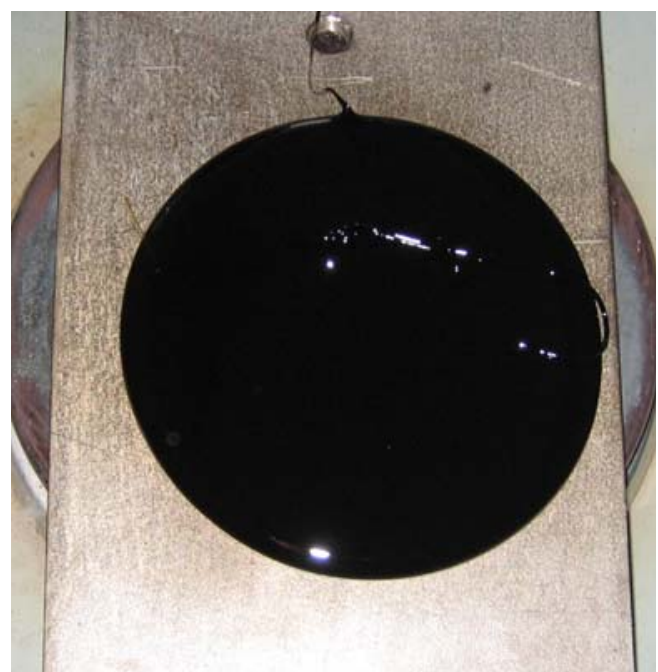

Fig.2. Quenched glass

\section{Table 2. Compositions of Synthesized Glasses}

\begin{tabular}{|c|c|c|c|c|c|c|c|c|c|c|c|c|c|c|c|c|c|c|c|c|c|}
\hline S-08 & & & & & & & & & & & & 001 & .099 & & 599 & & .000 & 1.000 & 0.000 & 0.000 & .000 \\
\hline S-19 & & 476 & 404 & 700 & 069 & & 777 & 638 & 333 & 988 & 804 & 209 & 070 & 277 & 333 & & 061 & .805 & 000 & 067 & 275 \\
\hline S-20 & & & & 700 & & & 0.777 & 638 & 333 & 988 & & 0.209 & 070 & 277 & 333 & & 061 & 805 & 000 & 067 & 275 \\
\hline JS-24 & & & 252 & 749 & & & 0.749 & .001 & .374 & .249 & & 0.501 & \begin{tabular}{|l|}
0.079 \\
\end{tabular} & 374 & 0.449 & & \begin{tabular}{|l|}
0.079 \\
\end{tabular} & 0.749 & 0.000 & \begin{tabular}{|l|}
0.079 \\
\end{tabular} & .379 \\
\hline S-35 & & & & 000 & & & 0.000 & & 0.500 & & & & 000 & & 000 & & & 0.000 & & 000 & \\
\hline & & & & & & & 540 & & 53 & & & & & & 267 & & & 0.465 & & 024 & \\
\hline & & & & 250 & & & \begin{tabular}{|l|}
0.750 \\
\end{tabular} & 000 & 380 & 000 & & .812 & 750 & 30 & 156 & & 750 & .750 & 000 & 500 & 000 \\
\hline & & & .337 & 250 & & & 0.250 & 3.000 & 0.380 & 1.000 & & 0.750 & 0.750 & & .156 & & 0.750 & 0.750 & 000 & 500 & .000 \\
\hline & & & 378 & 750 & & & 0.750 & 3.112 & 0.130 & & & 0.750 & 0.250 & & .156 & & 1.789 & 0.250 & 0.000 & 072 & .000 \\
\hline & & & & 251 & & & 47 & & & & & & & & 157 & & 751 & 0.251 & 000 & 501 & \\
\hline & & & & & & & & 75 & 30 & & & 370 & 250 & & 156 & & 250 & 250 & & 500 & \\
\hline & & & & 251 & & & 0.750 & 3.000 & 130 & 00 & & 0.750 & 750 & 80 & 456 & & 750 & .750 & 000 & 500 & 997 \\
\hline & & & & 750 & & & 0.547 & 3.521 & 131 & 31 & & 830 & 0.750 & & .456 & & 750 & 0.250 & & 500 & 000 \\
\hline & & & & 0.250 & & & 0.250 & 4.371 & 0.130 & 3.000 & & 1.032 & 0.250 & 30 & 0.156 & & 2.250 & 0.750 & 0.000 & 0.500 & 1.000 \\
\hline & & 404 & .791 & 0.750 & 22 & & 0.250 & 3.000 & 0.380 & 1.000 & 11.6 & 0.781 & 0.750 & 130 & 0.156 & & 2.250 & 0.250 & 0.000 & 0.500 & 1.000 \\
\hline & & & & 0.750 & & & 0.437 & 3.293 & 0.207 & 1.267 & & 1.050 & .470 & & 0.456 & & 0.820 & 0.320 & & 570 & 3.000 \\
\hline & & & & 250 & & & 0.250 & 5.000 & 0.1 & & & & 250 & & 456 & & .250 & 0.750 & & 500 & 000 \\
\hline & & & & 0.250 & & & 0.250 & 3.000 & 0.352 & 1.000 & & 0.751 & 0.250 & & 0.456 & & \begin{tabular}{|l|l|}
0.750 \\
\end{tabular} & 0.250 & & 1.500 & 1.000 \\
\hline & & & & 0.749 & & & \begin{tabular}{|l|}
0.749 \\
\end{tabular} & 3.767 & 0.131 & 1.883 & & 0.751 & \begin{tabular}{|l|}
0.749 \\
\end{tabular} & \begin{tabular}{|l|}
0.379 \\
\end{tabular} & 0.455 & 3.724 & \begin{tabular}{|l|}
1.177 \\
\end{tabular} & 0.607 & 0.000 & 1.198 & 2.999 \\
\hline & & .058 & .510 & 0.282 & .251 & 1.231 & 0.683 & 3.750 & 0.131 & 1.118 & & 0.751 & 0.251 & .187 & 0.224 & 785 & 0.751 & 0.251 & 0.000 & 0.501 & 1.001 \\
\hline & & & & 1.000 & & & 0.000 & 6.000 & 0.000 & 000 & & 3.000 & 0.000 & 00 & 0.600 & & 3.000 & 0.000 & 1.557 & 0.000 & 000 \\
\hline & & & & 1.000 & & & 0.000 & 5.733 & 0.500 & & & 0.468 & 1.000 & .500 & 0.600 & & 0.000 & 1.000 & 0.992 & 0.000 & 000 \\
\hline & & .349 & 6.126 & 0.000 & & & 0.000 & 5.718 & 0.000 & 3.854 & & 0.000 & 1.000 & 0.500 & 0.600 & & 0.000 & 1.000 & 2.429 & 0.000 & 0.000 \\
\hline & & 268 & & 0.000 & & & 1.000 & 2.000 & 0.500 & 0.000 & & 3.000 & 1.000 & 0.000 & 0.000 & & 2.203 & 1.000 & 3.000 & 0.000 & 0.000 \\
\hline & KRI-30 & 20.000 & 15.297 & 0.750 & 0.000 & 5.511 & 0.000 & 5.794 & 0.500 & 0.000 & 7.238 & 0.000 & 1.000 & 0.500 & 0.600 & 35.736 & 3.000 & 0.000 & 2.675 & 2.000 & 0.000 \\
\hline
\end{tabular}




\section{Research and Data Processing Methods}

\subsection{X-Ray Diffraction Analysis (XRDA)}

For XRD analysis, KRI used the DRON-UM1 diffractometer with $\mathrm{CuK}_{\alpha}$ irradiation. The X-ray tube had the voltage of $35 \mathrm{kV}$ and the current of $2 \mathrm{~mA}$. JCPDS data sets were used to assure a high quality analysis.

KRI used the PDWIN software application for a quantitative analysis of crystalline phases, i.e., sample analysis with the known mass absorption coefficient. Magnetite $\left(\mathrm{Fe}_{3} \mathrm{O}_{4}\right)$ with the spinel structure, $\alpha$-quartz $\left(\mathrm{SiO}_{2}\right)$, and nepheline $\left(\mathrm{NaAlSiO}_{4}\right)$ were used as standard phases.

Since the sensitivity of the XRD method for identifying spinel was, in our case, 1 mass \%, we used SEM photographs for evaluating the spinel content in those glass samples that contained less than 1 mass \% of spinel. This analysis is shown below using KRI-15 sample as an example.

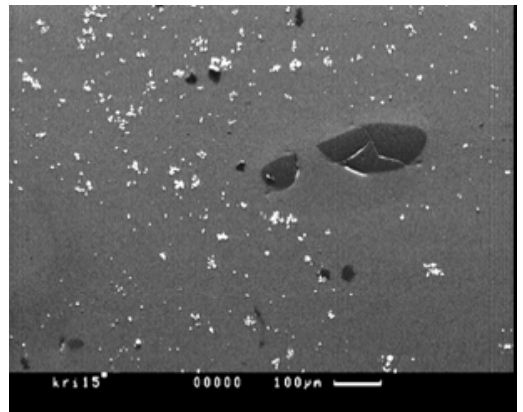

Fig. 3. Original image. White inclusions - spinel. Magnification 1000x

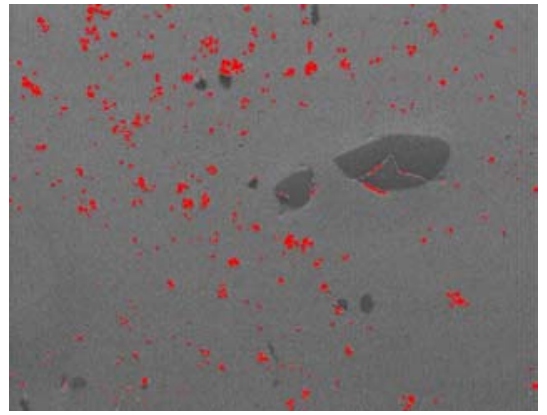

Fig. 4. Digitized image. Red dots are spinel.

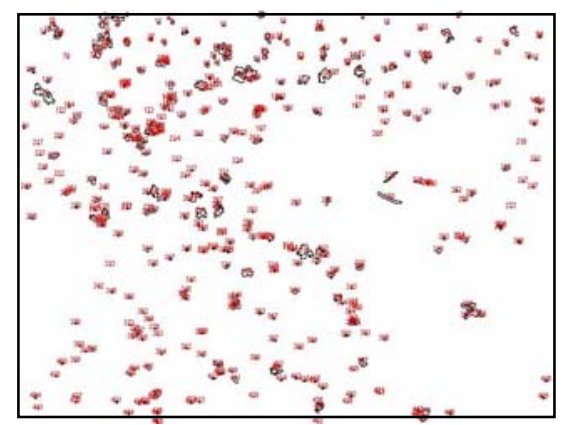

Fig. 5. Processed image with each spinel particle recorded and numbered

Image Analysis Results: quantity of particles: 453; total Area: 16,102.97 $\mu^{2}$; average size: $35.55 \mathrm{\mu m}^{2}$; area fraction: $2.14 \%$. For statistics: processing of another photograph of the same sample gives $2.11 \%$ of the area fraction. We used a similar technique for evaluating all compositions that contained crystalline phase spinel inclusions.

\subsection{Chemical and Phase Composition Glass Homogeneity Studies}

For glass homogeneity studies, we applied scanning microscopy and electron probe microanalysis (EPMA) using CAMSCAN-4DV with the semiconductor spectrometer LINK AN10000 (UK). The studies included the following phases:

1. Prepare glass samples. We polished glass sample fragments and coated them with carbon to create a conductive layer using a carbon rod thermal evaporation method.

2. Evaluate glass sample homogeneity and inclusions by analyzing scanning microscopy images in back scattered electrons. The phases with a higher atomic number could be seen as lighter 
phases, e.g., spinel inclusions in glass. Obtained images could be recorded as colored photographs with magnification from x20 to x10000.

3. Perform electron probe microanalysis to determine glass chemical composition with the detection limit of about 0.1 mass \% for most elements. The area analysis with the minimal magnification of x20 gives an average glass composition in the area of a few $\mathrm{mm}^{2}$. To analyze sample homogeneity, we analyzed 5 sample areas, $0.01 \mathrm{~mm}^{2}$ each. For major macroelements, we calculated mean square deviations associated with non-homogeneity (Er). We determined the non-homogeneity coefficient as the ratio of $\mathrm{Er}$ and the measurement error ( $\mathrm{Re})$. If $\mathrm{Er} / \mathrm{Re}<2$, the element distribution in the sample bulk was considered homogenous. 


\section{Chemical and Phase Composition Glass Homogeneity Studies}

Using XRD, SEM, and EPMA, KRI studied the following characteristics of the synthesized glasses: homogeneity, chemical and phase compositions. Attachments 1, 2, and 3 contain data obtained by using EPMA, SEM, and XRD for the KRI matrix glasses from KRI-1 through KRI30 .

Table 3 provides phase compositions of the synthesized glasses and their crystalline phases determined by using XRD and SEM. Table 4 contains chemical compositions of the spinel in glass samples KRI-05, KRI-08, KRI-09, KRI-10, and KRI-11. As KRI-08 and KRI-09 shows, the same glass can have spinels of various chemical compositions.

Table 3. Phase Compositions of Quenched KRI Glasses

\begin{tabular}{|c|c|c|c|c|}
\hline Sample \# & Crystalline phase, XRD & Content, mass \%, XRD & Content, vol.\%, SEM & $\begin{array}{l}\text { Size, } \\
\mu \mathrm{m}\end{array}$ \\
\hline KRI-01 & $\mathrm{n} / \mathrm{d}$ & $\mathrm{n} / \mathrm{d}$ & - & - \\
\hline KRI-02 & $\mathrm{n} / \mathrm{d}$ & $\mathrm{n} / \mathrm{d}$ & - & - \\
\hline KRI-03 & $\mathrm{n} / \mathrm{d}$ & $\mathrm{n} / \mathrm{d}$ & - & - \\
\hline KRI-04 & $\mathrm{n} / \mathrm{d}$ & $\mathrm{n} / \mathrm{d}$ & - & - \\
\hline KRI-05 & spinel & 3.5 & 2.8 & 1.2 \\
\hline KRI-06 & $\mathrm{n} / \mathrm{d}$ & $\mathrm{n} / \mathrm{d}$ & - & - \\
\hline KRI-07 & $\mathrm{n} / \mathrm{d}$ & $\mathrm{n} / \mathrm{d}$ & - & - \\
\hline KRI-08 & spinel & 1.5 & 6.6 & 11 \\
\hline KRI-09 & spinel & 2.4 & 1.5 & 13 \\
\hline KRI-10 & $\mathrm{n} / \mathrm{d}$ & $\mathrm{n} / \mathrm{d}$ & 0.9 & 2.2 \\
\hline KRI-11 & spinel & 3 & 2.5 & 4.3 \\
\hline KRI-12 & spinel & 2 & 1.2 & 15.8 \\
\hline KRI-13 & spinel & 2 & 2.2 & 11.3 \\
\hline KRI-14 & spinel & 2.5 & 1.5 & 12.3 \\
\hline KRI-15 & spinel & 3.5 & 2,7 & 9.5 \\
\hline KRI-16 & $\mathrm{n} / \mathrm{d}$ & $\mathrm{n} / \mathrm{d}$ & 0,5 & 2.8 \\
\hline KRI-17 & $\mathrm{n} / \mathrm{d}$ & $\mathrm{n} / \mathrm{d}$ & 0,91 & 2.8 \\
\hline KRI-18 & spinel & 2.5 & 2.3 & 10.0 \\
\hline KRI-19 & $\mathrm{n} / \mathrm{d}$ & $\mathrm{n} / \mathrm{d}$ & 0,2 & 10 \\
\hline KRI-20 & $\mathrm{n} / \mathrm{d}$ & $\mathrm{n} / \mathrm{d}$ & 0,34 & 5.9 \\
\hline KRI-21 & $\mathrm{n} / \mathrm{d}$ & $\mathrm{n} / \mathrm{d}$ & 0,6 & 8.5 \\
\hline KRI-22 & $\mathrm{n} / \mathrm{d}$ & $\mathrm{n} / \mathrm{d}$ & 0,2 & 7.3 \\
\hline KRI-23 & spinel & 3.2 & 2,8 & 7.6 \\
\hline KRI-24 & $\mathrm{n} / \mathrm{d}$ & $\mathrm{n} / \mathrm{d}$ & 0,58 & 7.7 \\
\hline KRI-25 & $\mathrm{n} / \mathrm{d}$ & $\mathrm{n} / \mathrm{d}$ & 0,78 & 6.1 \\
\hline KRI-26 & $\mathrm{n} / \mathrm{d}$ & $\mathrm{n} / \mathrm{d}$ & $\mathrm{n} / \mathrm{d}$ & - \\
\hline KRI-27 & spinel & 1 & 0.8 & 4.2 \\
\hline KRI-28 & $\mathrm{n} / \mathrm{d}$ & $\mathrm{n} / \mathrm{d}$ & - & - \\
\hline KRI-29 & $\mathrm{n} / \mathrm{d}$ & $\mathrm{n} / \mathrm{d}$ & - & - \\
\hline KRI-30 & $\mathrm{n} / \mathrm{d}$ & $\mathrm{n} / \mathrm{d}$ & - & - \\
\hline
\end{tabular}


Table 4. Spinel Chemical Compositions (mass \%) in Quenched KRI Matrix Glasses (EPMA Data)

\begin{tabular}{|c|c|c|c|c|c|c|c|c|c|}
\hline & $\mathrm{Al} 2 \mathrm{O} 3$ & Cr2O3 & $\mathrm{FeO}$ & $\mathrm{Fe} 2 \mathrm{O} 3$ & $\mathrm{MnO}$ & $\mathrm{NiO}$ & $\mathrm{TiO} 2$ & $\mathrm{ZnO}$ & Total \\
\hline KRI-05 SP1 & 10.09 & 17.81 & \multicolumn{2}{|c|}{41.70} & 16.53 & 0.00 & 0.00 & 13.57 & 99.79 \\
\hline KRI-08 SP1 & 1.13 & 7.89 & 1.63 & 60.09 & 17.83 & 0.00 & 0.00 & 11.45 & 100.03 \\
\hline KRI-08 SP2 & 10.09 & 17.81 & \multicolumn{2}{|c|}{42.29} & 16.04 & 0.00 & 0.00 & 13.57 & 99.79 \\
\hline KRI-09 SP1 & 2.08 & 13.01 & 1.19 & 50.43 & 4.13 & 21.00 & 0.67 & 6.72 & 99.24 \\
\hline KRI -09 SP2 & 2.27 & 25.15 & 2.60 & 38.86 & 3.49 & 16.80 & 0.00 & 9.83 & 98.99 \\
\hline KRI-09 SP3 & 3.84 & 7.35 & \multicolumn{2}{|c|}{54.96} & 5.25 & 20.97 & 0.71 & 6.66 & 99.74 \\
\hline KRI -09 SP4 & 3.97 & 8.04 & \multicolumn{2}{|c|}{54.36} & 4.52 & 20.36 & 0.83 & 7.22 & 99.30 \\
\hline KRI -09 SP5 & 3.78 & 3.51 & \multicolumn{2}{|c|}{58.72} & 4.91 & 21.63 & 0.83 & 5.35 & 98.73 \\
\hline KRI-10 SP1 & 1.32 & 28.95 & 2.14 & 36.08 & 4.13 & 20.23 & 0.00 & 5.73 & 98.59 \\
\hline KRI-10 SP2 & 1.51 & 35.09 & 2.42 & 30.90 & 4.52 & 18.33 & 0.00 & 7.84 & 100.62 \\
\hline KRI-11 SP1 & 3.02 & 2.34 & \multicolumn{2}{|c|}{65.29} & 3.10 & 20.36 & 0.00 & 2.24 & 96.35 \\
\hline
\end{tabular}

The content of the "critical elements" in the generated spinels with the gross formula (Fe,Ni,Mn,Zn)(Fe,Cr,Al $)_{2} \mathrm{O}_{4}$ depends on the glass composition [1]. The presence of spinel crystals of various compositions in one glass can be explained by their crystallization at various temperatures during cooling of the original melt [2]. 


\section{CCC Testing of KRI Matrix Glasses}

\subsection{Testing and Determination of Phase Compositions for KRI Matrix Glasses}

All synthesized glasses were tested for their resistance to crystallization using heat treatment to simulate cooling along the centerline of a Defense Waste Processing Facility (DWPF)-type canister. This cooling regime is commonly referred to as the canister centerline cooled (CCC) curve.

According to the recommendations, we placed the Pt crucibles with about $25 \mathrm{~g}$ glass samples in each crucible into the muffle furnace with an adjustable cooling/heating rate. Fig. 6 compares a temperature curve that we used in our experiments with the recommended full-scale canister cooling curve showing that our cooling curve matches the baseline fairly well.

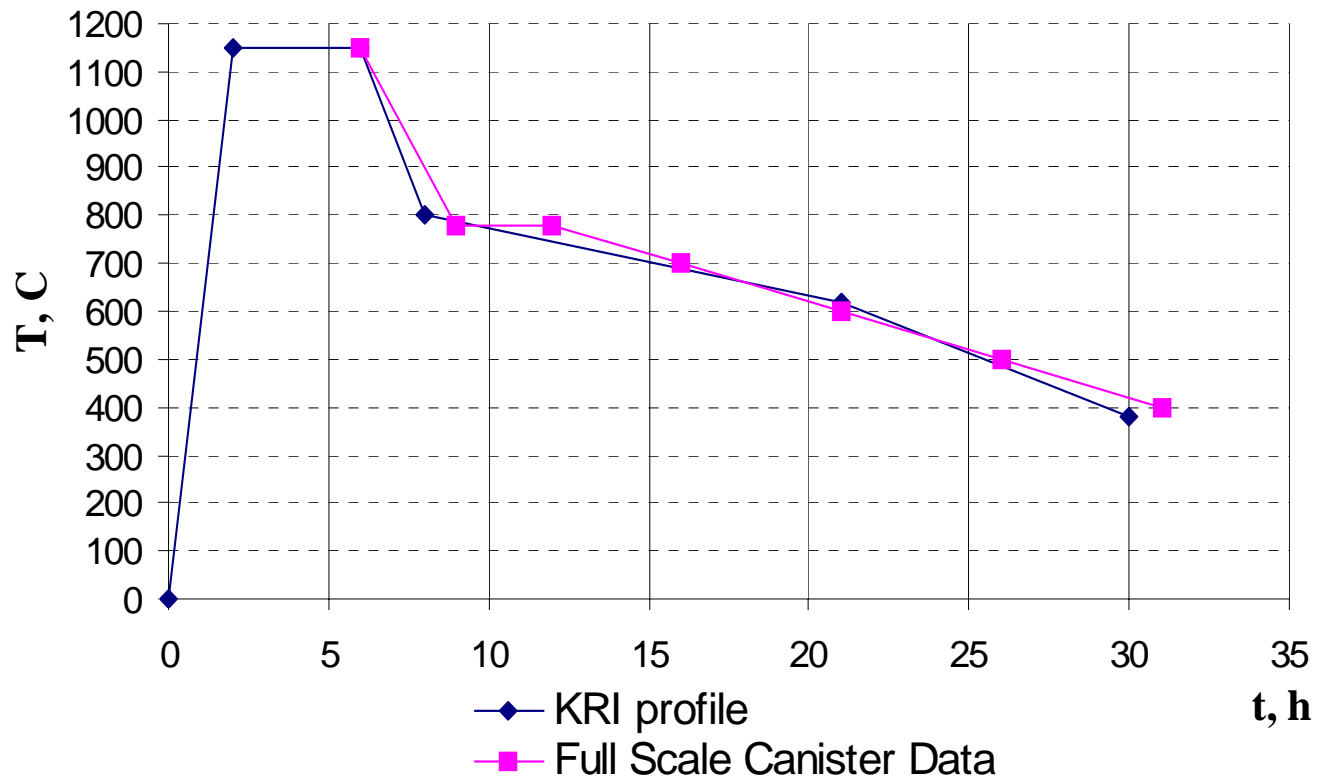

Fig.6. Thermal Heat Treatment Schedule to Simulate CCC Curve

Attachment 4 contains photographs of the glass sample surfaces after the CCC treatment. Table 5 provides visual descriptions of glass samples surfaces after the CCC treatment. Table 6 illustrates phase compositions of the CCC treated glasses, and Table 7 gives data on chemical compositions of the spinels resulting from the CCC treament. Attachment 5 contains microphotogprahs of the same samples. Attachment 6 provides data on the chemical homogeneouty obtained by EPMA. Attachment 7 contains XRD patterns of the CCC treated glasses. 
SRNS-STI-2008-00057

Revision 0

Appendix C (SRNS-OS-2008-00001)

\section{Table 5. Description of CCC Treated Glass Samples}

\begin{tabular}{|c|c|c|}
\hline $\begin{array}{l}\text { Sample } \\
\quad \#\end{array}$ & Surface & Bulk \\
\hline KRI-01 & $\begin{array}{l}\text { Smooth surface, semi-dull gloss, several star-shaped silver } \\
\text { white crystals from } 0.1 \text { to } 0.3 \mathrm{~mm}\end{array}$ & $\begin{array}{l}\text { Glassy shine, conchoidal transparent cross- } \\
\text { section, several star-shaped silver white } \\
\text { crystals from } 0.1 \text { to } 0.3 \mathrm{~mm}\end{array}$ \\
\hline KRI-02 & $\begin{array}{l}\text { Cell-like surface, semi-dull gloss, a few star-shaped silver } \\
\text { white crystals from } 0.1 \text { to } 0.2 \mathrm{~mm} \text { мM (more than in KRI- } \\
01 \text { ) }\end{array}$ & $\begin{array}{l}\text { Glassy shine, non-transparent cross-section, } \\
\text { brown unevenly distributed color }\end{array}$ \\
\hline KRI-03 & $\begin{array}{c}\text { Large cell-like surface, semi-dull gloss, silver white crystals } \\
\text { throughout almost the entire surface }\end{array}$ & $\begin{array}{l}\text { Glassy shine, no crystals, conchoidal } \\
\text { transparent cross-section }\end{array}$ \\
\hline KRI-04 & $\begin{array}{l}\text { Large cell-like surface, semi-dull gloss, silver white crystals } \\
\text { from } 0.1 \text { to } 0.4 \mathrm{~mm} \text { throughout the entire surface, a thin torn } \\
\text { gray film }\end{array}$ & $\begin{array}{l}\text { Glassy shine, non-transparent cross-section, } \\
\text { uneven brown color. No new formations }\end{array}$ \\
\hline KRI-05 & Small cell-like even surface, matte gloss, no new formations & $\begin{array}{l}\text { Dull gloss, non-transparent cross section, no } \\
\text { new formations }\end{array}$ \\
\hline KRI-06 & $\begin{array}{l}\text { Large cell-like surface, semi-dull gloss, silver white crystals } \\
\text { from } 1 \text { to } 2 \mathrm{~mm}\end{array}$ & $\begin{array}{l}\text { Glassy shine, conchoidal transparent cross- } \\
\text { section, no new formations }\end{array}$ \\
\hline KRI-07 & $\begin{array}{c}\text { Large cell-like surface, semi-dull gloss, silver white crystals } \\
\text { throughout almost the entire surface }\end{array}$ & $\begin{array}{l}\text { Conchoidal transparent cross-section, semi- } \\
\text { dull gloss, no new formations }\end{array}$ \\
\hline KRI-08 & $\begin{array}{l}\text { Small cell-like even surface, semi-dull gloss, tiny silver } \\
\text { white crystals }(<0.01 \mathrm{~mm}) \text { throughout the entire surface }\end{array}$ & $\begin{array}{l}\text { Non-transparent cross section, a radial seam on } \\
\text { the cross-section, semi-dull gloss, tiny }(<0,01 \\
\text { mm) crystals }\end{array}$ \\
\hline KRI-09 & $\begin{array}{l}\text { Small cell-like surface, semi-glassy shine, tiny }(<0.01 \mathrm{~mm}) \\
\text { silver white crystals throughout the entire surface }\end{array}$ & $\begin{array}{l}\text { Conchoidal non-transparent cross-section, } \\
\text { semi-dull gloss, tiny }(<0,01 \mathrm{~mm}) \text { crystals }\end{array}$ \\
\hline KRI-10 & $\begin{array}{l}\text { Fibrous surface, glassy shine, few silver white crystals (0.1- } \\
\qquad 0.2 \mathrm{~mm})\end{array}$ & $\begin{array}{c}\text { Conchoidal cross-section with a radial seam. } \\
\text { Tiny }(<0.01 \mathrm{~mm}) \text { silver white crystals on the } \\
\text { cross section radius }\end{array}$ \\
\hline KRI-11 & $\begin{array}{l}\text { Small cell-like even surface, semi-glassy shine, tiny }(<0.01 \\
\text { mm) silver white crystals on the entire surface }\end{array}$ & $\begin{array}{c}\text { Semi-dull gloss, small cell-like non- } \\
\text { transparent cross-section, tiny }(<0.01 \mathrm{~mm}) \\
\text { silver white crystals are distributed throughout } \\
\text { the entire bulk }\end{array}$ \\
\hline KRI-12 & $\begin{array}{l}\text { Large cell-like surface, semi-dull gloss. Silver white crystal } \\
\text { rounded agglomerations up to } 8 \mathrm{~mm} \text { in size are observed on } \\
\qquad 40 \% \text { of the surface }\end{array}$ & $\begin{array}{l}\text { Semi-dull gloss, uneven color of the cross } \\
\text { section (black inclusions in clear brown glass). } \\
\text { Long (up to } 50 \mathrm{~mm} \text { ) needle-like randomly } \\
\text { oriented agglomerations }\end{array}$ \\
\hline KRI-13 & $\begin{array}{l}\text { Small cell-like surface, semi-glassy shine, tiny }(<0.01 \mathrm{~mm}) \\
\text { silver white crystals on the entire surface }\end{array}$ & $\begin{array}{l}\text { Small cell-like surface of the cross-section, } \\
\text { tiny }(<0.01 \mathrm{~mm}) \text { silver white crystals are } \\
\text { distributed throughout the entire bulk }\end{array}$ \\
\hline KRI-14 & $\begin{array}{l}\text { Small cell-like surface, semi-dull gloss, tiny }(<0.01 \mathrm{~mm}) \\
\text { silver white crystals on the entire surface }\end{array}$ & $\begin{array}{c}\text { Semi-dull gloss, small cell-like non- } \\
\text { transparent cross-section, tiny }(<0.01 \mathrm{~mm}) \\
\text { silver white crystals are distributed throughout } \\
\text { the entire bulk }\end{array}$ \\
\hline KRI-15 & $\begin{array}{l}\text { Most surface smooth, glassy shine, a thin gray film near the } \\
\text { edge of the crucible, tiny }(<0.01 \mathrm{~mm}) \text { silver white crystals } \\
\text { on the entire surface }\end{array}$ & $\begin{array}{c}\text { Semi-dull gloss, small cell-like non- } \\
\text { transparent cross-section, tiny }(<0.01 \mathrm{~mm}) \\
\text { silver white crystals are distributed throughout } \\
\text { the entire bulk }\end{array}$ \\
\hline KRI-16 & $\begin{array}{l}\text { Mlarge cell-like surface, uneven color of the glass, black } \\
\text { dented inclusions in the brown glass, tiny crystals in the } \\
\text { dents. } 20 \% \text { of the surface is covered with silver white } \\
\text { crystalline round agglomerates up to } 50 \mathrm{~mm} \text { in size }\end{array}$ & $\begin{array}{l}\text { Glassy shine, conchoidal transparent cross } \\
\text { section, no new formations observed }\end{array}$ \\
\hline KRI-17 & $\begin{array}{l}\text { Uneven surface, dull gloss, practically all surface is covered } \\
\text { with a gray film }\end{array}$ & $\begin{array}{c}\text { Non-transparent small cell-like cross-section, } \\
\text { no crystals observed }\end{array}$ \\
\hline
\end{tabular}


SRNS-STI-2008-00057

Revision 0

Appendix C (SRNS-OS-2008-00001)

\begin{tabular}{|c|c|c|}
\hline KRI-18 & $\begin{array}{l}\text { Large cell-like surface, semi-glassy shine, } 10-15 \% \text { of the } \\
\text { surface is covered with unevenly distributed separate white } \\
\text { rounded crystalline agglomerates }\end{array}$ & $\begin{array}{l}\text { Conchoidal cell-like cross-section, crystals } \\
(<0.01 \mathrm{~mm}) \text { are evenly distributed in the bulk } \\
\text { and located in the cells }\end{array}$ \\
\hline KRI-19 & $\begin{array}{c}\text { Large cell-like surface, glassy shine, tiny crystals are } \\
\text { observed on the bottom of the cells. } 10 \% \text { of the surface is } \\
\text { covered with few silver white crystalline rounded } \\
\text { agglomerates }\end{array}$ & $\begin{array}{l}\text { Glassy shine, conchoidal cell-like cross- } \\
\text { section, Crystals }(<0.01 \mathrm{~mm}) \text { are evenly } \\
\text { distributed in the bulk. }\end{array}$ \\
\hline KRI-20 & $\begin{array}{l}\text { Cell-like surface, semi-dull gloss, tiny }(<0.01 \mathrm{~mm}) \text { silver } \\
\text { white crystals on the surface . }\end{array}$ & $\begin{array}{l}\text { Semi-glassy shine, small cell-like transparent } \\
\text { cross-section, tiny }(<0.01 \mathrm{~mm}) \text { silver white } \\
\text { crystals are distributed in the entire bulk }\end{array}$ \\
\hline KRI-21 & $\begin{array}{c}\text { Large cell-like surface, semi-dull gloss, tiny crystals on the } \\
\text { bottom of the cells, } 40 \% \text { of the surface is covered with few } \\
\text { silver white round crystalline agglomerates ( } 10 \mathrm{~mm} \text { in size), } \\
\text { Uneven color of the glass. Black inclusions on the brown } \\
\text { glass }\end{array}$ & $\begin{array}{l}\text { Semi-glassy shine, small cell-like cross- } \\
\text { section, tiny }(<0.01 \mathrm{~mm}) \text { silver white crystals } \\
\text { are distributed throughout the entire bulk }\end{array}$ \\
\hline KRI-22 & $\begin{array}{l}\text { Large cell-like surface, semi-dull gloss, small crystals on the } \\
\text { bottom of the cells. Dense crystalline agglomerates }(10 \mathrm{~mm} \\
\text { in size) occupy } 50 \% \text { of the surface }\end{array}$ & $\begin{array}{l}\text { Semi-glassy shine, small cell-like transparent } \\
\text { cross-section, tiny }(<0.01 \mathrm{~mm}) \text { silver white } \\
\text { crystals are distributed in the entire bulk }\end{array}$ \\
\hline KRI-23 & $\begin{array}{l}\text { Most of the surface is smooth, glassy shine, a torn thin gray } \\
\text { film near the edge of the crucible. Tiny }(<0.01 \mathrm{~mm}) \text { silver } \\
\text { white crystals are evenly distributed on the surface }\end{array}$ & $\begin{array}{l}\text { Semi-glassy shine, small cell-like transparent } \\
\text { cross-section, tiny }(<0.01 \mathrm{~mm}) \text { silver white } \\
\text { crystals are distributed in the entire bulk }\end{array}$ \\
\hline KRI-24 & $\begin{array}{l}\text { Large cell-like surface, glassy shine, tiny crystals on the } \\
\text { bottom of the cells, } 20 \% \text { of the surface is covered with a few } \\
\text { silver white crystalline round agglomerates }\end{array}$ & $\begin{array}{l}\text { Semi-glassy shine, small cell-like transparent } \\
\text { cross-section, tiny }(<0.01 \mathrm{~mm}) \text { silver white } \\
\text { crystals are distributed in the entire bulk }\end{array}$ \\
\hline KRI-25 & $\begin{array}{l}\text { Small cell-like surface. Glassy shine, crystals of various } \\
\text { sizes on the bottom of the cells }\end{array}$ & $\begin{array}{l}\text { Semi-glassy shine, small cell-like transparent } \\
\text { cross-section, tiny }(<0.01 \mathrm{~mm}) \text { silver white } \\
\text { crystals are distributed in the entire bulk }\end{array}$ \\
\hline KRI-26 & $\begin{array}{c}80 \% \text { of the surface is covered with silver white crystals of } \\
\text { various shapes. Needle-like } 0.01-1 \mathrm{~mm} \text { long round } \\
\text { crystalline agglomerates }\end{array}$ & $\begin{array}{c}\text { Semi-glassy shine, small cell-like transparent } \\
\text { and uneven cross-section, tiny }(<0.01 \mathrm{~mm}) \\
\text { silver white crystals are distributed in the entire } \\
\text { bulk }\end{array}$ \\
\hline KRI-27 & $\begin{array}{l}\text { Glassy shine, silver white unevenly distributed crystals (0.01 } \\
\qquad-0.5 \mathrm{~mm})\end{array}$ & $\begin{array}{l}\text { Semi-glassy shine, small cell-like transparent } \\
\text { cross-section, tiny }(<0.01 \mathrm{~mm}) \text { silver white } \\
\text { crystals are distributed in the entire bulk }\end{array}$ \\
\hline KRI-28 & $\begin{array}{l}\text { Homogenous dense silver-colored film covers the surface } \\
\text { from the edge to the center of the crucible. There is an } \\
\text { irregularly shaped area }(0.8-0.5 \mathrm{~cm}) \text { in the center covered } \\
\text { with a less dense film. Greenish crystals are observed on the } \\
\text { border of the area. Caverns are observed in the area, with } \\
\text { greenish crystals on the edges. }\end{array}$ & Dull gloss, uneven color \\
\hline KRI-29 & $\begin{array}{l}\text { Uneven surface completely covered with a loose murky } \\
\text { film. }\end{array}$ & $\begin{array}{l}\text { Dull gloss, small }(0.01 \mathrm{~mm}) \text { and large }(10 \mathrm{~mm}) \\
\text { tan inclusions are observed. }\end{array}$ \\
\hline KRI-30 & $\begin{array}{l}\text { Large cell-like surface, with the cells evenly distributed on } \\
\text { the surface. There is a three-pointed star-shaped crystal ( } 0.1 \\
-0.5 \mathrm{~mm}) \text { on the bottom of each cell. Dark gray and brown } \\
\text { spots are observed in the bulk in the center of the crucible }\end{array}$ & Glassy shine, uneven color (brown and black) \\
\hline
\end{tabular}


Table 6. Phase Compositions of CCC Treated KRI Matrix Glasses

\begin{tabular}{|c|c|c|c|c|}
\hline Sample \# & $\begin{array}{c}\text { Crystalline phase, } \\
\text { XRD }\end{array}$ & $\begin{array}{c}\text { Content, mass \%, } \\
\text { XRD } \\
\end{array}$ & $\begin{array}{c}\text { Content, vol. .\%, } \\
\text { SEM }\end{array}$ & Size, $\mu \mathrm{m}$ \\
\hline KRI-01 & -- & $<1$ & 0.3 & \\
\hline KRI-02 & spinel & 1 & 0.8 & \\
\hline KRI-03 & -- & $<1$ & 0.2 & \\
\hline KRI-04 & -- & $<1$ & 0.3 & \\
\hline KRI-05 & spinel & 8 & 7.3 & 8.8 \\
\hline KRI-06 & spinel & 1.5 & 1.2 & \\
\hline KRI-07 & spinel & $<1$ & 0.2 & \\
\hline KRI-08 & spinel & 15 & 15 & 15 \\
\hline KRI-09 & spinel & 4 & 3.1 & 5.1 \\
\hline KRI-10 & spinel & $<1$ & 0.94 & 4.1 \\
\hline KRI-11 & spinel & 6 & 5.3 & 3.7 \\
\hline KRI-12 & spinel & 3 & 2.5 & 1.0 \\
\hline KRI-13 & spinel & 3.5 & 2.8 & 11.3 \\
\hline KRI-14 & spinel & 4.5 & 3.8 & 12.3 \\
\hline KRI-15 & spinel & 5 & 4.3 & 9.5 \\
\hline KRI-16 & spinel & 1 & 0.6 & 3.3 \\
\hline KRI-17 & spinel & 3 & 2.0 & 3.2 \\
\hline KRI-18 & spinel & 3.5 & 3.6 & 10.2 \\
\hline KRI-19 & spinel & 3 & 2.3 & 32.5 \\
\hline KRI-20 & spinel & 3 & 2.6 & 17.7 \\
\hline KRI-21 & spinel & 3.5 & 2.5 & 14.0 \\
\hline KRI-22 & spinel & 3 & 2.8 & 4.7 \\
\hline KRI-23 & spinel & 3 & 2.6 & 11.1 \\
\hline KRI-24 & spinel & 4 & 3.5 & 5.0 \\
\hline KRI-25 & spinel & 2 & 2.2 & 25.0 \\
\hline KRI-26 & spinel & 6 & 4.5 & 16.5 \\
\hline KRI-27 & spinel & 2 & 1.9 & 27.0 \\
\hline KRI-28 & $\begin{array}{c}\text { nepheline } \mathrm{NaAlSiO}_{4}(\# 19-1176) \\
\mathrm{Li}_{2} \mathrm{SiO}_{3}(\# 29-828) \\
\mathrm{Na}_{8} \mathrm{Al}_{6} \mathrm{Si}_{6} \mathrm{O}_{24} \mathrm{SO}_{4}(\# 21-1099) \\
\text { spinel }\end{array}$ & $\begin{array}{c}40 \\
33 \\
24 \\
3\end{array}$ & & \\
\hline KRI-29 & nepheline $\mathrm{NaAlSiO}_{4}$ & 10 & 2.0 & 10.2 \\
\hline KRI-30 & spinel & 3 & 1.4 & 10.9 \\
\hline
\end{tabular}


SRNS-STI-2008-00057

Revision 0

Appendix C (SRNS-OS-2008-00001)

Table 7. Chemical Compositions of Spinels (mass \%) in CCC Treated KRI Matrix Glasses (EPMA Data)

\begin{tabular}{|c|c|c|c|c|c|c|c|c|c|}
\hline Sample & TiO2 & $\mathrm{Al} 2 \mathrm{O} 3$ & $\mathrm{FeO}$ & $\mathrm{Fe} 2 \mathrm{O} 3$ & Cr2O3 & $\mathrm{NiO}$ & $\mathrm{ZnO}$ & $\mathrm{MnO}$ & Total \\
\hline KRI-11 SP & nd & 2.08 & 8.58 & 44.36 & 19.44 & 16.67 & 3.24 & 3.10 & 97.48 \\
\hline $2 \mathrm{SP}$ & nd & nd & 7.84 & 51.34 & 14.47 & 16.29 & 2.99 & 3.75 & 96.67 \\
\hline KRI-12 SP & nd & nd & 0.00 & 50.47 & 15.20 & 21.25 & 1.87 & 7.88 & 96.67 \\
\hline $2 \mathrm{SP}$ & nd & nd & 0.00 & 58.62 & 6.43 & 22.78 & 1.49 & 7.24 & 96.56 \\
\hline KRI-13 SP & nd & nd & 0.00 & 39.32 & 23.68 & 15.02 & 7.34 & 9.69 & 95.05 \\
\hline $2 \mathrm{SP}$ & 0.67 & nd & 0.00 & 38.03 & 24.27 & 14.38 & 7.84 & 9.82 & 95.01 \\
\hline KRI-14 SP & 0.50 & 2.08 & 0.66 & 41.44 & 21.20 & 19.34 & 5.48 & 6.85 & 97.55 \\
\hline $2 \mathrm{SP}$ & nd & 2.46 & 1.84 & 37.99 & 25.00 & 16.93 & 6.47 & 6.72 & 97.40 \\
\hline KRI-16 SP & 3.17 & 6.43 & 0.00 & 52.18 & 1.02 & 24.94 & 5.85 & 5.43 & 99.03 \\
\hline $2 \mathrm{SP}$ & 1.67 & nd & 0.00 & 58.19 & 3.36 & 28.00 & 3.61 & 2.97 & 97.80 \\
\hline KRI-17 SP & 0.67 & 1.89 & 53.80 & 0.00 & 1.46 & 25.45 & 1.99 & 7.11 & 92.37 \\
\hline $2 \mathrm{SP}$ & nd & 2.84 & 2.19 & 51.18 & 12.13 & 22.78 & 1.62 & 5.17 & 97.91 \\
\hline KRI-18 SP & nd & 2.08 & 0.00 & 55.90 & 6.29 & 23.42 & 1.37 & 8.27 & 97.32 \\
\hline $2 \mathrm{SP}$ & 0.50 & 1.89 & 0.00 & 53.19 & 7.89 & 23.29 & 1.87 & 8.40 & 97.03 \\
\hline KRI-19 SP & 0.50 & 1.70 & 0.00 & 53.90 & 9.80 & 23.16 & 1.74 & 7.49 & 98.30 \\
\hline $2 \mathrm{SP}$ & 0.67 & 1.89 & 0.00 & 57.62 & 3.22 & 24.56 & 1.37 & 8.40 & 97.72 \\
\hline $3 \mathrm{SP}$ & 1.17 & nd & 0.00 & 57.76 & 3.51 & 23.67 & 1.62 & 8.79 & 96.51 \\
\hline KRI-20 SP & nd & 2.08 & 3.71 & 43.20 & 20.91 & 22.40 & 2.86 & 2.97 & 98.13 \\
\hline $2 \mathrm{SP}$ & nd & nd & 3.39 & 42.13 & 23.83 & 22.14 & 2.86 & 2.97 & 97.33 \\
\hline KRI-21 SP & nd & nd & 1.15 & 49.33 & 16.96 & 24.94 & 1.74 & 3.49 & 97.62 \\
\hline $2 \mathrm{SP}$ & nd & nd & 0.28 & 59.59 & 7.02 & 27.11 & 0.87 & 2.97 & 97.84 \\
\hline $3 \mathrm{SP}$ & nd & nd & 1.25 & 51.65 & 14.62 & 25.07 & 1.87 & 3.10 & 97.56 \\
\hline KRI-22 SP & 0.83 & nd & 0.00 & 59.91 & 4.97 & 28.25 & 1.74 & 2.45 & 98.16 \\
\hline $2 \mathrm{SP}$ & 2.50 & 2.08 & 0.00 & 56.19 & 1.46 & 31.56 & 1.37 & 3.10 & 98.26 \\
\hline KRI-23 SP & nd & 3.02 & 1.88 & 47.66 & 15.50 & 22.78 & 3.61 & 3.88 & 98.33 \\
\hline $2 \mathrm{SP}$ & nd & 3.21 & 3.24 & 48.16 & 14.62 & 21.76 & 3.11 & 3.88 & 97.98 \\
\hline KRI-24 SP & 0.67 & nd & 0.00 & 51.90 & 11.26 & 23.03 & 4.23 & 5.68 & 96.77 \\
\hline $2 \mathrm{SP}$ & 0.83 & 1.51 & 0.00 & 57.05 & 4.39 & 25.32 & 2.99 & 5.68 & 97.78 \\
\hline KRI-25 SP & nd & 1.70 & 5.33 & 47.69 & 17.40 & 21.00 & 2.37 & 3.23 & 98.71 \\
\hline $2 \mathrm{SP}$ & nd & nd & 3.26 & 57.14 & 8.63 & 22.78 & 2.12 & 2.71 & 96.63 \\
\hline
\end{tabular}


SRNS-STI-2008-00057

Revision 0

Appendix C (SRNS-OS-2008-00001)

\section{Liquidus Temperature $\left(T_{L}\right)$ Measurements for KRI Matrix}

\subsection{Muffle Furnace Calibrations and $T_{L}$ Measurements for SRM-773 Reference Standard}

We used furnace UMEGA SNOL 7.2/1300 for liquidus $\left(T_{L}\right)$ temperature measurements of the glass samples. This furnace was fabricated in December, 2006, and it meets the low-voltage (73/23/EEC) and electromagnetic compatibility (89/336/EEC) requirements of the Directives of the European Union. The dimensions of the working chamber are 284 × $208 \times 112 \mathrm{~mm}$. The furnace has five heating surfaces. Prior to $T_{L}$ measurements, we calibrated the furnace consistence between the required heating temperature $\left(t_{1}\right)$ and the actual temperature in the heat area $\left(\mathrm{t}_{0}\right)$.

For verification that the actual temperature in the work zone of the heat area of the furnace $\left(\mathrm{t}_{0}\right)$ matched the required heating temperature $\left(\mathrm{t}_{1}\right)$ (the temperature setting of the furnace controller), the certified reference standard thermocouple was placed inside the furnace along the axis of the heat area, with the hot junction point of the thermocouple being placed down from the axis (FIg. 7). The differences in the furnace temperature readings and the temperature values measured by the reference standard thermocouple $\left(\mathrm{t}_{0}\right)$ were recorded. In the temperature range of 850$1,200^{\circ} \mathrm{C}$, the $\mathrm{t}_{0} \mathrm{t}_{1}$ value remained constant and was equal to $8{ }^{\circ} \mathrm{C}$. Upon reaching the required temperature, the holding time was approximately 10 hours.

To reduce the temperature gradient in the furnace, we used a Pt boat with the volume of approximately $~ 300 \mathrm{~mL}$ with a tightly fitting lid. The temperature gradient in the Pt boat was measured using temperature rings. For the temperatures of $850^{\circ} \mathrm{C}$ and $1050^{\circ} \mathrm{C}$, we used rings PTCR-ETH manufactured by KEMA, dmc ${ }^{2}$, For the temperature of $1150^{\circ} \mathrm{C}$, we used rings PTCR-STH manufactured by KEMA, Ferro received from Dr. Alexander Fluegel, PNNL. Upon completion of the experiments, we measured the diameters of the rings and determined the temperature difference. The temperature field homogeneity inside the Pt boat was $\pm 2^{\circ} \mathrm{C}$, which was confirmed for different temperatures by the temperature rings placed into the Pt boat. Up to 8 Pt alloy crucibles with studied glass samples can be placed into the Pt boat.

The next phase of the furnace calibration was to determine $T_{L}$ for the SRM773 glass reference standard. Fig. 7 shows Pt crucibles with lids containing the previously prepared standard samples placed into the Pt boat.

To prepare the standard samples, they were crushed, and a piece of the sample of approximately 3 grams with the particle size of $0.425-4.0 \mathrm{~mm}$ was taken for the measurements. This $3 \mathrm{~g}$ piece was triple washed with ethanol in the ultrasound bath and then dried. After drying, the glass was placed into the Pt crucible, and then into the Pt boat with a lid where the glass was held for 24 hours at the required temperature. 


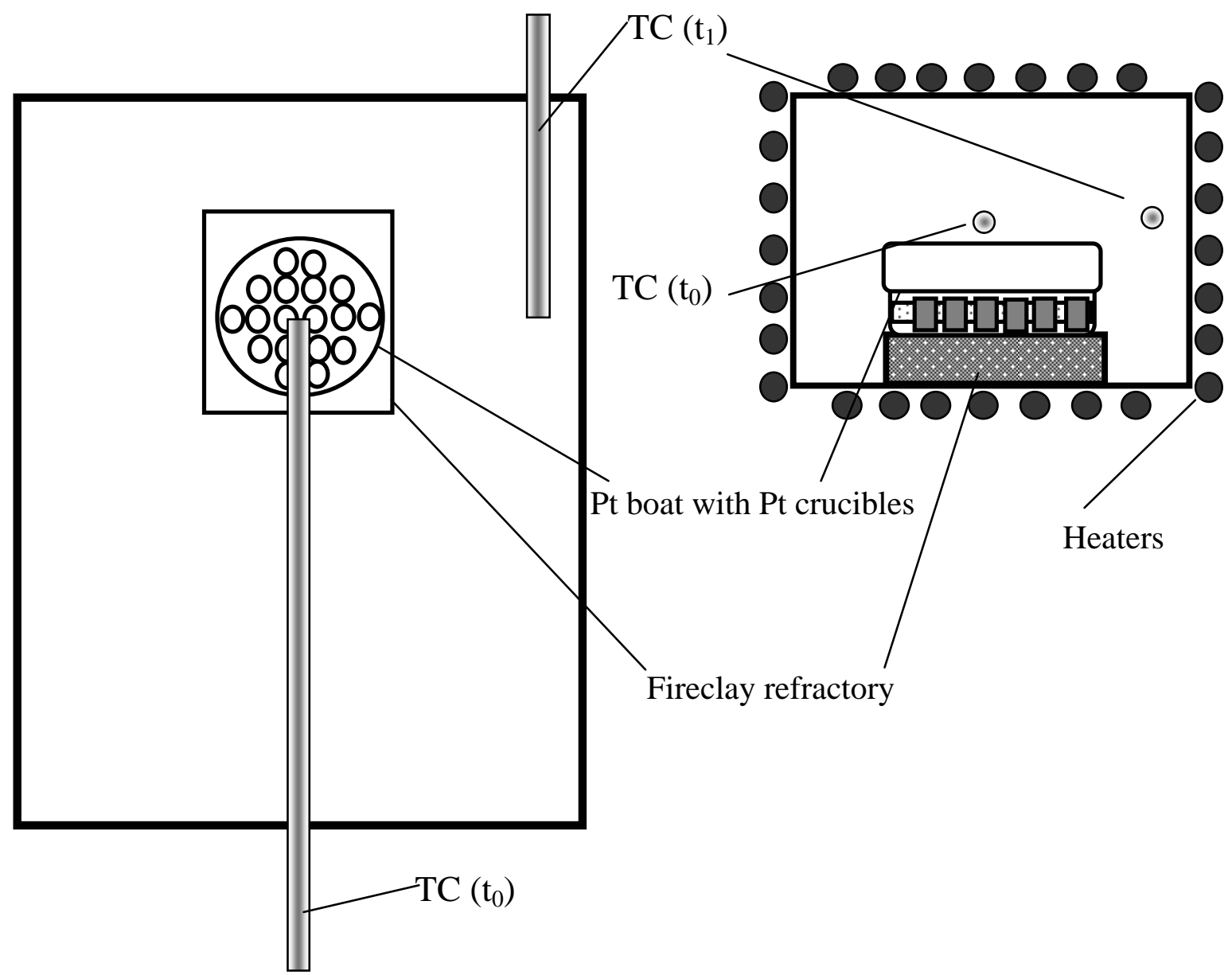

Fig. 7. Liquidus Temperature Measurements Using Pt Boat

\begin{tabular}{|c|c|}
\hline & \begin{tabular}{c} 
Pt crucible \\
$\mathrm{D}=23 \mathrm{~mm}$ \\
$\mathrm{H}=8 \mathrm{~mm}$ \\
Glass mass $=3$ grams \\
\hline
\end{tabular} \\
\hline
\end{tabular}

After holding for 24 hours followed by quenching, the glass samples were analyzed to determine the crystalline phase volume using SEM. To measure $\mathrm{T}_{\mathrm{L}}$, the SRM773 standard samples were held at the following temperatures: $1008^{\circ} \mathrm{C}, 989^{\circ} \mathrm{C}$, and $981^{\circ} \mathrm{C}$. 


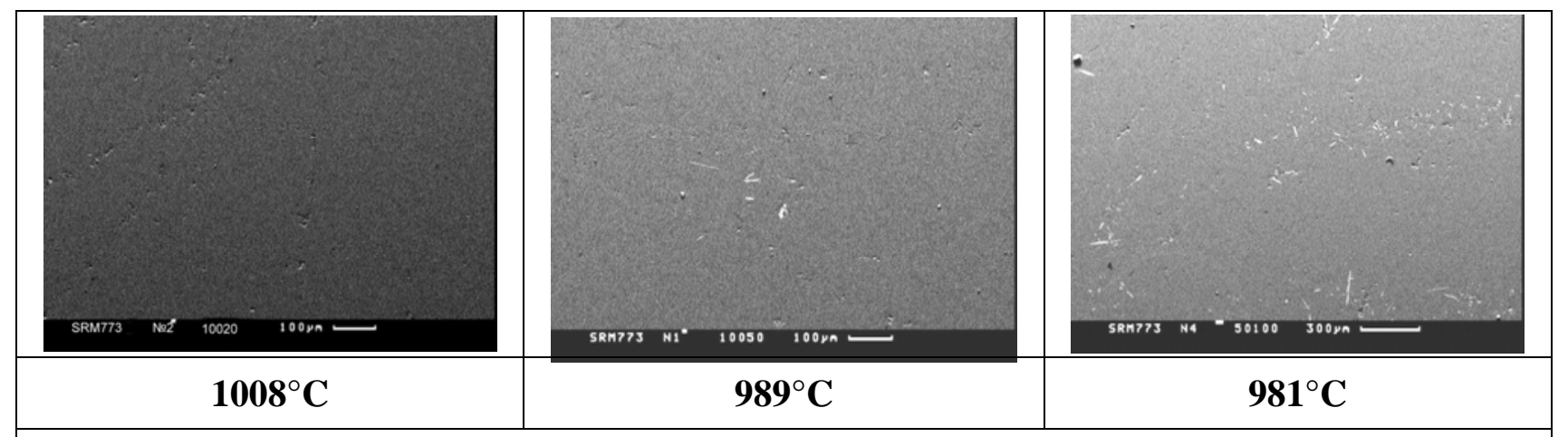

Fig. 9 Microphotos of SRM773 Standard Glasses Held at Various Temperatures

The SEM and XRD data showed that the SRM773 glass sample held at $1008^{\circ} \mathrm{C}$ had no inclusions and the glass was X-ray amorphous. According to the SEM, the SRM773 glass sample held at $989^{\circ} \mathrm{C}$ contained less than 0.1 vol.\% of wollastonite. According to the SEM and XRD data, the SRM773 glass sample held at $981^{\circ} \mathrm{C}$ contained the crystalline phase of wollastonite, $\mathrm{CaSiO}_{3}$. The average content of wollastonite was 0.7 vol.\%.

Therefore, according to our experimental results, the liquidus temperature for the SRM773 standard glass was $989 \pm 2^{\circ} \mathrm{C}$.

\section{$5.2 \mathrm{~T}_{\mathrm{L}}$ Measurements for KRI Matrix Glasses}

Table 8 provides SEM data on quantities of the primary crystalline phase (vol. \%) at various heat treatment temperatures for KRI matrix glasses. The obtained data were used to establish the occurrence temperature interval and minimum temperature interval for $\mathrm{T}_{\mathrm{L}}$ measurements. The minimum temperature interval (occurrence interval) was determined as the difference between two temperatures: the first is the temperature at which the glass has a crystalline phase, and the second is the temperature at which the glass is amorphous. Then the temperature occurrence interval was narrowed. For this purpose, the volume percentage of the crystalline phase formed within 24 hours of heat treatment at a certain temperature was measured. The analysis of quantities and types of crystalline particles in the glass at the minimal volume percentage of the generated crystalline phase made it possible to narrow the temperature interval to $10^{\circ} \mathrm{C}$. (the minimum temperature interval). The liquidus temperature is defined as the temperature at which 1-3 crystalline particles are formed in the glass sample volume at the minimal volume percentage of the primary crystalline phase (less than 0.1 vol. \%). The liquidus temperatures are given in Table 9.

The data show that for KRI-01, KRI-02, KRI-03, KRI-06, KRI-07, KRI-28,, and KRI-30, the $\mathrm{T}_{\mathrm{L}}$ values are in the range of $958-1100^{\circ} \mathrm{C}$. Samples KRI-05, KRI-08, KRI-09, KRI-10, KRI-13, $\mathrm{KRI}-15$, and $\mathrm{KRI}-23$ have high $\mathrm{T}_{\mathrm{L}}$ values in the range of $1180-1270^{\circ} \mathrm{C}$. For the remaining compositions, $\mathrm{T}_{\mathrm{L}}$ values exceeded the melting temperature $\left(1150^{\circ} \mathrm{C}\right)$ by $10-20$ degrees $\mathrm{C}$. 
SRNS-STI-2008-00057

Revision 0

Appendix C (SRNS-OS-2008-00001)

Table 8. Primary Crystalline Phase in KRI Matrix Glasses after CCC Treatment

\begin{tabular}{|c|c|c|c|c|c|c|c|c|c|c|c|}
\hline & \multicolumn{11}{|c|}{ Spinel content, vol. \%, SEM } \\
\hline & $1258^{0} \mathrm{C}$ & $1208^{0} \mathrm{C}$ & $1183^{0} \mathrm{C}$ & $1158^{0} \mathrm{C}$ & $1133^{0} \mathrm{C}$ & $1108^{0} \mathrm{C}$ & $1083^{0} \mathrm{C}$ & $1058^{0} \mathrm{C}$ & $1033^{0} \mathrm{C}$ & $1008^{0} \mathrm{C}$ & $958^{0} \mathrm{C}$ \\
\hline KRI-01 & - & - & - & - & - & 0.0 & - & 0.0 & 0.0 & 0.04 & 0.23 \\
\hline KRI-02 & - & - & - & - & - & 0.0 & 0.0 & 0.07 & 0.69 & - & - \\
\hline KRI-03 & - & - & - & - & - & 0.0 & 0.0 & 0.03 & 0.37 & - & - \\
\hline KRI-04 & - & - & - & 0.0 & 0.0 & 0.13 & 0.58 & 0.66 & - & - & - \\
\hline KRI-05 & 0.17 & 0.24 & - & 0.31 & - & - & - & - & - & - & - \\
\hline KRI-06 & - & - & - & 0.0 & - & 0.0 & 0.14 & 0.73 & 1.12 & - & - \\
\hline KRI-07 & - & - & - & 0.0 & - & 0.0 & 0.0 & 0.08 & 0.24 & - & - \\
\hline KRI-08 & - & 0.0 & 0.0 & 0.28 & - & - & - & - & 1.12 & - & - \\
\hline KRI-09 & - & 0.0 & 0.15 & 0.70 & - & - & - & - & - & - & - \\
\hline KRI-10 & 0.0 & 1.12 & - & 1.25 & - & 1.50 & 1.61 & - & - & - & - \\
\hline KRI-11 & - & 0.0 & 0.0 & 0.06 & - & 0.65 & - & 0.68 & - & - & - \\
\hline KRI-12 & - & 0.0 & 0.0 & 0.13 & - & - & - & - & 1.95 & - & - \\
\hline KRI-13 & - & 0.0 & 0.04 & 0.42 & - & - & - & 1.55 & - & - & - \\
\hline KRI-14 & - & 0.0 & 0.0 & 0.38 & - & - & - & - & 1.42 & - & - \\
\hline KRI-15 & - & 0.0 & 0.10 & 0.52 & - & 2.1 & - & - & - & - & - \\
\hline KRI-16 & - & 0.0 & 0.0 & 0.16 & - & 0.55 & - & 0.77 & - & - & - \\
\hline KRI-17 & - & 0.0 & 0.0 & 0.02 & - & 0.80 & - & - & - & - & - \\
\hline KRI-18 & - & 0.0 & 0.0 & 0.09 & - & - & - & - & 1.52 & - & - \\
\hline KRI-19 & - & 0.0 & 0.0 & 0.32 & - & 1.45 & - & - & - & - & - \\
\hline KRI-20 & - & 0.0 & 0.0 & 0.13 & - & 1.05 & - & - & - & - & - \\
\hline KRI-21 & - & 0.0 & 0.0 & 0.17 & - & 0.80 & - & - & - & - & - \\
\hline KRI-22 & - & 0.0 & - & 0.0 & 0.05 & 0.95 & - & - & - & - & - \\
\hline KRI-23 & - & 0.0 & 0.0 & 0.50 & - & 0.65 & - & - & - & - & - \\
\hline KRI-24 & - & - & 0.0 & 0.07 & - & 0.75 & - & - & - & - & - \\
\hline KRI-25 & - & 0.0 & 0.0 & 0.20 & - & 0.80 & - & - & - & - & - \\
\hline KRI-26 & - & 0.0 & - & 0 & 0.0 & 0.50 & - & - & - & - & - \\
\hline KRI-27 & - & 0.0 & 0.0 & 0.07 & - & 0.8 & - & - & 1.16 & - & - \\
\hline KRI-28 & - & - & - & 0.0 & - & 0.0 & - & 0.0 & 1.1 & - & - \\
\hline KRI-29 & - & - & - & - & 0.0 & 0.05 & 0.08 & 0.21 & 0.23 & - & - \\
\hline KRI-30 & - & - & - & - & - & 0.0 & - & 0.0 & - & 0.0 & 0.03 \\
\hline
\end{tabular}


Table 9. Liquidus Temperatures Determined by SEM

\begin{tabular}{|c|c|c|c|}
\hline \multirow{2}{*}{ Glass } & \multicolumn{3}{|c|}{ Temperatures, ${ }^{\mathbf{0}} \mathbf{C}$} \\
\cline { 2 - 4 } & Occurrence interval & Minimum temperature interval & $\mathbf{T}_{\mathbf{L}}$ \\
\hline KRI-01 & $1008-1033$ & 1008 & 1008 \\
\hline KRI-02 & $1058-1083$ & $1058-1068$ & 1063 \\
\hline KRI-03 & $1058-1083$ & 1058 & 1058 \\
\hline KRI-04 & $1108-1133$ & $1108-1118$ & 1113 \\
\hline KRI-05 & $1258-1283$ & $1258-1268$ & 1263 \\
\hline KRI-06 & $1083-1108$ & $1093-1098$ & 1095 \\
\hline KRI-07 & $1058-1083$ & $1068-1073$ & 1071 \\
\hline KRI-08 & $1158-1183$ & $1173-1183$ & $1173-1183$ \\
\hline KRI-09 & $1183-1208$ & $1183-1193$ & $1183-1193$ \\
\hline KRI-10 & $1208-1258$ & $1248-1258$ & $1248-1258$ \\
\hline KRI-11 & $1158-1183$ & $1158-1168$ & $1158-1168$ \\
\hline KRI-12 & $1158-1183$ & $1168-1173$ & $1168-1173$ \\
\hline KRI-13 & $1158-1208$ & 1183 & 1183 \\
\hline KRI-14 & $1158-1183$ & $1173-1183$ & $1173-1183$ \\
\hline KRI-15 & $1183-1208$ & $1183-1193$ & $1183-1193$ \\
\hline KRI-16 & $1158-1183$ & $1158-1163$ & $1158-1163$ \\
\hline KRI-17 & $1158-1183$ & 1158 & 1158 \\
\hline KRI-18 & $1158-1183$ & $1168-1178$ & $1168-1178$ \\
\hline KRI-19 & $1158-1183$ & $1173-1183$ & $1173-1183$ \\
\hline KRI-20 & $1158-1183$ & $1158-1168$ & $1158-1168$ \\
\hline KRI-21 & $1158-1183$ & $1168-1173$ & $1168-1173$ \\
\hline KRI-22 & $1133-1158$ & 1133 & 1133 \\
\hline KRI-23 & $1158-1183$ & $1168-1173$ & $1168-1173$ \\
\hline KRI-24 & $1158-1183$ & $1158-1168$ & $1158-1168$ \\
\hline KRI-25 & $1158-1183$ & $1168-1178$ & $1168-1178$ \\
\hline KRI-26 & $1108-1133$ & $1118-1123$ & $1118-1123$ \\
\hline KRI-27 & $1158-1183$ & $1158-1168$ & $1158-1168$ \\
\hline KRI-28 & $1033-1058$ & $1043-1048$ & $1043-1048$ \\
\hline KRI-29 & $1108-1133$ & 1108 & 1108 \\
\hline KRI-30 & $958-1108$ & 958 & 958 \\
\hline
\end{tabular}

The data in Table 9 show that the increase of $\mathrm{Cr}_{2} \mathrm{O}_{3}, \mathrm{NiO}, \mathrm{Al}_{2} \mathrm{O}_{3}$, and $\mathrm{Fe}_{2} \mathrm{O}_{3}$ content in the KRI matrix glasses leads to increase of the liquidus temperatures. The increase of $\mathrm{Na}_{2} \mathrm{O}$ and $\mathrm{Li}_{2} \mathrm{O}$ concentrations reduce the liquidus temperature [2]. Boron and silicon oxids do not significantly affect $\mathrm{T}_{\mathrm{L}}$. For KRI-05, KRI-08, KRI-09, KRI-10, KRI-13, KRI-15, and KRI-23, the $\mathrm{T}_{\mathrm{L}}$ values are significantly higher than the recommended temperature for synthesis, i.e., $1150^{\circ} \mathrm{C}$, which makes it impossible to use these glasses on a larger scale. KRI-11, KRI-12, KRI-16, KRI-17, KRI-18, KRI-20, KRI-24, and KRI-26 have crystalline inclusions over 1 vol. \% at the synthesis temperature. The liquidus temperatures for KRI-01, KRI-02, KRI-03, KRI-06, KRI-07, and KRI30 are $50-100^{\circ} \mathrm{C}$ lower than the glass melting temperature. These compositions can be considered promising for subsequent testing at SMK and EP-5 systems. 


\section{PCT Evaluations of Quenched and CCC Treated KRI Matrix Glasses}

We used PCT-A procedure for leaching KRI matrix glasses. A sample of each glass was crushed in a titanium mill (Fig. 10) and sieved the powders through Fritsch sieves (Fig.11). One gram of the appropriate glass (0.074-0.149 mm fraction sluiced in water and alcohol and then purified by ultrasound (Fig.12) and dried) was placed into a stainless steel or polytetrafluoroethylene vessel (Figs. 13 and 14) where 10 grams of deionized water were added. We used apyrogenic deeply demineralized water (brand name "Ultraaqua") with the resistance of $22.7 \mathrm{M} \Omega / \mathrm{cm}$.

Each series of testing included three samples of each KRI glass, three samples of the ARM standard glass and one blank sample. The airtight vessels were held for 7 days at $90^{\circ} \mathrm{C} .0 .445 \mu \mathrm{m}$ membrane filters were used for sampling leachates to measure elements concentrations using ICP-AE.

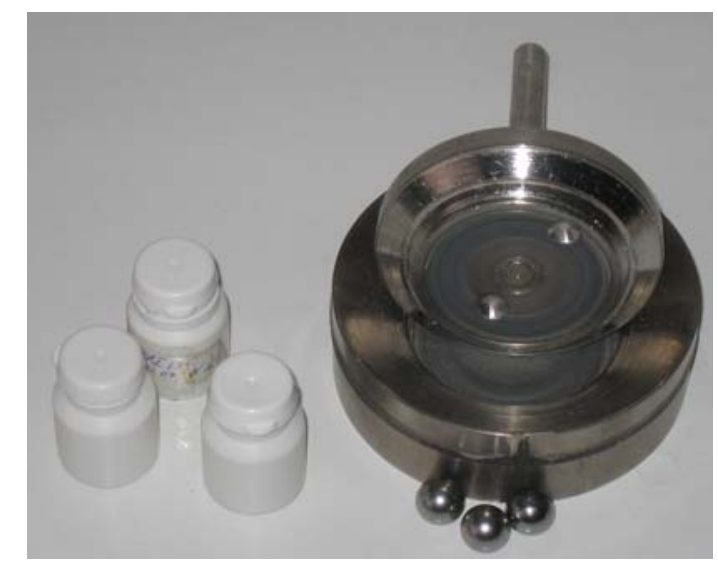

Fig. 10. Mill for Glass Crushing

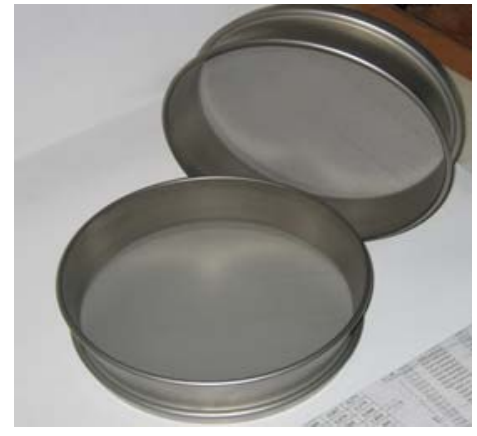

Fig. 11 Set of Sieves FRITSCH TEST SIEVE ASTM E 11

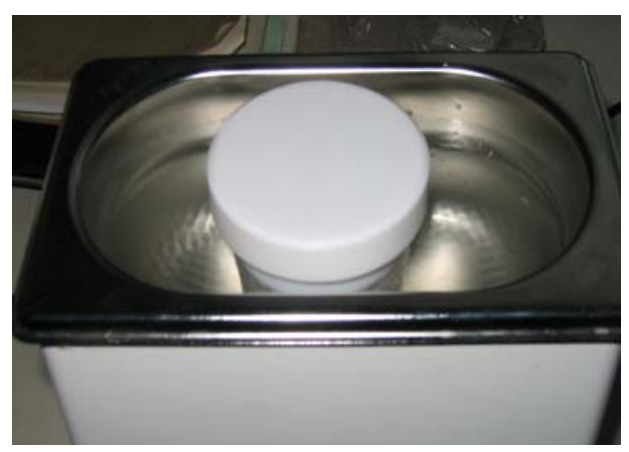

Fig.12 Ultrasound Bath UZV7-0.063/37 


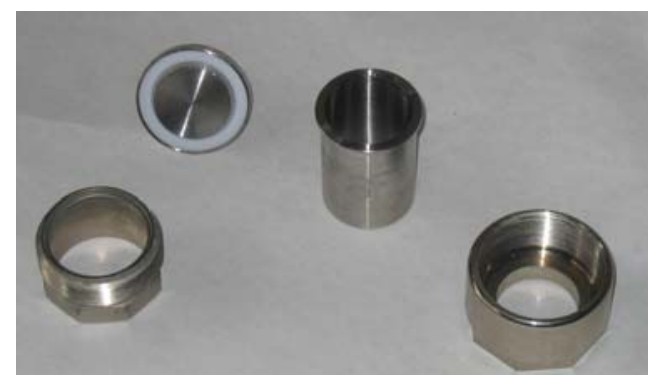

Fig.13. Stainless steel vessel, disassembled

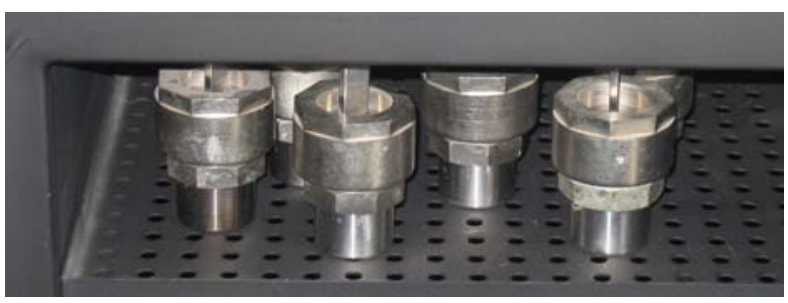

Fig. 14. Vessels in Oven

Table 10 provides data on tuning ICP-AE spectrometer using multi-element ICP standard (manufactured in the U.S.). Tables 11 and 12 provide elements concentrations in the blank samples and ARM glass standard leachates.

Table 10. Tuning ICP-AE Spectrometer Using ICP Standard

\begin{tabular}{|c|c|c|c|c|}
\hline \multirow{2}{*}{ Series } & \multicolumn{4}{|c|}{$\mathrm{mg} / \mathrm{L}$} \\
\cline { 2 - 5 } & $\mathrm{B} \mathrm{(20)}$ & $\mathrm{Li} \mathrm{(10)}$ & $\mathrm{Na}(81)$ & $\mathrm{Si}(50)$ \\
\hline \multirow{3}{*}{1} & 20 & 10 & 81 & 50 \\
\cline { 2 - 5 } & 20 & 10 & 81 & 50 \\
\cline { 2 - 5 } & 20 & 10 & 81 & 50 \\
\hline \multirow{2}{*}{2} & 19.9 & 10.1 & 80.9 & 49.7 \\
\cline { 2 - 5 } & 19.3 & 10.1 & 79.6 & 48.5 \\
\hline \multirow{2}{*}{3} & 19.5 & 10.0 & 80 & 49.5 \\
\cline { 2 - 5 } & 19 & 10.0 & 79.4 & 49.0 \\
\hline \multirow{2}{*}{4} & 19 & 10 & 84 & 47 \\
\hline \multirow{2}{*}{5} & 19 & 9.9 & 77 & 50 \\
\cline { 2 - 5 } & 18 & 9.8 & 75 & 48 \\
\hline \multirow{2}{*}{6} & 20 & 9.9 & 82 & 49 \\
\cline { 2 - 5 } & 20 & 9.7 & 82 & 48 \\
\hline
\end{tabular}

Table 11. ARM Standard Leaching

\begin{tabular}{|c|c|c|c|c|c|c|}
\hline \multirow{4}{*}{ Series } & \multirow{4}{*}{$\begin{array}{c}\text { Vessel } \\
\text { material }\end{array}$} & \multirow{4}{*}{$\begin{array}{c}\text { PH } \\
\text { of leachates }\end{array}$} & \multicolumn{4}{|c|}{ Elements concentrations in leachates (average values), mg/L } \\
\hline & & & $\mathrm{B}$ & $\mathrm{Li}$ & $\mathrm{Na}$ & $\mathrm{Si}$ \\
\hline & & & \multicolumn{4}{|c|}{ Reference values, mg/L } \\
\hline & & & 10.80- 16.32 & $\begin{array}{l}12.87- \\
22.65\end{array}$ & 28.86- 43.58 & $49.03-74.43$ \\
\hline 1 & Teflon & 9.61 & 15 & 12 & 34 & 52 \\
\hline 2 & steel & 10.14 & 16 & 12.8 & 34.5 & 56.2 \\
\hline 3 & Teflon & 9.59 & 14 & 13 & 32 & 51 \\
\hline 4 & Teflon & 9.55 & 13 & 13 & 33 & 49.6 \\
\hline 5 & Teflon & 9.96 & 16 & 14 & 35 & 51 \\
\hline 6 & steel & 10.33 & 16 & 13 & 34 & 53 \\
\hline
\end{tabular}


Table 12. Elements Concentrations in Blank Samples

\begin{tabular}{|c|c|c|c|c|c|}
\hline \multirow{2}{*}{ Series } & \multirow{2}{*}{$\begin{array}{l}\mathrm{pH} \text { upon } \\
\text { completion } \\
\text { of test }\end{array}$} & \multicolumn{4}{|c|}{ Concentrations upon completion of test, $\mathrm{mg} / \mathrm{L}$} \\
\hline & & B & $\mathrm{Li}$ & $\mathrm{Na}$ & Si \\
\hline 1 & 5.80 & $<0.01$ & $<0.01$ & $<0.10$ & $<0.064$ \\
\hline 2 & 6.68 & 0.033 & $<0.01$ & 0.16 & 0.12 \\
\hline 3 & 6.65 & 0.012 & 0.029 & 0.18 & 0.085 \\
\hline 4 & 6.28 & 0.029 & 0.013 & 0.24 & 0.13 \\
\hline 5 & $\mathrm{H} / \mathrm{a}$ & $<0.01$ & $<0.001$ & 0.05 & $<0.05$ \\
\hline 6 & 6.52 & $<0.01$ & $<0.0037$ & 0.12 & 0.11 \\
\hline
\end{tabular}

Table 13 provides elements concentrations in water normalized for weight percentage of this element in the glass $(\mathrm{NL}, \mathrm{g} / \mathrm{L})$, as well as $\log \mathrm{NL}\left[\mathrm{El}_{\mathrm{i}}(\mathrm{g} / \mathrm{L})\right.$. 
SRNS-STI-2008-00057

Revision 0

Appendix C (SRNS-OS-2008-00001)

Table 13. KRI Glasses Leaching Data

\begin{tabular}{|c|c|c|c|c|c|c|c|c|c|c|c|c|c|c|c|c|c|}
\hline \multirow{3}{*}{ Glass \# } & \multicolumn{9}{|c|}{ NL, g/L } & \multicolumn{8}{|c|}{$\log N L\left[\mathrm{El}_{\mathrm{i}}(\mathrm{g} / \mathrm{L})\right]$} \\
\hline & \multicolumn{4}{|c|}{ quenched } & \multicolumn{5}{|c|}{ CCC treated } & \multicolumn{4}{|c|}{ quenched } & \multicolumn{4}{|c|}{ CCC treated } \\
\hline & $\mathrm{B}$ & $\mathrm{Li}$ & $\mathrm{Na}$ & $\mathrm{Si}$ & $\mathrm{B}$ & $\mathrm{Li}$ & $\mathrm{Na}$ & $\mathrm{Si}$ & $\mathrm{U}$ & B & $\mathrm{Li}$ & $\mathrm{Na}$ & $\mathrm{Si}$ & B & $\mathrm{Li}$ & $\mathrm{Na}$ & $\mathrm{Si}$ \\
\hline KRI-01 & 0.39 & 0.53 & 0.32 & 0.32 & 0.42 & 0.55 & 0.32 & 0.36 & - & -0.41 & -0.28 & -0.50 & -0.50 & -0.38 & -0.30 & -0.50 & -0.44 \\
\hline KRI-02 & 0.53 & 0.65 & 0.37 & 0.30 & 0.40 & 0.53 & 0.31 & 0.29 & - & -0.28 & -0.19 & -0.43 & -0.52 & -0.40 & -0.28 & -0.51 & -0.54 \\
\hline KRI-03 & 0.36 & 0.52 & 0.30 & 0.26 & 0.40 & 0.53 & 0.33 & 0.31 & - & -0.45 & -0.28 & -0.52 & -0.59 & -0.40 & -0.28 & -0.48 & -0.51 \\
\hline KRI-04 & 0.37 & 0.53 & 0.36 & 0.28 & 0.41 & 0.55 & 0.35 & 0.30 & - & -0.43 & -0.27 & -0.44 & -0.55 & -0.39 & -0.26 & -0.46 & -0.52 \\
\hline KRI-05 & 0.87 & 0.96 & 0.70 & 0.23 & 2.95 & 3.31 & 2.57 & 0.23 & - & -0.06 & -0.02 & -0.15 & $\begin{array}{l}-0.65 \\
\end{array}$ & 0.47 & 0.52 & 0.41 & -0.64 \\
\hline KRI-06 & 0.47 & 0.52 & 0.47 & 0.28 & 0.47 & 0.56 & 0.42 & 0.27 & - & -0.33 & -0.28 & -0.33 & -0.55 & -0.33 & -0.25 & -0.38 & -0.57 \\
\hline KRI-07 & 0.74 & 0.74 & 0.68 & 0.33 & 0.47 & 0.55 & 0.42 & 0.27 & - & -0.13 & -0.13 & -0.17 & -0.49 & -0.33 & -0.26 & -0.38 & -0.57 \\
\hline KRI-08 & 2.34 & 1.44 & 1.19 & 0.16 & 3.11 & 2.94 & 2.03 & 0.17 & - & 0.37 & 0.16 & 0.08 & -0.79 & 0.49 & 0.47 & 0.31 & -0.77 \\
\hline KRI-09 & 0.37 & 0.60 & 0.29 & 0.23 & 0.42 & 0.51 & 0.36 & 0.22 & - & $\begin{array}{l}-0.43 \\
\end{array}$ & -0.22 & -0.54 & -0.64 & -0.38 & $\begin{array}{l}-0.29 \\
\end{array}$ & -0.44 & $\begin{array}{l}-0.66 \\
\end{array}$ \\
\hline KRI-10 & 0.30 & 0.46 & 0.33 & 0.22 & 0.28 & 0.44 & 0.39 & 0.20 & - & -0.53 & -0.34 & -0.48 & -0.66 & -0.55 & -0.36 & -0.41 & -0.70 \\
\hline KRI-11 & 0.40 & 0.54 & 0.42 & 0.27 & 0.48 & 0.58 & 0.52 & 0.27 & - & -0.39 & -0.27 & $\begin{array}{l}-0.38 \\
\end{array}$ & -0.57 & -0.32 & -0.24 & -0.28 & -0.57 \\
\hline KRI-12 & 0.45 & 0.58 & 0.32 & 0.21 & 0.43 & 0.56 & 0.34 & 0.29 & - & -0.34 & -0.23 & -0.50 & -0.69 & -0.37 & -0.25 & -0.47 & -0.54 \\
\hline KRI-13 & 0.39 & 0.58 & 0.35 & 0.26 & 0.28 & 0.46 & 0.29 & 0.22 & - & $\begin{array}{l}-0.41 \\
\end{array}$ & -0.24 & -0.45 & $\begin{array}{l}-0.59 \\
\end{array}$ & -0.55 & $\begin{array}{l}-0.34 \\
\end{array}$ & $\begin{array}{l}-0.54 \\
\end{array}$ & $\begin{array}{l}-0.66 \\
\end{array}$ \\
\hline KRI-14 & 0.58 & 0.67 & 0.39 & 0.31 & 0.27 & 0.48 & 0.21 & 0.19 & - & -0.23 & -0.18 & -0.41 & -0.51 & -0.57 & -0.32 & $\begin{array}{l}-0.68 \\
\end{array}$ & -0.72 \\
\hline KRI-15 & 0.40 & 0.62 & 0.28 & 0.23 & 0.39 & 0.65 & 0.22 & 0.22 & - & $\begin{array}{l}-0.40 \\
\end{array}$ & -0.21 & -0.55 & -0.64 & -0.41 & $\begin{array}{l}-0.19 \\
\end{array}$ & $\begin{array}{l}-0.66 \\
\end{array}$ & $\begin{array}{l}-0.66 \\
\end{array}$ \\
\hline KRI-16 & 0.34 & 0.55 & 0.32 & 0.22 & 0.28 & 0.44 & 0.25 & 0.18 & - & -0.47 & -0.26 & -0.49 & -0.66 & -0.55 & -0.36 & -0.60 & -0.75 \\
\hline KRI-17 & 0.36 & 0.53 & 0.38 & 0.19 & 0.34 & 0.54 & 0.36 & 0.20 & - & $\begin{array}{l}-0.44 \\
\end{array}$ & $\begin{array}{l}-0.28 \\
\end{array}$ & $\begin{array}{l}-0.42 \\
\end{array}$ & -0.71 & -0.47 & $\begin{array}{l}-0.27 \\
\end{array}$ & $\begin{array}{l}-0.44 \\
\end{array}$ & $\begin{array}{l}-0.70 \\
\end{array}$ \\
\hline KRI-18 & 0.38 & 0.63 & 0.33 & 0.18 & 0.42 & 0.56 & 0.29 & 0.21 & - & -0.42 & -0.20 & -0.48 & -0.75 & -0.38 & -0.25 & -0.54 & -0.68 \\
\hline KRI-19 & 0.39 & 0.45 & 0.47 & 0.24 & 0.38 & 0.47 & 0.50 & 0.28 & - & $\begin{array}{l}-0.41 \\
\end{array}$ & -0.35 & -0.33 & $\begin{array}{l}-0.62 \\
\end{array}$ & -0.42 & $\begin{array}{l}-0.33 \\
\end{array}$ & $\begin{array}{l}-0.30 \\
\end{array}$ & -0.55 \\
\hline KRI-20 & 0.36 & 0.53 & 0.38 & 0.19 & 0.38 & 0.55 & 0.39 & 0.21 & - & -0.44 & -0.27 & -0.42 & -0.72 & -0.42 & -0.26 & -0.41 & -0.68 \\
\hline KRI-21 & 0.38 & 0.54 & 0.43 & 0.22 & 0.43 & 0.62 & 0.52 & 0.26 & - & $\begin{array}{l}-0.42 \\
\end{array}$ & -0.27 & -0.37 & -0.66 & -0.37 & -0.21 & $\begin{array}{l}-0.29 \\
\end{array}$ & $\begin{array}{l}-0.58 \\
\end{array}$ \\
\hline KRI-22 & 0.36 & 0.52 & 0.35 & 0.23 & 0.97 & 1.07 & 0.57 & 0.25 & - & $\begin{array}{l}-0.44 \\
\end{array}$ & $\begin{array}{l}-0.29 \\
\end{array}$ & $\begin{array}{l}-0.46 \\
\end{array}$ & $\begin{array}{l}-0.63 \\
\end{array}$ & $\begin{array}{l}-0.01 \\
\end{array}$ & 0.03 & $\begin{array}{l}-0.24 \\
\end{array}$ & -0.60 \\
\hline KRI-23 & 0.41 & 0.67 & 0.22 & 0.23 & 0.39 & 0.79 & 0.27 & 0.35 & - & $\begin{array}{l}-0.37 \\
\end{array}$ & -0.17 & -0.65 & -0.63 & -0.41 & -0.05 & $\begin{array}{l}-0.57 \\
\end{array}$ & -0.46 \\
\hline KRI-24 & 0.32 & 0.52 & 0.30 & 0.22 & 0.34 & 0.51 & 0.31 & 0.23 & - & -0.50 & -0.29 & $\begin{array}{l}-0.53 \\
\end{array}$ & -0.66 & -0.47 & -0.29 & -0.51 & -0.64 \\
\hline KRI-25 & 0.39 & 0.56 & 0.38 & 0.26 & 0.40 & 0.54 & 0.39 & 0.27 & - & -0.41 & -0.25 & -0.42 & -0.59 & -0.40 & -0.27 & -0.41 & -0.57 \\
\hline KRI-26 & 0.63 & 0.73 & 0.86 & 0.40 & 0.64 & 0.82 & 0.87 & 0.41 & 0.12 & -0.20 & -0.13 & $\begin{array}{l}-0.66 \\
\end{array}$ & $\begin{array}{l}-0.40 \\
\end{array}$ & $\begin{array}{l}-0.19 \\
\end{array}$ & $\begin{array}{l}-0.09 \\
\end{array}$ & $\begin{array}{l}-0.06 \\
\end{array}$ & $\begin{array}{l}-0.39 \\
\end{array}$ \\
\hline KRI-27 & 1.04 & 1.11 & 0.72 & 0.29 & 1.29 & 1.28 & 0.93 & 0.34 & 0.05 & 0.02 & 0.04 & -0.14 & -0.54 & 0.11 & -0.11 & -0.03 & -0.47 \\
\hline KRI-28 & 0.74 & 0.70 & 1.20 & 0.55 & 6.99 & 1.19 & 2.40 & 2.75 & 0.30 & -0.13 & -0.16 & 0.08 & -0.26 & 0.84 & 0.08 & 0.38 & 0.44 \\
\hline KRI-29 & 0.71 & 0.49 & 0.77 & 0.32 & 7.99 & 6.86 & 4.27 & 0.85 & 0.07 & -0.15 & -0.31 & -0.11 & $\begin{array}{l}-0.49 \\
\end{array}$ & 0.90 & 0.84 & 0.63 & -0.07 \\
\hline KRI-30 & 0.34 & 0.48 & 0.27 & 0.22 & 0.42 & 0.41 & 0.80 & 0.27 & 0.42 & -0.47 & -0.32 & -0.57 & -0.65 & -0.38 & $\begin{array}{l}-0.39 \\
\end{array}$ & -0.10 & -0.57 \\
\hline
\end{tabular}


The NL(i) values show that all glasses leach incongruently. Practically for all glasses, with the exception of KRI-28 and KRI-29, all NL(i) values are in the following row: $\mathrm{NL}(\mathrm{Li})>\mathrm{NL}(\mathrm{B})>\mathrm{NL}(\mathrm{Na})>\mathrm{NL}(\mathrm{Si})$. In a number of cases, we observed deviations from the overall similar leaching pattern. For example, glasses KRI-05, KRI-08, and KRI-27, with the highest $\mathrm{B}_{2} \mathrm{O}_{3}$ concentration (19.96 wt.\%, $16.25 \mathrm{wt} . \%$, and $17.20 \mathrm{wt} . \%$ ), are characterized by a higher dissolution rate for all three elements, i.e., boron, lithium, and sodium. If the alkaline metal content $\left(\mathrm{R}_{2} \mathrm{O}\right)$ increases in the glasses KRI-08 and KRI-27, NL of these elements further increases. A high $\mathrm{R}_{2} \mathrm{O}$ content in the glasses KRI-26, KRI-28, and KRI-29 leads to the same results.

Tables 14 and 15 show NL of elements for glasses US-08, US-19, US-20, US-24, US-35, US-44, and US-45 with the compositions identical to glasses KRI-01, KRI-02, KRI-03, KRI-04, KRI-05, KRI-06, and KRI-07. The glass leaching results obtained in the United States and at KRI are fairly close.

Table 14. Leach Data for KRI and US Quenched Glasses with Identical Compositions

\begin{tabular}{|c|c|c|c|c|c|c|c|c|c|}
\hline \multirow{2}{*}{$\begin{array}{c}\text { Glasses with } \\
\text { identical } \\
\text { compositions }\end{array}$} & \multicolumn{9}{|c|}{ KRI } \\
\cline { 3 - 10 } & $\mathrm{B}$ & $\mathrm{Li}$ & $\mathrm{Na}$ & $\mathrm{Si}$ & $\mathrm{B}$ & $\mathrm{Li}$ & $\mathrm{Na}$ & $\mathrm{Si}$ \\
\hline KRI-01 & US-08 & -0.41 & -0.28 & -0.50 & -0.50 & -0.36 & -0.22 & -0.49 & -0.42 \\
\hline KRI-02 & US-19 & -0.28 & -0.19 & -0.43 & -0.52 & -0.35 & -0.24 & -0.46 & -0.49 \\
\hline KRI-03 & US-20 & -0.44 & -0.28 & -0.52 & -0.59 & -0.35 & -0.23 & -0.45 & -0.51 \\
\hline KRI-04 & US-24 & -0.43 & -0.28 & -0.44 & -0.55 & -0.40 & -0.25 & 0.47 & -0.54 \\
\hline KRI-05 & US-35 & -0.60 & -0.02 & -0.16 & -0.64 & -0.10 & -0.04 & -0.20 & -0.68 \\
\hline KRI-06 & US-44 & -0.33 & -0.28 & -0.33 & -0.55 & -0.28 & -0.25 & -0.30 & -0.55 \\
\hline KRI-07 & US-45 & -0.13 & -0.13 & -0.17 & -0.49 & -0.32 & -0.25 & -0.31 & -0.54 \\
\hline
\end{tabular}

Table 15. Leach Data for KRI and US CCC Treated Glasses

\begin{tabular}{|c|c|c|c|c|c|c|c|c|c|}
\hline \multirow{3}{*}{\multicolumn{2}{|c|}{$\begin{array}{l}\text { Glasses with } \\
\text { identical } \\
\text { compositions }\end{array}$}} & \multicolumn{8}{|c|}{$\log N L\left[\mathrm{El}_{\mathrm{i}}(\mathrm{g} / \mathrm{L})\right]$} \\
\hline & & \multicolumn{4}{|c|}{ KRI } & \multicolumn{4}{|c|}{ US } \\
\hline & & B & $\mathrm{Li}$ & $\mathrm{Na}$ & $\mathrm{Si}$ & B & $\mathrm{Li}$ & $\mathrm{Na}$ & $\mathrm{Si}$ \\
\hline KRI-01 & US-08 & -0.38 & -0.30 & -0.50 & -0.44 & -0.39 & -0.27 & -0.49 & -0.45 \\
\hline KRI-02 & US-19 & -0.40 & -0.28 & -0.51 & -0.54 & -0.38 & -0.27 & -0.47 & -0.53 \\
\hline KRI-03 & US-20 & -0.40 & -0.28 & -0.48 & -0.51 & -0.38 & -0.26 & -0.46 & -0.52 \\
\hline KRI-04 & US-24 & -0.39 & -0.26 & -0.46 & -0.52 & -0.39 & -0.26 & -0.45 & -0.53 \\
\hline KRI-05 & US-35 & 0.47 & 0.52 & 0.41 & -0.64 & 0.60 & 0.55 & 0.40 & -0.66 \\
\hline KRI-06 & US-44 & -0.33 & -0.25 & -0.38 & -0.57 & -0.29 & -0.25 & -0.31 & -0.54 \\
\hline KRI-07 & US-45 & -0.33 & -0.26 & -0.38 & -0.57 & -0.34 & -0.26 & -0.34 & -0.55 \\
\hline
\end{tabular}




\section{TCLP Leaching}

\subsection{Selection of Appropriate Extraction Fluid}

Glasses KRI-02, KRI-05, KRI-07, KRI-10, KRI-28, and KRI-30 were tested using TCLP Procedure, method 1311. The extraction fluid selection was performed in accordance with Section 7.1.4 of the TCLP Procedure. According to the data given in Table 14, for extraction of the toxic elements from all tested glasses, extraction fluid \# 1 (Buffer \#1 acidity) should be used. The buffer prepared according to Section 5.7.1 of the TCLP procedure had the $\mathrm{pH}$ value of $4.91 \pm 0.05$.

Table 16. Selection of Appropriate Extraction Fluid

\begin{tabular}{|c|c|c|c|}
\hline Glass ID & $\begin{array}{c}\mathrm{pH} \\
\text { (section 7.1.4.1- } \\
\text { 7.1.4.2 of TCLP } \\
\text { test) }\end{array}$ & $\begin{array}{c}\mathrm{pH} \\
\text { (section 7.1.4.3- } \\
\begin{array}{c}7.1 .4 .4 \text { of TCLP } \\
\text { test) }\end{array}\end{array}$ & $\begin{array}{c}\text { Type (number) of } \\
\text { extraction fluid }\end{array}$ \\
\hline KRI-02 & 8.92 & 1.68 & $\# 1$ \\
\hline KRI-05 & 7.04 & 1.63 & $\# 1$ \\
\hline KRI-07 & 8.83 & 1.72 & $\# 1$ \\
\hline KRI-10 & 8.49 & 1.76 & $\# 1$ \\
\hline KRI-28 & 9.70 & 1.80 & $\# 1$ \\
\hline KRI-30 & 8.74 & 1.78 & \\
\hline
\end{tabular}

\subsection{TCLP Glass Testing}

The glasses of six compositions were crushed. For each glass, we leached glass fractions below $10 \mathrm{~mm}$ placing $2 \mathrm{~g}$ of the glass followed by $40 \mathrm{ml}$ of the extraction fluid \#1 into polystyrene vessels. After sealing the vessels, we agitated them for 18 hours. Upon completion of the extraction, the TCLP extracts were filtered using a $0.45 \mu \mathrm{m}$ membrane filter. The average $\mathrm{pH}$ values of the TCLP extracts were recorded as 4.87 .

The TCLP extract samples were prepared for measuring concentrations of toxic elements using mass spectrometry with inductively coupled plasma (MS-ICP) as follows: for each glass, $10 \mathrm{~mL}$ of the filtered extract were acidified by $0.1 \mathrm{~mL}$ of nitric acid (reagent grade). We observed for all tested glasses that, upon acidification of the extracts, no precipitation occurred. The blank sample was prepared directly from $10 \mathrm{~mL}$ of extraction fluid \# 1 acidified by $0.1 \mathrm{~mL}$ of $\mathrm{HNO}_{3}$. MS-ICP toxic concentrations values for the extracts $\left(\mathrm{X}_{\text {unspiked }}\right)$ were given in Table 17. All concentrations values are adjusted relatively to the blank values. Table 17 shows that none of the toxic concentrations in the TCLP-extracts exceeds the allowable limits. If a concentration of a toxic element in the glass increases, its concentration also increases in the TCLP-extract.

Table 17. Concentrations of Toxic Elements in TCLP-Extracts

\begin{tabular}{|c|c|c|c|c|}
\hline \multirow{2}{*}{$\#$} & \multicolumn{4}{|c|}{$\mathrm{X}_{\text {unspiked }}, \mathrm{mg} / \mathrm{L}$} \\
\cline { 2 - 4 } & $\mathrm{Cr}$ & $\mathrm{Mn}$ & $\mathrm{Pb}$ & $\mathrm{Zn}$ \\
\hline US EPA's TCLP limit & 5 & $\mathrm{~N} / \mathrm{L}$ & 5 & 25 \\
\hline (Blank) & $(0.0018)$ & $(0.0026)$ & $(0.0068)$ & $(0.015)$ \\
\hline KRI-02 & 0.0092 & 0.4370 & 0.0880 & 0.037 \\
\hline KRI-05 & 0.0082 & 0.7774 & $\mathrm{~N} / \mathrm{A}$ & 0.365 \\
\hline KRI-07 & 0.0051 & 0.1270 & 0.0410 & 0.095 \\
\hline KRI-10 & 0.0112 & 0.0574 & 0.1130 & 0.060 \\
\hline
\end{tabular}


Appendix C (SRNS-OS-2008-00001)

\begin{tabular}{|c|c|c|c|c|}
\hline KRI-28 & 0.6380 & 2.8970 & 1.0930 & N/A \\
\hline KRI-30 & N/D & N/A & 1.393 & 1.985 \\
\hline
\end{tabular}

\subsection{TCLP Testing Quality Assurance}

According to Section 5.8 of the TCLP Procedure, we prepared an analytical chromium and lead standard that was used as standard additives for the TCLP-extracts samples.

First of all, we prepared a reference standard. For this purpose, $0.03312 \mathrm{~g}$ of the analytical standard was added to extraction fluid \# 1 acidified with $0.1 \mathrm{~mL} \mathrm{HNO}_{3}$. $\mathrm{Cr}$ and $\mathrm{Pb}$ concentrations were measured in this reference standard using MS-ICP. Table 18 provides calculated and MS-ICP measured $\mathrm{Cr}$ and $\mathrm{Pb}$ concentrations in the reference standard, as well as their average values.

Table 18. $\mathrm{Cr}$ and $\mathrm{Pb}$ Concentrations in the Reference Standard

\begin{tabular}{|c|c|c|c|c|c|}
\hline \multicolumn{3}{|c|}{ Cr } & \multicolumn{3}{c|}{ Pb } \\
\hline \multicolumn{3}{|c|}{ Concentration, mg/L } & \multicolumn{3}{c|}{ Concentration, mg/L } \\
\hline $\begin{array}{c}\text { Calculated } \\
\text { value }\end{array}$ & $\begin{array}{c}\text { MS-ICP } \\
\text { measured } \\
\text { value }\end{array}$ & Average value & $\begin{array}{c}\text { Calculated } \\
\text { value }\end{array}$ & $\begin{array}{c}\text { MS-ICP } \\
\text { measured } \\
\text { value }\end{array}$ & Average value \\
\hline 0.0556 & 0.0452 & $0.0504 \pm 10.3 \%$ & 0.126 & 0.0922 & $0.109 \pm 15.5 \%$ \\
\hline
\end{tabular}

Then we used a method of precise additions, adding exact portions of $\mathrm{Cr}$ and $\mathrm{Pb}$ analytical standard to $10 \mathrm{~mL}$ of the TCLP-extracts acidified by $0.1 \mathrm{~mL} \mathrm{HNO}_{3}$. We calculated $\mathrm{Cr}$ and $\mathrm{Pb}$ concentrations in the TCLP-extracts that will correspond to the introduced exact portions ( $K_{\text {known, }}$ Table 19). After the analytical standard was added, samples of the TCLP-extracts wer analyzed for toxic elements using MS-ICP. The MS-ICP data for $\mathrm{Cr}$ and $\mathrm{Pb}$ concentrations ( $\left.\mathrm{X}_{\text {spiked }}\right)$ are given in Table 19.

Table 19 contains \%R (\% recovery) values. The following equation was used for \%R calculations:

$\% \mathrm{R}=100\left(\mathrm{X}_{\text {spiked }}-\mathrm{X}_{\text {unspiked }}\right) / \mathrm{K}_{\text {known }}$

Table 19. TCLP Testing Quality Assurance Using a Method of Precise Additions

\begin{tabular}{|c|c|c|c|c|c|c|c|c|c|}
\hline \multirow{2}{*}{ Glass \# } & \multirow{2}{*}{$\begin{array}{l}\text { Analytical } \\
\text { standard, g }\end{array}$} & \multicolumn{4}{|c|}{ [Cr], mg/L } & \multicolumn{4}{|c|}{ [Pb], mg/L } \\
\hline & & $\begin{array}{c}X \\
\text { spiked }\end{array}$ & $\begin{array}{c}\text { X } \\
\text { unspiked }\end{array}$ & $\begin{array}{c}\mathrm{K} \\
\text { known }\end{array}$ & $\begin{array}{c}\% \\
\text { release }\end{array}$ & $\begin{array}{c}\mathrm{X} \\
\text { spiked }\end{array}$ & $\begin{array}{c}\text { X } \\
\text { unspiked }\end{array}$ & $\begin{array}{c}\mathrm{K} \\
\text { known }\end{array}$ & $\begin{array}{c}\% \\
\text { release }\end{array}$ \\
\hline $\begin{array}{l}\text { Reference } \\
\text { standard }\end{array}$ & 0.03312 & $(0.0452)$ & - & 0.0452 & 100.0 & $(0.0922)$ & - & 0.0922 & 100.0 \\
\hline KRI-02 & 0.03240 & 0.0552 & 0.0092 & 0.0442 & 104.1 & 0.193 & 0.088 & 0.0902 & 116.4 \\
\hline KRI-05 & 0.03080 & 0.0552 & 0.0082 & 0.0420 & 111.9 & N/A & N/A & N/A & - \\
\hline KRI-07 & 0.03432 & 0.0592 & 0.0051 & 0.0468 & 115.6 & 0.183 & 0.041 & 0.0955 & 148.7 \\
\hline KRI-10 & 0.03573 & 0.0632 & 0.0112 & 0.0488 & 106.6 & 0.233 & 0.113 & 0.0995 & 120.6 \\
\hline KRI-28 & 0.03513 & 0.6680 & 0.6380 & 0.0479 & 62.6 & 1.193 & 1.093 & 0.0978 & 102.2 \\
\hline KRI-30 & 0.03410 & 0.0563 & N/D & 0.0465 & 121.1 & 1.493 & 1.393 & 0.0949 & 105.4 \\
\hline
\end{tabular}

Table 19 makes it possible to conclude that the TCLP testing of the glasses provided a sufficient level of data validity since the average release of the toxic elements of concern, i.e., $\mathrm{Cr}$ and $\mathrm{Pb}$, is $111.5 \%$ (with the exception of Pb in KRI-07 and $\mathrm{Cr}$ in KRI-28). A low Cr release in KRI-28 is 
SRNS-STI-2008-00057

Revision 0

Appendix C (SRNS-OS-2008-00001)

related to the fact that, for that glass, $\mathrm{K}_{\text {known }}$ value is in the range of the $\mathrm{X}$ spiked measurement uncertainty. 


\section{Uranium and Iron Oxidation States}

For melting of glasses that contain a large number of elements with variable oxidation states, the equilibrium between their oxidation states is very important. Some elements can be present in the glass in different oxidation states functioning as modifiers or entering the glass array, for example, $\mathrm{Mn}\left(3^{+}\right)$and $\mathrm{Mn}(2+)$; $\mathrm{Fe}\left(3^{+}\right)$and $\mathrm{Fe}(2+)$; $\mathrm{Cr}(6+)$ and $\mathrm{Cr}\left(3^{+}\right)$; $\mathrm{U}\left(4^{+}\right)$and $\mathrm{U}(6+)$. In case of iron, we need to consider reversibility of the $\mathrm{Fe}_{2} \mathrm{O}_{3} \leftrightarrow 2 \mathrm{FeO}+1 / 2 \mathrm{O}_{2}$ reaction at high temperatures. Higher manganese oxides $\left(\mathrm{MnO}_{2}\right)$ contribute to converting $\mathrm{FeO}$ into $\mathrm{Fe}_{2} \mathrm{O}_{3}$ due to its decomposition associated with heating and oxygen release. In addition, the presence of such compounds as carbonates and sulfates in the feed may affect the oxidation states of multivalent cations.

Taking into account these factors, we studied the oxidation states of iron using Mossbauer spectroscopy and the oxidation states of uranium using optical spectrometry in quenched glasses. The results of these preliminary studies are described below.

To study the oxidation states of iron and uranium, we selected those glasses (Table 20), the feed for which contained various concentrations of carbonates and did not contain the crystalline phase (spinel).

Table 20. Glass Compositions Evaluated by Mossbauer Spectroscopy

\begin{tabular}{|l|c|c|c|c|c|c|c|c|c|c|c|c|c|c|c|c|c|c|c|c|}
\hline & $\mathrm{Al}_{2} \mathrm{O}_{3}$ & $\mathrm{~B}_{2} \mathrm{O}_{3}$ & $\mathrm{CaO}$ & $\mathrm{Cr}_{2} \mathrm{O}_{3}$ & $\mathrm{Fe}_{2} \mathrm{O}_{3}$ & $\mathrm{~K}_{2} \mathrm{O}$ & $\mathrm{Li}_{2} \mathrm{O}$ & $\mathrm{MgO}$ & $\mathrm{MnO}$ & $\mathrm{Na}_{2} \mathrm{O}$ & $\mathrm{NiO}$ & $\mathrm{PbO}$ & $\mathrm{SO}_{3}$ & $\mathrm{SO}_{4}$ & $\mathrm{SiO}_{2}$ & $\mathrm{SrO}$ & $\mathrm{TiO}_{2}$ & $\mathrm{ZnO}^{2}$ & $\mathrm{ZrO}_{2}$ & $\mathrm{UO}_{3}$ \\
\hline KRI-01 & 15.19 & 9.55 & 0.00 & 0.00 & 9.74 & 1.00 & 4.86 & 0.00 & 2.43 & 9.81 & 0.001 & 0.09 & 0.49 & 0.59 & 45.78 & 0.00 & 1.00 & 0.00 & 0.00 & 0.00 \\
\hline KRI-06 & 12.80 & 12.58 & 0.55 & 0.305 & 8.86 & 0.64 & 4.05 & 0.25 & 1.83 & 12.73 & 0.359 & 0.66 & 0.22 & 0.26 & 39.55 & 1.62 & 0.46 & 1.02 & 1.46 & 0.00 \\
\hline KRI-28 & 18.34 & 6.12 & 0.00 & 1.00 & 5.00 & 0.00 & 5.71 & 0.00 & 3.85 & 18.52 & 0.00 & 1.00 & 0.50 & 0.60 & 36.50 & 0.00 & 1.00 & 0.00 & 0.00 & 2.42 \\
\hline KRI-30 & 20.00 & 15.29 & 0.75 & 0.00 & 5.51 & 0.00 & 5.79 & 0.50 & 0.00 & 7.23 & 0.00 & 1.00 & 0.50 & 0.60 & 35.73 & 3.00 & 0.00 & 0.00 & 2.00 & 2.67 \\
\hline
\end{tabular}

\subsection{Uranium Oxidation State in KRI-28 and KRI-30}

Uranium oxidation state in glasses KRI-28 and KRI-30 was determined using spectrometric and luminescent methods. Uranium was introduced as $\mathrm{U}_{3} \mathrm{O}_{8}$ into the feed. Since the glasses contained elements with a high absorption (U,Fe,Cr), they were practically non-transparent in the visible and shorter wavelength region of the spectrum. Therefore, we chemically dissolved the samples without interfering with the uranium oxidation state. We performed the dissolution as described below.

We placed $~ 1 \mathrm{~g}$ samples of the KRI-28 and KRI-30 glasses into Teflon bottles with plugs and added $0.5 \mathrm{~mL}$ concentrated phosphoric acid and $0.3 \mathrm{~mL}$ of $40 \%$ hydrofluoric acid. Then the bottles were simmered in "sand bath" for 5 hours at $80-100^{\circ} \mathrm{C}$, followed by HF distillation. We added $1 \mathrm{~mL}$ of water to the sample and sampled $0.6 \mathrm{~mL}$ of the generated solution that was placed into a standard quartz vial. The spectrum was taken in the $300-800 \mathrm{~nm}$ range using the spectrometer UV-3101. As a reference standard, we used the similarly prepared standardized $\mathrm{U}_{3} \mathrm{O}_{8}$ solution.

Similar treatment of uranium dioxide did not result in generation of $\mathrm{U}\left(6^{+}\right)$. $\mathrm{U}\left(4^{+}\right)$and $\mathrm{U}\left(6^{+}\right)$ absorptions do not overlap in the visible part of the spectrum. As analytical bands, we selected the following: $420 \mathrm{~nm}$ for uranyl and $640 \mathrm{~nm}$ for $\mathrm{U}(4+)-640 \mathrm{~nm}$. From the $\mathrm{U}_{3} \mathrm{O}_{8}$ spectrum, we determined absorption coefficients calculated for $1 \mathrm{mg}$ of uranium $\left(\mathrm{K}_{\mathrm{i}}\right)$, and these coefficients 
were 0.0161 and 0.0155 for $420 \mathrm{~nm}$ and $640 \mathrm{~nm}$, respectively. The $A_{i} / K_{i}$ ratio gives the content of this oxidation state of uranium in the sample in mg. Table 21 provides the measurements results.

Table 21. U(6+) and U(4+) Content ion Glasses KRI-28 and KRI-30

\begin{tabular}{|c|c|c|c|c|c|}
\hline \multirow{2}{*}{ Composition } & \multicolumn{2}{|c|}{ Absorption, $\mathrm{A}_{\mathrm{i}}$} & \multicolumn{3}{c|}{ Content } \\
\cline { 2 - 6 } & $420 \mathrm{~nm}$ & $640 \mathrm{~nm}$ & $\mathrm{U}(6+), \mathrm{mg}$ & $\mathrm{U}(4+), \mathrm{mg}$ & $\mathrm{U}(\mathrm{IV}), \%$ \\
\hline $\mathrm{U}_{3} \mathrm{O}_{8}$ & 0.334 & 0.161 & 20.8 & 10.4 & 33.3 \\
\hline $\mathrm{KRI}-28$ & 0.30 & $\begin{array}{c}\text { Not } \\
\text { detectable }\end{array}$ & 19.0 & $\begin{array}{c}\text { Not } \\
\text { detectable }\end{array}$ & 0 \\
\hline KRI-30 & 0.140 & 0.037 & 8.7 & 2.4 & 21,5 \\
\hline
\end{tabular}

In the KRI-28 sample, the U(4+)- $640 \mathrm{~nm}$ absorption band overlapped with the chromium absorption band, therefore, we had to perform an additional luminescent analysis of the sample before and after its oxidation with strong nitric acid, to determine a relative uranium content.

Our studies showed an equal $\mathrm{U}(6+)$ intensity before and after the oxidation, which made us conclude that the KRI-28 glass sample had uranium in the oxidation state of $\mathrm{U}(6+)$. We plan to continue work with other uranium-containing glasses.

\subsection{Iron Oxidation State in Glasses KRI-01, KRI-06, and KRI-28}

We used Mossbauer spectroscopy to determine the oxidation state of iron in the glass samples KRI-01, KRI-06, and KRI-28. Mossbauer spectra were taken using the spectro-photometer VS $1101 \mathrm{E}$ in the mode of constant accelerations at room temperature. The gamma resonance pair ${ }^{57} \mathrm{Co}(\mathrm{Cr})$ source, detector RSD 112, and converter $\mathrm{K}_{2} \mathrm{Mg}^{57} \mathrm{Fe}(\mathrm{CN})_{6}$ were used to measure absorption spectra.

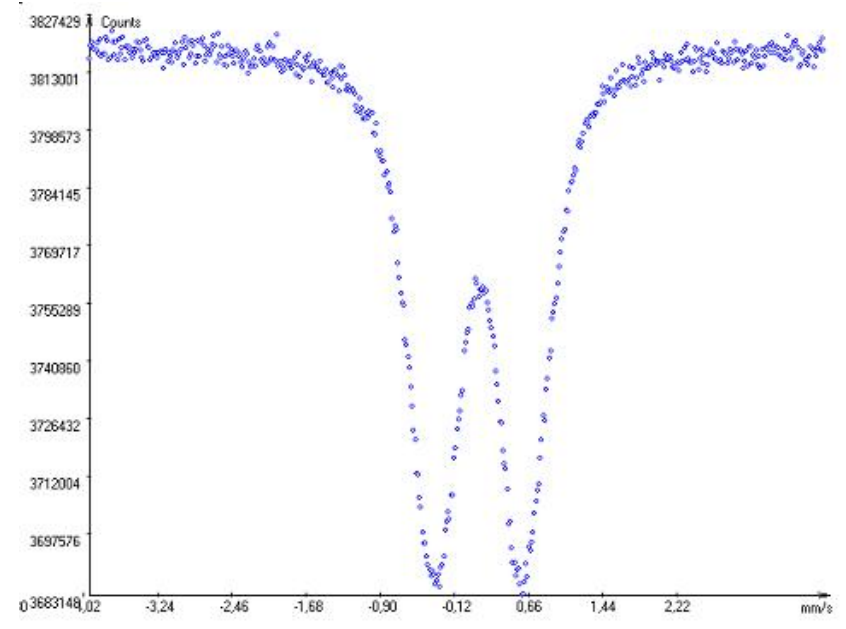

Fig. 15. Mossbauer Spectra for KRI-01 Glass

For data processing, we used a standard spectra Lorentz line shape processing code. The isomeric shift was given relatively to $\alpha$-Fe. We only determined $\mathrm{Fe}\left(3^{+}\right)$in the glasses. The KRI01, KRI-06, and KRI-28 glass samples had iron in one octahedral position, i.e., a doublet: isomeric shift (I.S) $=0.2 \mathrm{~mm} / \mathrm{s}$; quadruple split (Q.S.)=0.96 mm/s (Fig.15). 


\section{Viscosity Measurements for Selected KRI Matrix Glasses}

\subsection{Viscosity Measurement Procedure}

We measured viscosity of the melts using a vibration viscosimeter and also a viscosimeter that is based on a platinum ball immersion into the melt. Fig. 16 shows a schematic of the vibration viscosimeter. The principle of its operation and description are given below.

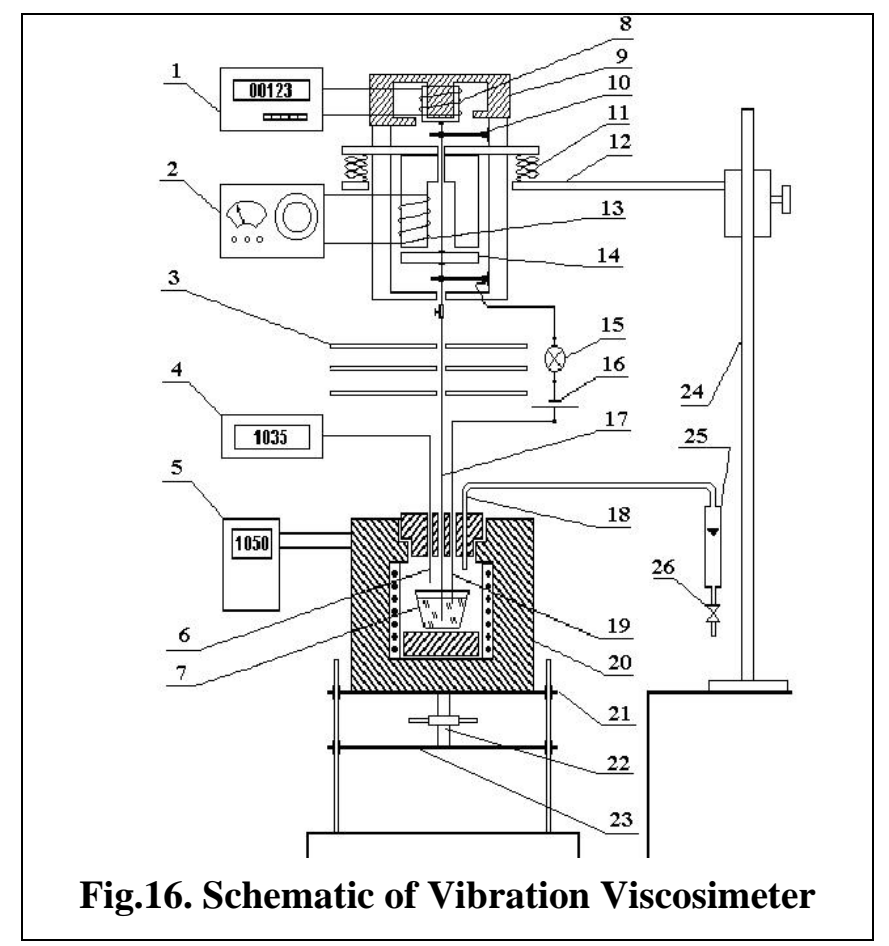

DC from the sound generator ZG-10 (2) is supplied to the vibrator coil (13). The variable magnetic field of the vibrator interacts with the anchor (14) and forces the mobile system of the viscosimeter to oscillate. Apart from the anchor, the mobile system of the viscosimeter includes the following: rod (17), springs (10), and coil (8). For viscosity measurements, the lower part of the rod is immersed into the crucible with liquid or melt (7). The buffers (11) between the body of the viscosimeter and the support bar (12) prevent transfer of oscillations from the viscosimeter to the support bar. When the mobile system oscillates, and an electromotive force is induced in the coil (8) that maximizes at the resonance, i.e., when the frequency of the viscosimeter oscillations coincides with the frequency of the forced oscillations.

The milli-voltmeter Sctch-4501 (1) records the electromotive force (E) induced in the coil (8).

When the rod is immersed into the liquid or melt, the oscillations amplitude will decrease with the viscosity increase, thereby making it possible to determine the viscosity by measuring the electromotive force $\mathrm{E}$.

To measure viscosity in the melts, scientists use platinum, molybdenum, or tungsten 1-2 mm diameter and 300-400 mm long wire for the rods, depending on the properties of the measured melt, as well as temperature and media of the measurements.

\section{Legend for Fig. 16}

1 - digital milli-voltmeter, 2 - sound generator ZG-10, 3 - heat screens,

4 - potentiometer PITs-5T, 5 - temperature regulator RIT-23, 6 - thermocouple,

7 - crucible with melt, 8 - measuring coil, 9 - permanent magnet,

10 - springs, 11 - buffers, 12 - support bar, 13 - vibrator coil,

14 - vibrator anchor, 15 - indicating light, 16 - power supply, 17 - tungsten wire rod,

18 - ceramic tube for inert gas supply,

19 - additional tungsten wire, 20 - shaft furnace,

21 - upper platform, 22 - fine-adjustment screw, 23 - lower platform,

24 - tripod, 25 - rotameter, 26 - vent for argon feed 
This viscosimeter uses a $350 \mathrm{~mm}$ long tungsten wire with the diameter of $1.2 \mathrm{~mm}$. The wire is immersed $15 \mathrm{~mm}$ deep into the liquid or melt. When the rod touches the melt surface, the indicating light (15) turns on, and, for this purpose, an additional tungsten rod (19) is immersed into the glass melt .

We use alundum or porcelain $25 \mathrm{~mm}$ high crucibles with the upper diameter of $35 \mathrm{~mm}$ and place them into the small-scale shaft furnace PP-1.2 (20). The temperature in the furnace is regulated by the temperature regulator RIT-23 (5). The chromel/alumel thermocouple (6) and the digital potentiometer PITs-5T (4) monitor the melt temperature.

Argon is fed into the furnace headspace through the ceramic tube (18) to prevent oxidation of the tungsten wire rods. The rotameter RM-A (25) monitors and regulates the argon flow rate. The body of the viscosimeter is installed on the tripod (21).

\subsection{Viscosity Parameters for Studied Glasses}

Fig. 17 shows data for comparison of viscosity values for the DWPG glass standard obtained by different methods. Fig 17 shows a satisfactory precision of the DWPF viscosity values measured by the vibrating viscosimeter in the temperature range of $850-1,000{ }^{\circ} \mathrm{C}$. Afterwards, in the 1,000 $1,150^{\circ} \mathrm{C}$ temperature range, the values start to significantly diverge, and at the temperature of $1,150^{\circ} \mathrm{C}$, our values significantly exceed the reference values.

For the viscosity values obtained by the platinum ball immersion based viscosimeter, the values diverge at the entire temperature range of $850-1,200{ }^{\circ} \mathrm{C}$. The immersion method measured data (KRI-2) have a temperature dependence similar to the round-robin results, but they are located slightly higher. It may likely be explained by the fact that different frit batches were used for our measurements and the round-robin measurements.

Viscosity DWPF start frit

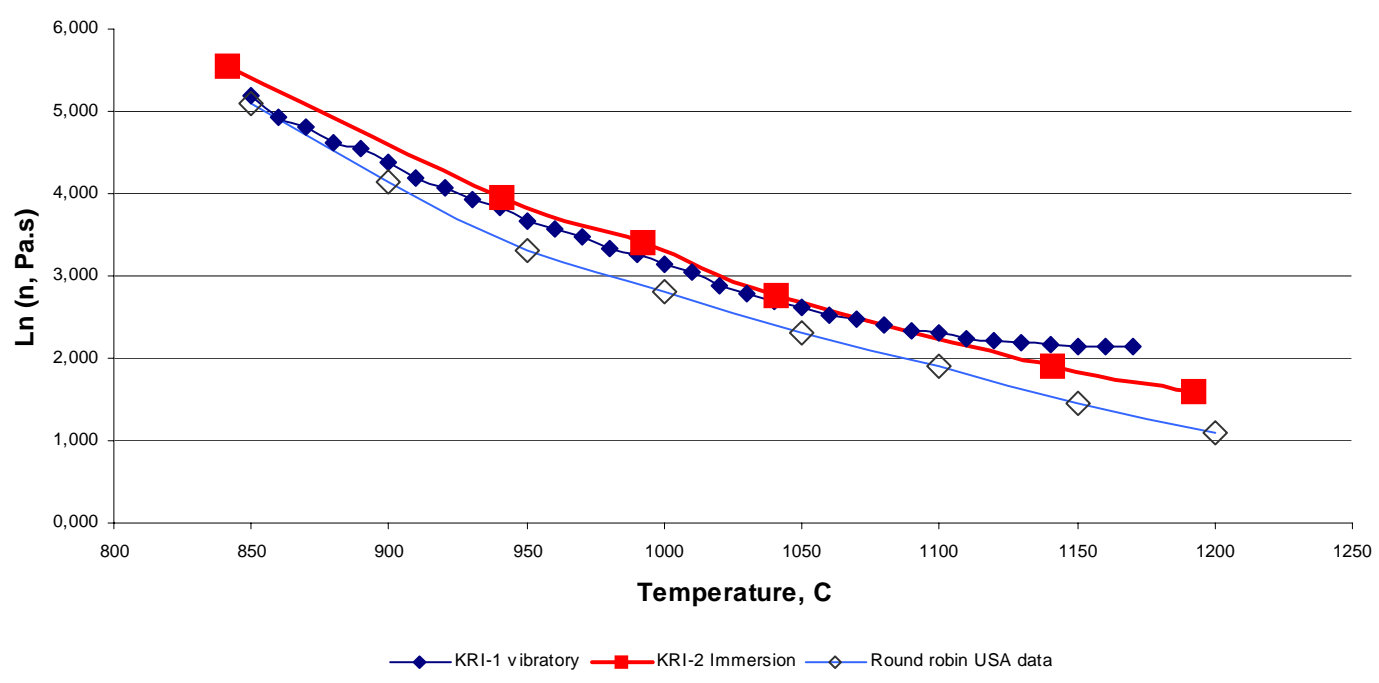

Fig. 17. Comparison of Viscosity Data for DWPF Standard vs Data Obtained Using Vibration and Immersion Viscosimeters

Since the viscosity data for the DWPF standard significantly differ from the U.S. data, the KRI viscosity measurements at KRI were only performed for several selected compositions, but not for all 30 compositions. Table 22 provides viscosity values for KRI-01, KRI-03, KRI-04, KRI07, KRI-11, KRI-20, KRI-24, and KRI-30 obtained using the vibration viscosimeter. Glasses 
KRI-01,KRI- 03, and KRI-07 correspond to US-08, US-20, and US-45. Previously submitted reports provided more detailed data on the viscosity of glasses. KRI-01, KRI-03, KRI-04, KRI07, KRI-11, KRI-20, KRI-24, and KRI- 30.

Table 22. Viscosity Values for KRI Matrix Glasses

\begin{tabular}{|c|c|c|c|c|c|c|c|c|}
\hline \multirow{2}{*}{$\mathrm{T},{ }^{\circ} \mathrm{C}$} & \multicolumn{9}{|c|}{ Viscosity, Pa s } \\
\cline { 2 - 10 } & KRI-01 & KRI-03 & KRI-04 & KRI-07 & KRI-11 & KRI-20 & KRI-24 & KRI-30 \\
\hline 950 & 135.83 & 70.45 & 92.02 & 19.70 & 93.32 & 62.99 & 155.08 & 33.09 \\
\hline 1000 & 74.00 & 40.61 & 47.70 & 10.88 & 43.92 & 33.22 & 65.87 & 19.31 \\
\hline 1050 & 41.61 & 24.53 & 30.33 & 6.31 & 23.70 & 21.49 & 35.95 & 13.18 \\
\hline 1100 & 26.30 & 16.39 & 21.10 & 4.36 & 15.23 & 16.22 & 21.27 & 10.01 \\
\hline 1150 & 15.36 & 11.72 & 17.01 & 3.60 & 12.35 & 13.45 & 16.28 & 8.51 \\
\hline
\end{tabular}




\section{Selection of Optimal KRI Matrix Glasses}

Table 23 provides all data on crystalline phases and leaching for KRI matrix glasses both quenched and CCC treated, as well as $\mathrm{T}_{\mathrm{L}}$ values. We used the following criteria to select glasses with the optical properties: NL values (PCT) are not supposed to exceed $1 \mathrm{~g} / \mathrm{L}, \mathrm{T}_{\mathrm{L}}$ values are not supposed to exceed $1050^{\circ} \mathrm{C}$, and the melt viscosity values are not supposed to exceed 5-7 Pas.

Table 23. Characterization Data Summary for KRI Matrix Glasses

\begin{tabular}{|c|c|c|c|c|c|c|c|c|c|c|c|}
\hline \multirow{3}{*}{ Glass \# } & \multicolumn{5}{|c|}{ Quenched KRI glasses } & \multicolumn{5}{|c|}{ CCC Treated KRI glasses } & \multirow{3}{*}{$\mathbf{T}_{\mathbf{L}}$} \\
\hline & \multirow{2}{*}{$\begin{array}{l}\text { Crystallinity, } \\
\text { vol. .\% }\end{array}$} & \multicolumn{4}{|c|}{ NL, g/L } & \multirow{2}{*}{$\begin{array}{c}\text { Crystallinity, } \\
\text { vol. .\% }\end{array}$} & \multicolumn{4}{|c|}{ NL, g/L } & \\
\hline & & B & $\mathrm{Li}$ & $\mathrm{Na}$ & $\mathrm{Si}$ & & B & $\mathrm{Li}$ & $\mathrm{Na}$ & Si & \\
\hline KRI-01 & $\mathrm{N} / \mathrm{d}$ & 0.39 & 0.53 & 0.32 & 0.32 & 0.3 & 0.42 & 0.55 & 0.32 & 0.36 & 1008 \\
\hline KRI-02 & $\mathrm{N} / \mathrm{d}$ & 0.53 & 0.65 & 0.37 & 0.30 & 0.8 & 0.40 & 0.53 & 0.31 & 0.29 & 1063 \\
\hline KRI-03 & $\mathrm{N} / \mathrm{d}$ & 0.36 & 0.52 & 0.30 & 0.26 & 0.2 & 0.40 & 0.53 & 0.33 & 0.31 & 1058 \\
\hline KRI-04 & $\mathrm{N} / \mathrm{d}$ & 0.37 & 0.53 & 0.36 & 0.28 & 0.3 & 0.41 & 0.55 & 0.35 & 0.30 & 1113 \\
\hline KRI-05 & 2.8 & 0.87 & 0.96 & 0.70 & 0.23 & 7.3 & 2.95 & 3.31 & 2.57 & 0.23 & 1263 \\
\hline KRI-06 & $\mathrm{N} / \mathrm{d}$ & 0.47 & 0.52 & 0.47 & 0.28 & 1.2 & 0.47 & 0.56 & 0.42 & 0.27 & 1095 \\
\hline KRI-07 & $\mathrm{N} / \mathrm{d}$ & 0.74 & 0.74 & 0.68 & 0.33 & 0.2 & 0.47 & 0.55 & 0.42 & 0.27 & 1071 \\
\hline KRI-08 & 6.6 & 2.34 & 1.44 & 1.19 & 0.16 & 15.0 & 3.11 & 2.94 & 2.03 & 0.17 & 1178 \\
\hline KRI-09 & 1.5 & 0.37 & 0.60 & 0.29 & 0.23 & 3.1 & 0.42 & 0.51 & 0.36 & 0.22 & 1188 \\
\hline KRI-10 & 0.9 & 0.30 & 0.46 & 0.33 & 0.22 & 0.94 & 0.28 & 0.44 & 0.39 & 0.20 & 1253 \\
\hline KRI-11 & 2.5 & 0.40 & 0.54 & 0.42 & 0.27 & 5.3 & 0.48 & 0.58 & 0.52 & 0.27 & 1162 \\
\hline KRI-12 & 1.2 & 0.45 & 0.58 & 0.32 & 0.21 & 2.5 & 0.43 & 0.56 & 0.34 & 0.29 & 1168 \\
\hline KRI-13 & 2.2 & 0.39 & 0.58 & 0.35 & 0.26 & 2.8 & 0.28 & 0.46 & 0.29 & 0.22 & 1183 \\
\hline KRI-14 & 1.5 & 0.58 & 0.67 & 0.39 & 0.31 & 3.8 & 0.27 & 0.48 & 0.21 & 0.19 & 1178 \\
\hline KRI-15 & 2.7 & 0.40 & 0.62 & 0.28 & 0.23 & 4.3 & 0.39 & 0.65 & 0.22 & 0.22 & 1183 \\
\hline KRI-16 & 0.5 & 0.34 & 0.55 & 0.32 & 0.22 & 0.6 & 0.28 & 0.44 & 0.25 & 0.18 & 1160 \\
\hline KRI-17 & 0.91 & 0.36 & 0.53 & 0.38 & 0.19 & 2.0 & 0.34 & 0.54 & 0.36 & 0.20 & 1158 \\
\hline KRI-18 & 2.3 & 0.38 & 0.63 & 0.33 & 0.18 & 3.6 & 0.42 & 0.56 & 0.29 & 0.21 & 1173 \\
\hline KRI-19 & 0.2 & 0.39 & 0.45 & 0.47 & 0.24 & 2.3 & 0.38 & 0.47 & 0.50 & 0.28 & 1178 \\
\hline KRI-20 & 0.34 & 0.36 & 0.53 & 0.38 & 0.19 & 2.6 & 0.38 & 0.55 & 0.39 & 0.21 & 1166 \\
\hline KRI-21 & 0.6 & 0.38 & 0.54 & 0.43 & 0.22 & 2.5 & 0.43 & 0.62 & 0.52 & 0.26 & 1171 \\
\hline KRI-22 & 0.2 & 0.36 & 0.52 & 0.35 & 0.23 & 2.8 & 0.97 & 1.07 & 0.57 & 0.25 & 1133 \\
\hline KRI-23 & 2.8 & 0.41 & 0.67 & 0.22 & 0.23 & 2.6 & 0.39 & 0.79 & 0.27 & 0.35 & 1170 \\
\hline KRI-24 & 0.58 & 0.32 & 0.52 & 0.30 & 0.22 & 3.5 & 0.34 & 0.51 & 0.31 & 0.23 & 1164 \\
\hline KRI-25 & 0.78 & 0.39 & 0.56 & 0.38 & 0.26 & 2.2 & 0.40 & 0.54 & 0.39 & 0.27 & 1175 \\
\hline KRI-26 & 0.5 & 0.63 & 0.73 & 0.86 & 0.40 & 4.5 & 0.64 & 0.82 & 0.87 & 0.41 & 1121 \\
\hline KRI-27 & $\mathrm{N} / \mathrm{d}$ & 1.04 & 1.11 & 0.72 & 0.29 & 1.9 & 1.29 & 1.28 & 0.93 & 0.34 & 1163 \\
\hline KRI-28 & N/d & 0.74 & 0.70 & 1.20 & 0.55 & 100 & 6.99 & 1.19 & 2.40 & 2.75 & 1045 \\
\hline KRI-29 & $\mathrm{N} / \mathrm{d}$ & 0.71 & 0.49 & 0.77 & 0.32 & 2.0 & 7.99 & 6.86 & 4.27 & 0.85 & 1108 \\
\hline KRI-30 & $\mathrm{N} / \mathrm{d}$ & 0.34 & 0.48 & 0.27 & 0.22 & 1.4 & 0.42 & 0.41 & 0.80 & 0.27 & 958 \\
\hline
\end{tabular}

Table 24 shows the glasses and their properties that can be considered the most appropriate for testing in SMK-1 and EP-5 systems. KRI-07 and KRI-30 are of a special interest. 
SRNS-STI-2008-00057

Revision 0

Appendix C (SRNS-OS-2008-00001)

Table 24. Optimal KRI Matrix Glass Compositions

\begin{tabular}{|c|c|c|c|c|c|c|c|c|c|c|c|c|}
\hline \multirow{3}{*}{ Glass \# } & \multicolumn{5}{|c|}{ Quenched KRI matrix glasses } & \multicolumn{5}{|c|}{ CCC treated KRI matrix glasses } & \multirow{3}{*}{$\mathrm{T}_{\mathrm{L}}$} & \multirow{3}{*}{$\begin{array}{c}\text { Viscosit } \\
\mathrm{y}, \mathrm{Pa} \mathrm{s} \\
\left(1150^{\circ} \mathrm{C}\right)\end{array}$} \\
\hline & \multirow{2}{*}{$\begin{array}{l}\text { Crystallinity, } \\
\text { vol. .\% }\end{array}$} & \multicolumn{4}{|c|}{ NL, g/L } & \multirow{2}{*}{$\begin{array}{l}\text { Crystallinity, } \\
\text { vol. .\% }\end{array}$} & \multicolumn{4}{|c|}{ NL, g/L } & & \\
\hline & & B & $\mathrm{Li}$ & $\mathrm{Na}$ & Si & & B & $\mathrm{Li}$ & $\mathrm{Na}$ & $\mathrm{Si}$ & & \\
\hline KRI-01 & $\mathrm{N} / \mathrm{d}$ & 0.39 & 0.53 & 0.32 & 0.32 & 0.3 & 0.42 & 0.55 & 0.32 & 0.36 & 1008 & 15.36 \\
\hline KRI-03 & $\mathrm{N} / \mathrm{d}$ & 0.36 & 0.52 & 0.30 & 0.26 & 0.2 & 0.40 & 0.53 & 0.33 & 0.31 & 1058 & 11.72 \\
\hline KRI-07 & $\mathrm{N} / \mathrm{d}$ & 0.74 & 0.74 & 0.68 & 0.33 & 0.2 & 0.47 & 0.55 & 0.42 & 0.27 & 1071 & 3.60 \\
\hline KRI-26 & 0.5 & 0.63 & 0.73 & 0.86 & 0.40 & 4.5 & 0.64 & 0.82 & 0.87 & 0.41 & 1121 & - \\
\hline KRI-30 & $\mathrm{N} / \mathrm{d}$ & 0.34 & 0.48 & 0.27 & 0.22 & 1.4 & 0.42 & 0.41 & 0.80 & 0.27 & 958 & 8.51 \\
\hline
\end{tabular}


SRNS-STI-2008-00057

Revision 0

Appendix C (SRNS-OS-2008-00001)

\section{Conclusions}

1. KRI completed crucible scale fabrication of $30 \mathrm{KRI}$ matrix compositions in the muffle furnace at $1150^{\circ} \mathrm{C}$. All KRI matrix glasses underwent CCC treatment experiments.

2. The synthesized glasses and CCC treated glasses were tested for chemical homogeneity and crystallization using SEM, EPMA, and XRD. The synthesized glasses KRI-01 - KRI-04, KRI06, KRI-07, KRI-26, and KRI-28 - KRI-30 are homogenous regarding their main components and free from crystalline inclusions. Glasses KRI-10, KRI-16, KRI-17, KRI-19, KRI-20, KRI21, KRI-22, KRI-24, KRI-25, and KRI-27 contain less than 1 vol. \% of the spinel crystalline phase.

3. The ARM glass standard was tested using the PCT procedure. The leaching parameters were also identified for KRI matrix quenched and CCC treated glasses. All studied glasses were shown to have a high chemical durability, wit the exception of KRI-05, KRI-08, KRI-22, and KRI-27- KRI-29. The NL(i) values fall in the following row: NL(Li) $>\mathrm{NL}(\mathrm{B})>\mathrm{NL}(\mathrm{Na})>\mathrm{NL}(\mathrm{Si})$. Glasses KRI-02, KRI-05, KRI-07, KRI-10, KRI-28, and KRI-30 were tested using the TCLP procedure. All toxic concentrations in the extracts do not exceed the allowable limits.

4. The liquidus temperature values were determined for the SRM 733 glass standard and all 30 KRI matrix compositions using the certified furnace SNOL 7.2/1300 manufactured by UMEGA. The increase of $\mathrm{Cr}_{2} \mathrm{O}_{3}, \mathrm{NiO}, \mathrm{Al}_{2} \mathrm{O}_{3}$ and $\mathrm{Fe}_{2} \mathrm{O}_{3}$ concentrations in the KRI matrix glasses leads to the increase of the liquidus temperatures. The increase of $\mathrm{Na}_{2} \mathrm{O}$ and $\mathrm{Li}_{2} \mathrm{O}$ concentrations decreases the liquidus temperature. Boron and silicon oxides do not significantly affect the liquidus temperature.

5. The viscosity as a function of temperature data were obtained for KRI-01, KRI-03, KRI-04, KRI-07, KRI-11, KRI-20, KRI- 24, and KRI-30.

6. Taking into account all physical and chemical characteristics of the KRI matrix glasses, KRI recommended compositions KRI-06 (KRI-07) and KRI-30 for further studies under the ongoing task. 


\section{References}

1 M. Mika, M.J. Schweiger, J.D. Vienna, P. Hrma. Liquidus Temperature of Spinel Precipitating High-Level Waste Glasses, Mat. Res. Symp. Proc.V.465, 1997, pp-71-78.

2 Pavel Hrma, John Vienna, Jarrod Crum, Greg Piepel, Martin Mika. Liquidus Temperature of High-Level Waste Borosilicate Glasses with Spinel Primary Phase”, Mat. Res. Symp. Proc.V.608, 2000, pp-671-676. 


\section{Attachment 1. EPMA Data on Chemical Homogeneity of Glasses}

Sample KRI 01

\begin{tabular}{|c|c|c|c|c|c|c|c|c|c|c|c|}
\hline & $\mathrm{SiO} 2$ & $\mathrm{TiO} 2$ & $\mathrm{Al} 2 \mathrm{O} 3$ & $\mathrm{Fe} 2 \mathrm{O} 3$ & $\mathrm{MnO}$ & $\mathrm{Na} 2 \mathrm{O}$ & $\mathrm{K} 2 \mathrm{O}$ & $\mathrm{SO} 3$ & B2O3 & Li2O & Total \\
\hline 1 & 44.60 & 0.91 & 15.74 & 8.78 & 2.47 & 11.61 & 0.88 & 0.59 & 9.50 & 4.90 & 100.00 \\
\hline 2 & 42.27 & 1.04 & 16.97 & 9.82 & 2.63 & 11.54 & 0.84 & 0.47 & 9.50 & 4.90 & 100.00 \\
\hline 3 & 42.69 & 1.11 & 15.67 & 10.36 & 2.58 & 11.83 & 0.90 & 0.47 & 9.50 & 4.90 & 100.00 \\
\hline 4 & 42.79 & 1.06 & 15.94 & 10.28 & 2.65 & 11.48 & 0.95 & 0.45 & 9.50 & 4.90 & 100.00 \\
\hline 5 & 43.08 & 1.03 & 16.08 & 9.81 & 2.58 & 11.62 & 0.89 & 0.50 & 9.50 & 4.90 & 100.00 \\
\hline Average & 43.09 & 1.03 & 16.08 & 9.81 & 2.58 & 11.62 & 0.89 & 0.50 & 9.50 & 4.90 & 100.00 \\
\hline
\end{tabular}

\section{Sample KRI 02}

\begin{tabular}{|c|c|c|c|c|c|c|c|c|c|c|c|c|c|c|c|}
\hline & Al2O3 & $\mathrm{CaO}$ & $\mathrm{Fe} 2 \mathrm{O} 3$ & $\mathrm{~K} 2 \mathrm{O}$ & $\mathrm{MgO}$ & $\mathrm{MnO}$ & $\mathrm{Na} 2 \mathrm{O}$ & $\mathrm{NiO}$ & $\mathrm{SO} 3$ & $\mathrm{SiO} 2$ & $\mathrm{TiO} 2$ & $\mathrm{ZrO} 2$ & $\mathrm{~B} 2 \mathrm{O} 3$ & $\mathrm{Li} 2 \mathrm{O}$ & \\
\hline 1 & 13.43 & 0.42 & 7.82 & 0.62 & 0.03 & 1.92 & 9.85 & 0.05 & 0.24 & 46.65 & 0.75 & 0.19 & 14.40 & 3.64 & 100.00 \\
\hline 2 & 15.57 & 0.61 & 8.82 & 0.64 & 0.00 & 2.24 & 10.82 & 0.05 & 0.32 & 41.72 & 0.88 & 0.28 & 14.40 & 3.64 & 100.00 \\
\hline 3 & 15.79 & 0.49 & 9.30 & 0.65 & 0.12 & 2.10 & 11.00 & 0.19 & 0.32 & 40.63 & 0.99 & 0.39 & 14.40 & 3.64 & 100.00 \\
\hline 4 & 15.70 & 0.64 & 8.79 & 0.68 & 0.11 & 2.34 & 11.09 & 0.12 & 0.38 & 40.89 & 0.97 & 0.25 & 14.40 & 3.64 & 100.00 \\
\hline 5 & 15.69 & 0.51 & 8.49 & 0.60 & 0.17 & 2.31 & 10.84 & 0.28 & 0.38 & 41.43 & 0.94 & 0.32 & 14.40 & 3.64 & 100.00 \\
\hline Average & 15.23 & 0.53 & 8.64 & 0.64 & 0.09 & 2.18 & 10.72 & 0.14 & 0.33 & 42.27 & 0.91 & 0.28 & 14.40 & 3.64 & 100.00 \\
\hline Er/Re & 4.00 & 4.40 & 2.80 & 0.50 & 0.80 & 1.7 & 1.0 & 0.7 & 2.10 & 3.40 & 1.40 & 1.50 & & & \\
\hline
\end{tabular}

\section{Sample KRI 03}

\begin{tabular}{|c|c|c|c|c|c|c|c|c|c|c|c|c|}
\hline & $\mathrm{Al} 2 \mathrm{O} 3$ & $\mathrm{CaO}$ & $\mathrm{Fe} 2 \mathrm{O} 3$ & $\mathrm{~K} 2 \mathrm{O}$ & $\mathrm{MnO}$ & $\mathrm{Na} 2 \mathrm{O}$ & $\mathrm{SO} 3$ & $\mathrm{SiO} 2$ & $\mathrm{TiO} 2$ & $\mathrm{~B} 2 \mathrm{O} 3$ & $\mathrm{Li} 2 \mathrm{O}$ & Total \\
\hline 1 & 16.38 & 0.577 & 8.99 & 0.7 & 2.39 & 10.85 & 0.51 & 40.44 & 1.032 & 14.4 & 3.64 & 100 \\
\hline 2 & 15.69 & 0.424 & 8.57 & 0.7 & 2.20 & 10.64 & 0.50 & 42.19 & 1 & 14.4 & 3.64 & 100 \\
\hline 3 & 11.85 & 0.532 & 7.46 & 0.6 & 2.09 & 10.11 & 0.47 & 48.22 & 0.633 & 14.4 & 3.64 & 100 \\
\hline 4 & 16.15 & 0.580 & 9.29 & 0.6 & 2.40 & 10.58 & 0.51 & 40.93 & 0.866 & 14.4 & 3.64 & 100 \\
\hline 5 & 16.47 & 0.554 & 8.91 & 0.6 & 2.42 & 11.87 & 0.49 & 39.64 & 0.99 & 14.4 & 3.64 & 100 \\
\hline Average & 15.31 & 0.533 & 8.64 & 0.7 & 2.30 & 10.81 & 0.50 & 42.28 & 0.904 & 14.4 & 3.64 & 100 \\
\hline Skin around $\mathrm{SiO}_{2}$ & 6.24 & 0.28 & 6.14 & 0.8 & 2.07 & 6.87 & 0 & 66.6 & 0.5 & & & \\
\hline Er/Re & 6.4 & 20.2 & 4.7 & 1 & 1.3 & 1.1 & 0.1 & 15.8 & 28.5 & & & \\
\hline
\end{tabular}

\section{Sample KRI 04}

\begin{tabular}{|c|c|c|c|c|c|c|c|c|c|c|c|c|c|c|c|}
\hline & $\mathrm{Al} 2 \mathrm{O} 3$ & $\mathrm{CaO}$ & $\mathrm{Fe} 2 \mathrm{O} 3$ & $\mathrm{~K} 2 \mathrm{O}$ & $\mathrm{MgO}$ & $\mathrm{MnO}$ & $\mathrm{Na} 2 \mathrm{O}$ & $\mathrm{NiO}$ & $\mathrm{SO} 3$ & $\mathrm{SiO} 2$ & $\mathrm{TiO} 2$ & $\mathrm{ZrO} 2$ & $\mathrm{~B} 2 \mathrm{O} 3$ & $\mathrm{Li} 2 \mathrm{O}$ & \\
\hline 1 & 14.37 & 0.64 & 7.84 & 0.69 & 0.03 & 2.20 & 11.2 & 0.7 & 0.2 & 45.81 & 0.71 & 0.37 & 12.25 & 3 & 100 \\
\hline 2 & 13.85 & 0.56 & 7.46 & 0.63 & 0.07 & 2.10 & 11.27 & 0.3 & 0.47 & 46.97 & 0.6 & 0.44 & 12.25 & 3 & 100 \\
\hline 3 & 11.36 & 0.39 & 7.54 & 0.63 & 0.09 & 2.33 & 10.26 & 0.6 & 0.21 & 50.42 & 0.5 & 0.45 & 12.25 & 3 & 100 \\
\hline 4 & 16.49 & 0.62 & 8.46 & 0.63 & 0.25 & 2.54 & 11.62 & 0.6 & 0.36 & 42.04 & 0.68 & 0.44 & 12.25 & 3 & 100 \\
\hline 5 & 16.63 & 0.67 & 8.72 & 0.67 & 0.04 & 2.77 & 11.83 & 0.5 & 0.49 & 41.03 & 0.88 & 0.47 & 12.25 & 3 & 100 \\
\hline Average & 14.54 & 0.58 & 8.01 & 0.65 & 0.09 & 2.39 & 11.23 & 0.5 & 0.35 & 45.26 & 0.68 & 0.43 & 12.25 & 3 & 100 \\
\hline Er/Re & 8.4 & 4.4 & 2.8 & 0.5 & 0.8 & 1.7 & 1 & 0.7 & 2.1 & 3.4 & 1.4 & 1.5 & & & \\
\hline
\end{tabular}


SRNS-STI-2008-00057

Revision 0

Appendix C (SRNS-OS-2008-00001)

Sample KRI 05

\begin{tabular}{|c|c|c|c|c|c|c|c|c|c|c|c|c|c|}
\hline & $\mathrm{Al} 2 \mathrm{O} 3$ & $\mathrm{CaO}$ & $\mathrm{Cr} 2 \mathrm{O} 3$ & $\mathrm{Fe} 2 \mathrm{O} 3$ & $\mathrm{~K} 2 \mathrm{O}$ & $\mathrm{MnO}$ & $\mathrm{Na} 2 \mathrm{O}$ & $\mathrm{SiO} 2$ & $\mathrm{SrO} *$ & $\mathrm{ZnO}$ & B2O3 & Li2O & Cyмма \\
\hline 1 & 13.81 & 0.91 & 0.32 & 12.62 & 1.84 & 5.07 & 9.70 & 38.99 & 2.70 & 2.04 & 10.00 & 2.00 & 100.00 \\
\hline 2 & 13.35 & 1.06 & 0.32 & 12.57 & 1.83 & 5.05 & 10.54 & 38.15 & 2.70 & 2.43 & 10.00 & 2.00 & 100.00 \\
\hline 3 & 12.67 & 0.89 & 0.31 & 12.46 & 1.79 & 4.54 & 9.61 & 40.78 & 2.70 & 2.25 & 10.00 & 2.00 & 100.00 \\
\hline 4 & 14.05 & 0.92 & 0.32 & 12.96 & 1.84 & 5.08 & 8.99 & 39.09 & 2.70 & 2.04 & 10.00 & 2.00 & 100.00 \\
\hline 5 & 13.45 & 0.90 & 0.47 & 12.93 & 1.82 & 5.29 & 9.15 & 39.00 & 2.70 & 2.28 & 10.00 & 2.00 & 100.00 \\
\hline Average in area & 13.46 & 0.94 & 0.35 & 12.71 & 1.83 & 5.01 & 9.60 & 39.21 & 2.70 & 2.21 & 10.00 & 2.00 & 100.00 \\
\hline Average & 11.08 & 0.82 & 0.29 & 11.03 & 1.53 & 4.42 & 8.16 & 40.38 & 2.70 & 2.31 & 10.00 & 2.00 & 100.29 \\
\hline Er/Re & 1.2 & & & 1.3 & 0 & 2 & 0.8 & 5.7 & & & & & \\
\hline
\end{tabular}

\section{Sample KRI 06}

\begin{tabular}{|c|c|c|c|c|c|c|c|c|c|c|c|c|c|c|c|c|c|}
\hline & $\mathrm{SiO} 2$ & $\mathrm{TiO} 2$ & $\mathrm{Al} 2 \mathrm{O} 3$ & $\mathrm{Cr} 2 \mathrm{O} 3$ & $\mathrm{Fe} 2 \mathrm{O} 3$ & $\mathrm{MnO}$ & $\mathrm{CaO}$ & $\mathrm{Na} 2 \mathrm{O}$ & $\mathrm{K} 2 \mathrm{O}$ & $\mathrm{NiO}$ & $\mathrm{ZnO}$ & $\mathrm{ZrO} 2$ & $\mathrm{PbO}$ & $\mathrm{SrO}$ & B2O3* & Li2O* & Total \\
\hline 1 & 40.24 & 0.43 & 13.30 & 0.35 & 8.73 & 1.86 & 0.37 & 13.90 & 0.47 & 0.35 & 1.02 & 1.12 & 0.61 & 0.58 & 12.58 & 4.10 & 100.00 \\
\hline 2 & 40.23 & 0.43 & 12.43 & 0.33 & 8.10 & 1.83 & 0.39 & 14.11 & 0.53 & 0.28 & 0.96 & 1.10 & 0.39 & 2.20 & 12.58 & 4.10 & 100.00 \\
\hline 3 & 40.45 & 0.43 & 13.25 & 0.34 & 8.33 & 1.93 & 0.40 & 13.94 & 0.53 & 0.31 & 1.09 & 1.03 & 0.66 & 0.64 & 12.58 & 4.10 & 100.00 \\
\hline 4 & 41.04 & 0.45 & 13.09 & 0.31 & 8.41 & 1.97 & 0.38 & 13.71 & 0.43 & 0.41 & 1.14 & 0.87 & 0.70 & 0.41 & 12.58 & 4.10 & 100.00 \\
\hline 5 & 39.30 & 0.52 & 13.75 & 0.31 & 8.68 & 1.96 & 0.43 & 14.02 & 0.58 & 0.32 & 1.07 & 1.25 & 0.48 & 0.66 & 12.58 & 4.10 & 100.00 \\
\hline Average & 40.51 & 0.45 & 13.14 & 0.32 & 8.40 & 1.89 & 0.40 & 13.74 & 0.50 & 0.34 & 1.04 & 1.06 & 0.62 & 0.91 & & & \\
\hline
\end{tabular}

\section{Sample KRI 07}

\begin{tabular}{|c|c|c|c|c|c|c|c|c|c|c|c|c|c|c|c|c|c|c|}
\hline & Al2O3 & $\mathrm{CaO}$ & $\mathrm{Cr} 2 \mathrm{O} 3$ & $\mathrm{Fe} 2 \mathrm{O} 3$ & $\mathrm{~K} 2 \mathrm{O}$ & $\mathrm{MgO}$ & $\mathrm{MnO}$ & $\mathrm{Na} 2 \mathrm{O}$ & $\mathrm{NiO}$ & $\mathrm{PbO}$ & $\mathrm{SiO} 2$ & $\mathrm{SrO} *$ & $\mathrm{TiO} 2$ & $\mathrm{ZnO}$ & $\mathrm{ZrO} 2$ & $\mathrm{~B} 2 \mathrm{O} 3 *$ & Li2O* & Total \\
\hline 1.00 & 12.31 & 0.13 & 0.40 & 7.47 & 0.67 & 0.00 & 2.96 & 9.52 & 0.70 & 0.88 & 37.52 & 2.70 & 0.77 & 1.37 & 0.62 & 20.00 & 2.00 & 100.00 \\
\hline 2.00 & 13.10 & 0.26 & 0.40 & 7.03 & 0.55 & 0.00 & 3.06 & 9.09 & 0.82 & 0.69 & 37.67 & 2.70 & 0.77 & 1.25 & 0.62 & 20.00 & 2.00 & 100.00 \\
\hline 3.00 & 14.14 & 0.00 & 0.39 & 7.51 & 0.65 & 0.00 & 3.10 & 10.44 & 0.68 & 0.86 & 34.51 & 2.70 & 0.75 & 1.44 & 0.85 & 20.00 & 2.00 & 100.00 \\
\hline 4.00 & 13.21 & 0.13 & 0.41 & 8.14 & 0.67 & 0.00 & 3.33 & 9.78 & 0.81 & 0.79 & 35.04 & 2.70 & 0.76 & 1.60 & 0.63 & 20.00 & 2.00 & 100.00 \\
\hline 5.00 & 10.13 & 0.12 & 0.39 & 6.40 & 0.53 & 0.00 & 2.61 & 8.40 & 0.79 & 0.67 & 42.88 & 2.70 & 0.59 & 1.20 & 0.60 & 20.00 & 2.00 & 100.00 \\
\hline $\begin{array}{c}\text { Average } \\
\text { in area }\end{array}$ & 11.75 & 0.25 & 0.40 & 6.82 & 0.54 & 0.00 & 2.91 & 8.37 & 0.80 & 0.97 & 39.55 & 2.70 & 0.75 & 1.46 & 0.73 & 20.00 & 2.00 & 100.00 \\
\hline Average & 12.58 & 0.13 & 0.40 & 7.31 & 0.61 & 0.00 & 3.01 & 9.45 & 0.76 & 0.78 & 37.53 & 2.70 & 0.73 & 1.37 & 0.66 & 20.00 & 2.00 & 100.00 \\
\hline Er/Re & 5.00 & 28.10 & & 4.40 & 0.90 & & 2.20 & 1.10 & & & 15.20 & & 13.60 & & 0.40 & & & \\
\hline
\end{tabular}

\section{Sample KRI 08}

\begin{tabular}{|c|c|c|c|c|c|c|c|c|c|c|c|c|c|c|c|c|c|c|}
\hline & $\mathrm{Al} 2 \mathrm{O} 3$ & $\mathrm{CaO}$ & $\mathrm{Cr} 2 \mathrm{O} 3$ & $\mathrm{Fe} 2 \mathrm{O} 3$ & $\mathrm{~K} 2 \mathrm{O}$ & $\mathrm{MnO}$ & $\mathrm{Na} 2 \mathrm{O}$ & $\mathrm{NiO}$ & $\mathrm{PbO}$ & $\mathrm{SiO} 2$ & $\mathrm{SrO}$ & $\mathrm{TiO} 2$ & $\mathrm{ZnO}$ & $\mathrm{ZrO} 2$ & $\mathrm{~B} 2 \mathrm{O} 3 *$ & Li2O* & Total \\
\hline 1 & 13.08 & 0.14 & 0.43 & 7.93 & 0.70 & 3.15 & 10.11 & 0.74 & 0.94 & 39.85 & 0.75 & 0.81 & 1.46 & 0.66 & 16.25 & 3.00 & 100.00 \\
\hline 2 & 13.92 & 0.27 & 0.42 & 7.47 & 0.58 & 3.25 & 9.66 & 0.86 & 0.73 & 40.03 & 0.75 & 0.81 & 1.33 & 0.65 & 16.25 & 3.00 & 100.00 \\
\hline 3 & 15.02 & 0.00 & 0.41 & 7.97 & 0.68 & 3.30 & 11.09 & 0.72 & 0.92 & 36.66 & 0.75 & 0.79 & 1.53 & 0.89 & 16.25 & 3.00 & 100.00 \\
\hline 4 & 14.03 & 0.14 & 0.43 & 8.65 & 0.71 & 3.53 & 10.40 & 0.87 & 0.84 & 37.23 & 0.75 & 0.82 & 1.70 & 0.66 & 16.25 & 3.00 & 100.00 \\
\hline 5 & 10.76 & 0.13 & 0.41 & 6.80 & 0.56 & 2.77 & 8.93 & 0.83 & 0.70 & 45.56 & 0.75 & 0.62 & 1.28 & 0.63 & 16.25 & 3.00 & 100.00 \\
\hline $\begin{array}{c}\text { Average in } \\
\text { area }\end{array}$ & 12.48 & 0.27 & 0.42 & 7.25 & 0.58 & 3.09 & 8.90 & 0.85 & 1.03 & 42.01 & 0.75 & 0.80 & 1.55 & 0.78 & 16.25 & 3.00 & 100.00 \\
\hline Average & 13.36 & 0.13 & 0.42 & 7.77 & 0.65 & 3.20 & 10.04 & 0.81 & 0.83 & 39.86 & 0.75 & 0.77 & 1.46 & 0.70 & 16.25 & 3.00 & 100.00 \\
\hline Er/Re & 5.0 & 28.1 & & 4.4 & 0.9 & 2.2 & 1.1 & & & 15.2 & & 14 & & 0 & & & \\
\hline
\end{tabular}


SRNS-STI-2008-00057

Revision 0

Appendix C (SRNS-OS-2008-00001)

Sample KRI 09

\begin{tabular}{|c|c|c|c|c|c|c|c|c|c|c|c|c|c|c|c|c|c|}
\hline & Al2O3 & $\mathrm{CaO}$ & $\mathrm{Cr} 2 \mathrm{O} 3$ & $\mathrm{Fe} 2 \mathrm{O} 3$ & $\mathrm{~K} 2 \mathrm{O}$ & $\mathrm{MnO}$ & $\mathrm{Na} 2 \mathrm{O}$ & $\mathrm{NiO}$ & $\mathrm{PbO}$ & $\mathrm{SiO}$ & $\mathrm{SrO} *$ & $\mathrm{TiO} 2$ & $\mathrm{ZnO}$ & $\mathrm{ZrO} 2$ & $\mathrm{~B} 2 \mathrm{O} 3$ & $\mathrm{Li2O}$ & Total \\
\hline 1 & 15.14 & 0.13 & 0.28 & 7.77 & 0.23 & 1.10 & 11.7 & 0.48 & 0.82 & 39.21 & 0.75 & 0.95 & 1.67 & 2.45 & 14.3 & 3 & 100 \\
\hline 2 & 16.09 & 0.13 & 0.27 & 8.24 & 0.23 & 1.22 & 12.5 & 0.48 & 0.82 & 37.08 & 0.75 & 0.79 & 1.53 & 2.56 & 14.3 & 3 & 100 \\
\hline 3 & 15.72 & 0.13 & 0.28 & 8.33 & 0.34 & 1.10 & 11.86 & 0.48 & 1.03 & 37.48 & 0.75 & 0.8 & 1.67 & 2.71 & 14.3 & 3 & 100 \\
\hline 4 & 16.06 & 0.27 & 0.28 & 7.63 & 0.23 & 1.10 & 12.22 & 0.6 & 1.03 & 37.42 & 0.75 & 0.95 & 1.55 & 2.58 & 14.3 & 3 & 100 \\
\hline 5 & 14.62 & 0.14 & 0.29 & 7.97 & 0.24 & 1.13 & 10.03 & 0.62 & 0.84 & 41.12 & 0.75 & 0.82 & 1.46 & 2.65 & 14.3 & 3 & 100 \\
\hline Average in area & 14.26 & 0.13 & 0.278 & 7.54 & 0.23 & 1.09 & 11.18 & 0.6 & 1.12 & 40.78 & 0.75 & 0.78 & 1.41 & 2.55 & 14.3 & 3 & 100 \\
\hline Average & 15.53 & 0.16 & 0.281 & 7.99 & 0.25 & 1.14 & 11.66 & 0.54 & 0.91 & 38.46 & 0.75 & 0.86 & 1.57 & 2.59 & 14.3 & 3 & 100 \\
\hline Er/Re & 2.1 & 18.3 & & 2.1 & 0.7 & 0.4 & 1.4 & & & 8 & & 14.3 & & 0.4 & & & \\
\hline Spinel 1 & 3.84 & 0 & 7.35 & 53.81 & 0 & 5.25 & 0 & 21 & 0 & 1.15 & 0 & 0.71 & 6.66 & 0 & 0 & 0 & 99.74 \\
\hline Spinel 2 & 3.97 & 0 & 8.04 & 53.29 & 0 & 4.52 & 0 & 20.4 & 0 & 1.07 & 0 & 0.83 & 7.22 & 0 & 0 & 0 & 99.3 \\
\hline Spinel 3 & 3.78 & 0 & 3.51 & 57.86 & 0 & 4.91 & 0 & 21.6 & 0 & 0.86 & 0 & 0.83 & 5.35 & 0 & 0 & 0 & 98.73 \\
\hline
\end{tabular}

\section{Sample KRI 10}

\begin{tabular}{|c|c|c|c|c|c|c|c|c|c|c|c|c|c|c|c|c|c|}
\hline & $\mathrm{Al} 2 \mathrm{O} 3$ & $\mathrm{CaO}$ & $\mathrm{Cr} 2 \mathrm{O} 3$ & $\mathrm{Fe} 2 \mathrm{O} 3$ & $\mathrm{~K} 2 \mathrm{O}$ & $\mathrm{MnO}$ & $\mathrm{Na} 2 \mathrm{O}$ & $\mathrm{NiO}$ & $\mathrm{PbO}$ & $\mathrm{SiO} 2$ & $\mathrm{SrO} *$ & $\mathrm{TiO} 2$ & $\mathrm{ZnO}$ & $\mathrm{ZrO} 2$ & $\mathrm{~B} 2 \mathrm{O} 3$ & $\mathrm{Li} 2 \mathrm{O}$ & Total \\
\hline 1 & 13.27 & 0.52 & 0.409 & 7.3931 & 0.67 & 1.197 & 12.32 & 0.82 & 0 & 47.67 & 1.79 & 0.31 & 1.15 & 0 & 9.38 & 3.11 & 100 \\
\hline 2 & 13.3 & 0.65 & 0.412 & 7.5083 & 0.68 & 1.094 & 12.9 & 0.71 & 0 & 47.01 & 1.8 & 0.16 & 1.28 & 0 & 9.38 & 3.11 & 100 \\
\hline 3 & 12.944 & 0.67 & 0.554 & 7.6109 & 0.69 & 1.23 & 12.18 & 0.6 & 0 & 48.05 & 1.8 & 0 & 1.18 & 0 & 9.38 & 3.11 & 100 \\
\hline 4 & 12.877 & 0.53 & 0.415 & 7.7042 & 0.57 & 1.225 & 12.24 & 0.72 & 0.61 & 46.8 & 1.81 & 0.31 & 1.06 & 0.64 & 9.38 & 3.11 & 100 \\
\hline 5 & 12.325 & 0.65 & 0.409 & 7.4527 & 0.56 & 1.086 & 12.42 & 0.71 & 0.6 & 47.66 & 1.78 & 0.31 & 0.93 & 0.63 & 9.38 & 3.11 & 100 \\
\hline Average in area & 13.111 & 0.54 & 0.427 & 7.7118 & 0.69 & 1.241 & 12.08 & 0.73 & 0.31 & 47.25 & 1.78 & 0.16 & 0.96 & 0.52 & 9.38 & 3.11 & 100 \\
\hline Average & 12.946 & 0.6 & 0.442 & 7.5267 & 0.63 & 1.164 & 12.41 & 0.71 & 0.24 & 47.44 & 1.8 & 0.22 & 1.12 & 0.25 & 9.38 & 3.11 & 100 \\
\hline Spinel & 1.89 & nd & 33.63 & 3.93 & 27.9 & nd & 4.78 & nd & 16.7 & nd & 0.86 & nd & nd & 7.97 & nd & 97.66 & \\
\hline Er/Re & 1.3 & & & 0.5 & 0.9 & 0.6 & 0.5 & & & 3.3 & & 24 & & 1.5 & & & \\
\hline
\end{tabular}

\section{Sample KRI 11}

\begin{tabular}{|c|c|c|c|c|c|c|c|c|c|c|c|c|c|c|c|c|c|c|}
\hline & Al2O3 & $\mathrm{CaO}$ & $\mathrm{Cr} 2 \mathrm{O} 3$ & $\mathrm{Fe} 2 \mathrm{O} 3$ & $\mathrm{~K} 2 \mathrm{O}$ & $\mathrm{MnO}$ & $\mathrm{Na} 2 \mathrm{O}$ & $\mathrm{NiO}$ & $\mathrm{SO} 3$ & $\mathrm{SiO} 2$ & $\mathrm{TiO} 2$ & $\mathrm{ZnO}$ & $\mathrm{ZrO} 2$ & $\mathrm{SrO}$ & $\mathrm{PbO}$ & $\mathrm{B} 2 \mathrm{O} 3$ & Li2O & Total \\
\hline 1 & 15.147 & 0 & 0 & 8.0231 & 0.4 & 0.849 & 11.13 & 0.32 & 0 & 43.16 & 0.29 & 0.41 & 1.12 & 1.08 & 0 & 13.99 & 4.08 & 100 \\
\hline 2 & 17.673 & 0.23 & 0.24 & 10.31 & 0.3 & 1.059 & 11.94 & 0.94 & 0.21 & 35.83 & 0.3 & 0.51 & 1.33 & 1.07 & 0 & 13.99 & 4.08 & 100 \\
\hline 3 & 15.866 & 0.14 & 0 & 8.7026 & 0.4 & 0.959 & 10.2 & 0.31 & 0.41 & 41.58 & 0.26 & 0.41 & 1.33 & 1.36 & 0 & 13.99 & 4.08 & 100 \\
\hline 4 & 16.128 & 0.11 & 0 & 8.8235 & 0.29 & 0.949 & 10.95 & 0.31 & 0.2 & 41.06 & 0.24 & 0.51 & 1.21 & 1.15 & 0 & 13.99 & 4.08 & 100 \\
\hline 5 & 17.649 & 0 & 0 & 9.4043 & 0.4 & 0.969 & 11.46 & 0.42 & 0 & 38.74 & 0.28 & 0.52 & 1.01 & 1.08 & 0 & 13.99 & 4.08 & 100 \\
\hline Average & 16.492 & 0.1 & 0.05 & 9.0501 & 0.36 & 0.959 & 11.14 & 0.46 & 0.16 & 40.07 & 0.28 & 0.47 & 1.2 & 1.15 & 0 & 13.99 & 4.08 & 100 \\
\hline Average in area & 15.969 & 0 & 0.299 & 9.0169 & 0.49 & 0.918 & 10.15 & 0.52 & 0 & 42.72 & 0.17 & 0 & 0.96 & 0.72 & 0 & 13.99 & 4.08 & 100 \\
\hline Er/Re & 3.41 & & & 3.43 & & & 0.54 & & & 7.15 & & & & & & & & \\
\hline
\end{tabular}


SRNS-STI-2008-00057

Revision 0

Appendix C (SRNS-OS-2008-00001)

Sample KRI 12

\begin{tabular}{|c|c|c|c|c|c|c|c|c|c|c|c|c|c|c|c|c|c|}
\hline & $\mathrm{Al} 2 \mathrm{O} 3$ & $\mathrm{CaO}$ & $\mathrm{Cr} 2 \mathrm{O} 3$ & $\mathrm{Fe} 2 \mathrm{O} 3$ & $\mathrm{~K} 2 \mathrm{O}$ & $\mathrm{MnO}$ & $\mathrm{Na} 2 \mathrm{O}$ & $\mathrm{NiO}$ & $\mathrm{SO} 3$ & $\mathrm{SiO} 2$ & $\mathrm{TiO} 2$ & $\mathrm{ZnO}$ & $\mathrm{ZrO} 2$ & $\mathrm{SrO}$ & B2O3 & Li2O & Totql \\
\hline 1 & 13.62 & 0.41 & 0.00 & 7.90 & 0.59 & 3.06 & 10.25 & 0.63 & 0.25 & 39.10 & 0.33 & 0.49 & 2.93 & 2.45 & 14.09 & 3.88 & 100.0 \\
\hline 2 & 11.93 & 0.40 & 0.14 & 7.10 & 0.58 & 2.72 & 9.28 & 0.73 & 0.00 & 43.63 & 0.32 & 0.60 & 2.46 & 2.15 & 14.09 & 3.88 & 100.0 \\
\hline 3 & 13.48 & 0.56 & 0.00 & 7.60 & 0.60 & 2.98 & 7.85 & 0.63 & 0.25 & 41.50 & 0.34 & 0.38 & 3.12 & 2.73 & 14.09 & 3.88 & 100.0 \\
\hline 4 & 13.68 & 0.41 & 0.29 & 8.66 & 0.59 & 3.29 & 9.48 & 1.12 & 0.00 & 38.73 & 0.33 & 0.73 & 2.64 & 2.08 & 14.09 & 3.88 & 100.0 \\
\hline 5 & 12.29 & 0.40 & 0.00 & 7.24 & 0.58 & 2.72 & 8.50 & 0.60 & 0.00 & 44.21 & 0.16 & 0.71 & 2.71 & 1.92 & 14.09 & 3.88 & 100.0 \\
\hline Average & 13.00 & 0.44 & 0.09 & 7.70 & 0.58 & 2.95 & 9.07 & 0.74 & 0.10 & 41.44 & 0.30 & 0.58 & 2.77 & 2.27 & 14.09 & 3.88 & 100.0 \\
\hline $\begin{array}{c}\text { Average } \\
\text { in area }\end{array}$ & 13.07 & 0.55 & 0.00 & 7.37 & 0.58 & 2.89 & 7.61 & 0.62 & 0.25 & 40.24 & 0.33 & 0.37 & 3.02 & 2.65 & 14.09 & 3.88 & - \\
\hline Er/Re & 2.58 & & & 2.54 & & & 0.79 & & & 6.17 & & & & & & & \\
\hline
\end{tabular}

\section{Sample KRI 13}

\begin{tabular}{|c|c|c|c|c|c|c|c|c|c|c|c|c|c|c|c|c|c|}
\hline & $\mathrm{Al} 2 \mathrm{O} 3$ & $\mathrm{Cr} 2 \mathrm{O} 3$ & $\mathrm{Fe} 2 \mathrm{O} 3$ & $\mathrm{~K} 2 \mathrm{O}$ & $\mathrm{MnO}$ & $\mathrm{Na} 2 \mathrm{O}$ & $\mathrm{NiO}$ & $\mathrm{PbO}$ & $\mathrm{SO} 3$ & $\mathrm{SiO} 2$ & $\mathrm{TiO} 2$ & $\mathrm{ZnO}$ & $\mathrm{ZrO} 2$ & $\mathrm{SrO}$ & $\mathrm{B}_{2} \mathrm{O}_{3}$ & $\mathrm{Li}_{2} \mathrm{O}$ & $\mathrm{Total}$ \\
\hline 1 & 12.57 & 0.31 & 7.39 & 0.76 & 2.86 & 12.24 & 0.67 & 0.80 & 0.00 & 43.63 & 0.71 & 1.45 & 1.57 & 1.25 & 10.79 & 3.00 & 100.00 \\
\hline 2 & 13.37 & 0.16 & 7.77 & 0.66 & 3.09 & 12.47 & 0.55 & 1.17 & 0.00 & 41.72 & 0.73 & 1.35 & 1.62 & 1.54 & 10.79 & 3.00 & 100.00 \\
\hline 3 & 12.63 & 0.32 & 8.29 & 0.53 & 2.83 & 12.11 & 0.56 & 0.83 & 0.00 & 43.39 & 0.73 & 1.50 & 1.33 & 1.17 & 10.79 & 3.00 & 100.00 \\
\hline 4 & 13.61 & 0.31 & 7.52 & 0.52 & 2.78 & 12.46 & 0.55 & 0.93 & 0.54 & 42.34 & 0.72 & 1.47 & 1.45 & 1.02 & 10.79 & 3.00 & 100.00 \\
\hline 5 & 12.80 & 0.31 & 7.25 & 0.64 & 2.73 & 12.98 & 0.40 & 0.80 & 0.53 & 43.04 & 0.71 & 1.32 & 1.57 & 1.13 & 10.79 & 3.00 & 100.00 \\
\hline Average & 13.00 & 0.28 & 7.65 & 0.62 & 2.86 & 12.45 & 0.55 & 0.90 & 0.21 & 42.83 & 0.72 & 1.42 & 1.51 & 1.22 & 10.79 & 3.00 & 100.00 \\
\hline Average in area & 13.11 & 0.31 & 7.65 & 0.63 & 2.85 & 12.18 & 0.67 & 0.57 & 0.53 & 42.52 & 0.70 & 1.57 & 1.56 & 1.37 & 10.79 & 3.00 & 100.00 \\
\hline Er/Re & 1.2 & & 1.40 & & & 0.20 & & & & 1.60 & & & & & & & \\
\hline
\end{tabular}

\section{Sample KRI 14}

\begin{tabular}{|c|c|c|c|c|c|c|c|c|c|c|c|c|c|c|c|c|c|c|}
\hline & Al2O3 & $\mathrm{CaO}$ & $\mathrm{Cr} 2 \mathrm{O} 3$ & $\mathrm{Fe} 2 \mathrm{O} 3$ & $\mathrm{~K} 2 \mathrm{O}$ & $\mathrm{MnO}$ & $\mathrm{Na} 2 \mathrm{O}$ & $\mathrm{NiO}$ & $\mathrm{PbO}$ & $\mathrm{SO} 3$ & $\mathrm{SiO} 2$ & $\mathrm{TiO} 2$ & $\mathrm{ZnO}$ & $\mathrm{ZrO} 2$ & $\mathrm{SrO}$ & $\mathrm{B} 2 \mathrm{O} 3$ & $\mathrm{Li} 2 \mathrm{O}$ & $\mathrm{Total}$ \\
\hline 1 & 12.09 & 0.14 & 0.00 & 6.92 & 0.23 & 2.75 & 9.27 & 0.86 & 0.63 & 0.00 & 42.74 & 0.65 & 1.21 & 0.92 & 1.15 & 15.46 & 5.00 & 100.00 \\
\hline 2 & 13.33 & 0.14 & 0.00 & 7.45 & 0.25 & 3.04 & 10.61 & 0.78 & 0.88 & 0.00 & 38.74 & 0.85 & 1.27 & 1.10 & 1.09 & 15.46 & 5.00 & 100.00 \\
\hline 3 & 13.58 & 0.14 & 0.30 & 7.77 & 0.25 & 2.92 & 8.99 & 1.18 & 0.66 & 0.51 & 38.45 & 0.69 & 1.53 & 1.25 & 1.33 & 15.46 & 5.00 & 100.00 \\
\hline 4 & 13.45 & 0.14 & 0.30 & 7.41 & 0.24 & 3.02 & 9.59 & 0.91 & 0.77 & 0.00 & 39.62 & 0.68 & 1.52 & 0.69 & 1.20 & 15.46 & 5.00 & 100.00 \\
\hline 5 & 12.03 & 0.28 & 0.44 & 6.96 & 0.24 & 2.57 & 9.11 & 0.89 & 0.75 & 0.00 & 41.95 & 0.83 & 0.99 & 1.21 & 1.29 & 15.46 & 5.00 & 100.00 \\
\hline Average & 12.90 & 0.17 & 0.21 & 7.30 & 0.24 & 2.86 & 9.51 & 0.92 & 0.74 & 0.10 & 40.30 & 0.74 & 1.30 & 1.03 & 1.21 & 15.46 & 5.00 & 100.00 \\
\hline $\begin{array}{c}\text { Average in } \\
\text { area }\end{array}$ & 13.00 & 0.00 & 0.28 & 7.33 & 0.23 & 2.75 & 9.66 & 0.86 & 0.73 & 0.48 & 39.81 & 0.65 & 1.21 & 1.18 & 1.37 & 15.46 & 5.00 & 100.00 \\
\hline Er/Re & 1.9 & & & 1.2 & & & 1.7 & & & & 3.9 & & & & & & & \\
\hline
\end{tabular}

\section{Sample KRI 15}

\begin{tabular}{|c|c|c|c|c|c|c|c|c|c|c|c|c|c|c|c|c|c|}
\hline & $\mathrm{Al}_{2} \mathrm{O}_{3}$ & $\mathrm{Cr}_{2} \mathrm{O}_{3}$ & $\mathrm{Fe}_{2} \mathrm{O}_{3}$ & $\mathrm{~K}_{2} \mathrm{O}$ & $\mathrm{MnO}$ & $\mathrm{Na}_{2} \mathrm{O}$ & $\mathrm{NiO}$ & $\mathrm{PbO}$ & $\mathrm{SO}_{3}$ & $\mathrm{SiO}_{2}$ & $\mathrm{TiO}_{2}$ & $\mathrm{ZnO}_{2}$ & $\mathrm{ZrO}_{2}$ & $\mathrm{SrO}$ & $\mathrm{B}_{2} \mathrm{O}_{3}$ & $\mathrm{Li}_{2} \mathrm{O}$ & $\mathrm{Total}$ \\
\hline 1 & 17.03 & 0.00 & 8.77 & 0.49 & 1.19 & 10.48 & 0.78 & 0.66 & 0.26 & 39.67 & 0.17 & 0.64 & 1.38 & 1.33 & 13.68 & 3.32 & 100.00 \\
\hline 2 & 17.58 & 0.00 & 8.34 & 0.73 & 1.18 & 11.45 & 0.77 & 0.65 & 0.00 & 39.19 & 0.34 & 0.50 & 0.96 & 1.31 & 13.68 & 3.32 & 100.00 \\
\hline 3 & 19.17 & 0.31 & 9.95 & 0.63 & 1.22 & 10.71 & 0.67 & 0.00 & 0.00 & 37.38 & 0.00 & 0.65 & 0.85 & 1.48 & 13.68 & 3.32 & 100.00 \\
\hline 4 & 12.81 & 0.29 & 7.48 & 0.71 & 1.02 & 9.40 & 0.62 & 0.53 & 0.00 & 47.32 & 0.00 & 0.49 & 1.06 & 1.28 & 13.68 & 3.32 & 100.00 \\
\hline 5 & 17.96 & 0.00 & 8.65 & 0.49 & 1.04 & 12.39 & 0.51 & 0.00 & 0.25 & 38.30 & 0.34 & 0.50 & 1.23 & 1.19 & 13.68 & 3.32 & 100.00 \\
\hline Average & 16.91 & 0.12 & 8.64 & 0.61 & 1.13 & 10.89 & 0.67 & 0.37 & 0.10 & 40.37 & 0.17 & 0.56 & 1.10 & 1.32 & 13.68 & 3.32 & 100.00 \\
\hline $\begin{array}{c}\text { Average } \\
\text { in area }\end{array}$ & 15.57 & 0.29 & 8.44 & 0.61 & 1.17 & 11.24 & 0.64 & 0.00 & 0.50 & 41.52 & 0.00 & 0.50 & 1.09 & 1.43 & 13.68 & 3.32 & 100.00 \\
\hline Er/Re & 6.1 & & 3.0 & & & 0.7 & & & & 8.0 & & & & & & & \\
\hline
\end{tabular}


SRNS-STI-2008-00057

Revision 0

Appendix C (SRNS-OS-2008-00001)

\section{Sample KRI 16}

\begin{tabular}{|c|c|c|c|c|c|c|c|c|c|c|c|c|c|c|c|c|c|c|}
\hline & $\mathrm{Al} 2 \mathrm{O} 3$ & $\mathrm{CaO}$ & $\mathrm{Cr} 2 \mathrm{O} 3$ & $\mathrm{Fe} 2 \mathrm{O} 3$ & $\mathrm{~K} 2 \mathrm{O}$ & $\mathrm{MnO}$ & $\mathrm{Na} 2 \mathrm{O}$ & $\mathrm{NiO}$ & $\mathrm{PbO}$ & $\mathrm{SO} 3$ & $\mathrm{SiO} 2$ & $\mathrm{TiO}$ & $\mathrm{ZnO}$ & $\mathrm{ZrO} 2$ & $\mathrm{SrO} *$ & $\mathrm{~B} 2 \mathrm{O}$ * & Li2O* & Total \\
\hline 1 & 13.32 & 0.12 & 0.25 & 7.83 & 0.21 & 1.01 & 9.87 & 0.66 & 0.75 & 0.43 & 41.16 & 0.73 & 1.41 & 2.58 & 2.25 & 12.50 & 4.92 & 100.00 \\
\hline 2 & 13.25 & 0.14 & 0.00 & 8.27 & 0.12 & 1.18 & 9.24 & 0.65 & 0.77 & 0.51 & 41.11 & 0.85 & 1.64 & 2.61 & 2.25 & 12.50 & 4.92 & 100.00 \\
\hline 3 & 11.68 & 0.13 & 0.28 & 7.47 & 0.23 & 0.86 & 8.89 & 0.73 & 0.51 & 0.48 & 44.18 & 0.63 & 1.30 & 2.95 & 2.25 & 12.50 & 4.92 & 100.00 \\
\hline 4 & 12.77 & 0.13 & 0.27 & 7.51 & 0.23 & 0.97 & 10.53 & 0.72 & 0.71 & 0.47 & 41.20 & 0.63 & 1.40 & 2.79 & 2.25 & 12.50 & 4.92 & 100.00 \\
\hline 5 & 13.13 & 0.27 & 0.28 & 7.89 & 0.11 & 1.11 & 10.08 & 0.73 & 0.82 & 0.48 & 40.55 & 0.64 & 1.42 & 2.83 & 2.25 & 12.50 & 4.92 & 100.00 \\
\hline $\begin{array}{c}\text { Average } \\
\text { in area }\end{array}$ & 12.10 & 0.12 & 0.26 & 7.51 & 0.21 & 1.02 & 8.74 & 0.78 & 0.94 & 0.66 & 42.99 & 0.73 & 1.42 & 2.84 & 2.25 & 12.50 & 4.92 & 100.00 \\
\hline Average & 12.83 & 0.16 & 0.22 & 7.79 & 0.18 & 1.03 & 9.72 & 0.70 & 0.71 & 0.47 & 41.64 & 0.69 & 1.44 & 2.75 & 2.25 & 12.50 & 4.92 & 100.00 \\
\hline Er/Re & 2.2 & & & 1.4 & & & 0.5 & & & & 3.4 & & & & & & & \\
\hline
\end{tabular}

\section{Sample KRI-17}

\begin{tabular}{|c|c|c|c|c|c|c|c|c|c|c|c|c|c|c|c|c|}
\hline & $\mathrm{Al} 2 \mathrm{O} 3$ & $\mathrm{CaO}$ & $\mathrm{Cr} 2 \mathrm{O} 3$ & $\mathrm{Fe} 2 \mathrm{O} 3$ & $\mathrm{~K} 2 \mathrm{O}$ & $\mathrm{MnO}$ & $\mathrm{Na} 2 \mathrm{O}$ & $\mathrm{NiO}$ & $\mathrm{SiO} 2$ & $\mathrm{SrO} *$ & $\mathrm{TiO} 2$ & $\mathrm{ZnO}$ & $\mathrm{ZrO} 2$ & $\mathrm{~B} 2 \mathrm{O} *$ & Li2O* & Total \\
\hline 1 & 12.25 & 0.41 & 0.29 & 7.85 & 0.35 & 1.78 & 9.26 & 0.50 & 45.61 & 2.25 & 0.16 & 0.49 & 2.52 & 12.40 & 3.88 & 100.00 \\
\hline 2 & 11.48 & 0.41 & 0.00 & 7.56 & 0.35 & 1.65 & 9.77 & 0.50 & 46.58 & 2.25 & 0.16 & 0.49 & 2.51 & 12.40 & 3.88 & 100.00 \\
\hline 3 & 11.21 & 0.54 & 0.28 & 7.38 & 0.23 & 1.73 & 11.35 & 0.49 & 45.89 & 2.25 & 0.16 & 0.36 & 2.58 & 12.40 & 3.88 & 100.00 \\
\hline 4 & 13.67 & 0.56 & 0.15 & 9.04 & 0.36 & 2.08 & 10.01 & 0.51 & 41.71 & 2.25 & 0.17 & 0.50 & 2.71 & 12.40 & 3.88 & 100.00 \\
\hline 5 & 11.97 & 0.54 & 0.00 & 7.79 & 0.35 & 1.76 & 9.19 & 0.37 & 46.08 & 2.25 & 0.16 & 0.48 & 2.76 & 12.40 & 3.88 & 100.00 \\
\hline Average & 12.12 & 0.49 & 0.14 & 7.92 & 0.33 & 1.80 & 9.92 & 0.47 & 45.18 & 2.25 & 0.16 & 0.46 & 2.62 & 12.40 & 3.88 & \\
\hline $\begin{array}{c}\text { Average } \\
\text { in area }\end{array}$ & 13.34 & 0.564 & 0 & 8.5 & 0.24 & 1.82 & 10.32 & 0.4 & 43.17 & 2.25 & 0.17 & 0.5 & 2.45 & 12.40 & 3.88 & 100.00 \\
\hline Er/Re & 2.9 & & & 2.6 & & & 0.7 & & 5.9 & & & & & & & \\
\hline
\end{tabular}

\section{Sample KRI-18}

\begin{tabular}{|c|c|c|c|c|c|c|c|c|c|c|c|c|c|c|c|c|}
\hline & $\mathrm{Al} 2 \mathrm{O} 3$ & $\mathrm{CaO}$ & Cr2O3 & $\mathrm{Fe} 2 \mathrm{O} 3$ & K2O & $\mathrm{MnO}$ & $\mathrm{Na} 2 \mathrm{O}$ & $\mathrm{NiO}$ & $\mathrm{SiO} 2$ & $\mathrm{TiO} 2$ & $\mathrm{ZnO}$ & $\mathrm{ZrO} 2$ & $\mathrm{SrO}$ & B2O3 & $\mathrm{Li} 2 \mathrm{O}$ & Сумма \\
\hline 1 & 4.22 & 0.00 & 0.00 & 3.57 & 0.00 & 0.58 & 6.13 & 0.00 & 64.02 & 0.30 & 0.89 & 1.20 & 0.74 & 14.83 & 3.52 & 100.0 \\
\hline 2 & 14.79 & 14 & 0.30 & 7.84 & 0.25 & 1.05 & \begin{tabular}{|l|}
11.78 \\
\end{tabular} & 0.52 & 40.03 & 0.85 & 1.52 & 2.61 & 0.00 & 14.83 & 3.52 & 100.0 \\
\hline 3 & 5.79 & 14 & 0.30 & 8.00 & 0.12 & 1.05 & 12.21 & 0.64 & 37.07 & 0.85 & 1.64 & 2.75 & 1.08 & 14.83 & 3.52 & 100.0 \\
\hline 4 & 8.19 & 0.00 & 0.14 & 5.12 & 0.23 & 0.85 & 9.39 & 0.36 & 53.85 & 0.32 & 1.17 & 2.04 & 0.00 & 14.83 & 3.52 & 100.0 \\
\hline 5 & 16.35 & 0.14 & 0.31 & 8.18 & 0.25 & 1.08 & \begin{tabular}{|l|}
12.08 \\
\end{tabular} & 0.53 & 37.50 & 0.87 & 1.69 & 2.67 & 0.00 & 14.83 & 3.52 & 100.0 \\
\hline Average & 11.87 & 0.08 & 0.21 & 6.54 & 0.17 & 0.92 & 10.32 & 0.41 & 46.49 & 0.64 & 1.38 & 2.25 & 0.36 & 14.83 & 3.52 & \\
\hline $\begin{array}{c}\text { Average in } \\
\text { area }\end{array}$ & 14.81 & 0.14 & 0.30 & 8.00 & 0.25 & 1.05 & 10.84 & 0.64 & 39.25 & 0.85 & 1.64 & 3.03 & 0.84 & 14.83 & 3.52 & 100.0 \\
\hline $\mathrm{Er} / \mathrm{Re}$ & 16.25 & & & 8.3 & & & 2.16 & & 30.31 & & & & & & & \\
\hline
\end{tabular}

\section{Sample KRI 19}

\begin{tabular}{|c|c|c|c|c|c|c|c|c|c|c|c|c|c|c|c|c|}
\hline & $\mathrm{Al} 2 \mathrm{O} 3$ & $\mathrm{CaO}$ & $\mathrm{Cr} 2 \mathrm{O} 3$ & $\mathrm{Fe} 2 \mathrm{O} 3$ & $\mathrm{~K} 2 \mathrm{O}$ & $\mathrm{MnO}$ & $\mathrm{Na} 2 \mathrm{O}$ & $\mathrm{NiO}$ & $\mathrm{SiO} 2$ & $\mathrm{TiO} 2$ & $\mathrm{ZnO}$ & $\mathrm{ZrO} 2$ & $\mathrm{SrO}^{*}$ & B2O3* & Li2O* & Total \\
\hline 1 & 13.65 & 0.00 & 0.00 & 9.21 & 0.23 & 2.74 & 13.92 & 0.61 & 38.96 & 0.64 & 0.60 & 1.69 & 2.25 & 11.12 & 4.37 & 100.00 \\
\hline 2 & 13.24 & 0.14 & 0.28 & 9.03 & 0.23 & 3.02 & 13.70 & 0.99 & 39.79 & 0.65 & 0.00 & 1.18 & 2.25 & 11.12 & 4.37 & 100.00 \\
\hline 3 & 13.47 & 0.27 & 0.29 & 9.48 & 0.24 & 2.77 & 12.66 & 0.75 & 39.72 & 0.81 & 0.49 & 1.32 & 2.25 & 11.12 & 4.37 & 100.00 \\
\hline 4 & 13.78 & 0.00 & 0.14 & 9.01 & 0.24 & 2.80 & 13.76 & 0.88 & 39.67 & 0.66 & 0.00 & 1.33 & 2.25 & 11.12 & 4.37 & 100.00 \\
\hline 5 & 13.50 & 0.27 & 0.00 & 8.80 & 0.24 & 3.03 & 13.78 & 0.87 & 39.17 & 0.65 & 0.49 & 1.45 & 2.25 & 11.12 & 4.37 & 100.00 \\
\hline $\begin{array}{c}\text { Average in } \\
\text { area }\end{array}$ & 13.84 & 0.14 & 0.14 & 9.33 & 0.24 & 2.94 & 12.82 & 0.76 & 39.18 & 0.66 & 0.62 & 1.60 & 2.25 & 11.12 & 4.37 & 100.00 \\
\hline Average & 13.53 & 0.14 & 0.14 & 9.11 & 0.23 & 2.87 & 13.56 & 0.82 & 39.46 & 0.68 & 0.31 & 1.40 & 2.25 & 11.12 & 4.37 & 100.00 \\
\hline Er/Re & 0.6 & & & 1.1 & & & 0.4 & & 0.8 & & & & & & & \\
\hline
\end{tabular}


SRNS-STI-2008-00057

Revision 0

Appendix C (SRNS-OS-2008-00001)

\section{Sample KRI-20}

\begin{tabular}{|c|c|c|c|c|c|c|c|c|c|c|c|c|c|c|c|c|c|}
\hline & Al2O3 & $\mathrm{CaO}$ & $\mathrm{Cr} 2 \mathrm{O} 3$ & $\mathrm{Fe} 2 \mathrm{O} 3$ & $\mathrm{~K} 2 \mathrm{O}$ & $\mathrm{MnO}$ & $\mathrm{Na} 2 \mathrm{O}$ & $\mathrm{NiO}$ & $\mathrm{PbO}$ & $\mathrm{SiO} 2$ & $\mathrm{SrO} *$ & $\mathrm{TiO} 2$ & $\mathrm{ZnO}$ & $\mathrm{ZrO} 2$ & $\mathrm{~B} 2 \mathrm{O} *^{*}$ & Li2O* & Total \\
\hline 1 & 16.85 & 0.56 & 0.29 & 9.30 & 0.24 & 1.29 & 11.20 & 0.51 & 0.54 & 38.16 & 2.25 & 0.17 & 0.00 & 0.85 & 14.79 & 3.00 & 100.00 \\
\hline 2 & 16.61 & 0.57 & 0.15 & 9.34 & 0.25 & 1.19 & 11.01 & 0.65 & 0.00 & 38.94 & 2.25 & 0.34 & 0.00 & 0.91 & 14.79 & 3.00 & 100.00 \\
\hline 3 & 16.14 & 0.42 & 0.29 & 9.36 & 0.24 & 1.28 & 11.11 & 0.63 & 0.53 & 38.27 & 2.25 & 0.33 & 0.49 & 0.85 & 14.79 & 3.00 & 100.00 \\
\hline 4 & 15.70 & 0.41 & 0.14 & 9.04 & 0.24 & 1.15 & 11.86 & 0.50 & 0.00 & 40.03 & 2.25 & 0.00 & 0.00 & 0.87 & 14.79 & 3.00 & 100.00 \\
\hline 5 & 16.41 & 0.42 & 0.15 & 9.37 & 0.24 & 1.30 & 11.29 & 0.51 & 0.00 & 38.70 & 2.25 & 0.17 & 0.50 & 0.88 & 14.79 & 3.00 & 100.00 \\
\hline Average & 16.34 & 0.47 & 0.2 & 9.28 & 0.24 & 1.24 & 11.3 & 0.56 & 0.21 & 38.82 & 2.3 & 0.2 & 0.2 & 0.873 & 14.79 & 3.00 & \\
\hline Average in area. & 16.17 & 0.54 & 0.00 & 9.44 & 0.12 & 1.13 & 11.79 & 0.62 & 0.00 & 38.71 & 2.25 & 0.32 & 0.36 & 0.77 & 14.79 & 3.00 & 100.00 \\
\hline Er/Re & 1.1 & & & 0.5 & & & 0.4 & & & 2.4 & & & & & & & \\
\hline
\end{tabular}

\section{Sample KRI 21}

\begin{tabular}{|c|c|c|c|c|c|c|c|c|c|c|c|c|c|c|c|c|c|}
\hline & $\mathrm{Al} 2 \mathrm{O} 3$ & $\mathrm{CaO}$ & $\mathrm{Fe} 2 \mathrm{O} 3$ & $\mathrm{~K} 2 \mathrm{O}$ & $\mathrm{MnO}$ & $\mathrm{Na} 2 \mathrm{O}$ & $\mathrm{NiO}$ & $\mathrm{PbO}$ & $\mathrm{SO} 3$ & $\mathrm{SiO} 2$ & $\mathrm{TiO} 2$ & $\mathrm{ZnO}$ & $\mathrm{ZrO} 2$ & $\mathrm{SrO} *$ & $\mathrm{~B} 2 \mathrm{O} *$ & Li2O* & Total \\
\hline 1 & 14.84 & 0.56 & 8.03 & 0.36 & 1.30 & 13.18 & 0.90 & 0.43 & 0.50 & 39.44 & 0.34 & 0.50 & 3.67 & 0.82 & 11.83 & 3.29 & 100.00 \\
\hline 2 & 14.31 & 0.54 & 7.40 & 0.35 & 0.99 & 14.82 & 0.73 & 0.62 & 0.00 & 39.61 & 0.32 & 0.60 & 3.50 & 0.82 & 11.83 & 3.29 & 100.00 \\
\hline 3 & 14.53 & 0.55 & 7.61 & 0.48 & 1.27 & 14.25 & 0.88 & 0.53 & 0.49 & 39.05 & 0.33 & 0.49 & 3.59 & 0.82 & 11.83 & 3.29 & 100.00 \\
\hline 4 & 14.22 & 0.41 & 7.79 & 0.35 & 1.39 & 14.37 & 0.87 & 0.53 & 0.49 & 38.90 & 0.33 & 0.73 & 3.69 & 0.82 & 11.83 & 3.29 & 100.00 \\
\hline 5 & 14.54 & 0.55 & 7.99 & 0.36 & 1.40 & 14.50 & 0.88 & 0.74 & 0.00 & 38.42 & 0.33 & 0.61 & 3.73 & 0.82 & 11.83 & 3.29 & 100.00 \\
\hline Average in area & 14.17 & 0.55 & 7.99 & 0.36 & 1.27 & 14.38 & 1.00 & 0.53 & 0.49 & 38.64 & 0.33 & 0.61 & 3.73 & 0.82 & 11.83 & 3.29 & 100.00 \\
\hline Average & 14.49 & 0.52 & 7.77 & 0.38 & 1.27 & 14.22 & 0.85 & 0.57 & 0.30 & 39.08 & 0.33 & 0.59 & 3.64 & 0.82 & 11.83 & 3.29 & \\
\hline Er/Re & 0.8 & & 1.1 & & & 0.5 & & & & 1.1 & & & & & & & \\
\hline
\end{tabular}

\section{Sample KRI 22}

\begin{tabular}{|c|c|c|c|c|c|c|c|c|c|c|c|c|c|c|c|c|c|}
\hline & $\mathrm{Al} 2 \mathrm{O} 3$ & $\mathrm{CaO}$ & $\mathrm{Cr} 2 \mathrm{O} 3$ & $\mathrm{Fe} 2 \mathrm{O} 3$ & $\mathrm{~K} 2 \mathrm{O}$ & $\mathrm{MnO}$ & $\mathrm{Na} 2 \mathrm{O}$ & $\mathrm{NiO}$ & $\mathrm{SO} 3$ & $\mathrm{SiO} 2$ & $\mathrm{TiO} 2$ & $\mathrm{ZnO}$ & $\mathrm{ZrO} 2$ & $\mathrm{SrO}^{*}$ & B2O3* & Li2O* & Total \\
\hline 1 & 15.47 & 0.13 & 0.00 & 7.66 & 0.11 & 0.97 & 12.17 & 0.84 & 0.47 & 38.47 & 0.79 & \begin{tabular}{|l|l|}
0.59 \\
\end{tabular} & 3.56 & 2.25 & 11.52 & 5.00 & 100.00 \\
\hline 2 & 16.38 & 0.13 & 0.00 & 8.21 & 0.22 & 1.06 & 13.15 & 0.58 & 0.46 & 36.14 & \begin{tabular}{|l|}
0.76 \\
\end{tabular} & 0.57 & 3.57 & 2.25 & 11.52 & 5.00 & 100.00 \\
\hline 3 & 16.17 & 0.27 & 0.00 & 8.14 & 0.23 & 1.10 & 12.93 & 0.85 & 0.48 & 36.22 & \begin{tabular}{|l|}
0.79 \\
\end{tabular} & 0.47 & 3.59 & 2.25 & 11.52 & 5.00 & 100.00 \\
\hline 4 & 16.34 & 0.26 & 0.27 & 8.23 & 0.22 & 1.08 & 13.02 & 0.83 & 0.46 & 35.42 & 0.62 & 0.46 & 4.02 & 2.25 & 11.52 & 5.00 & 100.00 \\
\hline 5 & 16.01 & 0.26 & 0.27 & 8.29 & 0.11 & 1.07 & 13.40 & 0.59 & 0.46 & 36.28 & 0.77 & 0.00 & 3.73 & 2.25 & 11.52 & 5.00 & 100.00 \\
\hline Average in area & 15.29 & 0.26 & 0.27 & 7.92 & 0.23 & 1.09 & 10.55 & 0.72 & 0.47 & 39.66 & 0.63 & 0.70 & 3.43 & 2.25 & 11.52 & 5.00 & 100.00 \\
\hline Average & 16.07 & 0.21 & 0.11 & 8.11 & 0.18 & 1.06 & 12.94 & 0.74 & 0.47 & 36.50 & \begin{tabular}{|l|l|}
0.75 \\
\end{tabular} & \begin{tabular}{|l|}
0.42 \\
\end{tabular} & \begin{tabular}{|l|}
3.69 \\
\end{tabular} & 2.25 & 11.52 & 5.00 & 100.00 \\
\hline $\mathrm{Er} / \mathrm{Re}$ & 1.2 & & & 1.1 & & & 0.4 & & & 2.8 & & & & & & & \\
\hline
\end{tabular}

\section{Sample KRI 23}

\begin{tabular}{|c|c|c|c|c|c|c|c|c|c|c|c|c|c|c|c|c|c|}
\hline & $\mathrm{Al} 2 \mathrm{O} 3$ & $\mathrm{CaO}$ & $\mathrm{Fe} 2 \mathrm{O} 3$ & $\mathrm{~K} 2 \mathrm{O}$ & $\mathrm{MnO}$ & $\mathrm{Na} 2 \mathrm{O}$ & $\mathrm{NiO}$ & $\mathrm{PbO}$ & $\mathrm{SO} 3$ & $\mathrm{SiO} 2$ & $\mathrm{TiO} 2$ & $\mathrm{ZnO}$ & $\mathrm{ZrO} 2$ & $\mathrm{SrO}$ & B2O3* & Li2O & Total \\
\hline 1 & 18.20 & 0.00 & 7.57 & 0.70 & 1.12 & 11.64 & 0.37 & 0.52 & 0.72 & 40.21 & 0.32 & 0.72 & 1.56 & 0.75 & 12.60 & 3.00 & 100.00 \\
\hline 2 & 19.12 & 0.27 & 7.84 & 0.70 & 1.12 & 12.13 & 0.49 & 0.62 & 0.48 & 38.16 & 0.32 & 0.96 & 1.43 & 0.75 & 12.60 & 3.00 & 100.00 \\
\hline 3 & 18.83 & 0.14 & 7.89 & 0.71 & 1.15 & 11.31 & 0.50 & 0.74 & 0.49 & 39.49 & 0.33 & 0.74 & 1.33 & 0.75 & 12.60 & 3.00 & 100.00 \\
\hline 4 & 18.58 & 0.13 & 7.71 & 0.81 & 1.12 & 12.61 & 0.61 & 0.73 & 0.48 & 38.38 & 0.32 & 0.72 & 1.43 & 0.75 & 12.60 & 3.00 & 100.00 \\
\hline 5 & 18.26 & 0.00 & 7.89 & 0.71 & 1.15 & 11.31 & 0.50 & 0.74 & 0.25 & 40.74 & 0.16 & 0.61 & 1.33 & 0.75 & 12.60 & 3.00 & 100.00 \\
\hline Average in area & 17.40 & 0.13 & 8.36 & 0.69 & 1.24 & 10.40 & 0.61 & 0.72 & 0.24 & 40.86 & 0.32 & 0.84 & 1.55 & 0.75 & 12.60 & 3.00 & 99.72 \\
\hline Average & 18.60 & 0.11 & 7.78 & 0.73 & 1.13 & 11.80 & 0.49 & 0.67 & 0.49 & 39.40 & 0.29 & 0.75 & 1.42 & 0.75 & 12.60 & 3.00 & 100.00 \\
\hline Er/Re & 1.3 & & 0.6 & & & 0.5 & & & & 2.6 & & & & & & & \\
\hline
\end{tabular}


SRNS-STI-2008-00057

Revision 0

Appendix C (SRNS-OS-2008-00001)

\section{Sample KRI24}

\begin{tabular}{|c|c|c|c|c|c|c|c|c|c|c|c|c|c|c|c|c|}
\hline & $\mathrm{Al} 2 \mathrm{O} 3$ & $\mathrm{CaO}$ & $\mathrm{Cr} 2 \mathrm{O} 3$ & $\mathrm{Fe} 2 \mathrm{O} 3$ & $\mathrm{~K} 2 \mathrm{O}$ & $\mathrm{MnO}$ & $\mathrm{Na} 2 \mathrm{O}$ & $\mathrm{NiO}$ & $\mathrm{SiO} 2$ & $\mathrm{SrO} *$ & $\mathrm{TiO} 2$ & $\mathrm{ZnO}$ & $\mathrm{ZrO} 2$ & $\mathrm{~B}^{2 O}{ }^{*}$ & Li2O & Total \\
\hline 1 & 13.65 & 0.42 & 0.15 & 8.88 & 0.60 & 2.07 & 11.08 & 0.64 & 43.38 & 1.18 & 0.50 & 1.00 & 2.98 & 9.69 & 3.77 & 100.00 \\
\hline 2 & 14.16 & 0.57 & 0.00 & 8.68 & 0.61 & 2.22 & 9.96 & 0.52 & 44.23 & 1.18 & 0.68 & 1.01 & 2.74 & 9.69 & 3.77 & 100.00 \\
\hline 3 & 13.92 & 0.42 & 0.15 & 8.22 & 0.61 & 2.09 & 10.74 & 0.51 & 44.72 & 1.18 & 0.51 & 0.88 & 2.59 & 9.69 & 3.77 & 100.00 \\
\hline 4 & 13.48 & 0.57 & 0.00 & 8.00 & 0.61 & 1.97 & 9.33 & 0.39 & 46.45 & 1.18 & 0.51 & 1.01 & 3.03 & 9.69 & 3.77 & 100.00 \\
\hline 5 & 12.52 & 0.43 & 0.00 & 7.86 & 0.74 & 1.84 & 8.79 & 0.52 & 48.42 & 1.18 & 0.34 & 0.89 & 3.03 & 9.69 & 3.77 & 100.00 \\
\hline Average & 13.55 & 0.48 & 0.06 & 8.33 & 0.63 & 2.04 & 9.98 & 0.51 & 45.44 & 1.18 & 0.51 & 0.96 & 2.87 & 9.69 & 3.77 & 100.00 \\
\hline Average in area & 12.21 & 0.42 & 0.29 & 7.81 & 0.72 & 2.05 & 9.78 & 0.51 & 47.66 & 1.18 & 0.50 & 0.74 & 2.68 & 9.69 & 3.77 & 100.00 \\
\hline Er/Re & 2.3 & & & 2 & & & & 0.8 & & & & 4.5 & & & & \\
\hline
\end{tabular}

\section{Sample KRI 25}

\begin{tabular}{|c|c|c|c|c|c|c|c|c|c|c|c|c|c|c|c|c|}
\hline & Al2O3 & $\mathrm{CaO}$ & $\mathrm{Cr} 2 \mathrm{O} 3$ & $\mathrm{Fe} 2 \mathrm{O} 3$ & $\mathrm{~K} 2 \mathrm{O}$ & $\mathrm{MnO}$ & $\mathrm{Na} 2 \mathrm{O}$ & $\mathrm{NiO}$ & $\mathrm{SiO} 2$ & $\mathrm{SrO} *$ & $\mathrm{TiO} 2$ & $\mathrm{ZnO}$ & $\mathrm{ZrO} 2$ & $\mathrm{~B} 2 \mathrm{O} *$ & $\mathrm{Li} 2 \mathrm{O}$ & $\mathrm{Total}$ \\
\hline 1 & 16.02 & 0.28 & 0.15 & 10.68 & 0.60 & 1.16 & 11.56 & 0.38 & 40.78 & 0.75 & 0.00 & 0.37 & 0.00 & 13.51 & 3.75 & 100.00 \\
\hline 2 & 13.77 & 0.14 & 0.00 & 9.56 & 0.71 & 1.14 & 9.42 & 0.50 & 45.52 & 0.75 & 0.16 & 0.00 & 1.06 & 13.51 & 3.75 & 100.00 \\
\hline 3 & 15.65 & 0.00 & 0.00 & 11.11 & 0.48 & 1.16 & 11.83 & 0.00 & 40.79 & 0.75 & 0.17 & 0.00 & 0.81 & 13.51 & 3.75 & 100.00 \\
\hline 4 & 15.88 & 0.14 & 0.00 & 10.41 & 0.61 & 1.18 & 10.36 & 0.26 & 42.03 & 0.75 & 0.17 & 0.00 & 0.96 & 13.51 & 3.75 & 100.00 \\
\hline 5 & 14.66 & 0.14 & 0.00 & 10.38 & 0.47 & 1.14 & 11.51 & 0.37 & 42.25 & 0.75 & 0.00 & 0.00 & 1.06 & 13.51 & 3.75 & 100.00 \\
\hline Average & 15.19 & 0.14 & 0.03 & 10.43 & 0.58 & 1.16 & 10.94 & 0.30 & 42.28 & 0.75 & 0.10 & 0.07 & 0.78 & 13.51 & 3.75 & 100.00 \\
\hline Average in area & 15.60 & 0.14 & 0.00 & 10.82 & 0.59 & 1.13 & 11.78 & 0.37 & 40.76 & 0.75 & 0.32 & 0.48 & 0.00 & 13.51 & 3.75 & 100.00 \\
\hline Er/Re & 2.80 & & & 2.30 & & & & & 0.90 & & & 5.40 & & & & \\
\hline
\end{tabular}

Sample KRI 26

\begin{tabular}{|c|c|c|c|c|c|c|c|c|c|c|c|c|}
\hline & Al2O3 & $\mathrm{CaO}$ & $\mathrm{Fe} 2 \mathrm{O} 3$ & $\mathrm{Na} 2 \mathrm{O}$ & $\mathrm{NiO}$ & $\mathrm{SO} 3$ & $\mathrm{SiO} 2$ & $\mathrm{SrO}$ & $\mathrm{UO} 3$ & $\mathrm{~B} 2 \mathrm{O} 3$ & Li2O & Total \\
\hline 1 & 10.05 & 0.64 & 10.47 & 13.59 & 3.15 & 0.46 & 40.43 & 3.03 & 1.98 & 10.19 & 6.00 & 100.00 \\
\hline 2 & 10.15 & 0.79 & 10.50 & 13.46 & 3.00 & 0.71 & 41.16 & 2.34 & 1.70 & 10.19 & 6.00 & 100.00 \\
\hline 3 & 10.64 & 0.77 & 10.28 & 14.30 & 2.94 & 0.46 & 40.12 & 2.73 & 1.55 & 10.19 & 6.00 & 100.00 \\
\hline 4 & 10.30 & 0.76 & 10.50 & 13.21 & 2.54 & 0.68 & 40.62 & 3.33 & 1.85 & 10.19 & 6.00 & 100.00 \\
\hline 5 & 10.18 & 0.64 & 10.69 & 13.77 & 3.02 & 0.68 & 40.25 & 2.59 & 1.97 & 10.19 & 6.00 & 100.00 \\
\hline Average in area & 10.52 & 0.77 & 10.17 & 13.90 & 2.67 & 0.46 & 40.65 & 2.80 & 1.86 & 10.19 & 6.00 & 100.00 \\
\hline Average & 10.26 & 0.72 & 10.49 & 13.67 & 2.93 & 0.60 & 40.52 & 2.80 & 1.81 & 10.19 & 6.00 & 100.00 \\
\hline Er/Re & 0.7 & & 0.8 & 0.5 & & & 1.3 & & & & & \\
\hline
\end{tabular}

Sample KRI 27

\begin{tabular}{|c|c|c|c|c|c|c|c|c|c|c|c|c|c|c|c|c|}
\hline & $\mathrm{Al} 2 \mathrm{O} 3$ & $\mathrm{CaO}$ & $\mathrm{Cr} 2 \mathrm{O} 3$ & $\mathrm{Fe} 2 \mathrm{O} 3$ & $\mathrm{MnO}$ & $\mathrm{Na} 2 \mathrm{O}$ & $\mathrm{NiO}$ & $\mathrm{PbO}$ & $\mathrm{SO} 3$ & $\mathrm{SiO} 2$ & $\mathrm{TiO} 2$ & $\mathrm{UO} 3$ & $\mathrm{ZrO} 2$ & $\mathrm{~B} 2 \mathrm{O} 3$ & $\mathrm{Li} 2 \mathrm{O}$ & $\mathrm{Total}$ \\
\hline 1 & 10.63 & 0.75 & 0.26 & 9.69 & 0.81 & 10.82 & 0.00 & 1.44 & 0.00 & 36.49 & 0.89 & 0.86 & 4.46 & 17.20 & 5.73 & 100.00 \\
\hline 2 & 10.54 & 0.75 & 0.66 & 10.02 & 0.58 & 10.06 & 0.34 & 0.87 & 0.67 & 35.63 & 1.05 & 1.30 & 4.62 & 17.20 & 5.73 & 100.00 \\
\hline 3 & 9.71 & 0.84 & 0.50 & 9.05 & 0.66 & 10.62 & 0.44 & 1.01 & 0.86 & 35.39 & 0.86 & 1.34 & 4.97 & 17.20 & 5.73 & 100.00 \\
\hline 4 & 10.22 & 0.64 & 0.54 & 9.56 & 0.71 & 10.63 & 0.00 & 1.38 & 0.00 & 37.89 & 1.07 & 0.00 & 4.46 & 17.20 & 5.73 & 100.00 \\
\hline 5 & 10.91 & 0.76 & 0.66 & 9.92 & 0.58 & 9.85 & 0.46 & 1.17 & 0.68 & 35.93 & 1.05 & 0.87 & 4.26 & 17.20 & 5.73 & 100.00 \\
\hline $\begin{array}{c}\text { Average } \\
\text { in area }\end{array}$ & 10.53 & 0.73 & 0.64 & 9.70 & 0.67 & 10.68 & 0.66 & 1.13 & 0.44 & 35.42 & 1.31 & 0.84 & 4.35 & 17.20 & 5.73 & 100.00 \\
\hline Average & 10.40 & 0.75 & 0.52 & 9.65 & 0.67 & 10.39 & 0.25 & 1.18 & 0.44 & 36.27 & 0.99 & 0.87 & 4.55 & 17.20 & 5.73 & 100.00 \\
\hline Er/Re & 1.3 & & & 2.0 & & 0.5 & & & & 3.1 & & & & & & \\
\hline
\end{tabular}


SRNS-STI-2008-00057

Revision 0

Appendix C (SRNS-OS-2008-00001)

Sample KRI 28

\begin{tabular}{|c|c|c|c|c|c|c|c|c|c|c|c|c|c|c|c|c|}
\hline & $\mathrm{Al} 2 \mathrm{O} 3$ & $\mathrm{CaO}$ & $\mathrm{Cr} 2 \mathrm{O} 3$ & $\mathrm{Fe} 2 \mathrm{O} 3$ & $\mathrm{MnO}$ & $\mathrm{Na} 2 \mathrm{O}$ & $\mathrm{NiO}$ & $\mathrm{PbO}$ & $\mathrm{SO} 3$ & $\mathrm{SiO} 2$ & $\mathrm{SrO}$ & $\mathrm{TiO} 2$ & $\mathrm{UO} 3$ & $\mathrm{~B} 2 \mathrm{O} 3$ & Li2O & Total \\
\hline 1 & 18.03 & 0.00 & 0.90 & 5.25 & 4.18 & 18.16 & 0.00 & 1.04 & 0.66 & 36.54 & 0.00 & 0.88 & 2.52 & 6.13 & 5.72 & 100.00 \\
\hline 2 & 17.34 & 0.00 & 0.93 & 4.93 & 4.11 & 16.89 & 0.00 & 1.37 & 0.68 & 37.73 & 0.00 & 1.21 & 2.95 & 6.13 & 5.72 & 100.00 \\
\hline 3 & 17.59 & 0.00 & 0.50 & 4.92 & 4.23 & 18.93 & 0.00 & 1.02 & 0.65 & 36.71 & 0.00 & 1.01 & 2.59 & 6.13 & 5.72 & 100.00 \\
\hline 4 & 17.81 & 0.00 & 0.91 & 5.21 & 4.36 & 18.21 & 0.00 & 1.05 & 0.44 & 36.55 & 0.00 & 1.04 & 2.56 & 6.13 & 5.72 & 100.00 \\
\hline 5 & 17.78 & 0.00 & 1.21 & 4.72 & 4.14 & 18.38 & 0.00 & 1.00 & 0.52 & 36.51 & 0.00 & 1.04 & 2.86 & 6.13 & 5.72 & 100.00 \\
\hline $\begin{array}{c}\text { Average } \\
\text { in area }\end{array}$ & 17.67 & 0.00 & 0.92 & 5.39 & 4.41 & 17.44 & 0.00 & 1.07 & 0.45 & 37.15 & 0.00 & 1.05 & 2.59 & 6.13 & 5.72 & 100.00 \\
\hline Average & 17.71 & 0.00 & 0.89 & 5.01 & 4.20 & 18.11 & 0.00 & 1.10 & 0.59 & 36.81 & 0.00 & 1.03 & 2.70 & 6.13 & 5.72 & 100.00 \\
\hline Er/Re & 0.8 & & & 1.2 & & 1.0 & & & & 1.6 & & & & & & \\
\hline
\end{tabular}

Sample KRI 29

\begin{tabular}{|c|c|c|c|c|c|c|c|c|c|c|c|c|c|c|}
\hline & $\mathrm{Al} 2 \mathrm{O} 3$ & $\mathrm{Fe} 2 \mathrm{O} 3$ & $\mathrm{~K} 2 \mathrm{O}$ & $\mathrm{MgO}$ & $\mathrm{Na} 2 \mathrm{O}$ & $\mathrm{NiO}$ & $\mathrm{PbO}$ & $\mathrm{SiO} 2$ & $\mathrm{SrO}$ & $\mathrm{TiO} 2$ & $\mathrm{UO} 3$ & $\mathrm{~B} 2 \mathrm{O} 3$ & $\mathrm{Li} 2 \mathrm{O}$ & $\mathrm{Total}$ \\
\hline 1 & 14.47 & 5.61 & 0.87 & 0.00 & 20.21 & 3.03 & 1.00 & 35.43 & 1.59 & 1.04 & 3.36 & 11.38 & 2.00 & 100.00 \\
\hline 2 & 13.83 & 5.51 & 0.98 & 0.00 & 19.99 & 3.10 & 0.98 & 35.24 & 2.04 & 1.02 & 3.91 & 11.38 & 2.00 & 100.00 \\
\hline 3 & 13.21 & 5.64 & 0.85 & 0.00 & 19.25 & 3.22 & 1.31 & 35.14 & 2.16 & 1.18 & 4.63 & 11.38 & 2.00 & 100.00 \\
\hline 4 & 13.88 & 5.81 & 0.72 & 0.99 & 20.06 & 2.78 & 1.18 & 34.85 & 1.99 & 0.99 & 3.34 & 11.38 & 2.00 & 100.00 \\
\hline 5 & 14.22 & 5.89 & 0.87 & 0.00 & 19.03 & 3.28 & 1.00 & 35.75 & 1.95 & 1.03 & 3.59 & 11.38 & 2.00 & 100.00 \\
\hline $\begin{array}{c}\text { Average } \\
\text { in area }\end{array}$ & 14.27 & 5.91 & 0.87 & 0.00 & 17.98 & 3.16 & 0.89 & 36.31 & 2.20 & 1.04 & 3.98 & 11.38 & 2.00 & 100.00 \\
\hline Average & 13.92 & 5.69 & 0.86 & 0.20 & 19.71 & 3.08 & 1.09 & 35.28 & 1.95 & 1.05 & 3.76 & 11.38 & 2.00 & 100.00 \\
\hline Er/Re & 1.4 & 0.8 & & & 0.7 & & & 1.0 & & & & & & \\
\hline
\end{tabular}

Sample KRI 30

\begin{tabular}{|c|c|c|c|c|c|c|c|c|c|c|c|c|c|}
\hline & Al2O3 & $\mathrm{CaO}$ & $\mathrm{Fe} 2 \mathrm{O} 3$ & $\mathrm{Na} 2 \mathrm{O}$ & $\mathrm{PbO}$ & $\mathrm{SO} 3$ & $\mathrm{SiO} 2$ & $\mathrm{ZnO}$ & $\mathrm{SrO}$ & $\mathrm{UO} 3$ & $\mathrm{~B} 2 \mathrm{O} 3$ & $\mathrm{Li} 2 \mathrm{O}$ & \\
\hline 1 & 18.60 & 0.55 & 5.06 & 7.03 & 1.06 & 0.25 & 39.82 & 1.71 & 2.09 & 2.72 & 15.30 & 5.79 & 100.00 \\
\hline 2 & 19.61 & 0.55 & 5.22 & 7.85 & 1.06 & 0.49 & 37.65 & 1.97 & 1.63 & 2.85 & 15.30 & 5.79 & 100.00 \\
\hline 3 & 19.60 & 0.56 & 5.47 & 7.06 & 1.08 & 0.50 & 38.16 & 2.13 & 1.43 & 2.90 & 15.30 & 5.79 & 100.00 \\
\hline 4 & 20.03 & 0.55 & 5.38 & 7.48 & 1.07 & 0.74 & 36.48 & 1.97 & 2.46 & 2.74 & 15.30 & 5.79 & 100.00 \\
\hline 5 & 19.72 & 0.55 & 5.34 & 7.30 & 0.95 & 0.49 & 37.30 & 1.96 & 2.44 & 2.84 & 15.30 & 5.79 & 100.00 \\
\hline Average in area & 19.61 & 0.54 & 5.40 & 7.97 & 1.15 & 0.00 & 36.96 & 2.05 & 2.41 & 2.80 & 15.30 & 5.79 & 100.00 \\
\hline Average & 19.51 & 0.55 & 5.29 & 7.34 & 1.05 & 0.50 & 37.88 & 1.95 & 2.01 & 2.81 & 15.30 & 5.79 & 100.00 \\
\hline Er/Re & 4.15 & & 1.05 & 0.57 & & & 6.23 & & & & & & \\
\hline
\end{tabular}


Attachment 2. SEM Photographs of Quenched Glasses

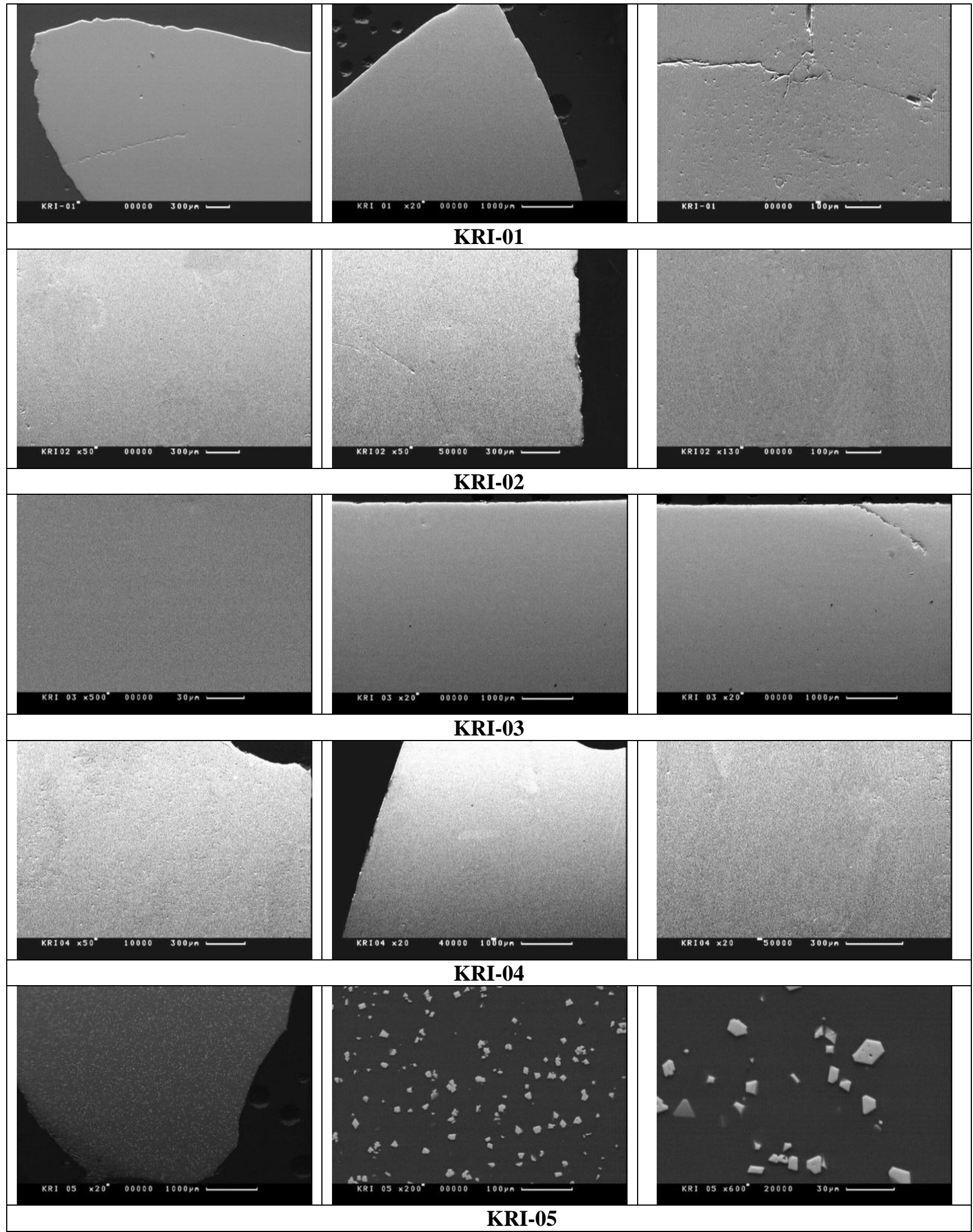


Appendix C (SRNS-OS-2008-00001)

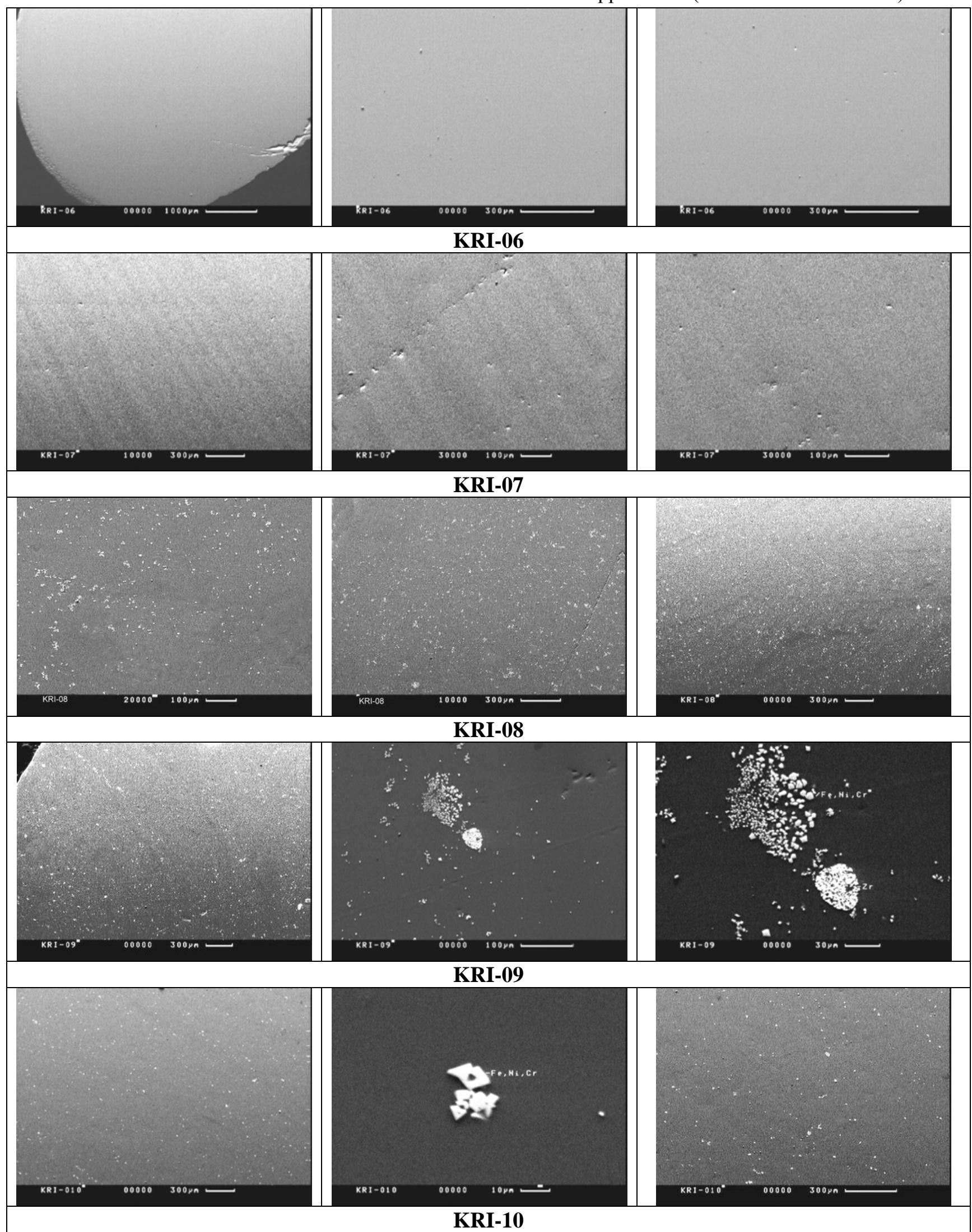


Appendix C (SRNS-OS-2008-00001)

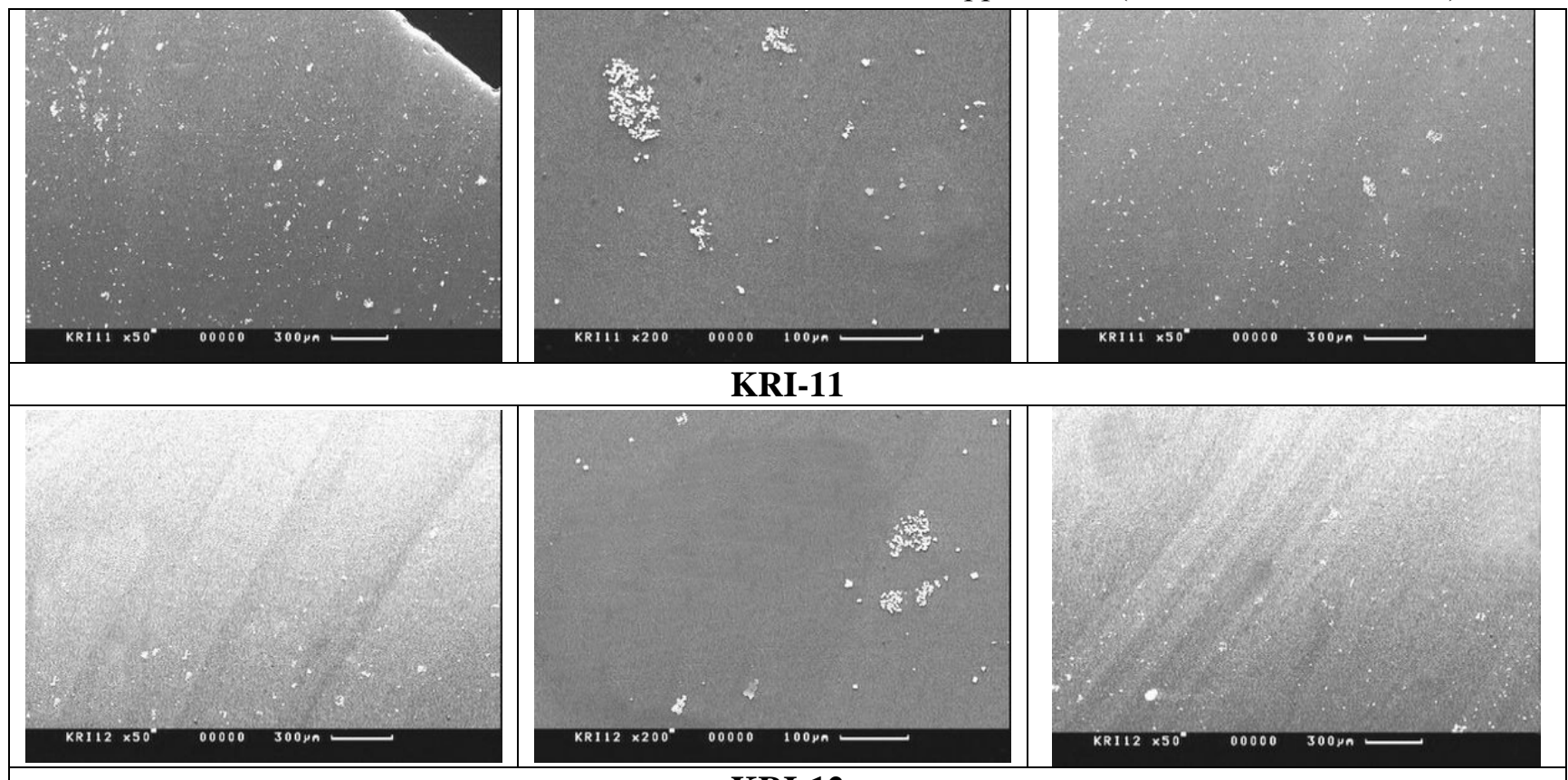

\section{KRI-12}

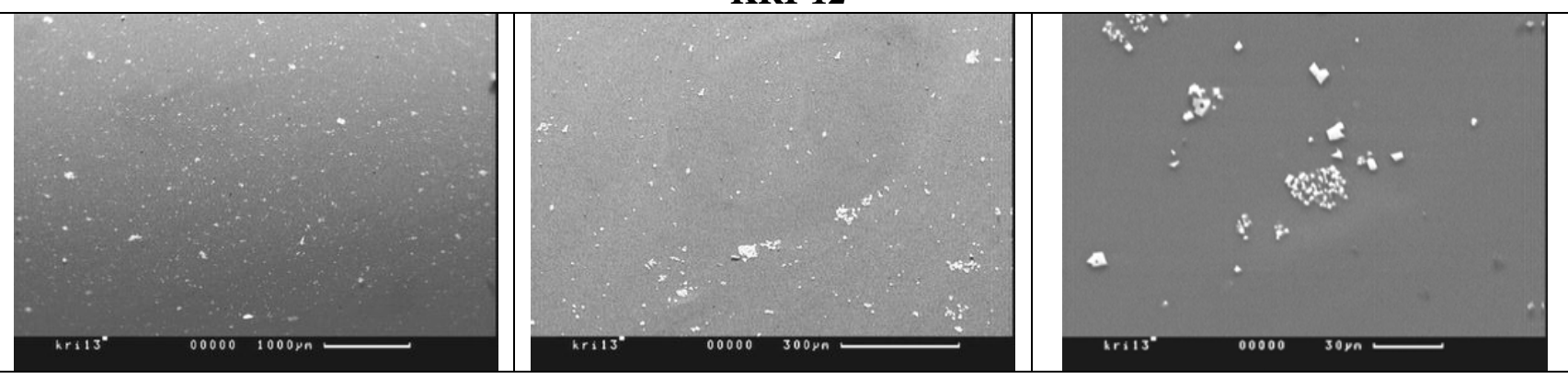

KRI-13

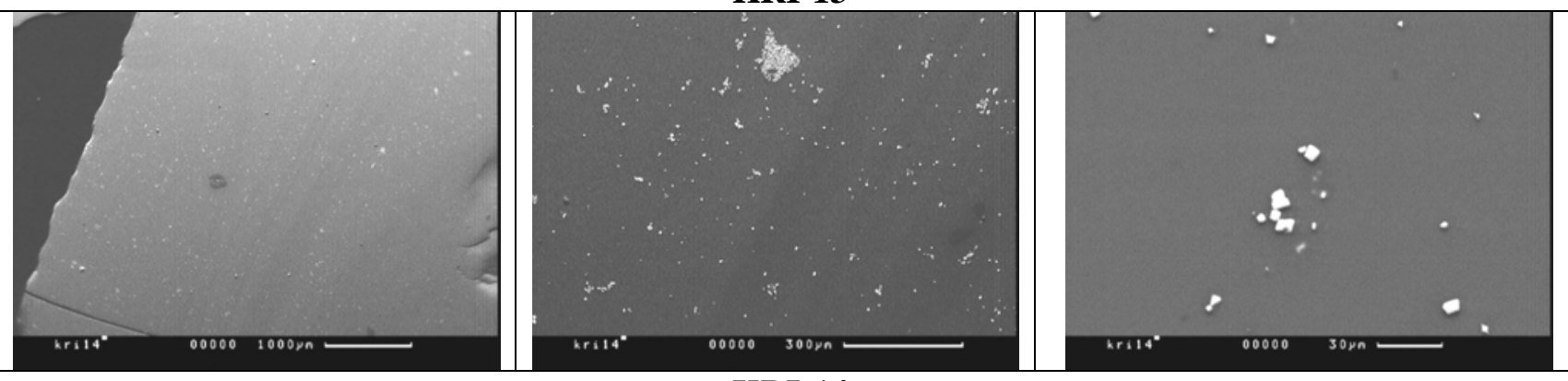

KRI-14
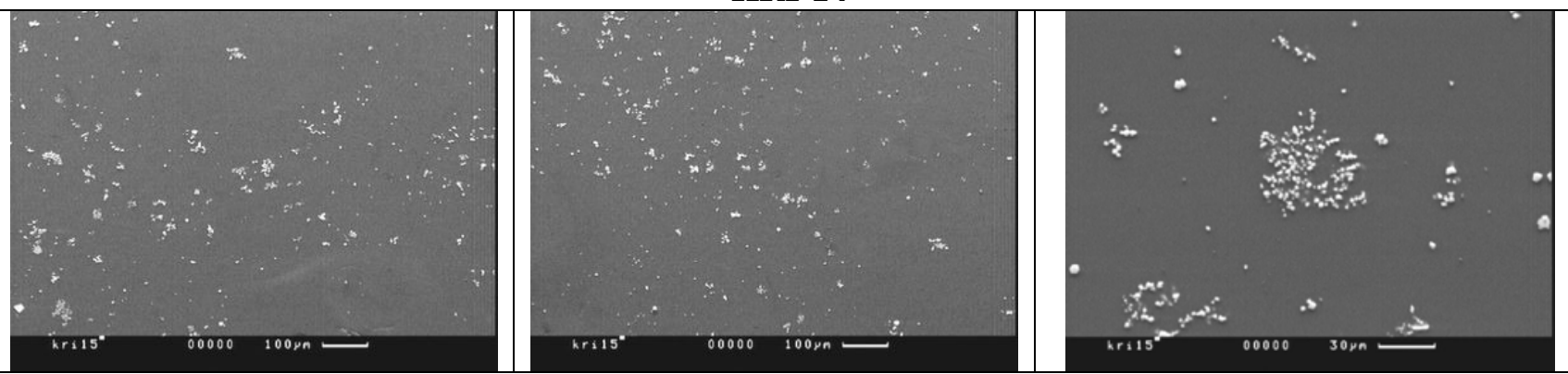

KRI-15 
Appendix C (SRNS-OS-2008-00001)

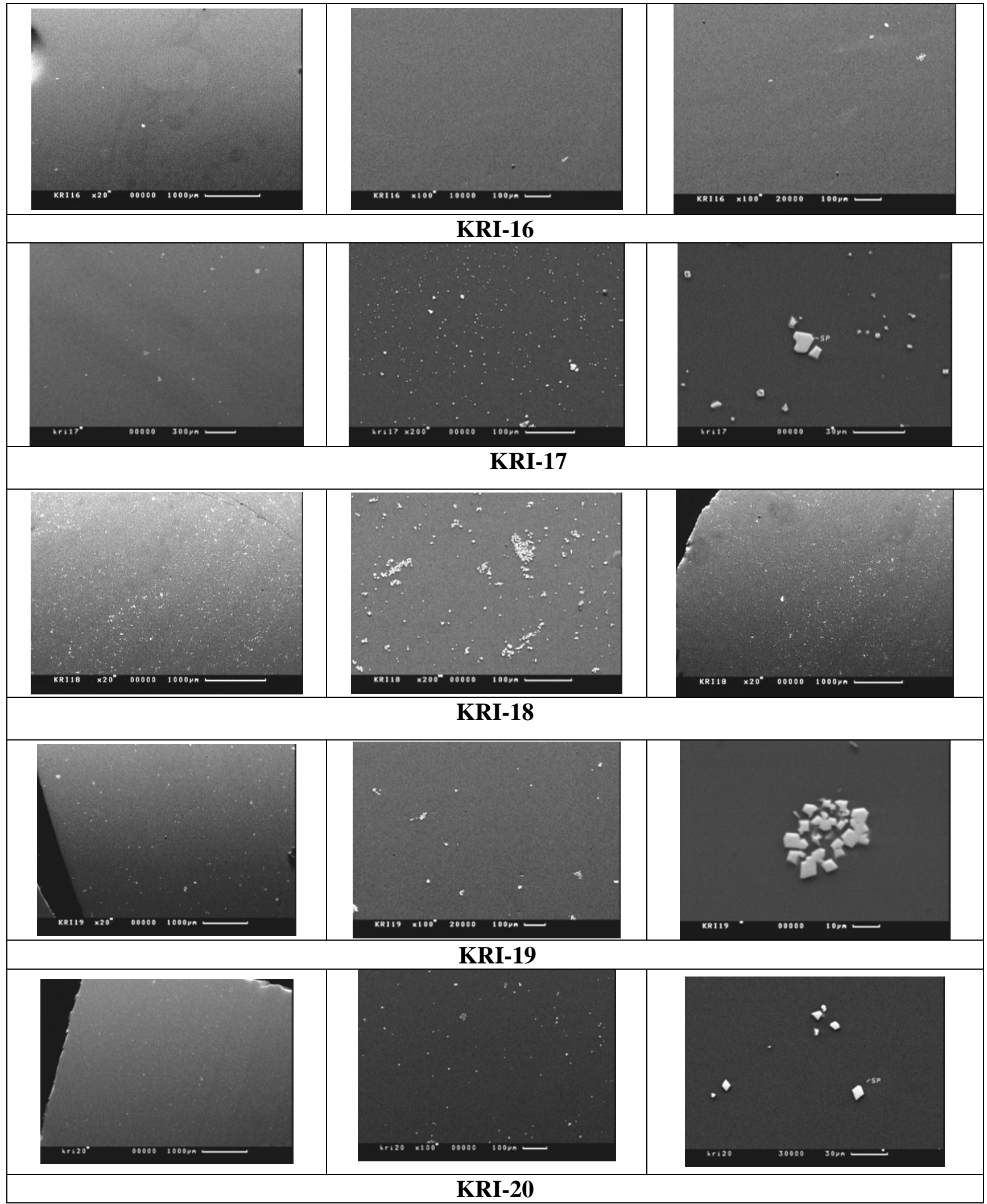




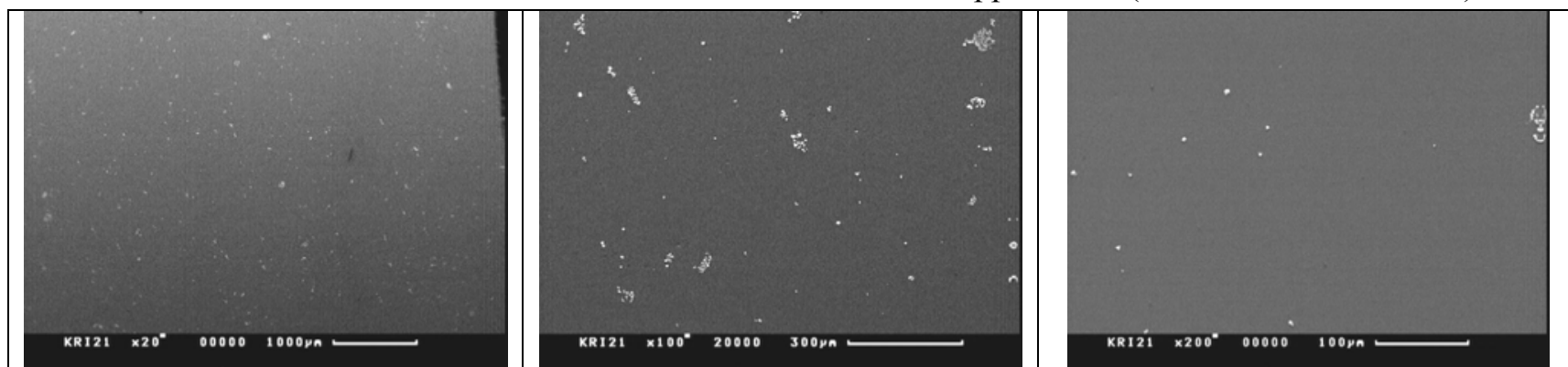

KRI-21

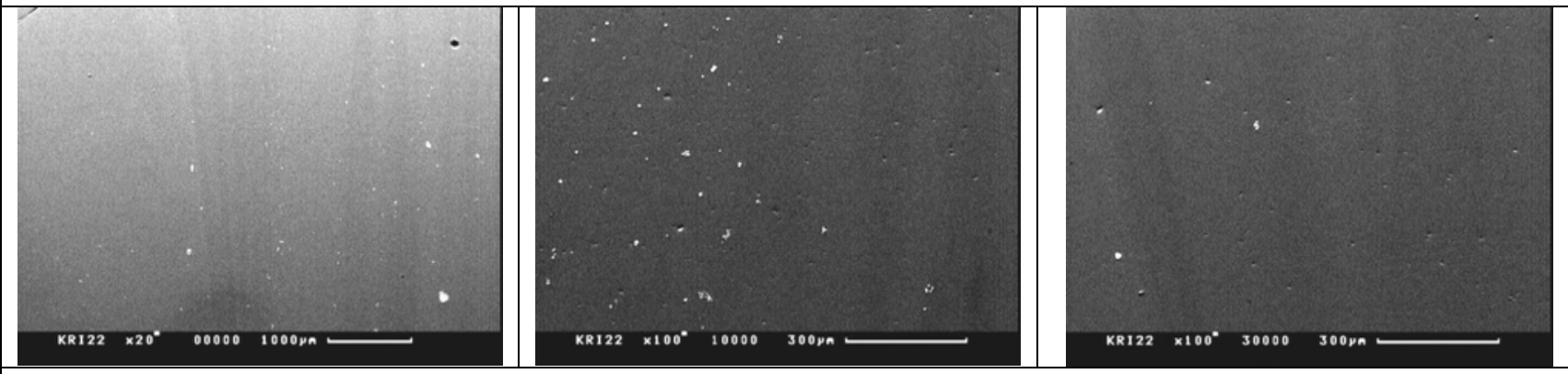

KRI-22

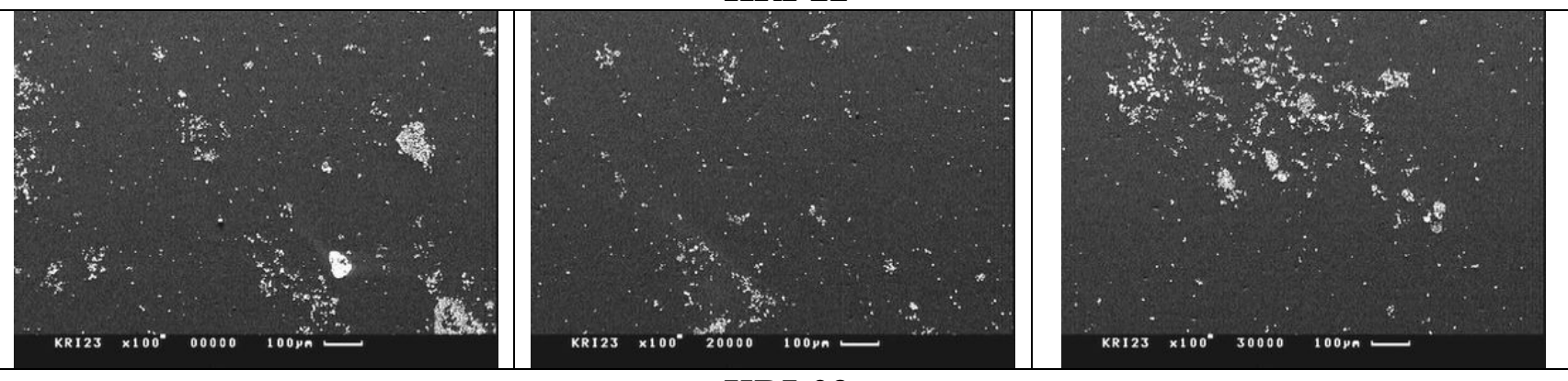

KRI-23

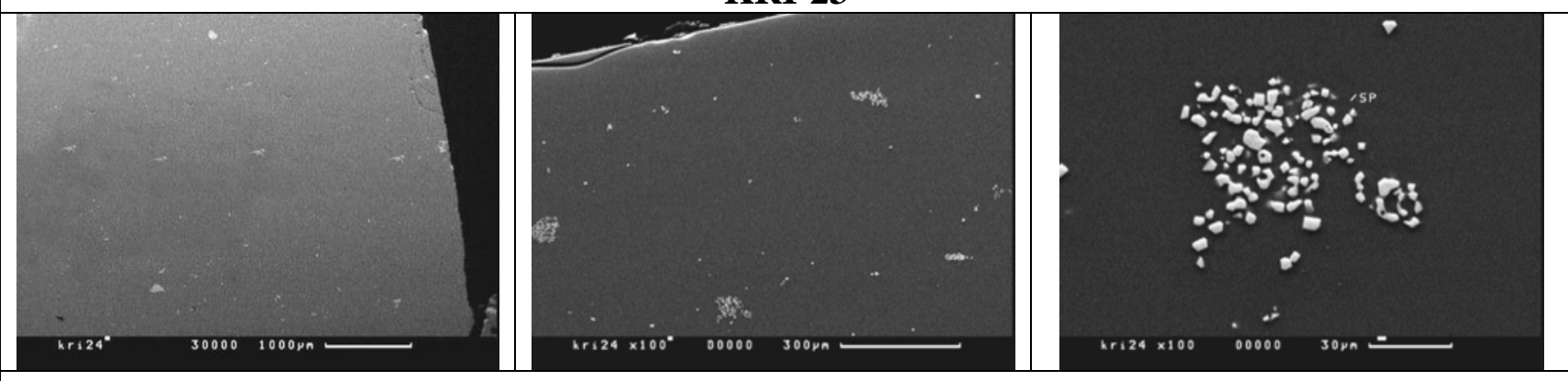

\section{KRI-24}

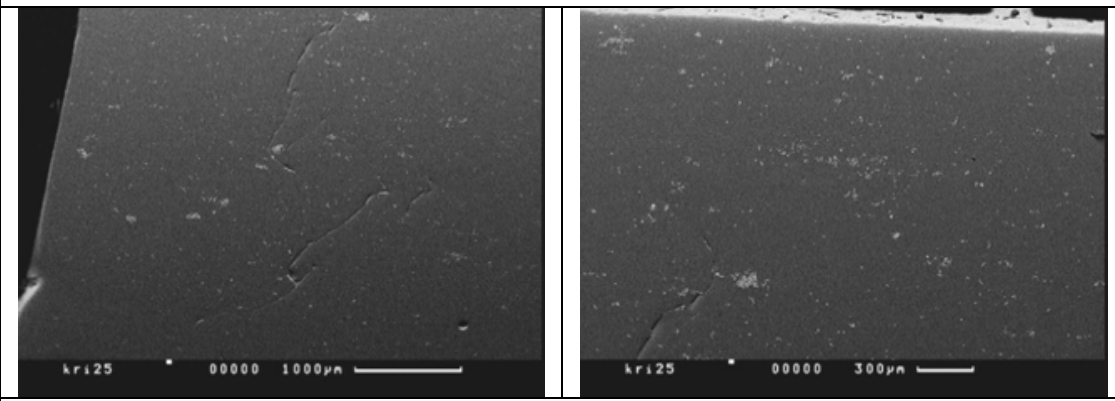

KRI-25 
Appendix C (SRNS-OS-2008-00001)

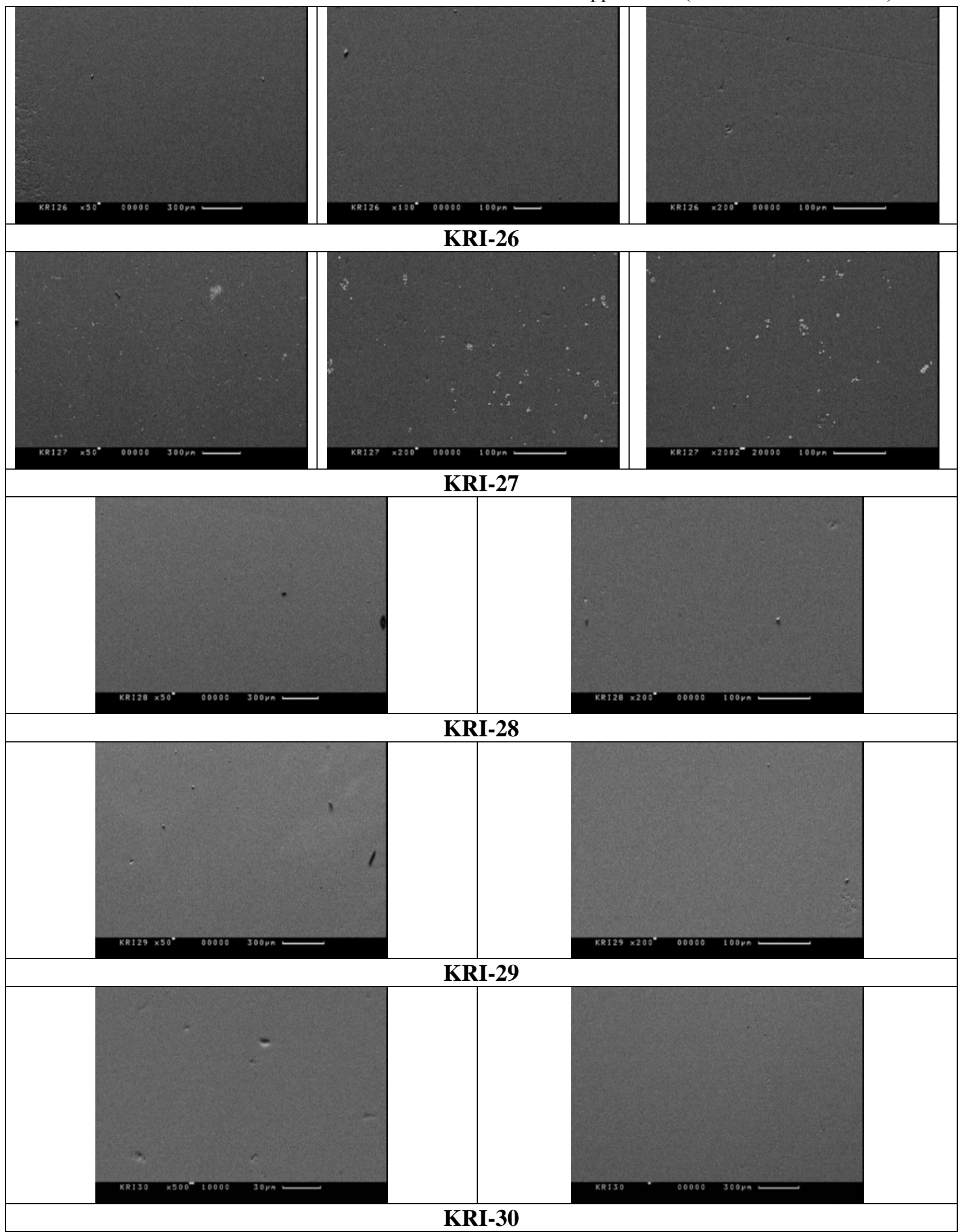


Attachment 3. XRD Data for Quenched Glasses

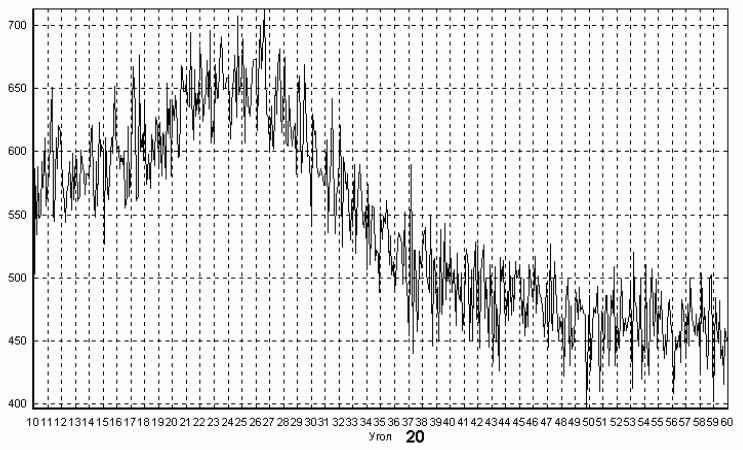

KRI-01

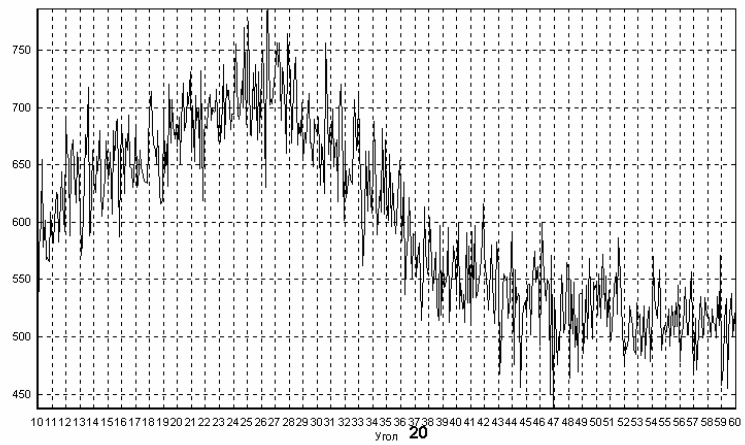

KRI-03

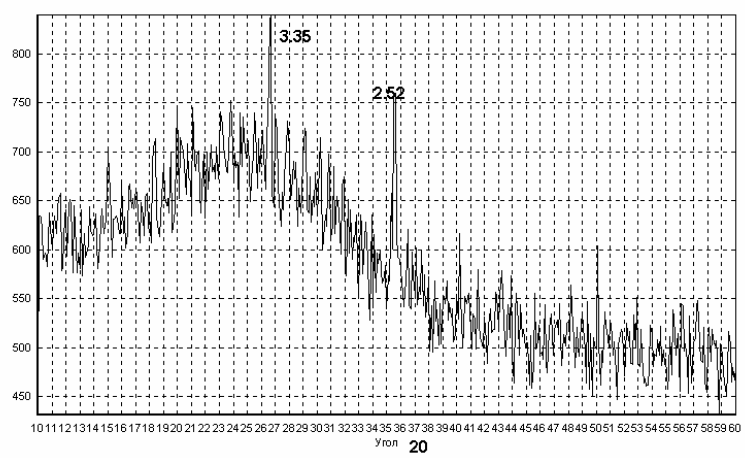

KRI-05

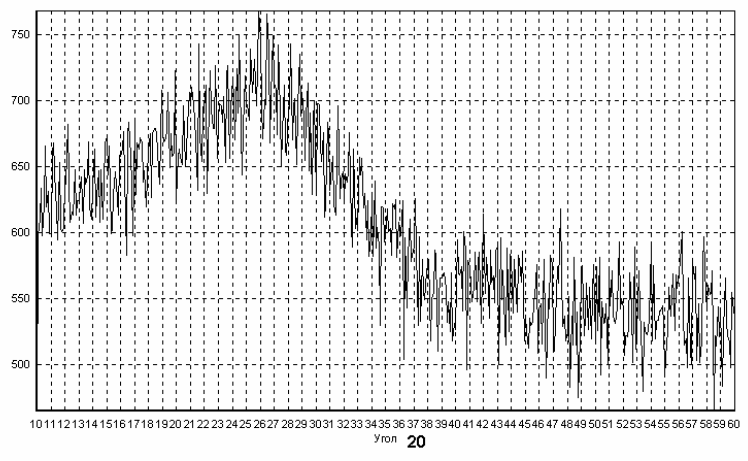

KRI-07

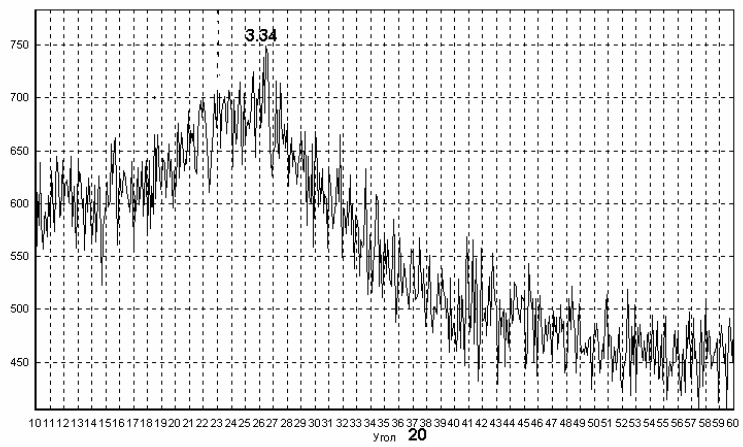

KRI-02

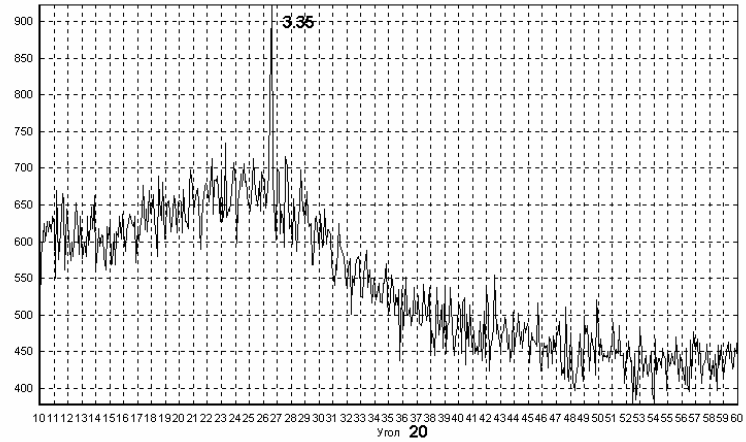

KRI-04

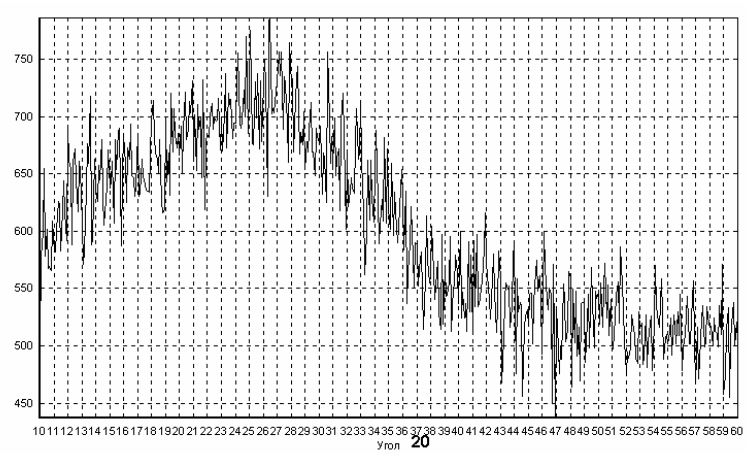

KRI-06

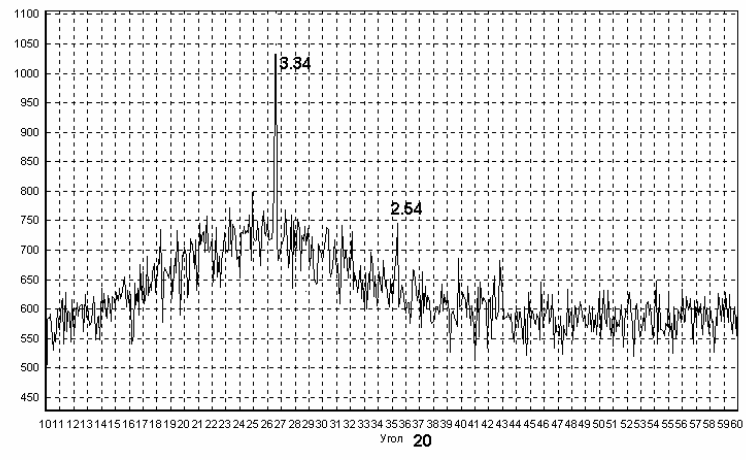

KRI-08 
SRNS-STI-2008-00057

Revision 0

Appendix C (SRNS-OS-2008-00001)
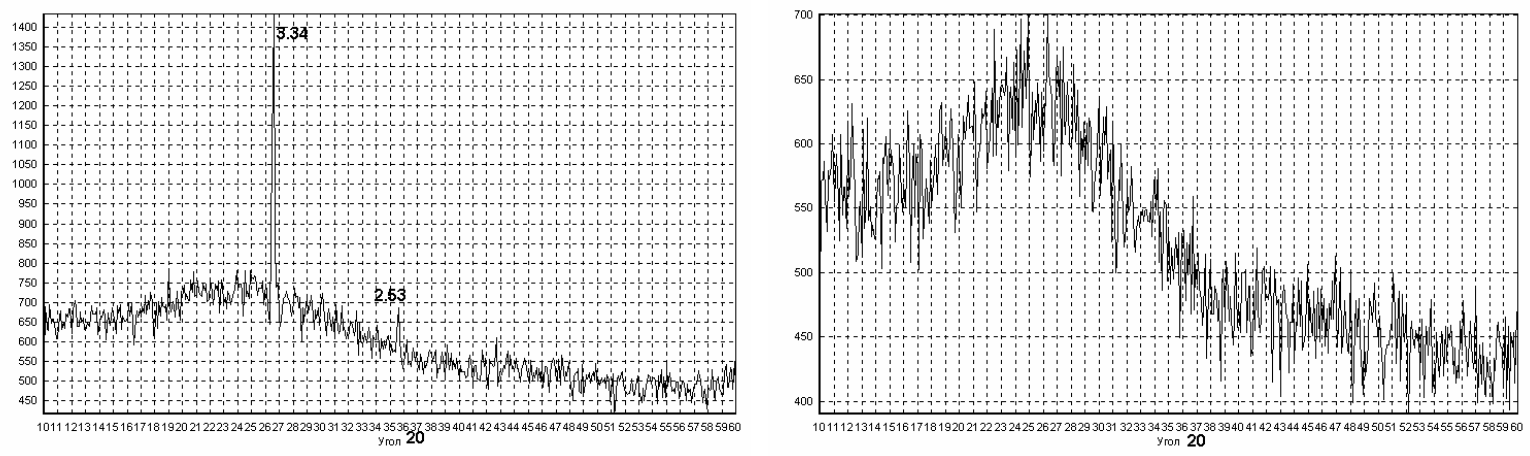

KRI-09

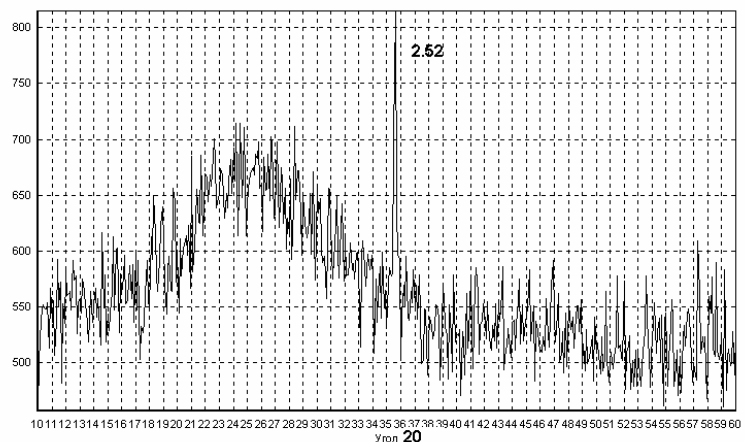

KRI-11

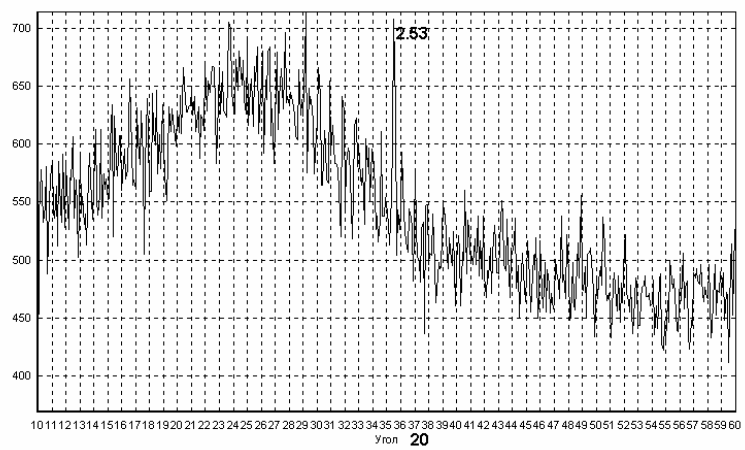

KRI-13

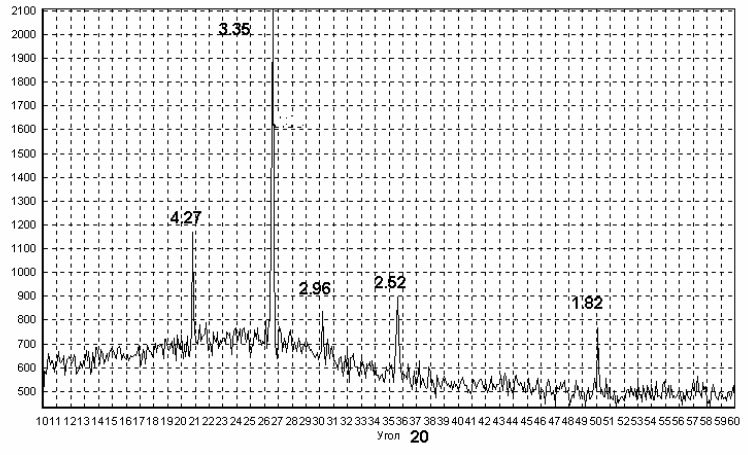

KRI-15

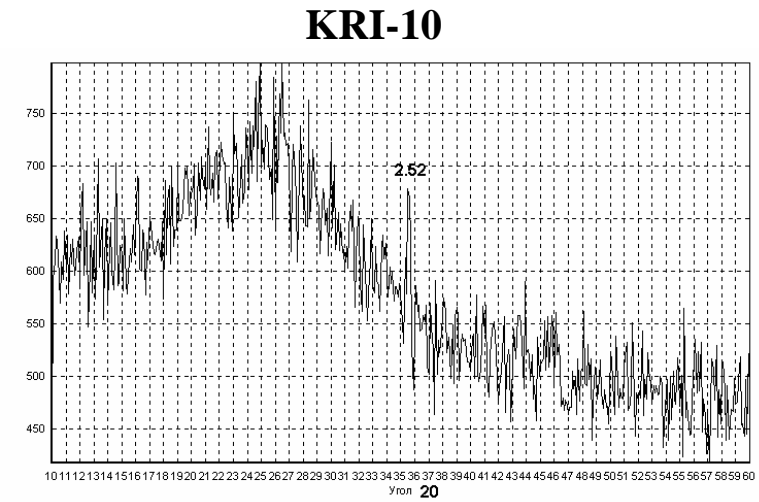

KRI-12

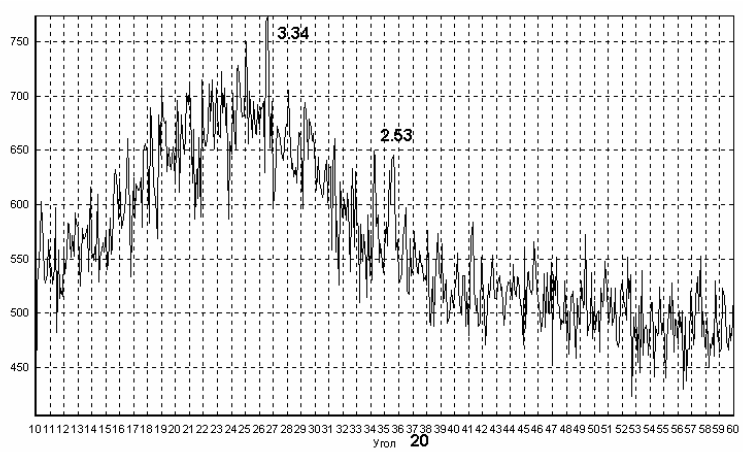

KRI-14

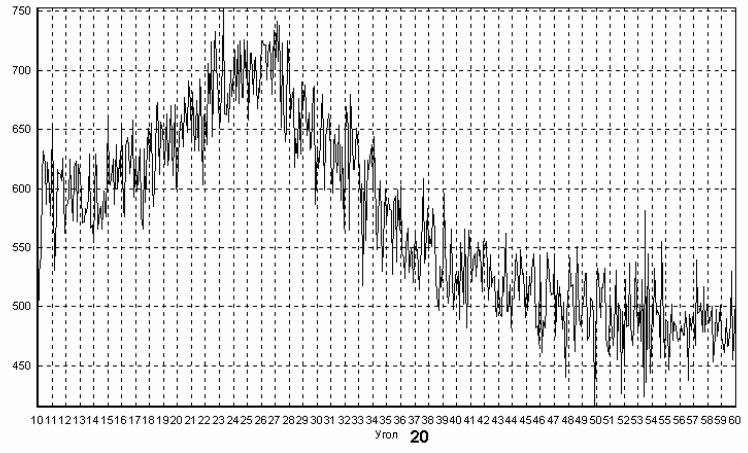

KRI-16 
SRNS-STI-2008-00057

Revision 0

Appendix C (SRNS-OS-2008-00001)
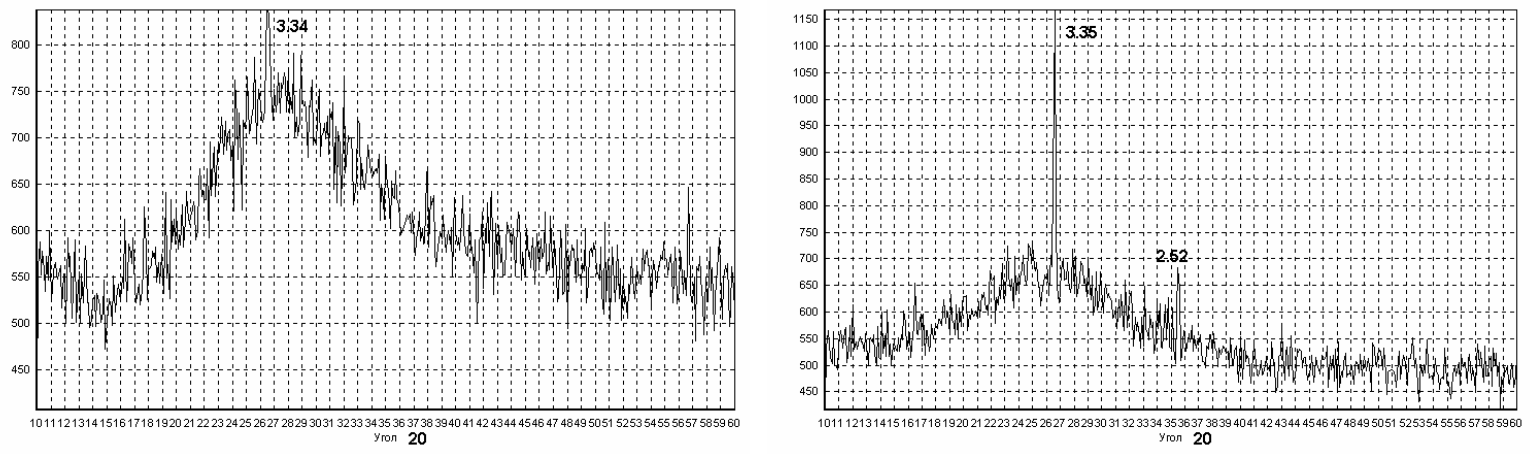

KRI-17

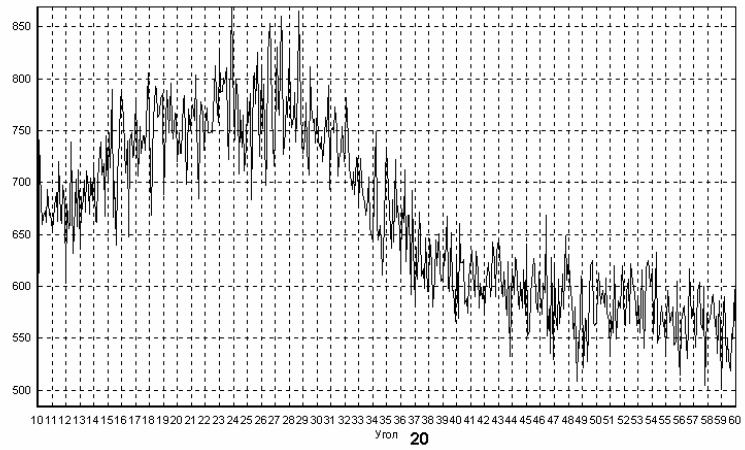

KRI-19

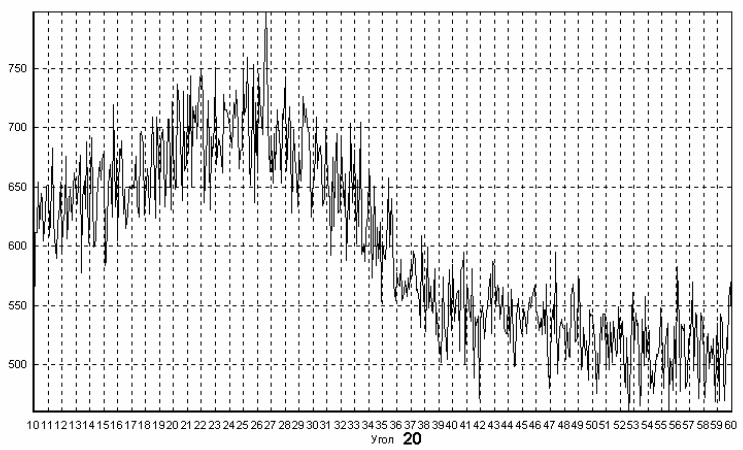

KRI-21

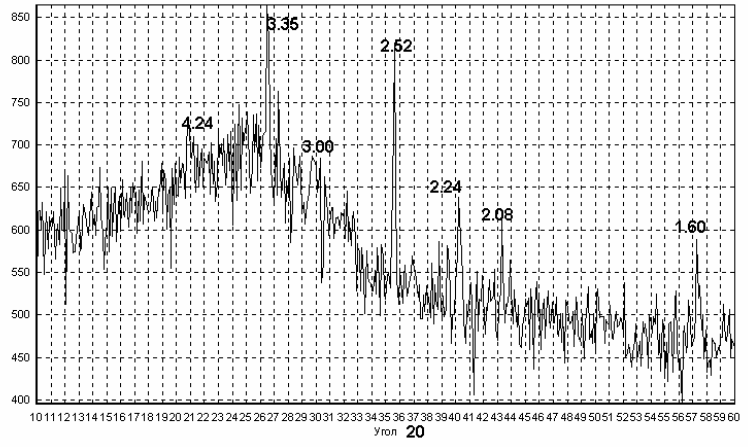

KRI-23
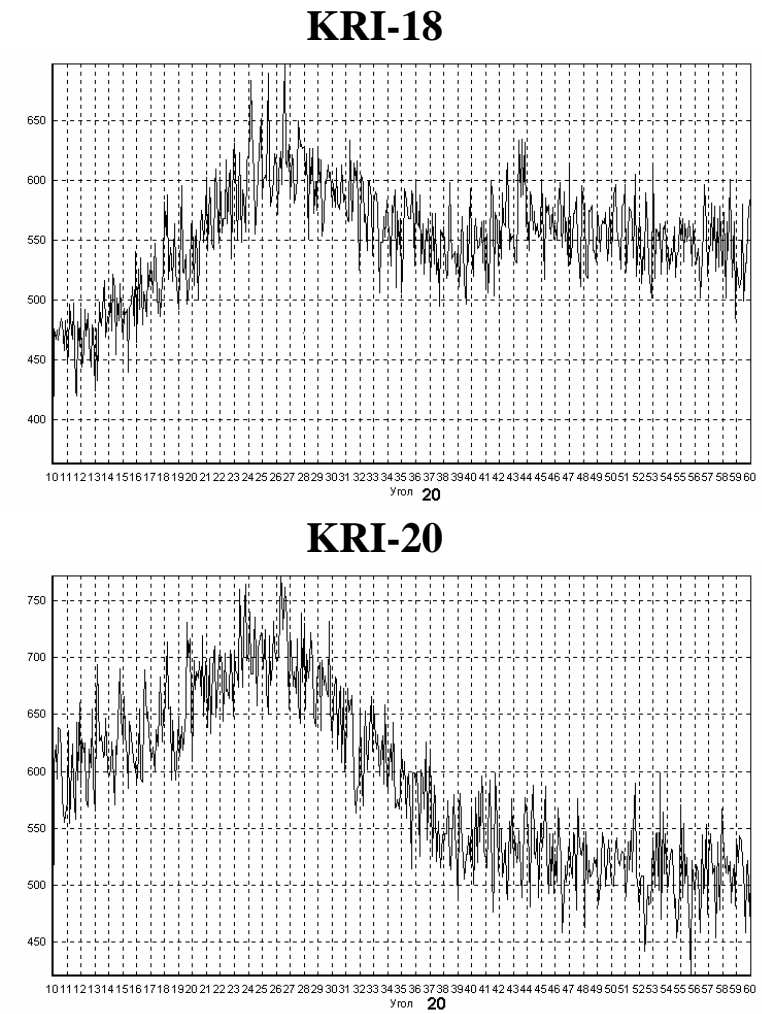

KRI-22

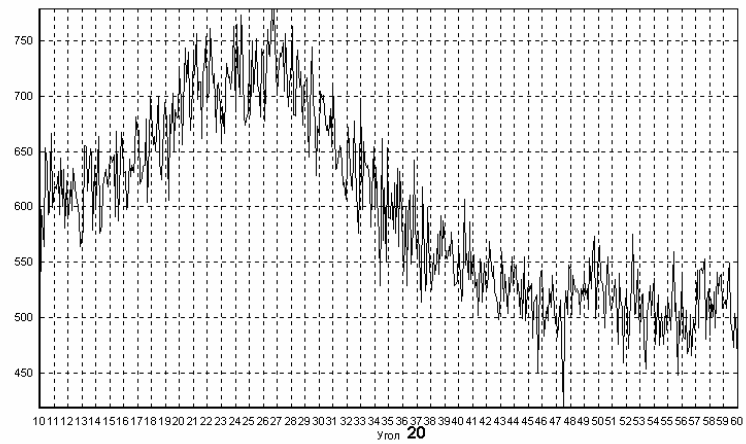

KRI-24 


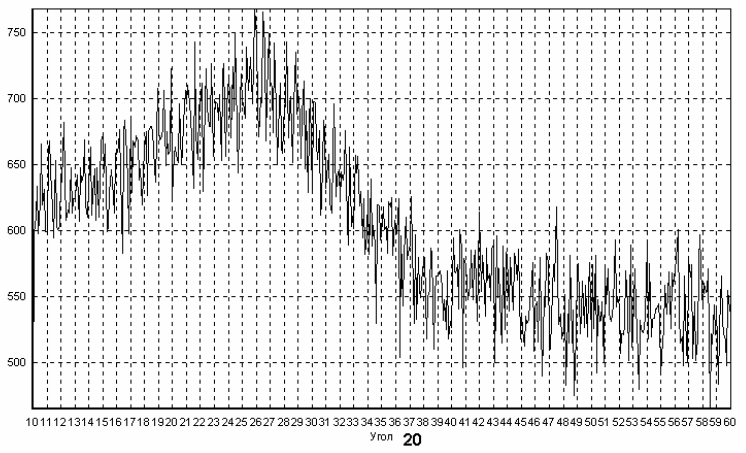

KRI-25

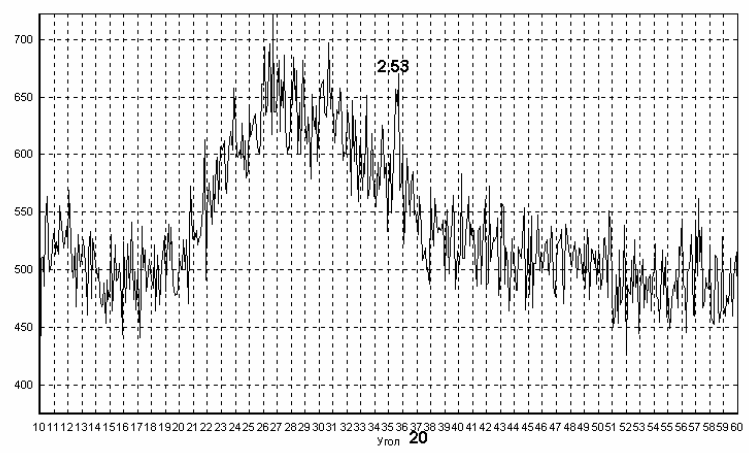

KRI-27

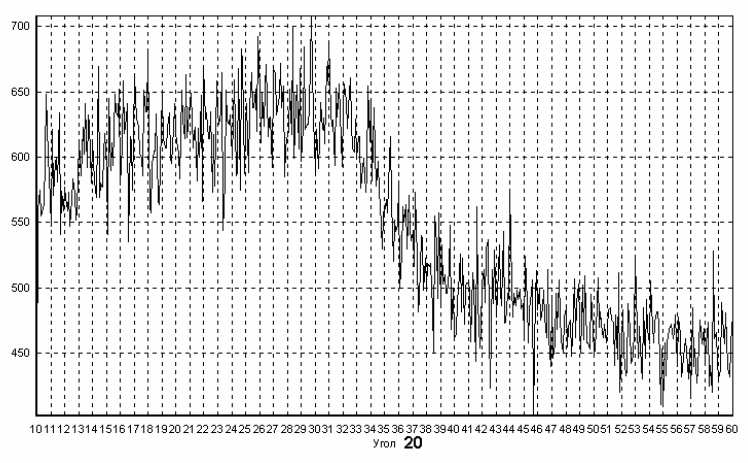

KRI-29

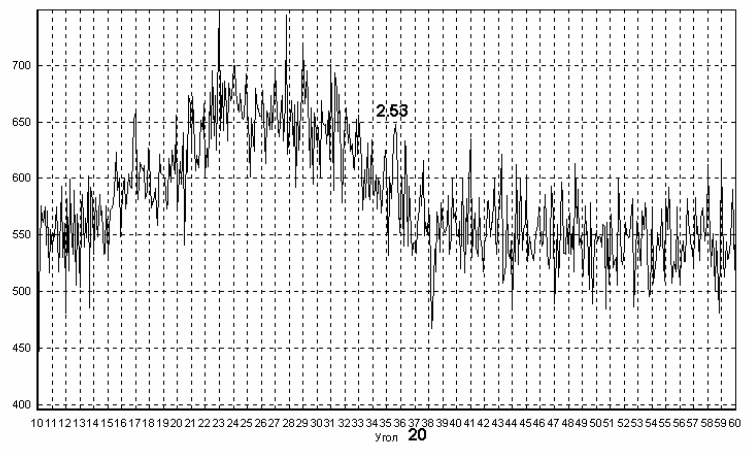

KRI-26

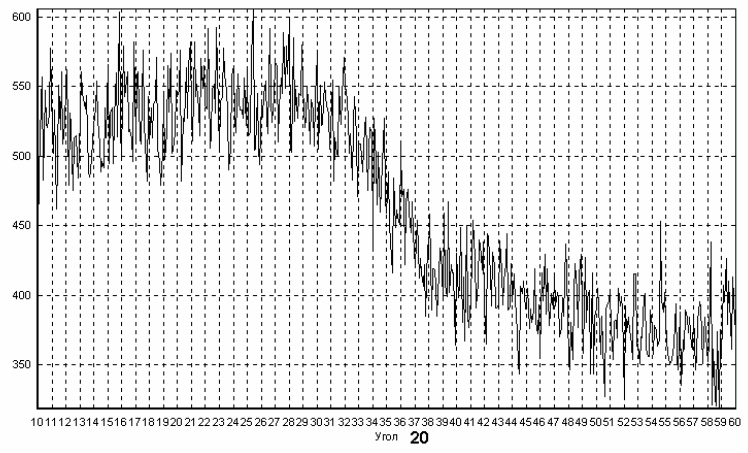

KRI-28

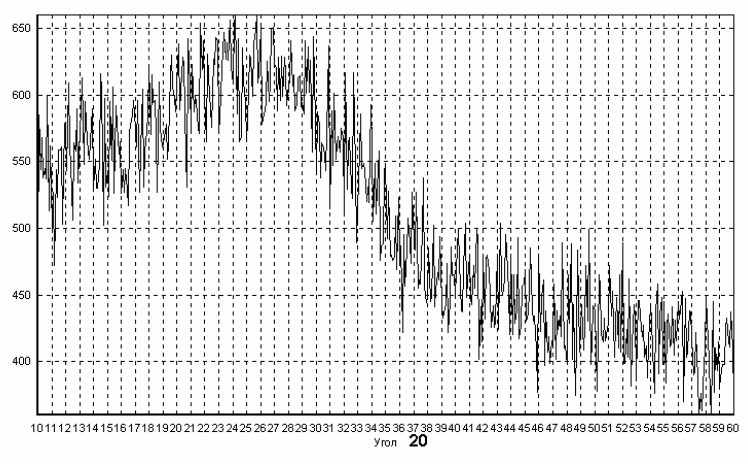

KRI-30 
Attachment 4 Surface of KRI Glasses after CCC Treatment

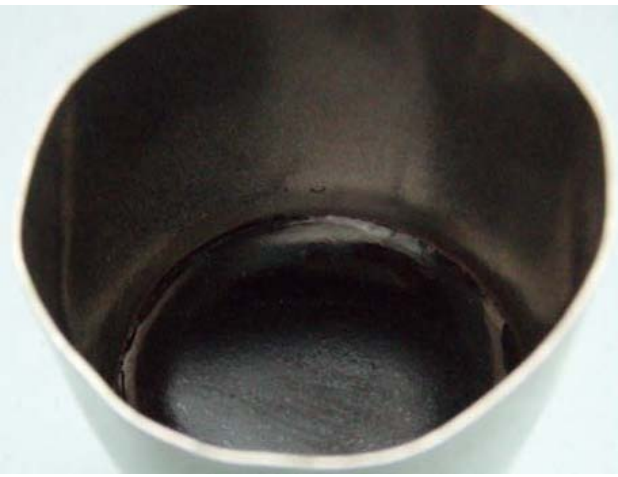

KRI-01 CCC

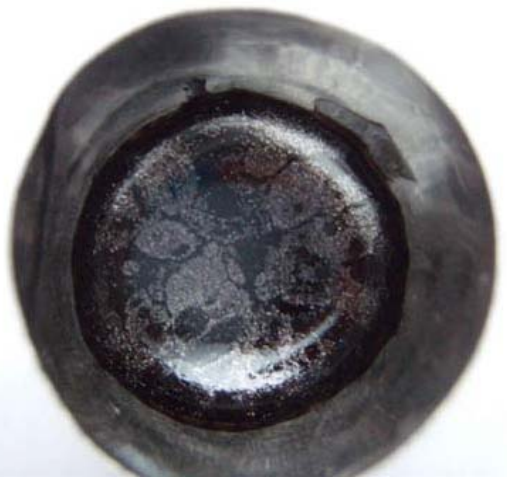

KRI-03 CCC

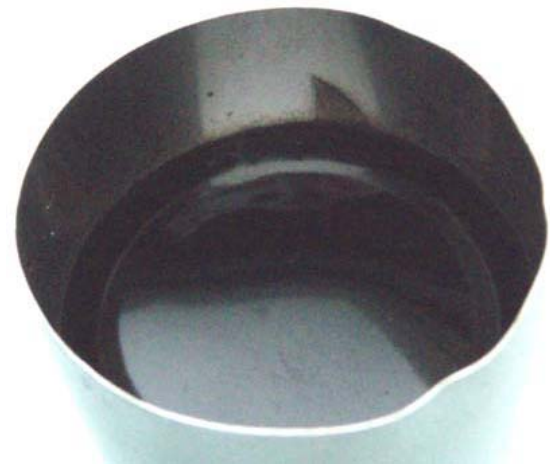

KRI-05 CCC

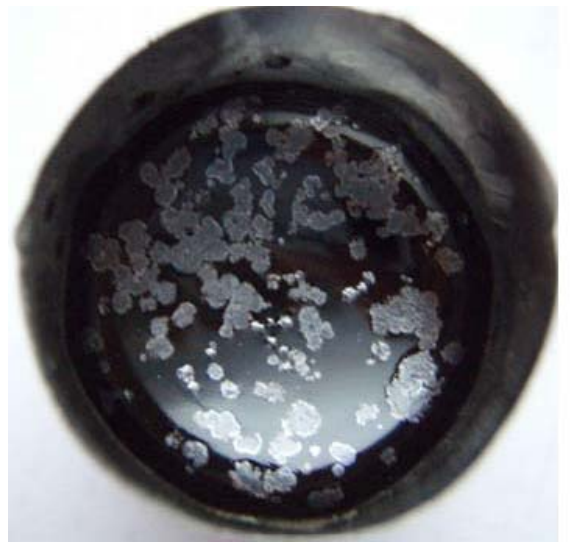

KRI-07 CCC

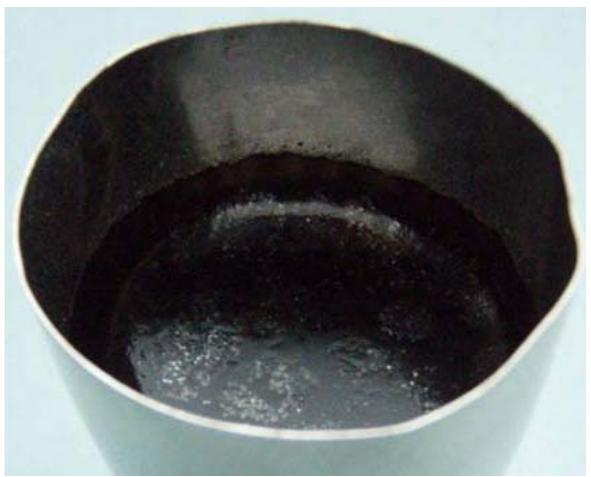

KRI-02 CCC
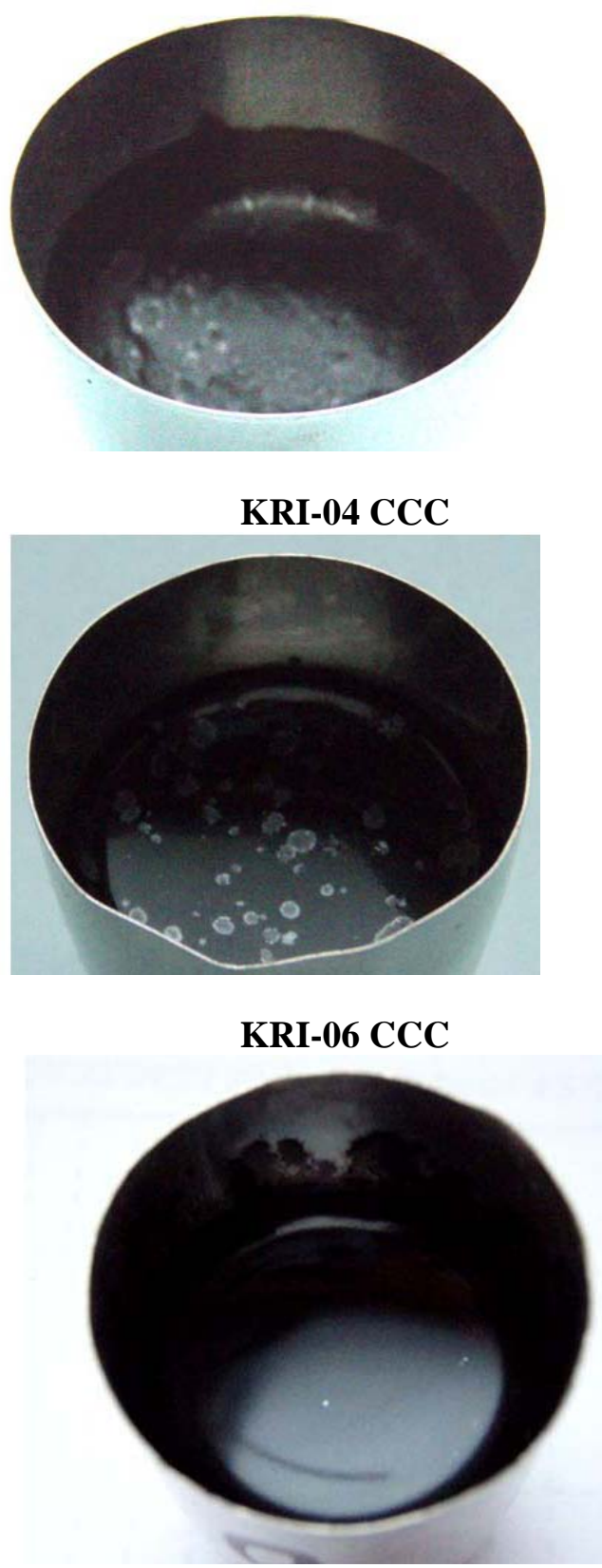

KRI-08 CCC 


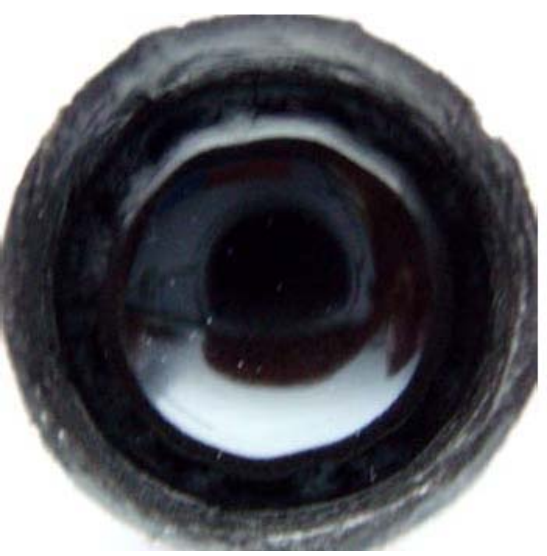

KRI-09 CCC

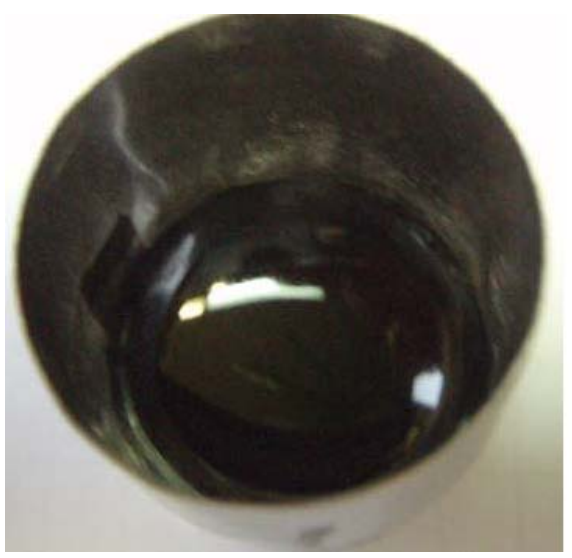

KRI-011 CCC

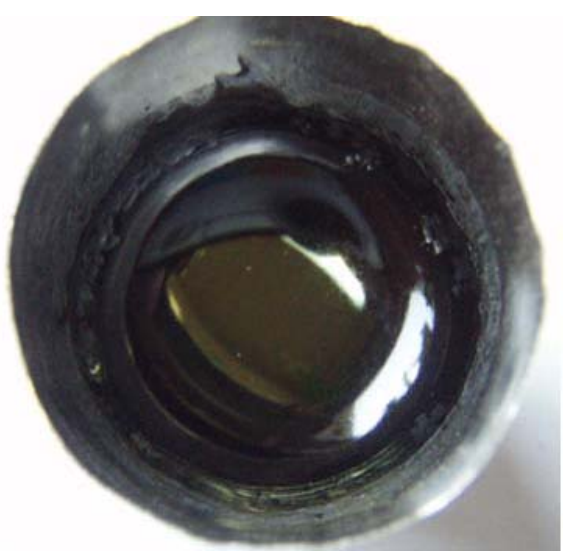

KRI-13 CCC

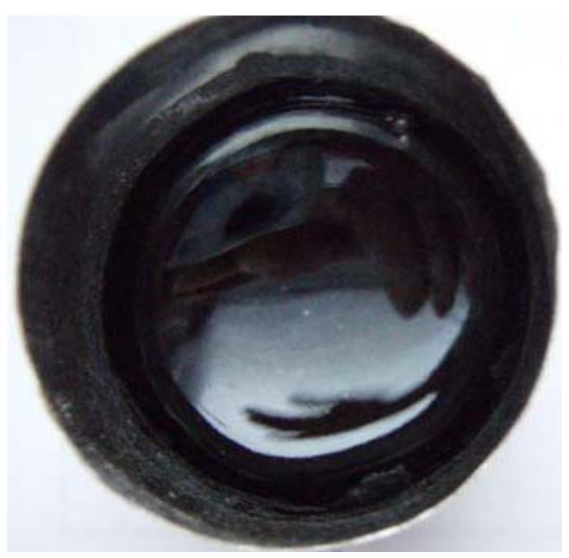

KRI-10 CCC

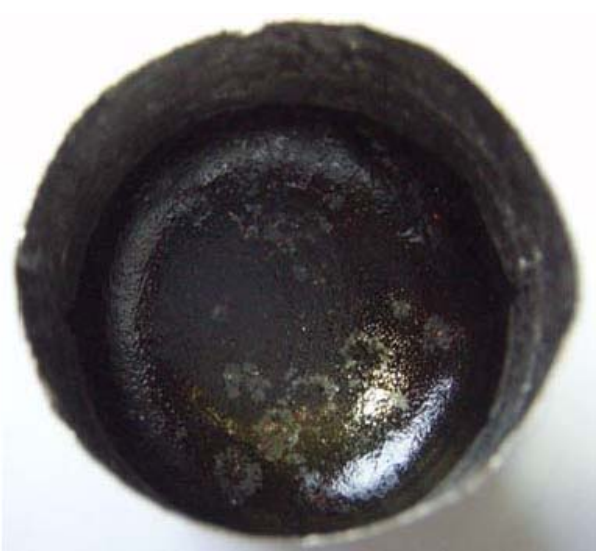

KRI-12 CCC

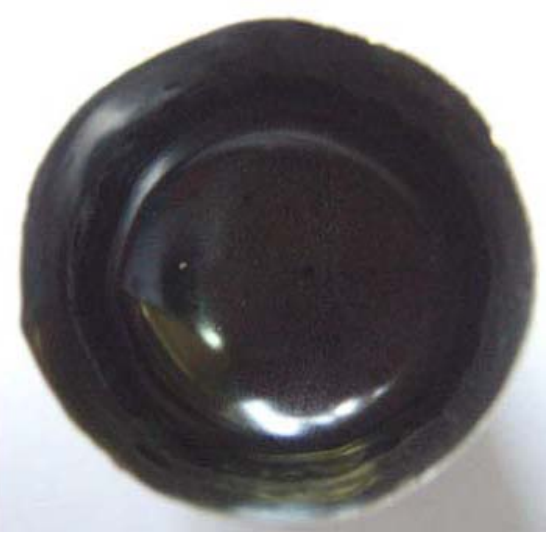

KRI-14 CCC 


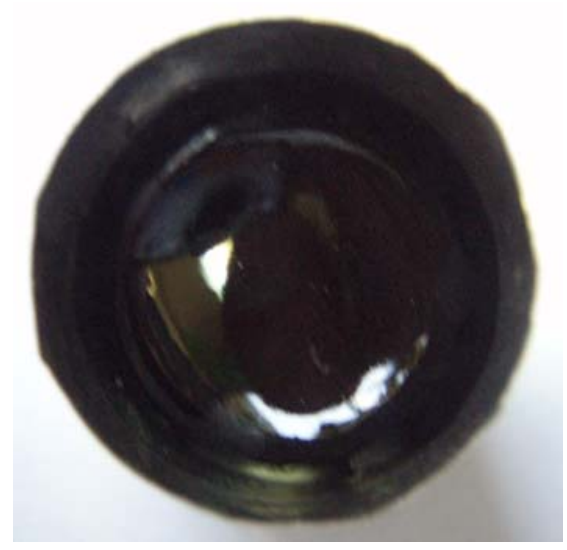

\section{KRI-15 CCC}

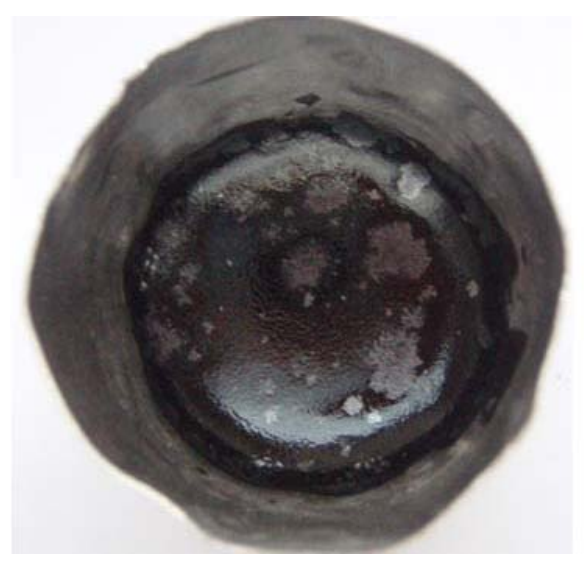

KRI-21 CCC

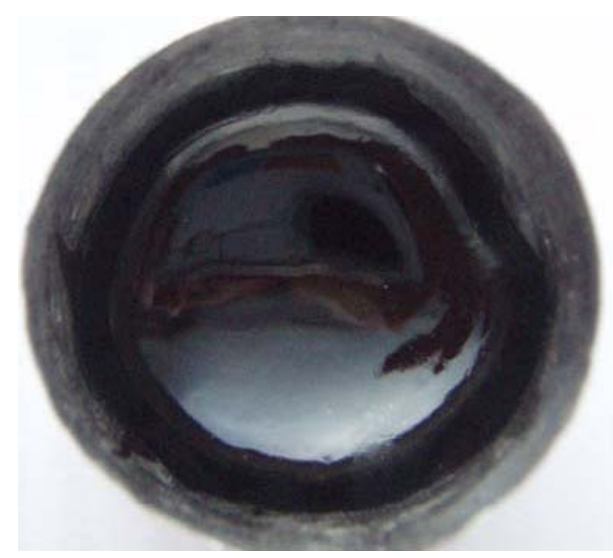

KRI-23 CCC

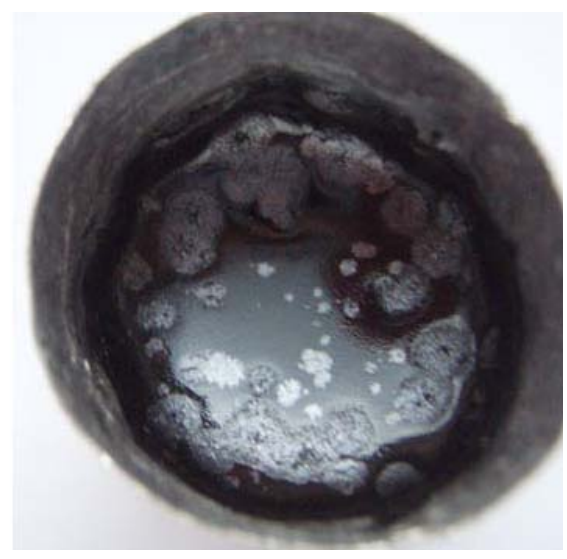

KRI-22 CCC

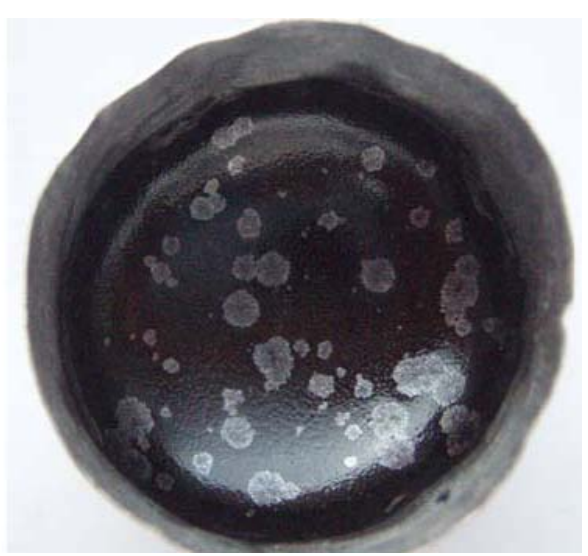

KRI-24 CCC 

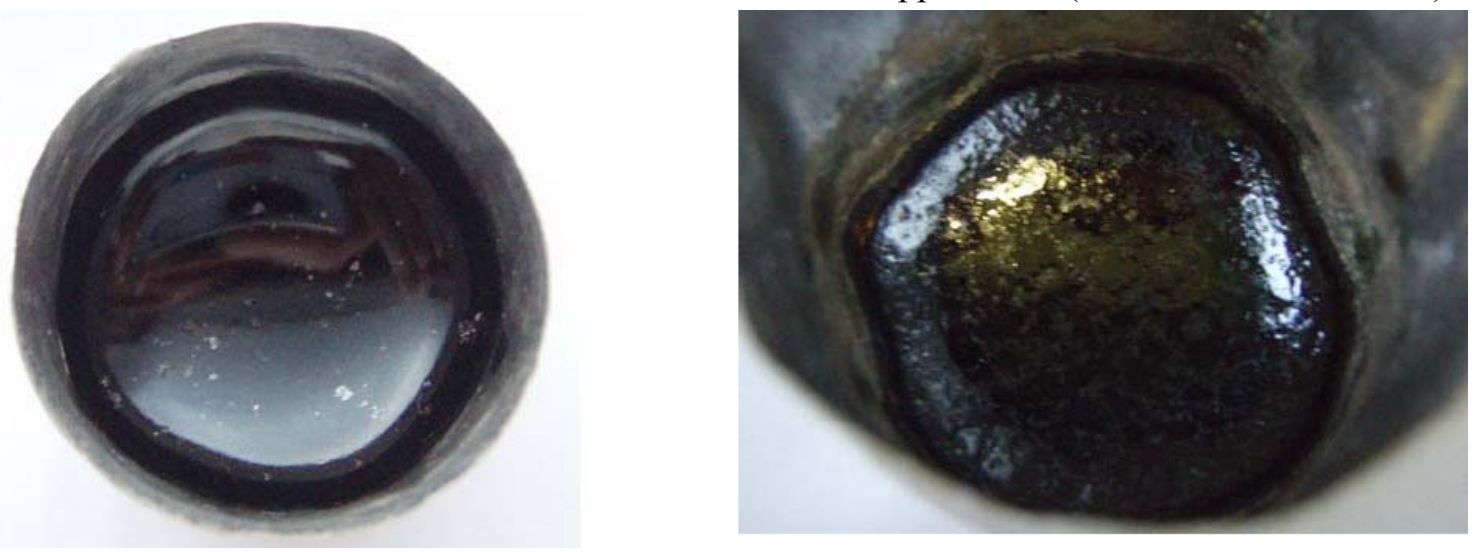

KRI-25 CCC

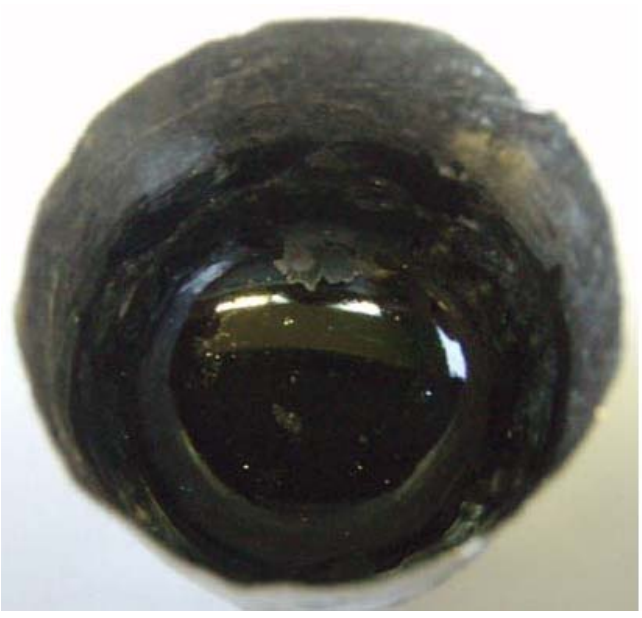

KRI-27 CCC

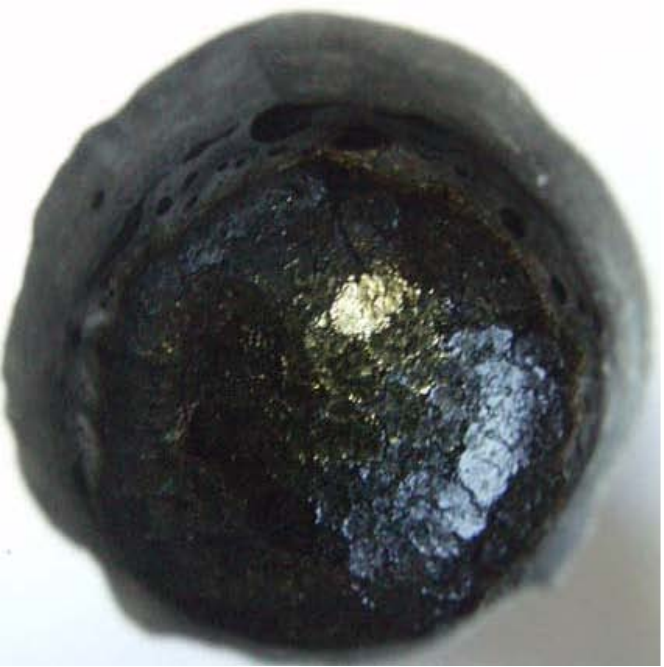

KRI-29 CCC

\section{KRI-26 CCC}
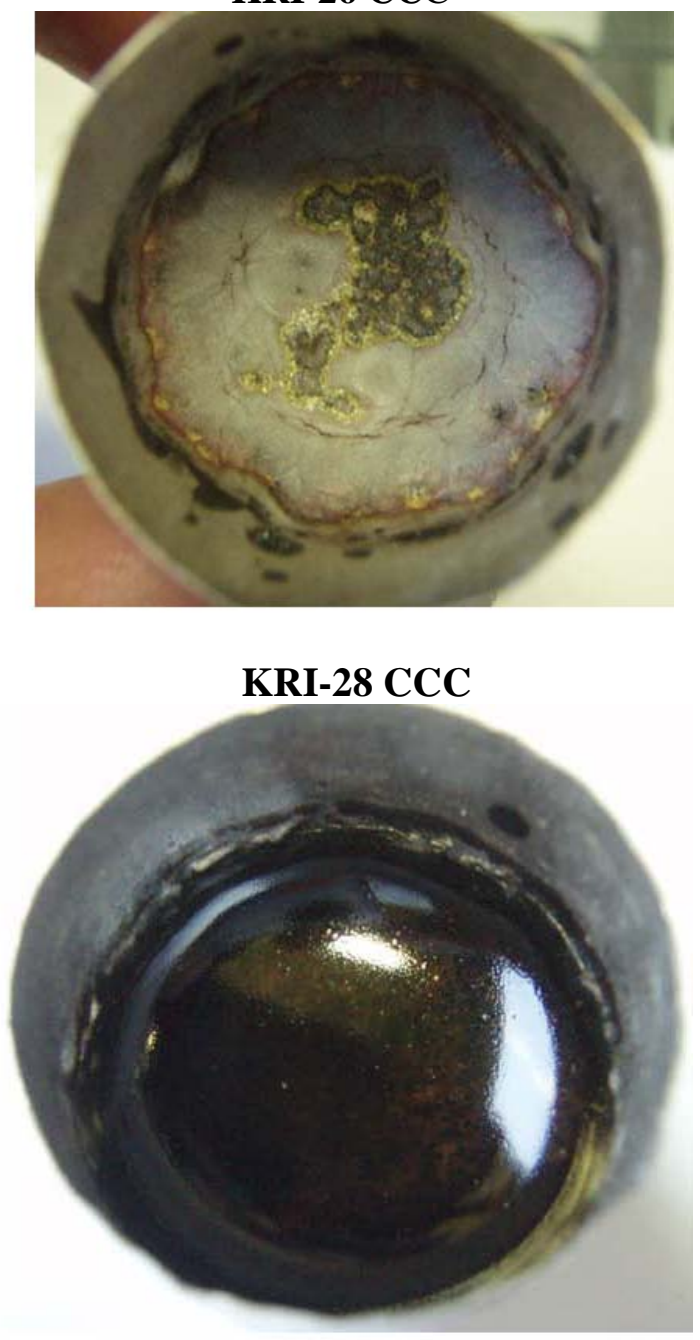

KRI-30 CCC 
Attachment 5. SEM Photographs of Glass Samples after CCC Treatment

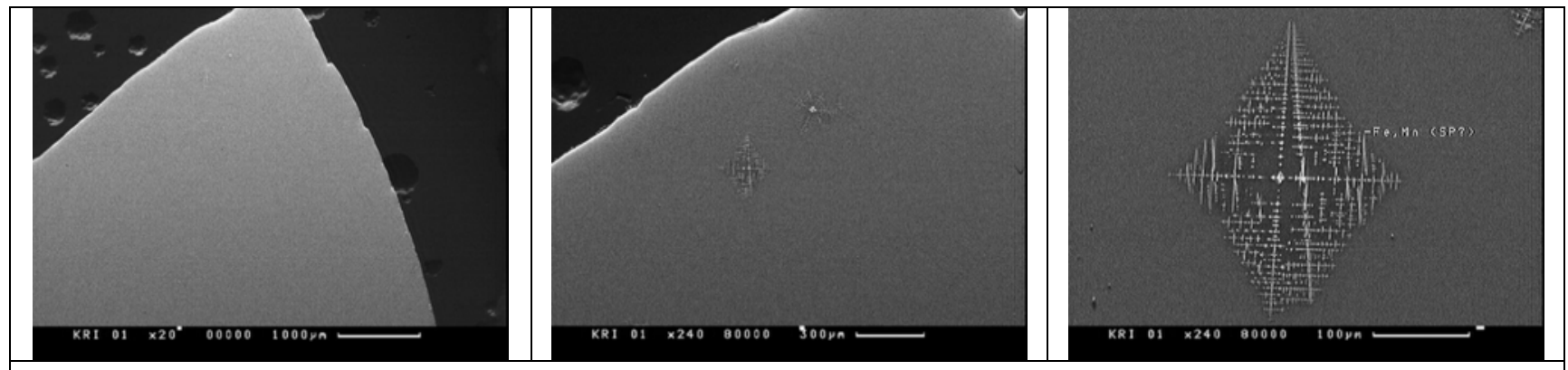

\section{KRI-01 CCC}

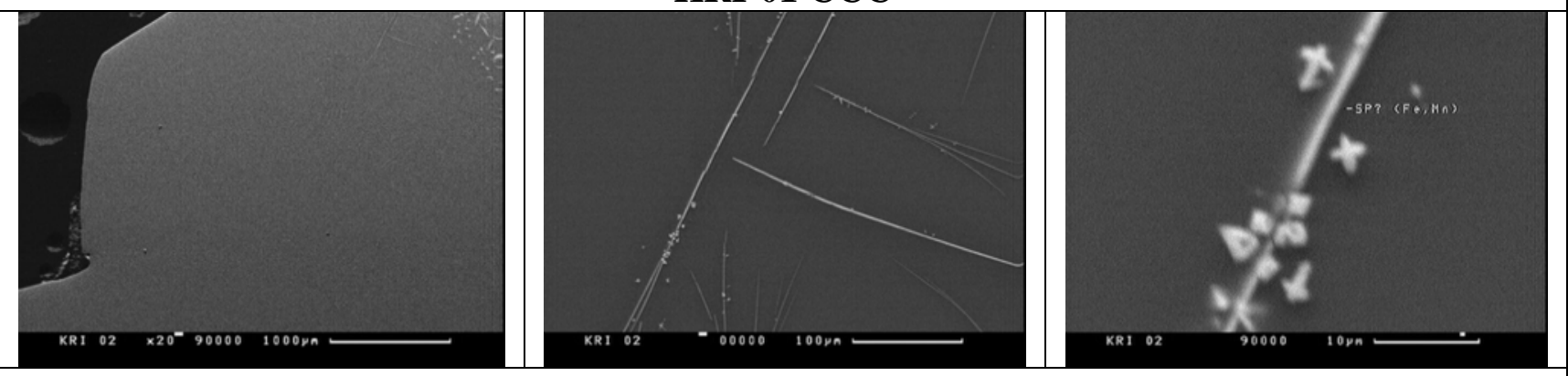

\section{KRI-02 CCC}

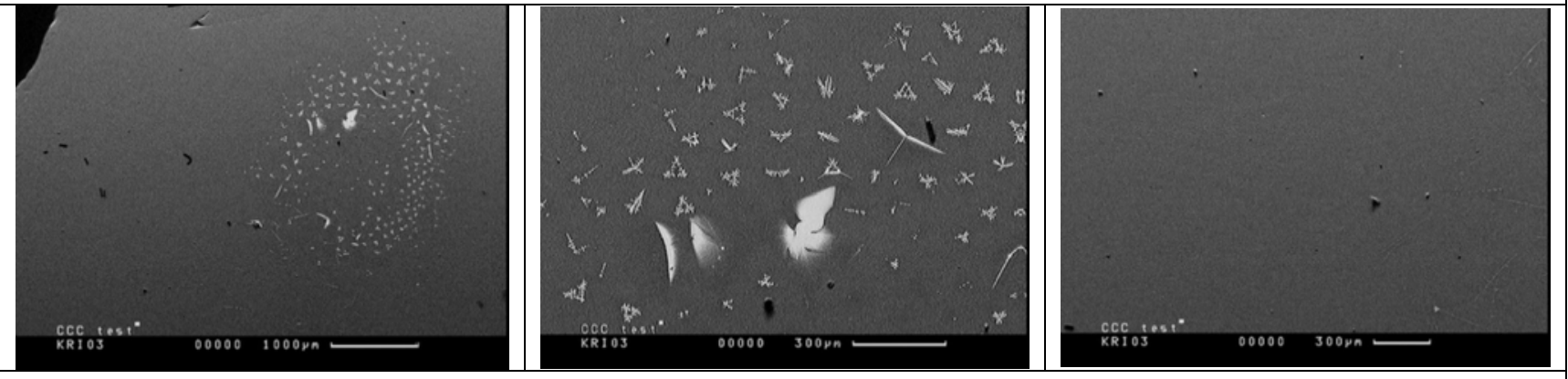

\section{KRI-03 CCC}

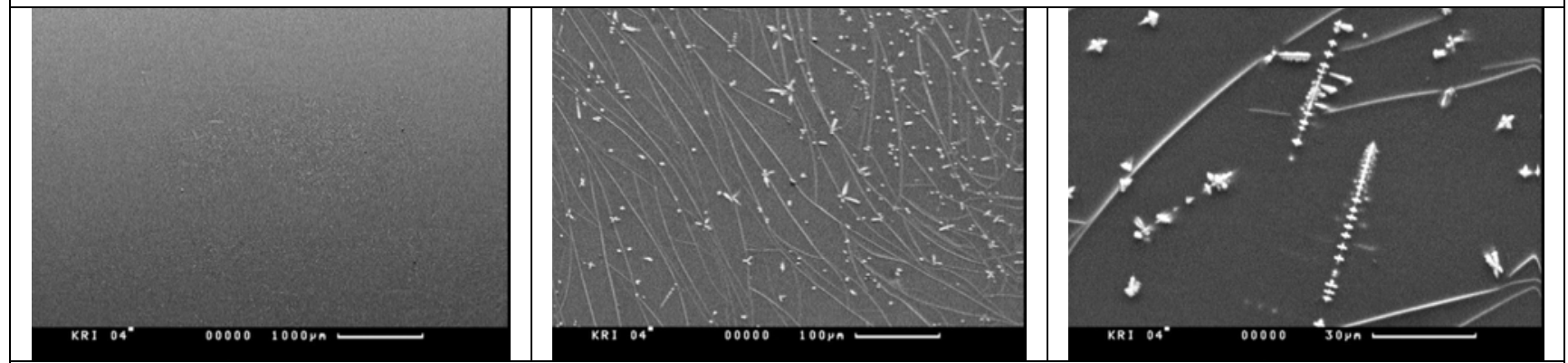

\section{KRI-04 CCC}
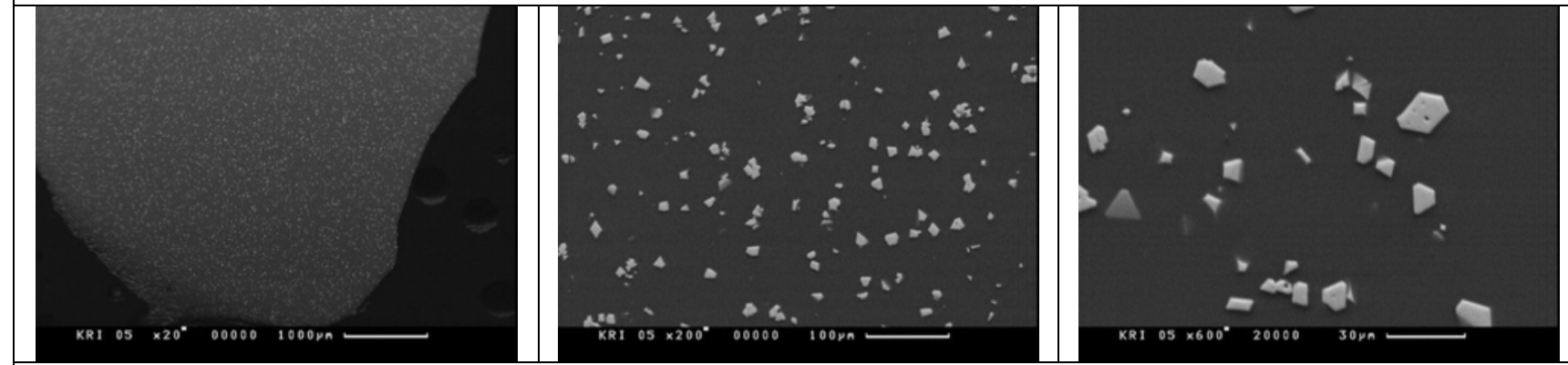

\section{KRI-05 CCC}



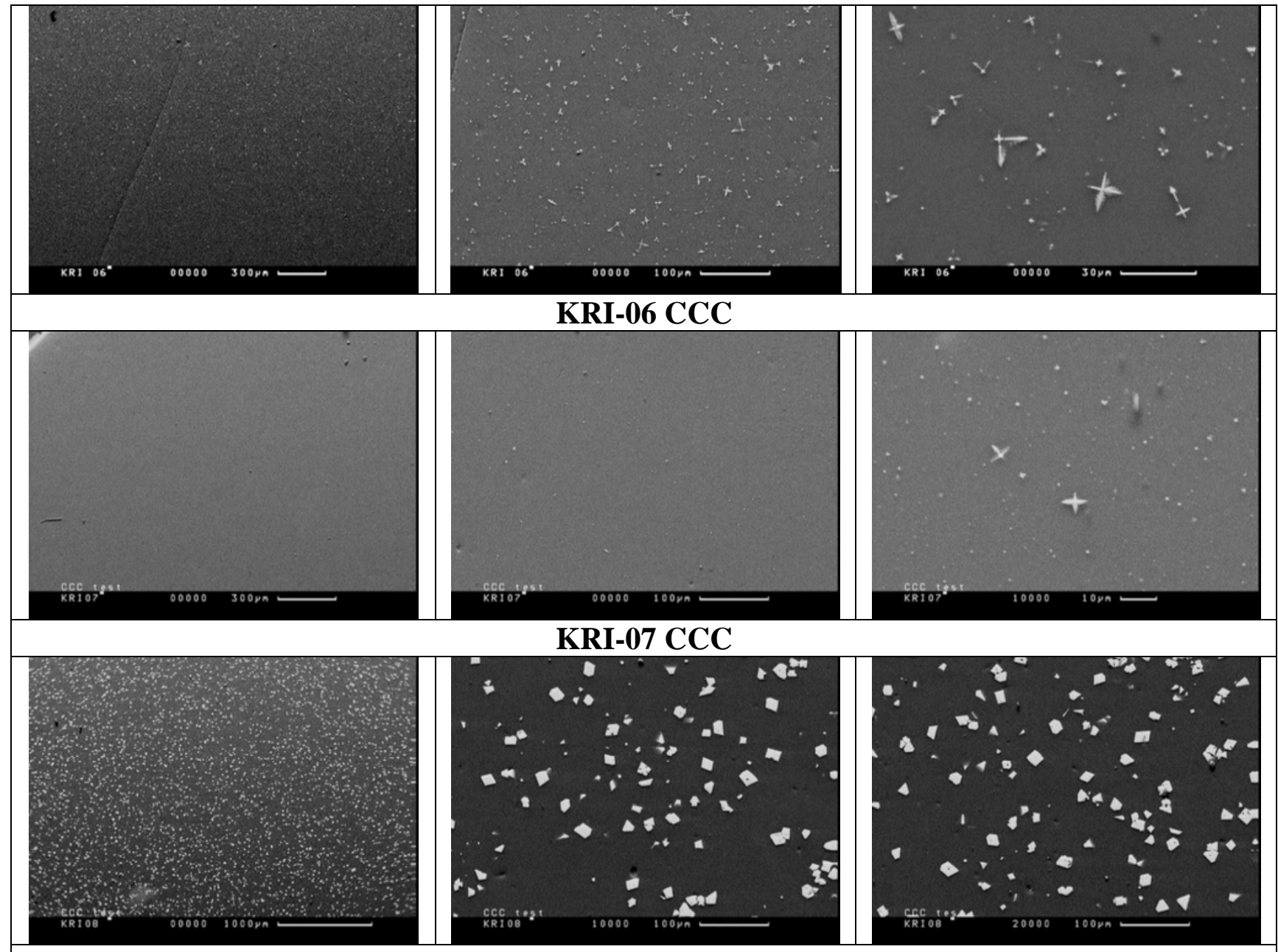

\section{KRI-08 CCC}

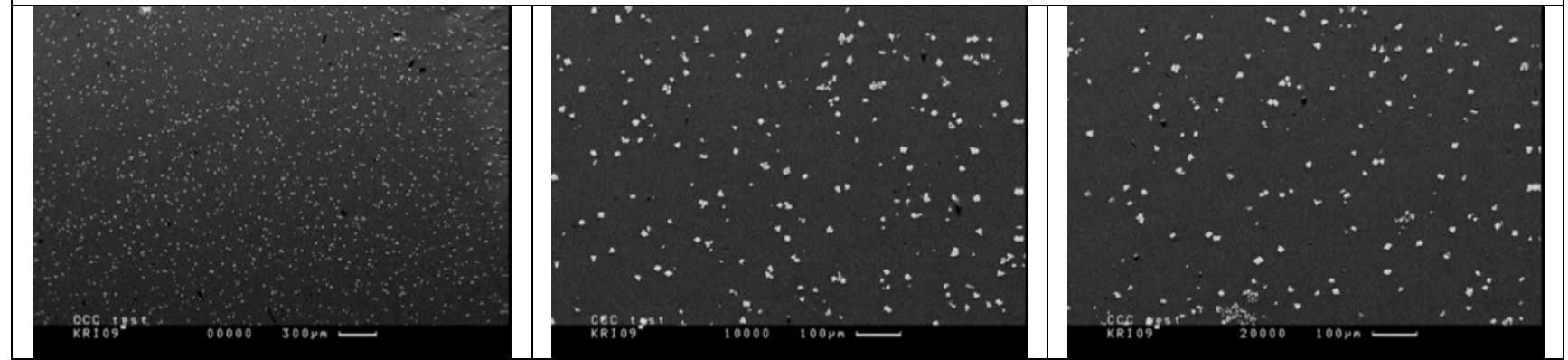

\section{KRI-09 CCC}
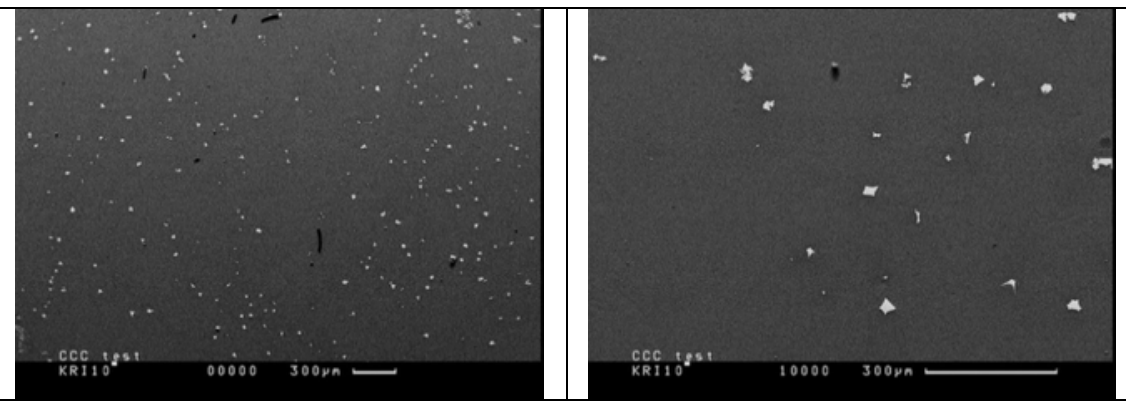

KRI-10 CCC 

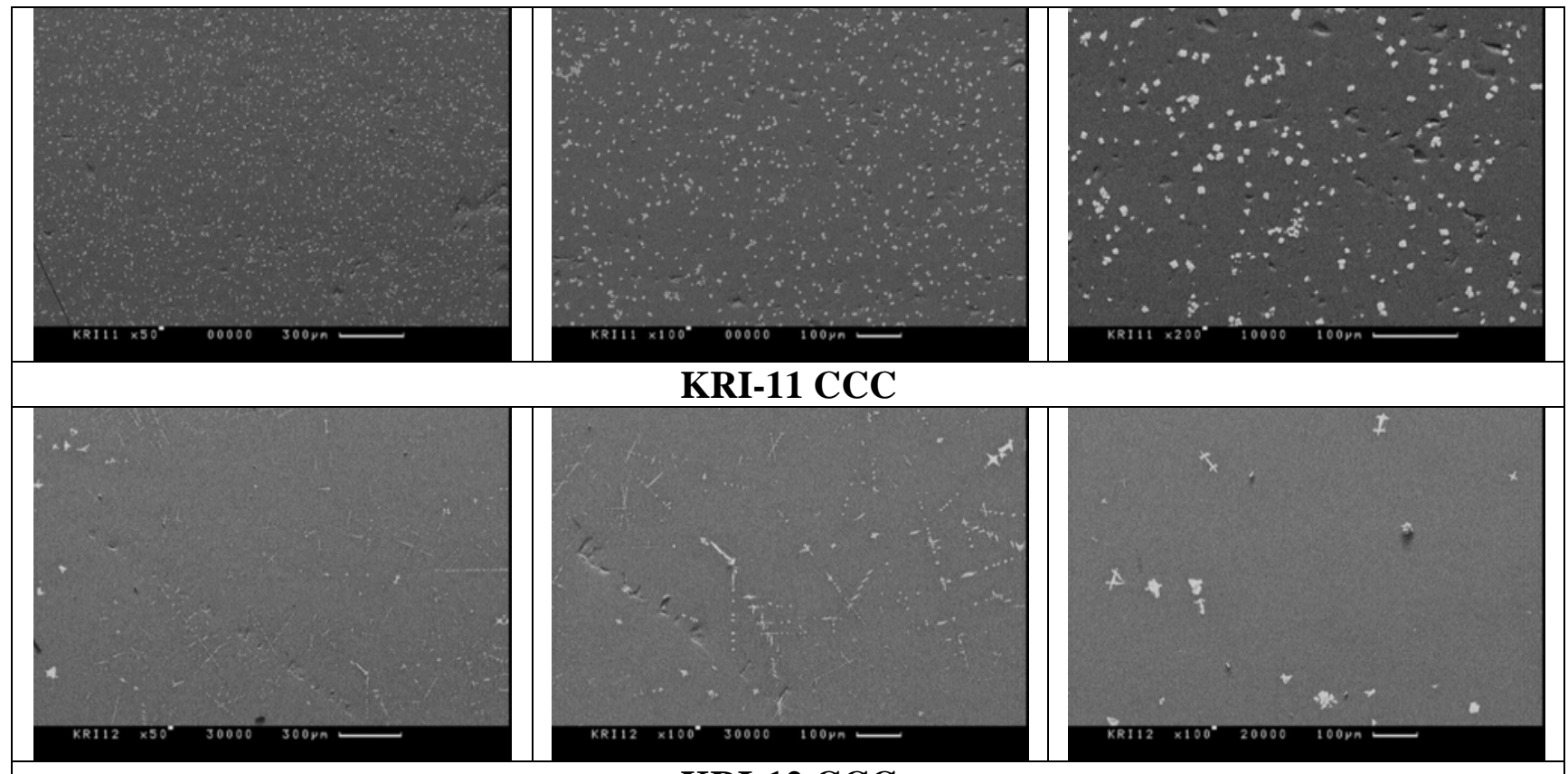

\section{KRI-12 CCC}

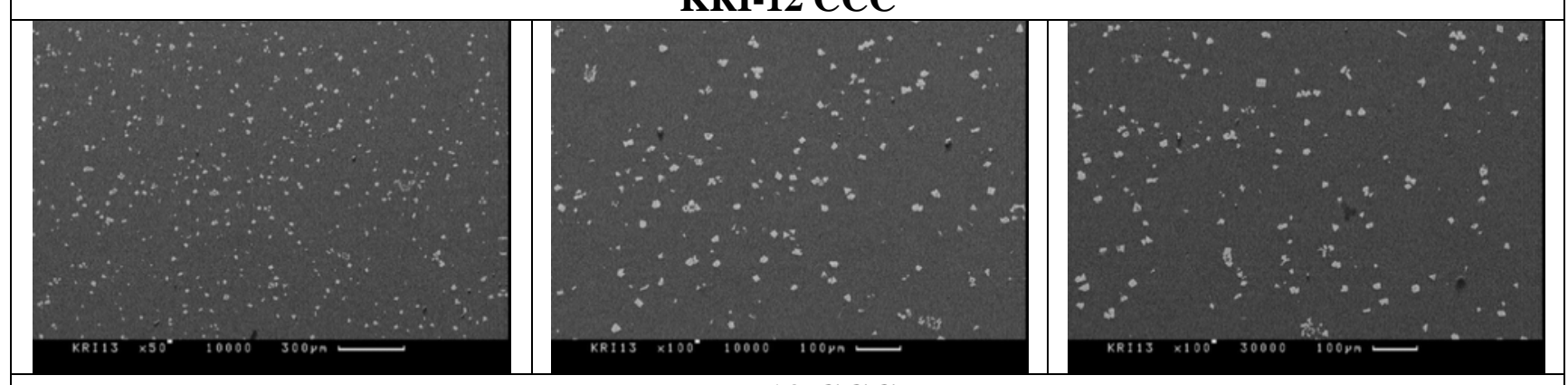

\section{KRI-13 CCC}

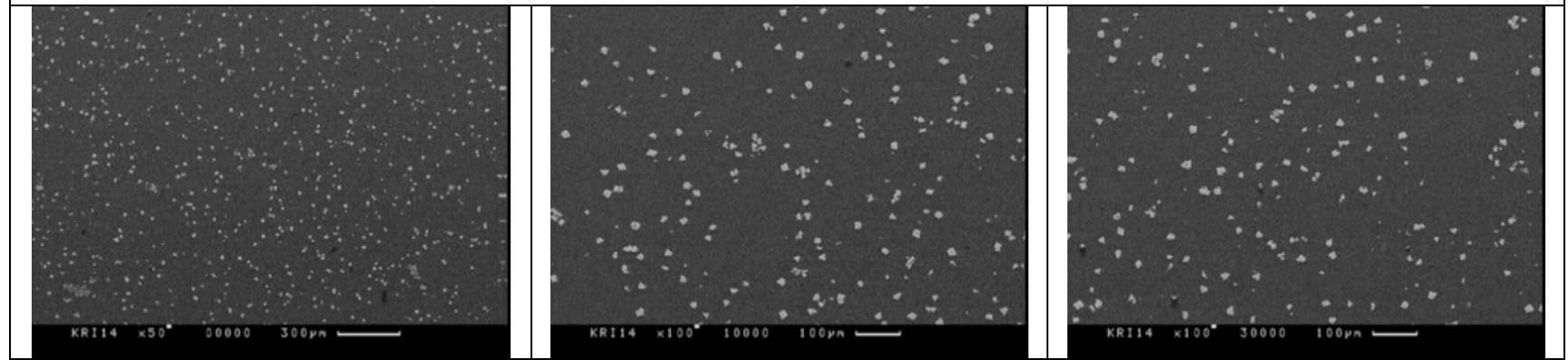

\section{KRI-14 CCC}
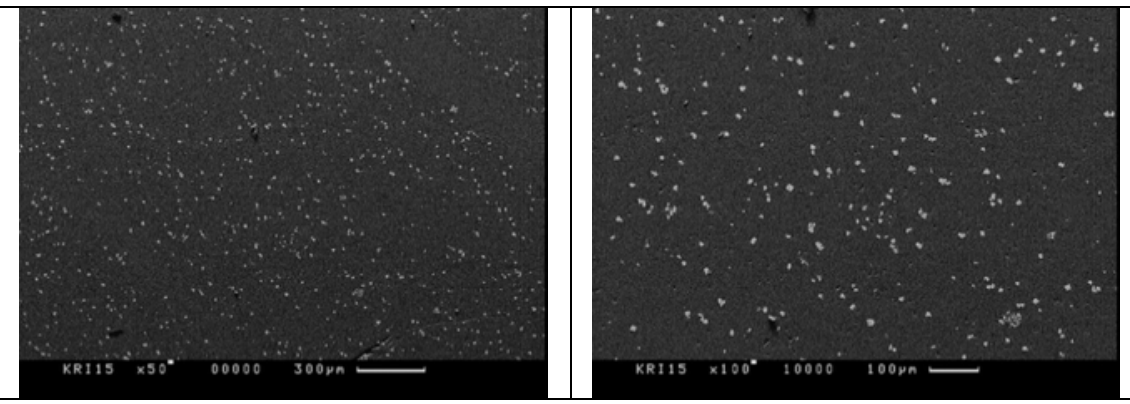

KRI-15 CCC 

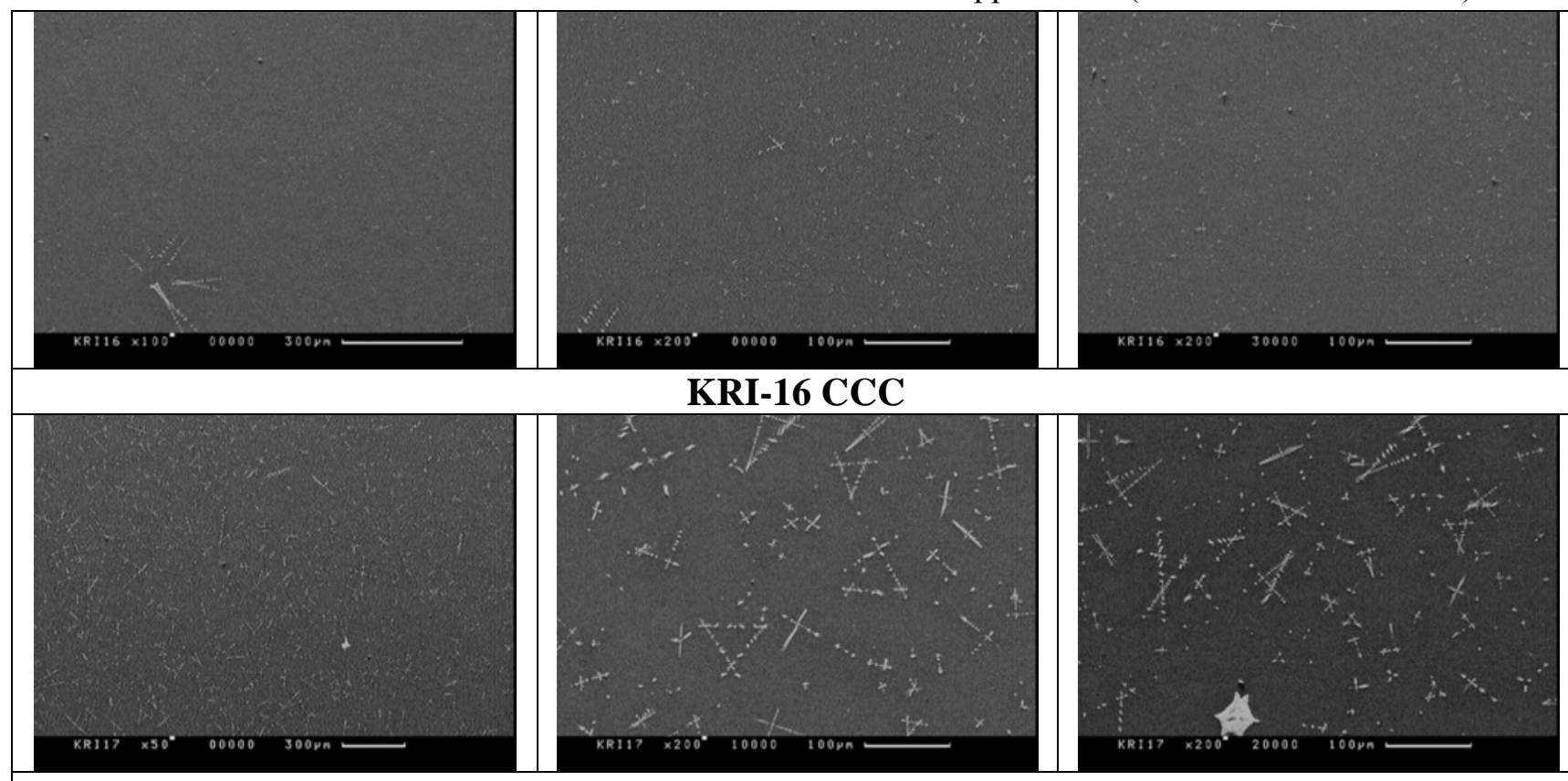

\section{KRI-17 CCC}

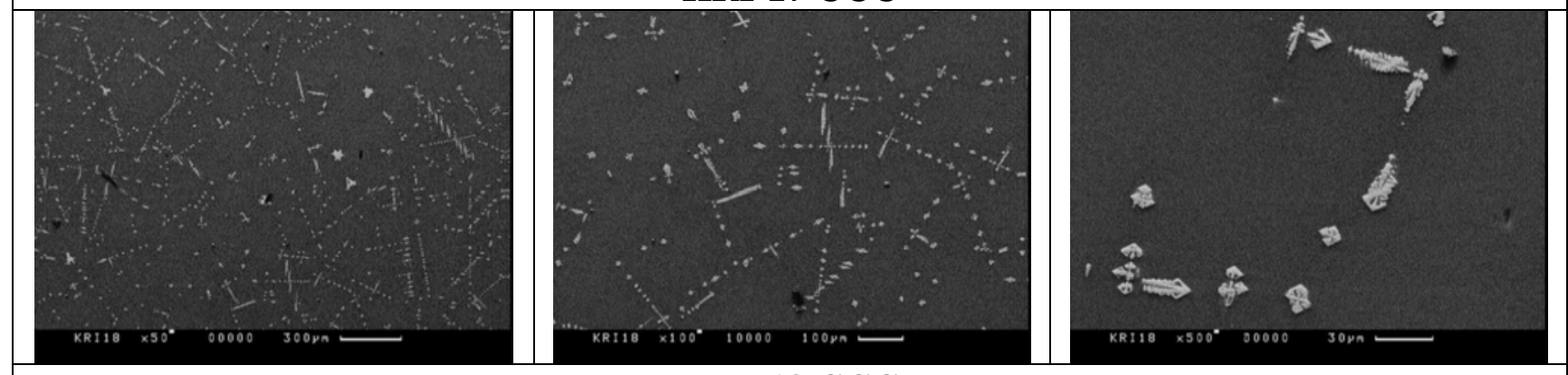

\section{KRI-18 CCC}

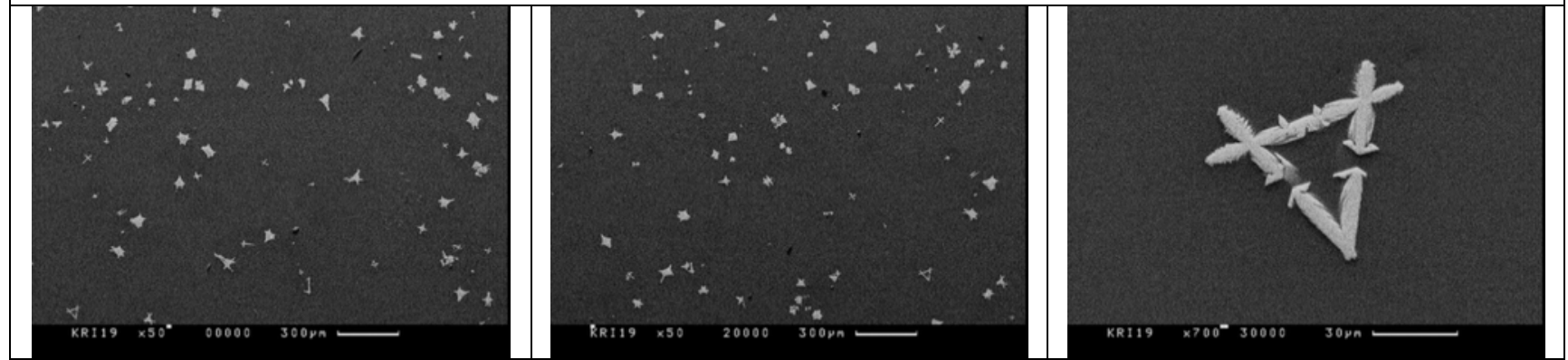

\section{KRI-19 CCC}
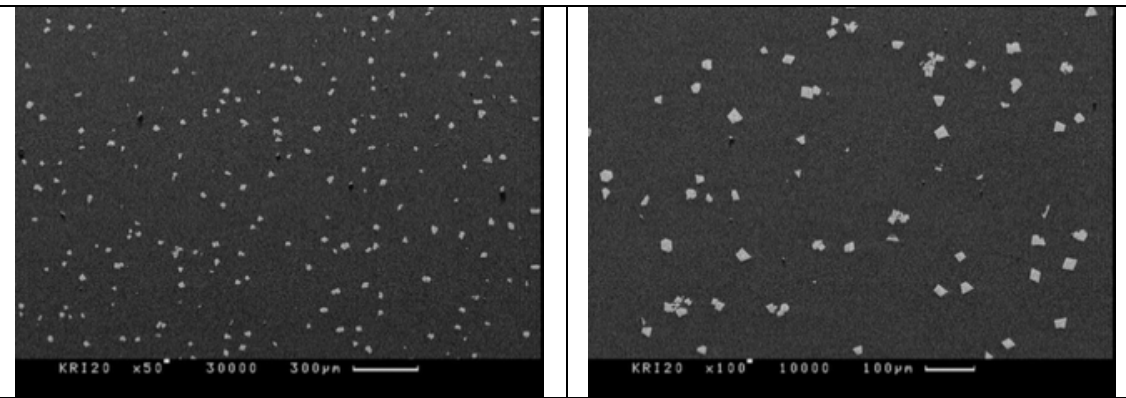

KRI-20 CCC 


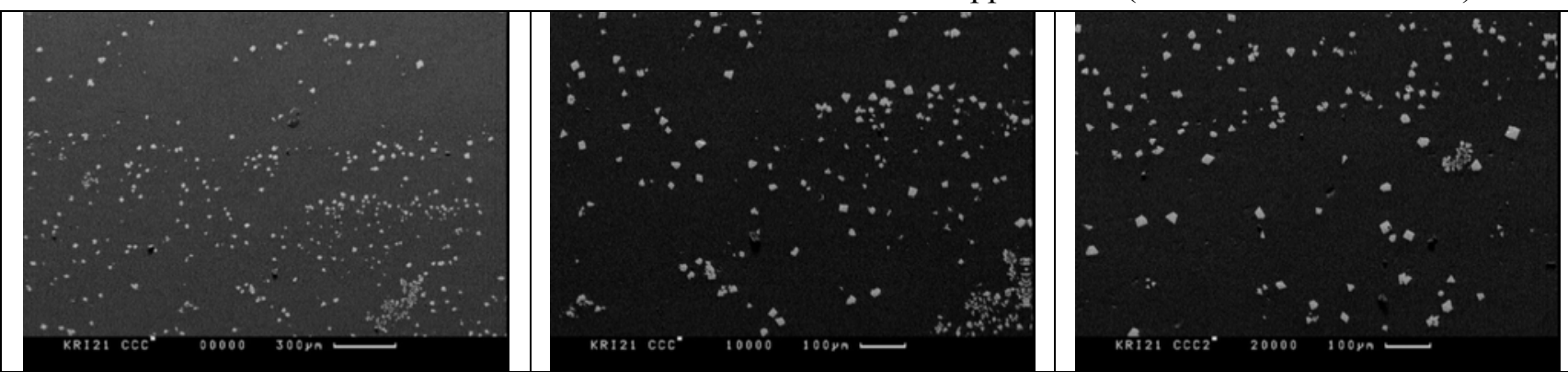

\section{KRI-21 CCC}

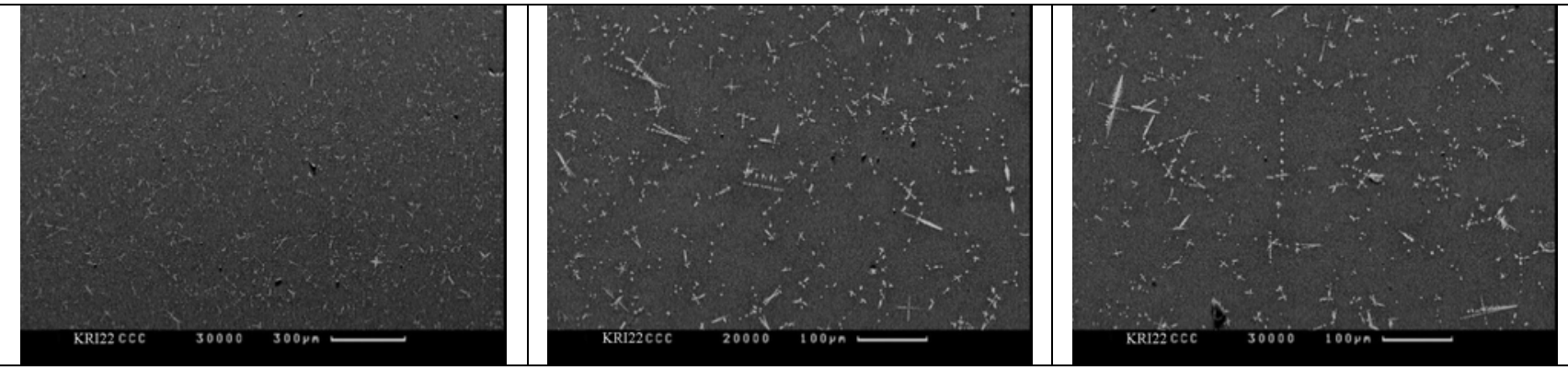

KRI-22 CCC

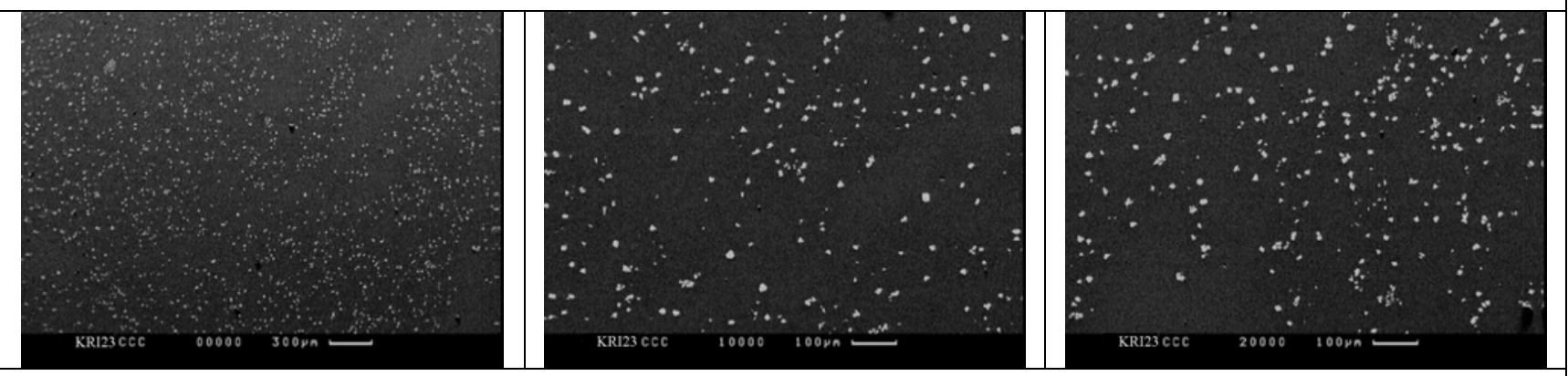

KRI-23 CCC

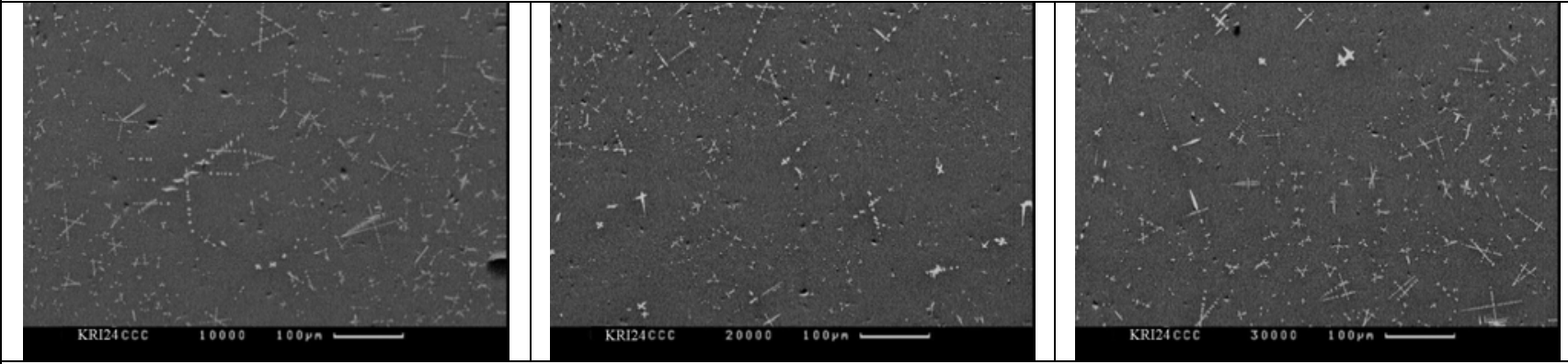

\section{KRI-24 CCC}

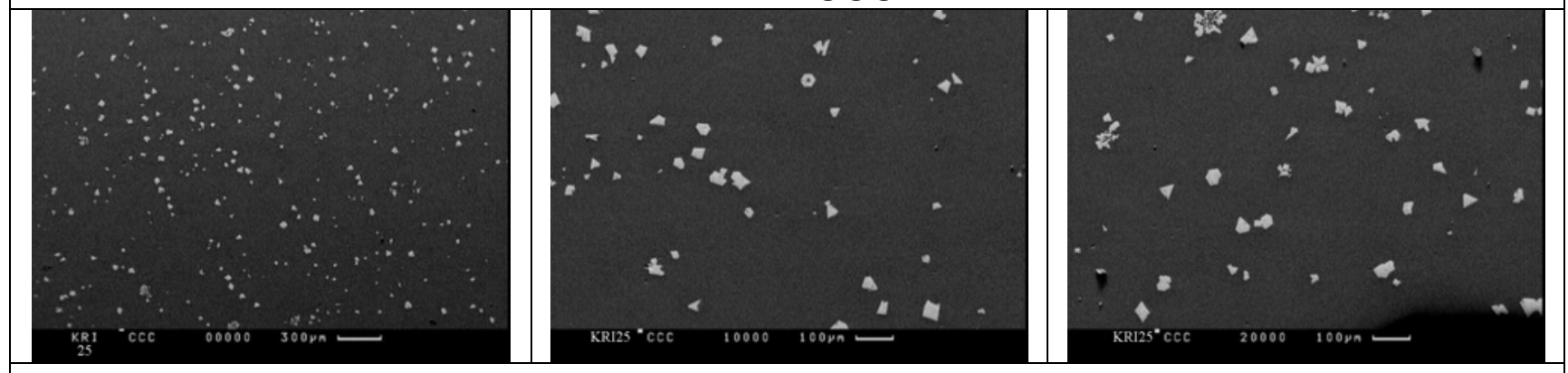

KRI-25 CCC 

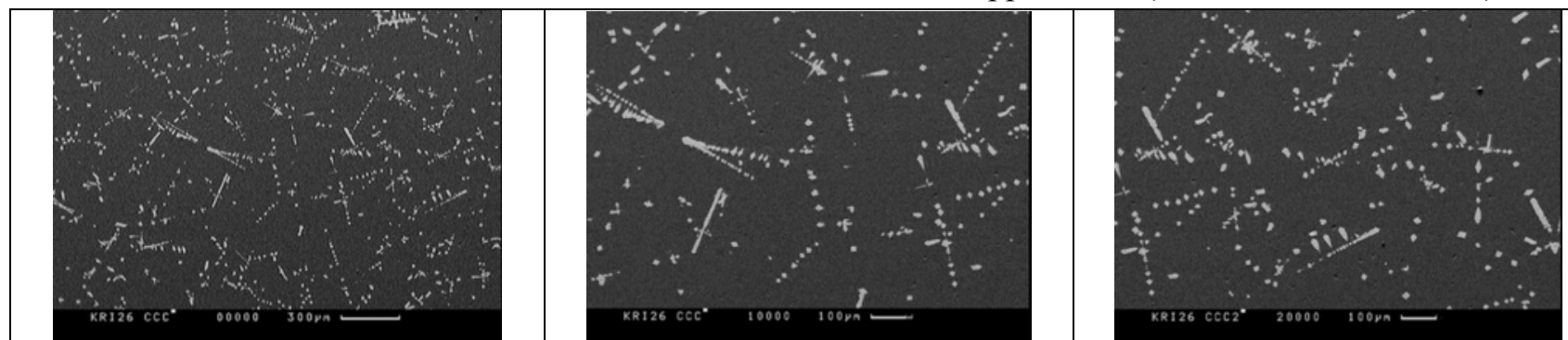

\section{KRI-26 CCC}
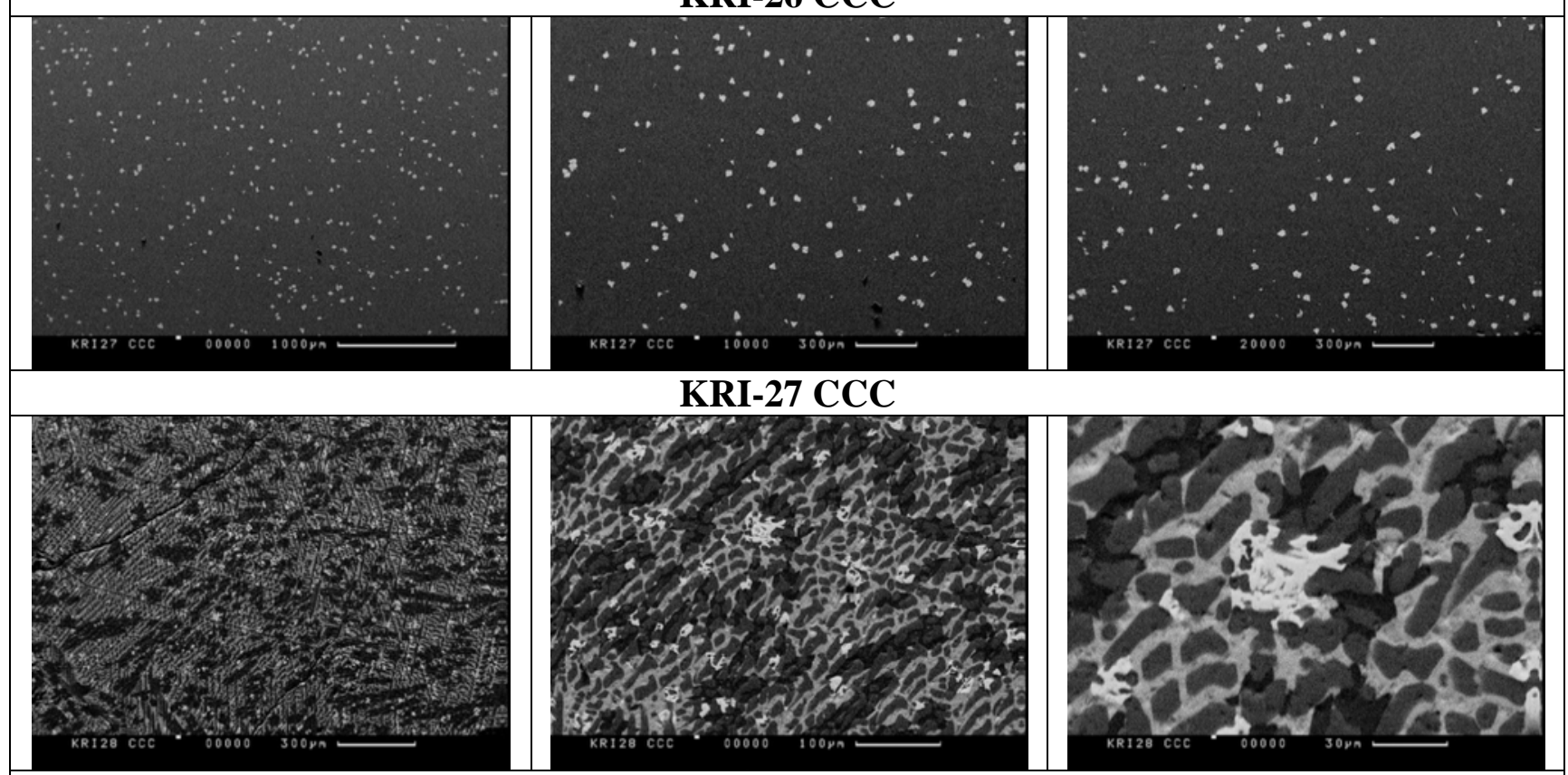

\section{KRI-28 CCC}
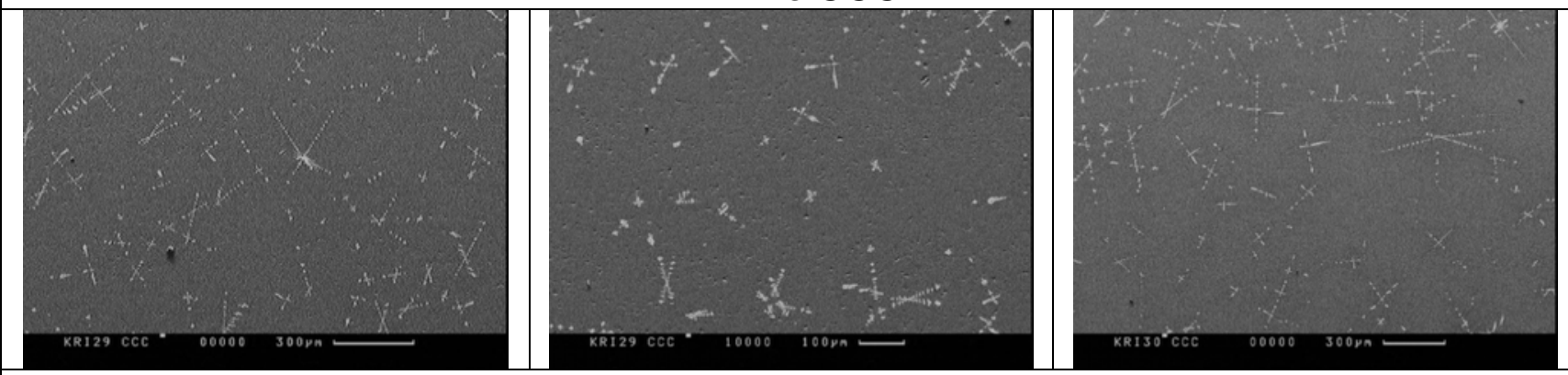

KRI-29 CCC
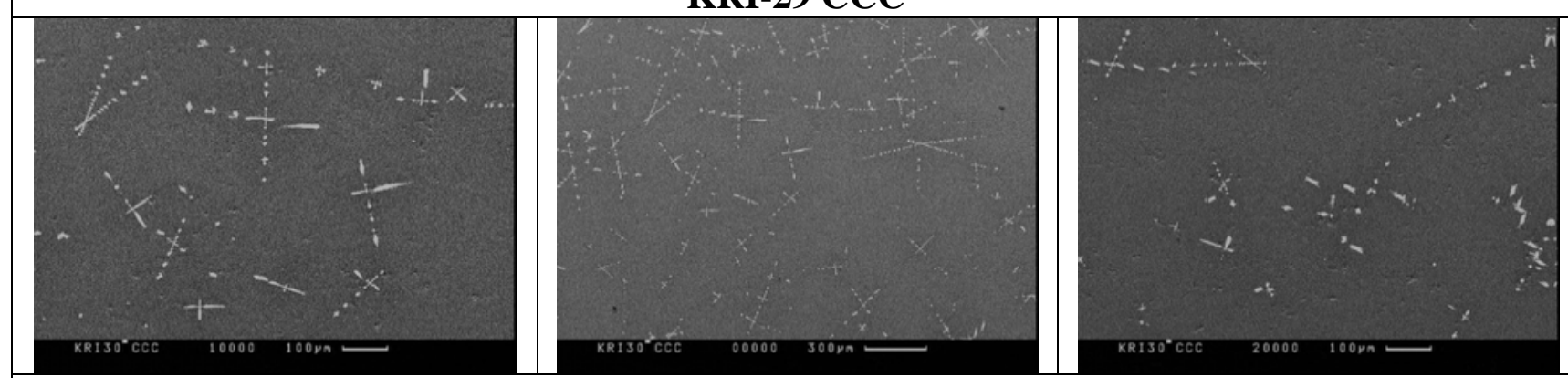

KRI-30 CCC 
SRNS-STI-2008-00057

Revision 0

Appendix C (SRNS-OS-2008-00001)

Attachment 6. EPMA Data on Chemical Homogeneity of Glasses After CCC Treatment

Sample KRI-01 CCC

\begin{tabular}{|c|c|c|c|c|c|c|c|c|c|c|c|}
\hline & $\mathrm{Al} 2 \mathrm{O} 3$ & $\mathrm{Fe} 2 \mathrm{O} 3$ & $\mathrm{~K} 2 \mathrm{O}$ & $\mathrm{MnO}$ & $\mathrm{Na} 2 \mathrm{O}$ & $\mathrm{SO} 3$ & $\mathrm{SiO} 2$ & $\mathrm{TiO} 2$ & $\mathrm{~B} 2 \mathrm{O} 3$ & $\mathrm{Li} 2 \mathrm{O}$ & $\mathrm{Total}$ \\
\hline 1 & 14.93 & 9.54 & 0.68 & 2.55 & 11.15 & 0.24 & 45.71 & 0.78 & 9.56 & 4.86 & 100.00 \\
\hline 2 & 14.91 & 10.04 & 0.69 & 2.33 & 10.24 & 0.24 & 46.18 & 0.95 & 9.56 & 4.86 & 100.00 \\
\hline 3 & 14.97 & 9.94 & 0.81 & 2.37 & 9.11 & 0.24 & 47.16 & 0.97 & 9.56 & 4.86 & 100.00 \\
\hline 4 & 15.04 & 9.76 & 0.68 & 2.66 & 10.59 & 0.23 & 45.69 & 0.94 & 9.56 & 4.86 & 100.00 \\
\hline 5 & 14.65 & 9.75 & 0.78 & 2.50 & 10.94 & 0.23 & 45.64 & 1.08 & 9.56 & 4.86 & 100.00 \\
\hline Average & 14.90 & 9.81 & 0.73 & 2.48 & 10.41 & 0.24 & 46.08 & 0.94 & 9.56 & 4.86 & 100.00 \\
\hline $\begin{array}{c}\text { Average } \\
\text { in area }\end{array}$ & 14.96 & 9.83 & 0.79 & 2.68 & 10.54 & 0.24 & 45.60 & 0.94 & 9.56 & 4.86 & 100.00 \\
\hline Er/Re & 0.43 & 0.71 & & & 0.64 & & 1.41 & & & & \\
\hline
\end{tabular}

Sample KRI-02 CCC

\begin{tabular}{|c|c|c|c|c|c|c|c|c|c|c|c|c|c|}
\hline & $\mathrm{Al} 2 \mathrm{O} 3$ & $\mathrm{CaO}$ & $\mathrm{Fe} 2 \mathrm{O} 3$ & $\mathrm{~K} 2 \mathrm{O}$ & $\mathrm{MnO}$ & $\mathrm{Na} 2 \mathrm{O}$ & $\mathrm{NiO}$ & $\mathrm{SO} 3$ & $\mathrm{SiO} 2$ & $\mathrm{TiO} 2$ & $\mathrm{~B} 2 \mathrm{O} 3$ & $\mathrm{Li} 2 \mathrm{O}$ & $\mathrm{Total}$ \\
\hline 1 & 14.38 & 0.40 & 8.26 & 0.58 & 1.99 & 10.26 & 0.25 & 0.48 & 44.76 & 0.64 & 14.40 & 3.64 & 100.00 \\
\hline 2 & 14.88 & 0.54 & 8.47 & 0.47 & 2.01 & 10.48 & 0.00 & 0.00 & 44.33 & 0.81 & 14.40 & 3.64 & 100.00 \\
\hline 3 & 14.23 & 0.41 & 8.66 & 0.59 & 2.02 & 10.02 & 0.00 & 0.49 & 44.60 & 0.98 & 14.40 & 3.64 & 100.00 \\
\hline 4 & 14.18 & 0.27 & 8.52 & 0.46 & 2.11 & 11.40 & 0.00 & 0.00 & 44.26 & 0.80 & 14.40 & 3.64 & 100.00 \\
\hline 5 & 14.33 & 0.27 & 8.61 & 0.70 & 1.88 & 11.13 & 0.00 & 0.00 & 44.11 & 0.97 & 14.40 & 3.64 & 100.00 \\
\hline Average & 14.40 & 0.38 & 8.50 & 0.56 & 2.00 & 10.66 & 0.05 & 0.19 & 44.41 & 0.84 & 14.40 & 3.64 & 100.00 \\
\hline $\begin{array}{c}\text { Average } \\
\text { in area }\end{array}$ & 14.47 & 0.41 & 8.86 & 0.58 & 2.13 & 10.06 & 0.00 & 0.48 & 44.20 & 0.81 & 14.40 & 3.64 & 100.00 \\
\hline Er/Re & 0.83 & & 0.57 & & & 0.47 & & & 0.58 & & & & \\
\hline
\end{tabular}

Sample KRI-03 CCC

\begin{tabular}{|c|c|c|c|c|c|c|c|c|c|c|c|c|}
\hline & $\mathrm{Al} 2 \mathrm{O} 3$ & $\mathrm{CaO}$ & $\mathrm{Fe} 2 \mathrm{O} 3$ & $\mathrm{~K} 2 \mathrm{O}$ & $\mathrm{MnO}$ & $\mathrm{Na} 2 \mathrm{O}$ & $\mathrm{NiO}$ & $\mathrm{SiO} 2$ & $\mathrm{TiO} 2$ & $\mathrm{~B} 2 \mathrm{O} 3$ & $\mathrm{Li} 2 \mathrm{O}$ & $\mathrm{Total}$ \\
\hline 1 & 14.79 & 0.53 & 8.35 & 0.68 & 1.83 & 10.67 & 0.00 & 43.81 & 0.79 & 14.40 & 3.64 & 100.00 \\
\hline 2 & 14.78 & 0.54 & 8.41 & 0.70 & 1.75 & 10.28 & 0.00 & 44.86 & 0.64 & 14.40 & 3.64 & 100.00 \\
\hline 3 & 14.75 & 0.53 & 8.29 & 0.69 & 1.84 & 10.90 & 0.36 & 43.80 & 0.79 & 14.40 & 3.64 & 100.00 \\
\hline 4 & 14.91 & 0.53 & 8.42 & 0.57 & 1.96 & 10.76 & 0.24 & 43.76 & 0.79 & 14.40 & 3.64 & 100.00 \\
\hline 5 & 14.49 & 0.56 & 8.11 & 0.60 & 1.93 & 9.39 & 0.00 & 46.05 & 0.83 & 14.40 & 3.64 & 100.00 \\
\hline Average & 14.75 & 0.54 & 8.32 & 0.65 & 1.86 & 10.41 & 0.12 & 44.44 & 0.77 & 14.40 & 3.64 & 100.00 \\
\hline $\begin{array}{c}\text { Average } \\
\text { in area }\end{array}$ & 14.70 & 0.52 & 8.43 & 0.68 & 1.94 & 10.48 & 0.00 & 43.53 & 0.94 & 14.40 & 3.64 & 100.00 \\
\hline Er/Re & 0.8 & & 0.6 & & & 1.0 & & 2.5 & & & & \\
\hline
\end{tabular}

Sample KRI-04 CCC

\begin{tabular}{|c|c|c|c|c|c|c|c|c|c|c|c|c|c|}
\hline & $\mathrm{Al} 2 \mathrm{O} 3$ & $\mathrm{CaO}$ & $\mathrm{Fe} 2 \mathrm{O} 3$ & $\mathrm{~K} 2 \mathrm{O}$ & $\mathrm{MnO}$ & $\mathrm{Na} 2 \mathrm{O}$ & $\mathrm{NiO}$ & $\mathrm{SO} 3$ & $\mathrm{SiO} 2$ & $\mathrm{TiO} 2$ & $\mathrm{~B} 2 \mathrm{O} 3$ & $\mathrm{Li} 2 \mathrm{O}$ & Total \\
\hline 1 & 14.27 & 0.54 & 7.60 & 0.58 & 2.25 & 12.39 & 0.25 & 0.48 & 45.58 & 0.81 & 12.25 & 3.00 & 100.00 \\
\hline 2 & 14.51 & 0.54 & 7.81 & 0.46 & 2.36 & 11.64 & 0.37 & 0.00 & 46.43 & 0.64 & 12.25 & 3.00 & 100.00 \\
\hline 3 & 14.30 & 0.68 & 7.90 & 0.47 & 2.26 & 11.37 & 0.00 & 0.00 & 47.14 & 0.65 & 12.25 & 3.00 & 100.00 \\
\hline 4 & 14.78 & 0.53 & 7.81 & 0.57 & 2.31 & 11.56 & 0.36 & 0.00 & 46.21 & 0.63 & 12.25 & 3.00 & 100.00 \\
\hline 5 & 14.20 & 0.41 & 8.36 & 0.59 & 2.27 & 10.92 & 0.37 & 0.00 & 46.81 & 0.81 & 12.25 & 3.00 & 100.00 \\
\hline Average & 14.41 & 0.54 & 7.90 & 0.53 & 2.29 & 11.57 & 0.27 & 0.10 & 46.43 & 0.71 & 12.25 & 3.00 & 100.00 \\
\hline $\begin{array}{c}\text { Average } \\
\text { in area }\end{array}$ & 14.41 & 0.52 & 7.30 & 0.56 & 2.28 & 12.78 & 0.47 & 0.00 & 45.18 & 0.62 & 12.25 & 3.00 & 100.00 \\
\hline $\mathrm{Er} / \mathrm{Re}$ & 0.70 & & 1.04 & & & 0.43 & & & 1.30 & & & & \\
\hline
\end{tabular}


SRNS-STI-2008-00057

Revision 0

Appendix C (SRNS-OS-2008-00001)

Sample KRI-05 CCC

\begin{tabular}{|c|c|c|c|c|c|c|c|c|c|c|c|c|c|c|c|}
\hline & $\mathrm{Al}_{2} \mathrm{O}_{3}$ & $\mathrm{CaO}$ & $\mathrm{Fe}_{2} \mathrm{O}_{3}$ & $\mathrm{~K}_{2} \mathrm{O}$ & $\mathrm{MnO}$ & $\mathrm{Na}_{2} \mathrm{O}$ & $\mathrm{PbO}$ & $\mathrm{SiO}_{2}$ & $\mathrm{TiO}_{2}$ & $\mathrm{ZnO}$ & $\mathrm{ZrO}_{2}$ & $\mathrm{SrO}$ & $\mathrm{B}_{2} \mathrm{O}_{3}$ & $\mathrm{Li}_{2} \mathrm{O}$ & Total \\
\hline 1 & 13.08 & 0.27 & 5.96 & 0.68 & 2.45 & 10.60 & 0.82 & 41.59 & 0.63 & 0.94 & 1.02 & 0.00 & 19.96 & 2.00 & 100.00 \\
\hline 2 & 13.62 & 0.26 & 4.14 & 0.68 & 2.18 & 10.34 & 0.60 & 42.88 & 0.62 & 0.93 & 1.01 & 0.77 & 19.96 & 2.00 & 100.00 \\
\hline 3 & 13.25 & 0.26 & 4.33 & 0.57 & 2.57 & 10.34 & 0.82 & 43.20 & 0.63 & 1.18 & 0.90 & 0.00 & 19.96 & 2.00 & 100.00 \\
\hline 4 & 13.17 & 0.26 & 4.04 & 0.57 & 2.43 & 11.42 & 0.71 & 42.75 & 0.63 & 1.17 & 0.89 & 0.00 & 19.96 & 2.00 & 100.00 \\
\hline 5 & 13.27 & 0.00 & 4.15 & 0.56 & 2.42 & 10.60 & 0.71 & 42.29 & 0.63 & 1.05 & 1.26 & 1.11 & 19.96 & 2.00 & 100.00 \\
\hline Average & 13.28 & 0.21 & 4.52 & 0.61 & 2.41 & 10.66 & 0.73 & 42.54 & 0.63 & 1.05 & 1.02 & 0.38 & 19.96 & 2.00 & 100.00 \\
\hline $\begin{array}{c}\text { Average } \\
\text { in area }\end{array}$ & 13.07 & 0.26 & 6.54 & 0.56 & 2.90 & 10.45 & 0.91 & 41.00 & 0.62 & 0.93 & 0.76 & 0.00 & 19.96 & 2.00 & 99.95 \\
\hline $\mathrm{Er} / \mathrm{Re}$ & 0.61 & & 2.96 & & & 0.36 & & 1.36 & & & & & & & \\
\hline
\end{tabular}

Sample KRI-06 CCC

\begin{tabular}{|c|c|c|c|c|c|c|c|c|c|c|c|c|c|c|c|c|c|c|}
\hline & $\mathrm{Al} 2 \mathrm{O} 3$ & $\mathrm{CaO}$ & $\mathrm{Cr} 2 \mathrm{O} 3$ & $\mathrm{Fe} 2 \mathrm{O} 3$ & K2O & $\mathrm{MnO}$ & $\mathrm{Na} 2 \mathrm{O}$ & $\mathrm{NiO}$ & $\mathrm{PbO}$ & SO3 & $\mathrm{SiO} 2$ & TiO2 & $\mathrm{ZnO}$ & $\mathrm{ZrO} 2$ & $\mathrm{SrO}$ & B2O3 & $\mathrm{Li} 2 \mathrm{O}$ & Total \\
\hline 1 & 12.40 & 0.26 & 0.27 & 8.70 & 0.45 & 1.69 & 12.50 & 0.00 & 0.50 & 0.00 & 41.53 & 0.31 & 0.93 & 2.03 & 1.77 & 12.59 & 4.05 & 100.00 \\
\hline 2 & 12.50 & 0.40 & 0.00 & 9.03 & 0.35 & 1.98 & 11.88 & 0.00 & 0.62 & 0.00 & 42.26 & 0.48 & 1.07 & 1.42 & 1.36 & 12.59 & 4.05 & 100.00 \\
\hline 3 & 12.53 & 0.40 & 0.28 & 8.64 & 0.46 & 1.74 & 12.68 & 0.00 & 0.41 & 0.00 & 42.37 & 0.48 & 0.72 & 1.17 & 1.48 & 12.59 & 4.05 & 100.00 \\
\hline 4 & 12.38 & 0.27 & 0.28 & 8.68 & 0.46 & 1.96 & 12.15 & 0.24 & 0.72 & 0.00 & 42.07 & 0.32 & 1.06 & 1.67 & 1.12 & 12.59 & 4.05 & 100.00 \\
\hline 5 & 12.69 & 0.27 & 0.28 & 8.63 & 0.46 & 1.86 & 11.89 & 0.00 & 0.52 & 0.00 & 42.30 & 0.48 & 0.95 & 1.55 & 1.47 & 12.59 & 4.05 & 100.00 \\
\hline Average & 12.50 & 0.32 & 0.22 & 8.74 & 0.44 & 1.85 & 12.22 & 0.05 & 0.55 & 0.00 & 42.11 & 0.41 & 0.95 & 1.57 & 1.44 & 12.59 & 4.05 & 100.00 \\
\hline $\begin{array}{c}\text { Average } \\
\text { in area }\end{array}$ & 12.50 & 0.39 & 0.27 & 8.52 & 0.45 & 1.93 & 12.30 & 0.24 & 0.60 & 0.00 & 40.89 & 0.47 & 1.04 & 1.89 & 1.87 & 12.59 & 4.05 & 100.00 \\
\hline $\mathrm{Er} / \mathrm{Re}$ & 0.37 & & & 0.62 & & & 0.29 & & & & 0.75 & & & & & & & \\
\hline
\end{tabular}

Sample KRI-07 CCC

\begin{tabular}{|c|c|c|c|c|c|c|c|c|c|c|c|c|c|c|c|c|c|}
\hline & $\mathrm{Al} 2 \mathrm{O} 3$ & $\mathrm{CaO}$ & Cr2O3 & Fe2O3 & $\mathrm{K} 2 \mathrm{O}$ & $\mathrm{MnO}$ & $\mathrm{Na} 2 \mathrm{O}$ & $\mathrm{NiO}$ & $\mathrm{PbO}$ & $\mathrm{SiO} 2$ & TiO2 & $\mathrm{ZnO}$ & ZrO2 & $\mathrm{SrO}$ & B2O3 & $\mathrm{Li} 2 \mathrm{O}$ & Total \\
\hline 1 & 12.76 & 0.39 & 0.27 & 8.85 & 0.45 & 1.91 & 12.58 & 0.35 & 0.80 & 39.79 & 0.46 & 0.92 & 1.75 & 2.08 & 12.60 & 4.05 & 100.00 \\
\hline 2 & 13.30 & 0.40 & 0.28 & 8.81 & 0.46 & 1.74 & 12.08 & 0.00 & 0.52 & 41.26 & 0.48 & 0.84 & 1.69 & 1.48 & 12.60 & 4.05 & 100.00 \\
\hline 3 & 13.05 & 0.40 & 0.00 & 9.04 & 0.58 & 1.86 & 11.37 & 0.00 & 0.72 & 41.28 & 0.48 & 1.19 & 1.55 & 1.81 & 12.60 & 4.05 & 100.00 \\
\hline 4 & 12.81 & 0.39 & 0.28 & 9.01 & 0.45 & 1.82 & 12.05 & 0.48 & 0.61 & 40.50 & 0.47 & 0.94 & 1.65 & 1.89 & 12.60 & 4.05 & 100.00 \\
\hline 5 & 12.93 & 0.39 & 0.14 & 8.57 & 0.56 & 1.94 & 12.63 & 0.00 & 0.81 & 40.34 & 0.47 & 1.05 & 1.52 & 1.99 & 12.60 & 4.05 & 100.00 \\
\hline Average & 12.97 & 0.40 & 0.19 & 8.86 & 0.50 & 1.85 & 12.14 & 0.17 & 0.69 & 40.63 & 0.47 & 0.99 & 1.63 & 1.85 & 12.60 & 4.05 & 100.00 \\
\hline $\begin{array}{c}\text { Average } \\
\text { in area }\end{array}$ & 13.16 & 0.40 & 0.28 & 8.99 & 0.57 & 1.72 & 12.60 & 0.00 & 0.72 & 40.84 & 0.48 & 0.83 & 1.29 & 1.47 & 12.60 & 4.05 & 100.00 \\
\hline $\mathrm{Er} / \mathrm{Re}$ & 1.1 & & & 0.9 & & & 0.8 & & & 1.6 & & & & & & & \\
\hline
\end{tabular}

\section{Sample KRI-08 CCC}

\begin{tabular}{|c|c|c|c|c|c|c|c|c|c|c|c|c|}
\hline & $\mathrm{Al} 2 \mathrm{O} 3$ & $\mathrm{CaO}$ & $\mathrm{Fe} 2 \mathrm{O} 3$ & $\mathrm{~K} 2 \mathrm{O}$ & $\mathrm{MnO}$ & $\mathrm{Na} 2 \mathrm{O}$ & $\mathrm{SiO} 2$ & $\mathrm{ZnO}$ & $\mathrm{SrO}$ & $\mathrm{B} 2 \mathrm{O} 3$ & $\mathrm{Li} 2 \mathrm{O}$ & Total \\
\hline 1 & 12.75 & 0.99 & 5.61 & 1.82 & 2.86 & 9.36 & 42.67 & 1.25 & 3.45 & 16.25 & 3.00 & 100.00 \\
\hline 2 & 12.76 & 1.00 & 5.41 & 1.85 & 2.91 & 9.51 & 42.92 & 1.02 & 3.38 & 16.25 & 3.00 & 100.00 \\
\hline 3 & 13.34 & 1.00 & 5.40 & 1.85 & 2.91 & 9.92 & 41.81 & 1.02 & 3.50 & 16.25 & 3.00 & 100.00 \\
\hline 4 & 13.35 & 1.00 & 5.26 & 2.10 & 2.64 & 9.79 & 42.07 & 1.27 & 3.27 & 16.25 & 3.00 & 100.00 \\
\hline 5 & 13.63 & 0.86 & 5.30 & 1.86 & 2.53 & 10.13 & 42.13 & 0.90 & 3.41 & 16.25 & 3.00 & 100.00 \\
\hline Average & 13.16 & 0.97 & 5.40 & 1.89 & 2.77 & 9.74 & 42.32 & 1.09 & 3.40 & 16.25 & 3.00 & 100.00 \\
\hline Average in area & 12.66 & 0.83 & 9.57 & 1.66 & 3.82 & 9.29 & 38.16 & 1.23 & 3.26 & 16.25 & 3.00 & 100.00 \\
\hline $\mathrm{Er} / \mathrm{Re}$ & 2.0 & & 0.7 & & & 0.5 & 1.1 & & & & & \\
\hline
\end{tabular}


SRNS-STI-2008-00057

Revision 0

Appendix C (SRNS-OS-2008-00001)

Sample KRI 09-CCC

\begin{tabular}{|c|c|c|c|c|c|c|c|c|c|c|c|c|c|c|c|}
\hline & $\mathrm{Al} 2 \mathrm{O} 3$ & $\mathrm{CaO}$ & $\mathrm{Fe} 2 \mathrm{O} 3$ & $\mathrm{~K} 2 \mathrm{O}$ & $\mathrm{MnO}$ & $\mathrm{Na} 2 \mathrm{O}$ & $\mathrm{PbO}$ & $\mathrm{SiO} 2$ & $\mathrm{TiO} 2$ & $\mathrm{ZnO}$ & $\mathrm{ZrO} 2$ & $\mathrm{SrO}$ & B2O3 & Li2O & Total \\
\hline 1 & 14.17 & 0.13 & 6.01 & 0.22 & 0.71 & 12.20 & 0.89 & 42.09 & 0.61 & 1.14 & 3.21 & 1.30 & 14.34 & 3.00 & 100.00 \\
\hline 2 & 14.42 & 0.13 & 5.65 & 0.23 & 0.73 & 11.42 & 0.91 & 43.35 & 0.63 & 1.17 & 2.80 & 1.22 & 14.34 & 3.00 & 100.00 \\
\hline 3 & 14.22 & 0.13 & 5.65 & 0.23 & 0.87 & 11.05 & 0.94 & 44.41 & 0.64 & 0.96 & 2.87 & 0.68 & 14.34 & 3.00 & 100.00 \\
\hline 4 & 14.44 & 0.00 & 5.32 & 0.23 & 0.86 & 11.58 & 0.93 & 43.54 & 0.80 & 0.95 & 3.22 & 0.79 & 14.34 & 3.00 & 100.00 \\
\hline 5 & 14.12 & 0.13 & 5.94 & 0.23 & 0.86 & 12.36 & 0.71 & 43.33 & 0.63 & 0.94 & 2.94 & 0.00 & 14.34 & 3.00 & 100.00 \\
\hline Average & 14.27 & 0.11 & 5.71 & 0.23 & 0.81 & 11.72 & 0.87 & 43.34 & 0.66 & 1.03 & 3.01 & 0.80 & 14.34 & 3.00 & 100.00 \\
\hline $\begin{array}{l}\text { Average in } \\
\text { area }\end{array}$ & 13.91 & 0.00 & 6.79 & 0.22 & 0.96 & 11.17 & 0.70 & 42.09 & 0.78 & 1.39 & 3.02 & 0.99 & 14.34 & 3.00 & 100.00 \\
\hline $\mathrm{Er} / \mathrm{Re}$ & 0.7 & & 1.4 & & & 0.9 & & 2.1 & & & & & & & \\
\hline
\end{tabular}

Sample KRI-10 CCC

\begin{tabular}{|c|c|c|c|c|c|c|c|c|c|c|c|c|c|c|c|c|}
\hline & $\mathrm{Al} 2 \mathrm{O} 3$ & $\mathrm{CaO}$ & Cr2O3 & $\mathrm{Fe} 2 \mathrm{O} 3$ & $\mathrm{~K} 2 \mathrm{O}$ & $\mathrm{MnO}$ & $\mathrm{Na} 2 \mathrm{O}$ & $\mathrm{NiO}$ & $\mathrm{SiO} 2$ & $\mathrm{TiO} 2$ & $\mathrm{ZnO}$ & $\mathrm{ZrO} 2$ & $\mathrm{SrO}$ & B2O3 & Li2O & Total \\
\hline 1 & 12.87 & 0.58 & 0.30 & 7.66 & 0.75 & 1.07 & 12.93 & 0.53 & 47.50 & 0.00 & 1.03 & 0.84 & 1.46 & 9.38 & 3.11 & 100.00 \\
\hline 2 & 12.62 & 0.56 & 0.29 & 7.54 & 0.60 & 1.16 & 13.56 & 0.63 & 46.30 & 0.17 & 1.12 & 1.08 & 1.88 & 9.38 & 3.11 & 100.00 \\
\hline 3 & 12.93 & 0.58 & 0.30 & 7.40 & 0.75 & 0.94 & 12.71 & 0.00 & 48.16 & 0.00 & 1.03 & 0.98 & 1.72 & 9.38 & 3.11 & 100.00 \\
\hline 4 & 12.99 & 0.57 & 0.45 & 7.77 & 0.62 & 0.93 & 12.44 & 0.26 & 47.45 & 0.34 & 1.02 & 0.97 & 1.70 & 9.38 & 3.11 & 100.00 \\
\hline 5 & 12.95 & 0.57 & 0.30 & 7.60 & 0.74 & 1.06 & 12.13 & 0.26 & 47.97 & 0.00 & 1.27 & 0.97 & 1.69 & 9.38 & 3.11 & 100.00 \\
\hline Average & 12.87 & 0.57 & 0.33 & 7.60 & 0.69 & 1.03 & 12.76 & 0.34 & 47.47 & 0.10 & 1.09 & 0.97 & 1.69 & 9.38 & 3.11 & 100.00 \\
\hline $\begin{array}{c}\text { Average in } \\
\text { area }\end{array}$ & 12.75 & 0.65 & 0.41 & 7.66 & 0.67 & 1.19 & 12.71 & 0.59 & 46.90 & 0.31 & 0.81 & 1.12 & 1.75 & 9.38 & 3.11 & 100.00 \\
\hline $\mathrm{Er} / \mathrm{Re}$ & 0.7 & & & 0.7 & & & 0.9 & & 1.8 & & & & & & & \\
\hline
\end{tabular}

Sample KRI-11 CCC

\begin{tabular}{|c|c|c|c|c|c|c|c|c|c|c|c|c|c|}
\hline & $\mathrm{Al} 2 \mathrm{O} 3$ & $\mathrm{CaO}$ & $\mathrm{Fe} 2 \mathrm{O} 3$ & $\mathrm{~K} 2 \mathrm{O}$ & $\mathrm{MnO}$ & $\mathrm{Na} 2 \mathrm{O}$ & $\mathrm{SiO} 2$ & $\mathrm{TiO} 2$ & $\mathrm{ZrO} 2$ & $\mathrm{SrO}$ & $\mathrm{B} 2 \mathrm{O} 3$ & $\mathrm{Li} 2 \mathrm{O}$ & $\mathrm{Total}$ \\
\hline 1 & 16.94 & 0.00 & 7.26 & 0.33 & 0.84 & 11.58 & 42.93 & 0.31 & 1.25 & 0.00 & 13.99 & 4.08 & 100.00 \\
\hline 2 & 17.58 & 0.14 & 5.09 & 0.24 & 0.64 & 10.53 & 44.91 & 0.00 & 1.60 & 1.17 & 13.99 & 4.08 & 100.00 \\
\hline 3 & 17.08 & 0.26 & 5.24 & 0.34 & 0.61 & 11.67 & 43.94 & 0.00 & 1.53 & 1.22 & 13.99 & 4.08 & 100.00 \\
\hline 4 & 17.17 & 0.27 & 5.38 & 0.35 & 0.62 & 11.72 & 44.89 & 0.32 & 1.17 & 0.00 & 13.99 & 4.08 & 100.00 \\
\hline 5 & 17.46 & 0.00 & 5.39 & 0.48 & 0.64 & 9.77 & 46.36 & 0.33 & 1.48 & 0.00 & 13.99 & 4.08 & 100.00 \\
\hline $\begin{array}{c}\text { Average } \\
\text { in area }\end{array}$ & 16.64 & 0.26 & 7.76 & 0.45 & 0.73 & 10.47 & 41.90 & 0.31 & 1.39 & 1.11 & 13.99 & 4.08 & 100.00 \\
\hline Average & 17.25 & 0.13 & 5.67 & 0.35 & 0.67 & 11.05 & 44.61 & 0.19 & 1.41 & 0.48 & 13.99 & 4.08 & 100.00 \\
\hline Er/Re & 1.33 & & 8.1 & & & 1.1 & 4.2 & & & & & & \\
\hline
\end{tabular}

Sample KRI-12 CCC

\begin{tabular}{|c|c|c|c|c|c|c|c|c|c|c|c|c|c|}
\hline & $\mathrm{Al} 2 \mathrm{O} 3$ & $\mathrm{CaO}$ & $\mathrm{Fe} 2 \mathrm{O} 3$ & $\mathrm{~K} 2 \mathrm{O}$ & $\mathrm{MnO}$ & $\mathrm{Na} 2 \mathrm{O}$ & $\mathrm{SiO} 2$ & $\mathrm{TiO} 2$ & $\mathrm{ZrO} 2$ & $\mathrm{SrO}$ & $\mathrm{B} 2 \mathrm{O} 3$ & $\mathrm{Li} 2 \mathrm{O}$ & $\mathrm{Total}$ \\
\hline 1 & 13.59 & 0.54 & 7.67 & 0.69 & 2.97 & 9.04 & 40.83 & 0.32 & 3.49 & 2.27 & 14.10 & 3.88 & 100.00 \\
\hline 2 & 13.22 & 0.39 & 7.62 & 0.66 & 2.85 & 10.04 & 39.79 & 0.31 & 3.73 & 3.04 & 14.10 & 3.88 & 100.00 \\
\hline 3 & 14.16 & 0.41 & 6.95 & 0.59 & 3.02 & 8.26 & 42.49 & 0.32 & 3.28 & 2.53 & 14.10 & 3.88 & 100.00 \\
\hline 4 & 14.47 & 0.40 & 5.88 & 0.69 & 2.47 & 9.55 & 42.22 & 0.32 & 3.62 & 2.38 & 14.10 & 3.88 & 100.00 \\
\hline $\mathbf{5}$ & 14.61 & 0.54 & 5.24 & 0.70 & 2.62 & 9.64 & 42.82 & 0.00 & 3.65 & 2.17 & 14.10 & 3.88 & 100.00 \\
\hline $\begin{array}{c}\text { Average } \\
\text { in area }\end{array}$ & 13.86 & 0.54 & 7.72 & 0.58 & 3.12 & 8.97 & 41.53 & 0.00 & 3.39 & 2.28 & 14.10 & 3.88 & 100.00 \\
\hline Average & 14.01 & 0.45 & 6.67 & 0.67 & 2.79 & 9.30 & 41.63 & 0.25 & 3.56 & 2.48 & 14.10 & 3.88 & 100.00 \\
\hline Er/Re & 2.97 & & 9.8 & & & 0.9 & 4.3 & & & & & & \\
\hline
\end{tabular}


SRNS-STI-2008-00057

Revision 0

Appendix C (SRNS-OS-2008-00001)

Sample KRI 13-CCC

\begin{tabular}{|c|c|c|c|c|c|c|c|c|c|c|c|c|c|c|c|}
\hline & Al2O3 & $\mathrm{CaO}$ & $\mathrm{Fe} 2 \mathrm{O} 3$ & $\mathrm{~K} 2 \mathrm{O}$ & $\mathrm{MnO}$ & $\mathrm{Na} 2 \mathrm{O}$ & $\mathrm{PbO}$ & $\mathrm{SiO} 2$ & $\mathrm{TiO} 2$ & $\mathrm{ZnO}$ & $\mathrm{ZrO} 2$ & $\mathrm{SrO}$ & $\mathrm{B} 2 \mathrm{O} 3$ & Li2O & Total \\
\hline 1 & 13.62 & 0.00 & 5.84 & 0.70 & 2.39 & 12.20 & 0.84 & 46.90 & 0.81 & 1.45 & 1.45 & 0.86 & 10.79 & 3.00 & 100.00 \\
\hline 2 & 13.54 & 0.00 & 6.17 & 0.59 & 2.41 & 12.56 & 0.74 & 46.86 & 0.66 & 1.34 & 1.33 & 0.86 & 10.79 & 3.00 & 100.00 \\
\hline 3 & 13.87 & 0.27 & 5.99 & 0.69 & 2.59 & 11.69 & 0.92 & 46.33 & 0.64 & 1.07 & 1.67 & 0.86 & 10.79 & 3.00 & 100.00 \\
\hline 4 & 13.93 & 0.00 & 6.51 & 0.58 & 2.63 & 11.37 & 0.73 & 46.93 & 0.49 & 1.45 & 1.57 & 0.86 & 10.79 & 3.00 & 100.00 \\
\hline 5 & 13.68 & 0.00 & 5.87 & 0.59 & 2.40 & 11.60 & 0.74 & 47.75 & 0.65 & 1.34 & 1.59 & 0.86 & 10.79 & 3.00 & 100.00 \\
\hline $\begin{array}{c}\text { Average } \\
\text { in area }\end{array}$ & 13.19 & 0.26 & 6.43 & 0.55 & 2.73 & 13.74 & 0.89 & 44.63 & 0.46 & 1.37 & 1.49 & 0.86 & 10.79 & 3.00 & 100.00 \\
\hline Average & 13.73 & 0.05 & 6.08 & 0.63 & 2.48 & 11.88 & 0.79 & 46.95 & 0.65 & 1.33 & 1.52 & 0.86 & 10.79 & 3.00 & 100.00 \\
\hline Er/Re & 0.84 & & 2.5 & & & 0.6 & & 1.7 & & & & & & & \\
\hline
\end{tabular}

Sample KRI-14 CCC

\begin{tabular}{|c|c|c|c|c|c|c|c|c|c|c|c|c|c|c|}
\hline & $\mathrm{Al} 2 \mathrm{O} 3$ & $\mathrm{Fe} 2 \mathrm{O} 3$ & $\mathrm{~K} 2 \mathrm{O}$ & $\mathrm{MnO}$ & $\mathrm{Na} 2 \mathrm{O}$ & $\mathrm{PbO}$ & $\mathrm{SiO} 2$ & $\mathrm{TiO} 2$ & $\mathrm{ZnO}$ & $\mathrm{ZrO} 2$ & $\mathrm{SrO}$ & $\mathrm{B} 2 \mathrm{O} 3$ & $\mathrm{Li} 2 \mathrm{O}$ & Total \\
\hline 1 & 14.08 & 4.15 & 0.23 & 2.63 & 8.35 & 0.83 & 44.54 & 0.65 & 1.32 & 1.44 & 1.14 & 15.46 & 5.00 & 100.00 \\
\hline 2 & 14.13 & 4.73 & 0.23 & 2.44 & 9.18 & 0.92 & 43.75 & 0.79 & 0.94 & 1.28 & 1.12 & 15.46 & 5.00 & 100.00 \\
\hline 3 & 14.30 & 4.24 & 0.35 & 2.72 & 9.42 & 0.93 & 44.70 & 0.48 & 1.19 & 1.16 & 0.00 & 15.46 & 5.00 & 100.00 \\
\hline 4 & 13.64 & 5.09 & 0.23 & 2.79 & 9.10 & 0.81 & 43.15 & 0.78 & 1.28 & 1.27 & 1.11 & 15.46 & 5.00 & 100.00 \\
\hline 5 & 13.85 & 4.08 & 0.23 & 2.58 & 8.98 & 1.02 & 44.62 & 0.64 & 1.07 & 1.54 & 0.90 & 15.46 & 5.00 & 100.00 \\
\hline $\begin{array}{c}\text { Average } \\
\text { in area }\end{array}$ & 13.44 & 6.46 & 0.11 & 2.62 & 8.83 & 0.89 & 42.50 & 0.77 & 1.03 & 1.12 & 0.98 & 15.46 & 5.00 & 100.00 \\
\hline Average & 14.00 & 4.46 & 0.25 & 2.63 & 9.00 & 0.90 & 44.15 & 0.67 & 1.16 & 1.34 & 0.85 & 15.46 & 5.00 & \\
\hline Er/Re & 1.28 & 4.0 & & & 0.5 & & 2.3 & & & & & & & \\
\hline
\end{tabular}

Sample KRI-15 CCC

\begin{tabular}{|c|c|c|c|c|c|c|c|c|c|c|c|c|c|c|}
\hline & Al2O3 & Fe2O3 & K2O & MnO & Na2O & PbO & SO3 & SiO2 & SrO & ZnO & ZrO2 & B2O3 & Li2O & Total \\
\hline 1 & 15.86 & 5.66 & 0.56 & 0.83 & 10.19 & 0.70 & 0.00 & 45.60 & 1.31 & 0.80 & 1.25 & 13.68 & 3.32 & 100.00 \\
\hline 2 & 15.44 & 6.84 & 0.57 & 1.09 & 10.00 & 0.71 & 0.70 & 45.04 & 1.33 & 0.00 & 1.27 & 13.68 & 3.32 & 100.00 \\
\hline 3 & 16.95 & 5.85 & 0.82 & 1.13 & 10.37 & 0.00 & 0.00 & 47.15 & 0.00 & 0.73 & 0.00 & 13.68 & 3.32 & 100.00 \\
\hline 4 & 16.02 & 5.60 & 0.55 & 0.94 & 10.32 & 0.69 & 0.00 & 46.05 & 1.29 & 0.57 & 0.98 & 13.68 & 3.32 & 100.00 \\
\hline 5 & 15.11 & 6.75 & 0.66 & 0.94 & 10.65 & 0.88 & 0.00 & 45.72 & 0.00 & 0.68 & 1.35 & 13.68 & 3.32 & 100.00 \\
\hline Average in area & 15.55 & 6.53 & 0.66 & 1.30 & 11.09 & 0.98 & 0.00 & 45.02 & 0.00 & 0.57 & 0.99 & 13.68 & 3.32 & 100.00 \\
\hline Average & 15.88 & 6.14 & 0.63 & 0.99 & 10.31 & 0.59 & 0.14 & 45.91 & 0.79 & 0.56 & 0.97 & 13.68 & 3.32 & 100.00 \\
\hline Er/Re & 2.0 & 3.3 & & & 0.3 & & & 2.5 & & & & & & \\
\hline
\end{tabular}

Sample KRI-16 CCC

\begin{tabular}{|c|c|c|c|c|c|c|c|c|c|c|c|c|c|c|c|c|c|c|}
\hline & $\mathrm{Al} 2 \mathrm{O} 3$ & $\mathrm{CaO}$ & $\mathrm{Cr} 2 \mathrm{O} 3$ & $\mathrm{Fe} 2 \mathrm{O} 3$ & $\mathrm{~K} 2 \mathrm{O}$ & $\mathrm{MnO}$ & $\mathrm{Na} 2 \mathrm{O}$ & $\mathrm{NiO}$ & $\mathrm{PbO}$ & $\mathrm{SO} 3$ & $\mathrm{SiO} 2$ & $\mathrm{TiO} 2$ & $\mathrm{ZnO}$ & $\mathrm{ZrO} 2$ & $\mathrm{SrO}$ & $\mathrm{B} 2 \mathrm{O} 3$ & $\mathrm{Li} 2 \mathrm{O}$ & $\mathrm{Total}$ \\
\hline 1 & 13.00 & 0.00 & 0.28 & 8.08 & 0.23 & 0.97 & 9.40 & 0.48 & 0.00 & 0.47 & 46.21 & 0.79 & 1.41 & 2.55 & 2.45 & 8.75 & 4.92 & 100.00 \\
\hline 2 & 12.97 & 0.13 & 0.00 & 8.08 & 0.00 & 0.84 & 9.88 & 0.59 & 1.10 & 0.46 & 45.48 & 0.77 & 1.04 & 2.76 & 2.19 & 8.75 & 4.92 & 100.00 \\
\hline 3 & 12.41 & 0.13 & 0.00 & 8.06 & 0.22 & 1.08 & 10.35 & 0.59 & 0.80 & 0.46 & 44.96 & 0.62 & 1.38 & 2.62 & 2.62 & 8.75 & 4.92 & 100.00 \\
\hline 4 & 12.64 & 0.13 & 0.41 & 7.80 & 0.23 & 0.85 & 10.28 & 0.72 & 1.01 & 0.00 & 46.39 & 0.94 & 1.06 & 2.16 & 1.67 & 8.75 & 4.92 & 100.00 \\
\hline 5 & 12.93 & 0.00 & 0.27 & 7.90 & 0.00 & 0.97 & 9.98 & 0.48 & 0.71 & 0.00 & 46.15 & 0.78 & 1.17 & 2.53 & 2.44 & 8.75 & 4.92 & 100.00 \\
\hline $\begin{array}{c}\text { Average } \\
\text { in area }\end{array}$ & 12.70 & 0.26 & 0.27 & 7.73 & 0.22 & 1.08 & 9.93 & 0.47 & 1.11 & 0.00 & 45.55 & 0.62 & 1.51 & 2.52 & 2.32 & 8.75 & 4.92 & 100.00 \\
\hline Average & 12.79 & 0.08 & 0.19 & 7.98 & 0.14 & 0.94 & 9.98 & 0.57 & 0.72 & 0.28 & 45.84 & 0.78 & 1.21 & 2.52 & 2.27 & 8.75 & 4.92 & 100.00 \\
\hline Er/Re & 1.28 & & & 1.1 & & & 0.5 & & & & 2.0 & & & & & & & \\
\hline
\end{tabular}


SRNS-STI-2008-00057

Revision 0

Appendix C (SRNS-OS-2008-00001)

Sample KRI-17 CCC

\begin{tabular}{|c|c|c|c|c|c|c|c|c|c|c|c|c|c|c|c|}
\hline & $\mathrm{Al} 2 \mathrm{O} 3$ & $\mathrm{CaO}$ & $\mathrm{Cr} 2 \mathrm{O} 3$ & $\mathrm{Fe} 2 \mathrm{O} 3$ & $\mathrm{~K} 2 \mathrm{O}$ & $\mathrm{MnO}$ & $\mathrm{Na} 2 \mathrm{O}$ & $\mathrm{NiO}$ & $\mathrm{SiO} 2$ & $\mathrm{TiO} 2$ & $\mathrm{ZrO} 2$ & $\mathrm{SrO}$ & $\mathrm{B} 2 \mathrm{O} 3$ & $\mathrm{Li} 2 \mathrm{O}$ & $\mathrm{Total}$ \\
\hline 1 & 13.68 & 0.53 & 0.00 & 8.16 & 0.23 & 1.72 & 11.42 & 0.00 & 42.62 & 0.00 & 2.96 & 2.36 & 12.40 & 3.88 & 100.00 \\
\hline 2 & 13.57 & 0.54 & 0.43 & 8.04 & 0.35 & 1.63 & 10.33 & 0.00 & 43.41 & 0.32 & 3.01 & 2.06 & 12.40 & 3.88 & 100.00 \\
\hline 3 & 13.52 & 0.51 & 0.00 & 8.12 & 0.33 & 1.90 & 12.85 & 0.58 & 40.83 & 0.00 & 3.22 & 1.84 & 12.40 & 3.88 & 100.00 \\
\hline 4 & 13.37 & 0.51 & 0.27 & 8.14 & 0.33 & 1.54 & 11.14 & 0.00 & 41.11 & 0.31 & 3.35 & 2.82 & 12.40 & 3.88 & 100.00 \\
\hline 5 & 13.40 & 0.65 & 0.00 & 7.86 & 0.45 & 1.57 & 11.69 & 0.00 & 41.56 & 0.00 & 3.28 & 2.54 & 12.40 & 3.88 & 100.00 \\
\hline $\begin{array}{c}\text { Average in } \\
\text { area }\end{array}$ & 13.49 & 0.52 & 0.27 & 8.21 & 0.22 & 1.68 & 11.24 & 0.47 & 41.07 & 0.31 & 3.25 & 2.52 & 12.40 & 3.88 & 100.00 \\
\hline Average & 13.51 & 0.55 & 0.14 & 8.06 & 0.34 & 1.67 & 11.49 & 0.12 & 41.91 & 0.13 & 3.16 & 2.33 & 12.40 & 3.88 & 100.00 \\
\hline Er/Re & 0.63 & & & 1.1 & & & 1.1 & & 3.6 & & & & & & \\
\hline
\end{tabular}

Sample KRI-18 CCC

\begin{tabular}{|c|c|c|c|c|c|c|c|c|c|c|c|c|c|c|c|}
\hline & $\mathrm{Al} 2 \mathrm{O} 3$ & $\mathrm{CaO}$ & $\mathrm{Fe} 2 \mathrm{O} 3$ & $\mathrm{~K} 2 \mathrm{O}$ & $\mathrm{MnO}$ & $\mathrm{Na} 2 \mathrm{O}$ & $\mathrm{NiO}$ & $\mathrm{SiO} 2$ & $\mathrm{TiO} 2$ & $\mathrm{ZnO}$ & $\mathrm{ZrO} 2$ & $\mathrm{SrO}$ & $\mathrm{B} 2 \mathrm{O} 3$ & $\mathrm{Li} 2 \mathrm{O}$ & $\mathrm{Total}$ \\
\hline 1 & 14.00 & 0.40 & 8.10 & 0.70 & 2.73 & 9.07 & 0.00 & 40.35 & 0.32 & 0.00 & 3.25 & 2.73 & 14.83 & 3.52 & 100.00 \\
\hline 2 & 14.12 & 0.55 & 7.02 & 0.71 & 2.79 & 9.14 & 0.00 & 42.08 & 0.33 & 0.00 & 3.05 & 1.86 & 14.83 & 3.52 & 100.00 \\
\hline 3 & 13.79 & 0.54 & 6.11 & 0.59 & 2.76 & 9.31 & 0.00 & 41.44 & 0.32 & 0.61 & 3.42 & 2.76 & 14.83 & 3.52 & 100.00 \\
\hline 4 & 14.30 & 0.54 & 6.70 & 0.69 & 2.60 & 8.91 & 0.00 & 41.83 & 0.00 & 0.60 & 3.10 & 2.38 & 14.83 & 3.52 & 100.00 \\
\hline 5 & 14.04 & 0.53 & 6.53 & 0.69 & 2.34 & 9.11 & 0.00 & 40.98 & 0.32 & 0.83 & 3.09 & 2.59 & 14.83 & 3.52 & 100.00 \\
\hline $\begin{array}{c}\text { Average in } \\
\text { area }\end{array}$ & 14.33 & 0.57 & 7.94 & 0.61 & 3.00 & 8.45 & 0.51 & 40.70 & 0.00 & 0.00 & 3.14 & 2.39 & 14.83 & 3.52 & 100.00 \\
\hline Average & 14.05 & 0.51 & 6.89 & 0.67 & 2.65 & 9.11 & 0.00 & 41.34 & 0.26 & 0.41 & 3.18 & 2.46 & 14.83 & 3.52 & 100.00 \\
\hline Er/Re & 0.93 & & 6.8 & & & 0.2 & & 2.3 & & & & & & & \\
\hline
\end{tabular}

Sample KRI-19 CCC

\begin{tabular}{|c|c|c|c|c|c|c|c|c|c|c|c|c|c|c|}
\hline & $\mathrm{Al} 2 \mathrm{O} 3$ & $\mathrm{CaO}$ & $\mathrm{Fe} 2 \mathrm{O} 3$ & $\mathrm{~K} 2 \mathrm{O}$ & $\mathrm{MnO}$ & $\mathrm{Na} 2 \mathrm{O}$ & $\mathrm{SiO} 2$ & $\mathrm{TiO} 2$ & $\mathrm{ZnO}$ & $\mathrm{ZrO} 2$ & $\mathrm{SrO}$ & $\mathrm{B} 2 \mathrm{O} 3$ & $\mathrm{Li} 2 \mathrm{O}$ & $\mathrm{Total}$ \\
\hline 1 & 13.68 & 0.00 & 7.59 & 0.33 & 2.49 & 14.32 & 41.39 & 0.61 & 0.57 & 1.24 & 2.27 & 11.12 & 4.37 & 100.00 \\
\hline 2 & 13.63 & 0.00 & 8.96 & 0.23 & 2.90 & 13.63 & 41.11 & 0.78 & 0.00 & 1.26 & 1.99 & 11.12 & 4.37 & 100.00 \\
\hline 3 & 14.05 & 0.27 & 8.99 & 0.23 & 2.71 & 12.59 & 41.63 & 0.48 & 0.00 & 1.42 & 2.14 & 11.12 & 4.37 & 100.00 \\
\hline 4 & 14.26 & 0.00 & 8.38 & 0.22 & 2.53 & 14.31 & 41.68 & 0.78 & 0.58 & 0.00 & 1.76 & 11.12 & 4.37 & 100.00 \\
\hline 5 & 14.22 & 0.27 & 8.82 & 0.23 & 2.49 & 12.87 & 42.33 & 0.64 & 0.00 & 0.91 & 1.71 & 11.12 & 4.37 & 100.00 \\
\hline $\begin{array}{c}\text { Average in } \\
\text { area }\end{array}$ & 14.32 & 0.26 & 8.52 & 0.23 & 2.69 & 11.49 & 41.98 & 0.79 & 0.00 & 1.02 & 2.58 & 11.12 & 4.37 & 100.00 \\
\hline Average & 13.97 & 0.11 & 8.55 & 0.25 & 2.62 & 13.54 & 41.63 & 0.66 & 0.23 & 0.97 & 1.98 & 11.12 & 4.37 & 100.00 \\
\hline Er/Re & 1.48 & & 5.3 & & & 1.0 & 1.5 & & & & & & & \\
\hline
\end{tabular}

Sample KRI-20 CCC

\begin{tabular}{|c|c|c|c|c|c|c|c|c|c|c|c|c|c|c|c|c|}
\hline & $\mathrm{Al} 2 \mathrm{O} 3$ & $\mathrm{CaO}$ & $\mathrm{Fe} 2 \mathrm{O} 3$ & $\mathrm{~K} 2 \mathrm{O}$ & $\mathrm{MnO}$ & $\mathrm{Na} 2 \mathrm{O}$ & $\mathrm{NiO}$ & $\mathrm{PbO}$ & $\mathrm{SiO} 2$ & $\mathrm{TiO} 2$ & $\mathrm{ZnO}$ & $\mathrm{ZrO} 2$ & $\mathrm{SrO}$ & $\mathrm{B} 2 \mathrm{O} 3$ & $\mathrm{Li} 2 \mathrm{O}$ & $\mathrm{Total}$ \\
\hline 1 & 15.14 & 0.53 & 7.08 & 0.23 & 0.99 & 13.36 & 0.00 & 0.82 & 39.80 & 0.00 & 0.59 & 1.29 & 2.37 & 14.79 & 3.00 & 100.00 \\
\hline 2 & 15.85 & 0.53 & 7.13 & 0.23 & 0.85 & 12.32 & 0.00 & 1.01 & 39.95 & 0.00 & 0.59 & 1.40 & 2.34 & 14.79 & 3.00 & 100.00 \\
\hline 3 & 15.95 & 0.69 & 7.57 & 0.24 & 0.89 & 11.77 & 0.00 & 0.85 & 40.77 & 0.33 & 0.00 & 1.06 & 2.09 & 14.79 & 3.00 & 100.00 \\
\hline 4 & 16.14 & 0.54 & 6.99 & 0.23 & 0.87 & 13.06 & 0.00 & 0.72 & 40.68 & 0.00 & 0.00 & 1.04 & 1.93 & 14.79 & 3.00 & 100.00 \\
\hline 5 & 15.53 & 0.54 & 7.32 & 0.23 & 1.00 & 12.51 & 0.00 & 1.15 & 40.16 & 0.32 & 0.00 & 1.04 & 2.40 & 14.79 & 3.00 & 100.00 \\
\hline Average in area & 15.68 & 0.53 & 8.21 & 0.34 & 0.97 & 12.20 & 0.36 & 1.02 & 39.76 & 0.31 & 0.00 & 1.15 & 1.67 & 14.79 & 3.00 & 100.00 \\
\hline Average & 15.72 & 0.56 & 7.22 & 0.23 & 0.92 & 12.60 & 0.00 & 0.91 & 40.27 & 0.13 & 0.24 & 1.17 & 2.22 & 14.79 & 3.00 & 100.00 \\
\hline Er/Re & 1.98 & & 2.1 & & & 0.8 & & & 1.4 & & & & & & & \\
\hline
\end{tabular}


SRNS-STI-2008-00057

Revision 0

Appendix C (SRNS-OS-2008-00001)

Sample KRI-21 CCC

\begin{tabular}{|c|c|c|c|c|c|c|c|c|c|c|c|c|c|c|c|c|c|}
\hline & Al2O3 & $\mathrm{CaO}$ & Fe2O3 & K2O & MnO & Na2O & NiO & PbO & SO3 & SiO2 & TiO2 & ZnO & ZrO2 & SrO & B2O3 & Li2O & Total \\
\hline 1 & 13.47 & 0.65 & 8.59 & 0.45 & 1.32 & 13.72 & 0.00 & 0.00 & 0.00 & 41.02 & 0.31 & 0.69 & 3.38 & 1.31 & 11.83 & 3.29 & 100.02 \\
\hline 2 & 14.17 & 0.67 & 7.14 & 0.35 & 1.12 & 13.86 & 0.00 & 0.00 & 0.00 & 43.01 & 0.00 & 0.00 & 3.12 & 1.48 & 11.83 & 3.29 & 100.02 \\
\hline 3 & 13.99 & 0.52 & 7.09 & 0.45 & 0.85 & 13.13 & 0.00 & 0.00 & 0.47 & 42.74 & 0.31 & 0.70 & 3.42 & 1.22 & 11.83 & 3.29 & 100.02 \\
\hline 4 & 13.64 & 0.54 & 7.70 & 0.35 & 1.24 & 12.84 & 0.00 & 0.62 & 0.00 & 42.44 & 0.32 & 0.00 & 3.51 & 1.71 & 11.83 & 3.29 & 100.02 \\
\hline 5 & 13.62 & 0.52 & 6.95 & 0.34 & 1.09 & 13.74 & 0.00 & 0.60 & 0.00 & 42.87 & 0.31 & 0.70 & 3.16 & 1.00 & 11.83 & 3.29 & 100.02 \\
\hline Average in area & 13.97 & 0.54 & 8.20 & 0.35 & 1.38 & 12.19 & 0.37 & 0.00 & 0.00 & 43.31 & 0.32 & 0.00 & 2.89 & 1.38 & 11.83 & 3.29 & 100.02 \\
\hline Average & 13.78 & 0.58 & 7.49 & 0.39 & 1.12 & 13.46 & 0.00 & 0.25 & 0.09 & 42.41 & 0.25 & 0.42 & 3.32 & 1.34 & 11.83 & 3.29 & 100.02 \\
\hline Er/Re & 1.5 & & 3.4 & & & 0.7 & & & & 4.0 & & & & & & & \\
\hline
\end{tabular}

Sample KRI-22 CCC

\begin{tabular}{|c|c|c|c|c|c|c|c|c|c|c|c|c|c|c|}
\hline & Al2O3 & CaO & Fe2O3 & K2O & MnO & Na2O & SiO2 & TiO2 & ZnO & ZrO2 & SrO & B2O3 & Li2O & Total \\
\hline 1 & 16.20 & 0.27 & 7.29 & 0.23 & 0.87 & 10.77 & 41.23 & 0.64 & 0.48 & 3.12 & 2.39 & 11.52 & 5.00 & 100.02 \\
\hline 2 & 15.86 & 0.27 & 7.25 & 0.24 & 0.76 & 10.78 & 41.57 & 0.81 & 0.00 & 3.43 & 2.54 & 11.52 & 5.00 & 100.02 \\
\hline 3 & 15.73 & 0.27 & 6.63 & 0.23 & 0.87 & 11.60 & 41.22 & 0.81 & 0.60 & 3.01 & 2.52 & 11.52 & 5.00 & 100.02 \\
\hline 4 & 15.95 & 0.27 & 7.26 & 0.00 & 0.87 & 11.11 & 41.07 & 0.64 & 0.00 & 3.50 & 2.83 & 11.52 & 5.00 & 100.02 \\
\hline 5 & 15.97 & 0.27 & 6.99 & 0.00 & 0.99 & 11.51 & 41.11 & 0.80 & 0.00 & 3.24 & 2.61 & 11.52 & 5.00 & 100.02 \\
\hline Average in area & 15.53 & 0.26 & 7.42 & 0.00 & 0.85 & 11.33 & 40.45 & 0.79 & 0.71 & 3.44 & 2.23 & 11.52 & 5.00 & 100.02 \\
\hline Average & 15.94 & 0.27 & 7.08 & 0.14 & 0.87 & 11.16 & 41.24 & 0.74 & 0.22 & 3.26 & 2.58 & 11.83 & 3.29 & 100.02 \\
\hline Er/Re & 0.86 & & 1.39 & & & 0.66 & 0.99 & & & & & & & \\
\hline
\end{tabular}

Sample KRI-23 CCC

\begin{tabular}{|c|c|c|c|c|c|c|c|c|c|c|c|c|c|c|c|c|}
\hline & Al2O3 & $\mathrm{CaO}$ & Fe2O3 & K2O & MnO & Na2O & PbO & SO3 & SiO2 & TiO2 & ZnO & ZrO2 & SrO & B2O3 & Li2O & Total \\
\hline 1 & 18.09 & 0.00 & 5.38 & 0.61 & 0.92 & 9.19 & 0.99 & 0.00 & 45.99 & 0.00 & 0.63 & 1.51 & 1.08 & 12.60 & 3.00 & 100.00 \\
\hline 2 & 18.11 & 0.14 & 5.78 & 0.59 & 0.89 & 11.44 & 0.74 & 0.00 & 43.13 & 0.33 & 0.00 & 1.60 & 1.63 & 12.60 & 3.00 & 100.00 \\
\hline 3 & 17.56 & 0.00 & 4.84 & 0.58 & 0.87 & 11.73 & 0.73 & 0.00 & 44.12 & 0.32 & 0.60 & 1.44 & 1.60 & 12.60 & 3.00 & 100.00 \\
\hline 4 & 17.99 & 0.00 & 5.55 & 0.82 & 1.13 & 10.73 & 0.00 & 0.49 & 43.46 & 0.32 & 0.60 & 1.71 & 1.61 & 12.60 & 3.00 & 100.00 \\
\hline 5 & 18.61 & 0.00 & 4.49 & 0.73 & 1.05 & 11.08 & 0.00 & 0.51 & 46.07 & 0.00 & 0.63 & 1.23 & 0.00 & 12.60 & 3.00 & 100.00 \\
\hline Average in area & 17.23 & 0.27 & 7.42 & 0.59 & 1.01 & 10.56 & 0.63 & 0.00 & 43.85 & 0.00 & 0.73 & 1.06 & 1.04 & 12.60 & 3.00 & 100.00 \\
\hline Average & 18.07 & 0.03 & 5.21 & 0.67 & 0.97 & 10.84 & 0.49 & 0.20 & 44.55 & 0.20 & 0.49 & 1.50 & 1.18 & 11.83 & 3.29 & 100.00 \\
\hline Er/Re & 1.87 & & 2.65 & & & 1.66 & & & 6.97 & & & & & & & \\
\hline
\end{tabular}

\section{Sample KRI-24 CCC}

\begin{tabular}{|c|c|c|c|c|c|c|c|c|c|c|c|c|c|c|c|c|}
\hline & $\mathrm{Al} 2 \mathrm{O} 3$ & $\mathrm{CaO}$ & $\mathrm{Fe} 2 \mathrm{O} 3$ & $\mathrm{~K} 2 \mathrm{O}$ & $\mathrm{MnO}$ & $\mathrm{Na} 2 \mathrm{O}$ & $\mathrm{NiO}$ & $\mathrm{PbO}$ & $\mathrm{SiO} 2$ & $\mathrm{TiO} 2$ & $\mathrm{ZnO}$ & $\mathrm{ZrO} 2$ & $\mathrm{SrO}$ & $\mathrm{B} 2 \mathrm{O} 3$ & $\mathrm{Li} 2 \mathrm{O}$ & $\mathrm{Total}$ \\
\hline 1 & 13.00 & 0.40 & 7.91 & 0.58 & 1.85 & 10.30 & 0.36 & 0.62 & 44.18 & 0.48 & 1.07 & 3.61 & 2.14 & 9.69 & 3.77 & 100.00 \\
\hline 2 & 13.03 & 0.40 & 7.11 & 0.58 & 1.48 & 11.22 & 0.00 & 0.82 & 44.88 & 0.48 & 0.83 & 3.62 & 2.04 & 9.69 & 3.77 & 100.00 \\
\hline 3 & 13.48 & 0.40 & 6.25 & 0.69 & 1.47 & 11.27 & 0.00 & 0.82 & 45.39 & 0.63 & 1.18 & 3.34 & 1.57 & 9.69 & 3.77 & 100.00 \\
\hline 4 & 13.02 & 0.54 & 6.93 & 0.58 & 1.63 & 9.93 & 0.00 & 0.94 & 46.31 & 0.65 & 0.97 & 3.28 & 1.72 & 9.69 & 3.77 & 100.00 \\
\hline 5 & 12.82 & 0.41 & 7.30 & 0.59 & 1.78 & 9.93 & 0.00 & 0.85 & 46.51 & 0.49 & 1.10 & 3.32 & 1.39 & 9.69 & 3.77 & 100.00 \\
\hline Average in area & 12.56 & 0.39 & 7.51 & 0.56 & 1.79 & 11.81 & 0.47 & 0.99 & 43.86 & 0.62 & 0.92 & 3.24 & 1.53 & 9.69 & 3.77 & 100.00 \\
\hline Average & 13.07 & 0.43 & 7.10 & 0.60 & 1.64 & 10.53 & 0.07 & 0.81 & 45.46 & 0.55 & 1.03 & 3.43 & 1.77 & 11.83 & 3.29 & 100.02 \\
\hline Er/Re & 1.22 & & 3.02 & & & 1.12 & & & 4.89 & & & & & & & \\
\hline
\end{tabular}


SRNS-STI-2008-00057

Revision 0

Appendix C (SRNS-OS-2008-00001)

Sample KRI-25 CCC

\begin{tabular}{|c|c|c|c|c|c|c|c|c|c|c|c|c|c|c|}
\hline & $\mathrm{Al} 2 \mathrm{O} 3$ & $\mathrm{CaO}$ & $\mathrm{Fe} 2 \mathrm{O} 3$ & $\mathrm{~K} 2 \mathrm{O}$ & $\mathrm{MnO}$ & $\mathrm{Na} 2 \mathrm{O}$ & $\mathrm{SiO} 2$ & $\mathrm{TiO} 2$ & $\mathrm{ZnO}$ & $\mathrm{ZrO} 2$ & $\mathrm{SrO}$ & $\mathrm{B} 2 \mathrm{O} 3$ & $\mathrm{Li} 2 \mathrm{O}$ & Total \\
\hline 1 & 16.24 & 0.26 & 6.80 & 0.68 & 1.21 & 12.84 & 40.99 & 0.31 & 0.00 & 1.77 & 1.66 & 13.50 & 3.75 & 100.00 \\
\hline 2 & 15.91 & 0.00 & 9.06 & 0.72 & 0.90 & 12.28 & 42.42 & 0.00 & 0.00 & 1.47 & 0.00 & 13.50 & 3.75 & 100.00 \\
\hline 3 & 15.84 & 0.27 & 9.05 & 0.59 & 1.13 & 12.08 & 41.53 & 0.33 & 0.61 & 1.32 & 0.00 & 13.50 & 3.75 & 100.00 \\
\hline 4 & 15.49 & 0.27 & 8.26 & 0.70 & 0.87 & 13.90 & 41.90 & 0.32 & 0.00 & 1.04 & 0.00 & 13.50 & 3.75 & 100.00 \\
\hline 5 & 15.87 & 0.27 & 8.82 & 0.58 & 1.00 & 11.32 & 41.54 & 0.32 & 0.00 & 1.43 & 1.60 & 13.50 & 3.75 & 100.00 \\
\hline Average in area & 15.76 & 0.26 & 9.66 & 0.67 & 1.08 & 12.86 & 39.47 & 0.31 & 0.00 & 1.38 & 1.31 & 13.50 & 3.75 & 100.00 \\
\hline Average & 15.87 & 0.21 & 8.40 & 0.65 & 1.02 & 12.48 & 41.68 & 0.26 & 0.12 & 1.41 & 0.65 & 11.83 & 3.29 & 100.02 \\
\hline Er/Re & 1.34 & & 4.74 & & & 1.60 & 2.63 & & & & & & & \\
\hline
\end{tabular}

Sample KRI-26 CCC

\begin{tabular}{|c|c|c|c|c|c|c|c|c|c|c|c|}
\hline & Al2O3 & $\mathrm{CaO}$ & $\mathrm{Fe} 2 \mathrm{O} 3$ & $\mathrm{Na} 2 \mathrm{O}$ & $\mathrm{SO} 3$ & $\mathrm{SiO} 2$ & $\mathrm{SrO}$ & $\mathrm{UO} 3$ & $\mathrm{~B} 2 \mathrm{O} 3$ & $\mathrm{Li} 2 \mathrm{O}$ & Total \\
\hline 1 & 11.35 & 0.79 & 6.30 & 15.94 & 0.23 & 44.61 & 2.66 & 1.92 & 10.19 & 6.00 & 100.00 \\
\hline 2 & 11.28 & 0.86 & 6.32 & 15.67 & 0.00 & 45.16 & 2.80 & 1.73 & 10.19 & 6.00 & 100.00 \\
\hline 3 & 11.63 & 0.85 & 6.34 & 14.82 & 0.50 & 45.58 & 2.15 & 1.94 & 10.19 & 6.00 & 100.00 \\
\hline 4 & 11.47 & 0.96 & 6.57 & 14.91 & 0.24 & 44.65 & 3.01 & 2.00 & 10.19 & 6.00 & 100.00 \\
\hline 5 & 11.47 & 0.83 & 6.54 & 14.61 & 0.25 & 45.37 & 2.70 & 2.03 & 10.19 & 6.00 & 100.00 \\
\hline Average in area & 11.04 & 0.77 & 9.13 & 14.64 & 0.46 & 42.02 & 2.27 & 1.87 & 10.19 & 6.00 & 100.00 \\
\hline Average & 11.44 & 0.86 & 6.41 & 15.19 & 0.25 & 45.07 & 2.66 & 1.92 & 10.19 & 6.00 & 100.00 \\
\hline Er/Re & 1.04 & & 0.86 & 0.96 & & 2.16 & & & & & \\
\hline
\end{tabular}

Sample KRI-27 CCC

\begin{tabular}{|c|c|c|c|c|c|c|c|c|c|c|c|c|c|c|}
\hline & Al2O3 & $\mathrm{CaO}$ & $\mathrm{Fe} 2 \mathrm{O} 3$ & $\mathrm{MnO}$ & $\mathrm{Na} 2 \mathrm{O}$ & $\mathrm{PbO}$ & $\mathrm{SiO} 2$ & $\mathrm{TiO} 2$ & $\mathrm{ZnO}$ & $\mathrm{ZrO} 2$ & $\mathrm{UO} 3$ & $\mathrm{~B} 2 \mathrm{O} 3$ & $\mathrm{Li} 2 \mathrm{O}$ & \\
\hline 1 & 10.97 & 0.83 & 7.45 & 0.51 & 10.74 & 0.95 & 38.97 & 1.31 & 0.00 & 4.39 & 0.95 & 17.20 & 5.73 & 100.00 \\
\hline 2 & 10.97 & 0.79 & 7.76 & 0.60 & 11.74 & 1.01 & 37.50 & 1.41 & 0.00 & 4.17 & 1.13 & 17.20 & 5.73 & 100.00 \\
\hline 3 & 11.29 & 0.84 & 7.40 & 0.51 & 11.00 & 1.18 & 38.16 & 1.33 & 0.00 & 4.17 & 1.20 & 17.20 & 5.73 & 100.00 \\
\hline 4 & 10.92 & 0.84 & 7.54 & 0.51 & 10.61 & 1.07 & 38.61 & 1.33 & 0.00 & 4.44 & 1.20 & 17.20 & 5.73 & 100.00 \\
\hline 5 & 10.96 & 0.84 & 7.42 & 0.52 & 10.77 & 1.18 & 38.52 & 1.33 & 0.00 & 4.32 & 1.20 & 17.20 & 5.73 & 100.00 \\
\hline Average in area & 10.74 & 0.79 & 8.66 & 0.61 & 10.72 & 1.02 & 37.50 & 1.26 & 0.00 & 3.96 & 1.02 & 17.20 & 5.73 & 100.00 \\
\hline Average & 11.02 & 0.82 & 7.51 & 0.53 & 10.97 & 1.08 & 38.35 & 1.34 & 0.00 & 4.30 & 1.13 & 17.20 & 5.73 & 100.00 \\
\hline Er/Re & 1.16 & & 0.98 & & 0.75 & & 2.80 & & & & & & & \\
\hline
\end{tabular}

Sample KRI 28 CCC

\begin{tabular}{|c|c|c|c|c|c|c|c|c|c|c|c|c|c|c|c|c|c|c|}
\hline \# in photo & & $\mathrm{Al} 2 \mathrm{O} 3$ & $\mathrm{CaO}$ & $\mathrm{Cr} 2 \mathrm{O} 3$ & $\mathrm{FeO}$ & $\mathrm{Fe} 2 \mathrm{O} 3$ & $\mathrm{MnO}$ & $\mathrm{Na} 2 \mathrm{O}$ & $\mathrm{NiO}$ & $\mathrm{PbO}$ & SO3 & $\mathrm{SiO} 2$ & $\mathrm{TiO} 2$ & $\mathrm{ZnO}$ & $\mathrm{ZrO} 2$ & $\mathrm{SrO}$ & UO3 & Total \\
\hline $\mathrm{T}$ & Average & 18.71 & nd & 0.44 & 3.86 & 0.00 & 2.97 & 18.46 & nd & 1.29 & 0.25 & 38.33 & 1.17 & nd & nd & nd & 2.40 & 87.89 \\
\hline 1 & spinel & 0.76 & nd & 14.77 & 0.00 & 37.46 & 43.28 & nd & nd & nd & nd & nd & 4.51 & nd & nd & nd & nd & 100.77 \\
\hline 5 & spinel & 1.13 & nd & 11.26 & 30.37 & 0.00 & 38.89 & nd & nd & nd & nd & nd & 4.17 & nd & nd & nd & nd & 85.83 \\
\hline 2 & white phase & 1.51 & nd & nd & 4.12 & 0.00 & 3.49 & 10.92 & nd & 3.88 & 1.75 & 20.56 & 3.34 & nd & nd & nd & 10.82 & 60.38 \\
\hline 6 & nepheline $\mathrm{H}$ & 31.00 & nd & nd & 2.57 & 0.00 & nd & 19.41 & nd & nd & nd & 39.61 & nd & nd & nd & nd & nd & 92.60 \\
\hline 7 & black - SiO2 & nd & nd & nd & 0.39 & 0.00 & 0.52 & nd & nd & nd & nd & 73.45 & nd & nd & nd & nd & nd & 74.35 \\
\hline
\end{tabular}


SRNS-STI-2008-00057

Revision 0

Appendix C (SRNS-OS-2008-00001)

Sample KRI-29 CCC

\begin{tabular}{|c|c|c|c|c|c|c|c|c|c|c|c|c|c|}
\hline & Al2O3 & Fe2O3 & K2O & Na2O & NiO & PbO & SiO2 & TiO2 & SrO & UO3 & B2O3 & Li2O & Total \\
\hline 1 & 14.89 & 5.56 & 0.84 & 20.56 & 0.51 & 0.86 & 37.58 & 1.17 & 1.65 & 3.00 & 11.38 & 2.00 & 100.00 \\
\hline 2 & 14.55 & 5.36 & 0.83 & 20.75 & 0.63 & 1.17 & 37.20 & 1.15 & 1.63 & 3.32 & 11.38 & 2.00 & 100.00 \\
\hline 3 & 14.89 & 5.49 & 0.83 & 20.44 & 0.88 & 1.06 & 36.69 & 1.15 & 1.86 & 3.31 & 11.38 & 2.00 & 100.00 \\
\hline 4 & 14.51 & 5.84 & 0.84 & 20.42 & 0.63 & 1.18 & 37.14 & 1.16 & 1.89 & 2.99 & 11.38 & 2.00 & 100.00 \\
\hline 5 & 14.97 & 5.45 & 0.82 & 20.42 & 0.62 & 1.16 & 36.84 & 1.31 & 1.50 & 3.52 & 11.38 & 2.00 & 100.00 \\
\hline Average in area & 15.12 & 5.44 & 0.82 & 19.59 & 1.74 & 0.74 & 36.77 & 1.14 & 1.73 & 3.52 & 11.38 & 2.00 & 100.00 \\
\hline Average & 14.76 & 5.54 & 0.83 & 20.52 & 0.65 & 1.09 & 37.09 & 1.19 & 1.71 & 3.23 & 11.38 & 2.00 & 100.00 \\
\hline Er/Re & 1.64 & 1.22 & & 0.24 & & & 1.72 & & & & & & \\
\hline
\end{tabular}

Sample KRI-30 CCC

\begin{tabular}{|c|c|c|c|c|c|c|c|c|c|c|c|c|c|c|c|c|}
\hline & Al2O3 & CaO & Fe2O3 & K2O & Na2O & NiO & PbO & SO3 & SiO2 & TiO2 & ZnO & SrO & UO3 & B2O3 & Li2O & Total \\
\hline 1 & 16.71 & 0.27 & 3.29 & 0.35 & 12.82 & 0.00 & 1.35 & 0.00 & 37.86 & 0.48 & 0.72 & 1.70 & 3.35 & 15.30 & 5.79 & 100.00 \\
\hline 2 & 16.85 & 0.39 & 4.38 & 0.34 & 13.01 & 0.00 & 1.00 & 0.00 & 36.18 & 0.62 & 1.04 & 2.08 & 3.01 & 15.30 & 5.79 & 100.00 \\
\hline 3 & 17.30 & 0.40 & 5.09 & 0.35 & 11.16 & 0.37 & 0.93 & 0.24 & 36.71 & 0.32 & 1.20 & 1.94 & 2.89 & 15.30 & 5.79 & 100.00 \\
\hline 4 & 16.96 & 0.26 & 5.13 & 0.34 & 12.47 & 0.84 & 1.12 & 0.00 & 35.39 & 0.47 & 1.06 & 2.01 & 2.84 & 15.30 & 5.79 & 100.00 \\
\hline 5 & 17.07 & 0.27 & 5.16 & 0.34 & 11.66 & 0.60 & 1.23 & 0.00 & 36.03 & 0.48 & 0.83 & 2.02 & 3.20 & 15.30 & 5.79 & 100.00 \\
\hline Average in area & 16.89 & 0.40 & 5.02 & 0.34 & 11.27 & 0.85 & 0.92 & 0.24 & 36.22 & 0.48 & 0.95 & 2.25 & 3.08 & 15.30 & 5.79 & 100.00 \\
\hline Average & 16.98 & 0.32 & 4.61 & 0.34 & 12.23 & 0.36 & 1.13 & 0.05 & 36.44 & 0.47 & 0.97 & 1.95 & 3.06 & 15.30 & 5.79 & 100.00 \\
\hline Er/Re & 1.71 & & 5.36 & & 1.31 & & & & 4.62 & & & & & & & \\
\hline
\end{tabular}


Attachment 7. XRD Data for Glasses after CCC Treatment

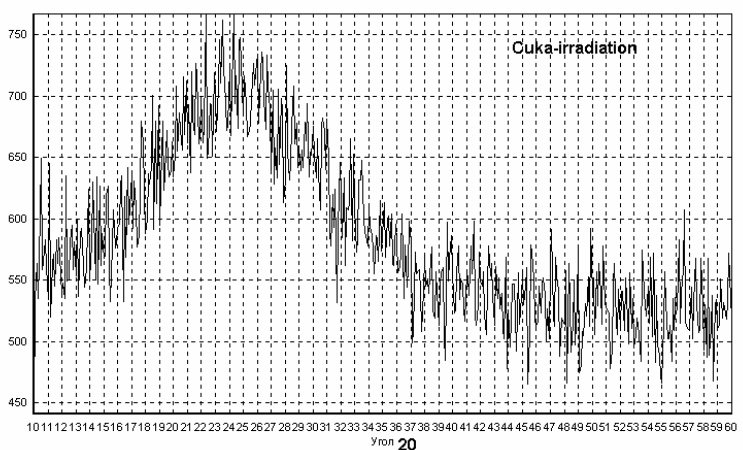

KRI-01 CCC

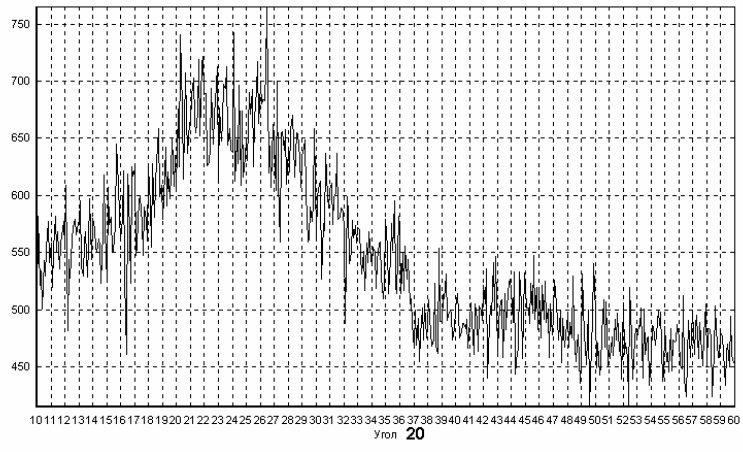

KRI-03 CCC

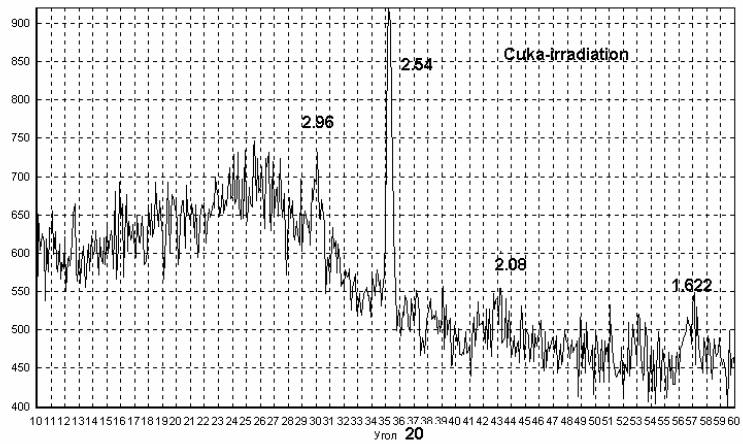

KRI-05 CCC

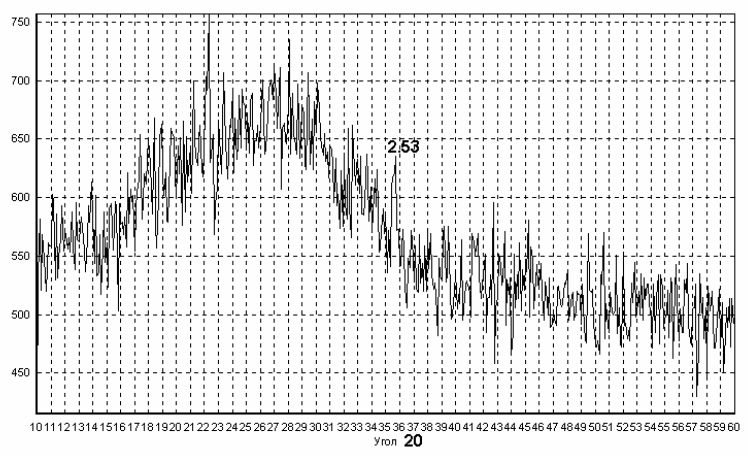

KRI-07 CCC

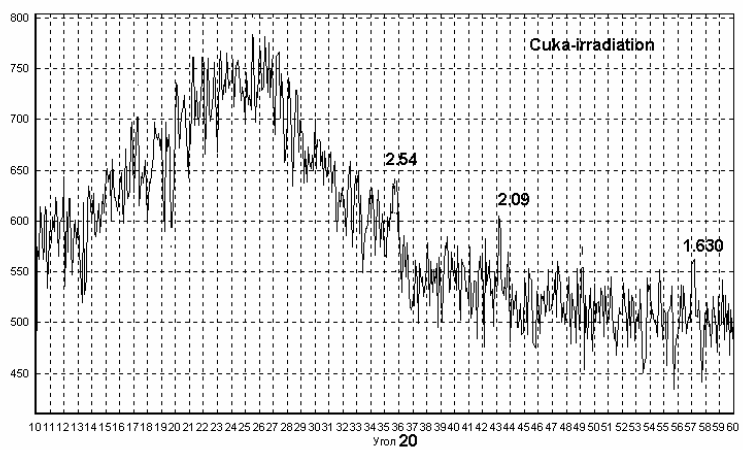

KRI-02 CCC

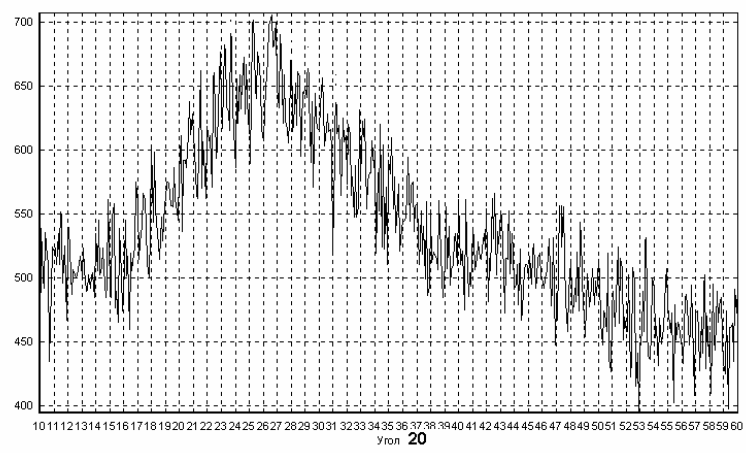

KRI-04 CCC

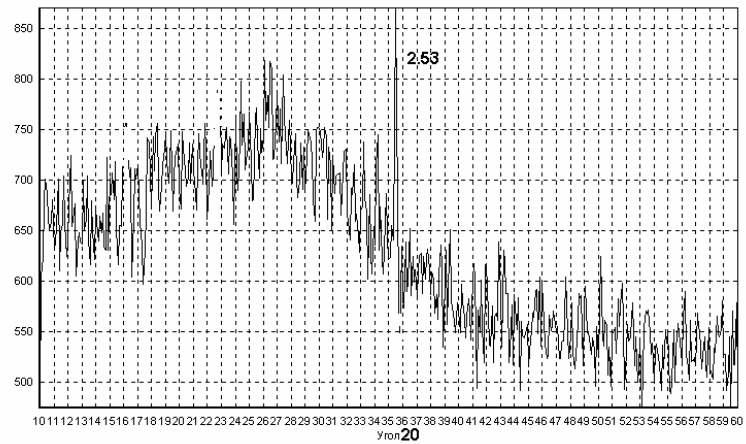

KRI-06 CCC

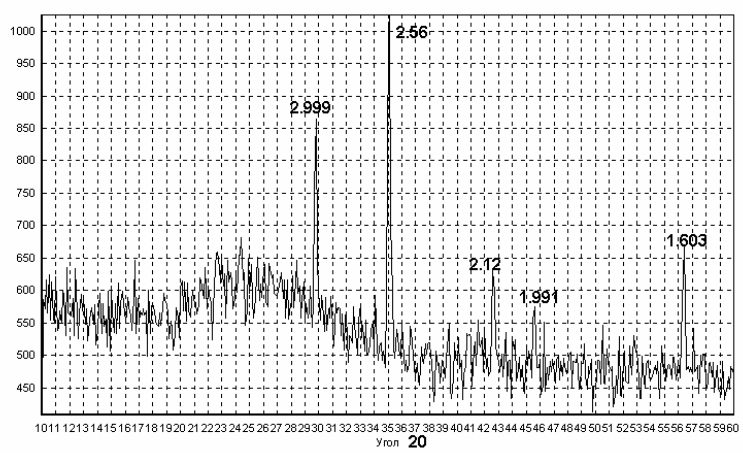

KRI-08 CCC 
Appendix C (SRNS-OS-2008-00001)

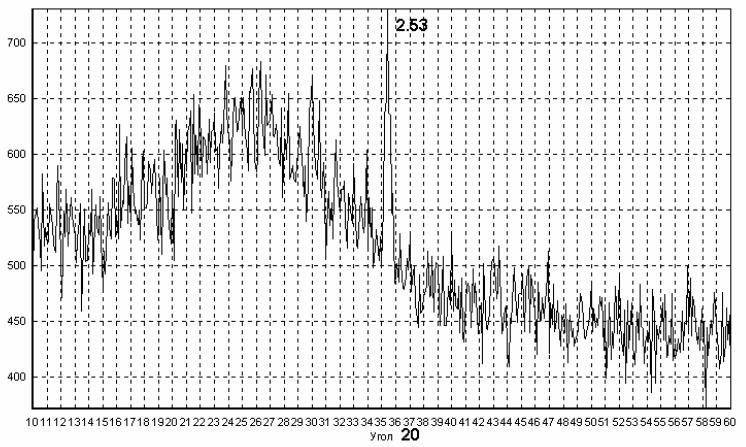

KRI-09 CCC

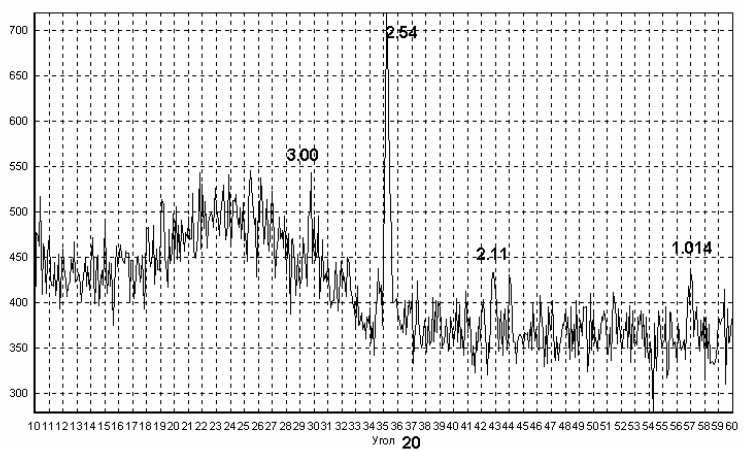

KRI-11 CCC

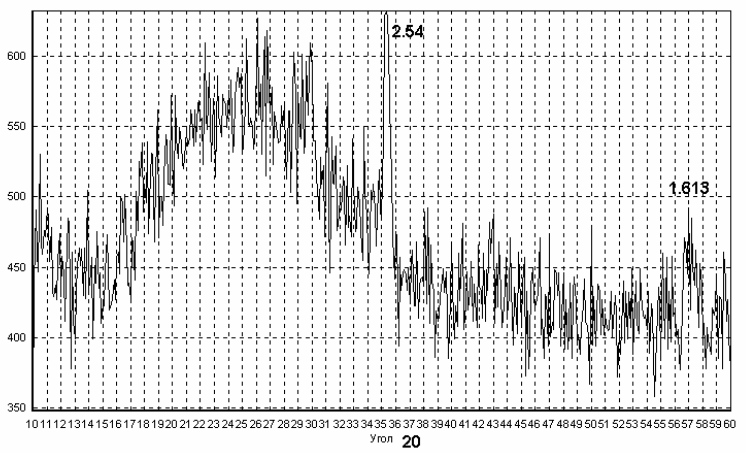

KRI-13-CCC

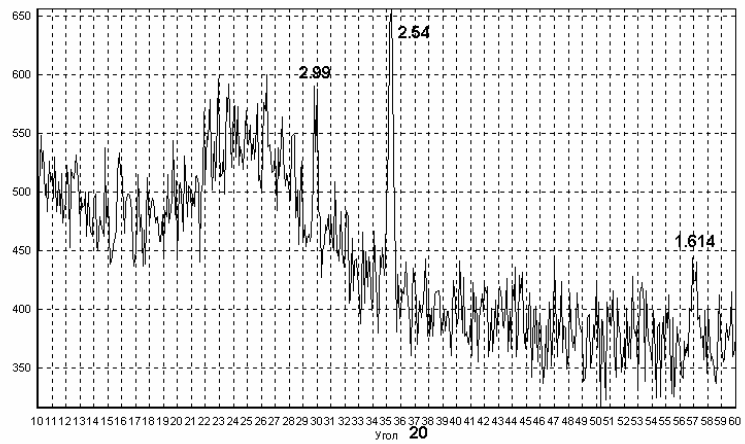

KRI-15 CCC

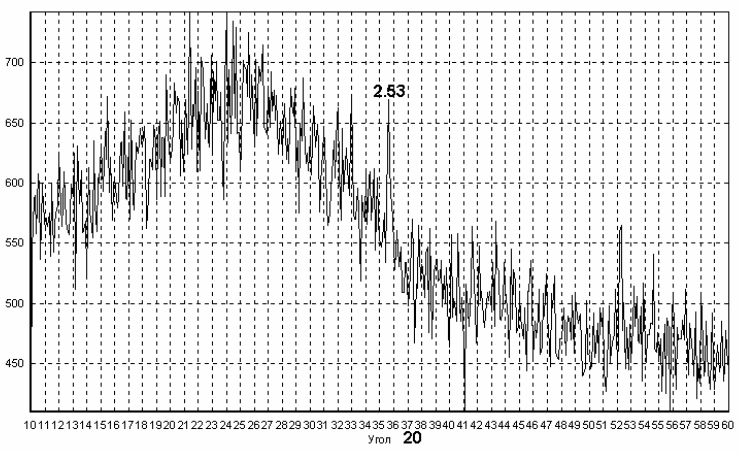

KRI-10 CCC

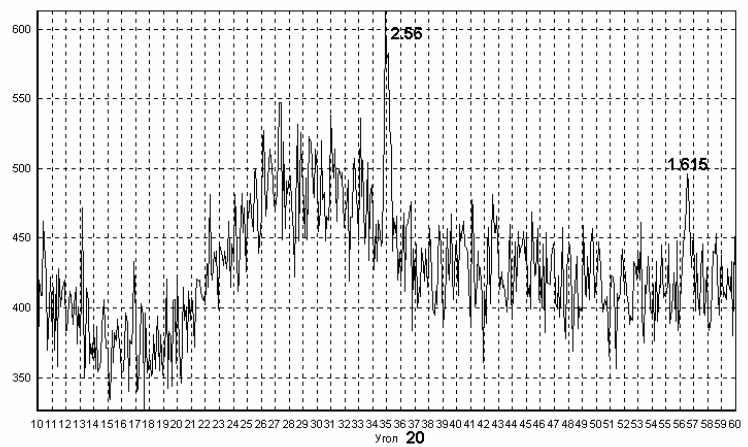

KRI-12 CCC

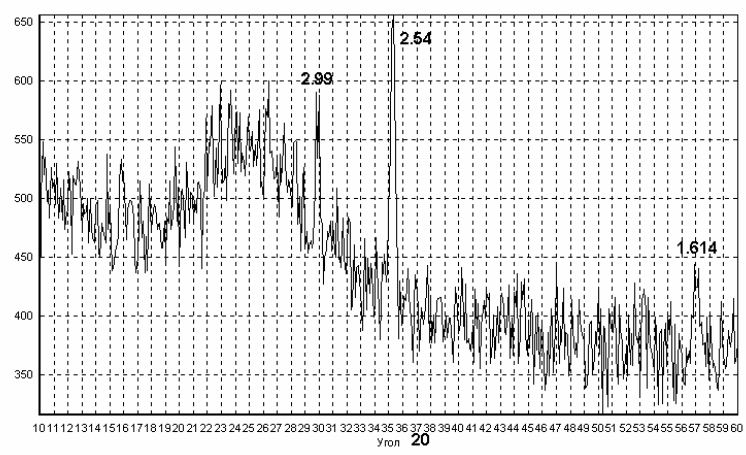

KRI-14-CCC

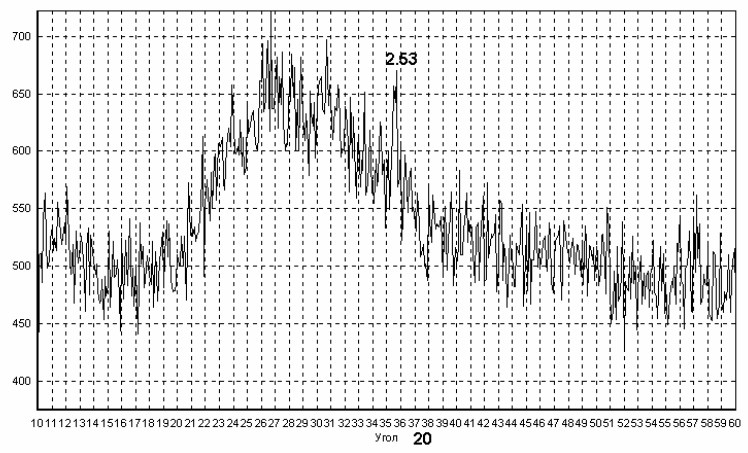

KRI-16 CCC 


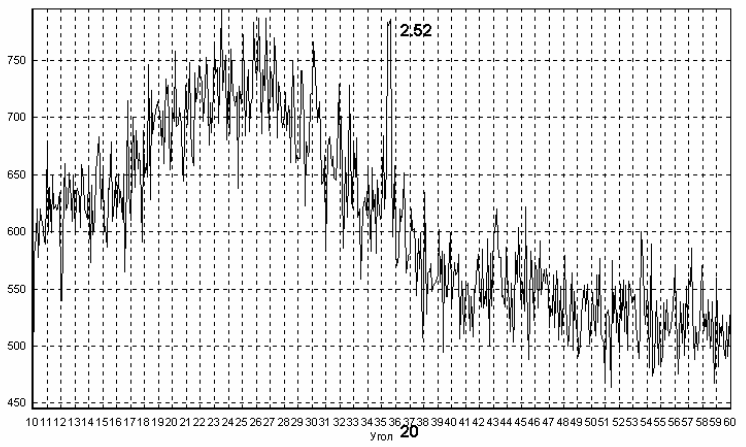

KRI-17 CCC

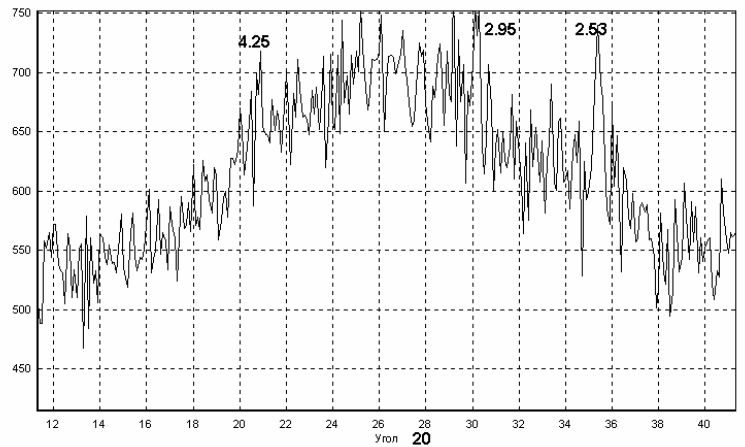

KRI-19 CCC

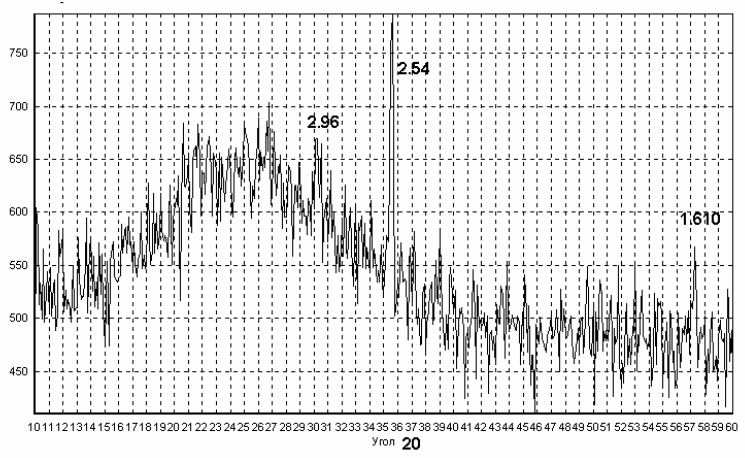

KRI-21 CCC

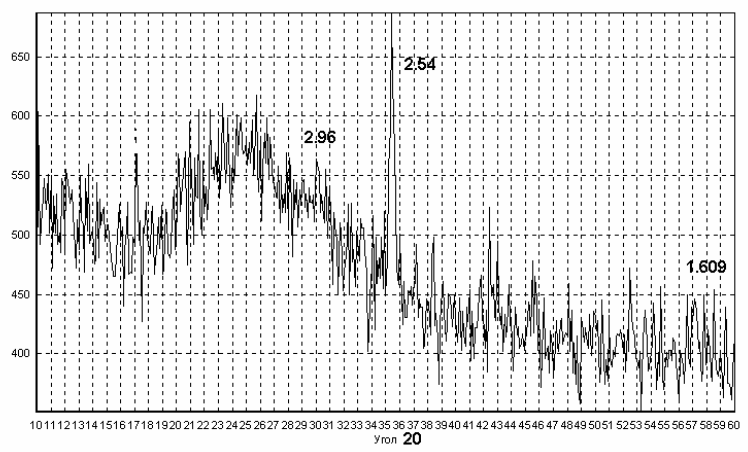

KRI-23 CCC

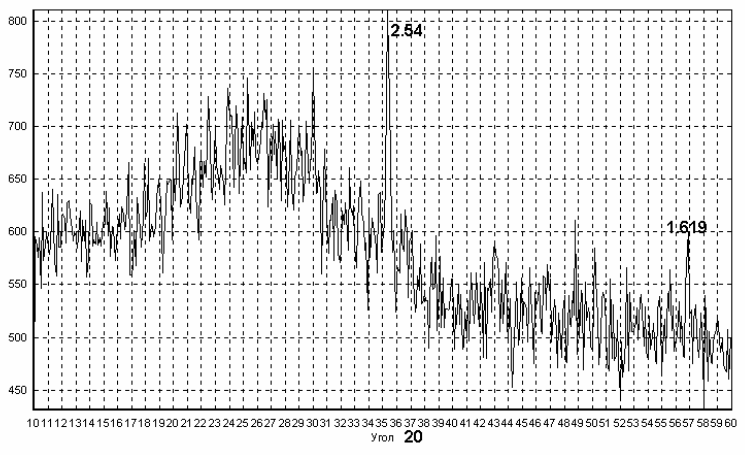

KRI-18 CCC

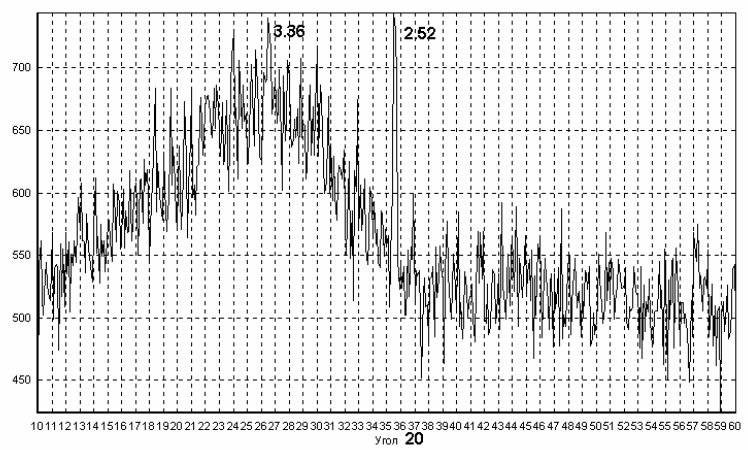

KRI-20 CCC

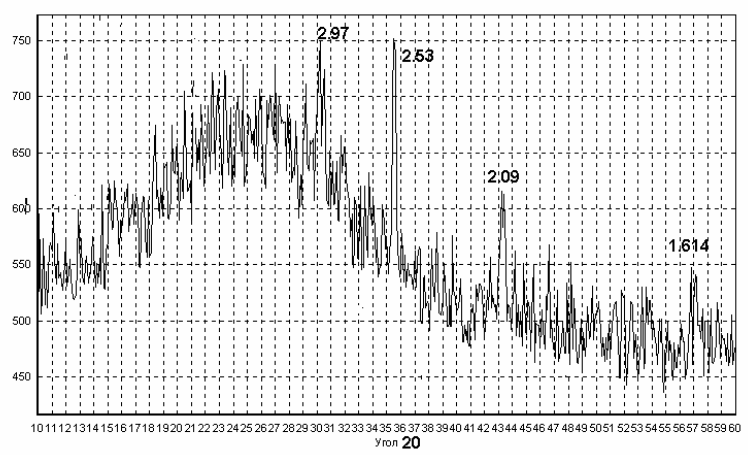

KRI-22 CCC

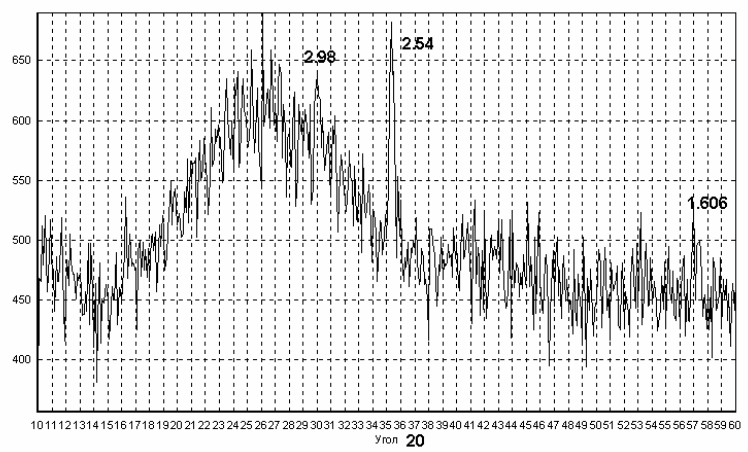

KRI-24 CCC 
SRNS-STI-2008-00057

Revision 0

Appendix C (SRNS-OS-2008-00001)
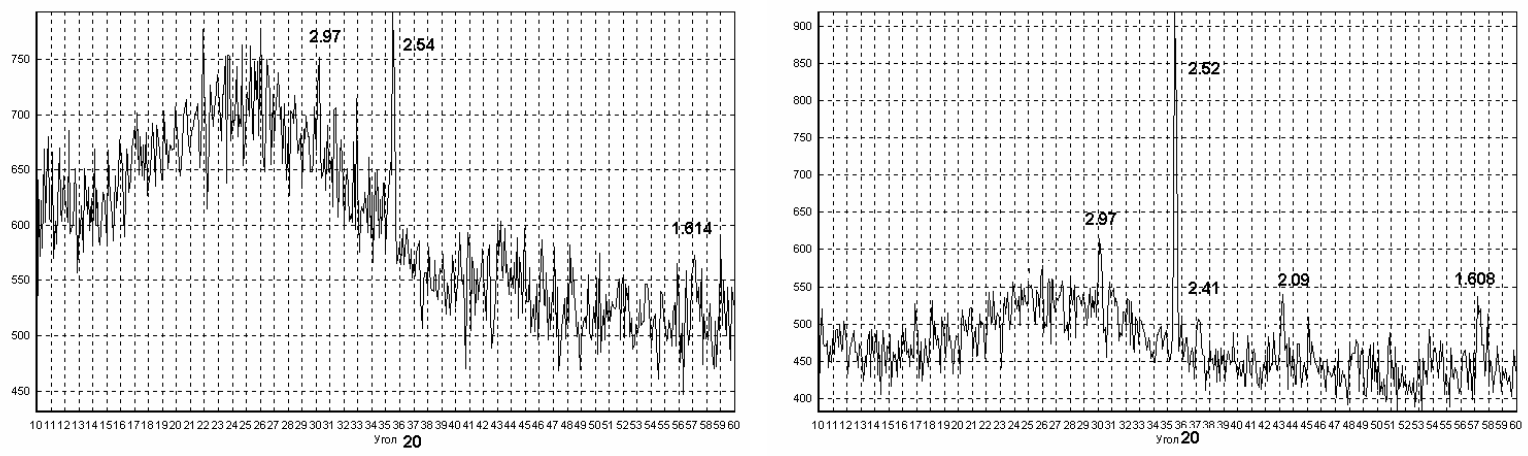

KRI-25 CCC

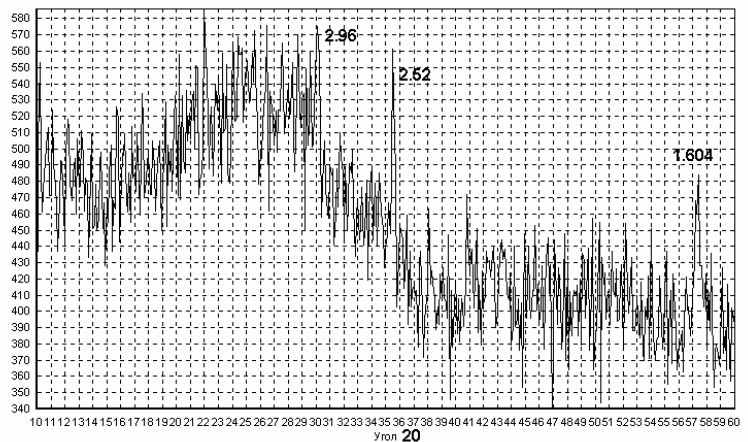

KRI-27 CCC

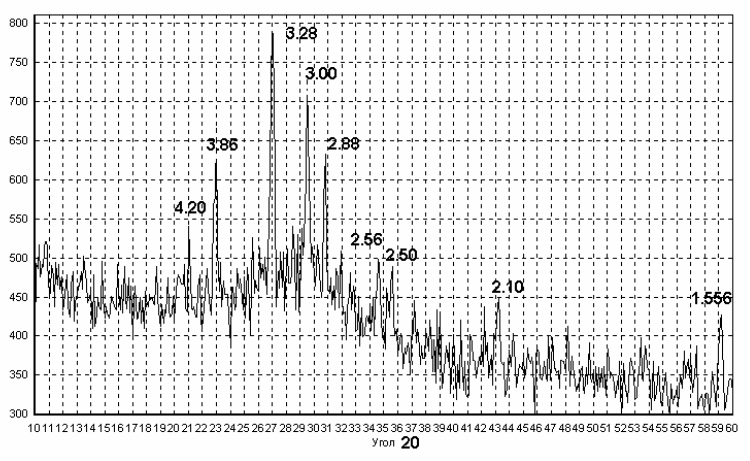

KRI-29 CCC

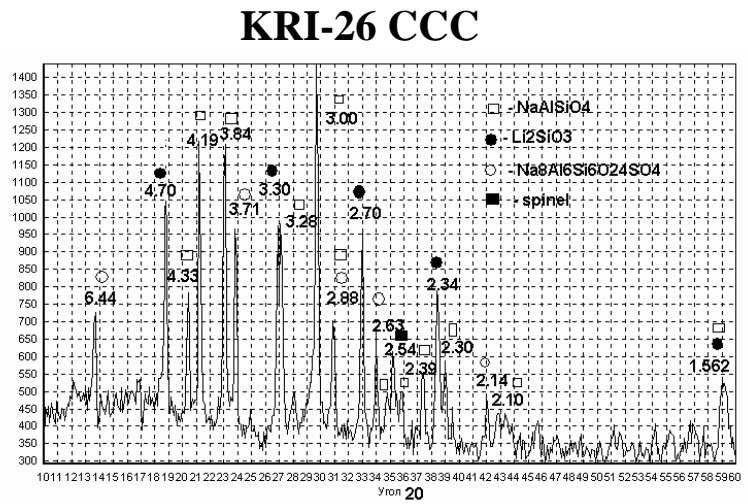

KRI-28 CCC

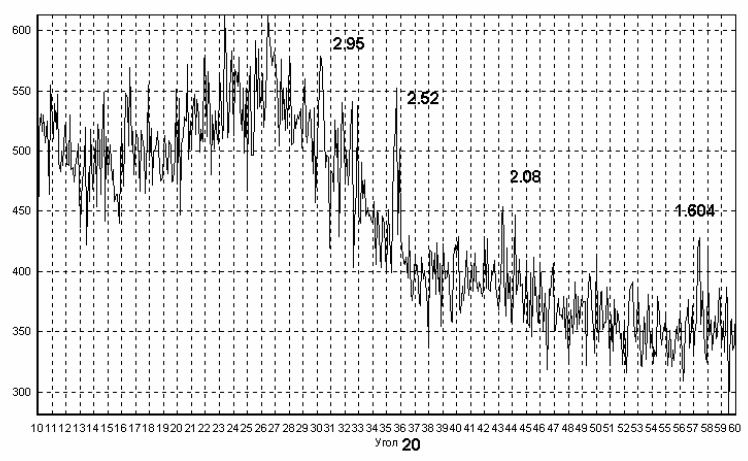

KRI-30 CCC 